\title{
La mise en oeuvre du droit applicable aux changements climatiques
}

Citation for published version (APA):

Yekini, A. (2022). La mise en oeuvre du droit applicable aux changements climatiques: le cas du Benin. [, Maastricht University]. ProefschriftMaken. https://doi.org/10.26481/dis.20220321ay

Document status and date:

Published: 01/01/2022

DOI:

10.26481/dis.20220321ay

Document Version:

Publisher's PDF, also known as Version of record

\section{Please check the document version of this publication:}

- A submitted manuscript is the version of the article upon submission and before peer-review. There can be important differences between the submitted version and the official published version of record.

People interested in the research are advised to contact the author for the final version of the publication, or visit the DOI to the publisher's website.

- The final author version and the galley proof are versions of the publication after peer review.

- The final published version features the final layout of the paper including the volume, issue and page numbers.

Link to publication

\footnotetext{
General rights rights.

- You may freely distribute the URL identifying the publication in the public portal. please follow below link for the End User Agreement:

www.umlib.nl/taverne-license

Take down policy

If you believe that this document breaches copyright please contact us at:

repository@maastrichtuniversity.nl

providing details and we will investigate your claim.
}

Copyright and moral rights for the publications made accessible in the public portal are retained by the authors and/or other copyright owners and it is a condition of accessing publications that users recognise and abide by the legal requirements associated with these

- Users may download and print one copy of any publication from the public portal for the purpose of private study or research.

- You may not further distribute the material or use it for any profit-making activity or commercial gain

If the publication is distributed under the terms of Article $25 \mathrm{fa}$ of the Dutch Copyright Act, indicated by the "Taverne" license above, 


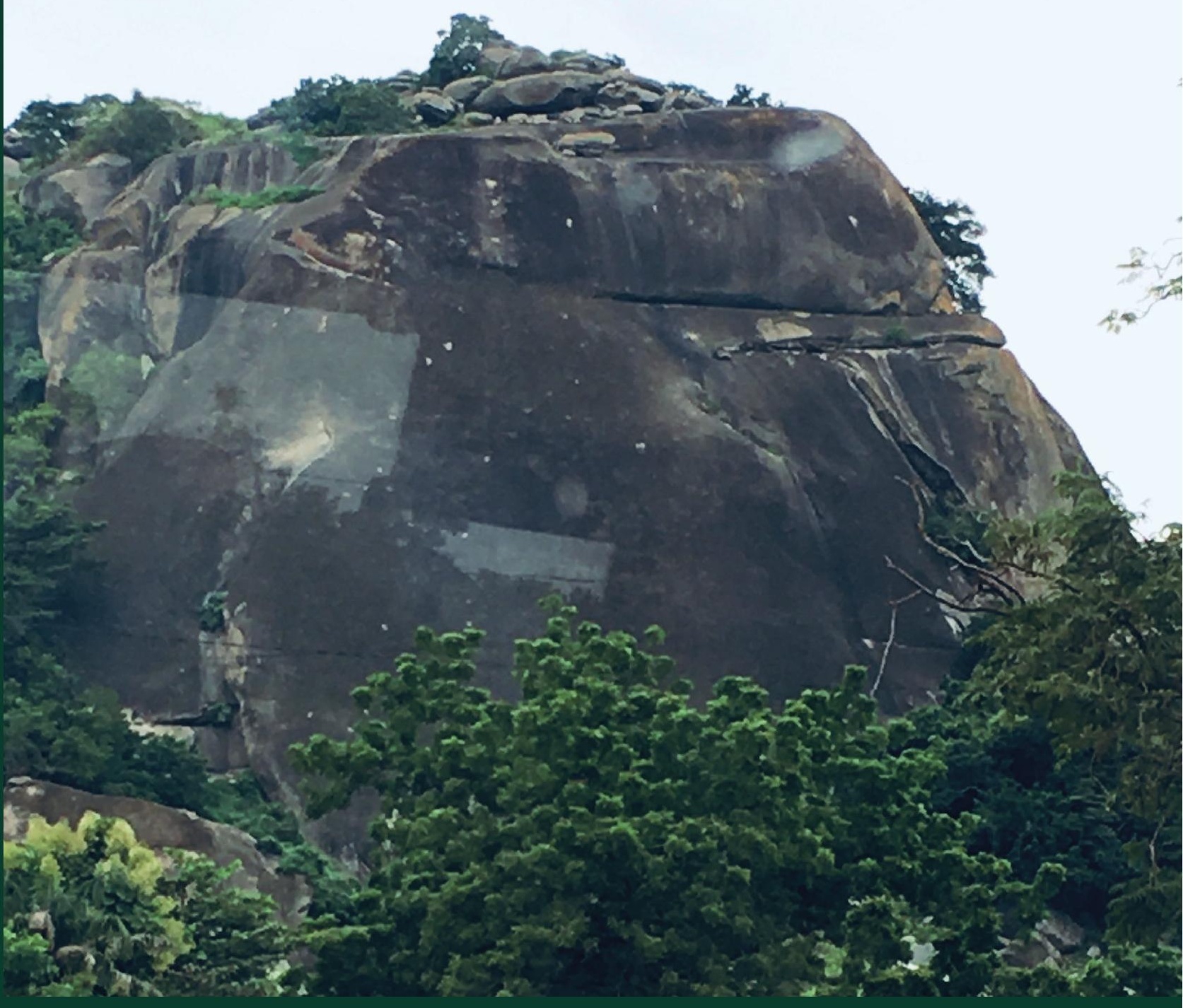

\section{LA MISE EN OEUVRE \\ DU DROIT APPLICABLE AUX CHANGEMENTS CLIMATIQUES:}

LE CAS DU BENIN

Amidou Yekini 



\section{LA MISE EN OEUVRE DU DROIT APPLICABLE AUX CHANGEMENTS CLIMATIQUES: LE CAS DU BENIN}

Amidou Yekini 


\section{Colophon}

(C) copyright Amidou Yekini, Maastricht 2022.

Printing: ProefschriftMaken | | www.proefschriftmaken.nl

ISBN 9789464237177

All rights reserved. No part of this publication may be reproduced, stored in a retrieval system or transmidded, in any form or by any means, electronic, mechanical, photocopying, recording or otherwise, without prior permission of the autor or the copyright-owning journals for previous published chapters. 


\title{
LA MISE EN OEUVRE DU DROIT APPLICABLE AUX CHANGEMENTS CLIMATIQUES : LE CAS DU BENIN
}

\author{
DISSERTATION \\ to obtain the degree of Doctor at the Maastricht University, \\ on the authority of the Rector Magnificus, Prof. dr. Pamela Habibović \\ in accordance with the decision of the Board of Deans, \\ to be defended in public \\ on Monday 21 March 2022, at 10.00 hours
}

by

Amidou Yekini 


\section{Supervisor(s):}

Prof.dr. Michael G. Faure, Maastricht University

Prof.dr. Fréderic Joël Aivo, University Abomey-Calavi

\section{Assessment Committee:}

Prof.dr. G.E. van Maanen (chair)

Prof.dr. C.H. Born, Université Catholique de Louvain

Prof.dr. A.I. Ogus, Manchester University

Prof.dr. M.G.W.M. Peeters 


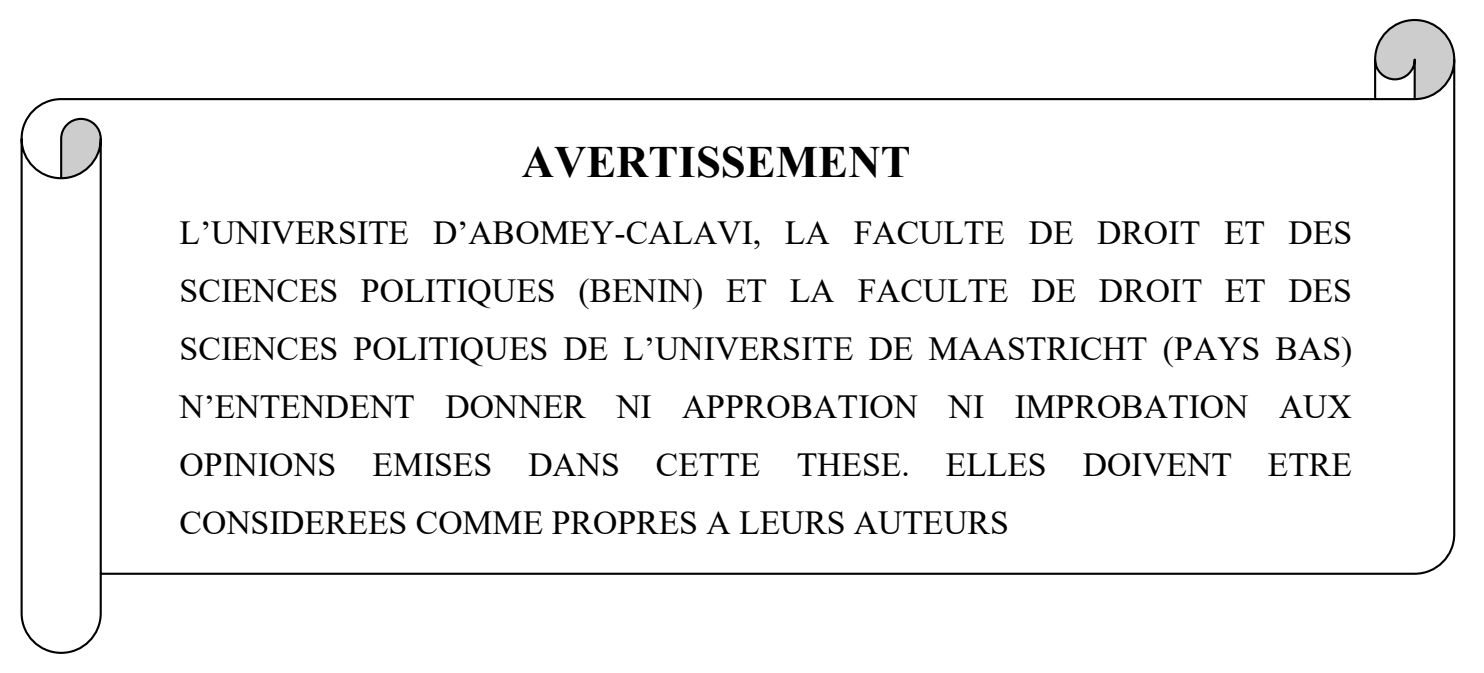




\section{Dédicaces}

A mon feu père, Salami YEKINI ;

A ma chère mère, Adégoun KEDJI ;

A mon fils ainé, Adio Mohamed Mouayad, ses frère et soeurs ;

A ma chère épouse Sabiratou O. LAWANI;

A mon ami et frère, Bernard G. ZINHOMEDE ;

Je dédie cette thèse. 


\section{REMERCIEMENTS}

Je remercie et témoigne ma gratitude à mon Directeur de recherche, le Professeur Michael G. FAURE de l'Université de Maastricht aux Pays-Bas, une référence intarissable qui m'a inspiré sur la richesse du Droit des Changements Climatiques et m'a été tout au long de la rédaction de cette thèse d'un soutien inestimable; qu'il trouve à travers cette œuvre, le couronnement de ses efforts.

Je dis également merci à tout son entourage notamment, l'équipe de l'Institut Metro (Institute for Transnational Legal Research) dont il a la charge et qui n'a ménagé aucun effort pour se mettre à mon service et à mon écoute tout au long de mes travaux de recherches ; je pense particulièrement aux mesdames Simonis Yleen et Jodogne Marina.

A Monsieur Frédéric Joël AÏVO, Agrégé des Facultés de Droit, Professeur de Droit public, Doyen Honoraire de la Faculté de Droit et de Science Politique de l'Université d'Abomey-Calavi, mon Codirecteur de recherche qui très tôt, m'a montré le chemin et encouragé à persévérer dans mes recherches; qu'il en soit remercié pour ses multiples conseils.

Monsieur Ibrahim David SALAMI, Agrégé des Facultés de Droit, Professeur de Droit public, Ancien Vice-Doyen de la Faculté de Droit et de Science Politique de l'Université d'Abomey-Calavi, qui m'a soutenu par ses conseils, qu'il en soit remercié.

Au Doyen de la Faculté de Droit et de Science Politique, Monsieur Roch GNANHOUI DAVID, Professeur Agrégé des Facultés de Droit et au Professeur Athanase LAWOGNI, Coordonnateur de la coopération scientifique interuniversitaire entre la Faculté de Droit et de Science Politique de l'Université d'Abomey-Calavi (République du Bénin) et la Faculté de Droit et de Science Politique de l'Université de Maastricht (Royaume des Pays-Bas), je dis merci et que vive cette coopération interuniversitaire.

Au Docteur Charles IBIKOUNLE, Enseignant Chercheur à la Faculté de Droit et de Science Politique de l'Université d'Abomey-Calavi, je dis merci. 


\section{LISTE DES SIGLES ET ABREVIATIONS}

ABE : $\quad$ Agence Béninoise pour l'Environnement

ABEC : $\quad$ Béninoise pour l'Environnement et du Climat

AFD : $\quad$ Agence Française de Développement

AGN : $\quad$ Groupe Africain de Négociation

ANCC : $\quad$ Autorité Nationale en charge des Changements Climatiques

ANCC : $\quad$ Agence Nationale des Changements Climatiques

ANPC : $\quad$ Agence Nationale de Protection Civile

AMCOW : Conférence des Ministres de l'Environnement, la Conférence ministérielle africaine surl'Eau

AME : $\quad$ Accords Multilatéraux sur l'Environnement

ANCC : $\quad$ Autorité Nationale des Changements Climatiques

ANPC : $\quad$ Agence Nationale de Protection Civile

BCEAO : Banque Centrale des Etats de l'Afrique de l'Ouest

BIRD : $\quad$ Banque Internationale pour la Reconstruction et le Développement

CADHP : Commission Africaine des Droits de l'Homme et des Peuples

CAJDH : Cour Africaine de Justice et des Droits de l'Homme

CAHOSCC : Comité des Chefs d'Etat et de Gouvernement sur les Changements Climatiques

CAPP : $\quad$ Cellule d'Appui au Partenariat Public Privé

CBCC : $\quad$ Conseil Béninois sur les Changements Climatiques

CCIB : $\quad$ Chambre de Commerce et d'Industrie du Bénin

CCNUCC : Convention Cadre des Nations Unies sur les Changements Climatiques

CDB : $\quad$ Convention sur la Diversité Biologique

CDN : $\quad$ Contribution déterminée au niveau national

CEA : $\quad$ Communauté Economique de l'Afrique de l'Est

CEDEAO : Communauté Economique des Etats de l'Afrique de l'Ouest

CEDH : $\quad$ Convention Européenne des Droits de l'Homme

CEDH: Cour Européenne des Droits de l'Homme

CEMAC : Communauté Economique et Monétaire des Etats de l'Afrique Centrale

CES : $\quad$ Conseil Economique et Social

CFC : Chlorofluorocarbone

CH4 : Méthane

CIJ : $\quad$ Cour Internationale de Justice

CICR : $\quad$ Comité Internationale de la Croix-Rouge 
CJCE : $\quad$ Cour de Justice de la Communauté Européenne

CJUA : $\quad$ Cour de Justice de l'Union Africaine

CMEIGB : Commission de Modélisation Economique des Impacts et de l'Intégration des Changements Climatiques dans le Budget Général de l'Etat

CNCC : $\quad$ Commission Nationale sur les Changements Climatiques

CNCC : $\quad$ Comité National sur les Changements Climatiques

CNDD : $\quad$ Commission Nationale pour le Développement Durable

CNULCD : Convention des Nations unies sur la Lutte Contre la Désertification

CO : $\quad$ Oxyde de carbone

CO2 : Dioxyde de Carbone

COP : $\quad$ Conférence des Parties à la Convention Cadre des Nations Unies sur les Changements Climatiques

CPDN : Contributions Prévues Déterminées au Niveau National

CPJI : $\quad$ Cour Permanente de Justice Internationale

CPI : $\quad$ Cour Pénale Internationale

DAO : $\quad$ Dossier d'Appel d'Offre

DC : $\quad$ Droit Constitutionnel

DGEFC : Direction Générale des Eaux Forets et Chasse

DI : $\quad$ Droit International

DICC : Droit International des Changements Climatiques

DIE : $\quad$ Droit International de l'Environnement

DIP : $\quad$ Droit International Public

DGEC : Direction Générale de l'Environnement et du Climat

DGEFC : Direction Générale des Eaux Forêts et Chasse

EC : $\quad$ Economie Circulaire

EIE : $\quad$ Etude d'Impact Environnementale

EKC : $\quad$ Courbe Environnementale de Kuznets

FAO : $\quad$ Organisation des Nations Unies pour l'alimentation et l'agriculture

FEM : $\quad$ Fonds pour l'Environnement Mondial

FNE : $\quad$ Fonds National pour l'Environnement

FNEC : $\quad$ Fonds National pour l'Environnement et le Climat

FVC : $\quad$ Fonds Vert pour le Climat

GATT : General Agreement on Tarifs and Trade, en français Accord général sur les tarifs douaniers et le commerce

GES : $\quad$ Gaz à Effet de Serre 
GIEC : $\quad$ Groupe Intergouvernemental d'Experts sur 1'Evolution du Climat

HCFC : Hydro Chlorofluorocarbone

IEC : Information Education Communication

IIED : Institut International pour l'Environnement et le Développement

MAAF : $\quad$ Ministère l'Agriculture de l'Alimentation et de la Forêt

MAEDI : Ministère des Affaires Etrangères et du Développement International

MCVDD : Ministère du Cadre de Vie et du Développement Durable

MEEM : $\quad$ Ministère de l'Environnement de l'Energie et de la Mer

MDP : Mécanisme pour le Développement Propre

MOC : $\quad$ Mise en Euvre Conjointe

MOM : $\quad$ Ministère des Outre-mer

MOP : $\quad$ Meetings of Parties

OIF : $\quad$ Organisation Internationale de la Francophonie

OHADA : Organisation pour l'Harmonisation des Droits d'Affaire en Afrique

ODD : Objectifs pour le Développement Durable

OMC : $\quad$ Organisation Mondiale pour le Commerce

OME : $\quad$ Organisation Mondiale de l'Environnement

OMM : Organisation Mondiale de la Météorologie

OMS : $\quad$ Organisation Mondiale de la Santé

ONCC : $\quad$ Organisation Nationale sur les Changements Climatiques

ONG : $\quad$ Organisation Non Gouvernementale

ONU : $\quad$ Organisation des Nations unies

ONUE : $\quad$ Organisation des Nations Unies pour l'Environnement

ORD : $\quad$ Organe de Règlement des Différends

ORSEC : Plan National d'Organisation des Secours en cas de Catastrophe

OTC : $\quad$ Accord sur les Obstacles Techniques au Commerce

OUA : $\quad$ Organisation de l'Unité Africaine

PAC : $\quad$ Port Autonome de Cotonou

PAE : $\quad$ Plan d'Action Environnemental

PANA : $\quad$ Plans Nationaux d'Adaptation

PANA-BENIN : Programme d'Action du Bénin aux fins de l'Adaptation aux Changements Climatiques

PDC : $\quad$ Plan de Développement Communal 
PIB : $\quad$ Produit Intérieur Brut

PMA : $\quad$ Pays Moins Avancés

PNUE : $\quad$ Programme des Nations Unies pour l'Environnement

PNUD : $\quad$ Programme des Nations Unies pour le Développement

PTF : $\quad$ Partenaires Techniques et Financiers

PREMI : Initiative pour la Réduction de la Pauvreté et la Gestion de l'Environnement

REP : $\quad$ Recours pour Excès de Pouvoir

SAD : $\quad$ Société Africaine de Dragage

SADC : $\quad$ Communauté de Développement de l'Afrique Australe

SBSTA : $\quad$ Organe Subsidiaire de Conseil Scientifique et Technologique

SBI : $\quad$ Organe Subsidiaire pour la mise en application

SDN : $\quad$ Société Des Nations

SNMO : $\quad$ Stratégie Nationale pour la Mise en Oeuvre de la Convention Cadre sur les Changements Climatiques

SPS : $\quad$ Mesures Sanitaires et Phytosanitaires

UA : Union Africaine

UCCC : Unité Communale pour les Changements Climatiques

UE : $\quad$ Union Européenne

UEMOA : Union Economique Monétaire Ouest Africaine

UICN : $\quad$ Union Mondiale pour la Nature

UNESCO : Organisation des Nations Unies pour l'Education, la Science et la Culture

USA : $\quad$ Etats-Unis d'Amérique 



\section{RESUME DE LA THESE}

Les changements climatiques constituent aujourd'hui un défi mondial qui préoccupe et mobilise la communauté internationale. L'aboutissement de cette mobilisation reste sans doute l'élaboration du régime juridique international de protection du climat avec pour pilier principal, la Convention Cadre des Nations Unies sur les Changements Climatiques. Ce régime juridique est élaboré sur la base de «l'équité et en fonction des responsabilités communes mais différenciées et des capacités respectives $»^{1}$ des Etats Parties, dans le « souci de préserver le système climatique dans l'intérêt des générations présentes et futures $»^{2}$. En effet, la lutte contre les changements climatiques fut engagée avec l'objectif « (...) de stabiliser conformément aux dispositions pertinentes de la Convention, les concentrations de gaz à effet de serre dans l'atmosphère à un niveau qui empêche toute perturbation anthropique dangereuse du système climatique $(\ldots) »^{3}$. En dépit de toute cette production normative, il est regrettable de constater aujourd'hui que le régime international de climat n'a pas permis de réaliser l'objectif d'atténuation des émissions de gaz à effet de serre et d'adaptation aux effets néfastes des changements climatiques. Il en est ainsi parce que, si la mobilisation des Etats pour la ratification de la Convention ne fait aucun doute, en revanche, les mêmes Etats qui ont volontairement accepté refusent délibérément d'honorer leurs engagements pour des raisons politiques, économiques et stratégiques. Ce travail ambitionne de lever le voile sur les causes de la faiblesse relevée dans l'effectivité du droit climatique suffisamment et massivement élaboré en faisant ressortir le caractère mitigé de la contribution dudit droit à la lutte contre les changements climatiques. La contribution limitative du droit est illustrée par le cas du Bénin, un Pays en développement exposé aux affres des changements climatiques où la volonté manifeste des autorités et leur engagement à combattre le fléau n'a pas suffit pour enrayer le fléau. Enfin, l'étude propose des perspectives pour une amélioration de la lutte contre les changements climatiques au Bénin.

\section{MOTS CLES:}

Accord de Paris-Adaptation-Atténuation-Changements climatiques-Contrôle du non respectConvention Cadre des Nations Unies sur les Changements Climatiques (CCNUCC)-Economie Circulaire- Développement durable -Gaz à Effet de Serre - Protocole de Kyoto- Organisation des Nations Unies pour l'Environnement (ONUE) -Union Africaine (UA).

\footnotetext{
${ }^{1}$ Article 3 paragraphe 1 de la Convention Cadre des Nations Unies sur les Changements Climatiques.

${ }^{2}$ Id.

${ }^{3}$ Article 2 de la Convention Cadre des Nations Unies sur les Changements Climatiques.
} 



\section{SUMMARY OF THE THESIS}

Climate change is now a global challenge that concerns and mobilizes the international community. The outcome of this mobilization undoubtedly remains the elaboration of the international legal regime for climate protection, with the United Nations Framework Convention on Climate Change as its main pillar. This legal regime is developed on the basis of equity and in accordance with common but differentiated responsibilities and respective capabilities of States Parties, with a view of preserving the climate system for the benefit of present and future generations. Indeed, the fight against climate change was undertaken with the objective" (...) to stabilize, in accordance with the relevant provisions of the Convention, greenhouse gas concentrations in the atmosphere at a level that prevents dangerous anthropogenic interference with the climate system (...)". Despite all this normative production, it is regrettable to note today that the international climate regime has not achieved the objective of mitigating greenhouse gas emissions and adapting to the adverse effects of climate change. This is because, while there is no doubt about the mobilization of States for the ratification of the Convention, the same States that have voluntarily accepted it, deliberately refuse to honor their commitments for political, economic and strategic reasons. This work aims to shed light on the causes of the weakness in the effectiveness of climate law, which is sufficiently and massively developed, by highlighting the mixed nature of the contribution of this right to the fight against climate change. The restrictive contribution of the law is illustrated by the case of Benin, a developing country exposed to the horrors of climate change where the authorities' manifest willingness and commitment to fight the scourge has not been sufficient to be up to date with these emission reduction commitments made by the ratification of the Climate Convention. Finally, the study proposes prospects for improving the fight against climate change in Benin.

\section{KEYWORDS:}

Paris Agreement-Adaptation-Adjustment-Climate Change-Compliance Monitoring-UN Framework Convention on Climate Change (UNFCCC) - Circular Economy- Sustainable Development-International Environmental Law-Greenhouse Gas-Protocol-Kyoto ProtocolUNEP-African Union (AU). 


\section{SOMMAIRE}

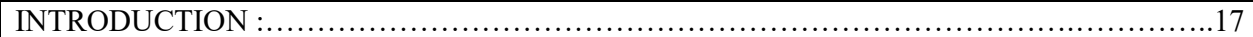

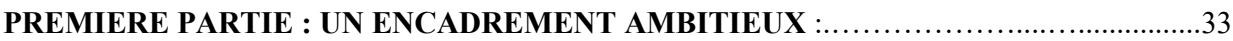

TITRE I : LES REGLES DE PROTECTION DU

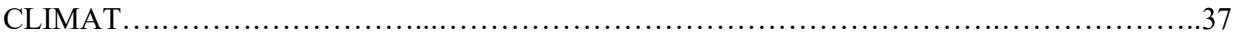

Chapitre 1: L'apport du droit

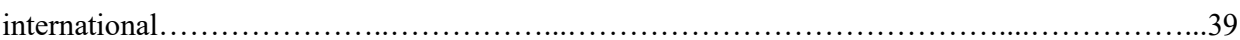

Chapitre 2 : Les mesures normatives

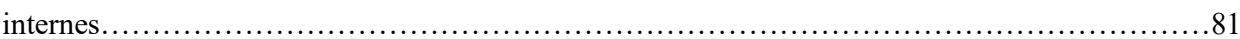

TITRE II : LES INSTITUTIONS DE PROTECTION DU

CLIMAT.

Chapitre1 : Le dispositif institutionnel

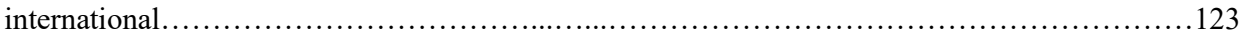

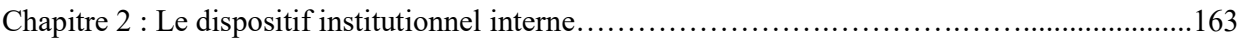

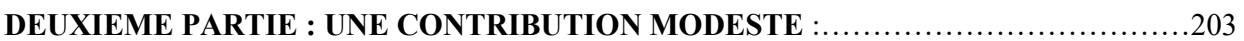

TITRE I: L'EFFECTIVITE DE LA

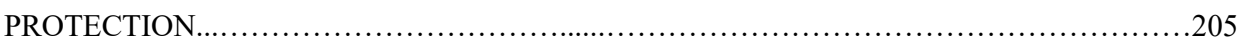

Chapitre1 : Les effets du droit

international.

Chapitre 2: La faible effectivité de la

protection

TITRE II: LES PERSPECTIVES DU DROIT CLIMATIQUE AU

BENIN

Chapitre 1: L'amélioration de l'encadrement

international

Chapitre 2 : Le renforcement de la gouvernance climatique

interne.

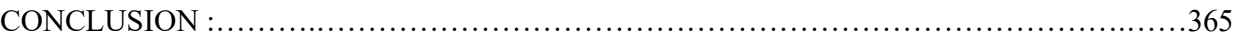

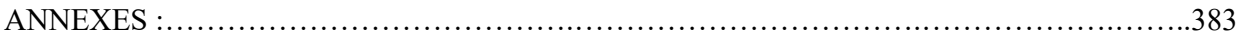

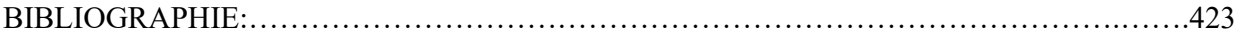

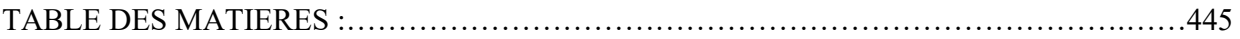




\section{INTRODUCTION}

L'instrument élaboré à l'occasion de la Conférence des Nations unies sur l'Environnement et le Développement organisée à Rio de Janeiro en $1992^{4}$, destiné à lutter contre les changements climatiques ${ }^{5}$ a défini le groupe de mot "changement climatique » comme des «modifications de l'environnement physique ou des biotes (...) » ${ }^{6}$. Cet instrument a indiqué les origines de ces modifications « (...) qui sont attribués directement ou indirectement à une activité humaine altérant la composition de l'atmosphère mondiale et qui viennent s'ajouter à la variabilité naturelle du climat observée au cours de périodes comparables» ${ }^{7}$. Cette définition n'est pas loin de celle préconisée par le droit climatique béninois qui dispose que les changements climatiques constituent des «(...) variations attribuées directement ou indirectement à une activité humaine altérant la composition de l'atmosphère mondiale $\|^{8}$.

Parfois, il est fait usage du pluriel du groupe de mot «changement climatique », qui devient «les changements climatiques», pour tenir compte des conséquences provoquées par les modifications de l'environnement physique ou des biotes. La plupart des traités internationaux en matière de protection du climat $^{9}$ font usage des deux (2) catégories de groupe de mot à savoir « le changement climatique » et «les changements climatiques » pour désigner le phénomène environnemental planétaire. A ces deux (2) catégories de groupe de mot s'ajoute une troisième $\left(3^{\mathrm{e}}\right)$, « les changements du climat ${ }^{10}$, pour nommer le même fléau planétaire à la seule différence que cette dernière est moins usité ${ }^{11}$.

Ces définitions établissent une relation de cause à effet entre le phénomène climatique diversement appelé, « changement climatique » ou «les changements climatiques » ou «les

\footnotetext{
${ }^{4}$ La Conférence des Nations unies sur l'Environnement et le Développement organisée à Rio de Janeiro en 1992 représente le troisième $\left(3^{\mathrm{e}}\right)$ Sommet de la Terre essentiellement consacré à la problématique des changements climatiques.

${ }^{5}$ Il s'agit de la Convention Cadre des Nations Unies sur les Changements Climatiques élaborée à New-York et puis adoptée à Rio de Janeiro en juin 1992.

${ }^{6}$ Cette définition est contenue dans l'article premier, paragraphe 1 de la Convention Cadre des Nations Unies sur les Changements Climatiques.

${ }^{7}$ Extrait de l'article premier paragraphe 2 de la Convention Cadre des Nations Unies sur les Changements Climatiques.

${ }^{8}$ Article premier $11^{\mathrm{e}}$ tiret de la loi $\mathrm{n}^{\circ} 2018-18$ portant règlementation des changements climatiques en République du Bénin.

${ }^{9}$ Notamment, la Convention Cadre des Nations Unies sur les Changements Climatiques, le Protocole de Kyoto et l'Accord de Paris.

${ }^{10}$ Ce groupe de mot est contenu dans le deuxième $\left(2^{\mathrm{e}}\right)$ paragraphe du préambule de la Convention Cadre des Nations Unies sur les Changements Climatiques.

${ }^{11}$ Dans le développement de la thèse, usage sera fait de ces deux (2) catégories de groupe de mot à savoir «le changement climatique » et « les changements climatiques » en tenant compte des contextes.
} 
changements du climat » et les « activités humaines », lesquelles peuvent ou sont à l'origine de la modification des paramètres environnementaux ${ }^{12}$ en ce qu'elles contribuent à l'émission des «gaz à effet de serre » ${ }^{13}$ dans l'atmosphère dans une proportion parfois prohibée par les règlementations internationales en la matière ${ }^{14}$. La plus caractéristique de ces gaz est le dioxyde de carbone, "due to human activities, primarily the burning of fossil fuel but also deforestation and other land use change» ${ }^{15}$.

L'assertion selon laquelle l'homme se trouve à la base de la dégradation de l'environnement et des changements climatiques est scientifiquement établie et récurrente dans tous les rapports ${ }^{16} \mathrm{du}$ Groupe d'Experts Intergouvernemental sur l'Evolution du Climat $(\text { GIEC })^{17}$. En effet, dans son premier rapport élaboré pour la période de1990 et 1992, il a été conclu que, «les émissions dues aux activités humaines accroissent sensiblement la concentration dans l'atmosphère des gaz à effet de serre» ${ }^{18}$, ce qui témoigne de l'influence

\footnotetext{
${ }^{12}$ S'agissant des causes du changement climatique, il a été consigné dans le cinquième $\left(5^{\mathrm{e}}\right)$ rapport d'évaluation du Groupe d'experts intergouvernemental sur l'évolution du climat (GIEC) paru en 2014, que «Les émissions anthropiques de gaz à effet de serre, qui ont augmenté depuis l'époque préindustrielle en raison essentiellement de la croissance économique et démographique, sont actuellement plus élevées que jamais, ce qui a entrâné des concentrations atmosphériques de dioxyde de carbone, de méthane et d'oxyde nitreux sans précédent depuis au moins 800000 ans. Leurs effets, associés à ceux d'autres facteurs anthropiques, ont été détectés dans tout le système climatique et il est extrêmement probable qu'ils aient été la cause principale du réchauffement observé depuis le milieu du XXe siècle. Voir page 4. du rapport du GIEC sur le site internet : https://www.ipcc.ch/reports. Consulté ce 30/05/2021.

${ }^{13}$ Selon l'article premier paragraphe 5 de la Convention Cadre des Nations Unies sur les Changements Climatiques, on entend par «gaz à effet de serre», les constituants gazeux de l'atmosphère, tant naturels qu'anthropiques, qui absorbent et réémettent le rayonnement infrarouge. Mais l'expression " effet de serre » provient de FOURRIER (J), «Remarques générales sur les températures du globe terrestre et des espaces planétaires », Annales de Chimie et de physique, 1824, vol.27, p. 136-167 qui a découvert le fléau qu'il a désigné ainsi par rapprochement avec le phénomène de captation de la chaleur par les vitres d'une serre.

${ }^{14} \mathrm{~A}$ titre indicatif, l'article 3 paragraphe 1 du Protocole de Kyoto a fixé des engagements chiffrés en matière de « limitation et de réduction des émissions en vue de réduire le total de leurs émissions d'au moins $5 \%$ par rapport au niveau de 1990 au cours de la période d'engagement», quant à l'article 2 paragraphe 1-a de l'Accord de Paris, le seuil d'émission de Gaz à effet de serre ne doit provoquer l'élévation de température moyenne de la planète audelà de $2{ }^{\circ} \mathrm{C}$ voire $1,5^{\circ} \mathrm{C}$ par rapport aux niveaux préindustriels. Tout dépassement de ce seuil n'est pas conforme à la règlementation internationale des changements climatiques.

${ }^{15}$ Michael Mastrandera et Stephen Schneider. "Climate Change Science Overview ", dans Climate Change: Science and Policy sous la dir. de Stephen Schneider. (Washington, D.C.: Island Press, 2010), 16.

${ }^{16}$ Le premier $\left(1^{\text {er }}\right)$ rapport concerne la période de 1990 et 1992 , le deuxième $\left(2^{\mathrm{e}}\right)$ rapport est celui de 1995 , le troisième $\left(3^{\mathrm{e}}\right)$ rapport est établi en 2001, le quatrième (4e) rapport est celui de 2007 et le cinquième $\left(5^{\mathrm{e}}\right)$ rapport est publié en 2014 sur le site internet : https://www.ipcc.ch/reports. Consulté ce 30/05/2021.

${ }^{17}$ Groupe d'Experts Intergouvernemental sur l'Evolution du Climat (GIEC) fut créé en 1988 conjointement par l'Organisation Métrologique Mondiale (OMM) et le Programme des Nations Unies pour l'Environnement (PNUE). Il est chargé de suivre l'évolution du climat.

${ }^{18}$ Premier rapport du GIEC, p.6. et le troisième ( $\left.3^{e}\right)$ Paragraphe du préambule de la Convention Cadre des Nations Unies sur les Changements Climatiques adoptée à Rio de Janeiro en 1992.
} 
perceptible de l'homme sur le climat global ${ }^{19}$. Cette assertion a été ensuite transcrite dans la Convention Cadre des Nations Unies sur les Changements Climatiques ${ }^{20}$.

Cependant, indépendamment de la pression que l'homme exerce sur l'environnement qui est qualifiée de pression anthropique ${ }^{21}$, il ressort également de la définition donnée à l'article premier paragraphe 2 de la Convention Cadre des Nations Unies sur les Changements Climatiques, que le changement climatique peut résulter des processus intrinsèques à la Terre ${ }^{22}$. Dans tous les cas, ces deux (2) scenarios conduisent à la production des gaz à effet de serre source de changement climatique.

Le débat sur le « changement climatique » est un sujet aussi vieil que l'humanité. Mais, il est devenu une préoccupation majeure pour la Communauté internationale ${ }^{23}$ à partir du moment où, la modification des paramètres climatiques provoquée par l'émission des gaz à effet de serre émanant de l'activité humaine a engendré un réchauffement supplémentaire de la planète Terre et a induit par ricochet des conséquences fâcheuses pour l'humanité. Le changement climatique est un phénomène susceptible d'engendrer des «catastrophes écologiques aux répercussions interétatiques» ${ }^{24}$. Il est également susceptible de plonger l'humanité dans un état de stress croissant et permanent parce que, "sa prévision recèle un grand nombre d'incertitude, notamment en ce qui concerne son déroulement dans le temps, son ampleur et ses caractéristiques régionales $»^{25}$. C'est un phénomène qui engendre «(...) des effets nocifs significatifs sur la composition, la résistance ou la productivité des écosystèmes naturels ou aménagés, sur le fonctionnement des systèmes socio-économiques ou sur la santé

\footnotetext{
${ }^{19}$ Cette assertion est contenue dans la seconde évaluation du GIEC, Changements climatiques 1995, p. 22. Sur le site internet : https://www.ipcc.ch/reports. Consulté ce 30/05/2021.

${ }^{20}$ Voir $3^{\mathrm{e}}$ paragraphe du préambule de la Convention Cadre des Nations Unies sur les Changements Climatiques. Par ailleurs, suivant la définition du « gaz à effet de serre » donnée au paragraphe 5 de l'article $1^{\text {er }}$ de la Convention Cadre des Nations Unies sur les Changements Climatiques, l'instrument international de lutte contre les changements climatiques situe la provenance des constituants gazeux de l'atmosphère qui absorbent et réémettent le rayonnement infrarouge que sont les gaz à effet de serre qui peuvent être tant de source, naturelle qu'anthropique.

${ }^{21}$ La pression anthropique résulte des effets générés par les activités humaines sur les ressources naturelles et les écosystèmes.

${ }^{22}$ Le paragraphe 5 de l'article premier de la Convention Cadre des Nations Unies sur les Changements Climatiques permet d'identifier des sources d'émission des gaz à effet de serre qui sont naturelles qui découlent d'un processus intrinsèque à la Terre.

${ }^{23}$ Le groupe de mot «Communauté internationale» est défini comme l'«ensemble des Etats pris dans leur universalité » ou un « ensemble plus vaste incluant à côté des Etats, les organisations internationales à vocation universelle, les particuliers et l'opinion publique internationale » selon le Dictionnaire de droit international public, élaboré sous la direction de Jean Salomon, Bruxelles, Bruylant/AUF, 2001, p. 205-206.

${ }^{24}$ Michel PRIEUR, actes du $1^{\text {er }}$ séminaire international de droit de l'environnement Rio de Janeiro +10 du 24 au 26 avril 2002 «Mondialisation et droit de l'environnement», Université de Limoges, 172p., p13.

${ }^{25}$ Sixième $\left(6^{\mathrm{e}}\right)$ paragraphe du préambule de la Convention Cadre des Nations Unies sur les Changements Climatiques.
} 
et le bien-être de l'homme $»^{26}$. Dominique Bourg, en mesurant la portée et l'ampleur des conséquences du phénomène climatique écrit que, "les changements climatiques nous laissent entrevoir la finitude planétaire ou, tout au moins, la finitude humaine» ${ }^{27}$. Cette assertion peut se justifier au vu des conséquences graves qu'induisent les changements climatiques, qui peuvent éventuellement provoquer le réaménagement des ressources hydriques, ainsi, « some areas will experience less annual rainfall; in others it will be less predictable, with seasonal rains failing to materialize» ${ }^{28}$, une situation qui peut compromettre l'existence de l'Homme dans certains Etats qui sont conditionnés par cette ressource naturelle. Néanmoins, les effets des changements climatiques sont déjà visibles dans certaines puissances pollueuses, à titre indicatif, «Desertification in China has been primarily caused by climate change ${ }^{29}$. Mais plus grave, les Experts du GIEC abordant dans leurs travaux les aspects concernant les risques et incidences futurs pouvant découler de l'évolution du climat, projette que «Les changements climatiques vont amplifier les risques existants et en engendrer de nouveaux pour les systèmes naturels et humains (...) $\rangle^{30}$.

En effet, lutter contre les changements climatiques, « (...) un problème d'environnement global (...) $»^{31}$ devient une nécessité impérative et constitue en même temps, un défi majeur pour les organisations internationales face aux conséquences imprévisibles, néfastes, voire irréversibles auxquelles est exposée l'humanité.

Autrefois, les préoccupations environnementales donnaient l'impression d'une question relevant du luxe uniquement à la charge des puissances occidentales, mais à partir de l'année $1992^{32}$, les données ont changé au point de pousser l'humanité à s'investir dans la recherche permanente de solutions pour conjurer le mal dont les effets « conduisent à la nécessaire solidarité $»^{33}$. La gestion du fléau d'envergure planétaire nécessite des actions convergées, pouvant se dérouler dans un cadre multilatéral. Car, au-delà de l'encadrement juridique, d'importants moyens notamment, financiers et la technologie sont indispensables pour mener

\footnotetext{
${ }^{26}$ Article premier, paragraphe 1 de la Convention Cadre des Nations Unies sur les Changements Climatiques.

${ }^{27}$ Dominique Bourg, « L'impératif écologique », Esprit, décembre 2009, 204p., p. 7.

${ }^{28}$ Dow, K., et Downing, T. (2011). The Atlas of Climate Change: Mapping the World's greatest Challenge(éd.3e). Los Angeles: University of California Press, Op. cit., 62.

${ }^{29}$ Joanna Lewis, " China», dans Climate Change and National Security: A Country-Leve/ Analysis, sous la dir. de Daniel Moran. (Washington, D.C.: Georgetown University Press, 2011), p.13.

${ }^{30}$ Le cinquième $\left(5^{\mathrm{e}}\right)$ rapport d'évaluation du Groupe d'experts intergouvernemental sur l'évolution du climat (GIEC) paru en 2014, p13. Sur le site internet : https://www.ipcc.ch/reports. Consulté ce 30/05/2021.

${ }^{31}$ BERTHAUD Pierre, CAVARD Denise, et CRIQUI Patrick, Le régime international pour le climat, vers la consolidation ou l'effondrement? In Revue française d'économie. Vol. 19 N², 2004. pp. 163-188.

${ }^{32}$ C'est l'année de l'adoption de la Convention Cadre des Nations Unies sur les Changements Climatiques.

${ }^{33}$ Michel PRIEUR, actes du $1^{\text {er }}$ séminaire international de droit de l'environnement Rio de Janeiro +20 du 24 au 26 avril 2002 «Mondialisation et droit de l'environnement », 172p., p13.
} 
à bien l'éradication du fléau. Considérant l'ampleur des conséquences planétaires des problèmes environnementaux, Michel PRIEUR, n'a-t-il pas indiqué « qu'il y a peu de place en droit de l'environnement pour les solutions purement nationales ${ }^{34}$ ?

Le paradoxe dans le déroulement de la manifestation des effets néfastes des changements climatiques ${ }^{35}$ est que, les pays en développement qui en subissent la rigueur ont une infirme responsabilité dans les facteurs qui contribuent à la dégradation de l'environnement ${ }^{36}$. Quant aux pays développés, qui sont pour la plupart de grands pollueurs de l'atmosphère ${ }^{37}$, sont réticents en ce qui concerne la mise à disposition des pays en voie de développement, des moyens pour atténuer les changements climatiques. Ce qui fait que, la thématique des changements climatiques est souvent abordée sur fond de clivage Nord-Sud.

Eu égard à ce qui précède, Etats développés et pays en voie de développement ont été conviés dans un cadre de coopération internationale pour lutter contre les changements climatiques et ses effets néfastes ${ }^{38}$.

Pour s'occuper de la gestion des changements climatiques, la Communauté internationale a fait recours au droit pour élaborer des moyens juridiques et mettre en place des institutions en vue de l'encadrement de la matière. C'est à juste titre que recours a été fait au droit, parce que cette matière « (...) intervient pour fixer, arrêter, stabiliser les relations humaines mouvantes et fluctuantes $(\ldots) »^{39}$, ainsi le droit constitue « $(\ldots)$ une réponse à l'incertitude du futur, c'est-à-dire un facteur de sécurisation pour l'homme vivant dans un univers instable et menaçant $(\ldots){ }^{40}$. Ainsi, les instruments juridiques internationaux notamment, convention ou traité, acte déclaratoire, charte, directive et autres moyens juridiques sont élaborés à l'occasion des « Sommets de la Terre» et au cours des rencontres internationales dédiées à la protection de l'environnement et à la lutte contre les changements climatiques et mis à contribution.

\footnotetext{
${ }^{34}$ Michel PRIEUR, « Pourquoi une revue juridique de l'environnement ? », RJE, n¹, 1976, p. 3.

${ }^{35} \mathrm{Au}$ terme de l'article premier paragraphe 1 de la Convention Cadre des Nations Unies sur les Changements Climatiques, on entend par "effets néfastes des changements climatiques» les modifications de l'environnement physique ou des biotes dues à des changements climatiques et qui exercent des effets nocifs significatifs sur la composition, la résistance ou la productivité des écosystèmes naturels et aménagés, sur le fonctionnement des systèmes socio-économiques ou sur la santé et le bien-être de l'homme.

${ }^{36}$ Voir paragraphe 4 du préambule de la Convention Cadre des Nations unies sur les Changements Climatiques.

${ }^{37} \mathrm{~A}$ titre d'exemple, l'ensemble des Etats de 1'Union Européenne, les Etats Unis d'Amérique et le Canada figurent parmi les plus grands pollueurs du monde. Information obtenue sur le site internet: https://climate.selectra.com/fr/. Consulté le 02/12/2021.

${ }^{38}$ Le cadre de la coopération internationale pour lutter contre les changements climatiques a été installé à l'occasion du troisième (3e) Sommet de la Terre tenu à Rio de Janeiro en 1992.

${ }^{39}$ Ellul, Jacques, Sur l'artificialité du droit et le droit d'exception, Archives de philosophie du Droit, 1963, p.26 et s.

${ }^{40}$ Id.
} 
Compte tenu de la complexité du phénomène et de son envergure planétaire, des instruments juridiques de protection de l'environnement élaborés en dehors des Sommets de la Terre, qui ont un lien avec la lutte contre les changements climatiques ${ }^{41}$ sont également mis à contribution.

Comment ces divers instruments élaborés peuvent-ils aider à contenir le fléau planétaire dans la limite des objectifs fixés dans la Convention Cadre des Nations Unies sur les Changements Climatiques?

Il s'agit ici de mette en évidence la capacité de l'instrument juridique face aux défis qu'imposent les changements climatiques. Evidemment, des performances et faiblesses seront relevées et des perspectives pour un meilleur encadrement du fléau seront envisagées. Il sera question dans la pratique de faire l'étude des conditions d'élaboration et de mise en œuvre du droit applicable aux changements climatiques à travers le sujet de thèse ainsi libellé : « La mise en ouvre du droit applicable aux changements climatiques : le cas du Bénin ».

La présentation qui suit renseigne sur les domaines du sujet, objet de la présente étude.

\section{Présentation du sujet}

Le sujet tel que formulé relève du domaine du Droit International (DI). Selon la doctrine majoritaire ${ }^{42}$, le Droit International peut être défini comme « le droit applicable à la société internationale ${ }^{43}$, c'est-à-dire "l'ensemble des règles qui régissent l'organisation et le fonctionnement de la société internationale $»^{44}$. Il convient alors de clarifier la notion de « société internationale » qui se définit comme étant, «l'ensemble des sujets régis par le droit international $»^{45}$. Dans le cas d'espèce, le sujet prend en compte la règlementation des droits et

\footnotetext{
${ }^{41}$ Il s'agit entre autres de la Convention de Vienne sur la protection de la couche d'ozone adoptée en 1985 et du Protocole de Montréal relatif à des substances qui appauvrissent la couche d'ozone tel qu'ajusté et amendé par la deuxième Réunion des Parties (Londres, 27-29 juin 1990) et la quatrième Réunion des Parties (Copenhague, 2325 novembre 1992), ajusté de nouveau par la septième Réunion des Parties (Vienne, 5-7 décembre 1995), et encore ajusté et amendé par la neuvième Réunion des Parties (Montréal, 15-17 septembre 1997) et par la onzième Réunion des Parties (Beijing, 29 novembre - 3 décembre 1999) et encore ajusté par la dix neuvième $\left(19^{\mathrm{e}}\right)$ Réunion des Parties (Montréal, 17- 21 septembre 2007) et encore amendé par la vingt-huitième (28 $)$ Réunion des Parties (Kigali, 10-15 octobre 2016).

${ }^{42}$ Par doctrine il faut comprendre les opinions émises, les enseignements des auteurs de droit international sur une question déterminée. Voir SALMON Jean, Dictionnaire de droit international public Bruxelles, Bruylant, 2001, p. 352 .

${ }^{43}$ DAILLIER Patrick, FORTEAU Mathias et PELLET Alain, Droit international Public, Paris, LGDJ, 8ème éd., 2009, p.43. ; voir aussi ROUSSEAU Charles, «Les principes du droit international », RCADI, 1958, p. 374.

${ }^{44}$ DUPUY Pierre-Marie et KERBRAT Yann, Droit international public, Paris, Dalloz, 10ème éd.,

2010, p.1. ; pour une autre définition du droit international qui ne le limite pas à la règlementation des relations purement interétatiques, voir ROLIN Henri, « Les principes du droit international public », RCADI, 1950, p. 311.

${ }^{45}$ SALMON Jean, Dictionnaire de droit international public, Bruxelles, Bruylant, 2001, p. 1037.
} 
obligations des Etats et Organisations internationales, les uns envers les autres dans le domaine de la protection internationale de l'environnement, c'est-à-dire le Droit International de l'Environnement (DIE). Mais il est plus orienté vers les objectifs de protection du climat, d'où la matière concernée est le Droit International des Changements Climatiques (DICC). Ce droit repose sur un arsenal juridique composé d'instruments conçus spécifiquement pour la protection du climat. Mais également, il est fait recours aux règles édictées pour la protection de l'environnement dans ce cadre. Il en existe à portée déclaratoire et à caractère universel, mais aussi des conventions, traités et accords internationaux. Ces instruments sont conçus à l'occasion des rencontres internationales notamment, les Sommets de la Terre et adoptés par les Etats et certaines organisations régionales d'intégration économique ${ }^{46}$ qui en assurent leur application. C'est ainsi que plusieurs instruments sur les changements climatiques sont élaborés et plus précisément, la Convention Cadre des Nations Unies sur les Changements Climatiques, l'un des piliers principaux du régime international de protection du climat. Ces instruments subissent par période des améliorations car le droit climatique est perfectible et évolutif.

Le droit des changements climatiques a une assise essentiellement scientifique qui s'explique par le fait que ses règles sont élaborées sur la base des connaissances scientifiques et techniques ${ }^{47}$. Puisque la « science est au cœur dudit droit ${ }^{48}$, la matière dépend des sciences exactes $^{49}$, de certaines sciences humaines ${ }^{50}$ et des sciences de la nature ${ }^{51}$. Le « droit» devra puiser des éléments dans plusieurs autres disciplines pour éclairer sa lanterne. C'est du reste ce qui fait du Droit International des Changements Climatiques un « droit » interdisciplinaire.

\footnotetext{
${ }^{46} \mathrm{Au}$ terme de l'article premier paragraphe 6 de la Convention Cadre des Nations Unies sur les Changements Climatiques, on entend par «Organisation régionale d'intégration économique» une organisation constituée par des Etats souverains d'une région donnée qui a compétence dans des domaines régis par la Convention ou ses protocoles et a été dûment autorisée, selon ses procédures internes, à signer, à ratifier, à accepter ou à approuver lesdits instruments ou à y adhérer. L'exemple de l'Union européenne qui a signé la Convention Cadre des Nations Unies sur les Changements Climatiques peut être cité dans ce cadre.

${ }^{47} \mathrm{Ce}$ droit s'élabore sur la base des recherches scientifiques effectuées par les Experts comme ceux du GIEC qui interviennent dans le cadre de l'élaboration et de la mise en œuvre de la Convention Cadre des Nations Unies sur les Changements Climatiques.

${ }^{48}$ Gutwirth, S., Waarheidsaanspraken in recht en wetenschap. Een onderzoek naar de verhouding tussen recht en wetenschap met bijzondere illustraties uit het informaticarecht, VUBPress/MAKLU, Bruxelles/Anvers, 1993, 846 p.

${ }^{49}$ Les sciences exactes sont définies selon le dictionnaire Larousse comme un «ensemble cohérent de connaissances relatives à certaines catégories de faits, d'objets ou de phénomènes obéissant à des lois et/ou vérifiés par les méthodes expérimentales». Disponible sur le site internet : https://www.larousse.fr.Consulté le 04/01/2022.

${ }^{50}$ Les sciences humaines se définissent selon le dictionnaire Larousse comme des « disciplines ayant pour objet l'homme et ses comportements individuels et collectifs, passés et présents». Disponible sur le site internet : https://www.larousse.fr.Consulté le 04/01/2022.

${ }^{51}$ Les sciences de la nature se définissent selon le dictionnaire Larousse comme une « étude scientifique des objets rencontrés dans la nature (animaux, plantes, roches et minéraux, etc.). (Cette expression est remplacée aujourd'hui par celle de science de la vie, dont la définition n'englobe pas les roches et les minéraux.)». Disponible sur le site internet : https://www.larousse.fr.Consulté le 04/01/2022.
} 
Mais à l'évidence, il y a de sérieux obstacles à l'atteinte des objectifs car, depuis le redéploiement de l'arsenal juridique et institutionnel, le constat est patent de ce que le phénomène climatique au lieu de reculer s'amplifie davantage. Même l'Accord de Paris récemment ${ }^{52}$ élaboré et adopté et sensé « (...) contribuer à la mise en œuvre de la Convention cadre des Nations Unies sur les Changements Climatiques $»^{53}$ et qui vise à renforcer la riposte mondiale à la menace des changements climatiques, dans le contexte du développement durable et de la lutte contre la pauvreté ${ }^{54}$ semble ne pas combler les attentes.

Par ailleurs, certains principes phares élaborés dans le cadre de la règlementation internationale des changements climatiques méritent d'être décortiqués pour en appréhender leur valeur juridique, notamment, «(...) le principe des responsabilités communes mais différenciées (...)» ${ }^{55}$ qui établit la part contributive de chaque acteur étatique dans la dégradation de l'environnement. Dans le sens de rétablir la justice climatique, l'Accord de Paris tout comme les traités précédents ${ }^{56}$, impose une charge financière et technologique aux pays développés ${ }^{57}$. Alors que, le non acquittement de cet engagement conventionnel risque d'évincer la mise en œuvre de la Convention Cadre des Nattions Unies sur les Changements Climatiques $^{58}$. Ce principe entre autres, récurrent, n'étant pas dénué de pertinence, il convient de s'appesantir sur l'étude de son objectivité pour élucider les conditions réelles de sa mise en œuvre.

La présente étude qui se veut objective, aborde la question de la mise en œuvre du droit applicable aux changements climatiques en s'appuyant sur le cas de la République du Bénin pour le fait que ce pays vulnérable aux effets néfastes des changements climatiques a ratifié la Convention Cadre des Nations Unies sur les Changements Climatiques ${ }^{59}$.

Dans le contexte où le mal planétaire dicte sa loi à la Communauté internationale et que les pays en voie de développement, tout comme l'Etat béninois et ses citoyens subissent de pleins fouets les conséquences fâcheuses des modifications climatiques, il urge d'empêcher le développement du fléau par la recherche des solutions idoines.

\footnotetext{
${ }^{52}$ A l'occasion de la COP21 à Paris en 2015.

${ }^{53}$ Article 2 paragraphe 1 de l'Accord de Paris de 2015.

${ }^{54}$ Article 2 paragraphe1 id.

${ }^{55}$ C'est le Principe 7 de la Déclaration de Rio sur l'Environnement et le Développement principes de gestion des forêts de 1992.

${ }^{56}$ Il s'agit de la Convention Cadre des Nations Unies sur les Changements Climatiques et du Protocole de Kyoto.

${ }^{57}$ Voir article 4 paragraphe 5 de l'Accord de Paris.

${ }^{58}$ V. Trien Lam, « Les enjeux juridiques de l'intégration des pays en développement dans le régime climatique post-Kyoto, 23 RQDI 31, 2010, pp. 88-89.

${ }^{59}$ Le Bénin a ratifié ladite Convention le 30 juin 1994. Etat de ratification de la Convention disponible sur le site internet: https://treaties.un.org. Consulté le 15/05/2021.
} 


\section{Justifications}

Le choix de ce sujet répond à une exigence académique liée à notre formation en Droit International de l'Environnement (DIE). Ce qui permet de capitaliser les acquis et de maîtriser une branche d'une matière qui relève d'un domaine vaste et riche en connaissance qu'est le Droit International des Changements Climatiques (DICC). Cette démarche a le mérite de renforcer notre initiation en thème de recherche en Droit International de l'Environnement et nous permet de mettre en exergue les connaissances théoriques apprises durant les années d'études antérieures en Droit International de l'Environnement (DIE).

En plus, depuis la tenue de la Conférence des Nations unies sur l'Environnement et le Développement organisée à Stockholm en 1972, les nombreux Sommets dits de la « Terre » et d'autres rencontres avec leurs corollaires d'instruments internationaux, régionaux, voire bilatéraux élaborés n'ont permis de régler de manière efficace et durable les innombrables questions qui se posent à l'humanité par rapport à la protection de l'environnement. Encore moins, les problèmes relatifs aux changements climatiques n'ont véritablement connu de solutions définitives qui permettent d'enrayer le fléau planétaire. La gravité des changements climatiques avec ses conséquences incommensurables, parfois irréversibles pour l'humanité, mérite qu'on s'y attarde car, si rien n'est fait de durable dans ce domaine, la Terre deviendra invivable et l'humanité en souffrira. Puisque l'humanité a besoin de la Terre, aucune recherche orientée dans le sens de la protection du climat ne sera de trop.

En République du Bénin, le Droit de l'environnement n'est pas encore bien connu dans les milieux universitaires ${ }^{60}$ et n'est pas non plus accessible au public ${ }^{61}$, alors que la protection de l'environnement a été élevée au rang de priorité par le Constituant béninois, ${ }^{62}$ qui au terme de l'article 27 de la constitution du 11 décembre 1990 révisée par la loi 2019-40 du 07 novembre 2019, établi que « Toute personne a droit à un environnement sain, satisfaisant et durable et a le devoir de le défendre. L'Etat veille à la protection de l'environnement ». Malgré la prise en compte de la dimension de la protection de l'environnement dans la loi fondamentale de l'Etat, cette matière demeure un tabou pour la population, ce qui témoigne de la preuve manifeste d'un besoin de vulgarisation des textes en vigueur pour faciliter une meilleure connaissance du Droit de protection de l'environnement et celui des changements climatiques, afin de garantir son

\footnotetext{
${ }^{60}$ Peu d'importance est accordée à l'enseignement du droit de l'environnement dans les facultés de droit. Il n'y a pas non plus une filière spécifique destinée à la protection de l'environnement.

${ }^{61}$ Les informations relatives à la protection de l'environnement demeurent encore la chasse gardée de certains intellectuels. La participation citoyenne à la protection de l'environnement n'est pas encore une réalité. La protection de l'environnement reste encore le domaine de monopole de l'Etat.

${ }^{62}$ Le constituant du 11 décembre 1990.
} 
accessibilité. La loi sur les changements climatiques intervenue après la consécration constitutionnelle du droit de protection de l'environnement au Bénin a mis l'accent sur la promotion des « $(\ldots)$ connaissances, savoir-faire et capacités endogènes ${ }^{63}$, par le financement des activités de la «formation et la recherche dans le domaine de l'environnement, en particulier des changements climatiques ${ }^{64}$. Cependant, la diffusion de ce droit ne peut se faire sans les outils didactiques, ouvrages, revues et thèses dont les sujets intègrent la thématique de la «lutte contre les changements climatiques ».

Telles sont les motivations pour lesquelles la recherche a été orientée dans le domaine du Droit International de l'Environnement et plus précisément, dans celui du Droit International des Changements Climatiques à travers l'étude d'un sujet qui ne manque pas d'intérêt.

\section{L'intérêt du sujet}

La question de l'étude portant sur les instruments juridiques de protection du climat, surtout celle qui concerne leur mise en œuvre au Bénin n'est pas dénuée d'intérêt. D’abord, s'agissant de l'objet de l'étude, c'est par le « droit » que sont fixées les conditions d'élaboration et de mise en œuvre des conventions relatives à la lutte contre les changements climatiques. Il est évident que c'est par ce même « droit» qu'il faudra résoudre les questions qui découlent des problèmes que posent les changements climatiques au Bénin.

Par ailleurs, il s'agit d'un sujet d'actualité car faut-il le rappeler, longtemps considérés comme un problème subsidiaire, les changements climatiques se sont imposés à l'humanité de par ses conséquences fâcheuses. La progression du phénomène inquiète plus d'un, compte tenu de son caractère irréversible, d'où le regain d'intérêt de la Communauté internationale au sujet de la protection du climat. Les Sommets de la Terre et les rencontres similaires qui ont suivi et celles qui sont en vue pour débattre des modalités d'encadrement des changements climatiques en sont les preuves.

C'est à travers le développement de ce sujet que sont mis en évidence l'effectivité et l'efficacité du droit applicable aux changements climatiques. L'étude de ce sujet permet de mettre en exergue les forces et faiblesses du droit applicable dans le cadre de la lutte contre les changements climatiques au Bénin. Elle permet également de révéler d'une part, la capacité de la Communauté internationale à maitriser les innombrables défis imposés par les changements

\footnotetext{
${ }^{63}$ Article 17 alinéa 2 de la loi nº2018-18 portant règlementation sur les changements climatiques en République du Bénin.

${ }^{64}$ Id.
} 
climatiques qui sont perçus comme un «problème d'environnement global puisqu'il concerne un bien collectif planétaire $»^{65}$ et d'autre part, de ressortir les moyens dont elle dispose ou qui peuvent être envisagés pour " le maintien d'un système climatique jugé acceptable pour la vie des hommes en société sur terre» ${ }^{66}$.

En somme, le développement du sujet permettra de proposer quelques solutions pour renforcer la lutte contre les changements climatiques dans le contexte international et au Bénin.

\section{Problématique}

Les changements climatiques existaient avant la tenue du Sommet de Stockholm de $1972^{67}$. Cependant, les préoccupations relatives à ce phénomène n'avaient pas été débattues au cours dudit Sommet, lequel avait accouché d'un instrument déclaratoire orienté vers les objectifs globaux de protection de l'environnement.

Paradoxalement, la période de 1972 à 1992 a révélé l'incapacité de l'outil juridique élaboré à Stockholm en 1972 et d'autres à régler les problèmes de protection de l'environnement auxquels venaient s'ajouter les changements climatiques qui fussent leur apparition brutale. Le Sommet de Rio de Janeiro de 1992 en est la preuve car, au cours de la rencontre, malgré le nombre important de traité de protection de l'environnement qui existait, il a été jugé opportun de faire adopter un instrument à caractère universel consacré essentiellement aux changements climatiques ${ }^{68}$. Mais en plus, compte tenu de la complexité des changements climatiques, certains instruments notamment, tendant à la protection de

\footnotetext{
${ }^{65}$ BERTHAUD Pierre, CAVARD Denise, et CRIQUI Patrick, Le régime international pour le climat, vers la consolidation ou l'effondrement? In Revue française d'économie. Vol. 19 N$^{\circ} 2$ 2, 2004. p. 163.

${ }^{66}$ BERTHAUD Pierre, CAVARD Denise, et CRIQUI Patrick, Le régime international pour le climat, vers la consolidation ou l'effondrement? In Revue française d'économie. Vol. 19 №2, 2004. p. 163.

${ }^{67}$ Concernant les changements observés dans le système climatique, il a été fait cas dans le rapport cinquième $\left(5^{\mathrm{e}}\right)$ rapport d'évaluation du Groupe d'experts intergouvernemental sur l'évolution du climat (GIEC), de ce que «Le réchauffement du système climatique est sans équivoque et, depuis les années 1950, beaucoup de changements observés sont sans précédent depuis des décennies voire des millénaires. L'atmosphère et l'océan se sont réchauffés, la couverture de neige et de glace a diminué et le niveau des mers s'est élevé ", p.2. Disponible sur www.ipcc.ch, consulté le 08/06/2021.

${ }^{68}$ Le texte de la Convention Cadre des Nations unies sur les Changements Climatiques a été adopté au siège des Nations Unies à New York, le 9 mai 1992. Conformément à l'article 20, il a été ouvert à la signature à Rio de Janeiro du 4 au 14 juin 1992 et par la suite au siège des Nations unies à New York, du 20 juin 1992 au 19 juin 1993.
} 
l'écosystème ${ }^{69}$, à la promotion du développement ${ }^{70}$ et à l'encadrement du milieu marin et côtier sont élaborés et mis à contribution pour conjurer le dérèglement climatique. A la Conférence de Rio de Janeiro de 1992, en plus de la Convention Cadre des Nations Unies sur les Changements Climatiques, autres instruments importants à caractère déclaratoire ${ }^{71}$ et convention $^{72}$ avaient été élaborés. C'était l'illustration parfaite de l'intérêt accordé par les acteurs du droit international à la lutte contre changements climatiques qui se sont engagés à doter la matière d'instruments juridiques adéquats. En effet, la Convention Cadre des Nations Unies sur les Changements Climatiques a ouvert la porte à des séries de rencontres consacrées uniquement à la résolution des problèmes causés par les changements climatiques.

Le sujet étant plus que d'actualité, l'important Sommet de Paris de 2015 a contribué au raffinement du système de lutte contre les changements climatiques. Et pour l'avenir, d'autres perspectives similaires sont en gestation. L'engagement de la Communauté internationale en face du fléau est notoire et semble-t-elle ne pas s'en lasser tant qu'une solution durable n'interviendrait pour conjurer définitivement le phénomène dans le but de léguer un héritage planétaire sain aux générations futures.

Mais après la tenue de l'historique Sommet de Rio de Janeiro de 1992 qui donnait l'espoir de conjurer les changements climatiques, le constat est visible de ce que le droit applicable aux changements climatiques ne fait pas encore suffisamment la preuve de ce qu'il constitue l'ultime recours pour lutter contre le fléau, car les changements climatiques évoluent à une vitesse de croisière et font rage dans bons nombres de pays. Pour mesurer l'ampleur des effets néfastes des changements climatiques dans le monde entier, «entre 1998 et 2017, les catastrophes climatiques et géophysiques ont provoqué la mort de 1,3 million de personnes et 4,4 milliards de blessés, sans abri, déplacés ou nécessitant une aide d'urgence. La majorité des décès était due à des événements géophysiques, en l'occurrence, des tremblements de terre et

\footnotetext{
${ }^{69} \mathrm{~L}$ 'instrument international de protection de l'écosystème est la Convention sur la diversité biologique adoptée à Rio de Janeiro le 05 juin 1992 entrée en vigueur le 29 décembre 1993 signée par cent soixante huit (168) Etats et compte cent quatre-vingt seize (196) Parties. Disponible sur le site internet : https://treaties.un.org/Pages, consulté le $15 / 05 / 2021$.

${ }^{70}$ Il existe entre autres «Les objectifs du développement durable (ODD) : Outils d'une stratégie mondiale à l'horizon 2030 ». Disponible sur le site internet: https://www.un.org/sustainabledevelopment/fr/. Consulté le $15 / 05 / 2021$.

${ }^{71} \mathrm{La}$ Déclaration de Rio de Janeiro sur l'environnement et le développement de 1992 et la déclaration de rio de Janeiro sur la protection de la forêt de 1992. Disponible sur le site internet : https://www.un.org/french/events/r. Consulté le 15/05/2021.

${ }^{72} \mathrm{La}$ Convention des Nations unies sur la biodiversité et la Convention des Nations unies sur la Désertification. Disponible sur le site internet : https:/www.unccd.int/sites/default/files/sessions/docum.Consulté le 15/05/2021.
} 
des tsunamis et toutes les catastrophes ont été causées par des inondations, des tempêtes, des sécheresses, des vagues de chaleur et d'autres événements météorologiques extrêmes ${ }^{73}$.

Les modifications climatiques représentent un « problème global » ${ }^{74}$, universel, « c'est un sujet de préoccupation pour toute l'humanité entière $»^{75}$ dont les effets impactent négativement plus les pays en développement. Les Etats pauvres qui n'ont que d'infirme responsabilité dans la pollution de l'atmosphère en payent malheureusement une lourde facture. Désormais, selon un rapport du GIEC, « les changements climatiques vont amplifier les risques existants et en engendrer de nouveaux pour les systèmes naturels et humains. Ces risques, qui ne sont pas répartis uniformément, sont généralement plus grands pour les populations et les communautés défavorisées de tous les pays, quelque soit leur niveau de développement ${ }^{76}$. C'est dans ces pays pauvres que persistent les fléaux tels que la déforestation, le dépérissement des forêts, la désertification, la dégradation des sols et les pollutions, conséquences des activités économiques entreprises par les Etats développés. Ils ne disposent pas de la technologie adéquate et de moyens financiers nécessaires à l'ascension des charges relatives aux projets de protection de l'environnement.

De l'observance de l'application du droit des changements climatiques et les constats qui sont faits de la persistance du phénomène, des inquiétudes apparaissent en ce qui concerne la capacité de ce droit à relever le défi de protection de l'environnement par l'éradication des changements climatiques. Ces inquiétudes suscitent une question importante :

\section{Question centrale}

Pourquoi le droit applicable aux changements climatiques ne permet pas en l'état d'atteindre l'objectif d'atténuation des Changements Climatiques?

Il en découle des questions suivantes:

- Quelle est la consistance du droit applicable aux changements climatiques ?

- Que retenir de son effectivité?

- Que faire pour relever les défis en la matière?

\footnotetext{
${ }^{73}$ Below (R) ; Wallemacq (P), Annual Disaster Statistical Review 201, CRED, 2018.

${ }^{74}$ PRIEUR Michel, Actes du $1^{\text {er }}$ séminaire international de droit de l'environnement : Rio+ 10 sur le thème «mondialisation et droit de l'environnement », Rio de Janeiro, du 24 au 26 avril 2002, pp. 13-22.

${ }^{75}$ Septième $\left(7^{\mathrm{e}}\right)$ considérant de la décision de la COP21.

${ }^{76}$ GIEC, 2014: Changements climatiques 2014: Rapport de synthèse. Contribution des Groupes de travail I, II et III au cinquième Rapport d'évaluation du Groupe d'experts intergouvernemental sur l'évolution du climat [Sous la direction de l'équipe de rédaction principale, R.K. Pachauri et L.A. Meyer]. GIEC, Genève, Suisse, 2.3 p.13. Disponible sur le site internet : https://www.ipcc.ch/reports. Consulté le 30/05/2021.
} 
Les divers éléments de réponse aux questionnements que dessus vont contribuer au développement du sujet qui se fera dans le cadre d'un objectif précis.

\section{Objectif}

L'objectif de cette recherche est de procéder à l'étude de l'effectivité du droit climatique dans un Etat en développement comme celui du Bénin. Une Etat d'Afrique occidentale qui illustre mieux la situation d'un pays en voie de développement assujetti aux effets néfastes des changements climatiques et engagé dans ce combat par son adhésion à la Convention Cadre des Nations Unies sur les Changements Climatiques.

Pour atteindre cet objectif, il est nécessaire de procéder à la vérification de certaines hypothèses. Dans le cas où elles sont avérées, leur analyse permettra de discuter objectivement $\mathrm{du}$ ou des problèmes que pose le sujet pour en ressortir les solutions adéquates.

\section{Hypothèses de recherche}

L'intérêt d'une étude en droit réside dans sa mise en œuvre. La question de la mise en œuvre en droit a été une préoccupation de la doctrine, des praticiens, des juristes et des théoriciens en matière des relations internationales. Ceux-ci ont entamés depuis les années 90, des réflexions sur les conditions d'élaboration des régimes internationaux de protection l'environnement et se préoccupent de leurs mise en œuvre ${ }^{77}$.

Pour toucher du doigt les problèmes liés à la mise en œuvre du droit applicable aux changements climatiques, la vérification de certaines hypothèses de recherche à travers des enquêtes permettront de juger de la pertinence et de la capacité du « droit » à relever les défis imposés par le fléau.

Dans le cas d'espèce, l'hypothèse prépondérante repose sur le constat de la persistance du phénomène des changements climatiques, qui fait croire que :

- Le foisonnement d'instruments juridique et institutionnel ne favorise pas la lutte contre les changements climatiques ;

- Le non respect des engagements constitue un obstacle à la lutte contre les Changements Climatiques.

\footnotetext{
${ }^{77}$ T. Risse, 2000, Rational choice, construction and the study of international institutions. In Political Science as discipline ? Reconsidering power, choice and the State at century's end. I. Katznelson, H. Milner eds.
} 
Ces hypothèses feront l'objet de vérification et l'agencement de tous ces éléments dans une thèse bien structurée doit suivre une démarche méthodologique et un plan.

\section{Démarche méthodologique et plan}

Pour cerner tous les contours du sujet, dans un premier temps, nous avons adopté une démarche qui a consisté à analyser le processus d'élaboration du droit applicable aux changements climatiques.

Cette analyse a révélé la richesse du droit climatique qui a été diversement et savamment élaboré au plan international, mais, moins laborieux et peu ingénieux pour ce qui concerne le Bénin.

Dans un premier temps, nous avons adopté une approche monographique qui est définie dans le Dictionnaire LE PETIT ROBERT $1^{78}$ comme une «étude complète et détaillée qui se propose d'épuiser un sujet précis relativement restreint». Cette méthodologie permet de faire une analyse exhaustive du sujet à travers la description du processus d'élaboration du droit applicable aux changements climatiques. Pour être cohérent dans notre démarche, nous avons également fait recours à l'approche fonctionnaliste pour faciliter la compréhension du fonctionnement des institutions impliquées dans le processus d'élaboration et de mise en œuvre du droit applicable aux changements climatiques afin d'évaluer leur performance.

Dans un second temps, nous avons procédé à l'étude de l'effectivité du droit applicable à la lutte contre les changements climatiques. Dans ce cas, il s'agit de faire l'étude du droit béninois des changements climatiques qui se construit sous l'influence du droit international. L'effectivité est une notion qui recèle une «vocation pratique dans la mesure où elle vise à évaluer les degrés d'application du droit, à préciser les mécanismes de pénétration du droit dans la société ${ }^{79}$. Cependant, la notion d'effectivité peut être diversement appréciée, mais elle doit être perçue dans le cadre de cette étude comme «l'instrument conceptuel d'évaluation du degré de réception de la norme, le moyen de mesurer des écarts entre pratique et droit» ${ }^{80}$. Il s'agit concrètement de faire l'étude de l'application des instruments juridiques diversement élaborés par le Droit international climatique au Bénin. La méthodologie empruntée dans ce

\footnotetext{
${ }^{78}$ Paul ROBERT, Dictionnaire Alphabétique et Analogique de la Langue Française, LE PETIT ROBERT 1, LE ROBERT, Paris, 1997, p.1222.

${ }^{79}$ RANGEON François, « Réflexions sur l'effectivité du droit », in CURAPP, Les usages sociaux du droit, PUF, 1989, p. 128.

${ }^{80}$ LASCOUMES Pierre et SÉVERIN Evelyne, « Théories et pratiques de l'effectivité du droit », Droit et Société, $\mathrm{n}^{\circ} 2,1986$, p. 127.
} 
cadre est fondée sur l'étude documentaire. La raison du choix d'une telle démarche consiste au fait que notre travail porte substantiellement sur l'étude du droit, ses instruments et les acteurs de sa mise en œuvre. Dans ces conditions, ce sont les instruments qui constituent ce droit qu'il faut interroger afin d'évaluer leur effectivité et leur efficacité. Parfois, l'évaluation des instruments juridiques requiert une méthode d'interprétation philosophique et doctrinale, raison pour laquelle nous avons adopté cette approche utile à tout travail juridique. L'étude documentaire est une méthode de "décortication des textes juridiques» ${ }^{81}$ qui facilite l'interprétation juridique et permet d'en saisir l'inspiration et l'esprit pour en dégager la substance afin d'en appréhender les lignes de force et de faiblesse ${ }^{82}$. Pour tenir compte de l'immensité et de la complexité des changements climatiques, il est nécessaire d'aller au-delà de l'interprétation juridique des textes. Pour cela, des approches politique, économique, historique et sociologique du droit ont été abordées dans une perspective multidisciplinaire et interdisciplinaire.

Eu égard à ce qui précède, les différentes idées générées à travers l'analyse du sujet de la thèse sont agencées dans un plan bipartite. Ce plan correspond à une démarche scientifique fondée sur la rationalité qui permet de ressortir les différends contours générés par l'analyse du sujet et la clarté qu'il doit offrir à travers l'exposition du droit applicable d'une part et puis son effectivité d'autre part. Ce qui nous a permis d'aborder dans la première partie de la thèse, l'aspect ambitieux de l'encadrement international des changements climatiques dont les normes ont été massivement et diversement élaborées (PREMIERE PARTIE).

Dans la deuxième partie, l'accent a été mis sur le caractère modeste de la contribution du droit à la réalisation des objectifs de la lutte contre les changements climatiques (DEUXIEME PARTIE).

\footnotetext{
${ }^{81}$ Yann TANGUY, La recherche documentaire en droit, PUF, Paris, 1991, p. 143.

${ }^{82} \mathrm{Id}$.
} 



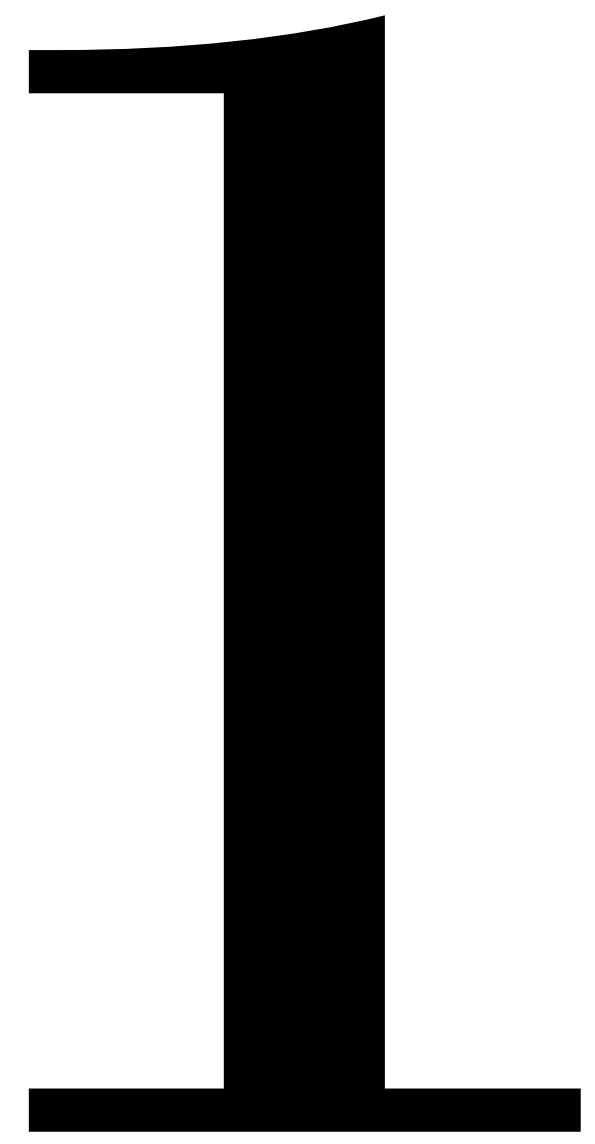




\section{PREMIERE PARTIE}

UN ENCADREMENT AMBITIEUX 

Le Secrétaire général des Nations unies Kofi Annan ${ }^{83}$, lors de la $12^{\mathrm{e}}$ Conférence des Nations unies sur le climat, n'avait-il pas déclaré que le changement climatique mondial constituait «une menace pour la paix et la sécurité» ${ }^{84}$. Une déclaration motivée par la crainte de ce que ce fléau peut engendrer « (...) des modifications du régime des pluies qui peuvent accroître la rivalité à laquelle donne lieu l'accès aux ressources et, partant, entraîner des tensions et des migrations potentiellement déstabilisantes, en particulier dans des pays fragiles ou des régions instables ${ }^{85}$. Des illustrations qui sont proches des situations comparables à « des troubles géopolitiques, particulièrement en termes de sécurité collective» ${ }^{86}$ tel que décrit par Patrick Allard et d'autres auteurs. Ce cri de cœur provenant de cette éminente personnalité représentant la Communauté internationale, ajouté aux nombreux autres appels à la conscientisation précédemment lancés par les scientifiques, chercheurs et organisations internationales, sur l'existence et la dangerosité des changements climatiques, ont fait déclencher une campagne sans relâche d'encadrement du fléau qui a commencé depuis 1972, année de la tenue de la première Conférence internationale sur l'Environnement et le Développement. Cette campagne de normalisation s'est amplifiée avec l'avènement de la tenue du Sommet de Rio de Janeiro en 1992 dont l'exclusivité des normes a été consacrée aux changements climatiques.

Malgré l'apparition récente de l'outil juridique, le droit s'est émergé et s'est manifesté par un encadrement ambitieux de la protection du climat.

Dans sa mission de protection du climat, le droit élabore des règles (TITRE I) et crée des institutions (TITRE II).

\footnotetext{
${ }^{83}$ Kofi Annan, septième $\left(7^{\mathrm{e}}\right)$ Secrétaire général de l'Organisation des Nations Unies. Il a entamé son premier $\left(1^{\mathrm{er}}\right)$ mandat le $1^{\text {er }}$ janvier 1997. Le 29 juin 2001, sur recommandation du Conseil de sécurité, l'Assemblée générale l'a réélu par acclamation pour un second mandat, commençant le $1^{\text {er }}$ janvier 2002 et s'achevant au 31 décembre 2006. Information disponible sur le site internet : https://www.un.org/sg/fr/content/kofi-annan, consulté le 07/06/2021. ${ }^{84}$ Extrait du neuvième $\left(9^{\mathrm{e}}\right)$ paragraphe du discours prononcé à l'occasion de la Conférence de Nairobi au Kenya par le Secrétaire général des Nations Unies, SG/SM/10739, du 15 novembre 2006. Disponible sur le site internet : https://www.un.org/press/fr/2006/SGSM10739.doc.htm. Consulté la 07/06/2021. ${ }^{85}$ Id.

${ }^{86}$ Sur l'étude du lien sécurité-changement climatique, voir Patrick Allard, "Malaise dans la climatisation, le changement climatique et la sécurité des États », Annuaire français des relations internationales, ${ }^{\circ} 6,2005$, pp. 942-951 ; Philippe Ambrosi et Stéphane Hallegate, "Changement climatique et enjeux de sécurité », Les cahiers de la sécurité, n 58, 2005, pp. 297-318; John T. Ackerman, « Climate Change, National Security, and the Quadrennial Defense Review», Strategic Studies Quarterly, printemps 2008, pp. 56-96. Le Center for Naval Analysis, groupe de recherche proche de l'Armée des États-Unis, considère le changement climatique comme « un multiplicateur de menace d'instabilité dans certaines des régions les plus instables du monde ». En fragilisant les institutions politiques et en détériorant le niveau de vie, ce phénomène pourrait provoquer «l'effondrement d'États »; Center for Naval Analysis Corporation, National Security and the Threat of Climate Change, 2007.
} 



\section{TITRE I:}

\section{LES REGLES DE PROTECTION DU CLIMAT}

Compte tenu de la complexité qui caractérise les changements climatiques, outre les normes qui relèvent de l'application de la Convention Cadre des Nations Unies sur les Changements Climatiques ${ }^{87}$, d'autres mesures sont édictées et mises à contribution. Ce qui fait qu'il existe une diversité de moyen juridique élaborée au plan international qui concoure à la lutte contre les changements climatiques (CHAPITRE I).

Les Parties souscrivent aux diverses Conventions dans l'objectif de les mettre à contribution pour lutter contre la dégradation de l'environnement. C'est pourquoi, l'ensemble des règles élaborées et destinées à la protection du climat sont déployées pour être mises en œuvre dans chaque Etat qui a souscrit à la lutte contre les changements climatiques dans le cadre intergouvernemental ${ }^{88}$. C'est ce qui justifie l'intervention de certains Etats Parties, tout comme la République du Bénin qui s’investit dans la mise en œuvre des dispositions applicables aux changements climatiques sur son territoire (CHAPITRE II).

\footnotetext{
${ }^{87}$ Ces normes sont constituées de l'ensemble des décisions et traités conclus dans le cadre de la Convention Cadre des Nations Unies sur les Changements Climatiques.

${ }^{88}$ C'est-à-dire, les Etats qui ont ratifié la Convention Cadre des Nations Unies sur les Changements Climatiques.
} 


\section{CHAPITRE I:}

\section{L'APPORT DU DROIT INTERNATIONAL}

L'ensemble des règles diversement élaborées et destinées à lutter contre les changements climatiques sont avérées comme de véritables œuvres architecturales provenant d'acteurs du Droit (SECTION I).

Cependant, ce droit qui s'élabore à foison n'est pas l'apanage des seuls acteurs du domaine, il provient également de la contribution d'acteurs extérieurs (SECTION II).

\section{SECTION I: L'GUVRE D'ACTEURS DU DROIT}

Le Droit international est un instrument juridique, une œuvre qui s'élabore par les acteurs que sont les Etats ${ }^{89}$. Le Droit international applicable aux changements climatiques qui est une émanation du droit international ne fait pas exception à cette règle. Seulement que, dans une certaine mesure, compte tenu de la particularité de la matière, en plus des Etats, les organisations régionales d'intégration économique sont autorisées à jouer le même rôle que ceux dévolues aux Etats dans l'encadrement de l'environnement et de la lutte contre les changements climatiques ${ }^{90}$. A ce titre, elles sont autorisées à intégrer les cadres conventionnels et peuvent participer aux négociations, à l'élaboration et à la mise en œuvre des instruments juridiques internationaux ${ }^{91}$. Ainsi, les Conventions internationales, les accords internationaux et les traités qui constituent les fondements légaux de ce droit sont négociés et ratifiés par non seulement les Etats mais aussi par d'organisation régionale d'intégration économique ${ }^{92}$ qui s'activent à construire progressivement cette matière (Paragraphe 1) jusqu' à l'édification d'un

\footnotetext{
${ }^{89}$ L'article 6 de la Convention de Vienne sur le droit des traités de 1969 établit la capacité des Etats de conclure des traités. Il s'ensuit que les principaux acteurs du droit international sont les Etats.

${ }^{90} \mathrm{Au}$ terme de l'article premier $\left(1^{\mathrm{er}}\right)$ paragraphe 6 de la Convention Cadre des Nations Unies sur les Changements Climatiques, l'«Organisation régionale d'intégration économique» qui est une organisation constituée par des États souverains d'une région donnée peut avoir compétence dans des domaines régis par la Convention Cadre des Nations Unies sur les Changements Climatiques ou ses protocoles subséquents si elle est dûment autorisée, selon ses procédures internes, à signer, à ratifier, à accepter ou à approuver lesdits instruments ou à y adhérer.

${ }^{91}$ Le cas de l'Union européenne qui a signé la Convention Cadre des Nations Unies sur les Changements Climatiques le 13 juin 1992 et l'a ratifiée le 21 décembre 1993. Information sur le site internet treaties.un.org. Consulté le 15/05/2021.

${ }^{92}$ Id.
} 
régime juridique international exclusif pour lutter contre les changements climatiques (Paragraphe 2).

\section{Paragraphe 1: Une construction progressive}

Les spécialistes de la question environnementale appuyés par certains acteurs du Droit international se sont réunis à Stockholm en 1972 à l'occasion de la Conférence des Nations unies sur l'Environnement et le Développement, pour réfléchir sur la question de la protection internationale de l'environnement ${ }^{93}$. C'était à cette occasion que les bases de la protection internationale de l'environnement avaient été projetées avec l'élaboration de quelques normes ${ }^{94}$ (A). Plus tard, ces normes ont été améliorées à l'occasion d'autres rencontres internationales notamment, au cours du troisième $\left(3^{\mathrm{e}}\right)$ Sommet de la Terre organisé à Rio de Janeiro en 1992 (B).

\section{A- Le commencement de l'encadrement}

Certains dirigeants mondiaux $^{95}$ en collaboration avec des Organisations internationales $^{96}$ et des Organisations associatives ${ }^{97}$ ainsi que certaines personnalités spécialistes de la question environnementale, frappés par l'ampleur des problèmes environnementaux, ont pris la résolution de s'engager dans la protection de l'environnement. Sur leur initiative, des rencontres sont organisées à l'échelle internationale pour se pencher sur la problématique de la protection internationale de l'environnement. Pour la cause, Etats et Experts intervenant dans le secteur environnemental se sont inscrits dans un creuset pour réfléchir ensemble et rechercher les voies et moyens pour juguler les problèmes environnementaux d'envergure planétaire. Cette rencontre qui a été organisée pour la toute première fois $^{98}$ à Stockholm en Suède en 1972 et a été rééditée dix (10) ans après à Nairobi au Kenya en 1982 et est devenue une tradition ${ }^{99}$. Cette rencontre périodique organisée par décennie

\footnotetext{
${ }^{93} \mathrm{La}$ Conférence de Stockholm de 1972 sur 1'Environnement et le Développement a été baptisée « premier Sommet de la Terre ». Il est devenu une tradition d'organiser à partir de cette année des rencontres décennales pour les dédier à la protection internationale de l'environnement.

${ }^{94}$ Il s'agit de la Déclaration de Stockholm de 1972 et le Plan d'action.

${ }^{95}$ Certains chefs d'Etats et responsables d'institutions internationales.

${ }^{96} \mathrm{Il}$ s'agit dans le cas d'espèce du système des Nations unies.

${ }^{97}$ Les Organisations Non Gouvernementales notamment, l’Union mondiale de la Nature (IUCN).

${ }^{98}$ Conférence des Nations unies sur l'Environnement et le Développement organisée à Stockholm en 1972.

${ }^{99}$ Désormais, les Sommets de la Terre s'organisent par décennie, après l'organisation du Sommet de Stockholm en 1972, plusieurs autres Sommets sont intervenus notamment, le Sommet de Nairobi en 1982 et le Sommet de Rio de Janeiro en 1992.
} 
entre dirigeants mondiaux et dédiée exclusivement à l'environnement est désignée sous le vocable « Sommet de la Terre ».

Le but essentiel de l'organisation de ces Conférences est de définir les moyens et politiques d'encadrement du développement durable à travers la protection de l'environnement.

$\mathrm{Au}$ total, cinq (5) Sommets de la Terre ${ }^{100}$ ont été organisés sur l'initiative de l'Organisation des Nations Unies à travers sa structure spécialisée qu'est, le Programme des Nations Unies pour l'Environnement (PNUE) ${ }^{101}$. Les rencontres les plus importantes qui ont contribué au renforcement des normes de protection de l'environnement et servi de fer de lance pour les actions de lutte contre les changements climatiques sont notamment, le Sommet de Stockholm et celui de Rio de Janeiro, tenus respectivement au cours des années 1972 et 1992. L'organisation de ces rencontres est la preuve de l'éclosion d'une culture mondiale dont le seul objectif, est l'encadrement de la protection de l'environnement. Les Sommets de la Terre sont certes importants, mais ne constituent pas une panacée pour l'éradication de tous les problèmes qui accablent l'intégrité de l'environnement. Ces rencontres sont organisées dans l'esprit de faire valoir la nécessité d'engager les dirigeants mondiaux dans la dynamique de contribuer collectivement au règlement des problèmes de dégradation de l'environnement à cause de leur dimension planétaire.

L'organisation des rencontres internationales pour se pencher sur la question environnementale se justifiait par, le contexte caractérisé par l'absence de normes devant aider à garantir l'intégrité de l'environnement mondial. Alors que, face à l'enjeu de protection universelle de l'environnement, il n'y avait pas un cadre de concertation où des initiatives pouvaient être engagées par la majorité de dirigeants mondiaux. Au cours des années 60, le système des Nations unies qui représentait la seule organisation mondiale qui avait la capacité d'engager des initiatives internationales visant à garantir la paix pour l'humanité, n'était non plus en mesure de s'orienter dans l'organisation administrative du secteur de l'environnement ${ }^{102}$. Le système des Nations unies n'était donc pas adapté à la résolution rapide

\footnotetext{
${ }^{100}$ Il s'agit du : Sommet de Stockholm de 1972 ; Sommet de Nairobi de 1982 ; Sommet de Rio de Janeiro de 1992 ; Sommet de Johannesburg de 2002 et Sommet Rio +20 de 2012.

${ }^{101}$ Le Programme des Nations Unies pour l'Environnement (PNUE), en anglais United Nations Environment Programme (UNEP) est une organisation dépendante de l'Organisation des Nations Unies, créé en 1972, et ayant pour but de coordonner les activités des Nations unies dans le domaine de l'environnement et d'assister les pays dans la mise en œuvre des politiques environnementales.

${ }^{102}$ Selon les dispositions de l'article 1 paragraphe 1 de sa charte, l'ONU a pour mission de «Maintenir la paix et la sécurité internationale ». La protection de l'environnement ne relève pas formellement de ses attributions. Charte des Nations unies (Version intégrale) disponible sur le site internet: https://www.un.org/fr/about-us/uncharter/full-text. Consulté le 19/01/2022.
} 
des questions qui résultent des progrès scientifiques et technologiques alors que l'environnement en subi les conséquences.

C'est pourquoi, certains Etats volontaristes se sont offerts pour proposer des orientations dans le sens de l'organisation de la protection internationale de l'environnement. C'est le cas de la Suède ${ }^{103}$ qui est parvenue à faire accepter à l'Assemblée générale des Nations unies l'idée de l'organisation d'une Conférence internationale essentiellement consacrée à la thématique environnementale ${ }^{104}$. L'initiative suédoise a été concrétisée par l'organisation du tout premier Sommet de la Terre qui s'est tenu à Stockholm en 1972 ${ }^{105}$. La Conférence a lieu dans un contexte caractérisé par la Guerre froide ${ }^{106}$ avec l'absence remarquée de l'Union Soviétique et ses alliés ${ }^{107}$. Ces derniers ont pourtant pris part au processus de préparation de ladite Conférence. Cependant, la Chine a saisi cette opportunité pour marquer son entrée sur la scène internationale en qualité de membre de l'ONU par sa participation audit Sommet ${ }^{108}$. Seulement que, pendant le déroulement de la Conférence, les négociations portant sur le projet de Déclaration ont achoppé avec la Chine qui ne voulait pas donner son accord à cause de la question de la pollution ${ }^{109}$. Malgré ces contradictions, les décisions issues du Sommet ont été adoptées $^{110}$.

Les motivations spécifiques qui ont conduit à l'organisation de l'historique Sommet de Stockholm à l'époque, se trouvaient dans les inquiétudes que suscitait le risque de bouleversement cosmique de la planète Terre. Ces mutations sont relayées par des opinions

\footnotetext{
${ }^{103}$ La Suède est un Etat européen membre de l'Organisation des Nations Unies.

${ }^{104} \mathrm{~A}$ la vingt-quatrième (24e) session de l'Assemblée générale des Nations unies, l'invitation du Gouvernement de la Suède à organiser une conférence dans ce pays courant juin 1972 a été entérinée. Mais il y avait déjà un rapport commandé par le Secrétaire général des Nations unies dans lequel des recommandations avaient été faites sur les principaux points à débattre au cours de la Conférence et a étudié les difficultés que posait la rédaction d'une Déclaration sur l'environnement.

${ }^{105} \mathrm{Ce}$ Sommet a projeté les bases de la protection internationale de l'environnement à travers l'élaboration de la Déclaration de Stockholm de 1972 suivie de son Plan d'action.

${ }^{106}$ La Guerre froide selon le dictionnaire Larousse est l'« Etat de tension qui opposa de 1945 à 1990, les ÉtatsUnis d'Amérique (USA), l'Union des Républiques Socialistes et Soviétiques (URSS) et leurs alliés respectifs qui formaient deux blocs dotés de moyens militaires considérables et défendant des systèmes idéologiques et économiques antinomiques.» Disponible sur le site internet : https://www.larousse.fr/encyclopedie/diht.Consulté le $04 / 01 / 2022$.

${ }^{107}$ Les alliés sont : Russie- Albanie-Bulgarie-Cuba-Hongrie-Pologne et Tchécoslovaquie.

${ }^{108} \mathrm{Ce}$ fut pour la première fois que la Chine en tant qu'Etat Membre de l'Organisation des Nations Unies participait à une rencontre internationale organisée par le système des Nations unies.

${ }^{109} \mathrm{La}$ Chine avait une position discordante de celle des autres participants à la Conférence parce qu'elle a jugé insuffisant l'article qui était consacré aux armes nucléaires et aux moyens de destruction massive. Mais la Déclaration n'avait pas fait cas des guerres impérialistes et colonialistes comme sources importantes de pollution. Malgré sa protestation le projet a été adopté. Kiss Alexandre Charles, Sicault Jean-Didier. La Conférence des Nations Unies sur l'Environnement (Stockholm, 5/16 juin 1972). In: Annuaire français de droit international, volume 18, 1972. pp. 603-628, p.611.

${ }^{110}$ Kiss Alexandre Charles, Sicault Jean-Didier. La Conférence des Nations Unies sur l'Environnement (Stockholm, 5/16 juin 1972). In: Annuaire français de droit international, volume 18, 1972. pp. 603-628, p. 611 et 614 .
} 
alarmistes. Il y avait des gémissements qui, loin de plonger dans les abimes du «pessimisme de l'intelligence $»^{111}$, ont plutôt engendré un phénomène contraire, c'est-à-dire « l'optimisme de la volonté $»^{112}$ qui s'est matérialisé par la sortie des dirigeants de leur léthargie pour se consacrer à la protection internationale de l'environnement. Cette conférence a été l'occasion saisie par les dirigeants mondiaux pour sensibiliser et définir des stratégies qui nécessitaient l'appui de la coopération internationale afin d'éradiquer les problèmes environnementaux ${ }^{113}$.

La première Conférence sur l'Environnement et le Développement tenue à Stockholm en Suède en 1972 a connu l'élaboration d'un important instrument de protection de l'environnement. Il s'agit de la «Déclaration de Stockholm de 1972», telle que son nom l'indique, ce n'est qu'un acte déclaratoire à portée universelle de protection de l'environnement ${ }^{114}$. L'adoption de cet instrument marque le commencement de l'édification d'un droit international qui a pour vocation la protection de l'environnement ${ }^{115}$.

Les dangers qui accablent la biosphère constituent les motifs qui ont contribué à la prise de conscience et à la nécessité de l'organisation de cette réunion mondiale. Fort heureusement, le premier Sommet environnemental est soldé par des résolutions qui ont débouché sur des « remèdes systématiques et institutionnels pour contenir les velléités de dégradation de l'environnement» ${ }^{116}$.

Le texte de la Déclaration est constitué de principes ${ }^{117}$ dont les plus importants sont, le droit à l'environnement et au développement ${ }^{118}$, le devoir de coopération ${ }^{119}$, la lutte légitime des peuples contre la pollution ${ }^{120}$, le principe de la responsabilité pour dommage écologique ${ }^{121}$, le principe pollueur-payeur, le principe de l'évaluation environnementale par des études d'impact et la promotion du développement économique et social ${ }^{122}$. La Déclaration a retracé

\footnotetext{
${ }^{111}$ Antonio Gramsci, Lettre à son frère Carlo écrite en prison, le 19 décembre 1929, Cahiers de prison, Gallimard, Paris, 1978-92.

112 Id.

${ }^{113}$ Cette initiative a été entreprise par Sverker Astrom, Représentant permanent de la Suède auprès des Nations unies.

${ }^{114}$ La Déclaration de Stockholm de 1972 n'est pas une convention ni un traité, donc il est peu contraignant.

${ }^{115}$ La Déclaration de Stockholm de 1972 fut le premier instrument international élaboré dans le cadre de la protection internationale de l'environnement.

${ }^{116}$ Le premier cadre institutionnel innové est celui de la Conférence des Nations unies dur l'Environnement et le Développement qui désormais devient une tradition qui s'occupe des questions de protection de l'environnement. La Déclaration qui en est issue a projeté des idées de création d'autres institutions devant intervenir dans la protection de l'environnement. Plus tard, le Programme des Nations Unies pour l'Environnement (PNUE) a été créé.

${ }^{117}$ Vingt-six (26) principes.

118 Principes 8 et 9 de la Déclaration de Stockholm de 1972.

119 Principes 22 et 24 id.

${ }^{120}$ Principe 6 ibid.

${ }^{121}$ Principe 22 ibid.

${ }^{122}$ Principe 8 de la Déclaration de Stockholm de 1972.
} 
des objectifs globaux et spécifiques essentiellement orientés vers la lutte contre la pollution ${ }^{123}$. A titre illustratif, la Déclaration prône l'encouragement de la «lutte légitime des peuples de tous les pays contre la pollution (...) ${ }^{124}$. Les questions écologiques ont été positionnées à l'échelle supérieure des préoccupations internationales, comme en témoigne la stipulation suivante: «Les ressources naturelles du globe, y compris l'air, l'eau, la terre, la flore et la faune, et particulièrement les échantillons représentatifs des écosystèmes naturels, doivent être préservés dans l'intérêt des générations présentes et à venir par une planification ou une gestion attentive selon que de besoin ${ }^{125}$. Cette Déclaration n'occulte pas l'impérieux devoir et obligation qui incombent à l'homme, de contribuer à l'amélioration de son cadre vie dans le but de léguer cet héritage dans un état satisfaisant aux générations futures ${ }^{126}$. Elle est accompagnée d'un Plan d'action qui n'est que l'assemblage d'une série de recommandations concrètes adoptées à l'occasion du Sommet ${ }^{127}$. Par ailleurs, le Sommet de Stockholm de 1972 a posé les jalons d'une coopération internationale nécessaire au développement du Droit international de l'Environnement, elle en a fait son épine dorsale en indiquant que «Les questions internationales se rapportant à la protection et à l'amélioration de l'environnement devraient être abordées dans un esprit de coopération par tous les pays grands ou petits sur un pied d'égalité. Une coopération par voie d'accords multilatéraux ou bilatéraux ou par d'autres moyens appropriés(...) ${ }^{128}$.

La rencontre de Stockholm a ouvert le secteur environnemental, autrefois occupé par les acteurs des Sciences sociales tels que, les écologistes, les scientifiques, les biologistes à l'univers des juristes spécialisés dans le domaine environnemental ${ }^{129}$. L'outil élaboré à l'occasion dudit Sommet a produit des résultats aux plans juridique et institutionnel, qualifiés de "globalement positif ${ }^{130}$, mais n'a pas suffit pour enrayer totalement les problèmes de détérioration de l'environnement. Cet instrument n'est pas non plus adapté pour contenir les

\footnotetext{
${ }^{123}$ Principes $6 ; 7$ et 22 de la Déclaration de Stockholm de 1972.

${ }^{124}$ Principe 6 de la Déclaration de Stockholm de 1972.

125 Principe 2 id.

126 Principe 1 ibid.

${ }^{127}$ Le Plan d'action est composé de cent neuf (109) résolutions séquencées en plusieurs rubriques à savoir : évaluation de l'environnement-gestion de l'environnement et puis mesures de soutien.

${ }^{128}$ Principe 24 de la Déclaration de Stockholm de 1972.

${ }^{129}$ La Déclaration de Stockholm de 1972, le plan d'action pour l'environnement et la résolution sur les essais d'armes nucléaires vont désormais servir d'instruments juridiques pour les juristes environnementalistes.

${ }^{130}$ Kiss Alexandre-Charles, Doumbe-Bille Stéphane. Conférence des Nations unies sur l'environnement et le développement (Rio de Janeiro-juin 1992). In: Annuaire français de droit international, volume 38, 1992. pp. 823843., p.824.
} 
problèmes en latence au moment de la Conférence ${ }^{131}$, lesquels ont émergé quelques années plus $\operatorname{tard}^{132}$.

Il est à rappeler que l'engagement pris par les Etats et organisateurs de la Conférence de Stockholm de 1972 à travers sa Déclaration, n'a pas été émoussé. Bien au contraire, il a été renouvelé et rénové vingt (20) ans plus tard à l'occasion du Sommet de Rio de Janeiro de 1992. Cette conférence est intervenue dans un contexte d'aggravation du réchauffement de la Terre et a fait progresser le système de protection de l'environnement par l'amélioration des normes.

\section{B-L'amélioration des normes de protection}

Depuis l'adoption de la Déclaration de Stockholm de 1972, trois (3) autres instruments déclaratoires ont suivi, il s'agit de la Charte mondiale de la nature adoptée en 1982 par l'Assemblée générale des Nations unies, de la Déclaration de Rio de Janeiro de 1992 sur l'Environnement et le Développement et puis la Déclaration de Rio de Janeiro de 1992 sur les principes, non juridiquement contraignants mais faisant autorité, pour un consensus mondial sur la gestion, la conservation et l'exploitation écologiquement viable de tous les types de forêts. En réalité, ces instruments constituent des « sources d'inspiration pour des législations d'Etats ou d'organisations régionales ou pour des conventions régionales $»^{133}$. Autrement dit, ce sont des «viviers juridiques $»^{134}$ qui jouent un « rôle de précurseur du Droit international de l'Environnement $\gg{ }^{135}$. Les Déclarations n'imposent aucune contrainte juridique aux acteurs seulement, "à force d'être consacrés, certains principes peuvent devenir des coutumes internationales ${ }^{136}$. Même dans ce cas, faudrait-il que les conventions admettent dans le principe, le recours systématique à l'utilisation des principes coutumiers. Par exemple la Cour internationale de justice est une juridiction compétente pour connaitre les différends nés de la gestion de l'application ou de l'interprétation de la Convention Cadre des Nations Unies sur les Changements Climatiques. Elle dispose comme source de droit, «Les conventions

\footnotetext{
${ }^{131}$ Il s'agit essentiellement des «changements climatiques».

${ }^{132} \mathrm{La}$ Conférence mondiale sur le climat de 1979 permet de conclure que les émissions anthropiques de dioxyde de carbone pourraient avoir un effet à long terme sur le climat. Disponible sur le site internet https://www.actualites-news-environnement.com/21729-1979-2009-histoire-trente-ans-lutte-changements climatiques.html. Consulté le16/05/202. L'organisation de ladite Conférence était déjà la preuve de l'émergence d'un phénomène nouveau appelé « changements climatiques ».

133 Agence Universitaire de la Francophonie/Université de Limoges, Cour de Jean-Marc LAVIEILLE «Les principes généraux du droit international de l'environnement ; Le principe de précaution»,18p, p3.

${ }^{134} \mathrm{Id}$.

135 Ibid.

${ }^{136}$ Agence Universitaire de la Francophonie/Université de Limoges, Cour de Jean-Marc LAVIEILLE «Les principes généraux du droit international de l'environnement; Le principe de précaution»,18p., p.3.
} 
internationales, soit générales, soit spéciales, établissant des règles expressément reconnues par les Etats en litige et les principes généraux de droit reconnus par les nations civilisées ${ }^{137}$, mais également, la haute juridiction fait recours à la « coutume internationale comme preuve d'une pratique générale acceptée comme étant le droit $»^{138}$.

Les acteurs du Droit international s'inspirent des instruments déclaratoires pour élaborer des conventions internationales, des accords internationaux et des traités. Cependant, les déclarations qui au départ ne sont pas contraignantes peuvent être revêtues de la force exécutoire après qu'elles soient incorporées dans les conventions. A l'évidence, la plupart des principes initiés et les concepts innovés dans les actes déclaratoires tendant à la protection internationale de l'environnement, sont pris en compte dans les accords internationaux. La preuve en est que, « La Conférence des Nations unies sur l'Environnement et le Développement de Rio de Janeiro de 1992 n'a vraiment pas introduit de principes totalement nouveaux $»^{139}$. Par exemple, le fait de rappeler dans la Convention Cadre des Nations Unies sur les Changements Climatiques, « les dispositions pertinentes de la Déclaration de la Conférence des Nations unies sur l'Environnement adoptée à Stockholm le 16 juin $1972 »^{140}$, dans le préambule de ladite convention, emporte des conséquences de droit. Désormais, la Déclaration de Stockholm de 1972 fait partie intégrante de la Convention Cadre des Nations Unies sur les Changements Climatiques ainsi, les normes non contraignantes de ladite Déclaration sont devenues des obligations contraignantes à la charge des Etats Parties à la Convention Cadre des Nations Unies sur les Changements Climatiques. Il s'ensuit que, les divers instruments déclaratoires pris en l'état ne peuvent constituer une solution définitive pour régler les problèmes environnementaux. La Déclaration de Stockholm, bien qu'elle soit pertinente doit être renforcée.

Conscient de la mollesse des actes déclaratoires, les acteurs du Droit international de l'Environnement s'activent pour rendre perfectible les normes environnementales existantes au point de les adapter aux réalités de l'époque. Cette réflexion permanente s'inscrit dans la ligne tracée par la Déclaration de Stockholm de 1972, qui insiste sur la nécessité de continuer la recherche ${ }^{141}$ en ce qui concerne l'environnement dans son état normal, ses détériorations et les

\footnotetext{
${ }^{137}$ Article 38 alinéa 1-3 du Statut de la Cour Internationale de Justice. Disponible sur le site internet: https://www.icj-cij.org/fr/statut. Consulté le 04/01/2022.

${ }^{138}$ Article 38 alinéa 2 id.

${ }^{139}$ Prieur Michel. Démocratie et droit de l'environnement et du développement. In: Revue Juridique de l'Environnement, ${ }^{\circ} 1,1993$. pp. 23-30.

${ }^{140}$ Paragraphe 8 du préambule de la Convention Cadre des Nations Unies sur les Changements Climatiques.

${ }^{141}$ Principe 20 de la Déclaration de Stockholm de 1972. Disponible sur le site internet : https://www.un.org/fr/c. Consulté le 04/01/2022.
} 
moyens de lutter contre celles-ci. Selon les termes de cette Déclaration, les connaissances peuvent faciliter la bonne compréhension du fonctionnement de l'environnement et les relations pouvant exister entre ses divers composants, par contre « l'ignorance ou la négligence peuvent causer des dommages considérables et irréversibles à l'environnement $»^{142}$.

Cependant, cet élan de considération et du respect d'une tradition en matière de gestion de la protection de l'environnement qu'a voulu véhiculer la Communauté internationale n'est pas unanimement suivi. Compte tenu de l'enjeu que revêt la protection de l'environnement et la perception différenciée des intérêts pour chaque Etat Partie, il existe des poches de résistance qui se développent et ne facilitent pas la bonne marche de l'initiative internationale de protection de l'environnement. Le projet de renforcement du droit international qui devrait être poursuivi à l'occasion du prochain Sommet de la Terre n'a pas eu lieu, parce que la rencontre « était organisée à Nairobi en 1982 dans une grande indifférence » ${ }^{143}$. Le climat provoqué par la « Guerre froide » ${ }^{144}$ ajouté au manque d'intérêt dont a fait preuve le Président des Etats-Unis d'Amérique d'alors ${ }^{145}$, ont fait capoter le Sommet qui n'a pu se tenir utilement. En effet, l'espoir engendré par l'historique Conférence de Stockholm de 1972 a laissé place à des désillusions au point de susciter des craintes quant à l'objectivité de l'instrument déclaratoire et les outils connexes élaborés à l'occasion du Sommet.

L'inquiétude est devenue croissante lorsque la Conférence mondiale sur le climat précédemment organisée à Genève en $1979^{146}$ avait révélé l'existence des «changements climatiques ». Un phénomène qui peut modifier les paramètres environnementaux au point de perturber l'existence de l'homme sur la Terre et engendrer des conséquences irréversibles. Ainsi donc, la Conférence de Genève appelait déjà tous les Etats à s'engager dans la prévention des probables conséquences fâcheuses que peuvent engendrer les changements climatiques. Ce fut pour la toute première fois que les représentants des Etats membres de l'Organisation Mondiale de la Météorologie (OMM) ont été sensibilisés sur les problèmes climatiques afin que des mesures soient prises pour conjurer le fléau. Les Etats ont été invités à converger leur

\footnotetext{
${ }^{142}$ Extrait du sixième $\left(6^{\mathrm{e}}\right)$ paragraphe du préambule de la Déclaration de Stockholm de 1972.

${ }^{143}$ MALJEAN-DUBOIS (Sandrine) et WEMAERE (Matthieu), La conférence des Nations unies sur le développement durable « Rio+20 », Centre d'études et de recherches internationales et Communautaires, AixMarseille Université, p.1.

${ }^{144}$ Selon le dictionnaire Larousse «État de tension qui opposa, de 1945 à 1990, les États-Unis, l'URSS et leurs alliés respectifs qui formaient deux blocs dotés de moyens militaires considérables et défendant des systèmes idéologiques et économiques antinomiques.» Disponible sur le site internet: https://www.larousse.fr/encyclopedie/dihttps://www.larousse.fr/encyclopedie/divers/guerre_froide/122564.Conu lté le 04/01/2022.

${ }^{145}$ Le Président en exercice était Ronald Reagan qui s'était fait représenté par sa fille.

${ }^{146}$ Première conférence mondiale sur le climat organisée par l'Organisation Métrologique Mondiale (OMM) et le Programme des Nations Unies pour l'Environnement (PNUE).
} 
efforts pour cerner les contours du changement climatique à travers des études et recherches. Eventuellement, des plans et stratégies ont été envisagés pour être mis à contribution.

Pour concrétiser les idées germées à l'occasion du Sommet mondial sur le climat tenu en 1979, l'Assemblée générale des Nations unies a pris certaines initiatives notamment, en adoptant le 06 décembre 1988 une résolution sur la protection du climat mondial ${ }^{147}$, dans laquelle il a été déclaré que l'ONU se préoccupait «par le fait que certaines activités humaines pourraient modifier les caractéristiques du climat mondial, faisant peser sur les générations présentes et futures la menace de graves conséquences économiques et sociales » ${ }^{148}$ et s'était aussi persuadée « que l'évolution du climat touche l'ensemble de l'humanité et que la question doit être abordée dans un cadre mondial, de manière à tenir compte des intérêts vitaux de l'humanité toute entière ${ }^{149}$. Elle a également entériné la création du Groupe Intergouvernemental d'Experts sur l'Evolution du Climat (GIEC) qui avait été institué par décision conjointe de l'Organisation Mondiale de la Météorologie (OMM) et du Programme des Nations Unies sur l'Environnement (PNUE) ${ }^{150}$. Le GIEC a pour mission, l'évaluation et le suivi scientifique des changements climatiques ${ }^{151}$. Aussitôt créé, le Groupe Intergouvernemental d'Experts sur l'Evolution du Climat (GIEC) a élaboré son premier rapport sur la période allant de 1990 à 1992. Ce rapport a révélé que, «l'un de ces mécanismes juridiques internationaux existants, la Convention de Vienne sur la protection de la couche d'ozone, appuyée du Protocole de Montréal sur les substances qui appauvrissent la couche d'ozone vise expressément à réduire les émissions des principaux gaz à effet de serre qui ont aussi pour effet d'appauvrir la couche d'ozone ${ }^{152}$. Toutefois, les Experts se sont accordés à estimer que les instruments juridiques et institutionnels qui concernent les changements climatiques doivent être pleinement utilisés et encore renforcés, mais qu'ils ne peuvent à eux seuls suffire pour faire face au problème $»^{153}$. Au cours de la quarante-quatrième (44 $)$ session de l'Assemblée générale

\footnotetext{
${ }^{147}$ La résolution 43/53 du 6 décembre 1988 de l'Assemblée générale des Nations unies relative à la « Protection du climat mondial pour les générations présentes et futures». Disponible sur le site internet : https://www.un.org/french/documents/ga/res/43/fres43.shtml. Consulté le 04/01/2022.

${ }^{148}$ Troisième $\left(3^{\mathrm{e}}\right)$ paragraphe de la résolution 43/53 du 6 décembre 1988 de l'Assemblée générale des Nations unies sur la «Protection du climat mondial pour les générations présentes et futures », située à la page $137 \mathrm{du}$ rapport des résolutions adoptées au cours de la quarante-troisième $\left(43^{\mathrm{e}}\right)$ session de l'Assemblée générale des Nations unies. ${ }^{149}$ Douzième $\left(12^{\mathrm{e}}\right)$ paragraphe de la résolution $43 / 53$ du 6 décembre 1988 de l'Assemblée générale des Nations unies sur la «Protection du climat mondial pour les générations présentes et futures», située à la page $138 \mathrm{du}$ rapport des résolutions adoptées au cours de la quarante-troisième $\left(43^{\mathrm{e}}\right)$ session de l'Assemblée générale des Nations unies.

${ }^{150}$ Douzième $\left(12^{\mathrm{e}}\right)$ paragraphe- 5 , id.

151 Ibid.

${ }^{152}$ Le paragraphe 7.5 .1 du premier rapport (1990 à 1992) du Groupe d'Experts Intergouvernemental sur l'Evolution du Climat. Page 156.

${ }^{153}$ Le paragraphe 7.5.1 du premier rapport (1990 à 1992) du Groupe d'Experts Intergouvernemental sur l'Evolution du Climat. Page 156.
} 
des Nations unies, il a été jugé de la nécessité d'élaborer en toute célérité une Convention Cadre sur les changements climatiques qui définira, quelques principes généraux et obligations ${ }^{154}$. Selon les termes du rapport du Groupe Intergouvernemental d'Experts sur 1'Evolution du Climat (GIEC), « 1' instrument en gestation doit comporter des dispositions qui suscitent une large adhésion des Etats ${ }^{155}$. Le diagnostic ainsi posé assorti de propositions concrètes entérinées par des négociations, le décor est planté pour la tenue d'un Sommet novateur en matière de lutte contre les changements climatiques, il s'agit du troisième Sommet, celui qui s'est tenu à Rio de Janeiro en 1992. Ce Sommet a «marqué une étape dans la perception des enjeux environnementaux $»^{156}$.

Les fruits du premier rapport du GIEC ont été exploités et ont donné naissance à un projet de Convention Cadre sur les changements climatiques qui a été élaboré à New-York en mai $1992^{157}$. La Convention a été adoptée à l'occasion du Sommet de Rio de Janeiro de 1992 puis entrée en vigueur le 21 mars $1994^{158}$. L'organisation du Sommet de Rio de Janeiro dans le contexte des changements climatiques a permis de porter sur la conscience des dirigeants mondiaux le caractère nuisible du phénomène climatique planétaire. Cet instrument élaboré sous « forme d'un traité cadre» ${ }^{159}$ a débouché sur de très bonnes perspectives en matière de lutte contre les changements climatiques, parce que d'abord, il a défini un objectif ultime, celui «(...) de stabiliser, (...), les concentrations de gaz à effet de serre dans l'atmosphère à un niveau qui empêche toute perturbation anthropique dangereuse du système climatique (...) ${ }^{160}$, pour «(...) que les écosystèmes puissent s'adapter naturellement aux changements climatiques (...) $»^{161}$ et pour «(...) que la production alimentaire ne soit pas menacée et que le

\footnotetext{
${ }^{154}$ Sixième $\left(6^{e}\right)$ paragraphe de la résolution 44/207 du 22 décembre 1989 de l'Assemblée générale des Nations unies sur la «Protection du climat mondial pour les générations présentes et futures, situé à la page 137 du rapport des résolutions adoptées au cours de la quarante-quatrième $\left(44^{e}\right)$ session de l'Assemblée générale des Nations unies. Disponible sur le site internet : https://www.un.org/french/documents/ga/res/43/fres43.shtml. Consulté le 04/01/2022.

${ }^{155}$ Selon les termes et l'aperçu général du premier rapport du GIEC, paragraphe 5.01, p.66.

${ }^{156}$ Cf. S. DOUMBE-BILLE / A. C. KISS, " La Conférence des Nations unies sur l'Environnement et le Développement », AFDI, 1992, pp. 823-843.

${ }^{157}$ Information consultée sur le site internet https://treaties.un.org., le 16/05/2021.

${ }^{158}$ Id.

${ }^{159}$ Un traité-cadre est défini comme « un instrument conventionnel qui énonce les principes devant servir de fondement à la coopération entre les États parties dans un domaine déterminé, tout en leur laissant le soin de définir, par des accords séparés, les modalités et les détails de la coopération, en prévoyant, s'il y a lieu, une ou des institutions adéquates à cet effet »; Alexandre KISS, "Les traités-cadres. Une technique juridique caractéristique du droit international de l'environnement », Annuaire français de droit international, 1993, p. 793. ${ }^{160}$ Article 2 de la Convention Cadre des Nations Unies sur les Changements Climatiques adoptée à Rio de Janeiro au cours du Sommet de la Terre en 1992.

${ }^{161}$ Article 2 de la Convention Cadre des Nations Unies sur les Changements Climatiques adoptée à Rio de Janeiro au cours du Sommet de la Terre en 199.
} 
développement économique puisse se poursuivre d'une manière durable.» ${ }^{162}$ En outre, pour tenir compte de la contribution de chaque Etat dans la crise climatique, l'instrument international a innové le principe phare des «responsabilités communes mais différenciées (...) ${ }^{163}$. Ce traité marque la reconnaissance des responsabilités des pays développés dans la pollution de l'atmosphère et l'adhésion de tous les Etats-Parties aux engagements d'atténuation des changements climatiques. Désormais, les Parties sont tenues de souscrire à des engagements de réduction de gaz à effet de serre, mais en tenant compte de la spécificité de leurs priorités nationales et régionales de développement. Dans ce cadre, les Parties, «établissent, mettent à jour périodiquement, publient et mettent à la disposition de la Conférence des Parties, conformément à l'article 12, des inventaires nationaux des émissions anthropiques par leurs sources et de l'absorption par leurs puits de tous les gaz à effet de serre non réglementés par le Protocole de Montréal, en recourant à des méthodes comparables qui seront approuvées par la Conférence des Parties $\gg{ }^{164}$. Le principe de précaution a été introduit dans le droit de protection de l'environnement et celui de la protection du climat en ces termes, «Il incombe aux Parties de prendre des mesures de précaution pour prévoir, prévenir ou atténuer les causes des changements climatiques et en limiter les effets néfastes(...) ${ }^{165}$. Le principe de précaution signifie que des mesures peuvent être prises en absence de risque pour empêcher le développement des risques apparents lors de la réalisation des projets. La mise en œuvre de ce principe a pour finalité de faire prendre par les Parties, des mesures « (...)Quand il y a risque de perturbations graves ou irréversibles, l'absence de certitude scientifique absolue ne doit pas servir de prétexte pour différer l'adoption de telles mesures, étant entendu que les politiques et mesures qu'appellent les changements climatiques requièrent un bon rapport coût-efficacité, de manière à garantir des avantages globaux au coût le plus bas possible $»^{166}$. Mais après quelques années d'application, des opinions ont commencé par s'interroger sur la nature de ce principe qui est devenu un sujet à controverse. Certains auteurs estiment qu'au bénéfice du « quasi consensus scientifique actuel » ${ }^{167}$ établi autour de la matière,

\footnotetext{
162 Ibid.

${ }^{163}$ Article 3 paragraphe 1 ibid.

${ }^{164}$ Article 4 paragraphe 1-a de la Convention Cadre des Nations Unies sur les Changements Climatiques adoptée à Rio de Janeiro au cours du Sommet de la Terre en 1992.

${ }^{165}$ Article 3 principe 3 de la Convention Cadre des Nations Unies sur les Changements Climatiques adoptée à Rio de Janeiro en 1992. Les mesures de précaution ont été également introduites dans la Déclaration de Rio de Janeiro sur l'Environnement et le Développement, adoptée à la même occasion au principe 15.

${ }^{166}$ Article 3 principe 3 de la Convention Cadre des Nations Unies sur les Changements Climatiques adoptée à Rio de Janeiro au cours du Sommet de la Terre en 1992.

${ }^{167} \mathrm{M}$. Torre-Schaub, Le principe de précaution dans la lutte contre le réchauffement climatique : entre croissance économique et protection durable, Revue européenne de droit de l'environnement, $\mathrm{n}^{\circ} 2,2002, \mathrm{p} .151$.
} 
« les changements climatiques », doivent relever du principe de prévention et non du principe de précaution.

En plus des instruments de protection du climat, «La Conférence des Nations Unies sur l'Environnement et le Développement a adopté une Déclaration qui a fait progresser le concept des droits et des responsabilités des pays dans le domaine de l'environnement. ${ }^{168}$. La Déclaration de Rio de Janeiro sur l'Environnement et le Développement a intégré les préoccupations apparues après la conférence de Stockholm de 1972, relatives à la dégradation continue de l'environnement.

Pendant que la Communauté internationale s'active autour de la question de la protection de l'environnement à travers le renforcement de l'arsenal juridique climatique par l'adoption de la Convention Cadre des Nations Unies sur les Changements Climatiques, l'épineuse question de réchauffement du globe continue d'agiter le monde entier. $\mathrm{Ce}$ phénomène est devenu « un défi mondial décisif qui, s'il n'est pas relevé d'urgence, mettra en péril non seulement l'environnement, mais aussi la prospérité économique, la réduction de la pauvreté, le développement durable et, plus généralement, la paix, la stabilité et la sécurité au niveau planétaire ${ }^{169}$. Pour ne pas rester indifférent à ce drame planétaire qui se profilait à l'horizon, des réflexions avaient continué dans le sens de la construction d'un régime juridique international du climat.

\section{Paragraphe 2: L'élaboration du régime international du climat}

L'adoption de la Convention Cadre des Nations Unies sur les Changements Climatiques a marqué le début d'un processus de codification essentiellement destiné à la lutte contre les changements climatiques.

L'application de cet instrument a servi de base à l'élaboration d'un cadre juridique international de gestion permanente et évolutive des changements climatiques (A) qui a été renforcé par l'apport de mesures diverses (B).

\footnotetext{
${ }^{168}$ Extrait du deuxième $\left(2^{\mathrm{e}}\right)$ paragraphe de l'introduction de la Déclaration de Rio sur l'Environnement et le Développement. Principes de gestion des forêts, adoptée au cours du Sommet planète terre de Rio de Janeiro, tenu au Brésil le 3-14 juin 1992.

${ }^{169}$ Stefan C. Aykut et Ami Dahan, 2014, Gouverner le climat ? 20 ans de négociations internationales, Paris, SciencesPo Les Presses, p.17.
} 


\section{A- L'application de la CCNUCC ${ }^{170}$}

Après l'adoption de la Convention Cadre des Nations Unies sur les Changements Climatiques entrée en vigueur en 1994, le second rapport du GIEC publié en 1995 avait révélé des situations catastrophiques en vue pour l'humanité. Les conclusions dudit rapport faisaient état des perturbations futures ${ }^{171}$. Selon les informations contenues dans ce rapport, l'aggravation des changements climatiques proviendrait d'une absence de politique rigoureuse d'atténuation des émissions des gaz à effet de serre ${ }^{172}$. Il s'ensuivrait un accroissement considérable de gaz à effet de serre dans l'atmosphère avec pour conséquence, « un forçage radiatif positif du climat qui tend à réchauffer la surface du globe et à produire d'autres changements climatiques $\gg{ }^{173}$. Cette tendance alarmiste d'aggravation du phénomène avait été confirmée par le Programme des Nations Unies pour le Développement (PNUD) ${ }^{174}$, qui avait aussi conclu dans son rapport qu' "à long terme, les changements climatiques représentent une menace grave pour le développement de l'humanité et à certains endroits ils mettent déjà en danger les efforts déployés par la Communauté internationale en vue de réduire la pauvreté extrême» ${ }^{175}$. Selon certains analystes, l'intensité des gaz à effet de serre émise dans l'atmosphère aura pour conséquence, «l'augmentation des températures qui va entraîner le renforcement du cycle hydrologique, d'où un risque d'aggravation des sécheresses et/ou des inondations à certains endroits et une possibilité de diminution de l'ampleur de ces phénomènes à d'autres endroits. Plusieurs modèles prévoient une augmentation de l'intensité des précipitations; ce qui pourrait conduire à des épisodes pluvieux plus extrêmes (...) ${ }^{176}$.

Face à ces informations gravissimes, les Parties à la Convention Cadre des Nations Unies sur les Changements Climatiques n'avaient de choix que de recourir à l'application rigoureuse de ladite Convention. La Convention Cadre des Nations Unies sur les Changements

\footnotetext{
${ }^{170}$ C'est un sigle qui signifie Convention Cadre des Nations Unies sur les Changements Climatiques.

${ }^{171}$ Deuxième $\left(2^{\mathrm{e}}\right)$ rapport du GIEC élaboré en 1995, document de synthèse des informations scientifiques et techniques relatives à l'interprétation de l'article 2 de la Convention Cadre des Nations Unies sur les Changements Climatiques, paragraphe 2.6, p.5. Disponible sur le site internet : www.ipcc.ch, consulté le 08/06/2021.

${ }^{172} \mathrm{Id}$.

${ }^{173}$ Deuxième $\left(2^{\mathrm{e}}\right)$ rapport du GIEC publié en 1995 : La concentration de Gaz à effet de serre continue d'augmenter, paragraphe 1, p.31. Disponible sur le site internet : www.ipcc.ch, consulté le 08/06/2021.

${ }^{174}$ Le Programme des Nations Unies pour le Développement est une institution spécialisée de l'Organisation des Nations Unies.

${ }^{175}$ PNUD, Rapport mondial sur le développement humain 2007/2008, La lutte contre le changement climatique : un impératif de solidarité humaine dans un monde divisé, Découverte, Paris, 2007, 382p., p.v.

${ }^{176}$ Rapport du GIEC sur l'évolution du climat, seconde évaluation du GIEC, 1995, document de synthèse des informations scientifiques et techniques relatives à l'interprétation de l'article 2 de la Convention Cadre des Nations Unies sur les Changements Climatiques, paragraphe 2.11, page 5. Disponible sur le site internet: www.ipcc.ch. Consulté le 08/06/2021.
} 
Climatiques a prévu des dispositions pour son amélioration. C'est pourquoi, elle a laissé une porte ouverte sur l'avenir pour faciliter la prise en compte et l'intégration des situations nouvelles en vue de parfaire l'œuvre de construction du régime climatique jusqu'à ce que les objectifs de lutte contre les changements climatiques soient atteints par la «consécration des résultats ${ }^{177}$. C'est pourquoi, la convention a initié le principe de négociations permanentes à travers l'organisation anale des Conférences des Parties $(\mathrm{COP})^{178}$ et a favorisé la tenue régulière des sessions sur des questions pertinentes et urgentes par la possibilité qu'elle a offerte aux Parties qui peuvent en formuler « la demande par écrit à condition que cette demande soit appuyée par un tiers (1/3) au moins des Parties, dans les six (6) mois qui suivent sa communication aux Parties par le secrétariat» ${ }^{179}$. Elle a également prévu des dispositions qui permettent, lorsque les négociations sont ouvertes de pouvoir les entériner soit, par voie d'amendement, car dispose-t-elle que, «Toute Partie peut proposer des amendements à la Convention $»^{180}$, soit par l'adoption et l'amendement d'annexes de la Convention ${ }^{181}$. La Communauté internationale qui, face à la nécessité de prendre des mesures nouvelles pour contrer les émissions de gaz à effet de serre suivant les perspectives projetées dans le second rapport du GIEC $^{182}$ a adopté à l'occasion de la $3^{\mathrm{e}}$ Conférence des Parties (COP3) tenue à Kyoto au Japon ${ }^{183}$ en décembre 1997 un protocole additionnel à la Convention.

Ce traité désigné sous le vocable «Protocole de Kyoto » est entré en vigueur le 16 février 2005 pour la première période d'engagement allant de 2008 à $2012^{184}$. Le Protocole de Kyoto a fixé des engagements chiffrés en matière de «limitation et de réduction des émissions en vue de réduire le total de leurs émissions d'au moins $5 \%$ par rapport au niveau de 1990 au cours de la période d'engagement» ${ }^{185}$. Le traité de Kyoto n'est pas revêtu « d'obligation de

\footnotetext{
${ }^{177}$ Michel PRIEUR, Droit de l'environnement, Paris, Dalloz, 8 éd., 2019, 1394p., p. 9.

${ }^{178}$ Selon les dispositions de la l'article 7 paragraphes 4 et 5 de la Convention Cadre des Nations Unies sur les Changements Climatiques, la Conférence des Parties, organe suprême de la Convention tient une session ordinaire par an. Mais elle peut tenir également des sessions extraordinaires à tout moment qu'elle juge nécessaire, ou lorsqu'une Partie en fait la demande par écrit appuyée par un tiers (1/3) au moins des Parties, dans les six (6) mois qui suivent sa communication aux Parties par le Secrétariat.

${ }^{179}$ Article 7 paragraphe 5 de la Convention Cadre des Nations Unies sur les Changements Climatiques.

${ }^{180}$ Article 15 paragraphe 1 de la Convention Cadre des Nations Unies sur les Changements Climatiques.

${ }^{181}$ L'adoption du protocole de Kyoto a été facilitée par la mise en œuvre des dispositions de l'article 16 de la Convention Cadre des Nations Unies sur les Changements Climatiques.

${ }^{182}$ Ces visions projetées sont inscrites aux pages 16 et 17 du deuxième $\left(2^{\mathrm{e}}\right)$ rapport du GIEC de 1995 . Disponible sur le site internet : www.ipcc.ch, consulté le 08/06/2021.

${ }^{183}$ Le Protocole de Kyoto a été adopté le 11 décembre 1997 à l'occasion de la COP3 par la Décision 1/CP.3. Disponible sur le site internet: https://unfccc.int/decisions?f\%5B0\%5D=session $\% 3 \mathrm{~A} 3607$. Consulté le 04/01/2022.

${ }^{184}$ La période d'engagement est fixée à l'article 3 du Protocole de Kyoto.

${ }^{185}$ Article 3 paragraphe 1 du Protocole de Kyoto.
} 
moyen ${ }^{186}$. Les Etats développés pollueurs qui ont la responsabilité de l'acquittement des charges de réduction des émissions de gaz à effet de serre sont libres de choisir les moyens convenables pour y parvenir. Pour faciliter la mise en œuvre du Protocole de Kyoto, il a été inventé des mécanismes dits de «flexibilité » tels que : la Mise en Oeuvre Conjointe ou l'Application conjointe (MOC) ${ }^{187}$ et le Mécanisme du Développement Propre (MDP) ${ }^{188}$. Ces divers mécanismes devront à terme, contribuer à la réduction des émissions des gaz à effet de serre dans les Etats développés par l'exécution dans les pays en voie de développement des projets d'absorption et de réduction des gaz à effet de serre. Malgré ces innovations contenues dans le Protocole de Kyoto, S. Maljean Dubois qui a fait l'analyse de son application en est venue à conclure que, c'est un « instrument qui contient des obligations plus précises et plus contraignantes que la Convention Cadre des Nations Unies sur les Changements Climatiques $»^{189}$.

Cependant, le Protocole de Kyoto établi par la Communauté internationale dans le but de renforcer le droit climatique n'a pas comblé les attentes. Il n'a pas reçu l'adhésion de certaines puissances pollueuses telles que les Etats-Unis d'Amérique qui l'ont signé le 12 novembre 1998, mais se sont abstenus de le ratifier ${ }^{190}$ au motif que les grands pollueurs tels que la Chine et l'Inde n'ont pas pris suffisamment d'engagement. Le Canada qui avait adhéré audit Protocole, s'est désengagé en $2011^{191}$. En somme, les pays qui sont assujettis au Protocole de Kyoto ne représentent qu'une infirme masse des émissions mondiales de gaz à effet de serre $^{192}$. Selon l'avis de certains chercheurs, « le Protocole de Kyoto à la Convention Cadre des Nations Unies sur les Changements Climatiques a introduit le régime international sans aucun

\footnotetext{
${ }^{186}$ De Perthuis, C., Et pour quelques degrés de plus ... Nos choix économiques face au risque climatique. Paris: Pearson Education France, 2009, p.63.

${ }^{187}$ Ce mécanisme est prévu par l'article 6 du Protocole de Kyoto.

${ }^{188}$ Ce mécanisme est prévu par l'article 12 du Protocole de Kyoto et mis en œuvre par Décision 17/CP.7, portant modalités et procédures d'application d'un mécanisme du développement propre tel que défini à l'article $12 \mathrm{du}$ Protocole de Kyoto, FCCC/CP/2001/13/Add.2.

${ }^{189}$ Maljean-Dubois Sandrine. La mise en route du Protocole de Kyoto à la Convention Cadre des Nations Unies sur les Changements Climatiques. In: Annuaire français de droit international, volume 51, 2005. pp. 433-463, p.434.

${ }^{190}$ Etat de ratification du Protocole de Kyoto au : 03-05-2020 05:01:03EDT disponible sur le site internet https://treaties.un.org. Consulté le 08/06/2021.

${ }^{191}$ Alors que la conformité de ce retrait avec le droit interne canadien a été confirmée dans l'affaire Daniel Turp c. Ministre de la Justice et Procureur Général du Canada, 2012 CF 893. Disponible sur le site internet https://climatecasechart-com.translate.goog/non-us-case/turp-v-minister-of-justice-and-attorney-general-ofcanada. Consulté le 04/01/2022.

192 Pour défaut de ratification par la Chine, la Russie, le Japon, le Canada et les Etats-Unis d'Amérique, seuls 15\% des émissions mondiales de gaz à effet de serre sont prises en compte par le Protocole de Kyoto. Information disponible sur le site internet https://www.geo.fr/environnement/protocole-de-kyoto-enjeux-et-bilan-193595. Consulté le 19/05/2021.
} 
doute le plus abouti et le plus original pour la gestion d'un bien commun ${ }^{193}$. Ce régime climatique selon le Professeur Alexandre Kiss, s'est révélé comme une « complexité déroutante $»{ }^{194}$. Alors que d'autres voix discordantes constataient que « le régime du climat composé de la Convention Cadre des Nations Unies sur les Changements Climatiques et le Protocole de Kyoto a montré son insuffisance ${ }^{195}$ malgré les prouesses réalisées.

Avant l'expiration de la première période d'engagement du Protocole de Kyoto prévue pour le 08 décembre $2012^{196}$, les négociations pour la conclusion d'un Accord pour la deuxième période d'engagement comptant pour la période de 2013 à 2020 ont été entreprises. Ces négociations entamées à Copenhague en $2009^{197}$ ont été officialisées au cours du Sommet de Cancun au Mexique en $2010^{198}$ et ce n'est qu'au cours du Sommet de Doha à Katar en décembre 2012 à l'occasion de la COP18 que l'Accord sur la deuxième période d'engagement a été adopté $^{199}$. L'aboutissement de ce processus a permis d'engager de nouvelles négociations entre les Parties à la Convention Cadre des Nations Unies sur les Changements Climatiques pour « élaborer au titre de la Convention un protocole, un autre instrument juridique ou un texte convenu d'un commun accord ayant valeur juridique, applicable à toutes les Parties» ${ }^{200}$ qui entrera en vigueur à partir de 2020. Ces négociations on été conduites par le « Groupe de travail spécial de la plate-forme de Durban pour une action renforcée ${ }^{201}$ et ont abouti à l'adoption de l'Accord de Paris le 12 décembre $2015^{202}$.

En face de l'enjeu des changements climatiques, l'adoption de l'Accord de Paris à la COP21 a été pour beaucoup une prouesse. Cet Accord contraint la Communauté internationale à s'engager dans une démarche de réduction substantielle des émissions de gaz à effet de serre.

\footnotetext{
${ }^{193}$ Maljean-Dubois Sandrine. Environnement, développement durable et droit international. De Rio à Johannesburg: et au-delà ?. In: Annuaire français de droit international, volume 48, 2002. pp. 592-623, p.595.

${ }^{194}$ A. Kiss, « Cinq années de droit international de l'environnement », RJE, n 4/2001, p. 573.

195 Sandrine Maljean-Dubois, Rajamani Lavanya. L'Accord de Paris sur les changements climatiques du 12 décembre 2015, Annuaire Français de Droit International, CNRS, 2017.

${ }^{196} \mathrm{La}$ première période d'engagement est fixée par les dispositions de l'article 3 paragraphe 1 du protocole de Kyoto. Ledit Protocole est entré en vigueur le 16 février 2005.

197 A l'occasion de la tenue de la COP15.

198 A l'occasion de la tenue de la COP16.

${ }^{199}$ Par décision 1/CMP.8 sur l'amendement de Doha au Protocole de Kyoto conformément au paragraphe 9 de son article 3. Disponible sur le site internet https://reaties.un.org/doc/Publication/MTDSG/Volume\%. Consulté le 08/06/2021.

${ }^{200}$ Nations Unies, Convention Cadre des Nations Unies sur les Changements Climatiques, Conférence des Parties Rapport de la Conférence des Parties sur sa dix-septième session, tenue à Durban du 28 novembre au 11 décembre 2011, Décisions adoptées par la Conférence des Parties, Décision 1/CP.17 Création d'un groupe de travail spécial de la plate-forme de Durban pour une action renforcée, paragraphe 2. Disponible sur le site internet: https://unfccc.int/resource/docs/2011/cop17/fre/09a01f.pdf. Consulté le 04/01/2022. ${ }^{201}$ Id.

${ }^{202}$ Décision 1/CP.21 relative à l'adoption de l'Accord de Paris. Disponible sur le site internet https://unfsss.int. Consulté le 08/06/2021.
} 
L'Accord de Paris conclu et ratifié par les cent quatre-vingt-dix (190) sur cent quatre vingt quatorze (194) Parties à la Convention Cadre des Nations Unies sur les Changements Climatiques $^{203}$, «vise à renforcer la riposte mondiale à la menace des changements climatiques $»^{204}$ en engageant tous les Etats à travailler pour contenir l'élévation de la température mondiale dans une proportion qui empêche toute perturbation du système planétaire. Cet accord est caractérisé par la flexibilité de ses dispositions ${ }^{205}$, car il met en évidence, « les systèmes d'engagements volontaires suffisamment ambitieux et respectueux de la souveraineté étatique $»^{206}$, contrairement aux engagements juridiquement contraignants du Protocole de Kyoto. L'Accord de Paris a remplacé dès 2020 le prolongement du Protocole de Kyoto $^{207}$. En effet, son application rigoureuse permettra de contenir le réchauffement climatique bien en dessous de $2^{\circ} \mathrm{C}$, voire le limiter à $1,5^{\circ} \mathrm{C}^{208}$. Pour atteindre cet objectif, l'Accord exige que toutes les Parties consacrent leurs efforts dans la réalisation des «contributions déterminées au niveau national» ${ }^{209}$. La COP21 a été l'occasion donnée aux Parties de prendre des engagements dans la limite de leur responsabilité.

En plus du bloc conventionnel sur les changements climatiques ${ }^{210}$, il y a des mesures diverses qui sont également élaborées et mises à contribution dans le cadre de la lutte contre les changements climatiques.

\section{B- Les diverses contributions}

Bien avant l'entrée en vigueur de la Convention Cadre des Nations Unies sur les Changements Climatiques, il apparaissait un phénomène nouveau de réchauffement planétaire. Ce n'était pas une élévation de température à laquelle était habituée l'humanité. Selon les recherches, l'augmentation de la température de la planète était provoquée par la dégradation

\footnotetext{
${ }^{203}$ L'état de ratification de l'Accord de Paris disponible sur le site internet : https://treaties.un.org/. Consulté le $19 / 05 / 21$

${ }^{204}$ Cinquième $\left(5^{\mathrm{e}}\right)$ paragraphe du préambule de l'Accord de Paris adopté en 2015.

${ }^{205}$ YVES Petit, Rapport introductif « La lutte contre le réchauffement climatique après l'Accord de Paris : quelles perspectives? ", La revue électronique en sciences de l'environnement, Volume 18 numéro1, mai 2018, p.2.

${ }^{206}$ YVES Petit, Rapport introductif « La lutte contre le réchauffement climatique après l'Accord de Paris : quelles perspectives? », VertigO - la revue électronique en sciences de l'environnement, Volume 18 numéro 1, mai 2018, p.5.

${ }^{207}$ Nations Unies, Convention Cadre des Nations Unies sur les Changements Climatiques, Conférence des Parties Rapport de la Conférence des Parties sur sa dix-huitième session, tenue à Doha du 26 novembre au 8 décembre 2012, Deuxième partie: Mesures prises par la Conférence des Parties à sa dix-huitième session, Décisions adoptées par la Conférence des Parties, Décision 2/CP.18: Faire avancer la plate-forme de Durban, Paragraphe 4. Disponible sur le site internet https://unfccc.int/resource/docs/2012/cop18/fre/08a01f.pdf. Consulté le 08/06/2021. ${ }^{208}$ Article 2 paragraphe 1-a de l'Accord de Paris de 2015.

${ }^{209}$ Article 3 de l'Accord de Paris.

${ }^{210}$ Il est constitué de la Convention Cadre des Nations Unies sur les Changements Climatiques et les accords subséquents.
} 
de la couche d'ozone ${ }^{211}$. Cette atteinte à la couche d'ozone se manifestait sous forme d'amincissement de ladite couche, causé par certaines substances qui contribuaient à son appauvrissement ${ }^{212}$. Les recherches entreprises ont révélé « l'influence des activités humaines sur la couche d'ozone stratosphérique qui s'est manifestée dès le début des années 1980, principalement au dessus de l'antarctique, où une baisse significative de la quantité totale d'ozone a été observée chaque printemps $»^{213}$. A la longue, il a été aussi établi que, «le changement climatique devrait aussi modifier durablement la couche d'ozone à l'échelle mondiale» ${ }^{214}$

A partir du moment où la preuve a été établie de l'imminente perturbation des conditions d'existence de l'homme sur Terre à cause des conséquences fâcheuses qui pourraient découler de la situation de la dégradation de la couche d'ozone, certains Etats se sont investis dans la recherche de solutions visant à sauvegarder l'intégrité de la couche d'ozone par l'élimination progressive des substances qui lui sont nuisibles. En effet, l'utilisation des $\mathrm{CFC}^{215}$ comme gaz propulseur dans les aérosols a été proscrite en 1978 par certains Etats tels que, le Canada, la Norvège et les Etats-Unis d'Amérique. Néanmoins, il fallait consentir plus d'effort en interdisant les CFC et même les hydro chlorofluorocarbones (HCFC) pour voir régler de manière durable le problème de la couche d'ozone. C'est pourquoi, le droit international avait

\footnotetext{
${ }^{211}$ Selon l'article 1 paragraphe 1 de la Convention de Vienne pour la protection de la couche d'ozone, par "couche d'ozone" on entend la couche d'ozone atmosphérique présente au-dessus de la couche limite de la planète.

${ }^{212}$ Ces substances dénommées «Substances Appauvrissant la Couche d'Ozone» sont réglementées par le Protocole de Montréal relatif à des substances qui appauvrissent la couche d'ozone tel qu'ajusté et amendé par la deuxième Réunion des Parties (Londres, 27-29 juin 1990) et la quatrième Réunion des Parties (Copenhague, 2325 novembre 1992), ajusté de nouveau par la septième Réunion des Parties (Vienne, 5-7 décembre 1995), et encore ajusté et amendé par la neuvième Réunion des Parties (Montréal, 15-17 septembre 1997) et par la onzième Réunion des Parties (Beijing, 29 novembre - 3 décembre 1999) et encore ajusté par la dix neuvième Réunion des Parties (Montréal, 17- 21 septembre 2007) et encore amendé par la vingt-huitième Réunion des Parties (Kigali, 10-15 octobre 2016). Il s'agit de : CFC (article 2A) ; Halons (article 2B); Autres CFC entièrement halogénés (article 2C); Tétrachlorure de Carbone (article 2D); trichloroéthane ou méthyle chloroforme (article 2E); Hydrochlorofluorocarbones (article 2F); Hydrobromofluorocarbones (article 2G) ; Bromure de méthyle (article 2H) ; Bromochlorométhane (article 2I) et Hydrofluorocarbones (article 2J).

${ }^{213}$ Cellule Etat de l'Environnement wallon (2007) : Rapport analytique sur l'état de l'environnement wallon 20062007, MRW, DGRNE, Namur, 736 p. Disponible sur le site internet: http://etat.environnement.wallonie.be/files/Publications/Rapport. Consulté le 08/06/2021.

${ }^{214}$ Staehelin ,J., F. Tummon, A. Stenke, C. Arndt et P. Bratfisch, « Modifications de la couche d'ozone suite au changement climatique », SPARC Faktenblatt, 2014, p.4.

${ }^{215}$ En 1974, Rowland et Molina publient dans Nature un article très remarqué sur la menace que représentent pour la couche d'ozone les chlorofluorocarbures (C.F.C.), composés employés en grandes quantités dans les circuits de réfrigération et dans les bombes à aérosols. Ils montrent que les C.F.C., après leur libération dans l'atmosphère, sont progressivement concentrés dans la couche d'ozone. Sous l'influence du rayonnement ultraviolet important, ils s'y décomposent en donnant notamment des atomes de chlore qui vont accélérer la destruction de la couche d'ozone stratosphérique. Les calculs de Molina et de Rowland montrent que, si aucun frein n'est apporté à l'utilisation des C.F.C., la couche d'ozone pourrait disparaître en quelques dizaines d'années. Leurs travaux ont été à la base des mesures de restrictions de l'usage des C.F.C. prises à la fin des années 1970 et au début des années 1980, puis concrétisées par l'adoption du protocole de Montréal en 1987.
} 
été sollicité pour mettre à disposition des normes d'envergure planétaire pour conjurer le mal. C'est dans ce contexte qu'a été élaborée la Convention sur la protection de la couche d'ozone, laquelle Convention a été adoptée à Vienne le 22 mars 1985 et entrée en vigueur le 22 septembre $1988^{216}$. Selon les dispositions conventionnelles, il était question que « les Parties prennent des mesures (...) pour protéger la santé humaine et l'environnement contre les effets néfastes résultant ou susceptibles de résulter des activités humaines qui modifient ou sont susceptibles de modifier la couche d'ozone $»^{217}$. De manière concrète, la Convention de Vienne a créé un cadre de coopération internationale pour contribuer à l'éradication des substances qui appauvrissent la couche d'ozone. Ces substances seront substituées par des «substances de remplacement $»^{218}$, c'est-à dire, des substances qui « réduisent, éliminent ou évitent les effets néfastes sur la couche d'ozone $»^{219}$. Ainsi les Parties pouvaient en application de ladite Convention, prendre des mesures législatives ou réglementaires pour encadrer les comportements humains susceptibles d'impacter négativement la couche d'ozone ${ }^{220}$.

Néanmoins, la Convention de Vienne sur la protection de la couche d'ozone adoptée en 1985 a présenté des limites et n'a pas permis de combler les attentes en ce qui concerne l'objectif de protection de la couche d'ozone par l'éradication des substances qui lui sont nuisibles. C'est dans le but de palier cette faiblesse qu'a été adopté le Protocole de Montréal relatif aux substances qui appauvrissent la couche d'ozone, un prolongement de la Convention de Vienne. Ce protocole contient des règles précises et contraignantes pour les Etats membres. Son objectif consiste à réduire et à terme à éliminer complètement les substances qui contribuent à la dégradation de la couche d'ozone. L'état des ratifications renseigne que cent quatre vingt-seize (196) pays sont signataires du Protocole de Montréal, entré en vigueur le $1^{\text {er }}$ janvier 1989. Néanmoins, ce Protocole a subi maintes ajustement en vue de son amélioration jusqu'au 15 octobre $2016^{221}$. Les adhésions massives à l'outil juridique ont permis d'élever ce Traité au rang du premier protocole environnemental à atteindre la ratification universelle ${ }^{222}$.

\footnotetext{
${ }^{216}$ Conformément au paragraphe 1 de l'article 17 de ladite Convention.

${ }^{217}$ Article 2 de la Convention de Vienne du 22 mars 1988.

${ }^{218}$ Article 1 paragraphe 4 de la Convention de Vienne sur la protection de la couche d'ozone. ${ }^{219} \mathrm{Id}$.

${ }^{220}$ Article 2 paragraphe 1 de la Convention de vienne sur la protection de la couche d'ozone.

${ }^{221}$ Le Protocole de Montréal relatif à des substances qui appauvrissent la couche d'ozone a été ajusté et amendé par la deuxième Réunion des Parties (Londres, 27-29 juin 1990) et la quatrième Réunion des Parties (Copenhague, 23-25 novembre 1992), ajusté de nouveau par la septième Réunion des Parties (Vienne, 5-7 décembre 1995), et encore ajusté et amendé par la neuvième Réunion des Parties (Montréal, 15-17 septembre 1997) et par la onzième Réunion des Parties (Beijing, 29 novembre - 3 décembre 1999) et encore ajusté par la dix neuvième Réunion des Parties (Montréal, 17- 21 septembre 2007) et encore amendé par la vingt-huitième Réunion des Parties (Kigali, 10-15 octobre 2016).

${ }^{222}$ Le Protocole de Montréal relatif à des substances qui appauvrissent la couche d'ozone adopté le, 16 septembre 1987 et entré en vigueur le, $1^{\text {er }}$ janvier 1989, conformément au paragraphe 1 de l'article 16 a été ratifié par cent
} 
Cet instrument qui est intervenu pour renforcer le dispositif de protection de la couche d'ozone à travers la règlementation de certaines substances ${ }^{223} \mathrm{a}$ «graduellement interdit l'usage des gaz fluorés de la famille des CFC et HCFC, utilisés dans les vaporisateurs, les climatiseurs et dans l'industrie du froid $»^{224}$.

La mise en auvre de la Convention de Vienne relative à la protection de la couche d'ozone et le Protocole de Montréal sur les substances appauvrissant la couche d'ozone, concoure-t-elle aux objectifs de la lutte contre les changements climatiques?

Pour démontrer l'importance du Protocole de Montréal sur les substances appauvrissant la couche d'ozone, Erik Solheim ${ }^{225}$, le chef de l'ONU Environnement ${ }^{226}$ affirmait que « Le protocole de Montréal est aussi important aujourd'hui qu'il l'était dans les années 80, il est important non seulement pour la couche d'ozone mais aussi comme partie intégrante des efforts pour freiner le changement climatique (...)»» ${ }^{227}$. Ce message dénote d'un lien qui existe entre la campagne d'éradication des substances appauvrissant la couche d'ozone et la lutte contre les changements climatiques. L'adoption de la Convention Cadre des Nations Unies sur les Changements Climatiques, dont l'objectif finaliste est d'empêcher le réchauffement de la planète, est un instrument qui complète d'autres instruments similaires qui déjà, sont mis à contribution pour protéger le climat. Le texte de la Convention Cadre des Nations Unies sur les Changements Climatiques en a fourni les preuves, car les dispositions de ladite Convention n'ont pas occulté l'existence d'anciens instruments juridiques qui concourent à la protection du climat notamment, la Convention de Vienne de 1985 sur la protection de la couche d'ozone et le Protocole de Montréal de 1987 relatif à des substances qui appauvrissent la couche d'ozone, ajusté et modifié le 29 juin 1990 qu'elle a rappelé dans certaines dispositions ${ }^{228}$. Mais en plus, la Convention Cadre des Nations Unies sur les Changements Climatiques en délimitant son champ d'application a précisé que les engagements auxquels les Parties sont astreintes dans le cadre de l'atténuation de l'émission des gaz à effet de serre ne concernent que ceux des gaz non

\footnotetext{
quatre vingt dix-huit (198) Etats Parties. Disponible sur le site internet : https://treaties.un.org/Pages/ViewDetais. Consulté le 05/01/2022.

${ }^{223}$ Ces substances sont inscrites aux annexes A, B, C, D, E et F du Protocole de Montréal relatif à des substances qui appauvrissent la couche d'ozone.

${ }^{224}$ De Perthuis, C. Et pour quelques degrés de plus ... Nos choix économiques face au risque climatique. Paris: Pearson Education France, 2009, 306p., p.80.

${ }^{225}$ Sixième $\left(6^{\mathrm{e}}\right)$ Directeur exécutif d'ONU Environnement et Secrétaire général adjoint de l'Organisation des Nations Unies. Disponible sur le site internet: https:/www.unep.org/fr/people/erik-solheim. Consulté le $05 / 01 / 2022$.

${ }^{226}$ Institution spécialisée du système des Nations unies en matière environnementale.

${ }^{227}$ C'était un extrait de discours prononcé par Erik Solheim à l'occasion des négociations, sur l'amendement au protocole de Montréal en 2016 à Kigali. Disponible sur le site internet : https://www.unep.org/es/node/19321 . Consulté le 05/01/2022.

${ }^{228}$ Paragraphe 14 du préambule de la Convention Cadre des Nations unies sur les Changement Climatiques.
} 
réglementés par le «Protocole de Montréal de $1987 »^{229}$. Il s'ensuit que ces textes qui ont précédés la Convention Cadre des Nations Unies sur les Changements Climatiques, concourent à l'objectif finaliste de lutte contre les changements climatiques.

Pour convenir de la nécessité d'une contribution plurielle à l'encadrement des changements climatiques, le GIEC a établi dans son rapport d'évaluation des changements climatiques que, "les Experts se sont accordés à estimer que les instruments juridiques et institutionnels qui concernent les changements climatiques doivent être pleinement utilisés et encore renforcés ${ }^{230}$, cependant, ces instruments " ne peuvent à eux seuls suffire pour faire face au problème $»^{231}$. D'où la nécessité de recourir à d'autres instruments élaborés en dehors du contexte de la Convention Cadre des Nations Unies sur les Changements Climatiques.

Dans ces conditions, sous un autre angle, l'importance de l'apport des aires protégées dans la lutte contre les changements climatiques est à rappeler. Selon une étude de l'Union Internationale pour la Conservation de la Nature (UICN), une organisation internationale à caractère associative qui intervient dans le domaine de la protection de l'environnement ${ }^{232}$, «Les aires protégées peuvent contribuer aux deux principales réponses aux changements climatiques. Pour ce qui concerne l'atténuation, les aires protégées contribuent au stockage et au captage du carbone, et s'agissant de l'adaptation, elles participent à la protection et au maintien de l'intégrité des écosystèmes» ${ }^{233}$. Il s'ensuit qu'elles ont une grande capacité d'emmagasinage de stock de carbone. La même étude révèle que, «Les aires protégées sont une partie intégrante de la réponse globale aux changements climatiques. Elles permettent d'attaquer la source des changements climatiques en réduisant les émissions de gaz à effet de serre. Elles aident la société à s'adapter aux effets des changements climatiques en sauvegardant les services essentiels dont les populations dépendent. Sans elles, le défi serait encore plus important et leur renforcement fournira une solution naturelle particulièrement efficace pour faire face à la crise du climat $»^{234}$. Elles participent à la conservation des ressources naturelles et leur permet de résister aux conséquences néfastes des changements

\footnotetext{
${ }^{229}$ Article 4 paragraphe1-a-b-c-d de la Convention Cadre des Nations Unies sur les Changements Climatiques.

${ }^{230}$ Le paragraphe 7.5.1 du premier rapport (1990 à 1992) du Groupe d'Experts Intergouvernemental sur l'Evolution du Climat. Disponible sur le site internet : https://www.ipcc.ch/site/assets. Consulté le 05/01/2022. ${ }^{231} \mathrm{Id}$.

${ }^{232}$ Voir infra, rubrique B- L'expérience de l’UICN, pp.68-72.

${ }^{233}$ Rapport : « Solutions Naturelles - Les aires protégées nous aident à faire face aux changements climatiques » produit par l'UICN-CMAP, TNC, le PNUD, WCS, la Banque mondiale et le WWF, p.15. Sur le site internet : https://www.iucn.org/sites/dev/files/import/downloads/natural_solutions_summary. Consulté le 08/06/2021.

${ }^{234}$ Rapport : "Solutions Naturelles - Les aires protégées nous aident à faire face aux changements climatiques » produit par l'UICN-CMAP, TNC, le PNUD, WCS, la Banque mondiale et le WWF, p.15. Sur le site internet : https://www.iucn.org/sites/dev/files/import/downloads/natural_solutions_summary. Consulté le 08/06/2021.
} 
climatiques. Par ces multiples fonctions, les aires protégées participent à l'atténuation des émissions de gaz à effet de serre et à l'adaptation aux effets des changements climatiques. Il s'ensuit que le domaine des changements climatiques bénéficiait d'un encadrement international qui ne date pas de maintenant comme en témoigne les plus anciennes conventions qui régissent cette matière. Selon Stéphane DOUMBE-BILLE, «le droit international applicable à la faune et aux aires protégées constitue sous ses multiples aspects, l'un des plus anciens, les plus riches et les plus caractéristiques du Droit International de l'Environnement ». ${ }^{235}$ Bien avant l'avènement de la conférence de Stockholm de 1972 sur l'environnement, il existait déjà des instruments juridiques internationaux de conservation des aires protégées. Par exemple, la Convention de Londres visant à garantir la conservation de diverses espèces d'animaux sauvages d'Afrique utiles à l'homme ou inoffensives, adoptée en 1900 et la Convention relative à la conservation de la faune et de la flore à l'état naturel, signée à Londres en 1933.

Le droit des aires protégées a été renforcé à l'occasion de la conférence de Stockholm de 1972. De nombreux engagements internationaux ont été pris pour le développement des aires protégées car la déclaration issue de cette Conférence a approuvé la protection des «échantillons représentatifs des écosystèmes naturels ${ }^{236}$ comme une exigence fondamentale des programmes nationaux de conservation. Plus tard, d'autres instruments non contraignants comme, la Charte Mondiale de la Nature de 1982, les Déclarations de Rio de 1992 et la Déclaration de Johannesburg de 2002 ont soutenu cet effort de protection.

En dépit des contributions massives et qualitatives à la lutte contre les changements climatiques, ce domaine ne saurait être la chasse gardée ou le domaine de monopole des seuls acteurs du Droit International des Changements Climatiques. Compte tenu de son immensité, et de sa complexité, d'autres voix plus autorisées, mais pas celles des professionnels du Droit de l'Environnement s'invitent dans le débat en apportant à leur manière des contributions à l'édification de ce « Droit ».

\footnotetext{
${ }^{235}$ Stéphane DOUMBE-BILLE, Droit international de la faune et des aires protégées : importance et implications pour l'Afrique, Etudes juridiques de la FAO en ligne, n²0, septembre 2001, p. 5.

${ }^{236}$ Principe 2 de la Déclaration de Stockholm de 1972.
} 


\section{SECTION II: LA CONTRIBUTION D'ACTEURS EXTERIEURS}

Le concept de « démocratie participe ${ }^{237}$ innové à l'occasion du Sommet de Rio de Janeiro en 1992 a permis d'ouvrir le secteur de protection de l'environnement aux acteurs extérieurs audit domaine. Cette possibilité d'intervention de nouveaux acteurs a été consacrée dans la Déclaration de Rio de Janeiro de 1992 sur l'Environnement et le Développement, qui stipule que «La meilleure façon de traiter les questions d'environnement est d'assurer la participation de tous les citoyens concernés, au niveau qui convient (...) » ${ }^{238}$. Le même texte concède à chaque individu, " (...) la possibilité de participer aux processus de prise de décision (...) $»^{239}$. Mais en plus, l'Accord de Paris, un cadre multilatéral renouvelé, de lutte contre les changements climatiques, a fait du Droit climatique, un « Droit de la totalité de son projet» ${ }^{240}$, parce que la mise en œuvre de ses dispositions « vise la totalité des Etats, la totalité des espèces et la totalité des éléments constitutifs du milieu de vie ${ }^{241}$. Désormais, aux côtés des Etats et Organisations internationales, se trouvent engagés dans le processus d'élaboration et de mise en œuvre du Droit international des Changements Climatiques, des acteurs non étatiques tels que les institutions internationales non gouvernementales, les organisations de la société civile, les acteurs du secteur privé et les organisations non gouvernementales. Ces nouveaux acteurs contribuent au développement du Droit international de l'Environnement avec les moyens dont ils disposent.

L'approche qui consiste à faire participer plusieurs catégories d'acteur à la conception et à la mise en cuvre du Droit international de l'Environnement n'est-elle pas une exigence de la matière?

Le Droit de l'Environnement apparaît aux yeux de nombreux chercheurs comme une notion complexe, c'est ce que confirme le Professeur Michel PRIEUR qui, en s'intéressant à l'aspect de la complexité de la matière déclare que, «compte tenu de son étroite dépendance par rapport aux sciences et à la technologie, sa compréhension exige un minimum de connaissance scientifique et toute réflexion critique à son propos impose une approche

\footnotetext{
${ }^{237}$ Principe 10 de la Déclaration de Rio de Janeiro sur l'Environnement et le Développement de 1992.

${ }^{238}$ Selon le principe 10 de Déclaration de Rio de Janeiro sur l'Environnement et le Développement, principes de gestion des forêts. SOMMET PLANETE TERRE Conférence des Nations unies sur l'environnement et le développement Rio de Janeiro, Brésil 3-14 juin 1992. Disponible sur le site internet: https://www.un.org/french/events/rio92. Consulté le 05/01/2022.

${ }^{239} \mathrm{Id}$.

${ }^{240}$ YVES Petit, Rapport introductif « La lutte contre le réchauffement climatique après l'Accord de Paris : quelles perspectives ? », La revue électronique en sciences de l'environnement, Volume 18 numéro1, mai 2018, p.8.

${ }^{241}$ Maurice Kamto, «Singularité du droit international de l'environnement », in Mélanges en hommage à Alexandre Kiss, les hommes et l'environnement, Editions Frison-Roche, 1998, p. 315 et s.
} 
pluridisciplinaire dans laquelle sont intégrées la biologie, la géographie, la géologie, la climatologie, l'océanologie, etc.» ${ }^{242}$ A plus forte raison, la gestion controversée des changements climatiques commande également que toute forme d'expertise et moyens adéquats soient mis à contribution pour enrayer le phénomène. Alors que ces nouveaux acteurs du Droit international de l'Environnement disposent de la compétence, des moyens techniques, mais en plus, des surfaces financières importantes qui peuvent être mis à contribution. En face de la complexité et de la densité que revêt la gestion des changements climatiques, les acteurs extérieurs sont recherchés dans le cadre de la mise en œuvre du Droit. Leur incursion dans le secteur environnemental a progressivement contribué à «craquer le moule traditionnel du Droit International classique ${ }^{243}$.

Actuellement, certains acteurs extérieurs au domaine environnemental participent à la mise en œuvre du Droit international du Climat (Paragraphe 2), de même, ils en existent qui influencent son élaboration (Paragraphe 1).

\section{Paragraphe 1: L'influence sur l'élaboration du droit}

Certaines institutions internationales sont présentes dans le domaine de la protection de l'environnement. C'est le cas de l'Organisation des Nations Unies (ONU) qui de par son pouvoir décisionnel intervient dans le processus d'encadrement des changements climatiques (A). De même sa structure technique, le Groupe d'Experts Intergouvernemental sur l'Evolution du Climat (GIEC) qui a compétence à évaluer les changements climatiques s'illustre par la qualité de son expertise qu'il apporte dans le cadre de la résolution des problèmes relatifs à la gestion des changements climatiques (B).

\section{A- L'intervention de l'ONU ${ }^{244}$}

L'Organisation des Nations Unies (ONU) a été créée en 1945 pour « maintenir la paix et la sécurité internationale» ${ }^{245}$ dans le monde entier. Elle suscite et utilise la coopération internationale pour réaliser ses objectifs. Son siège est à New-York.

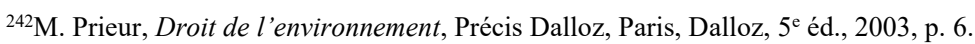

${ }^{243}$ D. Carreau, 1994. Droit international. Paris, Pedone, p. 31.

${ }^{244}$ Voir supra, liste des sigles et abréviations, p.7.

${ }^{245}$ Extrait de l'article 1 point 1 de la Charte des Nations Unies. Disponible sur le site internet: https://www.un.org/fr/about-us/un-charter.Consulté le 05/01/2022.
} 
La mission de l'ONU étant sans équivoque définie comme celle qui vise à établir la paix dans le monde entier, quelles sont les motivations pour lesquelles l'organisation mondiale marque son intervention dans le domaine de la protection de l'environnement?

En invitant l'homme à "faire le point de son expérience » ${ }^{246}$, la Déclaration de Stockholm de 1972 révèle les potentialités que l'Homme possède pour poser des actes utiles à l'humanité par « son pouvoir de transformer le milieu dans lequel il vit ${ }^{247}$, mais à la condition que ce pouvoir soit «(...) utilisé avec discernement $»^{248}$. En conséquence, ce pouvoir "peut apporter à tous les peuples les bienfaits du développement et la possibilité d'améliorer la qualité de la vie ${ }^{249}$. La Déclaration de Stockholm de 1972 a également permis de découvrir d'autres dimensions de l'homme à travers l'usage qu'il fait du pouvoir qu'il possède, qui lui permet de « continuer à découvrir, à inventer, à créer et à avancer $»^{250}$. Néanmoins ce pouvoir positivement décrit dans la Déclaration de Stockholm de 1972 peut s'avérer nuisible pour l'humanité s'il est « utilisé abusivement ou inconsidérément, il peut causer un mal incalculable aux êtres humains et à l'environnement» ${ }^{251}$. L'analyse de ces dispositions révèle la capacité de nuisance de l'homme, qui peut en absence de discernement entrainer l'humanité dans des situations anormales. Dans ce cas, le comportement de l'homme peut constituer une menace pour la paix.

L'ONU ayant pour mission de « Maintenir la paix et la sécurité (...)» ${ }^{252}$ dans le monde entier, se trouve dans l'obligation légitime de poser des actes pour empêcher tout comportement humain qui compromet l'existence de l'homme sur terre. En d'autres termes, l'ONU doit "prendre des mesures collectives efficaces en vue de prévenir et d'écarter les menaces à la paix» ${ }^{253}$. Dès lors, l'Organisation internationale s'investie désormais dans la mission de sécurité pour garantir la paix dans le monde. A la lumière de l'étendue de la mission qui lui est assignée, plusieurs motifs en relation avec la paix ou la sécurité internationale peuvent provoquer l'intervention de l'ONU dans le domaine de la protection de l'environnement. Par exemple, les actes attentatoires à l'environnement susceptibles de provoquer des « troubles géopolitiques, particulièrement en terme de sécurité collective $»^{254}$ peuvent justifier l'intervention de l'ONU

\footnotetext{
${ }^{246}$ Extrait du paragraphe 3 de la Déclaration de Stockholm de 1972.

247 Id.

248 Ibid.

249 Ibid.

${ }^{250}$ Ibid.

251 Ibid.

${ }^{252}$ Extrait du paragraphe1 de l'article 1 de la Charte des Nations unies de San Francisco de 1945.

${ }^{253} \mathrm{Id}$.

${ }^{254}$ Patrick ALLARD, « Malaise dans la climatisation, le changement climatique et la sécurité des États », Annuaire français des relations internationales, no 6, 2005, pp. 942-951.
} 
dans le secteur de la protection de l'environnement. Puisque, la protection de l'environnement est perçue comme un élément des Droits de 1'’Homme, l'ONU ayant pour mission la protection et la promotion des Droits de l'Homme ${ }^{255}$ est également qualifiée pour se saisir des questions relatives à la violation des règles de protection de l'environnement. Enfin, pour ce qui concerne les changements climatiques, il est clairement consigné dans certaines déclarations que les changements climatiques constituent «une menace pour la paix et la sécurité » ${ }^{256}$. La paix et la sécurité internationale relèvent des prérogatives de l'ONU. Puisque les effets pervers des changements climatiques peuvent induire la mise en cause des droits élémentaires des individus, le Conseil des Droits de l'Homme des Nations unies a reconnu que les changements climatiques peuvent produire dans l'avenir des « répercutions sur la jouissance effective des Droits de l'Homme» ${ }^{257}$. Tous ces éléments justifient l'intervention de l'ONU dans le domaine de la protection de l'environnement et celui de la lutte contre les changements climatiques. Mais en plus, les changements climatiques constituent, «l'un des plus grands défis $»^{258}$ pour la Communauté internationale. La Déclaration de Rio de Janeiro de 1992 a d'ailleurs établie une interconnexion entre les trois concepts « paix»; "développement» et «protection de l'environnement » par leurs caractères interdépendants et indissociables ${ }^{259}$.

En effet, l'Organisation des Nations Unies s'est d'abord intéressée à la question de la protection internationale de l'environnement puis après, au fléau que constitue le dérèglement climatique. L'ONU a été à l'origine des Sommets de la Terre à travers l'organisation par décennie des Conférences internationales sur l'Environnement et le Développement ${ }^{260}$. Elle a posé les bases de l'ordonnancement juridique du secteur de l'environnement par l'organisation de la Conférence de Stockholm de 1972. D'ailleurs, c'était la toute première initiative de l'ONU

\footnotetext{
${ }^{255} \mathrm{Au}$ termes de l'article 1 paragraphe 3 de la Charte des Nations Unies, l'ONU a pour mission de réaliser la coopération internationale en résolvant les problèmes internationaux d'ordre économique, social, intellectuel ou humanitaire, en développant et en encourageant le respect des droits de l'homme et des libertés fondamentales pour tous, sans distinctions de race, ce sexe, de langue ou de religion.

${ }^{256}$ Discours prononcé à la Conférence de Nairobi ; Nations Unies, Secrétaire général, SG/SM/10739, 15 novembre 2006. Disponible sur le site internet: https://www.un.org/press/fr/2006/SGT2522.doc.htm.Consulté le $05 / 01 / 2022$.

${ }^{257}$ NATIONS UNIES, Assemblée générale, Conseil des droits de l'homme, Droits de l'homme et changement climatique, A/HRC/7/L.21/Rev.1, 20 mars 2008.

${ }^{258}$ Nations Unies, Secrétaire général, SG/SM/10904, 9 mars 2007. La Convention Cadre des Nations Unies sur les Changements Climatiques reconnait les effets néfastes de ce phénomène sont « un sujet de préoccupation pour l'humanité tout entière ». Cette déclaration confirme la résolution 43/53 du 6 décembre 1988 de l'Assemblée générale des Nations unies qui déclara que le changement climatique constituait une préoccupation commune de l'humanité.

${ }^{259}$ Principe 25 de la Déclaration de Rio de Janeiro de 1992.

${ }^{260} \mathrm{~L}$ 'Organisation des Nations Unies (ONU) intervient par le biais du Programme des Nations Unies pour l'Environnement (PNUE).
} 
en matière de protection de l'environnement, puis sont intervenus après d'autres Sommets ${ }^{261}$ qui sont les chefs d'œuvre de l'ONU. C'est ainsi que, l'organisation des conférences destinées à la protection de l'environnement est devenue une tradition pour la Communauté internationale ${ }^{262}$. En organisant le Sommet de Stockholm de 1972, elle s'est imposée comme l'initiateur du Droit International de l'Environnement, puisque c'est à cette occasion que les actes qui ont consacré la naissance dudit droit ont été posés. Lorsque plus tard, l'humanité a été confrontée aux réalités liées aux changements climatiques, c'est également l'ONU qui a pris le devant de l'organisation de certaines rencontres notamment, la Conférence de Genève sur le Climat en 1979 et le Sommet de Rio de Janeiro sur l'Environnement et le Développement en 1992. Puis par la suite, les Conférences des Parties à la Convention Cadre des Nations Unies sur les Changements Climatiques ont pris le relai et ont fourni l'essentiel des arsenaux juridiques et institutionnels qui sont utilisés dans le cadre de la lutte contre les changements climatiques.

En vertu de quelles dispositions légales l'ONU est-elle parvenue à s'ingérer dans le domaine environnemental et plus particulièrement celui des changements climatiques?

La prestigieuse organisation internationale à travers son Assemblée générale ${ }^{263}$ et son Conseil de sécurité ${ }^{264} \mathrm{~s}$ 'investie dans l'organisation des conférences sur l'environnement et le développement et s'engage dans la résolution des problèmes liés aux changements climatiques, par la mobilisation de la Communauté internationale autour de cette problématique en vertu des dispositions contenues dans les articles 9 à 22 de la Charte des Nations unies. Elle dispose d'un pouvoir décisionnel qui l'autorise à prendre des initiatives dans le cadre de la protection de l'environnement ${ }^{265}$. En effet, la capacité de mobilisation de l'ONU lui a permis à travers

\footnotetext{
${ }^{261}$ Le Sommet de Nairobi au Kenya en 1982, le Sommet de Rio de Janeiro en 1992, le Sommet de Johannesburg en 2002 et puis le Sommet de Rio+20, tenu en 2012.

${ }^{262}$ Les Conférences consacrées à la protection de l'environnement sont organisées par décennie depuis la tenue de la Conférence de Stockholm en 1972 sont appelées Sommets de la Terre.

${ }^{263}$ Selon l'article 9 de la Charte de l'ONU, l'Assemblée générale se compose de tous les Membres des Nations unies. Chaque Membre a cinq (5) représentants au plus à l'Assemblée générale.

${ }^{264}$ Selon l'article 23 de la Charte des Nations unies le Conseil de sécurité se compose de quinze (15) Membres de l'Organisation. La République de Chine, la France, l'Union des Républiques Socialistes Soviétiques, le RoyaumeUni de Grande-Bretagne et d'Irlande du Nord, et les Etats-Unis d'Amérique sont membres permanents du Conseil de sécurité. Dix (10) autres Membres de l'Organisation sont élus, à titre de membres non permanents du Conseil de sécurité, par l'Assemblée générale qui tient spécialement compte, en premier lieu, de la contribution des Membres de l'Organisation au maintien de la paix et de la sécurité internationales et aux autres fins de l'Organisation, et aussi d'une répartition géographique équitable. Les membres non permanents du Conseil de sécurité sont élus pour une période de deux (2) ans. Lors de la première élection des membres non permanents après que le nombre des membres du Conseil de sécurité aura été porté de onze (11) à quinze (15), deux des quatre membres supplémentaires seront élus pour une période d'un an. Les membres sortants ne sont pas immédiatement rééligibles. Chaque membre du Conseil de sécurité a un représentant au Conseil.

${ }^{265}$ Voir les articles 9 à 22 de la Charte des Nations unies.
} 
l'Organisation Météorologique Mondiale (OMM) d'organiser la première Conférence mondiale sur le climat en 1979 à Genève. Ce fut au cours de cette rencontre qu'il a été conclu que les émissions anthropiques de dioxyde de carbone sont susceptibles de produire d'effet à long terme sur le climat. Mais plus tard, l'intervention de l'ONU dans le secteur environnemental a été entérinée par la résolution A/RES/63/281 en date du 03 juin 2009 de l'Assemblée générale des Nations unies sur la règlementation des «changements climatiques et leurs répercussions éventuelles sur la sécurité $»^{266}$.

Le facteur déterminant de tous ces aspects constitue la facilité que possède l'ONU à mettre en œuvre la coopération internationale sans quoi, il serait pratiquement illusoire de parler de la lutte contre les changements climatiques, un phénomène de nuisance planétaire. La capacité de mobilisation de l'ONU a permis d'obtenir l'adhésion de la quasi-totalité des Etats de la planète autour de la question concernant la lutte contre les changements climatiques ${ }^{267}$. Seulement que, lorsque le Protocole de Kyoto a été élaboré, certains Etats qui sont avérés comme des puissances pollueuses ont fait défection ${ }^{268}$ et ont rendu inefficace l'instrument, cependant, il a été ratifié par 192 Parties y compris une organisation économique régionale ${ }^{269}$. Dans ce cas, l'ONU ne dispose pas de moyens pour ramener les Etats rebelles dans le cadre conventionnel pour leur faire appliquer ses dispositions. Une situation que l'Accord de Paris a corrigée en suscitant une large adhésion de tous les pays y compris les puissances pollueuses ${ }^{270}$.

Sur un autre plan, l'intervention de l'ONU dans le cadre de la protection de l'environnement se manifeste sous forme de renforcement des capacités des institutions chargées de la mise en œuvre de la règlementation en matière de protection de l'environnement. Ainsi, le système des Nations unies s'intéresse au volet concernant le financement de la lutte contre les changements climatiques. La lutte contre les changements climatiques nécessite d'importants moyens financiers et l'apport de la technologie. Ces questions constituent l'un des aspects les plus prépondérants des efforts à déployer à l'échelle mondiale pour faire face aux

\footnotetext{
${ }^{266}$ Résolutions de la 63e session de l'Assemblée générale des Nations unies. Voir compte rendu de séance $\mathrm{n}^{\circ} \mathrm{A} / 63 / \mathrm{L} .8 /$ Rev.1 de 1'Assemblée Générale des Nations unies sur le site internet : https://www.un.org. Consulté le $19 / 05 / 2021$.

${ }^{267} \mathrm{~A}$ titre indicatif, la Convention Cadre des Nations Unies sur les Changements Climatiques regroupe actuellement cent soixante-cinq (165) Etats signataires et cent quatre vingt dix-sept (197) Parties. Disponible sur le site internet : https://treaties.un.org/pages.Consulté le 05/01/2022.

${ }^{268}$ Il s'agit des Etats Unis d'Amérique, la Chine et le Canada. Disponible sur le site internet : https://treaties.un.org. Consulté le 05/01/2022.

${ }^{269} \mathrm{La}$ seule organisation régionale signataire du traité est l'Union européenne qui ratifiée le Protocole le 31 mai 2002. Etat de ratification disponible sur le site internet : https://treaties.un.org. Consulté le 19/05/2021.

${ }^{270} \mathrm{~L}$ 'Accord de Paris a connu la participation de 191 Parties l'ont ratifié y compris certaines puissances qui avaient fait défection dans le cadre du Protocole de Kyoto, telles les Etats Unies d'Amérique, qui a fini par ratifié le Traité le 21 janvier 2021 et le Canada qui l'avait ratifié depuis le 05 octobre 2016. Information reçue du site internet: https://treaties.un.org. Consulté le 19/05/21.
} 
défis imposés par ce fléau. Alors que la mobilisation des ressources par les Etats individuellement pris est quasi impossible. L'ONU à travers le mécanisme financier ${ }^{271}$ de la Convention Cadre des Nations Unies sur les Changements Climatiques, contribue à la mise en place de certaines institutions pour financer les projets destinés à la lutte contre les changements climatiques et faciliter le transfert de la technologie. La Banque mondiale, une institution financière spécialisée de l'ONU dispose d'un département qui s'occupe de l'environnement et s'active à régler les problèmes de financement des changements climatiques ${ }^{272}$.

Par ailleurs, le cadre de l'Organisation des Nations Unies a servi à l'élaboration de mesures qui ont influencé le Droit international de l'Environnement. C'est l'Assemblée générale de l'ONU qui a adopté en 1982 la Charte mondiale de la nature ${ }^{273}$. De nombreuses résolutions de l'Assemblée générale des Nations unies sont consacrées aux questions des changements climatiques, notamment, celles qui ont contribué à la création du GIEC et à l'organisation des Sommets de la Terre, des COP et des rencontres dédiées à la protection de l'environnement et à la lutte contre les changements climatiques ${ }^{274}$.

D'autres organisations spécialisées dans le domaine des changements climatiques sont aussi présentes dans l'arène pour y apporter leur expertise comme c'est le cas du Groupe d'Experts Intergouvernemental sur l'Evolution du Climat (GIEC).

\section{B- L'expertise du GIEC ${ }^{275}$}

L'expertise définie comme l' «Examen de quelque chose en vue de son estimation, de son évaluation $»^{276}$ est perçue comme « la procédure destinée à éclairer une personne chargée de prendre une décision $»^{277}$. Elle permet de comprendre un phénomène et d'en avoir la certitude pour éviter des spéculations. Elle est sollicitée par des décideurs pour être utilisée dans le processus de prise de décision. Vu la complexité des changements climatiques et les controverses suscitées par les recherches scientifiques autour de la question, toute action pour

\footnotetext{
${ }^{271}$ Le mécanisme financier est institué à l'article 11 de la Convention-cadre des Nations unies sur les changements climatiques.

${ }^{272}$ Voir l'organigramme de la Banque Mondiale sur le site internet : https://www.banquemondiale.org/fr/abouts. Consulté le 08/06/2021.

${ }^{273}$ Cette charte a été adoptée par la résolution 37/7 de l'assemblée générale des Nations unies le 28 octobre 1982. Disponible sur le site internet : https://www.un.org/french. Consulté le 05/01/2022.

${ }^{274}$ Les résolutions adoptées à l'occasion des assemblées générales des Nations unies sont disponibles sur le site internet : https://reaties.un.org.

${ }^{275}$ Sigle qui se définit comme « Groupe d'Experts Intergouvernemental sur l'Evolution du Climat ».

${ }^{276}$ Dictionnaire Larousse. Disponible sur le site internet : https://www.larousse.fr/dictionnaires/francais. Consulté le $05 / 01 / 2021$.

${ }^{277}$ ENCINAS DE MUNAGORI (R.), « Expert et expertise », in Dictionnaire de la culture juridique, Paris, PUF, 2003. p. 686.
} 
les enrayer mérite d'être murement mijotée avant d'être entérinée par des actes officiels. Le juriste intervenant dans l'élaboration ou l'application du Droit climatique n'est pas forcément celui là qui a une connaissance scientifique de la manifestation du phénomène des changements climatiques. Dans le contexte où, la compréhension du déroulement du phénomène des changements climatiques peut être difficilement accessible, tout recours systématique à l'expertise sera d'une grande utilité en face d'un phénomène dont « la prévision (...) recèle un grand nombre d'incertitudes, notamment en ce qui concerne son déroulement dans le temps, son ampleur et ses caractéristiques régionales ${ }^{278}$. La non considération de la question relative à la recherche de la connaissance du phénomène climatique peut induire les acteurs à élaborer des normes peu efficaces. Au cas où ils sont en présence des normes de qualité, le défaut de connaissance peut les induire dans l'application erronée desdites normes.

La Communauté internationale a compris l'importance de l'expertise dans le processus d'élaboration et de mise en œuvre du Droit international de Protection du Climat, en prenant en considération, «les utiles travaux d'analyse menés par nombre d'Etats sur les changements climatiques et des contributions importantes apportées par l'Organisation Météorologique Mondiale, le Programme des Nations Unies pour l'Environnement et d'autres organes, organisations et organismes des Nations unies, ainsi que d'autres organismes internationaux et intergouvernementaux (...) ${ }^{279}$. Puis, elle s'est engagée à encourager les Parties à soutenir et à développer « (...) davantage les organisations ou les programmes et réseaux internationaux et intergouvernementaux dont le but est de définir, réaliser, évaluer et financer des travaux de recherche, de collecte de données et d'observation systématique, en tenant compte de la nécessité de limiter le plus possible les doubles emplois $»^{280}$.

Après l'organisation de la Conférence de Genève sur le climat en 1979, «les changements climatiques ont fait naître de vives discussions entre les scientifiques» ${ }^{281}$. Des controverses ont surgi sur plusieurs aspects, notamment la question concernant le caractère anthropique des émissions des gaz à effet de serre. Ces nombreuses contradictions que certains scientifiques ont qualifié d'《 univers controversé $»^{282}$, ont déterminé certaines institutions

\footnotetext{
${ }^{278}$ Sixième $\left(6^{\mathrm{e}}\right)$ paragraphe du préambule de la Convention Cadre des Nations Unies sur les Changements Climatiques.

${ }^{279}$ Quinzième $\left(15^{e}\right)$ paragraphe du préambule de la Convention Cadre des Nations Unies sur les Changements Climatiques.

${ }^{280}$ Article 5-a de la Convention Cadre des Nations Unies sur les Changements Climatiques.

${ }^{281}$ L'ouvrage : Cop21 La diplomatie climatique de Rio 1992 à Paris 2015 de Sandrine Maljean Dubois et Matthieu Wemaëre, p.10.

${ }^{282}$ O. Godard, «Stratégies industrielles et conventions d'environnement : de l'univers stabilisé aux univers controversés », Environnement, Économie, INSEE méthodes, n³9-40, pp. 145-174.
} 
appartenant au système des Nations unies à penser à la création d'autres institutions pour aider à clarifier des préoccupations liées aux changements climatiques. C'est dans ce cadre que la décision de la création du Groupe d'Experts Intergouvernemental sur 1'Evolution du Climat (GIEC) investi de la mission de procéder à l'évaluation des changements climatiques a été approuvée $^{283}$. La Communauté internationale s'était d'ailleurs saisie du premier rapport du GIEC $^{284}$, pour donner un cadre juridique au Droit des Changements Climatiques par l'élaboration et l'adoption de la Convention Cadre des Nations Unies sur les Changements Climatiques. Mais elle a continué à s'inspirer des résultats des rapports subséquents du GIEC pour perfectionner le Droit climatique. Le GIEC a déjà élaboré cinq (5) rapports sur l'évolution du climat $^{285}$ qu'il a mis à contribution dans le processus de la construction du régime international du climat. Grace à l'expertise apportée par le GIEC par l'élaboration des rapports, le Droit International des Changements Climatique existe aujourd'hui et continue d'être amélioré. La création du Groupe d'Experts Intergouvernemental sur l'Evolution du Climat (GIEC) en 1988 conjointement par l'Organisation Métrologique Mondiale (OMM) et le Programme des Nations Unies pour l'Environnement (PNUE) a été entérinée par une résolution de l'Assemblée générale des Nations unies ${ }^{286}$. Son rôle consiste à faire «des évaluations scientifiques, coordonnées à l'échelle internationale, de l'ampleur de la chronologie et des effets sur l'environnement et sur les conditions socio-économiques ${ }^{287}$, in fine, il «formule des stratégies pour agir sur ses effets ${ }^{288}$. Le GIEC à l'instar de toute institution spécialisée appartenant au système des Nations unies ne regroupe pas des personnes, ce n'est pas non plus un laboratoire de recherche, mais une institution ayant compétence à évaluer les fruits des recherches effectuées par d'autres ${ }^{289}$. Les rapports du GIEC sont périodiquement élaborés et

\footnotetext{
${ }^{283}$ La création de l'institution a été approuvée par la résolution 43/53 en date du 06 décembre 1988 relative à la « Protection du climat mondial pour les générations présentes et futures ». Voir paragraphe 2-5 de ladite résolution à la page 137 des résolutions adoptées au cours de la $43^{\mathrm{e}}$ session de l'Assemblée générale des Nations unies. Disponible sur le site internet : https://www.un.org/french/documents. Consulté le 05/01/2022.

284 Le rapport du GIEC comptant pour la période de 1990 et 1992 . Disponible sur le site internet :https://www.ipcc.ch.Consulté le05/01/2022.

${ }^{285}$ Le GIEC a publié cinq (5) rapports d'évaluation respectivement en 1990, 1995, 2001, 2007 et 2013/2014.

${ }^{286}$ La création du GIEC a été entérinée par la (A/RES/43/53) adoptée par l'Assemblée générale des Nations unies le 6 décembre 1988 relative à la " Protection du Climat mondial pour les générations présentes et futures ». Disponible sur le site: https://www.un.org/fr/sections/documents/general-assemblyresolutions.Consulté le $08 / 06 / 2021$.

${ }^{287}$ La création de l'institution a été approuvée par la résolution 43/53 en date du 06 décembre 1988 relative à la « Protection du climat mondial pour les générations présentes et futures ». Voir paragraphe 2-5 de ladite résolution à la page 137 des résolutions adoptées au cours de la 43 ${ }^{\mathrm{e}}$ session de l'Assemblée générale des Nations unies. Disponible sur le site internet : https://www.un.org/french. Consulté le 05/01/2022.

${ }^{288}$ Id.

${ }^{289}$ Le GIEC dans sa mission d'évaluation peut faire recours à d'autres institutions techniques telles que les laboratoires et institutions de recherche.
} 
les fruits des réflexions sont exploités dans le cadre des Sommets de la Terre et des Conférences des Parties à la Convention Cadre des Nations Unies sur les Changements Climatiques. Le Groupe d'Experts Intergouvernemental sur l'Evolution du Climat (GIEC) s'est illustré par ses rapports qui ont un impact positif sur la codification des normes internationales en matière de lutte contre les changements climatiques. Le GIEC est une institution d'aide à décision pour les institutions du système des Nations unies intervenant dans le domaine de la protection de l'environnement et dans le champ de la lutte contre les changements climatiques. Les fruits des réflexions menées au sein du groupe servent de repères et contribuent à la création des normes conventionnelles. Les rapports du GIEC ont servi de base à la constitution du régime juridique de lutte contre les changements climatiques.

L'expertise du GIEC a été expressément requise dans le cadre de l'application du Protocole de Kyoto. Cette institution est compétente pour « examiner régulièrement et réviser au besoin, les méthodologies permettant de contrôler l'observance des engagements des parties $»^{290}$. Dans le cadre de la mise en œuvre de la Convention Cadre des Nations Unies sur les Changements Climatiques, le GIEC a été convié à collaborer avec le Chef du Secrétariat provisoire ${ }^{291}$ de la Conférence des Parties pour « répondre aux besoins d'avis scientifiques et techniques objectifs» ${ }^{292}$.

Même si le fonctionnement du GIEC a été critiqué et que ses travaux ont été remis en cause par certains opposants « réfractaires ${ }^{293}$, la contribution du GIEC dans la lutte contre les changements climatiques a été déterminante au point de susciter l'attention de la Communauté scientifique qui l'a nominé «Prix Nobel de la Paix $2007 »^{294}$. Par ce couronnement, il a été reconnu à cette institution « le travail scientifique effectué » ${ }^{295}$ illustré par « ses rapports d'évaluation » ${ }^{296}$ dans lesquels, « le Groupe d'Experts Intergouvernemental sur l'Evolution du Climat (GIEC) a posé le diagnostic des changements climatiques à l'échelle

\footnotetext{
${ }^{290}$ Selon les dispositions de l'article 5 paragraphe 2 du Protocole de Kyoto.

${ }^{291}$ Le secrétariat créé par l'Assemblée générale des Nations unies dans sa résolution 45/212 du 21 décembre 1990 relative à la «Protection du climat mondial pour les générations présentes et futures ». Disponible sur le site internet : https://www.un.org. Consulté le 06/01/2022.

${ }^{292}$ Article 21 paragraphe 2 de la Convention Cadre des Nations Unies sur les Changements Climatiques.

${ }^{293}$ Sandrine Maljean-Dubois et Matthieu Wemaëre, COP21 : La Diplomatie climatique de Rio 1992 à Paris 2015 , Pedone, Paris, 2015, 332 p., p.12.

${ }^{294}$ Le Prix Nobel a été institué par Alfred Nobel de nationalité Suédoise, pour récompenser les meilleurs talents du monde dans les domaines notamment de la physique, la chimie, la médecine, la physiologie, la médecine, la littérature et la paix.

${ }^{295}$ Sandrine Maljean-Dubois et Matthieu Wemaëre, COP21 : La Diplomatie climatique de Rio 1992 à Paris 2015 , Pedone, Paris, 2015, 332 p., p.12.

${ }^{296}$ Sandrine Maljean-Dubois et Matthieu Wemaëre, COP21 : La Diplomatie climatique de Rio 1992 à Paris 2015, Pedone, Paris, 2015, 332 p., p.12.
} 
internationale et peu à peu a contribué à réduire la marge d'incertitude initiale» ${ }^{297}$. Néanmoins, le GIEC n'est pas la seule institution de la Convention Cadre des Nations Unies sur les Changements Climatiques qui contribue à la production de l'expertise pour le compte de la Conférence des Parties, il y a également l'Organe Subsidiaire de Conseil Scientifique et Technologique ${ }^{298}$ qui a pour mission, de « fournir en temps opportun à la Conférence des Parties et, le cas échéant, à ses autres organes subsidiaires des renseignements et des avis sur les aspects scientifiques et technologiques de la Convention ${ }^{299}$. Il existe aussi l'Organe Subsidiaire de Mise en œuvre ${ }^{300}$ qui se charge d'« aider la Conférence des Parties à assurer l'application et le suivi de la Convention.» ${ }^{301}$. Ces organes exercent leurs attributions sous le contrôle de la Conférence des Parties et travaillent en collaboration avec le GIEC.

Il existe aussi ceux de ces acteurs extérieurs au Droit des Changements climatiques que sont les acteurs non Etatiques qui participent à sa mise en œuvre.

\section{Paragraphe 2: La participation à la mise en œuvre du droit}

Au nombre d'acteurs extérieurs au domaine environnemental qui interviennent dans la mise en œuvre du Droit International des Changements Climatiques, se trouvent généralement les acteurs non étatiques (A) et spécifiquement, il existe une prestigieuse Organisation Internationale Non Gouvernementale dénommée « Union Mondiale pour la Nature (UICN) » qui y contribue avec son expérience $(\mathbf{B})$.

\section{A- Les acteurs non étatiques}

Au terme de la décision de l'Accord de Paris de 2015, les acteurs non étatiques sont définis comme des «entités non Parties à la Convention Cadre des Nations Unies sur les Changements Climatiques, y compris ceux de la société civile, du secteur privé, des institutions financières, des villes et des autorités infranationales qui s'engagent dans la lutte contre les changements climatiques $»^{302}$. Cette catégorie d'acteurs n'était pas impliquée dans

\footnotetext{
${ }^{297}$ Sandrine Maljean-Dubois et Matthieu Wemaëre, COP21 : La Diplomatie climatique de Rio 1992 à Paris 2015, Pedone, Paris, 2015, 332 p., p.12.

${ }^{298}$ Créé par l'article 9 de la Convention Cadre des Nations Unies sur les Changements Climatiques.

${ }^{299}$ Les attributions de l'Organe Subsidiaire de Conseil Scientifique et Technologique sont fixées par le Paragraphe 1 de l'article 9 de la Convention Cadre des Nations Unies sur les Changements Climatiques.

${ }^{300}$ Cette institution est créée par l'article 10 de la Convention Cadre des Nations Unies sur les Changements Climatiques.

${ }^{301}$ Le Paragraphe 1 de l'article 10 de la Convention Cadre des Nations Unies sur les Changements Climatiques fixe la mission de l'Organe Subsidiaire de Mise en œuvre.

302 Paragraphe 133 Décision 1/CP.21 sur l'Accord de Paris de 2015.
} 
l'organisation du Sommet de Stockholm de 1972. Elle n'a pas non plus été associée à la gestion de la protection de l'environnement pendant plusieurs décennies après l'organisation du Sommet de Stockholm de 1972. Il en a été ainsi parce qu'à l'origine, le Droit International de l'Environnement a été considéré comme l'apanage des seuls acteurs étatiques. Cette perception n'est pas absurde parce que, en se référant aux règles qui gouvernent à l'élaboration du Droit International de l'Environnement, cette matière emprunte ses règles du Droit International Public dont la principale source est fondée sur la Convention de Vienne sur les Traités de 1969. C'est dans cette Convention que le statut d'acteur devant participer à l'élaboration du Droit International Public a été défini. Selon les dispositions de ladite Convention, seuls les Etats ont la capacité de conclure des Traités ${ }^{303}$. Le Droit International de l'Environnement étant l'émanation du Droit International Public ne reconnait que le droit d'élaborer des conventions et traités internationaux qu'aux seuls acteurs étatiques. Dans ce cas, les organisations associatives participaient très rarement et exceptionnellement à l'action de protection de l'environnement ${ }^{304}$.

Néanmoins, à l'épreuve des faits, les réalités intrinsèques au domaine de l'environnement caractérisées par la complexité, la multi-dimensionnalité, la globalité et les charges colossales induites par la gestion de la matière, font révéler les limites que revêt le mode traditionnel d'élaboration et de mise en œuvre du Droit International Public. Dans ce cas, la mise à contribution d'autres acteurs s'avère indispensable. A titre indicatif, l'application du principe de la responsabilité commune mais différenciée contenu dans la Convention Cadre des Nations Unies sur les Changements Climatiques impose aux pays développés de mettre des ressources financières et de la technologie à la disposition des pays en voie de développement pour contribuer aux charges d'atténuation des gaz à effet de serre et celles concernant l'adaptation aux effets pervers des changements climatiques. Il s'ensuit que, la conception du droit par rapport à l'ascension des charges climatiques n'incombent qu'aux seuls Etats développés pour les motifs qu'ils sont non seulement pollueurs, mais également ce sont les acteurs principaux de l'animation du Droit international. Mais, la réalité est que, la mobilisation des ressources financières pose toujours des problèmes, de plus, le transfert des technologies n'est pas toujours effectif. Certains Etats sous le couvert de la souveraineté décident de l'application de la Convention Cadre des Nations Unies sur les Changements Climatiques selon l'intérêt que cela leur procure. Face à ces difficultés qui entravent la mise en œuvre de la

\footnotetext{
${ }^{303}$ Article 6 de la Convention de Vienne sur les Traités de 1969.

${ }^{304}$ Maljean-Dubois Sandrine et Lavanya Rajamani, La mise en œuvre du droit international de l'environnement, Académie de droit international de La Haye, La Haye, Martinus Nijhoff, 2011.
} 
Convention cadre, il convient de trouver des modes alternatifs de financement ou d'ascension des charges relatives à la gestion des changements climatiques. Ce facteur a contribué à l'occasion du Sommet de Rio de Janeiro de 1992 intervenu dans le contexte caractérisé par la manifestation des changements climatiques à craquer le moule traditionnel de la façon de faire le droit pour consacrer une ouverture au profit des acteurs non étatiques, qui sont autorisés à participer à l'œuvre de protection de l'environnement par l'initiation du principe de la démocratie participative ${ }^{305}$.

Seulement que, depuis 1992 que cette ouverture a été consacrée, à l'exception de l'UICN qui était déjà dans le domaine de protection de l'environnement avant l'avènement du Sommet de Stockholm de 1972, la présence des acteurs non étatiques n'a pas été remarquée dans l'arène des acteurs qui participent à l'animation de la lutte contre les changements climatiques au plan international. Ce fut à l'occasion de la COP21 tenue à Paris que le rôle de ces acteurs a été révélé. En effet, la décision de la COP21 reconnait « les droits et facultés d'agir des acteurs non étatiques en faveur du climat et leur offre un cadre d'action $»^{306}$.

Si les acteurs non étatiques n'ont pas droit délibérant au cours des processus d'élaboration des normes, ils peuvent toutefois influencer le processus en participant aux négociations. Il en est ainsi parce que l'enjeu du droit climatique se joue autour des négociations internationales. Ces négociations sont orientées à l'endroit de tous les acteurs étatiques à savoir les Etats, les Organisations Gouvernementales Internationales, de même que les acteurs non étatiques, notamment les Organisations Non Gouvernementales et le secteur privé. Les acteurs non étatiques dans leurs diversités, notamment, le secteur privé dispose de ressources financières, des moyens technologiques et de l'expertise qui peuvent servir à booster les charges qu'induit la gestion des changements climatiques. Le secteur privé qui est responsable de la majeure partie des émissions de gaz à effet de serre, source de dégradation de l'environnement est aussi capable de contribuer au processus de transition vers une société sans carbone. Les activités du secteur privé reposent pour la plupart, sur l'utilisation des produits de l'environnement, dont la gestion peut contribuer à sa dégradation. Il s'ensuit que les normes devant réglementer l'utilisation des ressources de l'environnement peuvent constituer parfois une barrière pour l'accomplissement des activités du secteur privé, éminemment présent dans le domaine du commerce.

\footnotetext{
305 Principe 10 de la déclaration de Rio de Janeiro sur l'environnement et le développement de 1992.

${ }^{306}$ Le Portail des acteurs non étatiques pour l'action climatique est disponible sur le site internet: http://climateaction.unfcc.int/.Consulté le 19/01/2022.
} 
Eu égard à ce qui précède, il y avait nécessité pour les acteurs du Droit international des Changements Climatiques et les acteurs non étatiques de collaborer pour faire aboutir les objectifs de lutte contre les changements climatiques. La nécessité d'une action concertée entre les acteurs traditionnels du Droit international des Changements Climatiques que sont notamment, les Etats et les Organisations Gouvernementales Internationales d'une part, et les acteurs non étatiques dans leur diversité d'autre part, en vue de créer un lien entre la Convention Cadre des Nations Unies sur les Changements Climatiques et les initiatives volontaires, a fait que les entités non parties ont été intégrées au «Groupe de travail spécial de la plateforme de Durban pour une action renforcée $»^{307}$. Un organe subsidiaire de la Convention Cadre des Nations Unies sur les Changements Climatiques dont la mission principale est, « d'élaborer au titre de la Convention un protocole, un autre instrument juridique ou un texte convenu d'un commun accord ayant valeur juridique, applicable à toutes les Parties $»^{308}$.

Ce fut à l'occasion de la COP20 tenue à Lima que les acteurs non étatiques se sont illustrés de part leur contribution volontaire à l'ascension des charges afférentes à la gestion des changements climatiques. La participation des acteurs non étatiques a l'action climatique a été légitimée par la Décision 1/CP.21 issue de la COP21 tenue à Paris en 2015 qui a reconnu les « efforts déployés par les entités non parties afin de développer leurs actions en faveur du climat $»^{309}$. C'est pourquoi, elles ont été invitées à « amplifier leurs efforts et à appuyer des mesures destinées à réduire les émissions et/ou renforcer la résilience et diminuer la vulnérabilité aux effets néfastes des changements climatiques» ${ }^{310}$. Cette catégorie d'acteur a d'ailleurs reçu les félicitations de la Conférence des Parties à l'occasion de la COP21 tenue à Paris en 2015, pour sa contribution au développement de ses actions en faveur de la lutte contre les changements climatiques ${ }^{311}$.

\footnotetext{
${ }^{307}$ Convention Cadre des Nations Unies sur les Changements Climatiques, Rapport de la Conférence des Parties sur sa dix-septième $\left(17^{\mathrm{e}}\right)$ session, tenue à Durban du 28 novembre au 11 décembre 2011, Deuxième $\left(2^{\mathrm{e}}\right)$ partie: Mesures prises par la Conférence des Parties à sa dix-septième $\left(17^{\mathrm{e}}\right)$ session, Décisions adoptées par la Conférence des Parties, Décision 1/CP.17 Création d'un groupe de travail spécial de la plate-forme de Durban pour une action renforcée, $1^{\text {er }}$ point. Disponible sur le site internet : https://unfccc.int/resource/docs/2011/cop17/fre/09a01f.pdf. Consulté le 06/01/2022.

${ }^{308}$ Convention Cadre des Nations Unies sur les Changements Climatiques, Rapport de la Conférence des Parties sur sa dix-septième session, tenue à Durban du 28 novembre au 11 décembre 2011, Deuxième partie: Mesures prises par la Conférence des Parties à sa dix-septième session, Décisions adoptées par la Conférence des Parties, Décision 1/CP.17 Création d'un groupe de travail spécial de la plate-forme de Durban pour une action renforcée, $2^{\mathrm{e}}$ point.

${ }^{309}$ Paragraphe 177 de ladite décision.

${ }^{310}$ Paragraphe134 id.

${ }^{311}$ Conférence des Parties sur sa vingt et unième $\left(21^{\mathrm{e}}\right)$ session, tenue à Paris du 30 novembre au 13 décembre 2015 Décision 1/CP.21 adoptée par la Conférence des Parties, point 117. Disponible sur le site internet: https://unfccc.int/sites/default/files/resource/docs/2015/cop21/fre/10a03f.pdf. Consulté le 06/01/2022.
} 
Les décisions ${ }^{312}$ de la dix-septième $\left(17^{\mathrm{e}}\right)$ Conférence des Parties à la Convention Cadre des Nations Unies sur les Changements Climatiques ont renforcé le rôle du secteur privé dans la gestion des changements climatiques. C'est à l'occasion de cette COP que le Fonds vert pour le climat a été institué. Le fonctionnement de ce Fonds est basé sur la contribution du secteur privé qui peut « financer directement et indirectement les activités de ce secteur en matière d'atténuation et d'adaptation aux niveaux national, régional et international $»^{313}$. Ce mode de fonctionnement est conçu suivant une approche participative, dont le seul objectif est d'exclure le mode de gestion unilatérale des Etats ${ }^{314}$. Selon les termes de l'Accord de Durban, le dispositif institutionnel «favorise la participation des acteurs du secteur privé dans les pays en développement, en particulier les acteurs locaux, y compris les petites et moyennes entreprises et les intermédiaires financiers locaux. Il soutient également des activités permettant au secteur privé de jouer un rôle dans les petits Etats insulaires en développement et les pays les moins avancés»» $^{315}$.

Parmi les acteurs non étatiques devant contribuer à l'action climatique, le rôle de l'UICN est à distinguer compte tenu de son l'influence sur l'élaboration du Droit International de l'Environnement à travers la qualité de l'expertise qu'elle apporte en s'inspirant de ses enrichissantes expériences.

\section{B-L'expérience de l'UICN}

L'Union Mondiale pour la Nature (UICN) est une organisation associative à caractère internationale créée, le 05 octobre 1948 suivant le droit suisse ${ }^{316}$. Elle a pour mission «d'influer les sociétés du monde entier, de les encourager et de les aider pour qu'elles conservent l'intégrité et la diversité de la nature et veillent à ce que toute utilisation des ressources naturelles soit équitable et écologiquement durable $»^{317}$. La conservation de l'intégrité et de la diversité de la nature dans l'esprit de justice des ressources naturelles, relève de son domaine de compétence. Elle est constituée d' « Etats et d'organismes gouvernementaux, d'organisations d'intégration politique et/ou économique, d'organisations nationales non

\footnotetext{
${ }^{312}$ Les trois (3) décisions issues de la COP de Durban. Disponible sur le site internet :https://unfccc.int/processand-meetings/conferences/past-conferences/durban. Consulté le 06/01/2022.

313 Point 41 de la Décision 3/CP.17 relative à la mise en place du Fonds vert pour le climat. Disponible sur le site internet : https://unfecc.int/resource/docs/2011/cop17/fre/09a01f.pdf. Consulté le 06/01/2022.

${ }^{314}$ Pont 42 id.

315 Paragraphe 43 ibid.

316 Première partie du Statut juridique point 1 des Statuts de l'UICN. Disponible sur le site internet: https://www.iucn.org/fr. Consulté le 06/01/2022.

${ }^{317}$ Deuxième partie des objectifs point 2 des Statuts de l'UICN. Id.
} 
gouvernementales, d'organisations internationales non gouvernementales, d'organisations des peuples autochtones et puis d'affiliés $\gg{ }^{318}$. Ce mélange hétérogène d'institutions internationales constitue la particularité qui caractérise cette organisation qui a rompu avec la conception traditionnelle des organisations associatives ${ }^{319}$. Selon certains auteurs, 1'UICN « est une organisation dépassant la dichotomie classique existante entre acteurs gouvernementaux et non gouvernementaux ${ }^{320}$. L'Union Mondiale pour la Nature (UICN) jouit d'un statut d'Organisation Non Gouvernementale (ONG) dont la reconnaissance se rapproche de celle d'autres institutions associatives internationales ayant privilège auprès du système des Nations unies notamment, le Comité Internationale de la Croix-Rouge (CICR) ${ }^{321}$ et a un mode de fonctionnement déconcentré. Elle dispose d'un réseau de compétence de scientifiques et est reconnue par les acteurs associatifs étatiques et interétatiques.

Comment l'UICN participe-t-elle au processus d'élaboration du Droit International de

\section{l'Environnement?}

La complexité des règles devant régir le domaine environnemental commande à ce que, recours soit fait à d'autres compétences utiles et nécessaires à leur élaboration. La recherche de la compétence voire, de l'expérience s'impose alors dans ce domaine sensible où le défaut de maitrise des paramètres environnementaux peut conduire à l'élaboration des mesures qui peuvent s'avérer calamiteuses pour l'encadrement de la matière. Pour ne pas tomber dans ces travers, les acteurs du Droit international ont suscité et bénéficié de la contribution d'autres acteurs qui y ont apporté leurs compétences, leurs techniques et leurs ressources pour accompagner le processus d'élaboration des normes. Ces nouveaux acteurs ${ }^{322}$ participent

\footnotetext{
${ }^{318}$ Troisième $\left(3^{\mathrm{e}}\right)$ partie membres point 4 des Statuts de l'UICN. Disponible sur le site internet: https://www.iucn.org/fr. Consulté le 06/01/2022.

${ }^{319}$ Une méthode selon laquelle il y a une séparation étanche des acteurs gouvernementaux de ceux de la société civile.

${ }^{320}$ Juliette Olivier, L'Union mondiale pour la nature (UICN) une organisation singulière au service du droit de l'environnement, Bruxelles, Bruylant, 2005. pour approche historique, voir aussi l'ouvrage de M. Martin Holdgate, Directeur général de l'UICN entre 1988 et 1994: Martin Holdgate, the Green Web A Union for World Conservation London, Earthscan, 1999.

${ }^{321}$ Selon l'article premier des Statuts du Comité international de la Croix-Rouge, Le CICR est une organisation consacrée par les Conventions de Genève, leurs Protocoles additionnels, les Statuts du Mouvement et les Conférences internationales de la Croix-Rouge et du Croissant-Rouge. Disponible sur le site internet: https://www.icrc.org/fr/document/statuts-du-comite-international-de-la-croix-rouge. Consulté le 06/01/2022.

${ }^{322}$ Groupe de mot utilisé pour désigner les acteurs du Droit International de l'Environnement autres que l'Etat et les Organisations d'intégrations régionales dans l'article élaboré par Juliette Olivier « Les nouveaux acteurs du Droit de l'Environnement. Le rôle de l'UNICN dans l'élaboration du Droit de l'Environnement », Revue européenne de Droit de l'Environnement, 2005, pp.274-296.
} 
désormais à « l'élaboration comme à la mise en œuvre des règles internationales $»^{323}$ et sont présents « dans les négociations des instruments classiques notamment, des conventions $»^{324}$.

L'Union Mondiale pour la Nature (UICN) de par son organisation impressionnante et son efficacité dans la gestion de la protection de l'environnement, contribue à trouver des solutions pratiques à certains problèmes pressants de l'environnement. Elle a fait ses preuves notamment, dans les domaines de la biodiversité et des changements climatiques, en consacrant l'essentiel de ses efforts en faveur d'une économie mondiale verte et en apportant son appui à la recherche scientifique. Selon Juliette Olivier, l'Union Mondiale pour la Nature (UICN) « bénéficie d'une place particulière dans le processus de création du Droit de l'Environnement lors des différentes phases de leur élaboration ${ }^{325}$. L'implication de 1'Union Mondiale pour la Nature (UICN) dans le processus d'élaboration de certains textes internationaux tels que, les traités à portée internationale ou régionale permet de se rendre compte de l'étendue des missions accomplies par la prestigieuse organisation. A titre illustratif, l'Organisation de l'Unité Africaine (OUA) devenue l'Union Africaine (UA) en collaboration technique avec l'Union Mondiale pour la Nature (UICN) avaient préparé la Convention sur la conservation de la nature et des ressources naturelles, qui avait été adoptée à Alger en septembre 1968 et entrée en vigueur le 07 mai $1969^{326}$. Par ailleurs, l'Union Mondiale pour la Nature (UICN) et la Communauté Economique des Etats de l'Afrique de l'Ouest (CEDEAO) ${ }^{327}$, participent conjointement à l'élaboration et à la mise en œuvre d'un programme régional sur les changements climatiques $^{328}$. Présentement, l'intervention des nouveaux acteurs dans le domaine de la protection de l'environnement est devenue coutume, comme en témoigne la contribution de l'UICN dans l'élaboration de plusieurs instruments internationaux, notamment, la Charte mondiale de la nature adoptée par l'Assemblée générale de l'ONU en $1982^{329}$.

\footnotetext{
${ }^{323}$ Maljean-Dubois (Sandrine), La mise en œuvre du droit international de l'environnement, Aix-en-Provence, Ceric, 2003, 65p., p20.

${ }^{324}$ Id.

${ }^{325}$ Juliette Olivier Ingénieur de recherche Centre d'étude de la recherche politique (CERPO) Université de Bourgogne: Les nouveaux acteurs du droit de l'environnement. Le rôle de l'UICN dans l'élaboration du droit de l'environnement. Revue Européenne de Droit de l'Environnement/année 2005/9-3/pp.274-296.

${ }^{326}$ Kamto Maurice. Les conventions régionales sur la conservation de la nature et des ressources naturelles en Afrique et leur mise en œuvre. In: Revue Juridique de l'Environnement, $\mathrm{n}^{\circ} 4,1991$. pp. 417-442.

${ }^{327}$ Selon l'article 2 paragraphe 1 du Traité révisé de la Communauté Economique des Etats de l'Afrique de l'Ouest (CEDEAO). la Communauté régionale sera à terme la seule Communauté Economique de la Région aux fins de l'intégration économique et de la réalisation des objectifs de la Communauté Economique Africaine. Disponible sur le site internet : https://www.ecowas.int/droit-de-la-cedeao/traites/?lang=fr. Consulté le 06/01/2022.

${ }^{328}$ La réalisation de ce programme est centrée sur les résultats produits dans le cadre d'un autre programme dénommé « Initiative pour la Réduction de la Pauvreté et la Gestion de l'Environnement (PREMI) ».

${ }^{329}$ Sandrine Maljean-Dubois, La mise en œuvre du droit international de l'environnement, Centre d'études et de recherches internationales et communautaires, Aix-en-Provence (Ceric) L e s n o te s d e 1 ' I d d r i , n ${ }^{\circ} 4$, Page 21.
} 
L’Union Mondiale pour la Nature (UICN) est impliquée dans la résolution des problèmes posés dans le cadre des changements climatiques par l'apport des solutions pratiques basées sur la nature. Elle préconise une meilleure gestion et la restauration des écosystèmes naturels. L'Union Mondiale pour la Nature (UICN) en collaboration avec d'autres organisations, œuvrent à l'intégration dans la politique environnementale, des approches qui reposent sur les écosystèmes pour remédier à l'atténuation des changements climatiques.

Le Professeur Alexandre Kiss, qualifie de période où les organisations scientifiques et de protection de la nature à l'instar de l'Union Mondiale pour la Nature (UICN) ont commencé par faire leur entrée dans le processus d'élaboration du Droit de protection de l'Environnement, de «l'ère écologique moderne» ${ }^{330}$.

Globalement, l'UICN de part ses approches de gestion des problèmes environnementaux a acquis beaucoup d'expérience.

En quoi cette expérience est utile dans le cadre de la lutte contre les changements climatiques?

L'UICN, imbue de son expérience influence les actions climatiques par les diverses activités qu'elle accompli en collaboration avec les institutions en charge de la gestion des changements climatiques notamment, le Groupe d'Experts Intergouvernemental sur l'Evolution du Climat (GIEC) et la Conférence des Parties à la Convention Cadre des Nations Unies sur les Changements Climatiques. Elle émet parfois des positions qu'elle relais dans des rapports qu'elle élabore d'initiative ou sur demande d'autres institutions, qu'elle destine aux institutions intervenantes dans la lutte contre les changements climatiques. Elle apporte la part de sa connaissance des sujets débattus dans les fora, sommets et autres lieux de rencontres destinés à la lutte contre les changements climatiques. Sa contribution permet d'éclairer la lanterne des décideurs et facilite la procédure de prise de décision pour un meilleur encadrement. Ses positions sont des propositions de solutions qui découlent des études sur des questions essentielles relatives à la protection de l'environnement et à la lutte contre les changements climatiques. A titre indicatif, à la veille de la préparation de la COP21 devant se tenir à Paris, l’UICN a élaboré un document intitulé, «Position de l'UICN sur les négociations de la CCNUCC en 2015» ${ }^{331}$. C'est un document qui a ressassé les points essentiels devant

\footnotetext{
${ }^{330}$ Cf. A. Kiss « Droit International de l'Environnement », 1 ère éd. Pedome, 1969, p.6 ; v à c et S. Doumbé-Billé « La genèse de l'ère écologique » in J. Fromageau et M Cornu (éd), " La genèse du Droit de l'environnement », L'Harmattan, coll. Droit du patrimoine culturel et naturel, 2001, vol 1, Fondements et enjeux internationaux, $p$ 165 et $\mathrm{s}$.

${ }^{331}$ UICN (Union Internationale pour la conservation de la nature): Document de position sur le site internet https://www.uicn.org. Consulté le 05/06/2021.
} 
figurer à l'ordre du jour pour les négociations en vue de la conclusion d'un accord qui sera issu de la COP21. Ce document contenait des propositions de positions d'amélioration d'un éventuel accord et a été destiné aux négociateurs. A l'issu de la rencontre, certaines propositions de l'UICN ont été prises en compte et intégrées dans l'Accord de Paris. A titre illustratif, le document de position de l'UICN a exhorté les Parties «à continuer les négociations de manière constructive, dans le but d'adopter un accord 2015 ambitieux, juste et équilibré lors de la $21^{e}$ Conférence des Parties à Paris, qui permettra progressivement de contenir l'augmentation de la température moyenne mondiale en dessous de $2^{\circ} \mathrm{C}$ ou $1,5^{\circ} \mathrm{C}$ au-dessus des niveaux préindustriels, en maintenant le plus haut niveau possible d'intégrité environnementale et en respectant l'égalité hommes/femmes et les droits humains.» Cet appel de l'UICN a prospéré en partie car, la COP21 en a tenu compte et a intégré cette proposition partiellement dans l'Accord de Paris de 2015 dans la rubrique qui concerne l'objectif de l'Accord de Paris ${ }^{332}$.

Les mesures normatives savamment et massivement élaborées seront transposées dans la règlementation de tous les Etats Parties de la Convention Cadre des Nations Unies sur les Changements Climatiques ${ }^{333}$. Le Bénin a également internalisé le Droit International de protection de l'Environnement et celui concernant les changements climatiques par la prise des mesures normatives internes.

\footnotetext{
332 Voir article paragraphe 1-a de l'Accord de Paris de 2015.

${ }^{333}$ Ainsi en ont disposé le $7^{\mathrm{e}}$ paragraphe du préambule de la Convention Cadre des Nations Unies sur les Changements Climatiques et le Principe 11 de la Déclaration de Rio de Janeiro de 1992 sur l'Environnement et le Développement, principes de gestion des forêts.
} 


\section{CHAPITRE II:}

\section{LES MESURES NORMATIVES INTERNES}

Les changements climatiques, avant d'être perçus comme un phénomène planétaire ont commencé par se manifester dans chaque région de la planète et plus précisément sur le territoire de chaque Etat de la planète. C'est un phénomène d'échelle dont les causes et manifestations prennent corps à partir du territoire des Etats. Les changements climatiques se trouvent en République du Bénin, Etat côtier d'Afrique occidentale étendue sur une superficie de $114763 \mathrm{~km}^{2}$ avec une population d'environ 10741458 habitants $^{334}$. Le territoire du Bénin débouche au sud sur l'océan Atlantique et a comme limitrophes, le Nigeria à l'est, le Togo à l'ouest, le Niger et le Burkina-Faso au nord ${ }^{335}$. Ce pays est confronté aux manifestations des changements climatiques qui se caractérisent par la montée de l'eau des courants marins, l'érosion côtière, l'inondation, la perturbation de la pluviométrie, la déforestation et la désertification $^{336}$. Suivant les prévisions, si rien n'est fait, «les changements climatiques pourraient entraîner des impacts significatifs pour son développement dans plusieurs domaines» ${ }^{337}$.

Comment faire pour contenir les effets des changements climatiques qui dictent leur loi au plan interne, afin d'éviter des souffrances à la population?

$\mathrm{Si}$, en dépit de toutes les connaissances et les recherches effectuées autour de ce fléau, l'outil juridique a été l'ultime recours pour la Communauté internationale, le Bénin n'a pas non plus hésité à emprunter ce chemin. En réalité, le «Droit» qui été élaboré à l'échelle internationale pour éradiquer les changements climatiques ne peut être efficace que s'il est déployé dans les régions, sur le territoire des Etats et des collectivités locales pour être mis en œuvre. Autrement dit, le Droit International des Changements Climatiques est en réalité élaboré pour être appliqué sur le territoire des Etats.

\footnotetext{
${ }^{334}$ Enquêtes de INSAE - Institut National de la Statistique et de l'Analyse Économique [archive], disponible sur le site internet : www.insae-bj.org. Consulté le 20 mai 2019.

${ }^{335}$ Information générale disponible sur le site internet officiel du Gouvernement béninois http://www.gouv.bj. Consulté le 05/06/2021.

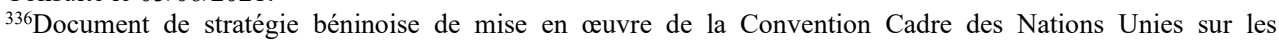
Changements Climatiques, paragraphe 2.5 sur le Diagnostic et solutions, pp.21-25. Disponible sur le site internet :https:/unfecc.int/sites/default/files/resource/Benin\%. Consulté le 02/06/2021.

${ }^{337}$ Le document de stratégie béninoise de mise en œuvre de la Convention Cadre des Nations unies sur les Changement Climatiques, p.10.
} 
Actuellement, le Bénin dispose d'une politique qui se soucie de plus en plus des problèmes environnementaux, car les autorités sont conscientes des risques que font courir les changements climatiques aux générations présentes et futures.

Préoccupé par cette situation, l'Etat béninois a adopté une démarche (SECTION I) qui a abouti à l'élaboration d'un cadre juridique quelque peu pertinent, mais inadéquat (SECTION II).

\section{SECTION I: LA DEMARCHE DU BENIN}

La consécration constitutionnelle du droit de protection de l'environnement au Bénin ${ }^{338}$ n'a pas suffit pour permettre au citoyen de jouir d'un environnement sain, satisfaisant et durable. Ce qui signifie qu'en réalité, l'intégration de la protection de l'environnement dans la constitution est une étape non moins importante dans le processus d'encadrement. Avec les complications engendrées par l'apparition des changements climatiques, le processus doit continuer dans le sens de l'amélioration de l'arsenal juridique de protection de l'environnement. Mais cette fois-ci, l'accent devra être mis sur les changements climatiques.

En effet, pour asseoir les bases d'un encadrement efficace de protection du climat, la démarche adoptée par le Bénin a consisté à engager l'Etat béninois dans les accords internationaux (Paragraphe 1) puis à procéder à l'élaboration d'une stratégie interne de lutte contre le fléau planétaire (Paragraphe 2).

\section{Paragraphe 1: L'engagement de l'Etat à l'international}

Le caractère global et planétaire que revêtent les changements climatiques nécessite des moyens d'actions dont la réalisation dépend plus, des solutions concertées provenant d'un cadre universel que des actions isolées dont l'efficacité serait redoutée. C'est un secret de polichinelle que le cadre international est un milieu de gestation par excellence de moyens juridicoinstitutionnels de protection de l'environnement et de lutte contre les changements climatiques. C'est encore le cadre international qui facilite la mobilisation des ressources financières importances pour prendre l'ascension des charges inhérentes à la lutte contre les changements climatiques. Ces motifs évoqués parmi tant d'autres, justifient l'engagement des Etats dans les accords internationaux de protection de l'environnement. C'est aussi pour ces motivations que la République du Bénin a marqué sa présence au plan international par son adhésion aux

\footnotetext{
${ }^{338}$ Article 27 de la constitution béninoise du 11 décembre 1990 révisée le 07 novembre 2019.
} 
instruments internationaux à caractère déclaratoire (A) et par la ratification d'un certain nombre d'accords internationaux (B).

\section{A- L'internalisation d'instruments déclaratoires}

Le Droit International de l'Environnement est né à partir de l'élaboration d'un important instrument déclaratoire ${ }^{339}$. Les instruments déclaratoires constituent des sources d'inspiration pour la communauté internationale qui s'en sert pour élaborer les Conventions et Traités en matière de protection de l'environnement et de lutte contre les changements climatiques. Les législations nationales s'en inspirent pour élaborer des lois et des actes réglementaires.

La toute première Déclaration qui a marqué la politique internationale de protection de l'environnement est celle de Stockholm de $1972^{340}$. Cette Déclaration a tracé le cadre juridique international de règlement des problèmes environnementaux en recommandant aux Etats, le renforcement de la coopération internationale pour aborder les questions internationales se rapportant à la protection et à l'amélioration de l'environnement ${ }^{341}$. Cet instrument a même reconnu l'état de pauvreté de certains pays qui ne sont pas en mesure de prendre les charges afférentes à la protection de l'environnement. Il invite par conséquent les Etats nantis qui pour la plupart, ont une part de responsabilité élevée dans la dégradation de l'environnement à mettre à la disposition des pays moins développés, la technologie et les moyens logistiques pour aider à faire face aux dépenses provoquées par l'accomplissement des besoins de protection de l'environnement ${ }^{342}$. Quant à la mise en œuvre des principes contenus dans la Déclaration de Stockholm de 1972, l'instrument renvoi les Etats et Organisations Internationales à prendre des mesures convenables dans le sens de la protection de l'environnement ${ }^{343}$. Il s'agira pour chaque Etat, de procéder à l'élaboration d'une législation interne aux fins d'internaliser les normes prescrites contenues dans ladite Déclaration, pour réaliser des objectifs en matière de protection de l'environnement.

En réalité, s'il n'est clairement énoncé nulle part dans la Déclaration de Stockholm de 1972 les thèmes relatifs à la lutte contre les changements climatiques, le contenu de cet instrument a un rapport avec le fléau comme en témoigne cette disposition qui stipule que,

\footnotetext{
${ }^{339}$ Il s'agit de la Déclaration de Stockholm sur l'environnement de 1972.

${ }^{340}$ Cet instrument normatif, le premier de l'histoire de l'humanité élaboré pour la protection internationale de l'environnement est issu du premier Sommet de la Terre sur l'Environnement tenu à Stockholm en 1972.

${ }^{341}$ Les Principe 22 et 24 de la déclaration de Stockholm de 1972.

${ }^{342}$ Principe 20 de la déclaration de Stockholm de 1972.

343 Principe 7 id.
} 
« (...) Les exemples de dommages des destructions et de dévastations provoquées par l'homme se multiplient sous ses yeux en de nombreuses régions du globe. On constate des niveaux dangereux de pollution de l'eau de la terre et des êtres vivants ; les perturbations profondes et regrettables de l'équilibre écologique de la biosphère; la destruction et l'épuisement des ressources irremplaçables, enfin de graves déficiences qui sont dangereuses pour la santé de l'homme, dans l'environnement qu'il crée et en particulier dans son milieu de vie et de travail $»^{344}$. En l'état, l'objet de cet instrument ne peut être analysé indépendamment du contexte de son élaboration. Pour rappel, au moment de l'adoption de la Déclaration de Stockholm de 1972, les changements climatiques n'étaient pas une préoccupation de l'heure. La situation qui prévalait n'a pas permis de définir les objectifs de la Déclaration par rapport aux changements climatiques. Cependant, la pertinence du contenu de cette Déclaration a fait de cet instrument un outil transversal et universel qui intéresse tous les domaines de l'environnement. C'est pourquoi il est en phase avec les réalités actuelles que sont les changements climatiques. Sa pertinence a été rappelée dans le préambule de la Convention Cadre des Nations Unies sur les Changements Climatiques ${ }^{345}$. Certains instruments élaborés à Rio ont aussi rappelé quelques thèmes de la Déclaration de Stockholm de $1972^{346}$. A partir de ce cloisonnement réalisé entre la Déclaration de Stockholm de 1972 et les instruments de lutte contre les changements climatiques, il peut être admis sans risque de se tromper que certains instruments de protection de l'environnement contribuent également à la lutte contre les changements climatiques.

Le Bénin n'était pas présent à la Conférence des Nations unies sur l'Environnement organisée à Stockholm en 1972, ainsi ce pays n'a pas participé à l'adoption de l'instrument déclaratoire élaboré au cours de la rencontre. Par contre, le Bénin était présent et a participé à l'adoption des Déclarations de Rio de Janeiro et à même ratifié la Convention Cadre des Nations Unies sur les Changements Climatiques. Par cet acte, le Bénin a souscrit indirectement à la Déclaration de Stockholm de 1972 qui fait partie intégrante de tous les instruments élaborés à l'occasion du Sommet de Rio de Janeiro de 1992. Parce que, en 1992, la Conférence des Nations

\footnotetext{
${ }^{344}$ Extrait du principe 3 de la Déclaration de la conférence de Stockholm de 1972. Disponible sur le site internet : https://www.un.org/fr/conferences/environment/stockholm1972. Consulté le 07/01/2022.

${ }^{345}$ Huitième paragraphe de la convention cadre sur les changements climatiques «Rappelant les dispositions pertinentes de la Déclaration de la Conférence des Nations unies sur l'environnement adoptée à Stockholm le 16 juin $1972 »$.

${ }_{346}$ Dans l'introduction de la Déclaration de Rio de Janeiro de 1992 « sur les principes de gestion des forets », il a été rappelé la Déclaration et ses principes élaborés au cours de la Conférence des Nations unies sur l'environnement organisée à Stockholm en 1972. Dans le $3^{\mathrm{e}}$ paragraphe de la même Déclaration, la Déclaration de la Conférence des Nations unies sur l'environnement adoptée à Stockholm le 16 juin 1972 a été réaffirmée.
} 
unies sur l'Environnement et le Développement a adopté deux importantes Déclarations dont l'une est spécifiquement consacrée à la gestion des forêts ${ }^{347}$. Les deux Déclarations élaborées dans le contexte des changements climatiques se veulent être le prolongement de la Déclaration de Stockholm de 1972. Le texte de l'une de ces Déclarations a réaffirmé intégralement les termes de « la Déclaration de la Conférence des Nations unies sur l'environnement adoptée à Stockholm le 16 juin $1972 »^{348}$ comme partie intégrante de ladite Déclaration.

La Déclaration de Rio de Janeiro de 1992 est constituée de vingt-sept (27) principes, articulés autour du concept «Développement durable » ${ }^{349}$, nouveau concept innové en droit dans le cadre de la conférence de Rio de Janeiro de 1992. Ce concept avait été érigé par une résolution de l'Assemblée générale des Nations unies en « principe directeur fondamental pour la Communauté internationale, les gouvernements, les institutions et entreprises privées $»^{350}$. Les principes de précaution ${ }^{351}$, d'évaluation environnementale ${ }^{352}$ et le concept de démocratie participative ${ }^{353}$ ont été réitérés dans ladite Déclaration.

Quant à la Déclaration de Rio de Janeiro de 1992 relative aux principes de gestion des forêts, elle a servi de tremplin aux concepts des droits et responsabilités des Etats dans le secteur de la protection de l'environnement. Cette déclaration est constituée d' « une série de principes sur la gestion écologiquement viable de tous les types de forêts» ${ }^{354}$ et a « pour but de contribuer à la gestion à la conservation et à l'exploitation écologiquement viable des forêts ${ }^{355}$. En faisant l'étude comparative des deux instruments déclaratoires, il y a des points de similitude entre la « Déclaration de Rio de Janeiro de 1992 sur les principes non juridiquement contraignants mais faisant autorité, pour un consensus mondial sur la gestion, la conservation et l'exploitation écologiquement viable de tous les types de forêts » et la « Déclaration de Stockholm de 1972 ». A titre indicatif, le droit concédé aux Etats d'exploiter leurs propres ressources selon leur politique, conformément aux prescriptions de la Charte des Nations unies, est consacré par les

\footnotetext{
${ }^{347}$ Il s'agit d'une part, de la Déclaration de Rio de 1992 et d'autre part, de la Déclaration de Rio sur l'Environnement et le Développement: principes de gestion des forêts. Disponible sur le site internet: https://www.un.org/french. Consulté le 07/01/2022.

348 Paragraphe 3 du préambule de la Déclaration de Rio de Janeiro de 1992.Id.

${ }^{349}$ Ce concept est véhiculé dans les principes $7 ; 8 ; 9 ; 12 ; 20 ; 21 ; 22 ; 24$ et 27 de la Déclaration de Rio de Janeiro de 1992. Id.

${ }^{350}$ La résolution 42/187 en date 11 décembre 1987 de l'Assemblée générale des Nations. Disponible sur le site internet : https://www.un.org/french. Consulté le 07/01/2022.

${ }^{351}$ Principe 15 de la Déclaration de Rio de Janeiro de 1992. Disponible sur le site internet : https://www.un.org. Consulté le 07/01/2022.

352 Principe 17 id.

${ }^{353}$ Contenue dans les principes $20 ; 21$ et 22 id.

${ }^{354}$ Extrait de l'introduction de la déclaration de Rio sur l'environnement et le développement : principes de gestion des forêts. Disponible sur le site internet : https:/www.un.org/french. Consulté le 07/01/2022.

${ }^{355}$ Principe 1-b de la déclaration de Rio de 1992 sur les forêts. Id.
} 
deux instruments ${ }^{356}$. Cette Déclaration est la preuve du consensus retrouvé au plan international sur l'encadrement international des forêts ${ }^{357}$.

Le Bénin ayant participé à l'adoption des Déclarations de Rio en 1992, s'évertue à se conformer à l'une des prescriptions qui stipulent que, «Les Etats doivent promulguer des mesures législatives efficaces en matière d'environnement ${ }^{358}$ mais dans ce cas, il faudra faire en sorte que «Les normes écologiques et les objectifs et priorités pour la gestion de l'environnement soient adaptés à la situation en matière d'environnement et de développement à laquelle elles s'appliquent. $\gg{ }^{359}$. Ce qui a engendré des réformes juridico-institutionnelles au plan interne qui ont conduit à l'adoption de la loi 93-009 du 02 juillet 1993, portant régime des forêts en République du Bénin et puis la création d'une importante institution dénommée, « Direction Générale des Eaux, Forêts et Chasse (DGEFC)» ${ }^{360}$ au sein du ministère en charge de l'Environnement ${ }^{361}$. Cet organe a pour attribution, « la protection et la gestion des forêts en République du Bénin $»^{362}$. Il procède à « l'élaboration des politiques et stratégies de l'Etat ainsi que les textes législatifs et règlementaires en matière de reboisement et de gestion durable des ressources naturelles notamment forestières, fauniques $»^{363}$. Il a également pour mission d'assurer « le suivi-évaluation de la mise en œuvre » ${ }^{364}$ et représente le « Point Focal National des conventions et accords multilatéraux en matière de ressources naturelles $»^{365}$. Enfin, il assure la « constitution et la préservation de l'intégrité du domaine forestier de l'Etat » ${ }^{366}$.

D'autres instruments internationaux ont été souscrits par le Bénin, ce qui rallonge la liste des moyens juridiques déployés par ce pays pour lutter contre les changements climatiques.

\footnotetext{
${ }^{356}$ Voir principe 21 de la Déclaration de Stockholm de 1972 et le principe 1-a de la déclaration de Rio de 1992 sur les forêts. Disponible sur le site internet : https://www.un.org/french. Consulté le 07/01/2022.

${ }^{357}$ Principe 1-d de la déclaration de Rio de 1992 sur les forêts. Id.

${ }^{358}$ Principe 11 de La déclaration de Rio sur l'environnement et le développement, principes de gestion des forêts. Id.

${ }^{359}$ Principe 11 de La déclaration de Rio de Janeiro de 1992 sur l'environnement et le développement, principes de gestion des forêts. Id.

${ }^{360}$ Voir article 12 du décret $n^{\circ} 2016-501$ du 11 aout 2016 portant attributions, organisation et fonctionnement du Ministère du Cadre de Vie et du Développement Durable en République du Bénin. Disponible sur le site internet : https://sgg.gouv.bj/doc/decret-2016-501. Consulté le 07/01/2022.

${ }^{361}$ Il s'agit du Ministère du Cadre de Vie et de Développement Durable (MCVDD).

${ }^{362}$ Voir article 13 du décret portant attributions, organisation et fonctionnement du Ministère du Cadre de Vie et du Développement Durable en République du Bénin. Disponible sur le site internet: https://sgg.gouv.bj/doc. Consulté le 07/01/2022.

${ }^{363}$ Id.

364 Ibid.

365 Ibid.

366 Ibid.
} 


\section{B- La ratification d'accords internationaux}

Trempé dans les méandres des changements climatiques qui menacent constamment de créer des distorsions pour la population, laquelle est déjà exposée à ses effets pervers qui se manifestent par de violentes pluies avec son corolaire d'inondation cyclique par endroit, l'avancée de l'érosion côtière dans la partie méridionale du pays, l'aggravation de la désertification dans la partie septentrionale ${ }^{367}$, le Bénin a adhéré aux diverses conventions qui concourent à la lutte contre les changements climatiques. Il a rejoint la Communauté internationale en lutte contre les changements climatiques par son adhésion à la Convention Cadre des Nations Unies sur les Changements Climatiques qu'il a ratifiée le 30 juin $1994^{368}$. Cet acte de haute portée politique fait propulser le Bénin dans le concert des Nations engagées pour éradiquer les changements climatiques. Cette ratification vaut pour l'Etat béninois, son engagement pour l'atténuation des émissions de gaz à effet de serre et sa responsabilité dans le développement des mesures d'adaptation des populations aux effets des changements climatiques.

Les enjeux de la Convention sur les changements climatiques pour le Bénin sont notamment, «la protection de la zone côtière, la réhabilitation des aires dégradées, le renforcement des puits d'absorption des gaz à effet de serre, la protection de l'atmosphère, la surveillance du système climatique et la promotion d'un développement durable ${ }^{369}$. Son engagement dans la Convention Cadre des Nations Unies sur les Changements Climatiques doit lui faire bénéficier des avantages contenus dans ladite convention. Car, cet instrument en créant des engagements à la charge des Parties $^{370}$ a inventé «le mécanisme financier » chargé de fournir des ressources financières sous forme de dons ou à des conditions de faveur notamment, pour le transfert de technologie ${ }^{371}$. Le Bénin participe également en tant qu'Etat partie à la Conférence des Parties, organe suprême créé par la Convention Cadre des Nations Unies sur les Changements Climatiques ${ }^{372}$ et jouit des privilèges dus à son statut.

\footnotetext{
${ }^{367}$ Document de stratégie béninoise de mise en œuvre de la Convention Cadre des Nations Unies sur les Changements Climatiques, 1. La Convention climat et le Protocole de Kyoto : opportunités et défis pour le Bénin, paragraphe 4, p.11. Disponible sur le site internet: https://unfccc.int/sites/default/files/resource/Benin\%. Page consultée le $02 / 06 / 2021$

${ }^{368}$ Par décret $n^{\circ} 93-304$ du 13 décembre 1993 portant ratification de la Convention-cadre des Nations unies sur les Changements climatiques en République du Bénin.

369 Selon le Programme d'Action National d'Adaptation aux Changements Climatiques (PANA-BENIN). Disponible sur le site internet : https://unfccc.int/resource/docs/napa/ben01f.pdf. Consulté le 07/01/2022.

${ }^{370}$ Le contenu de ces engagements est décliné dans l'article 4 de la Convention Cadre des Nations Unies sur les Changements Climatiques.

${ }^{371}$ Article 11 de la convention cadre sur les changements climatiques.

${ }^{372}$ Article 7 id.
} 
Le Bénin en adhérant à la Convention Cadre des Nations Unies sur les Changements Climatiques espérait au même titre que la Communauté internationale, relever les défis qu'imposent les changements climatiques aux Béninois. Malheureusement, cette attente n'est pas encore comblée tout comme pour bon nombres de pays ayant ratifiés la Convention Cadre des Nations Unies sur les Changements Climatiques. La volonté affichée par les autorités en entrainant le Bénin dans la ratification de la Convention n'a pas suffit pour empêcher le développement du fléau. L'aggravation du phénomène a fait virer ce pays du statut de puits à celui de source d'émission de gaz à effet de serre ${ }^{373}$. Les changements climatiques continuent à créer des désagréments à la population en rendant vulnérable les secteurs d'activité comme, l'agriculture, le tourisme, le littoral et la santé ${ }^{374}$. Néanmoins le Bénin n'a pas désamorcé, ce pays à continuer à prendre d'autres engagements.

En effet, le premier instrument juridiquement contraignant élaboré en application de la Convention Cadre des Nations Unies sur les Changements Climatiques, c'est-à-dire le Protocole de Kyoto a été ratifié par le Bénin, le 25 février $2002^{375}$. Ce traité international élaboré pour une première période d'engagement allant de 2008 à 2012, qui a suggéré une planification de réduction des émissions de gaz à effet de serre, a été adopté à l'occasion de la $3^{\mathrm{e}}$ conférence des Parties tenue à Kyoto le 29 novembre 1997 et entré en vigueur le 16 février $2005^{376}$. L' instrument additionnel à la Convention Cadre est intervenu pour repréciser les formes que doivent revêtir les engagements de réduction de gaz à effet de serre afin de faciliter l'atteinte des objectifs fixés dans le cadre de la lutte contre les changements climatiques ${ }^{377}$. Il a innové des mécanismes de flexibilité pour accompagner les pays développés à respecter leurs engagements d'atténuation des émissions de gaz à effet de serre ${ }^{378}$. C'est à partir de l'adoption

\footnotetext{
${ }^{373}$ Voir le $4^{\mathrm{e}}$ paragraphe de la page 213 de la Troisième Communication Nationale du Bénin à la Convention Cadre des Nations Unies sur les Changements Climatiques, élaborée par le Ministère du Cadre de Vie et du Développement Durable de la République du Bénin sur le site internet:https://www4.unfccc.int/sites/SubmissionsStaging/NationalReports/Documents/39685271_Benin-NC31-BENIN_TCN_2019.pdf, consultée le 08 décembre 2021.

${ }^{374}$ Voir $7^{-}$paragraphe, id.

${ }^{375}$ Voir état des ratifications du Protocole de Kyoto à la Convention Cadre des Nations Unies sur les Changements Climatiques adopté le 11 décembre 1997. Disponible sur le site internet https://treaties.un.org. Consulté le $07 / 01 / 2022$

${ }^{376}$ Voir état de ratification sur le site internet http://unfccc.int. Consulté le 07/01/2022.

${ }^{377}$ Il s'agit dans ce cadre des engagements chiffrés en matière de limitation et de réduction des gaz à effet de serre selon les dispositions de l'article 2 du Protocole de Kyoto.

${ }^{378}$ L'article 12 dudit Protocole dispose que «1. Il est établi un mécanisme pour un développement "propre"'. 2. L'objet du mécanisme pour un développement 'propr'" est d'aider les Parties ne figurant pas à l'annexe I à parvenir à un développement durable ainsi qu'à contribuer à l'objectif ultime de la Convention, et d'aider les Parties visées à l'annexe I à remplir leurs engagements chiffrés de limitation et de réduction de leurs émissions prévus à l'article 3. 3. Au titre du mécanisme pour un développement «propre»: a) Les Parties ne figurant pas à l'annexe I bénéficient d'activités exécutées dans le cadre de projets, qui se traduisent par des réductions d'émissions certifiées; b) Les Parties visées à l'annexe I peuvent utiliser les réductions d'émissions certifiées
} 
du Protocole de Kyoto que le Bénin a commencé par poser des actes majeurs dans le cadre de la mise en œuvre de la Convention Cadre des Nations Unies sur les Changements Climatiques notamment, par l'élaboration de la Stratégie nationale de mise en œuvre au Bénin de la Convention Cadre sur les Changements Climatiques (SNMO) ${ }^{379}$ et l'installation du Comité National sur les Changements Climatiques qui a reçu des attributions concernant la mise en œuvre de ladite Convention et ses accords subséquents ${ }^{380}$. Ce comité représente le point focal de la Convention au sein du ministère en charge de l'Environnement ${ }^{381}$.

Le Bénin a ratifié le 31 octobre $2016^{382}$ l'Accord de Paris intervenu à l'occasion de la $21^{\text {e }}$ Conférence des Parties organisée en 2015. C'est un Accord selon Laurent Fabius «juridiquement contraignant, universel et ambitieux ${ }^{383}$ qui énonce le prochain contexte d'actions contre les changements climatiques. L'Accord de Paris contribue à la mise en œuvre de la Convention Cadre des Nations Unies sur les Changements Climatiques ${ }^{384}$. Cet Accord qui a pour objectif finaliste de parvenir à la neutralité climatique renferme des approches holistiques pour contenir les émissions de gaz à effet de serre dans des proportions raisonnables ${ }^{385}$. L’Accord de Paris représente pour le Bénin, un instrument dont la mise en œuvre pourra faciliter le financement des projets d'atténuation des gaz à effet de serre et d'adaptation aux effets des changements climatiques.

Mais, bien avant l'adoption de la Convention Cadre des Nations Unies sur les Changements Climatiques, le Bénin avait déjà souscrit à certains instruments qui avaient été

obtenues grâce à ces activités pour remplir une partie de leurs engagements chiffrés de limitation et de réduction des émissions prévus à l'article 3, conformément à ce qui a été déterminé par la Conférence des Parties agissant comme réunion des Parties au présent Protocole (...).

${ }^{379} \mathrm{Ce}$ document a été réalisé par le Ministère de l'Environnement, de l'Habitat et de l'Urbanisme avec l'appui technique et financier du Programme des Nations Unies pour le Développement (PNUD) et l'appui technique de l'Institut des Nations Unies pour la Formation et la Recherche (UNITAR). La rédaction et la mise en forme définitive ont été assurées par une équipe d'experts nationaux sous la supervision de :Madame Jeanne Josette ACACHA AKOHA Point Focal Changements Climatiques Conseiller Technique à l'Environnement et Coordonnatrice $\mathrm{du}$ Programme $\quad$ CC:TRAIN $\quad$ Bénin 2003https://unfccc.int/sites/default/files/resource/Benin\%20INC_French_Addendum.pdf.

${ }^{380}$ Le Comité National sur les Changements Climatiques (CNCC) a pour mission le suivi de la mise en œuvre de la CCNUCC, l'appui à la définition des politiques et stratégies nationales et locales de lutte contre les effets néfastes des changements climatiques, la promotion des activités relatives à la mise en œuvre des directives politiques des programmes mondiaux, régionaux et nationaux de gestion des changements climatiques. Il est installé par le décret 2003-142 du 30 avril 2003 portant création, attributions et fonctionnement du CNCC.

${ }^{381}$ Article 7 alinéa 1 du décret $\mathrm{n}^{\circ} 2003-142$ du 30 avril 2003 Portant création, attributions et fonctionnement du Comité National de Lutte contre les Changements Climatiques. Disponible sur le site internet: https://sgg.gouv.bj/doc/decret-2003-142. Consulté le 07/01/2022.

${ }^{382}$ Accord de Paris - Etat des ratifications sur le site : https://unfccc.int/fr/node/513. Consulté le 09/06/2021.

${ }^{383}$ Déclaration du Ministre des affaires étrangères et du développement international, président de la COP21, lors de l'Événement. Disponible sur le site internet : https://www.vie-publique.fr/discours/195428-declaration-de-mlaurent-fabius-ministre-des-affaires-etrangeres. Consulté le 07/01/2022.

${ }^{384}$ Article 2 paragraphe 1 de l'Accord de Paris de 2015.

${ }^{385}$ Article 2 paragraphe 1-a id. 
élaborés au lendemain de la Conférence mondiale sur le climat tenue à Genève en 1979, où il avait été débattue de la question de la protection de la couche d'ozone et des substances qui contribuent à sa destruction. Les instruments internationaux élaborés à la suite de cette rencontre notamment, la Convention de Vienne pour la protection de la couche d'ozone adoptée le 22 mars 1985, entrée en vigueur le 22 septembre 1988 et son prolongement, le Protocole de Montréal sur les substances appauvrissant la couche d'ozone, adopté le 16 septembre 1987 et entré en vigueur le $1^{\text {er }}$ janvier 1989 ont tous reçu l'adhésion du Bénin qui les a ratifiés le $1^{\text {er }}$ juillet 1993.

D'autres instruments similaires sont conçus pour contribuer à la protection des forêts dans le cadre de la lutte contre la désertification. La désertification se définit comme "la transformation d'une région en désert, zone très sèche, aride et inhabitée, sous l'action de facteurs climatiques ou humains ${ }^{386}$. Selon Jean-GUILLAUMET, «La désertification apparaît sous le double jeu des agressions climatiques, sécheresse et dessèchement dans les régions, chaudes, tempérées ou froides, où le facteur déterminant est l'aridité» ${ }^{387}$. Elle provoque le démantèlement du couvert végétal jusqu'à sa dégradation totale. La désertification peut provenir des aléas naturels, mais aussi, elle peut découler de la mauvaise gestion des forêts. Dans cette optique, la lutte contre la désertification contribue à la protection des forêts et par ricochet participe à la protection du climat. La ratification par le Bénin de la Convention sur la Désertification, le 28 août 1996 induit le bénéfice de soutient financier au titre du Fonds pour l'Environnement Mondial (FEM) et le transfert de technologies ${ }^{388}$. Son impact s'est traduit par l'existence d'un ministère de l'Environnement chargé de coordonner toutes les activités à caractère environnemental. L'adoption de la Convention sur la désertification a permis d'élaborer le Plan d'Action Environnemental (PAE) ${ }^{389}$. Depuis son adhésion à la Convention sur la désertification, la République du Bénin a coutume d'élaborer son rapport national sur la mise en œuvre de ladite convention conformément aux prescriptions conventionnelles ${ }^{390}$.

Pour faciliter la mise en œuvre des accords internationaux ratifiés par le Bénin, des stratégies de lutte ont été élaborées.

\footnotetext{
${ }^{386}$ Voir Problème d'environnement $\mathrm{n}^{\circ} 3$ Désertification et déforestation (Note synthétique sur l'état des connaissances) JEAN-LOUIS GUILLAUMET Botaniste - Directeur de Recherche - ORSTOM, p.70. ${ }^{387}$ Id.

${ }^{388}$ BENIN IFDD www.ifdd.francophonie.org > pol_envir03 > Exp.pays participants > Benin.

389 Document de politique environnementale adopté en conseil des Ministres. Disponible sur le site internet: https://www.yumpu.com/fr/document/read/13719049/plan-daction-environnemental-du-benin-agence-beninoisepour-1-. Consulté le 07/01/2022.

${ }^{390}$ En application de l'article 26 de la Convention des Nations Unies sur la Désertification.
} 


\section{Paragraphe 2: L'élaboration de stratégie}

Le Gouvernement qui exécute la politique de l'Etat, assure la responsabilité de son élaboration. C'est une prescription de la Constitution béninoise du 11 décembre 1990 révisée le 07 novembre 2019, qui stipule que les « décisions déterminant la politique de l'Etat dans tous les secteurs sont délibérées par le Conseil des Ministres ${ }^{391}$. Ainsi, le Bénin avait élaboré des documents de stratégie, dont «Les Etudes Nationales de Perspectives à Long Terme, Bénin $2025 »^{392}$ en constituent l'épine dorsale. Les Etudes Nationales de Perspectives à Long Terme, Bénin 2025 est un instrument de planification élaboré pour la gestion de la politique générale de l'Etat jusqu'à l'horizon 2025. Selon ce document, le Bénin connaitra en 2025 un essor économique, une gouvernance qui respecte les normes où la promotion de la paix et du bien être social sera garantie. Pour réellement atteindre son objectif, les documents de stratégie sectoriels doivent être réalisés pour faciliter la mise en œuvre de la politique générale de l'Etat. Ce fut le cas en ce qui concerne la gestion du domaine des changements climatiques.

En matière de lutte contre les changements climatiques, malgré l'engagement lointain du Bénin dans ce domaine ${ }^{393}$, ce n'est qu'à l'occasion du Conseil des Ministres récemment organisé, qu'il a été approuvé par décret, le document intitulé, « Politique nationale de Gestion des Changements Climatiques » ${ }^{394}$. Le Gouvernement béninois préoccupé par les effets néfastes des changements climatiques et surtout de l'atteinte des objectifs fixés dans le cadre de sa politique générale a élaboré des stratégies $(\mathbf{A})$ et pris certaines initiatives pour contribuer à la lutte contre les changements climatiques $(\mathbf{B})$.

\section{A- Les stratégies béninoises}

La Communauté internationale à travers l'instrument de lutte contre les changements climatiques a recommandé " qu'il appartient aux Etats d'adopter une législation efficace en matière d'environnement, que les normes, objectifs de gestion et priorités écologiques doivent refléter les conditions d'environnement et de développement dans lesquelles ils s'inscrivent (...) $»^{395}$ et justifie ces recommandations par le fait « (...) que les normes appliquées par certains

\footnotetext{
${ }^{391}$ Article 54 alinéa 2 premier tiret de la constitution du 11 décembre 1990 révisée le 07 novembre 2019.

${ }^{392}$ Document de planification nationale de développement du Bénin.

${ }^{393}$ Le Bénin a ratifié la Convention Cadre des Nations Unies sur les Changements Climatiques le 30 juin 1994. Disponible sur le site internet : https://treaties.un.org. Consulté le 05/06/2021.

${ }^{394}$ Compte rendu du Conseil des Ministres du 03 février 2021 consultable sur le site https://sgg.gouv.bj.

395 Onzième $\left(11^{\mathrm{e}}\right)$ paragraphe du préambule de la Convention Cadre des Nations Unies sur les Changements Climatiques.
} 
pays risquent d'être inappropriées (...) $»^{396}$. L'analyse de ces dispositions révèle la nécessité pour les Etats Parties, de mettre en place un dispositif normatif et institutionnel en vue de faciliter l'application de la Convention Cadre des Nations Unies sur les Changements Climatiques. Mais surtout, ces mesures doivent être conformes aux réalités internes de chaque Etat. En d'autres termes, il est question de définir les modalités d'application efficace de la Convention Cadre des Nations Unies sur les Changements Climatiques sur les territoires des Etats Parties.

Comment appliquer le Droit International des Changements Climatiques au Bénin tout en ne s'éloignant pas des réalités politiques économiques socioculturelles du Pays?

Depuis la tenue du Sommet de Rio de Janeiro en juin 1992, il y a une meilleure prise de conscience de la nécessité d'adopter des stratégies plus structurées pour assurer le développement grâce à une gestion rationnelle de l'environnement. La prise de conscience des enjeux liés à la problématique des changements climatiques a favorisé l'élaboration et l'adoption de plusieurs politiques, stratégies et programmes de riposte par le Bénin à partir de l'année $1990^{397}$. Néanmoins, il sera tenu compte dans la présente étude des documents de stratégies les plus pertinents à cause de leur impact positif sur la lutte contre les changements climatiques et de leur relation avec la Convention Cadre des Nations Unies sur les Changements Climatiques.

En vue de l'application de l'instrument de lutte contre les changements climatiques, la «Stratégie béninoise pour la mise en œuvre de la Convention Cadre des Nations Unies sur les Changements Climatiques " a été élaborée en 2003 par le ministère en charge de l'Environnement ${ }^{398}$. Ce document décrit la stratégie de mise en œuvre de la Convention en trois (3) volets. Le premier volet fourni des explications pour la compréhension de certaines terminologies ou concepts ayant rapport avec les changements climatiques ${ }^{399}$. Ensuite, le deuxième volet ressasse les impacts possibles qu'engendreraient les modifications climatiques sur le développement du Bénin ${ }^{400}$. Enfin, le troisième volet propose des mesures à la mise en

\footnotetext{
${ }^{396}$ Onzième $\left(11^{\mathrm{e}}\right)$ paragraphe du préambule de la Convention Cadre des Nations Unies sur les Changements Climatiques.

${ }^{397} \mathrm{C}$ 'est au cours de cette année que la protection de l'environnement a été inscrite dans la constitution béninoise.

${ }^{398}$ A l'époque il est connu sous l'appellation de « Ministère de l'Environnement, de l'Habitat et de l'Urbanisme ». Suivant décret $n^{\circ}$ 97-194 portant attributions, organisation et fonctionnement du Ministère de l'environnement, de l'habitat et de l'urbanisme. Le décret est disponible sur le site internet : https://sgg.gouv.bj/doc/decret-1997-331. Consulté le 07/01/2022.

${ }^{399}$ Voir pages 7 à 10 du document de Stratégie béninoise pour la mise en œuvre de la Convention Cadre des Nations Unies sur les Changements Climatiques. Disponible sur le site internet : https://unfccc.int/sites/default. Consulté le 07/01/2022.

${ }^{400}$ Voir pages 13 à 21 id.
} 
œuvre de la Convention Cadre des Nations Unies sur les Changements climatiques ${ }^{401}$. Après avoir procédé à un inventaire des possibilités que dispose le Bénin pour lutter contre les changements climatiques ${ }^{402}$, les mesures d'application de la Convention ont été fixées de manière sectorielle. Les options prioritaires ont été arrêtées et consistent entre autres à, «Réaliser des recherches technologiques destinées à minimiser les émissions de gaz à effet de serre issues de l'utilisation continue de combustibles fossiles et mettre au point des sources combustibles non fossiles» ${ }^{403}$ et puis à « Mettre en ouvre des mesures efficaces à moindre coût pour la transition vers des sources d'énergie moins intensives en carbone et d'autres éléments qui ne contiennent pas de carbone telles que les énergies renouvelables» ${ }^{404}$. Ainsi à terme, le Bénin pourra atteindre l'autonomie énergétique par le développement des énergies renouvelables provenant des sources d'énergie telles que la biomasse ${ }^{405}$, la photovoltaïque ${ }^{406}$ ou la production du biogaz ${ }^{407}$. S'agissant des stratégies de réponse dans le cadre de l'adaptation, une application des mesures d'adaptation sur toute l'étendue du territoire est recommandée, mais l'accent a été mis sur les zones côtières du Bénin où des infrastructures de résilience sont prévues pour être réalisées afin d'arrêter la progression de la mer ${ }^{408}$.

Par ailleurs, le Gouvernement béninois s'est aussi doté au cours de l'année 2016, d'un autre document de stratégie intitulé : «document de stratégie de développement à faible

${ }^{401}$ Voir pages 52 à 71 du document de Stratégie béninoise pour la mise en œuvre de la Convention Cadre des Nations Unies sur les Changements Climatiques. Disponible sur le site internet : https://unfccc.int/sites/default. Consulté le 07/01/2022..

${ }^{402}$ Stratégie béninoise pour la mise en œuvre de la Convention Cadre des Nations Unies sur les Changements Climatiques, p.13. Disponible sur le site internet : https://unfecc.int/sites/default/files/resource/Benin. Consulté le 07/01/2022.

${ }^{403}$ Stratégie béninoise pour la mise en œuvre de la Convention Cadre des Nations Unies sur les Changements Climatiques, p.20. Id.

${ }^{404}$.Ibid.

${ }^{405}$ Selon le dictionnaire Larousse en ligne, biomasse se définit comme « Masse vivante, considérée du point de vue de l'énergie que l'on peut en obtenir par combustion ou fermentation (gaz de broussaille, gaz de fumier, feu de bois)». Disponible sur le site internet : https://www.larousse.fr/dictionnaires/francais/biomasse/9439. Consulté le $07 / 01 / 2022$.

${ }^{406}$ Selon le dictionnaire Larousse en ligne «Effet photovoltaïque, est un phénomène physique par lequel un rayonnement lumineux incident peut provoquer l'apparition d'une différence de potentiel entre les deux bornes d'une jonction semi-conductrice ». Id.

${ }^{407}$ Selon le dictionnaire Larousse, le biogaz est un «Gaz, principalement composé de méthane, produit par la décomposition de la matière organique et fournissant une énergie renouvelable». Ibid.

${ }^{408}$ Confère rubrique « Les politiques et mesures sectorielles d'adaptation » à la page 21 du document de Stratégie béninoise pour la mise en œuvre de la Convention Cadre des Nations Unies sur les Changements Climatiques. 
intensité de carbone et résilient au changement climatique $\gg{ }^{409}$. Ce document est en harmonie avec les directives du ministère en charge du Plan en matière d'élaboration de document de stratégie sectorielle ou thématique ${ }^{410}$. La stratégie de développement à faible intensité de carbone et résilient aux changements climatiques est un «document légal de planification intersectorielle thématique à moyen et court terme, validé par le Conseil des Ministres qui couvre la période 2016-2025 » ${ }^{411}$. Elle intègre la préoccupation du Bénin d'organiser une riposte en vue d'atténuer les changements climatiques et vient renforcer les acquis environnementaux en la matière. Ce document projette l'incorporation des dimensions climatiques dans les plans opérationnels, sectoriels, stratégiques du Bénin. C'est un document qui présente «l'état du climat au Bénin, analyse les défis et enjeux de l'adaptation et de l'atténuation. Il établit un diagnostic de la vulnérabilité des principaux secteurs de développement économique du pays, indique les fondements de la stratégie, le processus, le cadre institutionnel de mise en œuvre et de suivi-évaluation. Il devra accompagner le Bénin à faire face aux effets néfastes des changements climatiques notamment à travers des mesures d'adaptation aux changements climatiques d'une part, et, la contribution à la réduction des émissions de GES, d'autre part $»^{412}$.

Ce document comporte quatre (4) points fondamentaux dont, le premier est relatif au "renforcement de la résilience des communautés locales et des systèmes de production agricoles pour notamment, assurer la sécurité alimentaire en améliorant la productivité et en évitant les pertes de production et en développant les capacités des communautés locales dans la gestion d'un développement décentralisé résilient $\gg{ }^{413}$. A ce titre, cinq (5) sous programmes sont prévus pour être mis en œuvre, il s'agit: du «renforcement de la résilience des communautés et filières agricoles $»^{414}$; de la « mise en place des services financiers climatiques

\footnotetext{
${ }^{409} \mathrm{Ce}$ document a été réalisé par le Ministère du Cadre de Vie et du Développement Durable (Ex Ministère de l'Environnement chargé de la Gestion des Changements Climatiques, du Reboisement et de la Protection des Ressources Naturelles et Forestières - MECGCCRPRNF), avec l'appui financier de l'Agence Française de Développement (AFD) et le Programme des Nations unies pour le Développement (PNUD). L'appui technique a été assuré par les experts nationaux à travers six groupes de travail sectoriels, et coordonné par des experts internationaux mobilisés par Expertise France. Disponible sur le site internet : https://unfccc.int/files/focus/longterm_strategies/application/pdf/benin_long-term_strategy.pdf.Consulté le 07/01/2022.

${ }^{410} \mathrm{C}^{\prime}$ est l'outil référentiel en matière d'élaboration de documents de stratégie sectorielle ou thématique au Bénin. ${ }^{411}$ Voir page 11 dudit document. Disponible sur le site internet: https://unfccc.int/files/focus/longterm_strategies/application/pdf/benin_long-term_strategy.pdf. Consulté le 07/01/2022.

${ }^{412}$ Page 14 du document de Stratégie de développement à faible intensité de carbone et résilient aux changements climatiques 2016 - 2025. Id.

${ }^{413}$ Pilier 1, page 12 du document de Stratégie de développement à faible intensité de carbone et résilient aux changements climatiques $2016-2025$.

${ }^{414}$ Sous-programme 1 du Pilier 1 de la Stratégie de développement à faible intensité de carbone et résilient aux changements climatiques, page 12 .
} 
structurant ${ }^{415} ;$ du « renforcement de capacité des collectivités locales en appui à l'émergence de pôles régionaux de développement résilient face aux changements climatiques ${ }^{416}$; de la «construction des barrages à but multiple ${ }^{417}$ et puis de « forage, de construction de réservoirs, d'adductions d'eau potable et de gestion communautaire des ouvrages ${ }^{418}$. Le second point concerne la «Réduction des émissions de GES d'origine anthropique et l'amélioration du potentiel de séquestration de carbone, pour la réduction à la source des émissions de GES, ainsi que pour le renforcement $d u$ potentiel de séquestration de carbone des massifs forestiers. $»^{419}$. Trois (3) sous-programmes seront exécutés dans ce cadre à savoir l' « appui à la transition énergétique ${ }^{420}$; le « développement d'un mécanisme de contrôle/réglementation des émissions de GES » ${ }^{421}$ et le «renforcement des puits de carbone et de réduction des émissions dues à la déforestation et à la dégradation des forêts ${ }^{422}$. Enfin, le troisième point traite de la «Réduction des risques climatiques, la réduction de la vulnérabilité des communautés faces aux catastrophes naturelles ainsi qu'aux maladies sensibles au climat» ${ }^{423}$. Ce pilier sera mis en œuvre à travers quatre (4) sous-programmes que sont : la « mise en place et la restauration du réseau d'écoulement naturel et de drainage des eaux pluviales $\gg{ }^{424}$; le « renforcement du système d'alerte précoce de l'information climatique et de gestion des risques de catastrophes naturelles » ${ }^{425}$; la « protection des côtes d'Afrique de l'Ouest ${ }^{426}$ et la « lutte anti-vectoriel des maladies sensibles au climat ${ }^{427}$.

Néanmoins, les documents de stratégie, au-delà de leur aspect visionnaire, permettent de planifier le cadre de lutte contre les changements climatiques, d'en définir les modalités par des propositions à soumettre aux diverses institutions en charge de l'encadrement du fléau. L'Etat s'en inspire pour faire des prévisions de projets de règlementation. Ces documents n'ont

\footnotetext{
${ }^{415}$ Sous-programme 2 du Pilier 1 de la Stratégie de développement à faible intensité de carbone et résilient aux changements climatiques, page 12 .

${ }^{416}$ Sous-programme 8 du Pilier 1 ibid.

${ }^{417}$ Sous-programme 11 du Pilier 1 ibid.

${ }^{418}$ Sous-programme 12 du Pilier 1 de la Stratégie de développement à faible intensité de carbone et résilient aux changements climatiques, page 12 .

${ }^{419}$ Pilier 2, page 12 du document de Stratégie de développement à faible intensité de carbone et résilient aux changements climatiques $2016-2025$.

${ }^{420}$ Sous-programme 3 du Pilier 2 de la Stratégie de développement à faible intensité de carbone et résilient aux changements climatiques, page 12 .

${ }^{421}$ Sous-programme 4 id.

${ }^{422}$ Sous-programme 5 ibid.

${ }^{423}$ Pilier 3, page 13 du document de Stratégie de développement à faible intensité de carbone et résilient aux changements climatiques $2016-2025$.

${ }^{424}$ Sous-programme 6 du Pilier 3, Page 13, id.

${ }^{425}$ Sous-programme 7, id.

${ }^{426}$ Sous-programme 9, ibid.

${ }^{427}$ Sous-programme 10, ibid.
} 
pas force exécutoire, c'est pourquoi, la multiplicité des stratégies élaborées contrastent avec la réalité. A titre illustratif, en prévision d'un encadrement laborieux des changements climatiques, force est de constater qu'en dehors de l'unique loi élaborée pour réglementer le domaine des changements climatiques ${ }^{428}$ et l'adoption de document de politique de lutte contre les changements climatiques ${ }^{429}$, le cadre d'encadrement a été mitigé. C'est pourquoi, certaines mesures législatives ont été élaborées en vue de faciliter l'application des Stratégies. Ces dispositions tendant à rendre exécutoire l'application des mesures d'intégration des dimensions concernant les changements climatiques dans les documents de planification ${ }^{430}$ sont contenues dans la loi n 2018-18 relative aux changements climatiques en République du Bénin.

Les documents de stratégie ne sont que des outils de travail de référence, cela évite le pilotage à vue en matière de gouvernance climatique mais ne suffissent pas pour traduire en réalité les actions envisagées.

En plus des outils de planifications élaborés, le Gouvernement a pris un certain nombre d'initiative pour réglementer le domaine des changements climatiques.

\section{B-Les initiatives Gouvernementales}

En plus des stratégies élaborées pour planifier les actions et donner une orientation à la lutte contre les changements climatiques, l'Etat béninois a pris des initiatives provenant du Gouvernement pour contribuer à la lutte contre les changements climatiques. Il a procédé à la création d'une institution gouvernementale à compétence environnementale dénommée « Ministère du Cadre de Vie et du Développement Durable (MCVDD) », ${ }^{431}$ qui a en charge la gestion et la protection de l'environnement et la lutte contre les changements climatiques. Ce qui illustre l'option du Gouvernement du Bénin d'améliorer le bien-être de tous les citoyens et de préserver l'environnement au plan national. Ce ministère porte l'ambition de parvenir à un aménagement équilibré du territoire, à l'amélioration progressive mais effective du cadre de vie des populations, dans la promotion d'une gestion rationnelle et durable des ressources naturelles et forestières. Dans cette optique, la vision du secteur « cadre de vie et développement durable »

\footnotetext{
${ }^{428}$ Loi n²018-18 en date du 18 juin 2018 portant règlementation sur les changements climatiques au Bénin. Disponible sur le site internet : https://sgg.gouv.bj/doc/loi-2018-18. Consulté le 07/01/2022.

${ }^{429} \mathrm{Ce}$ document a reçu l'approbation du Conseil des Ministres du mercredi 03 février 2021. Voir compte rendu du Conseil des Ministres du 03 février 2021. Disponible sur le site internet : https://sgg.gouv.bj/cm/2021-02-03. Consulté le 07/01/2022.

${ }^{430} \mathrm{Ces}$ mesures sont contenues dans le chapitre III intitulé « Des obligations de prise en compte des changements climatiques dans les stratégies et planifications nationales et infranationales » de ladite loi.

${ }^{431}$ Décret n ${ }^{\circ} 2016-292$ du 17 mai 2016 fixant la structure -type des ministères en République du Bénin. Disponible sur le site internet: https://sgg.gouv.bj/cm/2021-02-03. Consulté le 07/01/2022.
} 
est « un développement inclusif et durable articulé autour de villes résilientes et sûres ${ }^{432}$ ». Pour en faire une préoccupation éminemment prioritaire, l'article $63 \mathrm{du}$ décret portant attributions organisation et fonctionnement du ministère en charge de l'Environnement a institué dans son organigramme, « la Direction Générale de l'Environnement et du Climat (DGEC) » avec une large attribution. Cette direction a entre autres pour mission, « d'élaborer et d'assurer la mise en œuvre ainsi que le suivi-évaluation de la politique et des stratégies de l'Etat en matière d'environnement et de la promotion de l'économie verte ${ }^{433}$. Elle s'occupe également de la «gestion des effets des changements climatiques, de la production des données environnementales climatiques, de l'économie verte à l'usage de tous les secteurs, puis propose au Gouvernement des mesures préventives de réduction de la pollution environnementale et d'émission de gaz à effet de serre $»^{434}$. Enfin, elle « impulse et coordonne la mise en œuvre des politiques, programmes et projets relatifs à la gestion de l'environnement, aux changements climatiques et à l'économie verte, mais aussi la définition de la vision à long terme de gestion de l'environnement et des effets des changements climatiques devant guider les politiques et mesures mises en œuvre pour faire face aux changements climatiques ${ }^{435}$. Cette réforme institutionnelle a respecté le cadre de stratégie qui a recommandé la prise en compte par le ministère en charge de l'Environnement, du volet concernant les changements climatiques ${ }^{436}$.

Sous un autre angle, malgré l'existence de la loi n 93-009 du 02 juillet 1993 portant protection des forêts en République du Bénin, le constat est alarmant quant à la vitesse à laquelle évolue la déforestation. Cela dénote des faiblesses que contient cette loi et de l'application peu orthodoxe qui en est faite. A titre indicatif, la loi autorise les multinationales à procéder à «l'exploitation des forêts» et à exercer «... le commerce et l'industrie des produits forestiers $\gg{ }^{437}$. Elles en profitent pour prendre d'assaut l'environnement forestier béninois en

\footnotetext{
${ }^{432}$ Programme d'Action du Gouvernement 2016-2021. Disponible sur le site internet : https://sgg.gouv.bj/cm/2021-02-03. Consulté le 07/01/2022.

${ }^{433}$ Article 63 deuxième alinéa deuxième tiret du décret n²016-501 du 11 août 2016 portant, attributions, organisation et fonctionnement du ministère du cadre de vie et du développement durable. Disponible sur le site internet : https://sgg.gouv.bj/cm/2021-02-03. Consulté le 07/01/2022.

${ }^{434}$ Article 63 premier alinéa du décret $n^{\circ} 2016-501$ du 11 août 2016 portant, attributions, organisation et fonctionnement du Ministère du Cadre de Vie et du Développement Durable. Disponible sur le site internet : https://sgg.gouv.bj/cm/2021-02-03. Consulté le 07/01/2022.

${ }^{435}$ Article 63 deuxième alinéa $5^{\mathrm{e}}$ et $8^{\mathrm{e}}$ tiret du décret $\mathrm{n}^{\circ} 2016-501$ du 11 août 2016 portant, attributions, organisation et fonctionnement du ministère du cadre de vie et du développement durable. Disponible sur le site internet : https://sgg.gouv.bj/cm/2021-02-03. Consulté le 07/01/2022.

${ }^{436}$ Voir Stratégie béninoise de mise en œuvre de la Convention Cadre des Nations Unies sur les Changements Climatiques, p.20 Disponible sur le site internet :https://unfccc.int/sites/default/files/resource/Benin\%. Page consultée le 02/06/2021 et Stratégie de développement à faible intensité de carbone et résilient aux changements climatiques 2016 - 2025, paragraphe 2, page 11. Disponible sur le site internet : https://unfccc.int/files/focus/longterm_strategies/application/pdf. Consulté le 08/06/2021.

${ }^{437}$ Article $1^{\text {er }}$ de la loi ${ }^{\circ} 93-009$ du 02 juillet 1993 portant protection des forêts en République du Bénin.
} 
mettant à sac les forêts béninoises. Elles utilisent le circuit du Port Autonome de Cotonou pour transporter les produits forestiers vers l'extérieur du pays, notamment l'Inde et la Chine ${ }^{438}$. Le problème est criard au point de susciter l'indignation des militants environnementalistes qui ne cessent de décrier le phénomène. Cette indignation populaire a provoqué la réaction du Gouvernement Béninois qui est intervenu en avril 2016 et a pris une mesure à polémique de suspension de l'exploitation des ressources forestières ${ }^{439}$. Il n'y avait point de doute sur la pertinence de la décision gouvernementale de suspendre l'exploitation forestière à des fins commerciales voire industrielles. Cette mesure est en phase avec les engagements pris par le Gouvernement béninois dans la Convention Cadre des Nations Unies sur les Changements Climatiques, lesquels consistent à œuvrer pour la création des puits d'absorption des gaz à effet de serre par la protection du domaine forestier, le domaine forestier étant des «pièges à carbone» ${ }^{440}$. Ces engagements ont été déclinés dans le document de Contributions Prévues Déterminées au niveau National (CPDN) comme stratégie devant emprunter le Gouvernement pour réduire les gaz à effet de serre. Cette mesure d'interdiction n'était qu'un moyen de mise en œuvre par voie réglementaire du CPDN, qui représente aussi un outil de stratégie de lutte contre les changements climatiques.

Cependant, l'opinion a été surprise par cette décision qui a créé beaucoup de problème pour la population en ce qui concerne l'assouvissement de leur besoin en combustible dans les ménages. La décision ne propose aucune solution alternative comme mesure d'accompagnement pour adoucir le choc provoqué par la rupture brutale en produits forestiers ${ }^{441}$. Par les moyens de pression, le Gouvernement avait été contraint de rapporter la mesure de suspension en autorisant à nouveau la « commercialisation sur le territoire national de bois d'œuvre, de bois de feu et de charbon de bois ${ }^{442}$. Ainsi, le Conseil des Ministres a décidé de la «suspension des permis de circulation exigés pour le transport de produits forestiers à des fins de consommation exclusivement nationale. Les opérations de débardage et de vidange de bois abattus aux fins de consommation exclusivement nationale ont été autorisées

\footnotetext{
${ }^{438}$ Ministère du Cadre de Vie et de Développement Durable, Direction Générale des Eaux, Forêts et Chasse, Rapport annuel d'activités 2016, DGEFC, page 47.

439 Par décision du Conseil des Ministres du 13 avril 2016, il a été décidé de la suspension provisoire de la délivrance des agréments aux usagers exploitants, commerçants et industriels du bois. Cette est mise en œuvre par l'arrêté n 53/MCVDD/SP du 15 avril 2016. Disponible sur le site internet : https://sgg.gouv.bj/cm/2016-04-13. Consulté le 08/06/2021.

${ }^{440}$ De Perthuis, C. Et pour quelques degrés de plus ... Nos choix économiques face au risque climatique. Paris: Pearson Education France, 2009, p.211.

${ }^{441}$ Besoins relatifs aux feux de bois ou de charbon pour la cuisine, ou dans les ateliers. La situation de chômage précoce dans laquelle ils se voient confrontés.

${ }^{442}$ Extrait de communiqué du Conseil hebdomadaire des Ministres du mercredi 11 mai 2016 disponible sur le site internet du Gouvernement au Bénin : https://sgg.gouv.bj. Consulté le 05/06/2021.
} 
à titre provisoire par le Gouvernement $\gg{ }^{443}$. C'est avec ce revirement intervenu dans la décision du Conseil des Ministres que le bal de la destruction des forets est à nouveau ouvert.

Face au revirement de position du Gouvernement qui a du rapporter sa décision d'interdiction suite à la pression populaire qu'il a subi, n'y a t-il pas lieu de s'interroger sur la pertinence ou non de la décision d'interdiction, de relever ses insuffisances et d'évaluer ses atouts en relation avec le protection des forêts?

Nonobstant la connaissance notoire des répercutions des effets des changements climatiques sur l'homme, il semblerait qu'opter pour des « mesures vertes drastiques » par les Gouvernements ne peut certainement pas fonctionner s'ils s'opposent fermement à d'autres priorités vitales» ${ }^{444}$. La décision d'interdiction de l'exploitation forestière à des fins commerciales est imbue d'objectivé mais manque de réalisme. L'analyse faite de la situation permet de déceler deux (2) problèmes potentiels qui sont à l'origine de la tension attisée par les exploitants, les commerçants et les consommateurs de produits forestiers lors de la survenance de la mesure de suspension de l'exploitation et de commercialisation des produits forestiers. Le premier est lié à l'absence d'une mesure alternative ou d'accompagnement pour atténuer les conséquences qui découleraient de la mise en œuvre de la décision de suspension. Car, après la survenance de la mesure d'interdiction, les populations se sont retrouvées subitement dans l'impossibilité d'accomplir certains besoins liés à l'exploitation forestière qui pour autant constituent une nécessité fondamentale qui conditionne leur existence. A titre illustratif, elles ne sont pas en mesure de produire le « feu » pourtant indispensable dans les ménages à cause de défaut de combustible. Les ménages ont souffert de cette décision, car aucune mesure alternative pour substituer le bois de feu ou le charbon de bois par l'utilisation des combustibles qui protègent l'environnement n'a été envisagée. Les commerçants et exploitants de produits forestiers n'étant pas préparés à cet évènement ont été surpris par la mesure d'interdiction qui les a privé d'intrants pourtant indispensable à l'accomplissement de leurs activités. Normalement, avant de prendre la décision de suspension, il faillait rendre accessible à la population les mesures alternatives appuyées de subventions et de sensibilisation qui atténueraient les effets néfastes induits par la mise en application de la décision de suspension. Le second problème est relatif à la situation sociale de la population assujettie au chômage, à la précarité de l'emploi. La couche de la population qui s'adonne à l'activité de commercialisation

\footnotetext{
${ }^{443}$ Extrait de communiqué du Conseil hebdomadaire des Ministres du mercredi 11 mai 2016 disponible sur le site internet du Gouvernement au Bénin : https://sgg.gouv.bj. Consulté le 05/06/2021.

${ }^{444}$ Genava Global Policy Prospects and Actions in Africa to cope with Climate Change Effects Kamal BENZAIDA $\mathrm{N}^{\circ} 3 / 2020$.
} 
et d'exploitation des produits forestiers a vu subitement sa situation s'aggraver parce qu'elle est exposée à la privation d'emploi ou d'activité génératrice de revenue, lui permettant d'assouvir ses besoins fondamentaux. La solution est toute simple, il fallait offrir une mesure alternative par la promotion de l'emploi et la contribution à l'élévation du niveau de vie de la population pour atténuer les difficultés qu'aurait engendrées la décision d'interdiction. La somme de ces faiblesses a contribué à la rétractation d'une mesure pourtant salutaire voire importante pour la protection du climat. Pour jauger de la pertinence de la mesure, selon les termes du rapport élaboré par la structure compétente, le volume total des exportations de bois en 2016 est évalué à $35137 \mathrm{~m} 3$, comparé au volume total de bois exporté en 2015, soit 83072 $\mathrm{m} 3^{445}$. Il s'ensuit que l'exportation du bois a connue une régression au cours de l'année d'entrée en vigueur de la mesure de suspension de l'exploitation des produits forestiers en $2016^{446}$. Cette régression peut se traduire en terme d'élévation du niveau de protection des forêts au cours de l'année 2016.

Par ailleurs, les conventions internationales élaborées à l'occasion du Sommet de Rio de Janeiro de 1992, qui contribuent à la lutte contre les changements climatiques, pour être mise en œuvre, nécessitent l'adoption de mesures internes d'application. Mais en absence de loi, le gouvernement a souvent usé de ces prérogatives constitutionnelles pour prendre quelques actes réglementaires contribuant à l'application desdites conventions. C'est dans cette optique que le décret n²003-142 du 30 avril 2003 a été pris pour instituer le Comité National sur les Changements Climatiques (CNCC). Un organe pluridisciplinaire chargé du suivi et de l'appui à la mise en œuvre de la Convention Cadre des Nations Unies sur les Changements Climatiques, ainsi que de l'étude de toutes questions scientifiques, technologiques et autres relatives à l'évolution du climat ${ }^{447}$. Cet organe est placé sous la tutelle du Ministre en charge de l'environnement et concentre dans son domaine de compétence toutes les attributions devant aider à la mise en application de la Convention Cadre des Nations Unies sur les Changements Climatiques $^{448}$. Il se charge entre autres, du suivi, du contrôle, du processus d'élaboration et de mise en œuvre de la Stratégie nationale sur les Changements Climatiques de même que, du suivi et le contrôle du processus d'élaboration des Communications Nationales du Bénin sur

\footnotetext{
${ }^{445}$ La preuve en est qu'au cours de cette année il y a régression du trafic portant sur les produits forestiers, comme certifie le Rapport annuel d'activités 2016, DGEFC, page 38, du Ministère du Cadre de Vie et de Développement Durable, Direction Générale des Eaux, Forêts et Chasse.

${ }^{446} \mathrm{Id}$.

${ }^{447}$ Article 2 du décret n ${ }^{\circ} 2003-142$ du 30 avril 2003 portant création, attributions et fonctionnement du Comité National sur les Changements Climatiques. Disponible sur le site internet : https://sgg.gouv.bj/doc/decret-2003142. Consulté le 07/01/2022.

${ }^{448}$ Article 3 du décret id.
} 
les Changements Climatiques, requises en application des dispositions de l'article 12.5 de la Convention $^{449}$.

En outre, la lutte contre les changements climatiques nécessite d'importants moyens de financement. S'inscrivant dans cette logique, le Gouvernement de la République du Bénin a fait constater par décret $n^{\circ}$ 2017-128 du 27 février 2017, la création du Fonds National pour l'Environnement et le Climat (FNEC), une institution qui était régie par la loi n 94-009 du 28 juillet 1994 portant création, organisation et fonctionnement des offices à caractères social, culturel et scientifique ${ }^{450}$. Le Fonds National pour l'Environnement et le Climat est un « établissement public doté de la personnalité morale et de l'autonomie financière. Il est placé sous la tutelle du ministère en charge du Cadre de Vie ${ }^{451}$. Le FNEC est une institution financière qui participe à la promotion des programmes et projets orientés vers les secteurs de protection de l'environnement ${ }^{452}$. Le Fonds intervient également dans le financement des activités relevant du domaine de la lutte contre les changements climatiques au Bénin ${ }^{453}$.

L'engagement du Bénin dans les accords internationaux de protection de l'environnement et de lutte contre les changements climatiques a facilité l'élaboration d'un encadrement juridique des changements climatiques pertinent mais inadéquat.

\section{SECTION II: UN ENCADREMENT JURIDIQUE PERTINENT, MAIS INADEQUAT}

Après avoir ratifié les instruments internationaux de lutte contre les changements climatiques, notamment, la Convention Cadre des Nations Unies sur les Changements Climatiques et ses instruments subséquents ${ }^{454}$ et participé à l'adoption des Déclarations de Rio de Janeiro de $1992^{455}$, le Bénin a élaboré son cadre juridique interne de protection de l'environnement et de lutte contre les changements climatiques. Mais à l'évidence,

\footnotetext{
${ }^{449}$ Article 3 points 5 et 6 du décret n ${ }^{\circ} 2003-142$ du 30 avril 2003 portant création, attributions et fonctionnement du Comité National sur les Changements Climatiques. Disponible sur le site internet: https://sgg.gouv.bj/doc/2. Consulté le 07/01/2022.

${ }^{450}$ Article $1^{\text {er }}$ du décret n ${ }^{\circ} 2003-142$ du 30 avril 2003 portant création, attributions et fonctionnement du Comité National sur les Changements Climatiques. Disponible sur le site internet : https://sgg.gouv.bj/doc/decret-2003142. Consulté le 07/01/2022.

${ }^{451}$ Article 2 du décret n ${ }^{\circ} 2017-128$ du 27 février 2017 constatant approbation de la création du Fonds National pour l'Environnement et le Climat (FNEC). Disponible sur le site internet : https://sgg.gouv.bj/doc/decret-2017-128.. Consulté le 07/01/2022.

${ }_{452}$ Article 5 id.

453 Ibid.

${ }^{454}$ Protocole de Kyoto et l'Accord de Paris.

${ }^{455}$ Déclaration de Rio sur l'environnement et le développement et la Déclaration de Rio sur l'environnement et le Développement : Principes de gestion des forêts.
} 
l'encadrement juridique mis en place quoique pertinent est inadéquat à la lutte contre les changements climatiques.

L'élément de pertinence qui caractérise cet encadrement juridique dénote de l'apport considérable du droit constitutionnel (Paragraphe 1).

Par contre, l'inadéquation du droit en gestation résulte de son caractère inadapté à la lutte contre les changements climatiques (Paragraphe 2).

\section{Paragraphe 1: L’apport du droit constitutionnel}

Pour développer les bases juridiques en matière d'encadrement de la protection de l'environnement, le Bénin a du opérer des réformes fondamentales au niveau de la Constitution pour consacrer la protection constitutionnelle de l'environnement (A) au point où l'environnement a gagné d'importance pour devenir objet de droit de l'homme (B).

\section{A- La protection constitutionnelle de l'environnement}

La constitutionnalisation ${ }^{456}$ du droit de protection de l'environnement est récente au Bénin par rapport à son histoire politique ${ }^{457}$. L'histoire constitutionnelle de ce pays révèle que, les constitutions antérieures ${ }^{458}$ étaient toutes restées silencieuses sur les préoccupations environnementales. Accédé à l'indépendance le $1^{\mathrm{er}}$ août 1960 , le « Dahomey » ${ }^{459}$ actuellement «Bénin ${ }^{460}$, « au regard de son histoire politique et constitutionnelle, assez bouleversée a connu sept (7) constitutions $»^{461}$ jusqu'en 1990, année de bouleversement de l'ordre

\footnotetext{
${ }^{456}$ Selon le dictionnaire Larousse en ligne, la «constitutionnalisation» signifie action conférant valeur constitutionnelle à un texte ou à un principe. Disponible sur le site internet https://www.larousse.fr/dictionnaires/francais/constitutionnalisation. Consulté le 07/01/2022.

${ }^{457}$ Depuis l'accession du Bénin à la souveraineté internationale le $1^{\text {er }}$ aout 1960 , ce n'est qu'en 1990 que la réforme constitutionnelle a intégrée les considérations concernant la protection de l'environnement à travers les articles 27,28,29, 74 et 98 lesquelles jettent les bases de la protection de l'environnement du Bénin.

${ }^{458}$ La constitution de 1959 ; la constitution du 26 novembre 1960 ; la constitution du 11 janvier 1964 ; la charte du $1^{\text {er }}$ septembre 1966 ; la constitution du 11 avril 1968 ; l'ordonnance 69-53 du 26 décembre 1969 portant charte du directoire ; l'ordonnance 70-34-CP du 07 mai 1970 portant charte du Conseil présidentiel ; l'ordonnance 74-68 du 18 novembre 1974 portant structure du pouvoir sous le GMR ; la loi fondamentale du 26 août 1977 amendée par la loi constitutionnelle nº 84003 du 6 mars 1984 ; la loi constitutionnelle n $90-022$ du 13 août 1990 portant organisation des pouvoirs durant la période de transition.

${ }^{459}$ Le 30 novembre 1975 , suite à un changement de régime politique, le Dahomey devient la République populaire du Bénin. Depuis le $1^{\text {er }}$ mars 1990, la République populaire du Bénin est devenue République du Bénin.

${ }^{460}$ Le Dahomey a changé de dénomination et est devenu République du Bénin depuis le 30 novembre 1975.

${ }^{461}$ De GAUDUSSON (Jean du Bois), CONAC (Gérard) et DESSOUCHES (Christine), in Les constitutions africaines, Tome1, édit. Bruylant, Bruxelles, 1997 il s'agit de : la constitution du 15 février1959, la constitution du 05 janvier 1964 ; la constitution du 31 mars 1968 ; la constitution du 07 mai 1970 ; la loi fondamentale du 26 août 1977 promulguée le 09 septembre 1977 et la constitution du 11 décembre 1990.
} 
constitutionnelle. Bien que le défi de protection de l'environnement fût agité, aucune mesure de protection de l'environnement n'était inscrite dans ces constitutions. Cette situation s'apparente à celle des Etats africains qui, dans leur majorité avaient des constitutions considérées essentiellement comme un « document déterminant le régime politique et décrivant le fonctionnement des institutions $»^{462}$. Ces pays redoutaient plus les impacts négatifs que pourrait induire une transformation constitutionnelle en vue de l'intégration des mesures de protection de l'environnement.

En revanche, ce n'est qu'à partir des années quatre-vingt-dix (90), que la constitution béninoise accordera une attention particulière à la protection de l'environnement. Ce fut à l'occasion du bouleversement politique ${ }^{463}$ intervenu dans ce pays que le «droit à un environnement sain, satisfaisant et durable $»^{464}$ a été introduit dans la constitution béninoise. La consécration constitutionnelle du droit à l'environnement est une réponse qui découle de l'application du Droit international, lequel oblige les Etats Parties à légiférer en tenant compte des préoccupations liées à la protection de l'environnement. La constitutionnalisation du droit de protection de l'environnement est un évènement majeur qui n'a pas laissé indifférent certains auteurs, dont François TCHOCA FANIKOUA qui, en appréciant la constitution du 11 décembre 1990, écrit dans son ouvrage que, «l'on peut affirmer aujourd'hui, eu égard à l'émergence des préoccupations environnementales dans la loi fondamentale sans risque de se tromper que la République du Bénin s'est dotée de l'une des constitutions les plus vertes ${ }^{465}$.

Cette réforme a été réalisée à cause de trois (3) précédents qui ont motivé le constituant béninois $^{466}$ à faire de la sauvegarde de l'environnement, un impératif constitutionnel. Pour rappel, après la survenance d'une affaire « supposée » ${ }^{467}$ de déversement de déchet radio actif et toxique en provenance de Tchernobyl en Ukraine sur le territoire béninois, puis la tentative repoussée d'importation en 1988 des déchets dangereux au Port Autonome de Cotonou (PAC) par le Gouvernement béninois et enfin, l'affaire concernant un dossier de 201.000 litres de

\footnotetext{
${ }^{461}$ FALLOUX (François) et TABOT (Lee), Crise et opportunité. Environnement et développement en Afrique, édit G. P. Maisonneuve \& La Rose. ACCT, Paris, 1992, p : 290-291.

${ }^{462}$ FALLOUX (François) et TABOT (Lee), Crise et opportunité. Environnement et développement en Afrique, édit G. P. Maisonneuve \& La Rose. ACCT, Paris, 1992, p : 290-291.

${ }^{463} \mathrm{La}$ conférence nationale des forces vives de la nation béninoise, tenue du, 11 au 28 février 1990 qui a permis de changer le régime dictatorial marxiste par un régime démocratique augurant ainsi l'ère du renouveau démocratique pour ce pays.

${ }^{464}$ Article 27 de la constitution béninoise du 11 décembre 1990 révisée le 07 novembre 2019.

${ }^{465} \mathrm{La}$ contribution du Droit pénal de l'environnement à la répression des atteintes à l'environnement au Bénin,Thèse de docteur François TCHOCA FANIKOUA Maastricht 2012 pp.26 et 27.

${ }^{466}$ Les acteurs de la Constitution béninoise du 11 décembre 1990.

${ }^{467}$ Elle est ainsi qualifiée parce que cette nouvelle provenait des rumeurs diffusées partout dans le pays sans aucune preuve.
} 
pesticides en litige en 1989 au Port Autonome de Cotonou, informée, l'opinion s'en était indignée et avait exigé la fin des pratiques rétrogrades qui violent la protection de l'environnement. Ces évènements ont coïncidé avec un bouleversement politique intervenu des suites d'une crise sociopolitique et économique sans précédent qui a entrainé le pays dans une ère politique qualifiée de «renouveau démocratique». La constitution élaborée dans ce contexte a pris en compte la protection de l'environnement comme un droit constitutionnel. Ce fut un évènement inédit et le premier de l'histoire du droit constitutionnel béninois. L'érection de l'environnement au rang de règle à valeur constitutionnelle est la preuve de l'expression de la volonté inébranlable des autorités de garantir aux populations, le respect des normes qui concourent à la protection de l'environnement. Cette volonté a été clairement affirmée dans la constitution béninoise ${ }^{468}$.

L'intégration du droit de protection de l'environnement à la constitution béninoise est un acte de haute portée politique, accompagnée par un encadrement juridique renforcé et constitué de dispositions qui garantissent, un environnement meilleur au profit des citoyens béninois. Ceux-ci peuvent espérer bénéficier d'un bon environnement comme le stipule l'article 27 de la constitution béninoise qui dispose que, « toute personne a droit à un environnement sain, satisfaisant et durable et a le devoir de le défendre. L'Etat veille à la protection de l'environnement». L'assurance de la protection de l'environnement a été affermie par les dispositions des articles 28 et 29 de la constitution béninoise qui énoncent respectivement, que, «Le stockage, la manipulation et l'évacuation des déchets toxiques ou polluants provenant des usines et autres unités industrielles ou artisanales installées sur le territoire national sont règlementées par la loi » et puis, «Le transit, l'importation, l'enfouissement, le déversement sur le territoire national des déchets toxiques ou polluants étrangers et tout accord y relatif constituent un crime contre la nation. Les sanctions applicables sont définies par la loi ». Toute une panoplie de dispositions constitutionnelles consacrées à la protection de l'environnement qui témoigne de l'attachement des Béninois aux préoccupations liées à leur existence dans leur cadre de vie.

Lorsque les balises du cadre réglementaire de protection de l'environnement sont posées, il convient de mettre en activité les différentes structures relevant du domaine qui vont désormais contribuer au redéploiement d'infrastructures juridiques et institutionnelles adéquates pour la mise en œuvre effective du droit de protection de l'environnement.

\footnotetext{
${ }^{468}$ Comme en témoigne les dispositions des articles 27, 28, 29, 74 et 98 inscrites dans la loi $\mathrm{n}^{\circ} 90-032 \mathrm{du} 11$ décembre 1990, portant constitution de la République du Bénin révisée le 07 novembre 2019.
} 
En effet, les instruments internationaux précurseurs de la notion du droit de jouir d'un environnement sain ont été intégrés au droit positif béninois dans l'objectif de renforcer l'engagement politique du Bénin dans le domaine de la protection de l'environnement. C'est d'abord la Déclaration universelle des droits de l'Homme qui dès 1948 énonçait dans son article 25 que, «toute personne a droit à un niveau de vie suffisant pour assurer sa santé et son bien être » qui a été faite partie intégrante de la Constitution béninoise ${ }^{469}$. Ensuite, sans avoir participé à la Conférence de Stockholm de 1972 où a été élaborée la Déclaration de Stockholm, dans laquelle il a été réaffirmé d'une manière directe que «l'homme a un droit fondamental à la liberté, à l'égalité et à des conditions de vie satisfaisantes, dans un environnement dont la qualité lui permette de vivre dans la dignité et le bien être (...).»» ${ }^{470}$, le Bénin a fait sienne ladite Déclaration. Il en est de même de la Charte Africaine des Droits de l'Homme et des Peuples adoptée à Nairobi au Kenya lors de la $18^{\mathrm{e}}$ conférence des Chefs d'Etat et de Gouvernement de l'Organisation de l'Unité Africaine (OUA) tenue le 18 juin 1981 qui a été ratifiée par le Bénin le 20 janvier 1986. Cette charte qui était élaborée une décennie avant le Sommet de Rio «se réfère aux valeurs de la civilisation africaine ${ }^{471}$ pour consacrer « dans la catégorie des droits de l'homme le droit de tous les peuples à un environnement satisfaisant et global propice à leur développement» ${ }^{472}$ a été également intégrée à la constitution béninoise ${ }^{473}$.

A partir de la consécration constitutionnelle du domaine de protection de l'environnement, l'Etat et ses démembrements se sont s'engagés à élaborer un encadrement juridique qui garantit le droit à un environnement sain aux citoyens. Pour cela, l'Etat a légiféré aux fins de réglementer le comportement des citoyens. Ces derniers ont des droits mais aussi des devoirs notamment, «le devoir de défendre ${ }^{474}$ et de maintenir durablement l'environnement. Afin de bien accomplir ce devoir, le constituant béninois a donné le droit de saisine directe de la Cour constitutionnelle au citoyen pour soit, soulever l'inconstitutionnalité d'une loi ou d'un règlement soit, pour soulever une exception d'inconstitutionnalité devant une juridiction en cas de procès relatif au contentieux environnemental. C'est l'article 122 de la

\footnotetext{
${ }^{469}$ Septième $\left(7^{\mathrm{e}}\right)$ paragraphe du préambule de la Constitution béninoise du 11 décembre 1990 , révisée le 07 novembre 2019.

${ }^{470}$ Principe 1 de la Déclaration de Stockholm de 1972.

${ }^{471}$ Extrait du cinquième $\left(5^{\mathrm{e}}\right)$ paragraphe du préambule de la Charte africaine des droits de l'Homme et des peuples adoptée par la $18^{\mathrm{e}}$ conférence des Chefs d'Etat et de Gouvernement de l'Organisation de l'Unité Africaine le 18 juin 1981.

${ }^{472}$ Article 24 de la Charte africaine des droits de l'Homme et des peuples adoptée par la $18^{\mathrm{e}}$ conférence des Chefs d'Etat et de Gouvernement de l'Organisation de l'Unité Africaine le 18 juin 1981.

${ }^{473}$ Septième $\left(7^{\mathrm{e}}\right)$ paragraphe du préambule de la constitution béninoise du 11 décembre 1990 , révisée le 07 novembre 2019.

${ }^{474}$ Article 27 de la constitution du 11 décembre 1990 révisée le 07 novembre 2019.
} 
constitution béninoise qui consacre cette évolution du droit constitutionnel béninois en disposant que « tout citoyen peut saisir la Cour constitutionnelle sur la constitutionnalité des lois (..)».

Quelles sont les implications juridiques qui découlent de la consécration constitutionnelle du droit de protection de l'environnement?

L'une des conséquences de la consécration constitutionnelle du droit de protection de l'environnement est la consolidation de l'ordonnancement juridique de protection de l'environnement. L'encadrement juridique est désormais élaboré non seulement par le législateur, mais il passe sous le contrôle du juge constitutionnel qui a la responsabilité d'assurer la cohérence et la progression de l'ordonnancement juridique. Il s'ensuit que les lois élaborées en application des dispositions constitutionnelles ne peuvent être en contradiction avec ellesmêmes. Ces lois seront déclarées anticonstitutionnelles et réputées nulles, donc inapplicables. Par conséquent, tout comportement de déviance écologique sera perçu comme une rébellion à l'égard de cette Constitution. Ceci permet de garantir la protection des acquis environnementaux. Le contrôle du Juge constitutionnel doit concourir à garantir in fine le principe de la non régression du droit de protection de l'environnement.

Fort de cette reconnaissance constitutionnelle, le droit à l'environnement ne serait il pas assimilable au droit de l'homme?

\section{B-L'environnement, objet de droit de l'homme}

Les recherches scientifiques menées depuis les années 90 mettent en cause l'Homme en relation avec son environnement. Selon certaines dispositions de la Convention Cadre des Nations Unies sur les Changements Climatiques, « l'activité humaine a augmenté sensiblement les concentrations de gaz à effet de serre dans l'atmosphère $»^{475}$ et engendre un réchauffement supplémentaire de la planète ${ }^{476}$. La plupart des rapports du GIEC se sont appesantis sur la question de la responsabilité de l'homme dans la dégradation de l'environnement et sont parvenus à la même conclusion que, "l'activité humaine contribue au renforcement de l'effet de serre et qu'il en résultera en moyenne un réchauffement supplémentaire de la surface

\footnotetext{
475 Paragraphe 3 du préambule de la Convention Cadre des Nations Unies sur les Changements Climatiques.

${ }^{476}$ Premier rapport d'évaluation du GIEC aperçu général et résumé destiné aux décideurs et supplément 1992 du GIEC.
} 
terrestre et de l'atmosphère, ce dont risquent de souffrir les écosystèmes naturels et l'humanité» ${ }^{477}$.

En réponse à cette situation aggravante, le Droit international a élaboré des normes sous diverses formes pour protéger l'environnement avec ses composants. Mais en réalité, la protection environnementale passe également par la reconnaissance des Droits humains. Cette reconnaissance a son fondement dans la Déclaration adoptée par la Conférence de Stockholm sur l'environnement humain tenue en juin 1972. Son premier principe dispose que, «l'homme a un droit fondamental à la liberté, à l'égalité et à des conditions de vie satisfaisantes, dans un environnement dont la qualité lui permette de vivre dans la dignité et le bien-être. Il a le devoir solennel de protéger et d'améliorer l'environnement pour les générations présentes et futures (...)»). De même, le préambule de la résolution 45/94 de l'Assemblée générale des Nations unies en date du 14 décembre 1990 a été consacré à la "Nécessité d'assurer un environnement salubre pour le bien-être de chacun». Ensuite, par un avis consultatif du 08 juillet $1996^{478}$, la Cour internationale de justice a montré l'importance que représente l'environnement pour l'homme en affirmant dans sa jurisprudence que, «l'environnement n'est pas une abstraction, mais bien au contraire, l'espace où vivent les êtres humains et dont dépendent la qualité de leur vie et leur santé, y compris pour les générations à venir» ${ }^{479}$. Il découle de ce qui précède, une relation entre l'Environnement et les droits fondamentaux de l'homme, de ce point de vue, la COP21 à travers le préambule de la décision 1-/CP.21, a innové sur les implications de ce lien en « considérant que les changements climatiques sont un sujet de préoccupation pour l'humanité tout entière ${ }^{480}$. Ainsi, « les parties devraient, lorsqu'elles prennent des mesures pour faire face à ces changements climatiques, respecter, promouvoir et prendre en considération leurs obligations respectives concernant les droits de l'homme... ${ }^{481}$. Au Brésil, une jurisprudence a été élaborée et a poussé en profondeur la question du droit de l'homme à un environnement sain, en établissant un cloisonnement entre le droit de protection de l'environnement et la protection de la santé des peuples. La décision du Juge brésilien prononcée contre le décret du Gouvernement a ordonné des mesures qui empêchent

\footnotetext{
${ }^{477}$ Troisième $\left(3^{\mathrm{e}}\right)$ paragraphe du préambule de la Convention Cadre des Nations Unies sur les Changements Climatiques.

${ }^{478}$ CIJ, Affaire de la licéité de la menace ou de l'emploi des armes nucléaires, avis consultatif, Rec., 1996, pp. 241-242, § 29. Disponible sur le site internet : https://www.icj-cij.org/public. Consulté le 08/01/2022. ${ }^{479} \mathrm{Id}$.

${ }^{480}$ Extrait du $8^{\mathrm{e}}$ paragraphe du préambule de la décision de la COP 21. Disponible sur le site internet: https://unfccc.int/process-and-meetings/conferences/past-conferences/paris-climate-change-conferencenovember-2015/cop-21/cop-21-decisions. Consulté le 08/01/2022. ${ }^{481}$ Id.
} 
l'application dudit décret en ce qu'il favorise la déforestation dans l'Amazonie ${ }^{482}$. Alors que la violation des règles de protection de l'environnement peut contribuer à la propagation du COVID $19^{483}$ et entamer la santé des Indiens, peuple autochtones de l'Amazonie ${ }^{484}$. Cette conception de la notion de droit de l'homme a été intégrée dans la Charte Africaine des Droits de l'Homme et des Peuples de 1981 en son article 24 qui dispose que, « Tous les peuples ont droit à un environnement satisfaisant et global, propice à leur développement». Cependant, si la Convention Européenne des Droits de l'Homme $(\mathrm{CEDH})^{485}$ a été muette sur la question de l'encadrement de l'environnement, fort curieusement, la juridiction Européenne compétente en matière des Droits de l'Homme a élaboré une jurisprudence importante qui a rapport avec la protection de l'environnement. Selon cette jurisprudence, «la pollution de l'environnement peut porter atteinte au bien-être des individus et ainsi violer certains droits garantis par la $C E D H »{ }^{486}$. Puisque l'objet principal qui caractérise l'existence de cette juridiction constitue les droits de l'Homme, la lecture de cette jurisprudence permet de conclure que la Juridiction Européenne a subordonné le bien être de l'homme au maintien d'un environnement non pollué. Il s'ensuit que, l'environnement peut être perçu comme objet de droit de l'homme et sa protection peut être appréhendée comme celle qui concoure à la protection des droits de l'Homme. Malgré ces prises de position convergente en ce qui concerne la corrélation qui existe entre l'Homme et l'environnement, dont l'une des conséquences est que, la protection de l'environnement contribue à la protection des droits de l'Homme, vice versa, l'opinion

\footnotetext{
${ }^{482}$ Halte au covid19 en Amazonie : le juge au secours des indiens et de la forêt par Michel Prieur, Professeur Emérite à l'Université de Limoges et Président du Centre International de Droit Comparé de l'Environnement publié initialement dans France Forum, ${ }^{\circ} 77$, juillet-aout 2020.

${ }^{483}$ Covid-19 fait référence à «Coronavirus Disease 2019 », la maladie provoquée par un virus de la famille des Coronaviridae, le SARS-CoV-2. Cette maladie infectieuse est une zoonose, dont l'origine est encore débattue, qui a émergé en décembre 2019 dans la ville de Wuhan, dans la province du Hubei en Chine. Elle s'est rapidement propagée, d'abord dans toute la Chine, puis à l'étranger provoquant une épidémie mondiale. Disponible sur le site internet: https://www.futura-sciences.com/sante/definitions/coronavirus-covid-19-18585/.Consulté le $13 / 08 / 2020$

${ }^{484}$ Covid-19 fait référence à «Coronavirus Disease 2019 », la maladie provoquée par un virus de la famille des Coronaviridae, le SARS-CoV-2. Cette maladie infectieuse est une zoonose, dont l'origine est encore débattue, qui a émergé en décembre 2019 dans la ville de Wuhan, dans la province du Hubei en Chine. Elle s'est rapidement propagée, d'abord dans toute la Chine, puis à l'étranger provoquant une épidémie mondiale. Tiré du site internet : https://www.futura-sciences.com/sante/definitions/coronavirus-covid-19-18585/.Consultéle 13/08/2020.

${ }^{485} \mathrm{La}$ Convention de sauvegarde des droits de l'homme et libertés fondamentales, plus connue sous le nom de Convention européenne des droits de l'homme a été ouverte à la signature à Rome le 4 novembre 1950 et est entrée en vigueur en 1953. Elle a été le premier instrument concrétisant et rendant contraignants certains des droits énoncés dans la Déclaration Universelle des Droits de l'Homme. Depuis son adoption en 1950, la Convention a été amendée plusieurs fois et enrichie de nombreux droits qui sont venus s'ajouter au texte initial. Disponible sur le site internet : https://www.echr.coe. Consulté le 08/01/2022.

${ }^{486}$ Voir à titre indicatif l'arrêt de la CEDH en date du 9 décembre 1994, Lopez Ostra c. Espagne (Requête no 16798/90), « la Cour a jugé que les bruits et les odeurs d'une station d'épuration pouvaient affecter le bien-être d'une personne et le droit au respect de son domicile. A un certain degré d'intensité, cela représente une violation du droit au respect de la vie privée et familiale, prévu à l'article 8 de la CEDH ». Disponible sur le site internet : https://echr.coe.int/Pages. Consulté le 04/06/2021.
} 
internationale et certains Etats, ne conçoivent pas que les droits de l'Homme soient pris en compte dans un traité international fut-il, sur les changements climatiques, cette question a suscité des controverses ${ }^{487}$.

Eu égard à ce qui précède, il existe une conséquence juridique qui découle du cloisonnement de la notion des droits de l'Homme et du droit de la protection de l'environnement qui est que, «l'obligation qui incombe aux Etats de protéger les droits de l'Homme peut être traduite comme l'obligation de prendre des mesures pour atténuer l'émission des gaz à effet de serre, car les impacts des changements climatiques entravent la jouissance de l'Homme» ${ }^{488}$. C'est par exemple l'une des leçons véhiculée par la jurisprudence élaborée par les juridictions des Pays-Bas dans l'affaire : Urgenda contre Pays-Bas, ${ }^{489}$ par laquelle il a été imposé à l'Etat Néerlandais le respect de ses engagements de réduction de l'émission des gaz à effet de serre souscrit dans la Convention Cadre des Nations Unies sur les Changements Climatiques.

A l'instar de la Communauté internationale, la constitution béninoise a établie un cloisonnement entre les concepts, « Environnement » et « Droits de l'homme ». Le Titre II de ladite constitution intitulé, «Des droits et devoirs de la personne humaine» est déjà évocateur et illustratif de la volonté du Constituant d'aligner au même rang, l'environnement et Droits fondamentaux de la personne humaine, notamment, le droit de tout individu à la vie, à la liberté, à la sécurité et à l'intégrité de sa personne ${ }^{490}$, le droit à la propriété ${ }^{491}$, le droit d'assurer sa défense $^{492}$. Plusieurs dispositions de la constitution béninoise, notamment les articles 27, 28, 29, 74, 98, 147 sont consacrées à la sauvegarde de l'environnement. Excepté l'article 147, tous les autres sont classés dans la même rubrique que celle qui a consacré le caractère sacré et inviolable de la personne humaine ${ }^{493}$. Par conséquent, le non respect desdites dispositions doit être considéré comme une violation des droits de l'Homme. Parfois, le contrevenant est susceptible de poursuite pour «haute trahison,(...) ${ }^{494}$, lorsqu'il s'agit du Président de la

\footnotetext{
${ }^{487}$ Les droits de l'Homme ont été mentionnés dans le paragraphe $11 \mathrm{du}$ préambule de la Convention Cadre des Nations Unies sur les Changements Climatiques.

${ }^{488}$ Benoit Mayer, « Un examen des lignes directrices de la Commission du droit international sur la protection des atmosphères », Melbourne Journal of International Law, 2019, p.2.

${ }^{489}$ Les références de l'arrêt de Cour suprême des Pays-Bas le 20 décembre 2019: ECLI:NL:HR:2019:2006.

Disponible sur le site internet : https:/www.urgenda.nl/en/themas/climate-case. Consulté le 20 avril 2020.

${ }^{490}$ Article 15 de la constitution béninoise du 11 décembre 1990 révisé le 07 novembre 2019.

491 Article 22, id.

492 Article 17, ibid.

${ }^{493}$ L'article 8 de la constitution du 11 décembre 1990 révisée le 07 novembre 2019 dispose que « La personne humaine est sacrée et inviolable (...) »

${ }^{494}$ Suivant les dispositions de l'article 74 de la constitution béninoise du 11 décembre 1990 révisée le 07 novembre 2019.
} 
République qui «(...), est reconnu auteur, co-auteur ou complice de violations graves et caractérisées des Droits de l'Homme, (...) ou d'acte attentatoire au maintien d'un environnement sain, satisfaisant, durable et favorable au développement $\gg{ }^{495}$. Autrement dit, «les enjeux environnementaux font désormais partie des droits de l’Homme» ${ }^{496}$ au Bénin.

La science a établi que l'existence humaine est rattachée à celle des autres éléments ${ }^{497}$ de l'environnement ${ }^{498}$. Il s'ensuit qu'en protégeant ces éléments, en les préservant des effets néfastes qui découlent de la dégradation de l'environnement, cette protection profite aussi directement ou indirectement 1 'homme ${ }^{499}$. En revanche, les actes qui concourent à la dégradation de l'environnement peuvent provoquer la destruction de l'homme. Dans ce contexte, l'homme peut être privé des conditions d'épanouissement et exposé à la privation de certaines ressources de la nature. La Déclaration de Rio de Janeiro de 1992 sur l'environnement et le développement reconnaît que, «la Terre constitue un tout marqué par l'interdépendance $»^{500}$. Si les préoccupations de l'homme sont inscrites au rang des priorités pour garantir un développement durable, le droit à une « vie saine et productive en harmonie avec la nature» ${ }^{501}$, devra lui être assuré. Il est clair donc que de tout point de vue, la protection de l'environnement est indissociable de la protection de $l^{\prime}$ homme ${ }^{502}$. La conséquence qui découle de l'entremêlement des domaines de l'environnement et droit de l'homme est d'une importance capitale du point de vue de la protection juridique de l'environnement.

Si l'environnement est défini comme objet des droits de l'homme, quel enjeu cela représente dans le cadre de la protection juridique de l'environnement?

L'environnement, ancré dans les droits de l'Homme, devient sur coup une matière transversale pouvant impliquer du point de vue de sa protection une matière présente dans plusieurs juridictions mais plus précisément dans le contexte du droit béninois, devant la juridiction constitutionnelle $\mathrm{e}^{503}$ et les juridictions de droit commun statuant en matière

\footnotetext{
${ }^{495}$ Suivant les dispositions de l'article 74 de la constitution béninoise du 11 décembre 1990 révisée le 07 novembre 2019.

${ }^{496}$ V. JAWORSKI, La Charte constitutionnelle de l'environnement face au droit pénal, in RJE n spécial 2005.

${ }^{497}$ Notamment, la faune, la flore et la biodiversité.

${ }^{498}$ Expression utilisée in PRIEUR M, Vers un droit de l'environnement renouvelée, Les Cahiers du Conseil constitutionnel, 2003, n 15, p. 219 .

499 Ibid.

${ }^{500}$ Extrait de l'introduction aux principes de gestion des forêts déclaration de Rio de Janeiro de juin 1992 sur l'environnement et le développement principes de gestion des forêts, Sommet planète terre conférence des Nations unies sur l'environnement et le développement.

${ }^{501}$ Extrait du principe 1 de la Déclaration de Rio de 1992 sur l'environnement et le développement principes de gestion des forêts Sommet planète.

502 Michel Prieur, « Vers un droit de l'environnement renouvelé », NCCC 2004, n 15.

${ }^{503}$ Aux termes de l'article 114 de la constitution béninoise du 11 décembre 1990, révisée le 07 novembre 2019 , « La Cour constitutionnelle est la plus haute juridiction de l'Etat en matière constitutionnelle. Elle est juge de la
} 
criminelle, administrative et civile. L'ancrage constitutionnel du droit de protection de l'environnement permet de déférer les conflits qui découlent de la gestion de la protection de l'environnement devant la plus haute juridiction de l'Etat béninois en matière de contrôle de constitutionnalité et des libertés fondamentales.

La violation des règles environnementales consacrées dans la constitution est justiciable de la Cour constitutionnelle béninoise. Cette institution a pour compétence de juger de la constitutionnalité de la loi et de garantir les droits fondamentaux de la personne ${ }^{504}$. Ainsi, le juge constitutionnel a des prérogatives tendant à apprécier la conformité des dispositions législatives et réglementaires élaborées dans le domaine de la protection de l'environnement au regard de la constitution. En cas de non-conformité, les dispositions querellées ne sont pas applicables. De la même manière, l'environnement vu sous l'angle de droit de l'homme, détermine également la compétence du Juge constitutionnel béninois. En cas de violation des règles environnementales, la Cour constitutionnelle en tant qu'institution garante des droits fondamentaux de l'Homme est aussi compétente pour en connaitre ${ }^{505}$. La mise en œuvre de ce droit ne souffre d'aucune insuffisance, car les voies de recours sont définies aussi bien pour les autorités administratives que pour les citoyens qui peuvent intervenir par voie d'action ${ }^{506}$ ou procéder à la saisine de la Juridiction constitutionnelle par voie d'exception ${ }^{507}$.

Par ailleurs, la source du droit de l'environnement est fondée sur la constitution. C'est sur cette base juridique que s'est développé un corps de règles de droit pour encadrer tous les domaines de l'environnement et puis spécifiquement, celui concernant les changements climatiques.

\section{Paragraphe 2: L'inadéquation du cadre juridique}

Au cours des deux (2) décennies qui ont précédé la constitutionnalisation du droit de protection de l'environnement au Bénin ${ }^{508}$ et la ratification de la Convention Cadre des Nations

\footnotetext{
constitutionnalité de la loi et elle garantit les droits fondamentaux de la personne humaine et les libertés publiques. Elle est l'organe régulateur du fonctionnement des institutions et de l'activité des pouvoirs publics».

${ }^{504}$ Suivant les dispositions de l'article 114 de la constitution béninoise du 11 décembre 1990 révisée le 07 novembre 2019.

${ }^{505} \mathrm{Id}$.

${ }^{506}$ Les autorités administratives et les citoyens peuvent saisir directement la Cour constitutionnelle lorsqu'il y a violation des règles environnementales.

${ }^{507}$ La saisine par voie d'exception de la Cour constitutionnelle est un recours qui se fait à l'issu d'une procédure pendante devant d'autres juridictions. Dans ce cas, ces juridictions sursoient à statuer jusqu'au prononcé de la décision par la Cour constitutionnelle.

${ }^{508}$ La consécration constitutionnelle du droit de protection de l'environnement est intervenue en 1990 à l'occasion de l'adoption de la constitution béninoise du 11 décembre 1990.
} 
Unies sur les Changement Climatiques ${ }^{509}$, le cadre juridique qui a été élaboré quoique pertinent, ne répond pas convenablement aux objectifs de lutte contre les changements climatiques.

L'analyse des circonstances d'élaboration de cet encadrement juridique révèle le désintérêt du Législateur béninois vis-à-vis du fléau (A). Par contre, il y a un accroissement de législation orientée vers les objectifs généraux de protection de l'environnement (B).

\section{$A$ - Le désintérêt du Législateur}

Le Droit International des Changements Climatiques est certes un droit planétaire, mais il n'est pas suffisamment ramifié. Ce droit est rendu universel par le nombre important d'Etats ayant adhérés la Convention Cadre des Nations Unies sur les Changements Climatiques ${ }^{510}$. En réalité, le lieu d'incubation de ce droit est le territoire des Etats Parties sur lesquels cette matière devra se déployer. Néanmoins, le développement de ce droit, c'est-à-dire, son internalisation dépend de l'engouement qu'éprouvent les institutions de l'Etat et leur capacité à réellement œuvrer pour sa mise en œuvre.

Le Bénin fait partie des Etats où le droit climatique n'a pas vite connu un véritable essor malgré son engagement précoce dans le domaine ${ }^{511}$. La ratification du traité international climatique le 30 juin 1994 vaut pour ce pays son engagement politique, qui le contraint à élaborer des mesures législatives et institutionnelles pour contribuer à l'atteinte des objectifs globaux de la Convention Cadre des Nations Unies sur les Changements Climatiques. Ainsi le Bénin s'est engagé pour atteindre les objectifs de réduction des émissions de gaz à effet de serre par la définition d'une stratégie globale d'intervention qui a abouti à l'élaboration en mai 2003 de la Stratégie Nationale de Mise en Euvre de la Convention Cadre des Nations Unies sur les Changements Climatiques (SNMO). Puis a suivi, la réalisation en 2008 du Programme d'Action du Bénin aux fins d'Adaptation aux changements climatiques (PANA-Bénin) ${ }^{512}$.

\footnotetext{
${ }^{509}$ Le Bénin a ratifié la Convention Cadre des Nations Unies sur les Changements Climatiques le 13 décembre 1993. Etat de ratification de la Convention Cadre des Nations Unies sur les Changements Climatiques, disponible sur le site internet : https://treaties.un.org/pages. Consulté le 09/01/2022.

${ }^{510} \mathrm{La}$ Convention Cadre des Nations Unies sur les Changements Climatiques regroupe actuellement 197 Parties. Soit 196 Etats et une organisation régionale d'intégration économique : l'Union européenne. Voir état de ratification de la Convention Cadre des Nations Unies sur les Changements Climatiques, disponible sur le site internet : https://treaties.un.org/pages. Consulté le 09/01/2022.

511 L'engagement précoce du Bénin dans la lutte contre les changements climatiques est matérialisé par la participation de ce pays au Sommet de Rio de Janeiro de 1992 et la ratification de la Convention Cadre des Nations Unies sur les Changements Climatiques le 30 juin 1994. Voir état de ratification de la Convention Cadre des Nations Unies sur les Changements Climatiques, disponible sur le site internet: https://reaties.un.org/pages. Consulté le 09/01/2022.

${ }^{512}$ En exécution de la Décision 28/CP.7 relative aux lignes directrices annotées pour l'établissement de programmes d'action nationaux aux fins de l'adaptation.
} 
Mais en réalité, la mise en œuvre des stratégies devrait aboutir à l'élaboration entre autres, des lois et règlements en matière de lutte contre les changements climatiques. Mais ce n'était pas le cas. La règlementation qui devrait faciliter la mise en œuvre n'a pas suivi.

Pourquoi ce déficit d'encadrement des changements climatiques dans le contexte de la gouvernance béninoise?

En vertu du principe de séparation des pouvoirs en vigueur en république du Bénin, les normes relevant du domaine de protection de l'environnement sont de la compétence exclusive $\mathrm{du}$ Parlement ${ }^{513}$. La constitution béninoise dispose que, «La loi détermine les principes fondamentaux de protection de l'environnement et de la conservation des ressources naturelles ${ }^{514}$. C'est sur ces fondements que le Parlement a été sollicité dans le cadre du processus de ratification d'instruments internationaux de protection de l'environnement en l'occurrence, la Convention Cadre des Nations Unies sur les Changements Climatiques et ses accords subséquents. Donc, se fondant sur ses attributions constitutionnelles, le parlement doit contribuer à asseoir un cadre juridique de lutte contre les changements climatiques constitué de textes législatifs avant l'intervention de tout autre acte juridique pouvant contribuer à leur application $^{515}$.

Mais force est de constater que, les mesures législatives ayant pour finalité de lutter contre les changements climatiques étaient rares $^{516}$. Le Parlement béninois a connu huit (8) législatures ${ }^{517}$, c'est seulement la septième législature qui a abordé la problématique de la nécessité d'un encadrement législatif des changements climatiques. L'Assemblée nationale ${ }^{518}$ s'est plus préoccupée à donner des autorisations de ratification des accords internationaux destinés à encadrer la lutte contre les changements climatiques ${ }^{519}$ sans beaucoup se soucier de leur modalité de mise en œuvre. Alors qu'en réalité, les outils de mise en œuvre des instruments

\footnotetext{
${ }^{513}$ Le Parlement est constitué au Bénin par l'Assemblée nationale unique dite Assemblée Nationale dont les membres portent le titre de député. Ainsi dispose l'article 79 de la constitution béninoise du 11 décembre 1990 révisée le 07 novembre 2019.

${ }^{514}$ Selon l'article 98 deuxième alinéa $10^{\text {e }}$ tiret de la constitution béninoise du 11 décembre 1990 révisée le 07 novembre 2019.

515 Généralement, les actes réglementaires tels que les décrets, les arrêtés contribuent à la mise en œuvre des dispositions législatives.

${ }^{516}$ Ce n'était qu'en juin 2019 que le Bénin s'est doté de la loi 2018-18 portant, règlementation sur les changements climatiques.

${ }^{517}$ Le mandat de la $7^{\mathrm{e}}$ législature est échu en avril 2019.

${ }^{518} \mathrm{~L}$ 'institution parlementaire béninoise est désignée au Bénin sous le vocable Assemblée Nationale. Voir l'article 79 de la constitution béninoise du 11 décembre 1990 révisée le 07 novembre 2019

519 Il s'agit de la Convention Cadre des Nations Unies sur les Changements Climatiques 13 juin 1993 et le 25 février 2002 et du Protocole de Kyoto. Elle a également souscrit aux autres conventions adoptées à l'occasion du Sommet de Rio de Janeiro de 1992 à savoir, la Convention des Nations Unies pour la Lutte contre la Désertification, qu'il a ratifiée le 14 août 1994, la Convention sur la Biodiversité qu'il a aussi ratifiée en juin 1994 et puis l'Accord de Paris ratifié en 2016.
} 
internationaux de lutte contre les changements climatiques devraient accompagner les actes de ratification. Ce n'était pas le cas et pourtant les risques que représentent les changements climatiques pour le Bénin étaient énormes.

Ce déficit d'encadrement climatique peut être justifié par le désintérêt du Législateur béninois, malgré qu'il a connaissance de la pertinence des risques qu'encourt la population qu'il est sensé représenté et protégé.

Qu'est ce qui peut être à l'origine du défaut d'engouement pour l'encadrement des changements climatiques?

En réalité, les actions législatives sont projetées par des documents de politique de l'Etat. C'est la mise en œuvre de la politique de l'Etat qui débouche sur des initiatives parlementaires qui sont concrétisées par l'élaboration des lois. Bien que les changements climatiques constituaient une priorité pour l'Etat béninois, le contexte caractérisé par l'élaboration tardive de document de politique ${ }^{520}$ et de stratégie en la matière n'ont pas favorisé l'émergence des mesures d'encadrement des changements climatiques. La Stratégie nationale de mise en œuvre au Bénin de la Convention Cadre des Nations Unies sur les Changements Climatiques n'a été réalisée par le ministère en charge de l'Environnement ${ }^{521}$ qu'environ une décennie après la ratification de la Convention Cadre des Nations Unies sur les Changements Climatiques ${ }^{522}$. Le second document intitulé « Stratégie de développement à faible intensité de carbone et résilient aux changements climatiques 2016 - $2025 »$ n'a été adopté qu'en 2016. Mais plus grave, ce n'est que le 03 février 2021 que le Bénin s'est doté d'un document de politique en matière de lutte contre les changements climatiques approuvé en Conseil des Ministres ${ }^{523}$.

Alors qu'en absence de ces outils de planification, il est difficile de développer un encadrement législatif cohérent et efficace.

Pour ne pas rester indifférent à ce tableau alarmant qui compromettrait dangereusement la vie des citoyens qu'ils représentent, certaines initiatives ont été entreprises pour inciter le

\footnotetext{
${ }^{520}$ Approbation en Conseil des Ministres le 03 février 2021 du document de politique de lutte contre les changements climatiques au Bénin. Disponible sur le site internet : https://sgg.gouv.bj/cm/2021-02-03. Consulté le $09 / 01 / 2022$.

${ }^{521}$ Il s'agit du Ministère de l'Environnement, de l'Habitat et de l'Urbanisme (MEHU).

${ }^{522}$ La Stratégie Nationale de Mise en Oeuvre au Bénin de la Convention Cadre des Nations Unies sur les Changements Climatiques a été élaborée en 2003. Disponible sur le site internet : https://unfccc.int/sites/defaue. Consulté le 09/01/2022.

${ }^{523}$ Le document est intitulé « document de politique de lutte contre les changements climatiques au Bénin ».
} 
Parlement à doter le Bénin de lois sur les changements climatiques ${ }^{524}$. C'est dans cette optique que le Fonds Vert pour le Climat (FVC) est intervenu pour soutenir l'effort du Gouvernement béninois en contribuant au renfoncement de capacité de certains acteurs, notamment, du parlement, de la société civile et du secteur privé en charge de la gestion des changements climatiques au Bénin ${ }^{525}$. Ces initiatives ont débouché à terme, sur l'adoption de la première et unique loi sur les changements climatiques ${ }^{526}$.

L'autre facteur qui ne favorise pas l'éclosion des mesures législatives relatives à la lutte contre les changements climatiques découle du contexte de sous développement dans lequel végète le Bénin. Alors que les charges colossales de gestion des activités afférentes à la mise en œuvre du droit applicable aux changements climatiques doivent être planifiées et exécutées suivant les facilités budgétaires. Il s'ensuit que le désintérêt du Législateur Béninois du domaine des changements climatiques n'est pas un comportement voulu. Ce comportement est motivé par l'insuffisance des ressources financières nécessaires à l'accomplissement des actions entrant dans le cadre de la mise en œuvre du droit applicable aux changements climatiques.

$\mathrm{Au}$ moment où les initiatives relevant du domaine des changements climatiques peinent à se développer, il y a un accroissement d'une législation orientée vers les objectifs généraux de protection de l'environnement.

\section{B- L'accroissement d'une législation aux objectifs généraux}

A travers la ratification de la Convention Cadre des Nations Unies sur les Changements Climatiques, le Bénin a pris des engagements politiques qui l'obligent à initier de mesures législatives et institutionnelles afin de mettre en œuvre des actions qui concourent à l'atteinte des objectifs relatifs à ses engagements souscrits dans la Convention Cadre des Nations Unies sur les Changements Climatiques ${ }^{527}$. Cette action de portée internationale devrait avoir pour conséquence, l'édification d'un arsenal juridique qui témoigne de l'ambition des autorités béninoises de conjurer les changements climatiques. Même si cette Convention est d'office

\footnotetext{
${ }^{524}$ Séminaire de formation des députés béninois sur les changements climatiques qui s'est tenu du 9 au 11 octobre à Porto-Novo. C'est une initiative du Réseau des parlementaires béninois pour le climat, l'environnement et le développement durable.

${ }^{525}$ Information disponible sur le site internet : http://www.bj.undp.org/content/benin. Consulté le 09/01/2022.

${ }^{526}$ Il s'agit de la loi n²018-18 portant règlementation des changements climatiques en République du bénin. Disponible sur le site internet : https://sgg.gouv.bj/doc/loi-2018-18.Consulté le 09/01/2022.

${ }^{527}$ Engagements d'atténuation et de réduction des émissions de gaz à effet de serre à travers la réalisation de sa Contribution Déterminée au Niveau National. Les engagements sont déclinés dans l'article 14 de la Convention Cadre des Nations Unies sur les Changements Climatiques.
} 
applicable au Bénin ${ }^{528}$, pour faciliter sa mise en œuvre efficace, un arsenal juridique composé de mesures législatives, ${ }^{529}$ et d'actes réglementaires devrait être élaboré.

Néanmoins, en lieu et place des initiatives tendant à l'encadrement de la lutte contre les changements climatiques, le parlement béninois s'est investi dans l'élaboration des textes de loi dont la plupart ont des objectifs orientés vers la protection de l'environnement. Dans ce cadre, nombreuses sont des lois qui sont adoptées et qui déterminent les conditions de conservation des écosystèmes tels que l'air et les sols, la gestion forestière et la conservation de la faune notamment, la faune terrestre, la faune aquatique et la pêche ${ }^{530}$.

Pour autant, le cadre législatif élaboré pour la protection de l'environnement n'en est pas un, car il ne protège pas suffisamment ce domaine. Dans ce contexte, il serait illusoire d'espérer le règlement des problèmes liés aux changements climatiques avec un encadrement juridique inapproprié. La plus importante loi concernant le domaine de l'environnement est la loi $\mathrm{n}^{\circ}$ 98-030 du 12 février 1999 portant loi cadre sur l'environnement au Bénin. Selon un auteur, la loi cadre est un outil juridique dont l'objectif peu être entre autre « d'encourager une gestion visant à centraliser et à coordonner les différentes interventions ou approches, ainsi que les régimes fonctionnelles des différents secteurs d'intervention pour protéger l'environnement $»^{531}$. A terme, cette catégorie de loi peut permettre de parvenir à « un système intégré de gestion environnementale en rassemblant des régimes juridiques souvent autonomes à chaque secteur, tout en établissant un système généralisé d'interfaces entre les secteurs ${ }^{532}$. Le législateur béninois l'a adoptée comme un instrument « qui définit les bases de la politique en matière d'environnement et qui organise sa mise en œuvre » ${ }^{533}$ à travers « les principes généraux qui régissent la gestion de l'environnement ${ }^{534}$. Mais également, la loi cadre a « créé le cadre institutionnel de gestion de l'environnement et a fixé leurs attributions, leur organisation et les modalités de leur fonctionnement de même que leur domaine d'intervention qui prend en compte des milieux récepteurs et naturels, la protection de la mise en valeur du

\footnotetext{
${ }^{528}$ L'article 147 nouveau de la Constitution du 11 décembre 1990 révisée le 07 novembre 2019 énonce le principe de l'applicabilité des conventions internationales ou traités régulièrement ratifiés après publication et sous réserve de leur entrée en vigueur.

${ }^{529}$ Le fondement en est l'article 98 de la constitution du 11 décembre 1990 révisée le 07 novembre 2019, qui range la protection de l'environnement et de la conservation des ressources naturelles dans le domaine de la loi.

${ }^{530}$ A titre illustratif, la loi nº8-030 du 12 février 1999 portant loi cadre sur l'environnement en République du Bénin à travers ses dispositions régie ces domaines de l'environnement.

${ }^{531}$ Emmanuel G. Moutondo, « Les lois-cadres environnementales dans les pays francophones d'Afrique », in Aspects contemporains du droit de l'environnement en Afrique de l'ouest et centrale, ouvrage coordonné par Laurent Granier, UICN, Droit et politique de l'environnement, nº69, 2008, P. 58.

532 Id.

${ }^{533}$ Article 1 de la loi n ${ }^{\circ} 98-030$ du 12 février 1999 portant loi cadre sur l'environnement en République du Bénin.

${ }^{534}$ Article 3 id.
} 
milieu naturel et de l'environnement humain et de la pollution et des nuisances » ${ }^{535}$. L'étude d'impact $^{536}$, l'audit environnemental ${ }^{537}$ et l'audience publique ${ }^{538}$ sur l'environnement, des plans d'urgence, des mesures d'incitation et enfin des sanctions pénales sont prévues par ladite loi ${ }^{539}$. Elle n'a qu'un caractère général et n'établie que des orientations fondamentales d'une politique à mettre en œuvre dans le domaine de protection de l'environnement. C'est une loi qui « définit les bases de la politique béninoise en matière de protection de l'environnement et organise la mise en auvre des dispositions des articles 27, 28, 29,74 et 98, de la constitution de la République du Bénin» ${ }^{540}$. Elle laisse cependant au pouvoir exécutif, le soin de les développer dans le détail et de les mettre en œuvre en faisant usage de ses prérogatives constitutionnelles. Les principes généraux de la gestion environnementale adoptés dans cette loi, placent en pôle de position l'environnement en tant que, «patrimoine commun de l'humanité ${ }^{541}$ dont la «protection et la mise en valeur doivent faire partie intégrante du plan de développement économique et social et de la stratégie de sa mise en æuvre ${ }^{542}$. Ces mesures tendent « à protéger l'environnement notamment, à prévenir et anticiper les actions de nature à avoir des effets immédiats ou futurs sur la qualité de l'environnement ${ }^{543}$. Ces mesures devraient contribuer « à faire cesser toute pollution et dégradation ou tout au moins, en limiter les effets négatifs sur l'environnement et promouvoir l'assainissement dans le but d'améliorer le cadre de vie et de surveiller étroitement et en permanence la qualité de l'environnement ${ }^{544}$. Elles ont aussi pour objectif, «de restaurer les zones et sites dégradés d'assurer l'équilibre entre l'environnement et le développement ${ }^{545}$.

Par ailleurs, des mesures d'incitation fiscales visant à associer le secteur privé et les entreprises publiques à la mise en œuvre de cette loi sont prévues pour être prises en compte par la loi des finances ${ }^{546}$.

\footnotetext{
${ }^{535}$ Article 7 à 14 de la loi nº8-030 du 12 février 1999 portant loi cadre sur l'environnement en République du Bénin.

${ }^{536}$ Est prévue par les dispositions de l'Article 87 de la loi nº8-030 du 12 février 1999 portant loi cadre sur l'environnement en République du Bénin.

${ }^{537}$ Est un prévu par les dispositions de l'Article 94 id.

${ }^{538}$ Est prévue par les dispositions de l'Article 96 ibid.

${ }^{539}$ Les articles 112 à 119 de la loi nº98-030 du 12 février 1999 portant loi cadre sur l'environnement en République du Bénin sont dédiés aux sanctions pénales.

${ }^{540}$ Selon l'article premier de la loi n ${ }^{\circ} 98-030$ du 12 février 1999 portant loi cadre sur l'environnement en République du Bénin.

${ }^{541}$ Article 3 paragraphe id.

${ }^{542}$ Ibid.

${ }^{543}$ Article 4-a de la loi n98-030 portant loi cadre sur l'environnement en République du Bénin.

${ }^{544}$ Article 4-a id.

${ }^{545}$ Article 4-b et c ibid.

${ }^{546}$ Article 105 ibid.
} 
En dehors de la loi cadre sur l'environnement, d'autres lois sont adoptées pour régir certains domaines environnementaux. La plus importante mesure en raison de sa relevance avec les changements climatiques est celle qui est orientée vers la protection des forêts. Le rôle que joue la forêt dans la protection du climat est indéniable de part sa capacité à absorber les gaz à effet de serre. Elle est d'ailleurs qualifiée de puits d'absorption de gaz à effet de serre et peut contribuer efficacement à la lutte contre les changements climatiques. Cependant, les forêts dont la nature a gratifié le Bénin sont en voie de disparition par le comportement de l'homme. La surexploitation de ces couverts végétaux à des fins économiques contribue à la destruction de ce patrimoine écologique. Pour les besoins de chauffage, les populations font recours au charbon de bois ou aux bois obtenus à partir de l'abattage des arbres provenant des forets. C'est pour éviter ce désastre écologique, que le Législateur Béninois a initié la loi n93-009 du 02 juillet 1993 portant régime des forêts en République du Bénin pour contribuer à la protection des forêts. Néanmoins, en lieu et place d'un instrument de protection, certaines dispositions de ladite loi autorisent implicitement la destruction des forêts par la consécration du principe de la commercialisation des produits forestiers. Le sacre du crime forestier par cette mesure dite, « de protection des forêts" a consisté à concéder le "statut de commerçants de produits forestiers ${ }^{547} » \mathrm{ou}$ « d'exploitant de produits forestiers à des fins commerciales ${ }^{548}$. Ce statut donne accès au commerce des produits forestiers, lequel commerce est autorisé par les dispositions de l'article $1^{\mathrm{er}}$ de ladite loi qui stipule que, «la gestion, la protection, l'exploitation des forets, le commerce et l'industrie des produits forestiers et connexes sont soumis aux dispositions de la présente loi ${ }^{549}$. Alors que, le commerce des produits de l'environnement provoque la mise en contact des règles de deux domaines à objectifs antagonistes car, le commerce des produits de l'environnement concourt à sa destruction, étant donné que les règles commerciales reposent sur la recherche de profit et fonctionnent sur la base de la libre concurrence.

Après avoir défini les domaines forestiers de l'Etat, ceux des particuliers et des coopératives et procédé à la description de la procédure de classement desdits domaines, leurs conditions d'aménagement et d'exploitation, la protection des forêts voulue a été également sacrifiée sur l'hôtel d'une autre disposition de la loi qui consacre le «droit d'usage ». Selon l'article 23 de la loi, «les droits d'usage sont ceux par lesquels des personnes physiques ou morales jouissent à titre temporaire ou définitif des produits de la forêt en vue de satisfaire un

\footnotetext{
${ }^{547}$ Article 45 de la loi n93-009 du 02 juillet 1993 portant régime des forêts en République du bénin.

${ }^{548}$ Article 52 id.

${ }^{549}$ Ibid.
} 
besoin individuel ou collectif ${ }^{550}$. Il en ressort que la loi établie un privilège au profit de certaines personnes qui peuvent jouir des produits de la forêt. Ces droits portent sur le sol forestier, les fruits et les produits de la forêt naturelle.

L'analyse des dispositions de la loi portant sur la protection de la forêt renvoie à la problématique de la qualité des normes législatives de protection de l'environnement au Bénin. C'est pour parer certaines éventualités relatives à la régression en matière de législation que la Cour constitutionnelle a été instituée au Bénin ${ }^{551}$. Mais cette juridiction ne parvient pas, parfois à arrêter certaines dérives législatives, car le contrôle de constitutionnalité ne permet pas toujours de protéger suffisamment l'environnement qui en réalité, bénéficie des règles qui sont essentiellement du domaine de la loi. Par exemple, l'article 32 alinéa premier de la loi portant régime des forets dispose que «Le domaine classé est exempt de tout droit d'usage portant sur le sol forestier. Tout défrichement y est interdit. (...) $»^{552}$. Cette disposition établit le principe de la prohibition du droit d'usage du sol forestier dans le domaine classé. Cependant, le second alinéa de la même disposition introduit une exception à la règle en disposant que, «... Toutefois, ce défrichement peut être spécialement autorisé par l'Administration Forestière (...)»» ${ }^{553}$. La dérogation introduite au côté des mesures d'interdiction, est la brèche qui est ouverte que les membres de l'Administration Forestière peuvent se saisir malicieusement pour organiser la destruction de la forêt parce que in fine la loi dispose que "Cette autorisation est temporaire ${ }^{554}$, mais que "les défrichements devront être exécutées de façon rationnelle sous le contrôle de l'Administration Forestière ${ }^{555}$. Ce régime d'exception peut être utilisé comme moyen de transaction illicite, voire de compromission, dommageable à l'environnement. Toutefois, cette règle pouvait être mieux exploitée dans une administration dont les cadres font preuve de conscience professionnelle et de probité irréprochable.

Mais, est-il important de reconnaitre que, nonobstant les faiblesses qui contrastent avec certains atouts, le droit de protection de l'environnement au Bénin est entrain d'être renforcé à travers certaines dispositions pénales qui y sont incorporées ${ }^{556}$. Le dispositif de sanction est

\footnotetext{
${ }^{550}$ Article 45 de la loi n93-009 du 02 juillet 1993 portant régime des forêts en République du bénin.

${ }^{551} \mathrm{La}$ Cour constitutionnelle est la plus haute juridiction de l'Etat en matière constitutionnelle. Elle est juge de la constitutionnalité de la loi (...). Suivant les dispositions de l'article 114 de la Constitution béninoise du 11 décembre 1990 révisée le 07 novembre 2019.

${ }^{552}$ Il s'agit de la loi n93-009 du 02 juillet 1993 portant régime des forêts en République du bénin

${ }_{553}$ Article 32 alinéa 2, id.

554 Ibid.

555 Ibid.

${ }^{55}$ Par exemple, la loi n²018-18 portant règlementation sur les changements climatiques en République du Bénin est munie d'un dispositif pénal contenu dans le TITRE IX intitulé « DU CONTENTIEUX, DES INFRACTIONS ET DES SANCTIONS »; de même la loi n98-030 portant loi cadre sur l'environnement en République du Bénin est munie d'un dispositif pénal contenu au TITRE VI intitulé « Des sanctions ».
} 
prévu par le Législateur dans les différentes lois destinées à la protection de l'environnement notamment, la loi portant régime de protection des forets en République du Bénin. Par ailleurs, ces mesures de protection de l'environnement bien que n'étant pas orientées vers les objectifs de lutte contre les changements climatiques ne manquent pas de pertinence, car leur application peut contribuer à la protection du climat. Il s'agit par exemple de la mise en œuvre des procédures d'étude d'impact, d'audit environnemental et d'audience publique ${ }^{557}$.

L'ensemble des règles élaborées par le « Droit» dans le cadre de la règlementation des changements climatiques ne peut qu'être mis en œuvre dans un cadre institutionnel créé pour les besoins d'encadrement de la matière.

\footnotetext{
${ }^{557}$ L'ensemble de ces procédures sont prévues dans: Chapitre I: Etude d'impact; Chapitre II : Audit environnemental; Chapitre III : Procédure d'audience publique, de la loi n $^{\circ} 98-030$ portant loi cadre sur l'environnement en République du Bénin.
} 


\section{TITRE II :}

\section{LES INSTITUTIONS DE PROTECTION DU CLIMAT}

Selon Ellul Jacques, l' «une des fonctions primordiales du Droit est de tracer un périmètre à l'intérieur duquel l'ordre imposé par l'homme, l'ordre juridique règne et à l'extérieur duquel se trouve livré d'autres forces, d'autres nécessités ${ }^{558}$. Quant à ce qui concerne le Droit international, cet ordre est établi par les Etats et les Organisations internationales à travers des normes qui sont élaborées pour réglementer les relations internationales. C'est suivant cette logique que le Droit International des Changements Climatiques qui est le chef d'œuvre de la Communauté internationale a fourni un arsenal juridique adéquat aux fins de contribuer à la lutte contre les changements climatiques. Cette protection pour être complète, est assurée par des institutions qui se chargent de la mise en œuvre des règles élaborées dans le but d'atteindre les objectifs de protection du climat. Il s'ensuit que, sans la contribution institutionnelle, la protection tant souhaitée de l'environnement ne peut être réalité.

N'est-ce pas ce qui fait dire au Professeur Maurice KAMTO, que «le Droit International de l'Environnement est l'ensemble des règles et techniques juridiques ainsi que des institutions relatives à la protection du milieu et à la conservation de la nature et des ressources naturelles ${ }^{559}$ ?

Dans le cas d'espèce, partant de la nécessité de mettre à contribution des institutions pour une gestion rationnelle et efficace des changements climatiques, la Communauté internationale a établi un dispositif institutionnel international (CHAPITRE I).

Cet élan d'encadrement institutionnel des changements climatiques par des institutions spécifiques impulsé au plan international, s'est propagé dans les Etats Parties à la Convention Cadre des Nations Unies sur les Changements Climatiques, qui se mirent à élaborer une organisation institutionnelle interne. Le Bénin n'est pas resté en marge de ce mouvement car,

\footnotetext{
${ }^{558}$ Ellul Jacques, Sur l'artificialité du droit et le droit d'exception, Archives de philosophie du Droit, 1963, p.26 et S.

${ }^{559}$ KAMTO (M.), Droit de l'environnement en Afrique, Paris, Edicef, 1996, p. 21.
} 
il a procédé à la mise en place des institutions aux fins d'encadrement des changements climatiques (CHAPITRE II). 


\section{CHAPITRE I:}

\section{LE DISPOSITIF INSTITUTIONNEL INTERNATIONAL}

L'évènement le plus considérable qui a marqué d'un sceau indélébile l'histoire du Droit International de l'Environnement a été la Conférence sur l'Environnement tenue à Stockholm en 1972. Depuis ce moment, les problèmes environnementaux sont devenus un enjeu majeur pour l'humanité et suscitent un intérêt croissant avec l'avènement du Sommet de Rio de Janeiro tenu en 1992.

La prise de conscience des préoccupations environnementales et les enjeux liés à la gestion des changements climatiques par la Communauté internationale a provoqué une émergence institutionnelle (SECTION I). Ce développement institutionnel a été accentué par l'incursion de certaines organisations internationales dans le domaine par la méthode d'appropriation de la question environnementale (SECTION II).

\section{SECTION I: L'EMERGENCE INSTITUTIONNELLE}

L'outil élaboré à l'occasion de la Conférence des Nations unies sur l'Environnement tenue à Stockholm en 1972 et le Plan d'action qui l'a accompagné560 ont recommandé la création d'institutions pour assurer la mise en œuvre des ses dispositions ${ }^{561}$.

Par ailleurs, la gestion des changements climatiques revêt des spécificités qui sont à l'origine des nombreuses institutions dont la création a été projetée dans le traité climatique afin de garantir la pérennité des acquis et ceux provenant éventuellement des rencontres qui seront organisées dans le cadre de la lutte contre les changements climatiques.

C'est l'intervention de l'Organisation des Nations Unies (ONU) dans le domaine de la protection de l'environnement qui a servi de déclic à ce mouvement de création institutionnelle

\footnotetext{
${ }^{560}$ Il s'agit de la Déclaration de Stockholm de 1972.

${ }^{561}$ En ce qui concerne la résolution sur les dispositions institutionnelles et financières, contenue dans le plan d'action de Stockholm de 1972, la Conférence s'est déclarée « Consciente de la nécessité urgente d'instituer dans le cadre des Nations unies, des arrangements institutionnels permanents pour la protection et l'amélioration de l'environnement et a décidé de la création de: 1- Conseil d'administration des programmes relatifs à l'environnement ; 2- Secrétariat de l'environnement ; 3- Fonds pour l'environnement et 4- Coordination ». Voir Rapport de la Conférence des Nations unies sur l'Environnement, Stockholm, 5-16 juin 1972.- Résolutions sur les dispositions institutionnelles et financières, Première Partie : Décision prise par la Conférence, aux pages 33 à 35 . Disponible sur le site internet: https://www.un.org/fr/conferences/environment/stockholm.Consulté le $10 / 01 / 2022$.
} 
(Paragraphe 1). Puis a suivi, le regain de création institutionnelle motivé par l'avènement des changements climatiques, dont l'aboutissement est la constitution d'un réseau institutionnel international essentiellement destiné à la protection du climat (Paragraphe 2).

\section{Paragraphe 1: L'encadrement par l'ONU}

L'encadrement institutionnel international de protection de l'environnement a commencé avec l'ONU. Cette dernière est apparue comme une organisation internationale pionnière en matière d'encadrement de la protection de l'environnement et de gestion des changements climatiques (A). Et puis, le cadre Onusien s'est révélé par la suite comme un espace de création d'institutions de protection de l'environnement et de lutte contre les changements climatiques(B).

\section{A- Une institution pionnière d'encadrement}

Le statut d'organisation pionnière d'encadrement du domaine de l'environnement s'est imposé à l'ONU compte tenu de l'envergure planétaire des conséquences qui découlent de la dégradation de l'environnement, mettant en cause la paix mondiale. En effet, en plus de la préservation de la paix, l'Organisation des Nations Unions (ONU) s'est illustrée à travers l'encadrement du domaine de protection de l'environnement, par l'organisation de Conférence internationale dédiée pour la cause ${ }^{562}$. Pour rappel, avant l'avènement du Sommet de Stockholm de 1972, il n'existait pas un cadre formel de protection de l'environnement à l'échelle internationale. N'avaient droit de citer avant 1972, que des initiatives éparses et unilatérales de chaque Etat de la planète, tendant à l'élaboration des législations orientées dans le sens de la protection de l'environnement notamment, par l'encadrement des éléments relavant de son domaine, telles que, la pollution des eaux, des océans et de l'air. Leur démarche consistait à créer des ministères ayant compétence en matière de protection de l'environnement, qui sont appuyés par des micros organisations avec pour mission d'assurer la préservation de la qualité de vie aux citoyens. Ce fut après, sous l'effet de la pression qui fusait aussi bien du rang des scientifiques que celle provenant des organisations internationales que le Sommet de Stockholm de 1972 avait été organisé avec l'implication effective de l'Organisation des Nations Unies qui

\footnotetext{
${ }^{562} \mathrm{C}$ 'est sur l'initiative de l'Organisation des Nations Unies que la Conférence de Stockholm sur l'Environnement a été organisée en 1972. Voir la résolution A/RES/2850 du 20 décembre 1971 sur la Conférence des Nations unies sur l'Environnement et la résolution A/RES/2849 du 20 décembre 1971 sur le Développement et l'Environnement. Disponible sur le site internet: https://www.un.org/french/documents/ga/m. Consulté le 10/01/2022. Cette conférence a généré des actes qui constituent le fondement du Droit International de l'Environnement.
} 
en était le maître d'œuvre. Cette incursion dans le domaine de la protection de l'environnement a été entérinée par l'Assemblée générale des Nations unies intervenue après la tenue du Sommet de Stockholm qui a eu lieu en 1972 qui reconnait à travers une résolution, que le domaine de protection de l'environnement, « incombe essentiellement aux gouvernants et peut être exercé plus efficacement, en premier lieu, aux nivaux national, et régional ${ }^{563}$ et indique que les «problèmes d'environnement de grande importance internationale relèvent de la compétence des organismes des Nations unies ${ }^{564}$.

Selon Peter Jackson, " $A u$ cours de la période qui a suivi sa création, les questions écologiques encore moins les changements climatiques n'étaient pas des sujets de préoccupation pour les Nations unies» ${ }^{565}$. Il n'y avait que l'Organisation Météorologique Mondiale (OMM), une institution spécialisée des Nations unies qui accomplissait certaines activités qui ont rapport avec la protection de l'environnement. C'était seulement, en 1968, que le Conseil Economique et Social des Nations unies a emboité le pas à l'Organisation Météorologique Mondiale $(\mathrm{OMM})$ en se préoccupant des questions relatives à l'environnement ${ }^{566}$. Leur démarche a été entérinée par la suite par certaines résolutions de l'Assemblée générale des Nations unies qui ont ordonné l'organisation de l'historique Conférence de Stockholm de 1972 consacrée à la problématique de la protection environnementale ${ }^{567}$. Cet engagement des Nations unies dans le domaine de la protection de l'environnement s'est concrétisé et a abouti à l'élaboration de quelques normes et principes fondamentaux de gestion et de protection de l'environnement.

Pour consolider ces acquis environnementaux, l'ONU a posé des actes pour enraciner profondément le Droit international de l'environnement par la réglementation de la

\footnotetext{
${ }^{563}$ Troisième paragraphe de la résolution 2997 du 15 décembre 1972 de l'assemblée générale des Nations unies relatives aux dispositions institutionnelles et financières concernant la coopération internationale dans le domaine de l'environnement. Disponible sur le site internet: https://www.un.org/french/documents. Consulté le $10 / 01 / 2022$.

${ }^{564}$ Quatrième paragraphe de la résolution 2997 du 15 décembre 1972 de l'assemblée générale des Nations unies relatives aux dispositions institutionnelles et financières concernant la coopération internationale dans le domaine de l'environnement. Disponible sur le site internet: https://www.un.org/french/documents. Consulté le $10 / 01 / 2022$

${ }^{565}$ Déclaration de Peter Jackson rédacteur en chef de l'Annuaire de l'ONU, service relevant du Département de l'information, qui publie chaque année l'Annuaire des Nations Unies, l'ouvrage de référence le plus complet sur les activités de l'Organisation dans chronique ONU : De Stockholm à Kyoto : Un Bref Historique du Changement Climatique. Disponible sur le site https:/www.un.org/fr/chronicle/article/de-stockholm-kyoto-un-bref-historiquedu-changement-climatique. Consulté le 10/01/2022.

${ }^{566}$ Le Conseil Economique et Social (ECOSOC), est un des six (6) organes principaux de l'ONU créé d'après le chapitre $\mathrm{X}$ de la Charte des Nations unies. Il est placé sous l'égide de l'Assemblée générale des Nations unies et a un rôle consultatif concernant les questions de coopération économique et sociale internationale.

${ }^{567}$ Voir la résolution A/RES/2850 du 20 décembre 1971 sur la Conférence des Nations unies sur l'Environnement et la résolution A/RES/2849 du 20 décembre 1971 sur le Développement et l'Environnement. Disponible sur le site internet : https://www.un.org/french/documents/ga/res/27/fres27.shtml. Consulté le 10/01/2022.
} 
« coopération internationale dans le domaine de l'environnement en matière de ressources naturelles partagées par deux ou plusieurs Etats ${ }^{568}$. L'ONU a également élaboré un régime de prohibition des activités anthropiques professionnelles pouvant impacter l'environnement. A titre indicatif, puisque les activités militaires non conventionnées peuvent être nuisibles à l'environnement, l'organisation internationale a décrété l'«Interdiction d'agir sur l'environnement et le climat à des fins militaires et autres incompatibles avec le maintien de la sécurité internationale, le bien-être et la santé de l'être humain ${ }^{569}$. Dans ce cadre, la «Convention sur l'interdiction d'utiliser des techniques de modification de l'environnement à des fins militaires ou toutes autres fins hostiles $»^{570}$ a été élaborée. Beaucoup de domaines ayant rapport avec l'environnement ont été encadrés par des résolutions de l'Assemblée générale des Nations unies.

Cet effort de protection de l'environnement dont fait preuve l'organisation faitière a été galvanisé par la survenance des changements climatiques qui menacent dangereusement la paix mondiale. Selon une étude de la Banque mondiale, ce phénomène planétaire développe des effets néfastes qui compliquent les conditions d'existence de l'homme sur la Terre et contribuent à la dégradation de ses droits fondamentaux ${ }^{571}$. L'ONU étant dépositaire de l'instrument international de protection des droits de l'Homme ${ }^{572}$ est interpelée par la violation massive de cet instrument provoquée par les changements climatiques.

L'ONU s'est engagée à nouveau à combattre le fléau planétaire en prenant plusieurs initiatives. A partir des années quatre-vingt (80), après avoir adopté une résolution sur la protection du climat mondial au profit des générations présentes et futures ${ }^{573}$, l'ONU s'est d'abord concentrée sur la protection de la couche d'ozone qui a été encadrée par la Convention de Vienne sur la protection de la couche d'ozone ${ }^{574}$ et par le Protocole de Montréal qui en est

\footnotetext{
${ }^{568}$ Confère la résolution de l'Assemblée Générale des Nations unies A/RES/3129 en date du 13 décembre 1973. Disponible sur le site internet: https://www.un.org/french/documents/ga/res/27/fres27.shtml. Consulté le $10 / 01 / 2022$.

${ }^{569}$ Voir la résolution A/RES/3264 du 09 décembre 1974 de l'Assemblée Générale des Nations Unies. Id.

${ }^{570}$ Voir la résolution A/RES/3172 du 10 décembre 1976 de l'Assemblée Générale des Nations Unies. Id.

${ }^{571}$ Siobhan McInerney-Lankford, Mac Darrow, and Lavanya Rajamani,Humanrights andclimatechange:areview of theinternationallegaldimensions, Banque Mondiale, 2011. Stephen Humphreys(dir.),Humanrights andclimatechange, CambridgeUniversityPress, 2010; Christel Cournil et CatherineColard-Fabregoule (dir.), Changements environnementaux globaux et droits de l'Homme,Bruylant,Bruxelles,2012.

${ }^{572} \mathrm{La}$ Déclaration universelle des droits de l'Homme de 1948. Disponible sur le site internet: https://www.un.org/fr/universal-declaration-human-rights/. Consulté le 08/06/2021.

${ }^{573}$ Voir la résolution de l'Assemblée Générale des Nations unies A/RES/44/207 du 22 décembre 1989 relative à la protection du climat mondial pour les générations présentes et futures. Disponible sur le site internet: https://www.un.org/french/documents/ga/res/27/fres27.shtml. Consulté le 10/01/2022.

${ }^{574} \mathrm{La}$ Convention de Vienne sur la protection de la couche d'ozone a été adoptée en 1985. Disponible sur le site internet : https://treaties.un.org. Consulté le 10/01/2022.
} 
le prolongement ${ }^{575}$ et qui est destinée à renforcer la protection de la couche d'ozone par la règlementation de certaines substances qui provoquent sa dégradation.

L'ONU, préalablement prédestinée pour l'accomplissement des missions internationales de paix pour le compte de la Communauté internationale au profit de l'humanité, s'est retrouvée par la suite concomitamment avec ses attributions originelles, comme une institution créatrice du Droit International de l'Environnement et initiatrice des bases du Droit International de Protection du Climat. L'ONU participe à l'émergence des ces droits par la mise en œuvre de la coopération internationale qui se matérialise par la mobilisation des Etats autour des objectifs de protection de l'environnement et de lutte contre les changements climatiques. Cette institution a œuvré pour le développement de ces droits à travers son élaboration, sa mise en œuvre et continue d'être présente dans le domaine malgré certaines faiblesses qui jalonnent ses interventions.

En tant qu'institution d'encadrement, elle participe non seulement à l'élaboration du cadre normatif, mais elle contribue également à la création d'institutions d'encadrement et de gestion de protection de l'environnement et de lutte contre les changements climatiques.

\section{B- Un cadre de création d'institutions}

La Conférence de Stockholm de 1972 a amorcé des approches de solutions aux questions relatives à l'encadrement institutionnel en recommandant à travers le Plan d'Action pour l'Environnement que, "l'Assemblée générale crée un Conseil d'administration des programmes relatifs à l'environnement(...) ${ }^{576}$. La mise en œuvre des recommandations décidées à Stockholm en 1972, par l'Assemblée générale de l'ONU a abouti à la création du Programme des Nations Unies pour l'Environnement (PNUE), une institution relevant de l'organigramme de l'ONU ${ }^{577}$. C'est la toute première organisation appartenant au système des Nations unies qui a compétence à coordonner et à promouvoir l'action de la Communauté internationale dans le domaine environnemental ${ }^{578}$. La création du PNUE a été rendue possible

\footnotetext{
${ }^{575}$ Le Protocole de Montréal a été adopté en 1987. Disponible sur le site internet : https://treaties.un.org. Consulté le 10/01/2022.

${ }^{576}$ Voir Rapport de la Conférence des Nations unies sur l'Environnement Stockholm, 5 au 16 juin 1972, Première recommandation relative aux « Résolutions sur les dispositions institutionnelles et financières », p.33. Disponible sur le site internet : https://undocs.org/pdf?symbol=fr/A/CONF.48/14/Rev.1. Consulté le 08/06/2021.

${ }^{577}$ Cette institution a été créée par la Résolution 2997 en date du 15 décembre 1972 de l'Assemblée Générale des Nations unies relative aux « Dispositions institutionnelles et financières concernant la coopération internationale dans le domaine de l'environnement ». Disponible sur le site internet : https://www.un.org/french/documents. Consulté le 10/01/2022.

${ }^{578}$ Le domaine de compétence de l'institution est contenu dans le rapport de la Conférence des Nations Unies sur l'Environnement Stockholm, 5 au 16 juin 1972, deuxième recommandation relative aux « Résolutions sur les
} 
grâce à l'adoption de la résolution 2997 de l'Assemblée générale de l'ONU en date du 15 décembre $1972^{579}$, relative aux «Dispositions institutionnelles et financières concernant la coopération internationale dans le domaine de l'environnement $»$. Le Programme des Nations Unies pour l'Environnement est l'institution au sommet en matière de protection internationale de l'environnement incorporée au système des Nations unies . Le Programme des Nations Unies pour l'Environnement est établi à Nairobi au Kenya. C'est un organe subsidiaire permanent des Nations unies «qui a été conçu à l'origine comme un catalyseur devant stimuler l'action des autres institutions $»^{580}$ intervenant dans le domaine de la protection de l'environnement.

Le PNUE a été institué pour « la nécessité d'une mise en œuvre rapide et efficace, par les gouvernements et la Communauté internationale, des mesures conçues pour sauvegarder et améliorer l'environnement au bénéfice des générations actuelles et futures » ${ }^{581}$. Plus tard, «l'action $21 »^{582}$ à renforcer ses attributions en lui conférant la mission de coordination « des tâches qui seront déterminées par des instruments juridiques de plus en plus nombreux notamment, le fonctionnement du secrétariat des conventions internationales en tenant compte de la nécessité d'utiliser aux mieux les ressources, en regroupant éventuellement les secrétariats qui viendront à être créés ${ }^{583}$. Cette institution a pour but, d'assurer la coordination des activités des Nations unies et d'accompagner les pays membres dans la mise en œuvre des politiques environnementales. C'est pourquoi, elle représente le siège de plusieurs conventions de protection de l'environnement ${ }^{584}$.

\footnotetext{
dispositions institutionnelles et financières », p.33. Disponible sur le site internet : https://undocs.org/pdf?symbol=fr/A/CONF.48/14/Rev.1. Consulté le 10/01/2022.

${ }^{579}$ Résolution adoptée par l'assemblée générale au cours de la vingt-septième session 2997 (XXVII) 15 décembre 1972, relatives aux dispositions institutionnelles et financières concernant la coopération internationale dans le domaine de l'environnement. Disponible sur le site internet: https:/www.un.org/french/documents/ga/res. Consulté le 10/01/2022.

${ }^{580} \mathrm{Voy}$. Ph. Sands, 1995. Principles of international environmental law. Manchester University Press, Manchester, New York, vol. 1, Framework, standards and implementation, pp. 72-73.

${ }^{581}$ Premier paragraphe de la résolution 2997 du 15 décembre 1972, dispositions institutionnelles et financières concernant la coopération internationale dans le domaine de l'environnement. Disponible sur le site internet : https://www.un.org/french/documents/ga/res/27/fres27.shtml. Consulté le 10/01/2022.

${ }^{582}$ Le plus substantiel des cinq (5) documents importants issus de la conférence des Nations-Unies sur l'Environnement et le Développement à Rio en 1992. À partir d'Action 21, le concept de développement durable est en train maintenant d'être appliqué dans des plans d'action locaux, régionaux et nationaux, et dans divers secteurs de l'environnement comme l'aménagement urbain, le transport, l'habitation, la foresterie, l'agriculture, les pêcheries, l'énergie, etc. Après avoir décrit brièvement les origines lointaines et prochaines du concept de développement durable, ce texte centrera l'attention sur la mise en œuvre d'Action 21, surtout au plan local.

${ }^{583}$ Voir Action 21 Chapitre 38, paragraphe 622. Disponible sur le site internet : https://www.un.org/french/ga/s. Consulté le 08/06/2021.

${ }^{584}$ Notamment, le Secrétariat de l'Ozone, le Fonds multilatéral du Protocole de Montréal, les Secrétariats de la Convention sur le commerce international des espèces de faune et de flore sauvages menacées d'extinction, la Convention sur la diversité biologique, la Convention sur les espèces migratoires ainsi qu'un nombre important d'accords liés aux substances chimiques, dont la Convention de Bâle sur le contrôle des mouvements transfrontières des déchets dangereux et la Convention de Stockholm sur les polluants organiques persistants.
} 
Après sa création, le PNUE a contribué à consolider les normes environnementales en obligeant les institutions Etatiques à diverses échelles à l'observance des règles de protection de l'environnement. En procédant ainsi, le PNUE a aidé à l'amélioration de la qualité de vie à travers la promotion de la coopération internationale en matière de protection de l'environnement. Le PNUE est composé du Conseil d'administration, du Forum Global des Ministres de l'Environnement, du Secrétariat et du Fonds pour l'Environnement Mondial ${ }^{585}$. Il emploie environ six cents (600) personnes de nationalités diverses et collabore avec les agences de l'environnement ou de l'énergie, scientifiques, Experts et ONG dans le monde ${ }^{586}$. Les questions environnementales ont pris alors de l'ampleur au sein cette organisation avec l'adoption de la résolution des Nations unies relative à «l'étude des perspectives en matière d'environnement jusqu'à l'an 2000 et au-delà» ${ }^{587}$. Cependant, la « complexité, la lourdeur de la structure institutionnelle du PNUE en plus de la précarité de ses moyens » ${ }^{588}$ ne permettent pas à l'organisation d'étendre son autorité sur les Etats et les organisations internationales qui relèvent de sa compétence. En effet, «Il ne dispose pas de pouvoir de surveillance ni de sanction ${ }^{589}$ pour contraindre à l'accomplissement des engagements souscrits par des institutions dans les conventions internationales de protection de l'environnement. Par ailleurs, il lui est reproché le manque de transparence dans la gestion de ses ressources. Tant de facteurs qui entravent l'efficacité des actions du PNUE dans le domaine de la protection de l'environnement.

Malgré son intervention, les problèmes environnementaux s'amplifient et plus grave, viennent s'ajouter les changements climatiques avec ses effets pervers. C'est pourquoi, l'Organisation des Nations Unies à travers la résolution 42/184 en date du 11 décembre 1987 relative à la coopération internationale dans le domaine de l'environnement, adoptée par son Assemblée générale a invité les autorités du Programme des Nations Unies pour l'Environnement (PNUE) $)^{590}$ à se concentrer de concert avec d'autres institutions telles que, l'Organisation Météorologique Mondiale (OMM) et le Conseil International des Unions

\footnotetext{
${ }^{585} \mathrm{La}$ résolution 2997 du 15 décembre 1972, qui prend acte de cette volonté d'institutionnaliser la protection et la gestion de l'environnement par le biais d'arrangements institutionnels permanents, établit un conseil d'administration, un secrétariat, un fonds et un comité administratif de coordination.

${ }^{586}$ Information disponible sur le site internet du Programme des Nations Unies pour l'Environnement https://www.unenvironment.org/fr. Consulté le 05/06/2021.

${ }^{587}$ La résolution de l'Assemblée Générale des Nations unies A/RES/42/186 adoptée à l'occasion de sa $42^{\mathrm{e}}$ session tenue le 11 décembre 1987. Disponible sur le site internet : https://www.un.org/dept. Consulté le 10/01/2022.

588 MALAJEAN-DUBOIS(Sandrine), WEMAERE (Matthieu), La Conférence des Nations unies sur le développement durable Rio +20 . Annuaire français de relations internationales, Paris : La Documentation française, 2014, pp.721-735, p.732.

${ }^{589} \mathrm{Id}$.

${ }^{590}$ Le Conseil d'administration.
} 
Scientifiques $^{591}$, autour de la résolution des problèmes créés par l'évolution du climat dans le cadre du «Programme Climatologique Mondial ${ }^{592}$. Lorsque des preuves des conséquences négatives du changement climatique étaient perçues avec la survenance des pluies acides sur certains continents, des programmes avaient été élaborés par le PNUE en collaboration avec l'OMM pour assurer la veille sur ce phénomène ${ }^{593}$.

Cette collaboration agissante a permis d'asseoir les bases d'une institution internationale technique compétente pour produire de l'expertise dans le domaine des changements climatiques par la création du GIEC dont l'existence juridique a été consacrée à l'occasion de l'Assemblée générale de l'ONU tenue en 1989, sanctionnée par la résolution 44/207 relative à la protection du climat mondial pour les générations présentes et futures ${ }^{594}$.

La Conférence de Stockholm de 1972 avait recommandé que, dans la perspective de l'assouvissement des besoins de financement des programmes environnementaux, « un fonds de contributions volontaires soit constitué conformément aux procédures financières de l'Organisation des Nations unies ${ }^{595}$. Ce fonds est destiné au financement des «initiatives nouvelles qui seront prises en matière d'environnement dans le cadre des organismes des Nations unies. Elles comprendront en particulier les initiatives envisagées dans le Plan d'action adopté par la Conférence des Nations unies sur l'Environnement, notamment en ce qui concerne les projets intégrés, et les autres activités relatives à l'environnement dont pourrait décider le Conseil d'administration» ${ }^{596}$. En exécution des recommandations institutionnelles et financières retenues dans le cadre de la Conférence de Stockholm en 1972, l'Organisation des

\footnotetext{
${ }^{591} \mathrm{Ce}$ Conseil, fondé pour la promotion de l'activité scientifique dans différentes branches et pour des applications ayant pour portée le bien de l'humanité, représente l'expansion et l'évolution de l'Association Internationale des Académies (IAA, 1899 - 1914) et du Conseil International de Recherche (IRC 1919 - 1931). Le secrétariat de l'ICSU se trouve à Paris. Disponible sur le site internet : http://cths.fr/an/societe.php. Consulté le 10/01/2022.

${ }^{592}$ Ce programme est créé en 1979 par l'OMM, le PNUE, la COI et le CIUS. Le PCM aide les Etats à mettre en œuvre l'information climatologique relative aux activités humaines et contribue à l'amélioration des mesures tout en facilitant la compréhension des processus climatiques planétaires.

${ }^{593}$ De Stockholm à Kyoto : Un bref historique du Changement Climatique par Peter Jackson. Disponible sur le site http://www.un.org/french/pubs/chronique/2007/numero2/0207p06.html. Consulté le 10/01/2022.

${ }^{594} \mathrm{La}$ position de l'Assemblée générale des Nations unies sur la création du GIEC et les missions qui lui sont affectées transparaissent à travers les points 1 à 12 du paragraphe 10 de la résolution 44/207 en date du 22 décembre 1989 relative à la «protection du climat mondial pour les générations présentes et futures », transcrite à la page 136 des Résolutions adoptées au cours de la $44^{\mathrm{e}}$ session de l'assemblée générale des Nations unies. Rapport de l'Assemblée générale disponible sur le site internet: https://documents-ddsny.un.org/doc/RESOLUTION/GEN/NR. Consulté le 10/01/2022.

${ }^{595} \mathrm{La}$ sixième recommandation relative aux fonds pour l'environnement contenue dans le rapport de la conférence des Nations unies sur l'environnement Stockholm, 5-16 juin 1972 Nations unies, p. 34. Rapport de la Conférence des Nations unies sur l'Environnement tenue à Stockholm en 1972 disponible sur le site internet: https://undocs.org/pdf?symbol=fr/A/CONF.48/14/Rev.1. Consulté le 10/01/2022.

${ }^{596} \mathrm{La}$ septième recommandation relative aux fonds pour l'environnement contenue dans le rapport de la conférence des Nations unies sur l'environnement Stockholm, 5-16 juin 1972 NATIONS UNIES, p. 34. Le rapport de la Conférence des Nations unies sur l'Environnement tenue à Stockholm en 1972 disponible sur le site internet : https://undocs.org/pdf?symbol=fr/A/CONF.48/14/Rev.1. Consulté le 10/01/2022.
} 
Nations Unies a décidé et créer le Fond Mondial pour l'Environnement (FEM), une institution financière pour appuyer le Programme des Nations Unies pour l'Environnement (PNUE). Ce fonds est alimenté par les ressources provenant des Etats membres de l'Organisation et du budget général de l'ONU ${ }^{597}$.

Par ailleurs, l'application de la Convention Cadre des Nations Unies sur les Changements Climatiques a généré un cadre institutionnel essentiellement climatique

\section{Paragraphe 2 : L'existence d'un cade institutionnel climatique}

La dynamique de création d'institutions de protection de l'environnement provoquée par la tenue de la Conférence de Stockholm de 1972, s'est intensifiée avec l'avènement des changements climatiques. Après la création d'institutions typiquement environnementales ${ }^{598}$, l'ONU s'est investie dans l'élaboration d'un cadre juridique qui a généré des institutions qui ont essentiellement pour vocation, « la lutte contre les changements climatiques ».

L'élaboration et l'application de la Convention Cadre des Nations Unies sur les Changements Climatiques a contribué à la construction d'un réseau institutionnel international essentiellement dédié à la lutte contre les changements climatiques, composé d'un cadre permanent de gestion des changements climatiques (A) et d'un cadre de financement climatique (B).

\section{A- Le cadre permanent de gestion}

La Communauté internationale a fait inscrire dans la Convention Cadre des Nations Unies sur les Changements Climatiques des dispositions qui créent des institutions permanentes pour la mise en œuvre de ladite Convention. En effet, au terme de 1'article 7.-1. de la Convention, un organe dénommé « Conférence des Parties» a été institué. Le protocole de Kyoto défini la «Conférence des Parties» comme l'institution de la Convention Cadre des Nations Unies sur les Changements Climatiques ${ }^{599}$. L'Accord de Paris a repris la même définition que celle du Protocole de Kyoto ${ }^{600}$ mais avec la nuance que le mot « Partie » désigne dans ce contexte les signataires de l'Accord de Paris ${ }^{601}$. Il s'ensuit que l'institution dénommée

\footnotetext{
${ }^{597}$ Maljean- Dubois (Sandrine), Quel droit pour l'environnement? Espagne, UNIGRAF, 2008, p. 115.

598 Telles que le Programme des Nations Unies pour l'Environnement (PNUE) et le Fonds Mondial pour l'Environnement (FME).

${ }^{599}$ Article premier dudit Protocole.

${ }^{600}$ Article premier-b dudit Accord.

${ }^{601}$ Article premier-c id.
} 
«Conférence des Parties» est commune à tous les traités relatifs à la Convention Cadre des Nations Unies sur les Changements Climatiques. La Conférence des Parties a pour mission, de «faire régulièrement le point de l'application de la Convention et de tous autres instruments juridiques connexes qu'elle pourrait adopter et de prendre dans les limites de son mandat, les décisions nécessaires pour favoriser leur application effective» ${ }^{602}$. Pour rendre permanente la mission de cette institution, la même Convention a défini la périodicité des sessions ${ }^{603}$ en ces termes, " la Conférence des Parties, (...), tient des sessions ordinaires une fois par an » ${ }^{604}$, toutes fois que le besoin se fait sentir ou qu'il y a urgence, «La Conférence des Parties tient des sessions extraordinaires à tout autre moment qu'elle juge nécessaire (...) ${ }^{605}$. Ces dispositions conventionnelles combinées, consacrent l'institutionnalisation permanente de l'encadrement des changements climatiques.

La Conférence des Parties (COP) est l'organe au plus haut niveau qui intervient pour les négociations internationales devant se dérouler sous l'emprise de la Convention Cadre des Nations Unies sur les Changements Climatiques ${ }^{606}$. Cette institution est une représentation de l'association de tous les Etats et organisations internationales gouvernementales membres de la Convention ${ }^{607}$. La Conférence des Parties, entre autres fonctions, « examine périodiquement les obligations des Parties et les arrangements institutionnels découlant de la Convention, en fonction de l'objectif de la Convention, de l'expérience acquise lors de son application et de l'évolution des connaissances scientifiques et techniques. Elle encourage et facilite l'échange d'informations sur les mesures adoptées par les Parties pour faire face aux changements climatiques et à leurs effets, en tenant compte de la diversité de situations, de responsabilités et de moyens des Parties ainsi que de leurs engagements respectifs au titre de la Convention ${ }^{608}$. Se fondant sur ces informations, la Conférence des Parties évalue les effets des mesures prises par les Parties et les progrès accomplis pour atteindre l'objectif ultime de la Convention. La présidence de la Conférence des Parties est rotative entre régions de l'ONU que

\footnotetext{
${ }^{602}$ Selon la décision 6/CP.1 et les dispositions de l'article 7-2 de la Convention Cadre des Nations Unies sur les Changements Climatiques.

${ }^{603}$ Les rencontres périodiques sont organisées à intervalle de temps (un an).

${ }^{604}$ Article 7 -4 de la Convention Cadre des Nations Unies sur les Changements Climatiques.

${ }^{605}$ Article 7 id.

${ }^{606}$ Article 7 paragraphe 2 ibid.

${ }^{607}$ Au terme de l'article premier-6 de la Convention Cadre des Nations Unies sur les Changements Climatiques, l'«organisation régionale d'intégration économique» constituée par des Etats souverains d'une région donnée peut avoir compétence dans des domaines régis par la Convention ou ses protocoles lorsqu'elle est dûment autorisée, selon ses procédures internes à, signer, ratifier, accepter, approuver lesdits instruments ou y adhérer.

${ }^{608}$ Article 7 paragraphe 2 a et b de la Convention Cadre des Nations Unies sur les Changements Climatiques.
} 
sont, l'Afrique, l'Amérique et les Caraïbes, l'Asie, l'Europe Centrale et Orientale et, l'Europe de l'Ouest.

La conférence des Parties fonctionne avec une organisation interne dont le Secrétariat de la Convention ${ }^{609}$ à la tête de laquelle se trouve le Secrétaire général qui nomme un Secrétaire exécutif $^{610}$. La conférence des Parties, appuyée de son secrétariat n'est qu'un simple organe politique. L'efficacité de ses actions dépend de la contribution des structures telles que, l'Organe Subsidiaire de Conseil Scientifique et Technologique (SBSTA) $)^{611}$ et l'Organe Subsidiaire pour la mise en application $(\mathrm{SBI})^{612}$ qui font office d'organe d'aide à décision pour la Conférence des Parties $^{613}$ et donnent leur avis à la $\mathrm{COP}^{614}$. Ces institutions sont constituées des représentants des Etats et les Experts et se concertent sur des sujets transversaux concernant l'ensemble de leurs domaines d'expertise ${ }^{615}$.

Comme son nom l'indique, la tâche du SBSTA est de fournir en temps opportun, de conseil à la Conférence des Parties sur les questions scientifiques, technologiques et méthodologiques ${ }^{616}$. Il intervient dans le cadre de la promotion du développement et du transfert de technologies et appuie techniquement les Parties dans le cadre de l'élaboration de leurs communications nationales ${ }^{617}$. Quant à l'Organe Subsidiaire pour la mise en application (SBI), il conseille la Conférence des Parties sur toutes questions relatives à la mise en œuvre de la Convention ${ }^{618}$. Le SBI procède à l'examen des communications nationales et inventaires d'émissions de gaz à effet de serre soumis par les Parties dans le cadre de l'évaluation de l'effectivité de la Convention ${ }^{619}$. Il intervient comme Conseiller de la Conférence des Parties

\footnotetext{
${ }^{609}$ Article 8 de la Convention Cadre des Nations Unies sur les Changements Climatiques.

${ }^{610}$ La nomination du Secrétaire exécutif conformément au point 2 de la décision 14/CP.1 du 07 avril 1995. Disponible sur le site internet https:/unfccc.int/sites/default/files/resource/docs/french. Consulté le 10/01/2022.

${ }^{611}$ Cet organe est créé par l'article 9 de la Convention Cadre des Nations Unies sur les Changements Climatiques.

${ }^{612}$ L'Organe Subsidiaire pour la mise en application est créé par l'article 10 de la Convention Cadre des Nations Unies sur les Changements Climatiques.

613 Suivant les stipulations de l'article 9 de la Convention Cadre des Nations Unies sur les Changements Climatiques.

${ }^{614}$ Article 9 paragraphe 1 de la Convention Cadre des Nations Unies sur les Changements Climatiques.

${ }^{615} \mathrm{La}$ composition des membres de ces institutions est définie respectivement à l'article 9 paragraphe 1 alinéa 2 et à l'article 10 paragraphe 1 de la Convention Cadre des Nations Unies sur les Changements Climatiques.

${ }^{616}$ Les dispositions de l'article 9 paragraphel de la Convention Cadre des Nations Unies sur les Changements Climatiques définissent les attributions du SBSTA.

617 Suivant les dispositions de l'article 9 de la Convention Cadre des Nations Unies sur les Changements Climatiques définissent les attributions du SBSTA.

${ }^{618}$ L'article 10 paragraphe 1 de la Convention Cadre des Nations Unies sur les Changements Climatiques définit les attributions de l'Organe Subsidiaire pour la mise en application (SBI).

${ }^{619}$ L'article 10 paragraphe 2-a de la Convention Cadre des Nations Unies sur les Changements Climatiques définit également les attributions de l'Organe Subsidiaire pour la mise en application (SBI).
} 
sur les questions relatives à la gestion du mécanisme financier géré par le Fonds pour l'Environnement Mondial (FEM) et sur les préoccupations budgétaires ${ }^{620}$.

Depuis son institutionnalisation, plusieurs COP ont été organisées dont, trois (3) ont marqué la progression de l'histoire du droit de protection du climat de part leur importance. La COP1 tenue à Berlin en 1995 est la toute première conférence des Parties organisée après l'adoption de la Convention Cadre des Nations Unies sur les Changements Climatiques. Son importance découle de son caractère inaugural, c'est à cette occasion que les décisions de mise en œuvre de l'instrument international de lutte contre changements climatiques ont été adoptées $^{621}$. Ensuite, la COP3 tenue en 1997, ne manquait pas de pertinence pour l'encadrement juridique du climat. C'est au cours de cette rencontre que le Protocole de Kyoto a été adopté. Ce rendez-vous a été la preuve de la consécration de la consolidation du régime international climatique pour contrer la progression du réchauffement climatique. Cette fois-ci, la Communauté internationale a mis à contribution un instrument qui se distingue légèrement de la Convention de part la méthodologie dans la fixation des objectifs de limitation des gaz à effet de serre, qui seront désormais chiffrés et quantités ${ }^{622}$. Mais en plus, c'est un accord qui présente beaucoup de contrainte de réduction d'émissions pour les Parties ${ }^{623}$. Enfin, la rencontre qui ne manque pas non plus d'intérêt pour la lutte contre les changements climatiques est la COP21, tenue à Paris en $2015^{624}$. L'une des plus importantes décisions prises à ce Sommet est l'adoption de l'Accord de Paris qui représente un protocole additionnel à la Convention Cadre des Nations Unies sur les Changements Climatiques de $1992^{625}$. C'est un accord qui contient des mesures obligatoires mais ne réprime pas ceux d'entre les Etats Parties qui ne respectent pas leurs engagements ${ }^{626}$. Cet Accord a pour objectif de «contenir le réchauffement climatique

\footnotetext{
${ }^{620}$ Secrétariat de la Convention, A Guide to the Climate Change Convention Process, version préliminaire de la deuxième édition, 2002, p.21. Disponible sur le site internet : https://unfccc.int/resource/process/guideprocessp.pdf. Consulté le 10/01/2022.

${ }^{621}$ Voir rapport de la Conférence des Parties à la Convention Cadre des Nations Unies sur les Changements Climatiques, première session tenue à Berlin du 28 mars au 7 avril 1995. Disponible sur le site internet: https://unfccc.int/sites/default/files/resource/docs/french/cop1/g9561656.pdf. Consulté le 08/06/2021.

622 Suivant les dispositions de l'article 2 du Protocole de Kyoto.

${ }^{623}$ Selon l'article 3 paragraphe 1 du Protocole de Kyoto, il convient pour les Parties de « réduire le total de leurs émissions de ces gaz d.au moins $5 \%$ par rapport au niveau de 1990 au cours de la période d'engagement allant de 2008 à $2012 »$.

${ }^{624} \mathrm{Du} 30$ novembre au 12 décembre 2015 s'est tenue à Paris sur le site du Bourget, la 21ème Conférence des Parties de la Convention Cadre des Nations Unies sur les Changements Climatiques (COP21/CMP11).

${ }^{625}$ Le 12 décembre 2015, les 196 parties (195 pays et l'Union européenne) de la Convention Cadre des Nations unies sur les Changements Climatiques (CCNUCC) ont adopté par consensus l'Accord de Paris. Voir état de ratification de l'Accord de Paris sur le site internet : https://treaties.un.org/Pages. Consulté le 30/12/2015.

${ }^{626} \mathrm{~L}$ 'Accord de Paris est un protocole additionnel à la « Convention Cadre des Nations Unies sur les Changements Climatiques » de 1992. Il est contraignant, mais ne prévoit pas de sanctions pour les pays qui y contreviendraient. Disponible sur le site internet : https://www.planete-energies.com/fr/medias/decryptages. Consulté le 11/01/2022.
} 
nettement en dessous de $2{ }^{\circ} \mathrm{C}$ par rapport aux niveaux préindustriels et de poursuivre l'action menée pour limiter l'élévation des températures à $1,5^{\circ} \mathrm{C} \gg{ }^{627}$. L'Accord de Paris est entré en vigueur le 04 novembre 2016, signé par cent quatre-vingt-quinze (195) Etats et ratifié par cent quatre-vingt-onze (191) Parties, y compris les vingt-huit (28) Etats membres de l'Union Européenne (UE) totalisant ainsi 96, 92\% des émissions mondiales de gaz à effet de serre ${ }^{628}$ en plus des deux grandes puissances pollueuses, à savoir la Chine et les Etats-Unis d'Amérique. Seulement que le 04 novembre 2019, les Etats-Unis d'Amérique ont marqué leur désaccord en se retirant de 1'Accord de Paris ${ }^{629}$. Cette décision de retrait des Etats-Unis d'Amérique a pris effet le 04 novembre 2020. Mais aussitôt que l'exécutif a changé de tête à l'issu des élections présidentielles de novembre 2020, la position des Etats-Unis d'Amérique a aussitôt vacillé avec le Président élu, Joe Biden qui a engagé à nouveau avec plus de regain son Pays dans l'Accord de Paris en le ratifiant le 20 janvier $2021^{630}$. Le nouveau Président positionne son pays au cœur de la lutte contre les changements climatiques en nommant un Conseiller spécial aux Changements Climatiques ${ }^{631}$ et en organisant le 22 avril 2021 un Sommet virtuel climatique entre les puissances pollueuses et plusieurs autres Etats de la planète. Les Etats-Unis d'Amérique se sont engagés à réduire de moitié leur taux de pollution à l'horizon 2030. Quant à la Fédération de la Russie qui représente 7,53\% des émissions mondiales de $\mathrm{CO}_{2}{ }^{632}$, elle intègre le Traité de Paris mais déclare « qu'il est inacceptable de se servir de l'Accord et de ses mécanismes pour entraver le développement socioéconomique durable des Parties» ${ }^{633}$.

L'encadrement institutionnel développé dans le contexte de la Convention Cadre est diversifié, car la matière à encadrer présente des subtilités liées à son caractère complexe et universel.

Pour que les objectifs de protection de l'environnement et les défis imposés par les changements climatiques soient relevés, l'encadrement institutionnel a été également orienté dans le sens du financement des changements climatiques.

\footnotetext{
${ }^{627}$ Selon les dispositions de l'article 2 de l'Accord de Paris de 2015.

628 Statistiques concernant l'état de ratification de l'Accord de Paris, disponible sur le site internet: https://treaties.un.org. Consulté le 23 mai 2021.

${ }^{629}$ Voir décision du Secrétaire Général des Nations unies sur le site internet : https://reaties.un.org/doc/Publicat. Consulté le 23/05/2021.

${ }^{630}$ Etat de ratification de l'Accord de Paris disponible sur le site internet : https://treaties.un.org. Consulté le 23/05/2021.

${ }^{631}$ John Kerry.

${ }^{632}$ Selon le décompte officiel utilisé dans le cadre de l'Accord.

633 Voir Paragraphe 3 de la Déclaration de la Fédération de la Russie. Disponible sur le site internet: https://treaties.un.org/Pages/View. Consulté le 11/01/2022.
} 


\section{B- Le cadre de financement}

La mise en œuvre du principe des « responsabilités communes mais différenciées » ${ }^{634}$, de la Convention Cadre des Nations Unies sur les Changements Climatiques oblige les Etats Parties à souscrire à des engagements réciproques. Les pays développés pour la plupart pollueurs, sont commis à l'avant-garde de la lutte contre les changements climatiques et sont assujettis à l'obligation de réduction de l'émission des gaz à effet de serre ${ }^{635}$. Ils sont par conséquent indexés pour apporter le maximum de financement au profit des pays en voie de développement, vulnérables aux effets néfastes des changements climatiques par l'application du principe «pollueur payeur ${ }^{636}$. Ces Etats ont été identifiés en raison de leur contribution massive aux changements climatiques, car grâce aux études scientifiques et statistiques, la Convention a indiqué que « la majeure partie des gaz à effet de serre émise dans le monde par le passé et dans le présent ont leur origine dans les pays développés ${ }^{637}$. Ces Etats sont connus et inscrits sur les listes annexes I et II de la Convention. Dans le cadre de l'acquittement de leurs engagements conventionnels, les Etats- Parties doivent «établir et mettre à jour périodiquement des inventaires nationaux des émissions gaz à effet de serre non réglementés par le Protocole de Montréal » ${ }^{638}$. De même, ils sont invités «à établir à mettre en œuvre à publier et à mettre régulièrement à jour des programmes nationaux et le cas échéant, régionaux contenant des mesures visant à atténuer les changements climatiques en tenant compte des émissions anthropiques par leurs sources et de l'absorption par leurs puits de tous les gaz à effet de serre non réglementés par le Protocole de Montréal, ainsi que des mesures visant à faciliter l'adaptation voulue aux changements climatiques » ${ }^{639}$. Ils sont également invités «à encourager et à soutenir par leur coopération la mise au point, l'application et la diffusion notamment, par voie de transfert de technologies, pratiques et procédés qui permettent de maîtriser, de réduire ou de prévenir les émissions anthropiques des gaz à effet de serre dans tous les secteurs pertinents, en particulier y compris ceux de l'énergie, des transports, de

\footnotetext{
${ }^{634}$ C'est le Principe 1 de l'article 3 de la Convention Cadre des Nations Unies sur les Changements Climatiques. ${ }^{635}$ C'est ce que dispose le Principe $3-1$ de la Convention Cadre des Nations Unies sur les Changements Climatiques.

${ }^{636}$ Le principe pollueur-payeur a été adopté par le Conseil de l'OCDE le 14 novembre 1974 sur proposition du Comité de l'environnement. Voir: OCDE, Recommandation du Conseil sur la mise en ouvre du Principe Pollueur-Payeur, OECD/LEGAL/0132, p.5. Disponible sur le site internet : https://legalinstruments.oecd.org. Consulté le 11/01/2022.

${ }^{637} \mathrm{C}$ 'est ce qui est indiqué dans le quatrième paragraphe du préambule de la Convention Cadre des Nations Unies sur les Changements Climatiques.

${ }^{638}$ Suivant les dispositions de l'article 4 paragraphe 1-a de la Convention Cadre des Nations Unies sur les Changements Climatiques.

${ }^{639}$ Suivant les dispositions de l'article 4 paragraphe 1-b relatif aux engagements dans la Convention Cadre des Nations Unies sur les Changements Climatiques.
} 
l'industrie, de l'agriculture, des forêts et de la gestion des déchets $\gg{ }^{640}$. A l'opposé, les pays en développement sont tenus de développer les puits d'absorption de gaz à effet de serre aux fins d'atténuation de leurs émissions.

Paradoxalement, la réalisation de ces engagements conventionnels, multiples et multiformes sur une grande échelle nécessite la mobilisation d'importantes ressources financières que chaque Etat individuellement pris ne possède. Ces charges constituent un fardeau pour les pays en voie de développement victimes des changements climatiques. Dès lors que la question du financement est apparue comme le nœud gordien pour le règlement des problèmes qui découlent du réchauffement climatique, la complexité du phénomène qui impose la limitation des émissions de gaz à effet de serre à l'échelle planétaire oblige à préconiser des mesures rigoureuses notamment, la mise en place d'un système de financement gigantesque pour assurer les charges induites par la gestion des changements climatiques. Il convient à travers ce financement, de faciliter la mise à disposition de la technologie d'atténuation et d'adaptation pour maitriser les changements climatiques.

A partir du moment où le diagnostic posé révèle que l'exécution des engagements mis à la charge des Etats Parties à la Convention Cadre des Nations Unies sur les Changements Climatiques est conditionnée par la mobilisation d'importantes ressources financières, il importe de trouver une formule pour assurer la disponibilité de moyens financiers dans le cadre de la lutte contre les changements climatiques.

La préoccupation qui apparait en ce moment est de savoir comment mobilier les ressources financières nécessaires et gérer le financement de la lutte contre les changements climatiques?

La Convention Cadre des Nations Unies sur les Changements Climatiques a innové en instituant le «Mécanisme Financier ${ }^{641}$. Son développement devra permettre de mettre à la disposition des pays en voie de développement, d'importantes ressources financières nécessaires pour faire en sorte que ces pays soient en mesure de relever les défis qu'imposent les changements climatiques, tout en développant leur propre économie de façon durable.

En effet, selon les dispositions de la Convention Cadre des Nations Unies sur les Changements Climatiques, «le mécanisme financier est chargé de fournir des ressources financières sous forme de dons ou à des conditions notamment, pour le transfert de la

\footnotetext{
${ }^{640}$ Suivant les prescriptions de l'article 4-c de la Convention Cadre des Nations Unies sur les Changements Climatiques.

${ }^{641}$ Ce sont les dispositions de l'article 11 de la Convention Cadre des Nations Unies sur les Changements Climatiques adoptée à Rio de Janeiro en 1992 qui institutionnalisent le Mécanisme financier.
} 
technologie $»^{642}$. Ce mécanisme est placé sous le contrôle de la Conférence des Parties devant laquelle, il « est responsable et qui définit ses politiques, les priorités de son programme et les critères d'agrément liés à la Convention ${ }^{643}$. Son fonctionnement est confié à plusieurs institutions internationales existantes ${ }^{644}$, notamment, le Fonds pour l'Environnement Mondial du Programme des Nations Unies pour le Développement (PNUD), du Programme des Nations Unies pour l'Environnement (PNUE) et la Banque Internationale pour la Reconstruction et le Développement (BIRD) $)^{645}$.

La mise en œuvre du mécanisme de financement des changements climatiques de la Convention a donné lieu à la naissance d'un flot d'institutions financières, essentiellement qualifiées dans ce domaine créées à l'échelle mondiale, régionale, nationale.

A Copenhague, à l'occasion de la COP15, les pays développées ont adhéré à l'idée de mobilisation de cent (100) milliards de dollars jusqu'en 2020 à mettre à la disposition des pays en développement dans le cadre de la mise en œuvre des mesures d'atténuation des changements climatiques ${ }^{646}$. Ces financements devraient provenir des sources, publiques et privées, bilatérales et multilatérales et affectées en partie au Fonds Vert de Copenhague pour le climat $^{647}$. De même, la Communauté internationale a marqué son approbation pour le transfert de technologie au profit des pays en développement en vue de soutenir les efforts de protection des forêts pour réduire les émissions provenant du déboisement et de la dégradation des forêts. La solidarité internationale s'est manifestée et illustrée par «L'engagement collectif des pays développés qui consiste à fournir des ressources nouvelles et additionnelles, englobant le secteur forestier et des apports d'investissements par les institutions internationales, de l'ordre de 30 milliards de dollars pour la période 2010-2012» ${ }^{648}$. Les engagements convenus à l'occasion de la Conférence de Copenhague sont réalisés lors de la conférence de Cancun de

\footnotetext{
${ }^{642}$ Les dispositions de l'article 11 paragraphe 1 de la Convention Cadre des Nations Unies sur les Changements Climatiques adoptée à Rio de Janeiro en 1992, fixent les attributions du Mécanisme financier.

${ }^{643} \mathrm{Id}$.

644 Ibid.

${ }^{645}$ L'article 21 paragraphe 3 de la Convention Cadre des Nations Unies sur les Changements Climatiques définit les relations du Mécanisme financier avec d'autres institutions financières.

${ }^{646}$ Voir le rapport de la quinzième $\left(15^{\mathrm{e}}\right)$ session de la Conférence des Parties tenue à Copenhague du 7 au 19 décembre 2009. Deuxième partie relative au mesures prises par la Conférence des Parties à sa quinzième session Décision 2/CP.15, Paragraphe 8, p.7. Disponible sur le site internet :https://unfccc.int/resource/docs/2009/cop15. Consulté le 11/01/2022.

${ }^{647} \mathrm{Id}$.

${ }^{648}$ Rapport de la quinzième $\left(15^{\circ}\right)$ session de la Conférence des Parties tenue à Copenhague du 7 au 19 décembre 2009 Additif Deuxième partie relative au mesures prises par la Conférence des Parties à sa quinzième session Décision 2/CP.15, Paragraphe 8, p.7. Disponible sur le site internet : https://unfccc.int/resource/docs/2009/cop. Consulté le 11/01/2022.
} 
2010 par la création du « Fonds vert pour le climat » ${ }^{649}$, qui a démarré ses activités en 2011 lors de la Conférence de Durban sur les changements climatiques ${ }^{650}$. C'est un mécanisme financier dont le siège est établi à Incheon en Corée du Sud et placé sous la tutelle de la Convention Cadre des Nations Unies sur les Changements Climatiques. Le Fond Vert pour le Climat organise le transfert de fonds des Etats développés en direction des pays les moins avancés pour le financement des projets qui rentrent dans le cadre de la lutte contre les changements climatiques. Il est dirigé par un Conseil composé de vingt-quatre (24) membres provenant à égalité de pays avancés et de pays en développement, qui sont nommés pour un mandat de trois (3) ans renouvelable. Deux (2) Coprésidents sont élus par le Conseil parmi ses membres pour une période d'un (1) an ${ }^{651}$.

Les institutions à caractères environnementales aussi riches que variées soient-elles, apportent à leur manière leur contribution à la protection de l'environnement et à la lutte contre les changements climatiques. Elles sont également appuyées par des institutions étrangères au domaine, mais qui y font incursion par nécessité d'épargner l'humanité des effets néfastes qui découlent des changements climatiques, par la méthode d'«appropriation de la question environnementale».

\section{SECTION II: L'ENVIRONNEMENTALISATION D'INSTITUTIONS}

Le foisonnement institutionnel ne découle pas forcément de l'accumulation d'organisations ayant pour champ d'intervention le domaine de protection de l'environnement. Il peut être le fruit d'une accommodation des questions relevant du domaine de la protection de l'environnement ou de la lutte contre les changements climatiques par des institutions, qui à l'origine étaient conçues pour autre objectif. Ces diverses institutions deviennent «environnementales» par appropriation de la question de la protection de l'environnement qu'elles soumettent à leurs procédures.

Cette nouvelle fonction qu'elles accomplissent subsidiairement, vient s'ajouter à leurs attributions principales.

L'environnementalisation est une pratique que certaines organisations internationales (Paragraphe 1) et organisations d'intégration régionale développent pour apporter leur

\footnotetext{
${ }^{649}$ Présentation du Fonds Vert pour le Climat. Disponible sur le site internet : https://www.boad.org/generalitessur-le-fvc.Consulté le 11/01/2022.

${ }^{650}$ Dix-septième $\left(17^{\mathrm{e}}\right)$ Conférence des Parties ; COP17.

${ }^{651}$ Information disponible sur le site internet officiel du Fonds Vert pour le Climat : https://www.greenclimate.f. Consulté le 05/06/2021.
} 
contribution dans le cadre de la protection de l'environnement et de la lutte contre les changements climatiques (Paragraphe 2).

\section{Paragraphe 1: L'intervention d'organisations internationales}

$\mathrm{Au}$ lendemain de l'adoption de la Convention Cadre des Nations Unies sur les Changements Climatiques, il est rare de trouver encore d'organisations internationales qui n'ont pas étendue leur champ d'intervention au domaine de la protection de l'environnement. Nombreuses sont les institutions créées pour divers objectifs qui, en plus de leurs activités originelles, ont opté pour la protection de l'environnement. De nos jours, le phénomène d'environnementalisation d'organisations internationales foisonne.

Deux (2) exemples d'organisations internationales seront pris en compte dans le cadre de notre étude compte tenu de leur importance. Il s'agit en premier lieu, de l'exemple de l'Organisation Mondiale pour le Commerce (OMC) qui est une parfaite illustration du concept d'environnementalisation d'organisation internationale, car son importance n'est plus à démontrer dans l'arène d'institutions ayant pour vocation d'aider à conjurer les problèmes qui accablent l'environnement, alors qu'elle est créée pour réglementer le commerce mondial (A). En second lieu, il y a le cas de l'Organisation des Nations Unies pour l'Education, la Science et la Culture (UNESCO), une organisation à spécialité de protection du patrimoine culturel et naturel, qui joue un rôle non moins important au côté des acteurs originels du domaine de protection de l'environnement en s'investissant dans le domaine des changements climatiques (B).

\section{A- L'exemple de l'OMC}

Les règles qui régissent le commerce international et celles qui concourent à la protection de l'environnement relèvent de deux (2) domaines différents, dont les objectifs sont antagonistes, mais parfois interconnectés. Le commerce est fondé sur le principe de libre échange et peut être opéré sur les éléments de l'environnement. En effet, la mise en œuvre du principe de libre échange peut conduire à des conséquences graves sur l'environnement, car elle peut donner lieu à une exploitation effrénée des biens de l'environnement qui entrainera sa destruction. Alors que la protection de l'environnement conduit à l'élaboration et à l'application des mesures prohibitives ou permissives en vue de son encadrement pour sa préservation. Dans ce cas, la mise en œuvre des règles de protection de l'environnement peut parfois constituer un obstacle pour le principe de libre échange pratiqué dans le domaine du commerce. Eu égard à ce qui précède, la Déclaration de Rio de Janeiro de 1992 a prohibé « Les mesures de politique 
commerciale motivées par des considérations relatives à l'environnement ${ }^{652}$ qui constituent « un moyen de discrimination arbitraire ou injustifiable ${ }^{653}$ ou soit, représentent une « restriction déguisée aux échanges internationaux ${ }^{654}$. D'où l'importance de l'intervention d'une institution de régulation pour gérer les conflits d'incursion dans les domaines environnemental et commercial.

L'Organisation Mondiale du Commerce (OMC) a été créée pour «servir de cadre institutionnel commun pour la conduite des relations commerciales entre ses Membres (...) $)^{655}$. L'institution administre un certain nombre d'accords commerciaux, multilatéraux, qui font partie intégrante de l'Accord qui l'a institué ${ }^{656}$. Il s'agit des accords multilatéraux sur le commerce des marchandises ${ }^{657}$, le commerce des services ${ }^{658}$ et sur les aspects des droits de propriété intellectuelle qui touchent au commerce ${ }^{659}$, puis les accords commerciaux plurilatéraux portant sur le commerce des aéronefs civils, les marchés publics, le secteur laitier et la viande bovine ${ }^{660}$.

L'Organisation Mondiale pour le Commerce est l'unique institution mondiale à caractère commercial qui intervient dans le domaine de la protection de l'environnement. Les préoccupations liées à la règlementation du commerce international en liaison avec les domaines du développement durable, de la protection et la préservation de l'environnement sont consignées dans l'Accord instituant l'Organisation Mondiale du Commerce ${ }^{661}$. L'OMC intervient pour faciliter l'application des règles commerciales en intégrant les préoccupations liées à la protection et à la préservation de l'environnement. Dans l'optique où « commerce » et «environnement» sont vus comme deux (2) domaines à objectif complémentaire, le commerce international devrait aussi permettre de créer des richesses pour accompagner le bien être de l'humanité et assurer une meilleure protection de l'environnement.

\footnotetext{
${ }^{652}$ Ces mesures sont éditées par le principe 12 de la Déclaration de Rio de Janeiro de 1992.

${ }^{653} \mathrm{Id}$.

654 Ibid.

${ }^{655}$ C'est l'article 2 paragraphe 1 de Accord instituant l'Organisation Mondiale du Commerce qui détermine les attributions de l'Organisation.

${ }^{656} \mathrm{Id}$.

${ }^{657}$ L'annexe 1A à l'Accord instituant l'OMC comporte les accords administrés par l'Organisation commerciale internationale.

${ }^{658} \mathrm{~L}$ 'annexe $1 \mathrm{~B}$ id.

${ }^{659}$ L'annexe $1 \mathrm{C}$ ibid.

${ }^{660}$ Annexe 4 à l'Accord instituant l'OMC comporte les accords administrés par l'Organisation commerciale internationale.

${ }^{661}$ Deuxième paragraphe du préambule de l'Accord instituant l'OMC.
} 
A la lumière de la mission assignée à l'OMC, institution internationale qualifiée pour réglementer le domaine commercial, comment se manifeste le glissement de cette institution vers le domaine environnemental?

L'Organisation Mondiale du Commerce intervient dans le domaine de la protection de l'environnement de part la possibilité que les statuts offrent aux Membres de ladite organisation, qui peuvent élaborer des mesures commerciales qui protègent l'environnement ou participent à la lutte contre les changements climatiques. Quoi qu'elles n'aient pas été expressément indiquées dans les Accords de l'OMC, certaines dispositions peuvent être adoptées par les Membres de l'Organisation dans le sens de la protection de l'environnement ou de la lutte contre les changements climatiques. Cette progression a été consacrée par la jurisprudence élaborée par les différends organes de l'OMC qui sont intervenus pour préciser la portée et les limites de l'intervention de l'Organisation commerciale dans le domaine de la protection de l'environnement. Selon la source jurisprudentielle, cet élargissement du domaine d'intervention de l'OMC se justifie à travers les résultats d'un rapport provenant de son Organe d'appel, qui précise que le droit de cette institution n'est pas distinct du droit international et de ce fait, le droit commercial admet un lien avec le droit qui protège l'environnement ${ }^{662}$. Seulement que, la mise en œuvre de ce droit admet des conditions liées au caractère objectif et légitime que doit revêtir la mesure de protection de l'environnement, laquelle ne doit pas être discriminatoire, ni protectionniste ${ }^{663}$. Les mesures que les Membres de l'OMC sont autorisées à édicter ne doivent violer la règle de libre échange commercial qui représente un principe cardinal défendu par l'organisation commerciale. L'évolution jurisprudentielle de l'Organisation a été orientée dans ce sens et est conforme à l'objectif finaliste de l'Organisation commerciale, celui «de contribuer à la réalisation de ses objectifs par la conclusion d'accords visant, sur une base de réciprocité et d'avantages mutuels, à la réduction substantielle des tarifs douaniers et des autres obstacles au commerce et à l'élimination des discriminations dans les relations commerciales internationales $»^{664}$. Néanmoins, l'utilisation des mesures de protection de

\footnotetext{
${ }^{662}$ Voir OMC, Organe d'appel, Etats-Unis d'Amérique- Normes concernant l'essence nouvelle et ancienne formules (Plainte de la République Bolivarienne du Venezuela) (1996), OMC Doc WT/DS2/8 aux pp 17-19, disponible en ligne sur le site internet: OMC $<$ www.wto.org/french/tratop_f $/$ dispu_f $/$ cases_f $/$ ds 2 f f.htm $>[E$ tatsUnis - Essence].Consulté le 12/01/2022.

${ }^{663}$ Ces mesures sont prohibées par l'Accord sur les Obstacles Techniques au Commerce du 15 avril 1994, entré en vigueur le $1^{\text {er }}$ janvier 1995. Disponible sur le site internet : https://www.wto.org/french. Consulté le 12/01/2022. ${ }^{664}$ Quatrième paragraphe du préambule de l'Accord instituant l'Organisation Mondiale du Commerce.
} 
l'environnement à des fins protectionnistes n'est pas admise par la règlementation du commerce international $^{665}$.

A l'évidence, certaines mesures élaborées pour concrétiser les objectifs de préservation de l'environnement peuvent de par leur nature, restreindre le commerce en violation des droits d'autres membres. Ces mesures peuvent être prohibitives ou discriminatoires en contradiction avec les principes commerciaux établis à l'OMC. Dans ce cas, les mesures protectionnistes ou discriminatoires prises par les Membres de l'OMC pour protéger l'environnement ne sont pas en cas de contestation, toujours examinées devant l'Organisation commerciale comme un litige formel. Parfois, certaines exceptions visant à établir l'équilibre entre le droit des membres d'élaborer des mesures restrictives en contradiction avec le principe de libre échange commercial, pour atteindre les objectifs de protection légitime de l'environnement sont admises ${ }^{666}$.

Parmi les nombreux conflits déférés devant l'Organe de Règlement des Différends (ORD) de l'Organisation Mondiale du Commerce (OMC), les affaires suivantes: Brésilpneumatiques réchappés (vie et santé des personnes et des animaux et préservation des végétaux), Etats-Unis d'Amérique - Essence ${ }^{667}$ (lutte contre la pollution atmosphérique), EtatsUnis d'Amérique- Crevettes ${ }^{668}$ (tortues marines), et CE - Amiante ${ }^{669}$ (vie et santé des personnes) ont un lien avec la protection de l'environnement. La gestion de ces différends démontre l'importance du rôle de l'OMC, organe de régulation du commerce dans la protection de l'environnement. La jurisprudence de l'OMC offre la possibilité de s'apercevoir de la

\footnotetext{
${ }^{665} \mathrm{~L}$ 'article 2 du préambule de l'Accord sur les Obstacles Techniques au Commerce adopté le 15 avril 1994, entré en vigueur le $1^{\text {er }}$ janvier 1995 prohibe l'utilisation des mesures de protection de l'environnement à des fins protectionnistes. La même sujet a été abordé dans l'ouvrage de Gabrielle Marceau, "The New WTO TBT jurisprudence in US -Clove Cigarettes, WTO US-Tuna II, and US-COOL » (2013) 8:1 Asian J WTO \& Intl Health L \& Policy 1.

${ }^{666}$ Cette position est affirmée par la jurisprudence de l'OMC, Organe d'appel, Etats-Unis - Mesures affectant la production et la vente des cigarettes aux clous de girofle (Plainte de l'Indonésie) (2012), OMC Doc WT/DS406/AB/R. Disponible en ligne sur le site internet de : OMC <www.wto.org/french. Consulté le $12 / 01 / 2022$.

${ }^{667}$ Dans l'une des premières plaintes déposées à l'OMC par le Venezuela et ultérieurement par le Brésil contre les Etats-Unis d'Amérique, le rapport du Groupe spécial a été adopté en 1996 et l'affaire n'a pas remis en question le droit d'un pays à fixer des normes environnementales. La question centrale qui était étudiée concernait la discrimination, il s'agissait de savoir si la mesure imposée par les États-Unis d'Amérique établissait une discrimination à l'encontre de l'essence importée et en faveur des raffineries nationales. Disponible sur le site internet : https://docs.wto.org. Consulté le 13/01/2022.

${ }^{668}$ Plainte déposée par l'Inde, la Malaisie, le Pakistan et la Thaïlande contre les Etats-Unis d'Amérique. Les rapports du Groupe spécial et de l'Organe d'appel ont été adoptés en 1998. Disponible sur le site internet: https://www.wto.org/french. Consulté le 13/01/2022.

${ }^{669}$ Dans cette affaire, le Groupe spécial et l'Organe d'appel ont tous deux rejeté la plainte déposée par le Canada contre l'interdiction décrétée par la France d'importer de l'amiante et des produits en contenant, ce qui a renforcé l'idée que les Accords de l'OMC aident les Membres à protéger la santé et la sécurité des individus au niveau qu'ils jugent approprié. Disponible sur le site internet : https://www.wto.org/french. Consulté le 13/01/2022.
} 
pertinence des règles commerciales qui contribuent à la protection de l'environnement. Certaines règles élaborées en contradiction avec les principes de l'OMC peuvent être admises au titre des exceptions si tant est que, l'objectif finaliste est la protection de l'environnement, mais à la condition que leur mise en œuvre ne révèle pas d'élans protectionnistes. La justification des règles incompatibles aux principes $\mathrm{du}$ fonctionnement $\mathrm{du}$ commerce international est admise si ces mesures constituent une nécessité « (...) à la préservation des végétaux ou si elles se rapportent à la conservation de ressources naturelles épuisables » ${ }^{670}$. En définitif, les mesures relevant du domaine commercial élaborées dans le seul dessin de renforcer la protection de l'environnement sont admises, si elles sont conformes aux règles du GATT ou constituent une exception auxdites règles.

Les mesures applicables à l'OMC contribuent-elles à la lutte contre les changements climatiques?

L'Organisation Mondiale pour le Commerce n'a pas établi des règles spécifiques en vue de la protection de l'environnement. De la même manière, aucune règlementation de l'OMC n'a intégré spécifiquement les préoccupations concernant les changements climatiques. Néanmoins, certaines normes élaborées dans le cadre de la lutte contre les changements climatiques présentent des similitudes avec quelques principes applicables dans le cadre du règlement de différends soumis à l'OMC. A titre illustratif, la Convention Cadre des Nations Unies sur les Changements Climatiques a élevé au rang de principe conventionnel, la non discrimination dans le domaine des transactions ayant pour finalité la lutte contre les changements climatiques en interdisant « (...) que les mesures prises pour lutter contre les changements climatiques, y compris les mesures unilatérales, constituent un moyen d'imposer des discriminations arbitraires ou injustifiables sur le plan du commerce international (...)» ${ }^{671}$. Certes, la Convention Cadre adhère au principe de libre échange en disposant qu' « Il appartient aux Parties de travailler de concert à un système économique international qui soit porteur et ouvert et qui mène à une croissance économique et à un développement durable de toutes les Parties, en particulier des pays en développement parties, pour leur permettre de mieux s'attaquer aux problèmes posés par les changements climatiques ${ }^{672}$. Le principe de la non discrimination est également celui par lequel, l'OMC tranche certains différends commerciaux

\footnotetext{
${ }^{670} \mathrm{Sa}$ justification juridique se trouve dans l'article 20 du GAT qui institue l'exception environnementale dans les traités. En effet, en vertu de cette disposition les membres de l'OMC peuvent justifier des mesures prises qui contribuent à la protection de l'environnement et qui sont incompatibles avec les règles du GAT.

${ }^{671} \mathrm{~L}$ 'article 3 paragraphe 5 de la Convention Cadre des Nations Unies sur les Changements Climatiques interdit la prise de mesure tendant à lutter contre les changements climatiques comme moyen de discrimination dans les transactions commerciales. ${ }^{672} \mathrm{Id}$.
} 
internationaux. Dans cette optique, la compétence de l'OMC peut être requise lorsqu'il survient un litige commercial international relatif aux changements climatiques, dont la toile de fond est la dénonciation des mesures discriminatoires. A titre illustratif, le différend qui a opposé le Venezuela et le Brésil aux Etats-Unis d'Amérique (USA) suite à la plainte contre la modification apportée à la loi sur la lutte contre la pollution adoptée par les Etats Unis $\mathrm{d}^{\prime}$ Amérique ${ }^{673}$, une règlementation in fine, qui porterait des germes de la discrimination ${ }^{674}$ selon les requérants ${ }^{675}$ est porté devant l'OMC. La question centrale analysée par l'OMC concernait la discrimination. Il s'agissait de savoir si la mesure imposée par les Etats Unis d'Amérique établissait une discrimination à l'encontre de l'essence importée et en faveur des raffineries nationales ${ }^{676}$. Selon le jugement rendu le 20 mai 1996, le Groupe spécial de l'OMC ${ }^{677}$ a recommandé à l'Organe de Règlement des Différends « qu'il demande aux EtatsUnis d'Amérique d'appliquer le règlement sur l'essence conformément aux obligations qui leur incombent en vertu de l'Accord général ${ }^{678}$. Un différend dont l'objet s'apparente à la lutte contre les changements climatiques.

Par ailleurs, le Protocole de Kyoto qui est le prolongement de la Convention Cadre des Nations Unies sur les Changements Climatiques contient certaines dispositions qui ont rapport avec le commerce ${ }^{679}$. Ces mesures peuvent être source de conflit au niveau de l'Organisation Mondiale du Commerce, c'est pourquoi ce traité bénéficie d'une attention particulière auprès de l'institution qui y veille par l'entremise de son Secrétariat ${ }^{680}$. Il va s'en dire que l'Organe de

\footnotetext{
${ }^{673} \mathrm{Il}$ s'agit d'une loi modificative de la loi sur la lutte contre la pollution atmosphérique. L'Agence pour la protection de l'environnement (EPA) a promulgué la réglementation sur l'essence concernant la composition et les effets polluants de l'essence, afin de réduire la pollution atmosphérique aux États-Unis d'Amérique.

${ }^{674}$ Cette réglementation autorisait uniquement la vente d'essence présentant un certain degré de propreté dans les régions où la pollution atmosphérique était la plus élevée. Du coup, dans le reste du pays, la vente de l'essence qui n'était pas plus polluante que celle qui avait été vendue pendant l'année de base 1990 n'était plus possible.

675 Venezuela et le Brésil.

${ }^{676} \mathrm{C}$ 'est l'objet de la requête concernant le différends déféré devant l'OMC.

${ }^{677} \mathrm{Au}$ rang des organes de l'OMC intervenant dans le processus de règlement des différends se trouvent les groupes spéciaux qui sont des organes quasi-judiciaires, tout comme de tribunaux, chargés de rendre des décisions juridictionnelles pour régler les différends entre Membres en première instance. Ils se composent normalement de trois et exceptionnellement de cinq, experts sélectionnés au cas par cas. Ce qui veut dire qu'il n'y a pas de groupe spécial permanent à l'OMC, au contraire, un groupe spécial différent est composé pour chaque différend. Disponible sur le site internet : https://www.wto.org/french. Consulté le 13/01/2022.

${ }^{678} \mathrm{La}$ décision rendue dans le : différends7 Venezuela, Brésil/États-Unis: essence est disponible sur le site internet : https://www.wto.org/french. Consulté le 13/01/2022.

${ }^{679} \mathrm{Par}$ exemple, l'article 2, paragraphe 3 du Protocole de Kyoto à la Convention Cadre des Nations Unies sur les Changements Climatiques dispose que, «Les Parties visées à l'annexe I s'efforcent d'appliquer les politiques et les mesures prévues dans le présent article de manière à réduire au minimum les effets négatifs, notamment les effets néfastes des changements climatiques, les répercussions sur le commerce international et les conséquences sociales environnementales et économiques pour les autres Parties, surtout les pays en développement (...)». Il s'ensuit que certaines difficultés qu'engendrerait la gestion des changements climatiques sur le commerce international par un Etat Partie peuvent donner lieu à un différend qui peut être tranché par l'OMC.

${ }^{680}$ Voir page 1 de note du Secrétariat intitulée « Matrice des mesures commerciales prises au titre de divers accords environnementaux multilatéraux, Note du Secrétariat, Comité du Commerce et de l'environnement, OMC,
} 
Règlement des Différends de l'OMC peut intervenir dans la mise en œuvre du Protocole de Kyoto toutefois que l'application des mesures qui ont trait au commerce donne lieu à des contestations. Néanmoins, le cadre de l'OMC ne s'impose pas toujours aux Etats Parties du Protocole de Kyoto qui sont également membres de l'OMC. En règle générale, lorsqu'un différend survient dans le cadre de l'application des mesures commerciales entre les Etats membres de l'OMC ayant souscrits à un Accord Multilatéral Environnemental (AME), «(...) ceux-ci doivent envisager régler le différend par l'intermédiaire du mécanisme de règlement des différends prévu par l'AME ${ }^{681}$. Dans ce cas, la juridiction de l'OMC ne s'impose pas à ces Etats. Seulement que le cadre du Protocole de Kyoto n'a pas prévu un organe de règlement de différend outre que la Cour Internationale de Justice qui est le seul organe juridictionnel de la Convention Cadre des Nations Unies sur les Changements Climatiques qui dispose d'une compétence générale. Néanmoins, cette juridiction n'est pas spécialisée dans le domaine commercial, elle ne l'est non plus dans le domaine environnemental. Il s'ensuit qu'en cas de survenance de différend provoqué dans l'application des dispositions commerciales opposant les Etats Parties de la Convention Cadre des Nations Unies sur les Changements Climatiques, l'Organe de Règlement des Différends de l'OMC est mieux indiqué pour examiner ce conflit.

Paradoxalement, aucune jurisprudence ne témoigne de l'existence d'un différend qui découle des difficultés d'application de la Convention Cadre des Nations Unies sur les Changements Climatiques tranché par l'OMC. Inversement, il n'y a pas non plus de différend opposant les Etats membres de l'OMC qui sont Parties au Protocole de Kyoto qui soit porté devant la Cour Internationale de Justice ${ }^{682}$.

En dehors de l'OMC qui apporte déjà une contribution non négligeable à l'encadrement de la protection de l'environnement bien que n'étant pas du domaine, une autre organisation internationale importante ayant pour vocation essentiellement culturelle apporte sa pierre à l'édifice. Il s'agit de l'Organisation des Nations Unies pour l'Education, la Science et la Culture (UNESCO) qui s'approprie des attributions relevant du domaine de protection de l'environnement et s'investie également dans la lutte contre les changements climatiques.

WT/CTE/W/160/Rev.3, TN/TE/S/5/Rev.1, 16 février 2005» dans lequel il est ressassé les attributions du Secrétariat de l'OMC en matière de gestion des Accords commerciaux.

${ }^{681}$ Rapport (1996) du Comité du commerce et de l'environnement à la Conférence ministérielle de Singapour, WT/CTE/1, 12 novembre 1996, § 178. Disponible sur le site internet : https://www.wto.org/french. Consulté le 08/06/2021.

${ }^{682}$ Les recherches de jurisprudence pour illustrer ces deux cas ont été infructueuses. 


\section{B-L'exemple de l'UNESCO}

A l'occasion de la Conférence générale de l'Organisation des Nations Unies pour l'Education, la Science et la Culture tenue à Paris en 1972, le constat de ce « que le patrimoine culturel et le patrimoine naturel sont de plus en plus menacés de destruction non seulement par les causes traditionnelles de dégradation, mais encore par l'évolution de la vie sociale et économique qui les aggrave par des phénomènes d'altération ou de destruction encore plus redoutables» ${ }^{683}$, a été fait par la Communauté internationale. For de ce constat, les Etats membres de l'UNESCO avaient adopté en 1972 la «Convention relative à la protection du patrimoine culturel et naturel mondial ${ }^{684}$ afin de se doter d'un cadre normatif adapté à la conservation du patrimoine commun dans l'intérêt des générations actuelles et à venir. Cet instrument oblige les Etats Parties à procéder à l'identification, à la protection et à la conservation du patrimoine culturel et naturel considéré comme une valeur pour l'humanitét ${ }^{685}$. Après l'adoption de la Convention sur le patrimoine culturel et naturel, mil cent vingt et un (1121) sites répartis dans cent soixante-sept (167) Etats Parties sont inscrits sur la liste du patrimoine mondial ${ }^{686}$.

L'Organisation des Nations Unies pour l'Education la Science et la Culture (UNESCO), dont le siège est sis à Paris en France, est compétente pour administrer ce patrimoine mondial. Elle a pour objectif, de «contribuer au maintien de la paix et de la sécurité en resserrant par l'éducation, la science et la culture, la collaboration entre Nations, afin d'assurer le respect universel de la justice, de la loi, des droits de l'Homme et des libertés fondamentales pour tous, sans distinction de race, de sexe, de langue ou de religion, que la Charte des Nations unies reconnaît à tous les peuples $\gg{ }^{687}$.

$Y$ a-t-il un lien entre les objectifs de protection du patrimoine culturel et naturel administré par l'UNESCO et la lutte contre les changements climatiques?

\footnotetext{
${ }^{683}$ Deuxième paragraphe du préambule de la Convention pour la protection du patrimoine mondial, culturel et naturel de 1972. Disponible sur le site internet : https://whc.unesco.org/archive/convention-fr.pdf. Consulté le $13 / 01 / 2022$

${ }^{684}$ La Conférence générale de l'Organisation des Nations Unies pour l'Education, la Science et la Culture, réunie à Paris du 17 octobre au 21 novembre 1972, en sa dix-septième $\left(17^{\circ}\right)$ session a adopté la Convention : Disponible sur le site internet : https://whc.unesco.org/fr/conventiontexte. Consulté le 23/05/2021.

${ }^{685}$ Conformément à l'article 4 de la Convention pour la protection du patrimoine mondial, culturel et naturel adoptée à la Conférence générale de l'Organisation des Nations Unies pour l'Education, la Science et la Culture, réunie à Paris du 17 octobre au 21 novembre 1972, en sa dix-septième session. Disponible sur le site internet : https://whc.unesco.org/fr/conventiontexte. Consulté le 23/05/2021.

${ }^{686}$ Liste établie jusqu'au 02 février 2021. Disponible sur le site internet: https://whc.unesco.org/fr/list/st.Consulté le $02 / 02 / 2021$.

${ }^{687}$ L'objectif de l'UNESCO est fixé à l'article $1^{\text {er }}$ paragraphe1 de l'acte constitutif de ladite Organisation.
} 
Au moment de la création de l'UNESCO en 1945, l'institution avait des objectifs plus orientés dans le sens de la consolidation de la paix et l'épanouissement de l'Homme par la culture. Le contexte de sa création caractérisé par le climat d'après guerre ${ }^{688}$ justifiait les objectifs fixés dans la charte qui l'a institué. Au cours de la même période, les préoccupations liées à la protection internationale de l'environnement par le Droit étaient moins débattues par la Communauté internationale. De plus, les changements climatiques étaient moins connus comme un fléau pouvant troubler la paix planétaire au point de constituer une menace pour le patrimoine mondial culturel et naturel. Au moment de l'adoption de la Convention sur la protection du patrimoine mondial culturel et naturel en 1972, les risques de menace des effets des changements climatiques sur les sites de l'UNESCO n'étaient pas visibles.

Ce fut plusieurs décennies après la création de l'UNESCO que le Comité du patrimoine mondial avait indiqué que, «les effets des changements climatiques touchent de nombreux biens du patrimoine mondial et risquent d'en toucher bien davantage qu'ils soient culturels ou naturels, dans les années à venir» ${ }^{689}$. Par la suite, une étude commanditée par l'UNESCO, portant sur les changements climatiques en relation avec le patrimoine mondial réalisée plus tard, a révélé qu' "Un changement du climat quel qu'il soit déstabiliserait les équilibres environnementaux et sociaux autour du globe. Ces perturbations mettraient en péril la conservation des écosystèmes naturels et la durabilité des systèmes socio-économiques. Par conséquent, le changement climatique aura et a d'ores et déjà des effets néfastes sur la conservation des sites naturels et culturels inscrits au patrimoine mondial ${ }^{690}$, alors que "Le patrimoine est une source irremplaçable de vie et d'inspiration, le legs des civilisations passées, avec lequel nous vivons et que nous transmettrons aux générations futures» ${ }^{691}$. Il s'est avéré par la suite, que les modifications climatiques provoquaient des impacts négatifs sur les sites naturels et culturels inscrits au patrimoine mondial ${ }^{692}$. Par exemple, « Les glaciers fondent autour du monde et l'apparence de certains sites montagneux inscrits du fait de leur beauté esthétique exceptionnelle pourrait changer profondément ${ }^{693}$, de même, "la température de

\footnotetext{
${ }^{688}$ La deuxième guerre mondiale s'est déroulée en 1945.

${ }^{689}$ UNESCO. Comité du patrimoine mondial, « Rapports sur l'état de conservation de biens inscrits sur la liste du patrimoine mondial. Décision 29 COM 7B.a » dans Décisions adoptées lors de la $29^{\mathrm{e}}$ session du Comité du patrimoine mondial, (Durban, Afrique du Sud, 2005), WHC-05/29.COM/22, le 09 septembre 2005, p.41. Disponible sur le site internet:www.whc.unesco.org/archive/2005/whc 05-29 com-22f.pdf. Consulté le $15 / 06 / 2015$.

${ }^{690}$ Augustin Colette, Etudes de cas : Changements climatiques et patrimoine mondial. Organisation des Nations Unies pour l'Education la Science et la Culture, Convention du patrimoine mondial publié en juillet 2009 par le Centre du patrimoine mondial de l'UNESCO.

${ }^{691}$ Id.

${ }^{692}$ Ibid

693 Ibid.
} 
l'océan et l'augmentation de la concentration du carbone dissous rendent plus difficile la conservation des récifs coralliens, avec des événements de blanchissement plus fréquents pouvant conduire à une mortalité accrue des coraux ${ }^{694}$, enfin, "Le changement climatique conduira certaines espèces végétales et animales à se déplacer, lorsqu'elles deviendront incapables de s'adapter au changement de leur environnement, ce qui posera un problème pour la conservation des pôles de biodiversité inscrits en tant que sites naturels du patrimoine mondial $\gg{ }^{695}$. Comme preuve de la dégradation du patrimoine mondial, un rapport conjoint de l'UNESCO et de l'ONG Union of Concerned Scientists ${ }^{696}$, fait le point de trente-et-un (31) sites menacés par le changement climatique dans vingt-neuf (29) pays. Selon ce rapport, « Le changement climatique affecte les sites du patrimoine mondial partout dans le monde $»{ }^{697}$.

Ces menaces éventuelles sur le patrimoine mondial, nécessitent l'intervention urgente de l'UNESCO qui doit élaborer des stratégies en vue d'assurer sa protection. Lorsque les préoccupations environnementales ont commencé à surgir et se sont intensifiées, cette mutation obligea ainsi l'UNESCO à considérer la dimension des changements climatiques dans ses activités sans pour autant modifier les objectifs contenus dans l'Acte constitutif de l'Organisation ${ }^{698}$. Ainsi, le domaine de l'environnement et surtout ceux des changements climatiques sont devenus des matières qui se sont imposées à l'Organisation.

Par quels moyens l'UNESCO compte-t-elle protéger le patrimoine mondial culturel ou naturel contre les désastres des changements climatiques?

Dès lors que la Communauté scientifique s'est aperçue que les changements climatiques constituent de véritables problèmes pour la sauvegarde du patrimoine, elle a engagé une série d'actions contre le fléau dans le seul objectif de préserver le patrimoine mondial. L'une des plus importantes actions s'était déroulée à Copenhague à l'occasion de la $15^{\mathrm{e}}$ Conférence des Parties à la Convention Cadre des Nations Unies sur les Changements Climatiques, où

\footnotetext{
${ }^{694}$ Ibid.

${ }^{695}$ Ibid.

${ }^{696}$ L'Union of Concerned Scientists est la principale organisation scientifique à but non lucratif travaillant pour un environnement sain et un monde plus sûr. UCS combine la recherche scientifique indépendante et l'action citoyenne pour développer des solutions innovantes et pratiques et pour garantir des changements responsables dans la politique gouvernementale, les pratiques des entreprises et les choix des consommateurs. Disponible sur le site internet : https://www.ucsusa.org/about. Consulté le 14/01/2022.

${ }^{697}$ Rapport publié sur le site internet : https://www.consoglobe.com/sites-patrimoine-mondial-unesco-menaceschangement-climatique-cg. Consulté le 23/05/2021.

${ }^{698} \mathrm{La}$ Convention créant une Organisation des Nations unies pour l'éducation, la science et la culture, avait été adoptée à Londres le 16 novembre 1945 et modifiée par la Conférence générale lors de ses 2e, 3e, 4e, 5e, 6e, 7e, $8 \mathrm{e}, 9 \mathrm{e}, 10 \mathrm{e}, 12 \mathrm{e}, 15 \mathrm{e}, 17 \mathrm{e}, 19 \mathrm{e}, 20 \mathrm{e}, 21 \mathrm{e}, 24 \mathrm{e}, 25 \mathrm{e}, 26 \mathrm{e}, 27 \mathrm{e}, 28 \mathrm{e}, 29 \mathrm{e}, 31 \mathrm{e}$ et $40 \mathrm{e}$ sessions. L'Acte constitutif entre en vigueur dès 1946 et ratifié par vingt (20) Etats : l'Afrique du Sud, l'Arabie saoudite, l'Australie, le Brésil, le Canada, la Chine, le Danemark, l'Egypte, les Etats-Unis d'Amérique, la France, la Grèce, l'Inde, le Liban, le Mexique, la Norvège, la Nouvelle-Zélande, la République dominicaine, le Royaume-Uni, la Tchécoslovaquie et la Turquie. http://portal.unesco.org. Consulté le 23/05/2021
} 
«l'initiative de l'UNESCO pour contrer la lutte contre les changements climatiques »a été lancée ${ }^{699}$. Ensuite, l'Organisation des Nations Unies pour l'Education la Science et la Culture a adopté une résolution sur la «Stratégie de l'UNESCO pour faire face au changement climatique $»^{700}$, qui a été actualisée à l'occasion de la $39^{\mathrm{e}}$ session tenue à Paris en 2017. L'objectif ultime du document est de « permettre aux Etats membres de prendre d'urgence des mesures pour lutter contre le changement climatique et ses effets par l'éducation, la science, la culture, l'information et la communication, conformément à leurs contributions déterminées au niveau national, en application de l'Accord de Paris adopté par la COP-21 et dans le cadre général du Programme 2030 et de l'ODD13 qui y est défini $\gg{ }^{701}$. La réalisation de cette initiative requiert le renforcement des capacités des communautés vulnérables. La Stratégie de l'UNESCO pour contrer les changements climatiques consistera à la mise en œuvre des actions et activités prescrites par l'Organisation ${ }^{702}$ qui respectent un certain nombre de principe et critère généraux. Ces actions consistent entre autres à «répondre aux besoins des Etats Membres s'agissant de réaliser leurs Contributions Déterminées au niveau National dans le cadre de l'Accord de Paris, ainsi que l'ODD13 du Programme de développement durable à l'horizon 2030, dans le contexte général défini par la Stratégie à moyen terme de l'UNESCO pour 2014-2021 $»^{703}$ et puis à «mettre à profit les programmes, stratégies et plans d'action pertinents de l'UNESCO, les appuyer et être compatibles avec eux » ${ }^{704}$ et enfin à « faire mieux prendre conscience du caractère intersectoriel et interdisciplinaire du changement climatique en tant qu'enjeu s'inscrivant dans le cadre global du développement durable tout en prenant appui sur les points forts et les priorités de chacun des grands programmes de l'Organisation ${ }^{705}$.

L'implication de l'UNESCO dans la lutte contre les changements climatiques revêt un double intérêt pour les Etats Membres de l'Organisation et qui sont en même temps Parties à la

\footnotetext{
${ }^{699}$ Ce lancement a été fait par la Directrice générale de l’UNESCO, Irina Bokova.

${ }^{700} \mathrm{Il}$ s'agit de la résolution $38 \mathrm{C} / 21$ et à la décision $200 \mathrm{EX} / 5$.I.C, la Directrice générale soumet au Conseil exécutif une proposition finale d'actualisation de la Stratégie de l'UNESCO pour faire face au changement climatique. Disponible sur le site internet : https://unesdoc.unesco.org. Consulté le 14/01/2022.

${ }^{701}$ Sixième paragraphe de l'introduction du document de stratégie de l'UNESCO pour faire face au changement climatique. Disponible sur le site internet : https://unesdoc.unesco.org. Consulté le 14/01/2022.

${ }^{702}$ Les actions de mise en œuvre de la stratégie de l'UNESCO pour faire face au changement climatiques sont contenues dans la résolution 39C/5. Disponible sur le site internet : http://www.unesco.org/new/fr/bureau-ofstrategic-planning/resources/preparation. Consulté le 08/06/2021.

${ }^{703}$ Septième paragraphe point a du document de stratégie de l'UNESCO pour contre le changement climatique. Disponible sur le site internet : https://unesdoc.unesco.org. Consulté le 14/01/2022.

${ }^{704}$ Septième paragraphe point $b$ id.

${ }^{705}$ Septième paragraphe point $\mathrm{c}$ ibid.
} 
Convention Cadre des Nations Unies sur les Changements Climatiques. Ces Etats bénéficient de l'appui institutionnel, technique, scientifique voire financier de la part des deux institutions.

Par ailleurs, l'obligation qui incombe aux Etats Membres de l'UNESCO de protéger le patrimoine culturel et naturel aux fins d'assurer leur transmission aux générations futures, peut aussi être interprétée comme celle qui consiste à contribuer à la promotion des droits de l'Homme par la prise des mesures pour la réduction de l'émission des gaz à effet de serre ${ }^{706}$. Il s'ensuit qu'en protégeant le patrimoine culturel et naturel, le droit des changements climatiques peut être effectif. Autrement dit, le droit de protection du patrimoine culturel et naturel peut être appréhendé comme un élément d'effectivité du droit de protection du climat.

Les défis engendrés par les changements climatiques sont immenses. Il n'y a pas de limitation en ce qui concerne son encadrement. L'imminence des conséquences, imprévisibles, irrésistibles, voire dévastatrices ne laisse indifférent aucune institution. Elles sont devenues de par leur importance, les matières qui intéressent tout le monde et toute institution à l'instar des organisations régionales.

\section{Paragraphe 2: L'intervention d'organisations régionales}

La solidarité internationale est une exigence de la nouvelle gouvernance qui tienne compte du développement durable dans le contexte d'un monde globalisé assujetti aux effets néfastes des changements climatiques. La quasi-totalité d'instruments internationaux de protection de l'environnement a d'ailleurs mis l'accent sur la coopération internationale comme solution indispensable au règlement des problèmes environnementaux. N'est ce pas ce que stipule le principe 24 de la Déclaration de Stockholm de 1972 qui dispose que, «Les questions internationales se rapportant à la protection et à l'amélioration de l'environnement devraient être abordées dans un esprit de coopération par tous les pays grands ou petits sur un pied d'égalité. Une coopération par voie d'accords multilatéraux ou bilatéraux ou par d'autres moyens appropriés est indispensable pour limiter efficacement, prévenir, réduire et éliminer les atteintes à l'environnement ». Il s'agit de la mise à contribution de la coopération la plus large possible à diverses échelles au service de la protection de l'environnement.

Cependant, certains objecteurs de conscience indiquent que « La globalité du problème n'impose en rien une action globale et multilatérale soutenue par un traité ambitieux, universel

\footnotetext{
${ }^{706}$ Benoit Mayer, « Un examen des lignes directrices de la Commission du droit international sur la protection des atmosphères », Melbourne Journal of International Law, 2019, p.2.
} 
et formel $»^{707}$ à l'instar de la Convention Cadre des Nations Unies sur les Changements Climatiques. Il s'ensuit que les accords interétatiques, régionaux ou bilatéraux peuvent être mis à contribution ensemble avec la Convention Cadre des Nations Unies sur les Changement Climatiques. Malgré que le principe de la souveraineté qui a été réaffirmé dans la Convention, «doit présider à la coopération internationale destinée à faire face aux changements climatiques $»^{708}$, le dépassement des cadres nationaux pour la mise en œuvre du Droit de l'Environnement apparait comme élément d'efficacité dudit droit et a été recommandé par certains auteurs, tout comme Maurice KAMTO, qui indique que, «L'espace communautaire apparaît comme un niveau de création normative et d'impulsion de la mise en œuvre efficiente du droit de l'environnement $»^{709}$.

En conséquence, malgré leur souveraineté, les Etats ont ébauché des initiatives d'intégration et se sont constitués en des ensembles régionaux en convergeant leurs efforts, moyens et stratégies dans le but de bâtir une économie compétitive. Cependant, au cours de leurs existences, ces organisations régionales sont confrontées aux difficultés qui entre autres proviennent des problèmes environnementaux. Ainsi, elles ne peuvent s'empêcher de faire leur intrusion dans le domaine environnemental, car les réalités que constituent les changements climatiques risquent d'annihiler leurs efforts et les empêcher de réaliser leurs objectifs. C'est le cas de l'Union Africaine (UA) (A) et de la Communauté Economique des Etats de l'Afrique de l'Ouest (CEDEAO) qui s'investissent dans le domaine environnemental (B).

\section{A- Le cas de l'Union africaine}

En consécration de l'esprit de solidarité qui doit présider à la gestion des effets des changements climatiques qui découlent de la dégradation de l'environnement, des ensembles régionaux sont de plus en plus actifs dans le secteur environnemental. Les exemples les plus édifiants, sont ceux qui concernent l'Union Européenne (UE) et l'Union Africaine (UA), qui sont toutes des organisations régionales, relevant respectivement des continents européen et africain, lesquelles en plus de leurs attributions essentiellement orientées vers les objectifs économiques et stratégiques, participent à la protection de l'environnement et s'engagent dans

\footnotetext{
${ }^{707}$ Abbas, M., Economie politique globale des changements climatiques. Grenoble: Presse Universitaires de Grenoble, 2010, Op., p.97.

${ }^{708}$ Dixième $\left(10^{\mathrm{e}}\right)$ paragraphe du préambule de la Convention Cadre des Nations Unies sur les Changements Climatiques.

709 KAMTO (Maurice), La mise en œuvre du droit de l'environnement: Forces et faiblesses des cadres institutionnels, Revue Africaine de Droit de l'Environnement n ${ }^{\circ} 01,2014$, p.34.
} 
la lutte contre les changements climatiques. Le cas d'organisation régionale qui intéresse la présente étude est celui de l'Union africaine ${ }^{710}$.

L'Union africaine a pour ressort territorial le continent africain, qui s'étend sur une superficie estimée à environ $30429202 \mathrm{~km}^{2}$, avec une population avoisinante plus de 1293 574394 habitants $^{711}$. L'Organisation continentale a été créée suite à l'adoption de son acte constitutif le 11 juillet 2000 à l'occasion du Sommet de Lomé au Togo. Ce fut le moment où l'Union africaine s'est vue devant le défi de « bâtir une Afrique intégrée, prospère et en paix, dirigée par ses citoyens et constituant une force dynamique sur la scène mondiale» ${ }^{712}$. Pour relever ce défi, l'Union africaine s'est fixée comme but, « d'œuvrer à la promotion de la démocratie, des droits de l'homme et du développement » ${ }^{713}$ et «d'accélérer le processus d'intégration sur le continent et de permettre à l'Afrique de jouer un rôle important dans l'économie mondiale, tout en déployant des efforts pour résoudre les problèmes sociaux, économiques et politiques, multiformes auxquels elle est confrontée $»^{714}$. L'objectif à terme de l'Union africaine est de réaliser le développement du continent africain ${ }^{715}$.

Néanmoins, ce développement voulu, prôné et projeté, risque d'être écorché par des obstacles majeurs qui proviendraient du caractère vulnérable du continent face aux effets néfastes des changements climatiques. La dégradation de l'environnement, la finitude des ressources naturelles, l'insécurité alimentaire généralisée, l'accroissement de la pauvreté, les risques sanitaires, les troubles sociaux et politiques, les mouvements migratoires etc., les conséquences du changement climatique, font l'objet des évaluations les plus alarmistes sur ce continent $^{716}$. Les Etats membres de l'organisation continentale ne sont pas épargnés des manifestations dramatiques des effets des changements climatiques. C'est le continent qui contribue le moins aux causes de ce désastre, parce que ses émissions de gaz à effet de serre

\footnotetext{
${ }^{710}$ Compte tenu de l'appartenance du Bénin à cette organisation continentale.

${ }^{711}$ Les données statistiques concernant la taille de la population africaine sont obtenues sur le site internet: https://www.populationdata.net/continents/afrique. Consulté le 24 mai 2021.

${ }^{712}$ Paragraphe 4 de, Agenda 2063, L'Afrique Que Nous Voulons, Edition finale, Version populaire, publié en avril 2015. Disponible sur le site internet : https://au.int/sites/default/files/documents. Consulté le 14/01/2022.

${ }^{713}$ Article 3 de l'acte constitutif de l'Union africaine. Disponible sur le site internet: https://au.int/sites/default. Consulté le 14/01/2022.

714 Article 3 id.

715 Ibid.

${ }^{716}$ Réponse au changement climatique en Afrique, une approche par la gouvernance des territoires. Disponible sur le site internet : www.afrique-gouvernance.net. Consulté le 14/01/2022.
} 
sont insignifiantes ${ }^{717}$, mais l'Afrique subie les conséquences négatives des changements climatiques pour le fait qu'elle est vulnérable ${ }^{718}$.

Les changements climatiques, étant identifiés comme l'une des entraves au processus du développement du continent africain, comment l'Union africaine peut lever ces obstacles afin de faciliter l'atteinte de ses objectifs?

Pour accompagner les objectifs entre autres, «d'intégration du continent et son positionnement dans l'économie mondiale $»^{719}$, les pères fondateurs ${ }^{720}$ de l'Union Africaine (UA) ont fait glisser l'organisation dans le domaine de la protection de l'environnement. Ils ont posé les bases juridiques devant servir à l'encadrement institutionnel du domaine de protection l'environnement par la définition des attributions environnementales au profit de certaines structures d'encadrement de l'Organisation ${ }^{721}$. Cet attachement manifesté pour la protection de l'environnement dans les actes fondateurs de l'UA s'est concrétisé en termes juridiques, par l'adoption du « texte révisé de la Convention africaine sur la conservation de la nature et des ressources naturelles» ${ }^{722}$.

Les dirigeants de l'Union africaine ayant perçus la portée des effets néfastes des changements climatiques pour le continent, sont allés au-delà des attentes, en faisant intervenir l'organisation faitière dans le domaine de la protection de l'environnement par son positionnement dans le cœur du système juridique international par la création d'une institution juridictionnelle internationale qui peut étendre ses compétences dans le domaine environnemental. Il s'agit de la Cour Africaine des Droits de l'Homme et des Peuples (CADHP) créée en vertu du Protocole relatif à la Charte africaine portant sur la création d'une Cour Africaine des Droits de l'Homme et des Peuples. Mais plus tard, cette juridiction a été remplacée par la Cour Africaine de Justice et des Droits de l'Homme (CAJDH) après une fusion réalisée avec la «Cour Africaine de Justice» ${ }^{723}$. Vraisemblablement, la compétence de cette

\footnotetext{
${ }^{717}$ Aux termes du quatrième paragraphe du préambule de la Convention Cadre des Nations Unies sur les Changements Climatiques, « la majeure partie des gaz à effet de serre émis dans le monde par le passé et à l'heure actuelle ont leur origine dans les pays développés ».

${ }^{718}$ L'Alliance pour Refonder la Gouvernance en Afrique, « Répondre au changement climatique en Afrique : une approche par la gouvernance des territoires ", Etude Académique, 2015. Disponible sur le site : https://www.wathi.org/repondre-au-changement-climatique-en-afrique-une-approche-par-la-gouvernance-desterritoires-arga. Consulté le 14/01/2022.

${ }^{719}$ Article 3 de l'acte constitutif de l'Union Africaine.

${ }^{720}$ Chefs d'Etat et de Gouvernement des Etats membres de l'Organisation de l'Unité Africaine (OUA) qui ont signé l'acte constitutif de l'Organisation régionale.

${ }^{721} \mathrm{Au}$ terme des articles 13-e et 14-d de l'Acte Constitutif de l'Union africaine, le Conseil exécutif et les Comités techniques spécialisés de l'Organisation sont compétentes en matière de protection de l'environnement.

$722 \mathrm{Au}$ cours de la deuxième session de la Conférence de l'UA, qui s'est tenue à Maputo le 11 juillet 2003.

${ }^{723}$ Selon l'article 2 du Protocole portant Statut de la Cour Africaine de Justice et des Droits de l'Homme, la Cour Africaine des Droits de l'Homme et Peuples et la Cour de Justice de l'Union africaine, créées respectivement par le Protocole relatif à la Charte africaine des droits de l'homme et des peuples et l'Acte constitutif de l'Union
} 
juridiction s'étend «sur toutes les affaires et à tous les différends d'ordre juridique et plus spécifiquement, elle intervient pour l'interprétation et l'application de la Charte africaine des droits de l'homme et des peuples, de la Charte africaine des droits et du bien être de l'enfant, du Protocole à la Charte des droits de l'homme et des peuples relatif aux droits de la femme, ou de tout autre instrument juridique relatif aux droits de l'homme auxquels sont parties les Etats concernés ${ }^{724}$. La compétence environnementale de la juridiction panafricaine a été définie dans la Charte africaine des droits de l'homme et des peuples de 1981 qui proclamait déjà que, « Tous les peuples ont droit à un environnement satisfaisant et global, propice à leur développement» ${ }^{725}$, ce qui faisait dire à Mohamed Ali Mekouar que, « La Charte africaine des droits de l'homme et des peuples a été l'un des premiers instruments juridiques régionaux de protection des droits de l'homme à consacrer formellement le droit à un environnement sain, qui à l'époque était un droit naissant ${ }^{726}$.

Mais en plus, la Cour Africaine de Justice et des Droits de l'Homme (CAJDH) entretient des relations étroites avec la Commission Africaine des Droits de 1'Homme et des Peuples ${ }^{727}$, une institution de l'Union africaine qui a presque les mêmes compétences en matière de violation des droits de 1'Homme ${ }^{728}$ à la seule différence, que la Cour africaine sanctionne les violations des droits de l'Homme, alors que la Commission Africaine des Droits de l'Homme et des Peuples a pour mission de promouvoir les droits de l'Homme ${ }^{729}$.

Cependant, en ce qui concerne l'effectivité de la protection de l'environnement par la mise en œuvre des actions juridictionnelles devant la Cour Africaine de Justice et des Droits de l'Homme, si les instruments ont consacré en dehors de la compétence des Etats Parties, le droit d'accès à ladite juridiction aux acteurs non étatiques ${ }^{730}$, il y a encore des entraves à l'effectivité

africaine, sont fusionnées en une Cour unique instituée et dénommée, « Cour africaine de justice et des droits de l'homme ».

${ }^{724}$ L'article 28 du Protocole portant statut de la Cour Africaine de Justice et des Droits de l'Homme défini les domaines de compétence l'institution juridictionnelle.

${ }^{725}$ Par l'article 24 de la Charte africaine des droits de l'homme et des peuples, il est attribué des compétences environnementales de la juridiction continentale.

${ }^{726}$ Mohamed Ali Mekouar, Le droit a l'environnement dans la charte africaine des droits de l'homme et des peuples, Etude juridique de la FAO en ligne, 16 avril 2001. Disponible sur le site internet: http://www.fao.org/Legal/default.htm. Consulté le 14/01/2022.

${ }^{727} \mathrm{~L}$ 'article 30 de la Charte africaine des droits de l'homme et des peuples « ... crée auprès de l'Organisation de l'Unité Africaine une Commission Africaine des Droits de l'Homme et des Peuples ci-dessous dénommée "la Commission", chargée de promouvoir les droits de l'homme et des peuples et d'assurer leur protection en Afrique ». En effet, l'article 4 de ladite Charte établi des relations entre la Cour et la Commission africaine des droits de l'homme et des peuples ainsi qu'il suit : "La Cour complète, conformément à la Charte et au présent Protocole, le mandat de protection de la Commission africaine des droits de l'homme et des peuples ».

${ }^{728} \mathrm{Id}$.

${ }^{729}$ Suivant les dispositions de l'article 45 de la Charte Africaine des Droits de l'Homme et des Peuples.

${ }^{730}$ Les recours contentieux peuvent s'exercer suivant deux (2) modalités : les saisines directes et les saisines indirectes. Les saisines directes tirent leur source de l'article 5-3 du Protocole de Ouagadougou qui énonce que 
de la participation de ceux-ci à l'action juridictionnelle, dans la mesure où le droit de saisine n'est reconnu qu'au profit des acteurs non étatiques qui ont un statut d'observateur auprès de l'Union Africaine ${ }^{731}$, contrairement à la Cour européenne des droits de l'homme (CEDH) qui reconnait le droit d'accès à « toute personne physique, toute organisation non gouvernementale ou tout groupe de particuliers ${ }^{732}$. Il s'ensuit que, dans le cadre de l'Union européenne, le droit de participation à la justice environnementale est inclusif.

La Cour Africaine de Justice et des Droits de l'Homme qui a compétence environnementale et qui représente un atout pour l'effectivité du droit de protection de l'environnement en Afrique, subi actuellement des situations qui peuvent entrainer sa disparition. Parce que, autrefois composée de dix (10) Etats que sont, le Burkina Faso, le Malawi, le Mali, la Tanzanie, le Ghana, le Rwanda, la Côte d'Ivoire, le Bénin, la Tunisie et la Gambie, les quatre (4) Etats que sont : la Tanzanie, le Rwanda, la Côte d'Ivoire et le Bénin se sont retirés de l'institution juridictionnelle panafricaine ${ }^{733}$. Seulement que ces retraits ne devraient en principe prendre effet qu'après une période de préavis d'un (1) an ${ }^{734}$.

Nonobstant le statut d'observateur de l'Union Africaine (UA) à la Conférence des Parties de la Convention Cadre des Nations Unies sur les Changements Climatiques, l'institution régionale organise les Etats Membres autour d'un objectif unique, celui de relever les défis des changements climatiques sur le continent africain ${ }^{735}$. Dans cette optique, elle a créé plusieurs institutions notamment, le Comité des Chefs d'Etat et de Gouvernement sur les Changements Climatiques (CAHOSCC), la Conférence des Ministres de l'Environnement, la

«La Cour peut permettre aux individus ainsi qu'aux organisations non-gouvernementales (ONG) dotées du statut d'observateur auprès de la Commission d'introduire des requêtes directement devant elle conformément à l'article 34-6 de ce Protocole».

${ }^{731}$ Selon les dispositions de l'article 36-4 du Protocole portant Statut de la Cour Africaine de Justice et des Droits de l' 'Homme.

${ }^{732}$ Voir les stipulations de l'article 34 du Protocole $\mathrm{n}^{\circ} 11$ à la Convention de sauvegarde des droits de l'homme et des libertés fondamentales portant restructuration du mécanisme de contrôle établi par la Convention, adopté le 11 mai 1994. Disponible sur le site internet : https://rm.coe.int/1680739dc8. Consulté le 14/01/2022.

${ }^{733}$ Information disponible sur le site internet de l'institution juridictionnelle: https://www.african-court.org. Consulté ce $24 / 05 / 2021$.

${ }^{734}$ Conformément à la jurisprudence de la Cour : Cour ADHP, Aff. Ingabire Victoire Umuhoza c. République du Rwanda, Req. $\mathrm{n}^{\circ}$ 003/2014, arrêt du 3 juin 2016. Para. 58. Disponible sur le site internet : https://www.unige.ch. Consulté le 14/01/2022.

${ }^{735}$ Gouvernance en Afrique réponse au changement climatique en Afrique Une approche par la gouvernance des territoires disponible sur le site internet : https://base.afrique-gouvernance.net/docs/cp_climat_final.Consulté le $14 / 01 / 2022$. 


\section{Conférence Ministérielle Africaine sur l'Eau (AMCOW) ${ }^{736}$ et le CLIM-DEV AFRIQUE ${ }^{737}$} pour contribuer à un meilleur encadrement des changements climatiques. En 2014, l'Union africaine a lancé le « document de stratégie africaine sur les changements climatiques ${ }^{738}$. Ce document présente la vision africaine pour contenir les changements climatiques et « définit des objectifs spécifiques pour chaque domaine thématique, l'adaptation et la gestion des risques, les mesures d'atténuation appropriées au niveau national et des questions transversales spécifiques. Pour chacun de ces objectifs, plusieurs actions sont identifiées et utilisées dans la définition d'un plan de mise en œuvre de la stratégie ${ }^{739}$.

Selon Baba HAMADY, «La gouvernance africaine de l'environnement est en construction et se manifeste à travers la diversification des organisations régionales et sousrégionales qui interviennent dans le domaine environnemental et le développement d'accords sectoriels sur l'environnement, ${ }^{740}$. La preuve en est celle de l'Union africaine qui s'est invitée dans le domaine de la protection de l'environnement et s'est engagée à combattre les changements climatiques. Ses pas sont emboités par la Communauté Economique des Etats de l'Afrique de l'Ouest (CEDEAO).

\section{B- Le cas de la CEDEAO}

L'acte constitutif créant l'Union africaine a fait un renvoi aux conventions sousrégionales en matière de réalisation des objectifs concernant son intervention dans le domaine de protection de l'environnement ${ }^{741}$. Sa justification se trouve dans le fait que, avant l'existence de l'Union Africaine (UA), certaines organisations sous-régionales du continent avaient déjà

\footnotetext{
${ }^{736}$ Le Conseil des Ministres Africains Chargés de l'Eau (AMCOW) a été créé en 2002 à Abuja au Nigeria, ayant principalement pour objectif de promouvoir la coopération, la sécurité, le développement social et économique et l'éradication de la pauvreté dans les États membres à travers la gestion efficace des ressources en eau du continent et la prestation de services d'approvisionnement en eau.

${ }^{737} \mathrm{Le}$ programme Climat pour le développement en Afrique (ClimDev-Afrique) est une initiative de la Commission de l'Union africaine (CUA), de la Commission économique des Nations unies pour l'Afrique (CEA) et de la Banque africaine de développement (BAD). Il est mandaté au plus haut niveau par les dirigeants africains (Sommet des Chefs d'Etat et de Gouvernement de l'UA). Le programme a été créé pour établir une base solide pour la réponse de l'Afrique au changement climatique. Au-delà du partenariat UA-CEA-BAD, le Programme travaille en étroite collaboration avec d'autres institutions et partenaires africains et non africains spécialisés dans le climat et le développement. Information disponible sur le site internet : https://www.afdb.org. Consulté le 14/01/2022.

${ }^{738} \mathrm{Ce}$ document est intervenu suite à l'adoption au Sommet de juillet 2009 à Syrte en Libye de la recommandation du Conseil exécutif sur le changement climatique en Afrique (EX.CL/Déc.500 (XV) Rev.1).

${ }^{739}$ Document de stratégie africaine de lutte contre les changements climatiques, p.10. Disponible sur le site internet : https://www.uneca.org. Consulté le 19/01/2022.

${ }^{740}$ Selon Baba HAMADY DEME, RIO+20 et l'avenir de la gouvernance de l'environnement en Afrique, RADE $\mathrm{N}^{\circ} 00,2012$, p.39.

${ }^{741}$ Selon les dispositions de l'article 3 paragraphe i de l'acte constitutif de l'Union africaine «Les objectifs de l'Union sont les suivants (...) coordonner et harmoniser les politiques entre les Communautés économiques régionales existantes et futures en vue de la réalisation graduelle des objectifs de l'Union (...) ».
} 
entamées une politique de protection de l'environnement ${ }^{742}$. A titre indicatif, les organisations sous-régionales, notamment, « La Communauté de Développement de l'Afrique Australe (SADC), la Communauté Economique et Monétaire de l'Afrique Centrale (CEMAC), la Communauté Economique de l'Afrique de l'Est (CEAE), l'Union Economique et Monétaire de l'Afrique de l'Ouest (UEMOA) ont toutes, des attributions environnementales ${ }^{743}$. Il s'ensuit que le droit communautaire de l'Union Africaine (UA) établi une interconnexion avec les organisations sous-régionales en vue de l'accomplissement de ses objectifs. C'est dans ce type de lien hiérarchique qu'intervient la CEDEAO, une Organisation sous-régionale de l'Afrique de l'ouest, la seule communauté économique de la sous-région, créée aux fins de « l'intégration économique et de la réalisation à terme, des objectifs de la Communauté Economique Africaine $»^{744}$ afin "d'élever le niveau de vie de ses peuples, de maintenir et d'accroitre la stabilité économique, de renforcer les relations entre les Etats membres et de contribuer au progrès et au développement du continent africain $»^{745}$. Les objectifs de l'organisation mettent en évidence le développement du contient africain, seulement qu'il y a des goulots qui ne sont pas favorables à l'atteinte de ces objectifs.

Autant que les changements climatiques constituent une préoccupation majeure du continent africain, au plan régional, les effets des changements climatiques entravent également la réalisation des objectifs que se sont fixés les organisations sous- régionales. Ce constat est patent dans les pays membres de la CEDEAO. En effet, depuis les années 70, l'évolution du climat en Afrique de l'ouest se caractérise par un accroissement des précipitations avec la recrudescence de fortes pluies et la résurgence des inondations et une élévation supplémentaire des températures. Les prévisions en matière d'évaluation des changements climatiques ont permis de classer l'Afrique de l'ouest parmi les deux grandes catégories de régions particulièrement vulnérables ${ }^{746}$. Les Experts ont estimés que les conséquences qui découleraient de la modification des paramètres météorologiques peuvent impacter

\footnotetext{
${ }^{742}$ François FALLOUX et Lee M. TABLOT, Crise et opportunité, environnement et développement en Afrique, Earthscan Publications Ltd, Londres, Page 88.

${ }^{743}$ Voir: B. Ouattara, «Le Rolle des organisations sous-régionales dans le développement du droit de l'environnement: l'exemple de l'UEMOA $\gg$, in L. Granier (coord.), Aspects contemporains du droit de l'environnement en Afrique de l'ouest et centrale, UICN, Gland, 2008, pp. 177-196.

${ }^{744}$ Article 2 paragraphe 1 du traité révisé de la CEDEAO signé à Cotonou le 24 juillet 1993 dispose que «Par le présent Traité, les Hautes Parties Contractantes réaffirment la création de la Communauté Economique des Etats de l'Afrique de l'Ouest (CEDEAO) et décident qu'elle sera à terme la seule Communauté Economique de la Région aux fins de l'intégration économique et de la réalisation des objectifs de la Communauté Economique Africaine ».

${ }^{745}$ Article 3 du traité révisé de la Communauté Economique des Etats de l'Afrique de l'Ouest (CEDEAO).

${ }^{746}$ Les évaluations du GIEC de 1990 à 1992, Régions et secteurs particulièrement vulnérables 2.1.3, p.102.
} 
dramatiquement cette région de l'Afrique ${ }^{747}$. En cas d'absence d'une action adéquate concertée, les effets pervers des changements climatiques peuvent évoluer au point où, « les rendements et la production des cultures diminueront considérablement et affecteront par la suite la sécurité alimentaire dans cette région $»^{748}$ et compromettre par ricochet les objectifs du développement harmonieux envisagés dans le cadre régional.

Dans un contexte régional particulièrement vulnérable aux effets néfastes des changements climatiques, comment la CEDEAO peut parvenir à accomplir son objectif d'intégration régionale?

Une réponse à cette interrogation se trouve dans les résultats d'un colloque organisé sur le renforcement de l'effectivité du Droit de l'Environnement en Afrique, lequel a recommandé de « Consolider le rôle des institutions régionales et sous régionales d'intégration existantes en Afrique dans le domaine de l'environnement, notamment en renforçant les structures existantes en charge de l'environnement dans ces organisations ou en les créant si elles n'existent pas ${ }^{749}$. Ce faisant, les préoccupations environnementales sont inscrites dans l'acte qui a créé la CEDEAO ${ }^{750}$. A l'opposé de certaines organisations internationales qui ne relèvent pas du domaine de protection de l'environnement, la CEDEAO a d'ores et déjà au moment de sa création pris des mesures normatives visant à réaliser les objectifs pertinentes de l'organisation par l'orientation de l'action de la communauté vers l'harmonisation des politiques en vue de la protection de l'environnement ${ }^{751}$ et a retenu comme principe fondamental, le respect, la promotion et la protection des droits de l'Homme et des Peuples ${ }^{752}$. A l'appui de cet encadrement, une commission de l'agriculture, de l'environnement et des ressources en eau, comportant une direction de l'Environnement chargée de l'élaboration de la politique environnementale de l'organisation a été installée ${ }^{753}$.

Lorsque les cadres normatif et institutionnel ont été mis en place, l'encadrement de la gestion de la protection de l'environnement par cette organisation a été centré sur la mise en

\footnotetext{
${ }^{747}$ Id p. 101.

748 BENZAIDA Kamal, Prospects and Actions in Africa to cope with Climate Change Effects, Géneva Global Policy Briefs n³/2020.

${ }^{749}$ Revue Africaine de Droit de l'Environnement $n^{\circ} 01-2014$, première recommandation sur l'effectivité du droit de l'environnement en Afrique à la page 17.

${ }^{750} \mathrm{Le}$ traité révisé de la CEDEAO. Disponible sur le site internet: https://www.ecowas.int/wpcontent/uploads/2015/02/Traite-Revise.pdf. Consulté le 08/06/2021.

${ }^{751}$ Article 3 paragraphe 1 du traité révisé de la CEDEAO.

${ }^{752}$ Article 4 du traité révisé de la CEDEAO.

${ }^{753}$ Communication de Baba HAMADY DEME RIO+20 ET L'AVENIR DE LA GOUVERNANCE DE L'ENVIRONNEMENT EN AFRIQUE, Revue africaine de droit de l'environnement $\mathrm{N}^{\circ} 00,2014$.
} 
œuvre de document de politique environnementale conçu ${ }^{754}$, et la réalisation des projets intégrés au profit des Etats membres. Les projets élaborés par cette organisation ont une dimension transnationale et cadrent avec la vision de la gestion planétaire des problèmes environnementaux. Elle a par ailleurs, « initié un cadre intergouvernemental pour s'occuper de la gestion des risques de catastrophe provenant des modifications des paramètres climatiques $»^{755}$.

Dans le but de renforcer la structure organisationnelle intervenant dans le domaine de la protection de l'environnement, une commission technique à l'environnement et ressources naturelles a été instituée ${ }^{756}$. Puis, l'organisation régionale est allée au delà des attentes car, pour jauger l'efficacité de ces principes ${ }^{757}$ désormais consacrés dans le traité, elle s'est dotée d'une institution autonome à caractère juridictionnel ${ }^{758}$ chargée de veiller au respect et à la bonne application des normes protectrices des droits de l'Homme. C'est à ce titre que la CEDEAO avec l'adoption du Protocole A/SP.1/01/05 du 19 Janvier 2005 a donné compétence à sa juridiction dénommée « Cour de justice de la CEDEAO » ${ }^{759}$ de connaitre des cas de violation des droits de l'Homme ${ }^{760}$. La notion des droits de l'Homme s'entend ici « le droit de l'Homme à un environnement sain», qu'il serait loisible d'en déférer les conflits qui découle de la violation des règles environnementales devant la juridiction compétente de la CEDEAO. Depuis l'apparition de cette réforme qui a élargi le champ de compétence de la Juridiction d'Abuja, les citoyens $^{761}$ ouest- africain ou les Etats Membres de la Communauté régionale victimes de violation des Droits humains y compris le droit à un environnement sain, de la part d'un Etat Membre de la Communauté peuvent désormais accéder au prétoire du juge communautaire qui est compétent pour trancher les conflits environnementaux.

Cette possibilité juridictionnelle offerte dans le cadre régional peut faciliter la mise en œuvre du principe de la responsabilité des Etats devant la juridiction internationale. Les Etats membres de la CEDEAO peuvent désormais ester en justice pour les cas de violation des règles

\footnotetext{
${ }^{754}$ La politique environnementale de la CEDEAO, disponible sur le site internet: website:www.ecowas.int. Consulté le 14/01/2022.

${ }^{755}$ Gouvernance en Afrique : Réponse au changement climatique en Afrique une approche par la gouvernance des territoires: Alliance pour refonder la gouvernance en Afrique- ARGO, p.8. Disponible sur le site internet: www.afrique-gouvernance.net. Consulté le 14/01/2022.

${ }^{756}$ Article 12 du traité révisé de la CEDEAO.

757 Article 4 id.

758 Article 15 ibid.

${ }^{759}$ Instituée par l'article 6 paragraphe 1-e et l'article 15 paragraphe 1 du Traité révisé de la CEDEAO.

${ }^{760}$ Article 9 du protocole A/P. 1/7/91 relatif à la Cour de Justice de la Communauté.

${ }^{761}$ Suivant les dispositions de l'article premier paragraphe 1 du protocole $n^{\circ}$ A/P3/82 relatif au Code de la citoyenneté de la Communauté adopté le 29 mai 1982, publié dans le journal officiel de la CEDEAO en juin 1982, est considéré comme citoyen de la communauté « toute personne qui, par la descendance, a la nationalité d'un Etat membre et qui ne jouit pas la nationalité d'un Etat non membre de la communauté».
} 
de protection de l'environnement parce que, la Convention Cadre des Nations Unies sur les Changements Climatiques, en ordonnant aux Etats Parties que, «Conformément à la Charte des Nations unies et aux principes du droit international, les Etats ont le droit souverain d'exploiter leurs propres ressources selon leur politique d'environnement et de développement (...) $»^{762}$, elle en délimitait également le domaine en prescrivant que les Etats Parties « (...) ont le devoir de faire en sorte que les activités exercées dans les limites de leur juridiction ou sous leur contrôle ne causent pas de dommage à l'environnement dans d'autres Etats ou dans des zones ne relevant d'aucune juridiction nationale $»^{763}$. Il s'ensuit que la violation de ce principe et beaucoup d'autres règles de protection environnementale peuvent donner lieu à des contestations entre Etats victime et pollueur, devant la juridiction de proximité qu'est la Cour de justice de la CEDEAO, qui de part ses décisions peut contribuer à la bonne application de la Convention climat à laquelle ont souscrit, tous les Etats Membres de ladite communauté.

L'un des atouts des textes applicables devant la juridiction Communautaire qui peut garantir l'effectivité du Droit Climatique est la possibilité de saisine de ladite juridiction établie au profit des citoyens de la Communauté régionale. Malheureusement, ce droit de saisine a commencé par être érodé dans certains Etats Membres. A titre d'exemple, le Bénin a opéré un retrait implicite du droit de saisine des citoyens béninois par une décision rendue par la Cour constitutionnelle de ce pays, par laquelle elle a établi que, « le protocole additionnel qui permet aux citoyens béninois de saisir directement la Cour de Justice de la CEDEAO n'est pas opposable à l'Etat béninois ${ }^{764}$. Bien entendu, cette décision n'emporte pas le retrait du Bénin de la Cour, mais ne permet plus au citoyen béninois d'avoir l'opportunité de faire valoir son droit au niveau de cette juridiction régionale.

L'efficacité du droit applicable aux changements climatiques, dépendra en grande partie de son redéploiement et de sa mise en œuvre sur le territoire des Etats Parties. C'est pourquoi, chaque Etat Partie se mit à élaborer son cadre institutionnel. Le Bénin a aussi développé son propre dispositif institutionnel de lutte contre les changements climatiques.

\footnotetext{
${ }^{762}$ Principe 2 de la Déclaration de Rio sur l'environnement. ${ }^{763} \mathrm{Id}$.

${ }^{764}$ Décision DCC N ${ }^{\circ} 20-434$ en date du 30 avril 2020 de la Cour constitutionnelle du Bénin. Disponible sur le site internet : https://courconstitutionnelle.bj/decisions-2. Consulté le 29/05/2021.
} 
TITRE II 


\section{CHAPITRE II:}

\section{LE DISPOSITIF INSTITUTIONNEL INTERNE}

Les préoccupations liées à l'implication des Etats Parties à la mise en œuvre des normes de Protection de l'Environnement, ont transparu dans le principe 17 de la Déclaration de Stockholm de 1972 qui stipule qu' "Il convient que des institutions nationales appropriées soient chargées de planifier, de gérer ou de réglementer l'utilisation des ressources de l'environnement dont disposent les Etats, en vue d'améliorer la qualité de l'environnement ». A travers ce principe, les Etats ayant participé à l'adoption de la Déclaration de Stockholm de 1972 ou ayant acceptés les termes de ladite Déclaration sont fondés à bâtir un cadre institutionnel en s'inspirant de leur propre législation pour s'occuper exclusivement de la question de la gestion de la protection de l'environnement sur leur territoire respectif.

Plus tard, le caractère pernicieux des effets des changements climatiques qui sont apparus après la tenue de la Conférence de Stockholm de 1972 a contribué à l'occasion du Sommet de Rio de Janeiro de 1992 à l'élaboration d'un cadre juridique international qui prend en compte, les préoccupations liées aux changements climatiques ${ }^{765}$. Lequel cadre juridique, incite maintenant les Etats Parties à l'installation d'un cadre institutionnel interne, devant s'occuper uniquement des questions relatives aux changements climatiques.

De toutes les manières, la problématique de l'encadrement institutionnel de la protection de l'environnement et des changements climatiques a été une préoccupation largement partagée par, l'ensemble des acteurs du Droit International de l'Environnement.

Au Bénin, pour faire suite à cette préoccupation, la constitution du 11 décembre 1990, révisée par la loi 2019-40 du 07 novembre 2019 a indiqué la portée de la responsabilité de l'Etat en matière d'encadrement institutionnel de l'environnement par son article 27 qui dispose que «(...) l'Etat veille à la protection de l'environnement». Cette disposition constitutionnelle oblige l'Etat béninois à construire une architecture institutionnelle pour contribuer à la protection de l'environnement.

Les autorités béninoises conscientes de ce que le pays n'est pas à l'abri des effets néfastes des changements climatiques, dans la perspective de se conformer aux engagements

\footnotetext{
${ }^{765}$ Mais avant la tenue du Sommet de Rio de Janeiro de 1992, l'organisation de la première Conférence mondiale sur le climat de 1979 a permis de mettre la lumière sur les problèmes liés aux changements climatiques et de conclure que les émissions anthropiques de dioxyde de carbone pourraient avoir un effet à long terme sur le climat. Disponible sur le site internet: https:/www.actualites-news-environnement.com/21729-1979-2009-histoiretrente-ans-lutte-changement-climatique. Consulté le 14/01/2022.
} 
souscrits dans le cadre des accords internationaux relatifs à la lutte contre les changements climatiques et aux dispositions constitutionnelles du Bénin en matière de gestion de la protection de l'environnement, ont engagé des réformes tendant à développer un cadre institutionnel qui s'est avéré à la fois pertinent (SECTION I) et adéquat à la lutte contre les changements climatiques (SECTION II).

\section{SECTION I: UN CADRE INSTITUTIONNEL PERTINENT}

L'encadrement institutionnel de l'environnement a réellement pris son envol en République du Bénin à partir de $1990^{766}$, année de la consécration constitutionnelle du droit de protection de l'environnement. Cette période a coïncidé avec l'avènement du Sommet de Rio de Janeiro de 1992, dédié à la lutte contre les changements climatiques, Sommet auquel le Bénin a pris part et a participé à l'adoption de tous les instruments de protection de l'environnement et ceux concernant la lutte contre les changements climatiques qui avaient été élaborés ${ }^{767}$.

L'organisation institutionnelle mise en place quoi que pertinente, a connu une évolution rapide et soutenue par une base légale fondée sur des dispositions éparses extraites notamment, de la constitution du 11 décembre 1990 révisée par la loi 2019-40 du 07 novembre 2019 et de la loi $n^{\circ}$ 98-030 portant loi cadre sur l'environnement en République du Bénin et puis la loi $\mathrm{n}^{\circ}$ 2018-18 relative à la règlementation des changements climatiques en République du Bénin. Néanmoins, certaines institutions de protection de l'environnement ont été créées par actes réglementaires, contrairement aux dispositions de l'article 98 de la constitution béninoise du 11 décembre 1990 révisée par la loi 2019-40 du 07 novembre 2019, qui range « dans le domaine de la loi, les règles concernant la protection de l'environnement et de la conservation des ressources naturelles ».

L'élément de pertinence que comporte l'encadrement institutionnel béninois réside dans le degré d'implication du Gouvernement dans le domaine de la protection de l'environnement et de la lutte contre les changements climatiques, (Paragraphe 1) ajouté au réseau institutionnel de protection de climat élaboré pour s'occuper essentiellement des questions relatives aux changements climatiques (Paragraphe 2).

\footnotetext{
${ }^{766}$ Année d'adoption de la nouvelle constitution après la tenue de la conférence des forces vives de la nation de février 1989 au PLM ALEDJO à Cotonou Bénin.

${ }^{767}$ Convention Cadre des Nations Unies sur les Changements Climatiques- Convention Cadre sur la Biodiversité, Déclaration de Rio de Janeiro de 1992 sur l'Environnement et le Développement et la Déclaration de Rio de 1992 sur l'Environnement et le Développement, principes de gestion des forets.
} 


\section{Paragraphe 1: La forte implication du Gouvernement}

Les constitutionnalistes conçoivent le concept de "fonctions régaliennes », comme celui qui est intrinsèque à l'existence de l'Etat. Autrement, les «fonctions régaliennes » sont des activités de souveraineté et comportent un certain nombre d'élément dont « la Sécurité ». Avec l'émergence du droit de l'environnement et surtout du droit climatique, la notion de sécurité a été élargie au «principe de précaution » ${ }^{768}$ dans les conventions internationales de protection de l'environnement. Il s'ensuit que les préoccupations de protection de l'Humanité contre les risques majeurs provoqués par la dégradation de l'environnement font partie intégrante des fonctions régaliennes dont la charge incombe à l'Etat.

Maintenant, qu'il est établi que la protection de l'environnement est une activité à caractère d'intérêt général ${ }^{769}$, relevant du domaine régalien de l'Etat, il n'y a pas d'obstacle à ce que les pouvoirs publics créent des services publics spécialisés chargés de la gérer.

Au-delà de l'aspect régalien que revêt la gestion de la protection de l'environnement, il y a plusieurs autres motivations pour lesquelles le Gouvernement est obligé de s'intéresser audit domaine. Le document de Stratégie BENIN ALAFIA 2025 a projeté des scenarios catastrophiques où toutes les hypothèses sont alarmistes. Spécifiquement, les études ont révélé que, « la situation environnementale au Bénin est préoccupante en raison des agressions perpétrées par l'homme sur les ressources naturelles ${ }^{770}$. C'est ce qui justifie même son élévation au rang des priorités dans le cadre de la règlementation environnementale ${ }^{771}$.

La loi n²018-18 sur les changements climatiques en République du Bénin a conféré beaucoup d'attributions à l'Etat. Selon les dispositions de ladite loi, c'est l'Etat, « (...) en tant que garant du droit des populations à un environnement sain, qui assure dans toutes les initiatives portant sur les changements climatiques, le respect des principes ${ }^{772}$. Il a pour responsabilité de « (...) préserver le système climatique contre les changements climatiques et leurs effets et conséquences négatifs dans tous les secteurs économiques et sociaux fragiles et

\footnotetext{
${ }^{768}$ Le principe 15 de la Déclaration de Rio de Janeiro sur l'Environnement et le Développement de 1992 impose l'application des mesures de précaution aux Etats. Ces mesures doivent être préconisées en cas de risque de dommages graves ou irréversibles. Ainsi, l'absence de certitude scientifique absolue ne doit pas servir de prétexte pour remettre à plus tard l'adoption de mesures effectives visant à prévenir la dégradation de l'environnement.

${ }^{769}$ Suivant une conception jurisprudentielle tirée de l'arrêt Fredin c/ Suède du 18 février 1991 a élevé la protection de l'environnement au rang d'intérêt général.

${ }^{770}$ Voir document de stratégie BENIN ALAFIA 2025, p. viii. Disponible sur le site internet: http://dsaflash.viabloga.com/files//BEN_2025_ALAFIA.pdf. Consulté le 08/06/2021.

${ }^{771} \mathrm{Il}$ s'agit de la protection constitutionnelle conférée au domaine de protection de l'environnement.

${ }^{772}$ Article 6 de la loi $n^{\circ} 2018$-18 portant sur la règlementation sur les changements climatiques en République du Bénin.
} 
vulnérables (...) ${ }^{773}$. L'Etat est également invité à prendre «des mesures de précaution pour prévoir, prévenir ou atténuer les causes des changements climatiques et en limiter les effets et conséquences négatifs (...) » ${ }^{774}$, y compris « (...) toutes les dispositions pour s'adapter au nouveau contexte climatique (...) ${ }^{775}$. Il doit « (...) xuvrer pour un développement durable en intégrant dans les programmes et projets nationaux de développement des mesures destinées à faire face aux changements climatiques (...) $»^{776}$ et « (...) rendre responsables, directement ou indirectement, l'auteur ou les auteurs de tout acte ou activité susceptible d'entrainer des perturbations du climat avec ses effets et conséquences négatifs ${ }^{777}$.

Pour accomplir efficacement cette mission, l'Etat béninois a procédé à la création d'un ministère chargé de la protection de l'Environnement qui intervient comme une plaque tournante de l'exécution de la politique environnementale de l'Etat (A). Mais surtout, la réussite de la mission assignée à ce Ministère dépend de la qualité de l'expertise que lui apportent certaines institutions techniques $(\mathbf{B})$.

\section{A- L'intervention du ministère de l'Environnement}

Après avoir prescrit la participation des institutions nationales dans les actions de protection de l'environnement ${ }^{778}$, la Conférence de Stockholm de 1972 s'était également penchée sur les questions relevant du domaine de compétence des autorités et des Gouvernements. Selon les dispositions conventionnelles, les autorités gouvernementales «(...) auront la responsabilité principale des politiques et de l'action à mener en matière d'environnement dans les limites de leur juridiction $\gg{ }^{779}$. En suivant ces prescriptions, le Bénin a emboité le pas à d'autres Nations ${ }^{780}$ en procédant à la création d'un cadre institutionnel destiné à la promotion des dispositions constitutionnelles ${ }^{781}$ en vigueur, en instituant le 29 juillet 1991 ,

\footnotetext{
773 Id.

774 Ibid.

${ }^{775}$ Ibid.

776 Ibid.

${ }^{777}$ Ibid

${ }^{778}$ Principe 17 de la déclaration de Stockholm de 1972.

${ }_{779}$ Proclame paragraphe 7 id.

${ }^{780} \mathrm{~A}$ l'instar à la République Française qui a créé le 07 janvier 1971 son premier département ministériel en charge de l'environnement dénommé, « Ministère délégué chargé de la Protection de la Nature et de l'Environnement » sous la présidence de Georges Pompidou. Disponible sur le site internet: https://www.francememoire.fr/dossiers/creation-du-ministere-de-la-protection-de-la-nature-et-de-lenvironnement. Consulté le $14 / 01 / 2022$.

${ }^{781}$ Il s'agit des articles 27, 28, 29, 74 et 98 de la constitution béninoise du 11 décembre 1990 révisée le 07 novembre 2019.
} 
le premier département ministériel de l'Environnement ${ }^{782}$. Le volet environnemental est devenu à partir de ce moment, une activité essentiellement à la charge de ce Ministère qui en assure la coordination $^{783}$. En procédant ainsi, le Bénin a donné une réponse aux préoccupations des citoyens en ce qui concerne la réglementation judicieuse en matière environnementale, puisque ces derniers « ont la conviction que l'élaboration des textes appropriés, s'adaptant au contexte et à l'évolution du pays, la mise en place d'institutions y afférentes et d'hommes compétents pour les animer permettront une meilleure gestion de l'environnement ${ }^{784}$.

Cependant, le ministère de l'Environnement à sa création n'avait pas exclusivement la charge de la mise en œuvre de la politique de l'Etat en matière de protection de l'environnement. En plus de ses attributions environnementales, il intervenait dans les domaines de l'habitat, de l'urbanisme et de la protection des ressources naturelles. En $2006,{ }^{785}$ les volets concernant l'habitat et l'urbanisme ont été détachés de ce département, pour lui imprimer l'efficacité indispensable à son action en lui faisant porter la dénomination, « Ministère de l'Environnement et de la Protection de la Nature (MEPN)» ${ }^{786}$. Ce n'est qu'en $2016^{787}$, que les attributions du Ministère ont été renforcées par l'intégration des données relatives aux changements climatiques $^{788}$, pour tenir compte des recommandations des Sommets consacrés aux changements climatiques. Il devint alors, « Ministère du Cadre de Vie et du Développement Durable» ${ }^{789}$. La mise en œuvre de la loi ${ }^{\circ} 2018-18$ portant règlementation des changements climatiques en République du Bénin va entrainer la mutation de cette institution qui deviendra, «Ministère des Changements Climatiques ${ }^{790}$, ce qui permettra de rompre avec toute équivoque que suscite les diverses appellations ${ }^{791}$. En effet, les attributions de ce Ministère sont revues autrement, en ce qu'il est « chargé de l'élaboration et de la mise en æuvre de la politique

\footnotetext{
${ }^{782} \mathrm{Par}$ décret $\mathrm{N}^{\circ}$ 91-176 portant composition Gouvernement. Disponible sur le site internet : https://sgg.gouv.bj/doc/decret-1991-176. Consulté le 14/01/2022.

${ }^{783}$ Selon les dispositions de l'article 6 de la loi n ${ }^{\circ} 98-030$ du 12 février 1999 portant loi cadre sur l'environnement en République du Bénin.

${ }^{784}$ Document de stratégie BENIN ALAFIA 2025, p.57.

${ }^{785} \mathrm{Au}$ cours de cette année un autre Gouvernement a succédé à l'ancien Exécutif.

${ }^{786}$ Voir Décret $\mathrm{n}^{\circ}$ 2007-493 du 02 novembre 2007, portant attributions, organisation et fonctionnement du Ministère de l'Environnement et de la Protection de la Nature. Disponible sur le site internet : https://www.francememoire.fr/dossiers/creation-du-ministere-de-la-protection-de-la-nature-et-de-lenvironnement. Consulté le $14 / 01 / 2022$.

${ }^{787}$ Année d'alternance à la tête de l'exécutif.

${ }^{788}$ Article 3 du décret $n^{\circ} 2016-501$ du 11 août 2016 portant, attributions, organisation et fonctionnement du ministère du cadre de vie et du développement durable.

${ }^{789}$ Article $1^{\text {er }}$ du décret $n^{\circ} 2016-264$ du 06 avril 2016 portant composition du Gouvernement en République du bénin.

${ }^{790}$ Article 77 de la loi ${ }^{\circ} 2018-18$ portant sur la règlementation en matière des changements climatiques au Bénin.

${ }^{791}$ Ces appellations sont : 1- Ministère de l'Environnement et de la Protection de la Nature ; 2 - Ministère de l'Environnement de l'Habitat et de l'Urbanisme ; 3- Ministère du Cadre de Vie et du Développement Durable.
} 
nationale en matière d'environnement, ainsi que de la coordination de son exécution (...) ${ }^{792}$. Cette disposition a entériné la compétence environnementale consacrée par acte règlementaire ${ }^{793}$ qui avait été pris à cette fin ${ }^{794}$. De même, cette institution « (...) a désormais en charge la définition, le suivi de la mise en œuvre et l'évaluation de la politique de l'Etat en matière (...) de gestion des effets des changements climatiques, reboisement, protection des ressources naturelles, forestières, préservation des écosystèmes et protection des berges et côtes $»^{795}$. Ses attributions en matière de lutte contre les changements climatiques ont été renforcées par la loi n²018-18 relative à la règlementation sur les changements climatiques en République du Bénin. Cette loi oblige « l'Etat qui adopte les politiques et stratégies appropriées pour la prévention, la réduction et la maîtrise des effets liés aux changements climatiques et aux catastrophes naturelles $(\ldots) \gg{ }^{796}$.

Comment le ministère en charge de l'Environnement, gère t-il, le volet concernant les changements climatiques?

Le changement d'appellation du ministère de l'Environnement cette fois-ci est accompagné de profondes réformes ${ }^{797}$ structurelles importantes qui ont contribué à la modification substantielle de son organigramme dans l'objectif de l'intégration des préoccupations liées aux changements climatiques. Pour faciliter la mise en œuvre de sa mission en ce qui concerne la lutte contre les changements climatiques, il est inclus dans l'organigramme du ministère de l'Environnement deux (2) importantes directions pour se charger uniquement du volet concernant les changements climatiques. La première est la Direction Générale de l'Environnement et du Climat (DGEC), une structure technique ${ }^{798}$ qui s'occupe exclusivement de la gestion des changements climatiques. Elle a en charge l'élaboration et la mise en œuvre de la politique et des stratégies de l'Etat en matière de protection de l'environnement ${ }^{799}$ et s'occupe également de la gestion des changements

\footnotetext{
${ }^{792}$ L'article 6 de la loi n 98-030 du 12 février 1999 portant loi cadre sur l'environnement en République du Bénin. ${ }^{793}$ Le décret $n^{\circ} 2016-292$ du 17 mai 2016, fixant la structure type des ministères et le décret $n^{\circ} 2016-501$ du 11 aout 2016 portant attributions, organisation et fonctionnement du ministère du cadre de vie et du développement durable.

${ }^{794}$ Le décret avait déjà créé le ministère avant l'existence de la loi qui l'a entériné.

795 Article 3 décret portant attributions, organisation et fonctionnement du Ministère du Cadre de Vie et du Développement Durable.

${ }^{796}$ Article 12 de la loi relative aux changements climatiques en République du Bénin.

${ }^{797} \mathrm{C}$ 'est le décret $\mathrm{n}^{\circ} 2016-501$ du 11 août 2016 portant, attributions, organisation et fonctionnement du ministère du cadre de vie et du développement durable, qui est porteur de ces réformes.

798 Article 62 du décret $\mathrm{n}^{\circ} 2016-501$ du 11 août 2016 portant, attributions, organisation et fonctionnement du ministère du cadre de vie et du développement durable.

${ }^{799}$ Article 63 du décret $n^{\circ} 2016-501$ du 11 août 2016 portant, attributions, organisation et fonctionnement du ministère du cadre de vie et du développement durable.
} 
climatiques $^{800}$. Pour concrétiser sa mission, la Direction Générale de l'Environnement et du Climat (DGEC) s'occupe entre autres activités, de la « conception des politiques et stratégies de l'Etat et la mise en place de la règlementation en matière environnementale, de gestion des effets des changements climatiques et en assure le suivi-évaluation de la mise en œuvre et fait des propositions au Gouvernement des mesures préventives de réduction de la pollution environnementale et d'émissions de gaz à effet de serre ${ }^{801}$.

La deuxième Direction la plus importante compte tenu de ses attributions en lien avec les changements climatiques, qui figure dans l'organigramme du ministère de l'Environnement est la Direction Générale des Eaux Forets et Chasse ${ }^{802}$. Une composante des forces de sécurité publique et assimilés qui a pour mission, « d'élaborer et d'assurer la mise en œuvre, ainsi que le suivi-évaluation de la politique et des stratégies de l'Etat en matière de gestion des eaux, forêts et chasse $»^{803}$. A ce titre, elle «participe à l'élaboration des politiques et stratégies de l'Etat en ce qui concerne le reboisement et la gestion des ressources naturelles et en assure la mise en œuvre ${ }^{804}$. Elle joue le rôle du «point focal national des conventions et accords multilatéraux en matière des ressources naturelles forestières ${ }^{805}$. Elle a mandat de « représenter le Bénin aux négociations des accords multilatéraux en matière de protection de l'environnement et puis, elle contribue à la sauvegarde de l'intégrité du domaine forestier de l'Etat ${ }^{806}$. Elle « développe et assure, la mise en œuvre des projets et programmes de lutte contre la désertification ${ }^{807}$. Elle constitue, gère et rends disponible aux divers acteurs, une base de données quantitatives, relatives aux ressources naturelles et forestières » ${ }^{808}$, « impulse et assure la mise en œuvre des politiques, programmes et projets ${ }^{809}$, «élabore les plans, programmes et projets de valorisation, de prévention et de lutte contre toutes les formes de dégradation des ressources naturelles forestières et faunique ${ }^{810}$. Elle «promeut le reboisement, la reforestation et autres méthodes de lutte contre la désertification ${ }^{811}$, élabore les

\footnotetext{
${ }^{800}$ Id.

801 Ibid.

${ }^{802}$ Ibid.

${ }^{803}$ Ibid.

${ }^{804}$ Ibid.

${ }^{805}$ Article 13 troisième tiret du décret $\mathrm{n}^{\circ} 2016-501 \mathrm{du} 11$ aout 2016, portant attributions, organisation et fonctionnement du Ministère du Cadre de Vie et du Développement Durable. ${ }^{806}$ Id.

807 Article 13 cinquième tiret du décret $\mathrm{n}^{\circ} 2016-501 \mathrm{du} 11$ aout 2016, portant attributions, organisation et fonctionnement du Ministère du Cadre de Vie et du Développement Durable.

${ }^{808}$ Article 13 sixième id.

${ }^{809}$ Article 13 huitième ibid.

${ }^{810}$ Article 13 neuvième tiret du décret $\mathrm{n}^{\circ} 2016-501 \mathrm{du} 11$ aout 2016, portant attributions, organisation et fonctionnement du Ministère du Cadre de Vie et du Développement Durable.

${ }^{811}$ Article 13 dixième tiret id.
} 
instruments et outils de gestion durable des ressources naturelles, participe à l'élaboration des politiques et stratégies de conservation des zones sensibles et la restauration des sites dégradés, assure la coordination, le pilotage du secteur forestier, la gestion des feux de végétation et suit la mise en œuvre de la politique, des stratégies, plans, programmes, projets, instruments et outils, en matière de gestion durable des ressources naturelles notamment, forestières et fauniques en liaison avec les structures spécialisées, puis gère et suit les conventions et accords internationaux relatifs à ses domaines de compétence et participe à l'élaboration des textes législatifs et réglementaires $»{ }^{812}$.

Les attributions du ministère en charge de l'Environnement en matière de lutte contre les changements climatiques sont nombreuses. Leur réalisation peut paraitre utopique si les compétences requises ne sont pas mises à contribution, c'est pourquoi l'encadrement juridique a prévu d'autres institutions techniques à caractère consultatif, pour accompagner le ministère de l'Environnement dans l'accomplissement de sa mission.

\section{B-L'accompagnement d'organes techniques}

«(...) Les mesures permettant de comprendre les changements climatiques et d'y faire face auront une efficacité pour l'environnement (...) si elles se fondent sur les considérations scientifiques, techniques et économiques appropriées et si elles sont constamment réévaluées à la lumière des nouveaux progrès réalisés dans ces domaines (...) ${ }^{813}$. Cet extrait du préambule de la Convention Cadre des Nations Unies sur les Changements Climatiques met en exergue la connaissance scientifique, comme un flambeau qui permet de mieux appréhender et de gérer efficacement le phénomène des changements climatiques. Cependant, le Ministère du Cadre de Vie et du Développement Durable ${ }^{814}$ qui a en charge la gestion des changements climatiques au Bénin, qui à la lumière de ses attributions ${ }^{815}$, constitue le pilier architectural le plus important de la politique de protection de l'environnement, ne dispose pas de toutes les compétences techniques orientées vers les objectifs d'encadrement de la protection de l'environnement et des changements climatiques. Cette situation est due au fait que l'administration publique béninoise ne regorge que de personnes ayant une compétence générale de la notion de la gestion

\footnotetext{
812 Ibid.

${ }^{813}$ Dix-septième paragraphe du préambule de la Convention Cadre des Nations Unies sur les Changements Climatiques.

${ }^{814}$ Dénomination du ministère en charge de l'environnement selon le décret $\mathrm{n}^{\circ} 2016-501 \mathrm{du} 11$ aout 2016 portant attributions, organisation et fonctionnement du ministère du cadre de vie et du développement durable.

${ }^{815}$ Ses attributions sont fixées dans la loi n 98-030 du 12 février 1999 portant loi-cadre sur l'environnement en République du Bénin.
} 
de l'administration publique. Les compétences spécialisées concernant les domaines de protection de l'environnement et la lutte contre les changements climatiques sont rares.

Quelle mesure l'Etat béninois a pris pour palier le déficit de compétence qui prévaut dans cette institution afin de l'amener à accomplir avec dextérité la mission de protection de l'environnement et celle concernant la lutte contre les changements climatiques?

Pour palier le déficit de compétence qui prévaut au sein de l'institution en charge de la gestion des changements climatiques, le Législateur béninois a prévu dans la loi cadre sur l'environnement ${ }^{816}$, la création de deux (2)organes consultatifs pour renforcer le ministère de l'Environnement en leur apportant leur expertise, dans le cadre de la mise en œuvre et de l'exécution de la politique de protection de l'environnement et de lutte contre les changements climatiques. Il s'agit de l'Agence Béninoise pour l'Environnement (ABE) et de la Commission Nationale pour le Développement Durable (CNDD).

\section{1- L'Agence Béninoise pour l'Environnement}

L'Agence Béninoise pour l'Environnement (ABE) en passe de devenir Agence Béninoise pour l'Environnement et du Climat (ABEC), pour tenir compte de l'intégration éventuelle de la dimension des changements climatiques à ses attributions, est un organe consultatif du ministère en charge de l'Environnement, créé par la loi nº $98-030$ du 12 février 1999 portant loi cadre sur l'environnement en République du Bénin, «pour servir d'institution d'appui à la politique nationale en matière de protection de l'environnement $»^{817}$. En d'autres termes, c'est une institution qui a en charge « la mise en œuvre de la politique environnementale définie par le Gouvernement dans le cadre du plan général de développement $»{ }^{818}$. Les modalités de son fonctionnement ont été déterminées par le décret $\mathrm{n}^{\circ} 2010-478$ du 05 novembre 2010 portant création, attributions, organisation et fonctionnement de l'Agence Béninoise pour l'Environnement. L'une des fonctions essentielles et importantes de l'ABE est l'évaluation environnementale, un processus visant à intégrer l'environnement dans l'élaboration d'un projet ou d'un document de planification pour éviter de faire subir des dommages éventuels à l'environnement ${ }^{819}$.

\footnotetext{
${ }^{816}$ La loi n 98-030 du 12 février 1999 portant loi-cadre sur l'environnement en République du Bénin. ${ }^{817}$ Article 11 de ladite loi.

${ }^{818}$ Article 12 du décret ${ }^{\circ} 95-47$ du 20 février 1995 portant organisation, attributions, et fonctionnement de l'Agence Béninoise pour l'Environnement.

${ }^{819}$ L'évaluation environnementale est prévue par l'article 5 du décret $n^{\circ} 2010-478$ du 05 novembre 2010 , portant création, attributions, organisation et fonctionnement de l'Agence Béninoise pour l'Environnement pour l'Environnement.
} 
Comment l'Agence Béninoise pour l'Environnement (ABE) appuie le ministère de l'Environnement dans le cadre de la lutte contre les changements climatiques?

L'Agence Béninoise pour l'Environnement (ABE) réalise des études d'impact environnemental pour le compte du ministère en charge de l'Environnement. Toute installation de projet d'envergure pouvant impacter l'environnement doit bénéficier de l'appui technique de l'ABE à travers ses avis sur la faisabilité du projet. L'évaluation environnementale « est un outil qui a été élaboré pour intégrer les impacts des changements climatiques et réduire ses risques ${ }^{820}$. Sa pertinence a été établie à travers plusieurs instruments internationaux de protection de l'environnement notamment, la Charte de la nature de $1982^{821}$ et la Déclaration de Rio de Janeiro sur l'Environnement et le Développement de $1992^{822}$. La Cour internationale de justice à travers sa jurisprudence a reconnu «l'obligation en droit international général d'entreprendre une étude d'impact environnementale en cas d'existence d'un risque que le projet proposé impacte négativement l'espace transfrontière ${ }^{823}$. En effet, la prise en compte dans l'évaluation environnementale, des dimensions concernant les changements climatiques peut contribuer à harmoniser les paramètres du projet avec le seuil des gaz à effet de serre admis dans les outils de planification en vigueur. C'est un outil qui permet d'élaborer et d'exécuter des projets en conformité avec les normes environnementales. Il empêche le déversement dans la nature des gaz à effet de serre dans les limites qui ne sont pas admises. L'Agence Béninoise pour l'Environnement (ABE) de part la réalisation des études d'impact environnemental au Bénin peut aider à minimiser le taux de pollution par l'émission des gaz à effet de serre. Seulement qu'au Bénin, les avis émis par les institutions techniques ne sont que facultatifs, malgré leurs pertinences, le Gouvernement peut passer outre et prendre une décision qui ne tient pas compte des réalités objectives contenues dans les rapports techniques.

La création de l'Agence Béninoise pour l'Environnement (ABE) est suivie de la Commission Nationale du Développement Durable (CNDD) dans le même cadre législatif.

\footnotetext{
${ }^{820}$ Hahn M \& Fröde A. Le Climate Proofing pour le Développement. S'adapter au changement climatique Réduire les risques. GIZ, Eschborn, 2011, 36 p.

${ }^{821}$ Adoptée par la résolution $37 / 7$ de l'Assemblée générale des Nations Unies, « Charte mondiale de la nature 1982 » paragraphe $11(\mathrm{c})$ et 16.

822 Principe 17 de ladite déclaration.

${ }^{823}$ CIJ, Arrêt de 2010 dans Pulp Mills, (n16) para 204. Disponible sur le site: https://www.icjcij.org/fr/decisions/judgment/2003/2021/desc. Consulté le 04/06/2021.
} 


\section{2- La Commission Nationale du Développement Durable}

La Commission Nationale du Développement Durable (CNDD) a été créée conformément à la loi nº99-030 du 12 février 1999 portant loi-cadre sur l'Environnement en République du Bénin. Son principal organe est l'Assemblée générale, composée de représentants des structures Gouvernementales, de l'Université, des Organisations Non Gouvernementales, de la Chambre du commerce et d'industrie, de la Chambre de 1'agriculture, des Associations de femmes et des Collectifs des Associations de Développement ${ }^{824}$. L'article 3 du décret qui l'a créé, la place sous l'autorité du Ministre de l'environnement ${ }^{825}$. Elle a compétence pour émettre des avis sur toute politique ou stratégie de développement susceptible d'affecter l'environnement, les ressources naturelles et la diversité biologique, préalablement à son adoption par le Gouvernement ${ }^{826}$. La CNDD a le statut d'organe consultatif pour le ministère en charge de l'Environnement, à ce titre elle « est chargée de l'intégration de la dimension environnementale dans les politiques, stratégies, programmes et projets sectoriels de développement $»^{827}$. Entre autres attributions, elle constitue un organe consultatif pour le Gouvernement en ce qui concerne l'élaboration des textes juridiques relatifs à l'environnement. Elle « favorise la technologie propre dans l'industrie, tout en contribuant à la maitrise de la pollution de l'air, de l'eau et des sols, de même qu'à l'amélioration de la qualité de vie ${ }^{828}$. La Commission Nationale du Développement Durable (CNDD) « initie des projets de textes en faveur de l'environnement et du développement et suit la mise en œuvre de la politique nationale du développement durable en coopérant le plus étroitement possible avec des organisations sous-régionales et internationales, le secteur privé, les pouvoirs locaux, les organisations non gouvernementales et les autres principaux groupes de la société intervenant pour le développement durable ${ }^{829}$. En plus de ses attributions, la Commission Nationale du Développement Durable (CNDD) « coordonne les actions de mise en synergie des conventions de la génération de Rio et joue le rôle de coordination en matière de préparation du rapport national de mise en œuvre de l'Agenda $21 »{ }^{830}$.

\footnotetext{
${ }^{824}$ Article 6 du décret $\mathrm{n}^{\circ} 99-641$ du 30 décembre 1999 portant attributions organisation et fonctionnement de la Commission Nationale du Développement Durable (CNDD).

${ }^{825}$ Décret $n^{\circ} 99-641$ du 30 décembre 1999 portant attributions organisation et fonctionnement de la Commission Nationale du Développement Durable (CNDD).

${ }^{826}$ Article 4 du décret $n^{\circ} 2005-068$ du 14 février 2005 portant attributions, organisation et fonctionnement de la commission nationale du développement durable.

${ }^{827}$ Article 2 id.

${ }^{828}$ Article 4 ibid.

${ }^{829}$ Article 4 ibid.

${ }^{830}$ Article 4 du décret $n^{\circ} 2005-068$ du 14 février 2005 portant attributions, organisation et fonctionnement de la commission nationale du développement durable.
} 
La Commission Nationale du Développement Durable a vu sa mission s'étendre au domaine des changements climatiques avec la loi $\mathrm{n}^{\circ}$ 2018-18 portant règlementation sur les changements climatiques en République du Bénin, laquelle loi prescrit à la charge de l'organe, que «Toute politique et toute stratégie de développement et leur déclinaison aux niveaux national, départemental, communal et local, intègrent la dimension changements climatiques sans occulter la durabilité environnementale et la réduction des risques de catastrophes naturelles (...) ${ }^{831}$. De même, « les stratégies et planifications existantes au niveau national et infranational devront être révisées pour intégrer la dimension changements climatiques (...) $»^{832}$. Des « dispositions réglementaires sont prises en vue de l'intégration des conclusions et modalités prévues dans les Plans nationaux d'adaptation à la stratégie de développement à faible intensité de carbone et résilient aux changements climatiques ${ }^{833}$.

Beaucoup d'autres organes à caractère technique sont créés et participent tous, à l'accomplissement de la politique de l'Etat en matière de protection de l'environnement. Néanmoins, l'Agence Béninoise pour l'Environnement (ABE) et la Commission Nationale du Développement Durable (CNDD) cités dans le cadre de cette étude, sont les plus importants organes techniques en raison du lien qui existe entre leur mission et les changements climatiques et le cadre juridique qui a consacré leur existence fait de ces organes des structures pérennes ${ }^{834}$.

Cependant, les spécificités que revêtent les changements climatiques et leurs effets néfastes sont les motifs devant entrainer l'Etat dans le processus de création d'institutions exclusivement orientées vers les objectifs de lutte contre les changements climatiques.

\section{Paragraphe 2: La création d'un réseau institutionnel de climat}

Pour engager la lutte contre les changements climatiques, le Bénin a procédé à la mise en place d'un réseau institutionnel climatique composé d'un organe relevant du ministère de l'Environnement (A) et d'un autre organe placé sous la tutelle du Ministre du plan (B).

\footnotetext{
${ }^{831}$ Article 7 alinéa 1 de la loi n 2018-18 relative aux changements climatiques en République du Bénin.

${ }^{832}$ Article 7 alinéa 2 id.

${ }^{833} \mathrm{Id}$.

${ }^{834}$ Il s'agit de la loi n98-030 du 19 février 1999 portant loi cadre sur l'environnement en République du Bénin.
} 


\section{A- Un organe relevant du ministère de l'Environnement}

Depuis la ratification par la République du Bénin de la Convention Cadre des Nations Unies sur les Changements Climatiques jusqu'à l'avènement de l'Accord de Copenhague en $2009^{835}$, les efforts d'encadrement institutionnel entrepris manquaient de pertinence, car il n'y avait pas un cadre juridique pouvant favoriser l'éclosion d'institutions spécialement destinées à la lutte contre les changements climatiques. Une situation qui contraste avec la volonté affichée par l'Etat béninois de lutter sans relâchement contre les changements climatiques.

Les compétences générales de protection de l'environnement attribuées au ministère de l'Environnement par la loi n $98-030$ du 12 février 1999 portant loi cadre sur l'environnement en République du Bénin ne répondent pas aux prescriptions de la Communauté internationale en matière de lutte contre les changements climatiques. En effet, le besoin de création d'institutions spécialisées en matière de lutte contre les changements climatiques est devenu manifeste après l'intégration des changements climatiques dans divers documents de politique, plan, projets, programmes de l'Etat, tel que recommandée par le document de stratégie nationale de mise en œuvre de la Convention Cadre des Nations Unies sur les Changements Climatiques ${ }^{836}$. Dans ce même document, il a été recommandé dans le volet de renforcement des capacités institutionnelles, de «créer effectivement le Comité National pour les Changements Climatiques (CNCC) pour faciliter les échanges de données et d'outils par l'organisation de séminaires et ateliers nationaux ${ }^{837}$. Malheureusement, le cadre juridique pouvant générer la création de cette institution est intervenu un peu plus $\operatorname{tard}^{838}$.

Mais avant la mise en place d'un encadrement juridique approprié à la lutte contre les changements climatiques ${ }^{839}$, la méthode jadis utilisée par les autorités béninoises pour suppléer le vide juridique et institutionnel, consistait à édicter des normes réglementaires ${ }^{840}$ pour créer

\footnotetext{
${ }^{835}$ Accord adopté à l'occasion de la quinzième $\left(15^{\mathrm{e}}\right)$ session de la Conférence des Parties tenue à Copenhague du 7 au 19 décembre 2009.

${ }^{836}$ Ce document a été réalisé par le Ministère de l'Environnement, de l'Habitat et de l'Urbanisme avec l'appui technique et financier du Programme des Nations unies pour le Développement (PNUD) et l'appui technique de l'Institut des Nations unies pour la Formation et la Recherche (UNITAR). La rédaction et la mise en forme définitive ont été assurées par une équipe d'experts nationaux sous la supervision de : Madame Jeanne Josette ACACHA AKOHA Point Focal Changements Climatiques Conseiller Technique à l'Environnement et Coordonnatrice du Programme CC:TRAIN au Bénin.

${ }^{837}$ Stratégie béninoise de mise en œuvre de la Convention Cadre des Nations Unies sur les Changements Climatiques, p.43. Disponible sur le site internet :https://unfccc.int/sites/default/files/resource/Benin\%. Page consultée le 02/06/2021.

${ }^{838} \mathrm{La}$ loi $\mathrm{n}^{\circ} 98-030$ du 12 février 1999 portant loi cadre sur l'environnement en République du Bénin pouvant générer le cadre institutionnel approprié est intervenu environ une décennie après l'entrée en vigueur de la Convention Cadre des Nations Unies sur les Changements Climatiques en 1994.

${ }^{839}$ Loi n²018-18 portant règlementation sur les changements climatiques en République du Bénin.

${ }^{840}$ Décrets et arrêtés.
} 
des institutions de protection de climat. C'est dans ces conditions que le Comité National sur les Changements Climatiques (CNCC) avait connu son existence ${ }^{841}$. Le Comité National sur les Changements Climatiques (CNCC) est un cadre institutionnel «pluridisciplinaire, multisectoriel et interinstitutionnel, chargé du suivi et de l'appui à la mise en œuvre de la Convention Cadre des Nations Unies sur les Changements Climatiques et de l'étude de toutes questions scientifiques technologiques et autres relatives à l'évolution du climat ${ }^{842}$. Il est composé de «représentants de départements ministériels, de représentant du secteur privé désigné par la Chambre de Commerce et d'Industrie du Bénin (CCIB) et de représentant du réseau des Organisations Non Gouvernementales au niveau départemental ${ }^{843}$. Il dispose d'une Assemblée générale, d'un Secrétariat permanent et des équipes techniques, comme organes pour son fonctionnement ${ }^{844}$.

Le Comité National sur les Changements Climatiques «s'occupe de l'étude des questions scientifiques, technologiques et autres relatives à l'évolution du climat ${ }^{845}$. Ses attributions sont entre autres, « la mise en exécution effective des décisions de la Conférence des Parties $»^{846}$. Enfin, le comité « participe à la coordination et à la définition de politiques et stratégies en vue de leur prise en compte par les secteurs public et privé dans différents domaines du climat ${ }^{847}$. «Les études et recherches sur les inventaires des gaz à effet de serre, la vulnérabilité et l'adaptation aux changements climatiques, ainsi que sur les options d'atténuation sont coordonnées par ledit Comité, qui organise des conférences, séminaires, ateliers, colloques et tables-rondes sur les changements climatiques $\gg{ }^{848}$. Le Comité National sur les Changements Climatiques dispose en son sein d'équipes techniques chargée de «l'élaboration, du suivi et de la mise en oeuvre des Communications nationales » ${ }^{849}$. Elle

\footnotetext{
${ }^{841}$ Ce Comité avait été créé par décret $n^{\circ} 2003-142$ du 30 avril 2003 portant attribution organisation et fonctionnement du Comité National sur les Changements Climatiques.

${ }^{842}$ Article 2 du décret $n^{\circ} 2003-142$ du 30 avril 2003 portant création, attributions et fonctionnement du Comité National de Lutte sur les Changements Climatiques.

${ }^{843}$ L'article 77 de la loi n²018-18 portant règlementation sur les changements climatiques au Bénin et l'article 4 du décret $n^{\circ} 2003-142$ du 30 avril 2003 portant attribution organisation et fonctionnement du Comité National sur les Changements Climatiques définissent la composition du Comité..

${ }^{844}$ Article 5 du décret $n^{\circ} 2003-142$ du 30 avril 2003 portant création, attributions et fonctionnement du Comité National de Lutte sur les Changements Climatiques.

${ }^{845}$ Article 2 du décret n ${ }^{\circ} 2003-142$ du 30 avril 2003 portant attribution organisation et fonctionnement du Comité National sur les Changements Climatiques.

${ }^{846}$ Article 3 du décret 2003-142 du 30 avril 2003 portant création, attributions et fonctionnement du comité national sur les changements climatiques.

${ }^{847}$ Article 3 du décret 2003-142 du 30 avril 2003 portant création, attributions et fonctionnement du comité national sur les changements climatiques.

${ }^{848}$ Id.

${ }^{849}$ Article 9 du décret $n^{\circ} 2003-142$ du 30 avril 2003 portant création, attributions et fonctionnement du Comité National de Lutte sur les Changements Climatiques.
} 
s'occupe également des «études, de l'élaboration, du suivi et de la mise en œuvre de Programmes d'Actions Nationaux aux fins d'Adaptation (PANA) et autres stratégies dans le domaine des changements climatiques ${ }^{850}$ et puis « du mécanisme pour un Développement Propre et autres mécanismes à mettre en place ${ }^{851}$. Par ailleurs, le CNCC intervient dans les activités d'information et de sensibilisation sur les changements climatiques ${ }^{852}$ et a pour responsabilité «le suivi, l'appui et l'évaluation de la mise en œuvre de la Convention Cadre des Nations Unies sur les Changements Climatiques et ses instruments juridiques subséquents ${ }^{853}$. Le Comité National sur les Changements Climatiques «appuie à la préparation de la participation du Bénin aux sessions des organes de la Convention et à la mise en exécution, effective des décisions de la Conférence des Parties à la Convention Cadre des Nations Unies sur les Changements Climatiques ${ }^{854}$. Il intervient dans « le suivi et le contrôle $\mathrm{du}$ processus d'élaboration des communications nationales du Bénin sur les changements climatiques, requises en application des dispositions de l'article 12.5 de la Convention Cadre sur les Changements Climatiques ${ }^{855}$, de la «mise en œuvre de la stratégie nationale sur les changements climatiques » ${ }^{856}$ et de la « coordination des études et recherches sur les inventaires des gaz à effet de serre, la vulnérabilité et l'adaptation aux changements climatiques, ainsi que sur les options d'atténuation ${ }^{857}$. Il «participe à l'évaluation des besoins technologiques nécessaires à la mise en œuvre de la Convention et en matière de renforcement des capacités ${ }^{858}$ et à « l'appui à la préparation et l'organisation de conférences, séminaires, ateliers, colloques, et tables-rondes sur des sujets relatifs aux changements climatiques ${ }^{859}$, de même que «l'émission d'avis sur les questions juridiques, scientifiques technologiques, méthodologiques et autres relatives à l'évaluation du climat ${ }^{860}$. Il « réalise l'étude des indicateurs nécessaires à la prévision des changements climatiques $»^{861}$. Le Comité National

\footnotetext{
${ }^{850} \mathrm{Id}$.

851 Ibid.

${ }^{852}$ Article 3 du décret $n^{\circ} 2003-142$ du 30 avril 2003 portant création, attributions et fonctionnement du Comité National de Lutte sur les Changements Climatiques.

853 Id.

${ }^{854}$ Article 8 du décret 2003-142 du 30 avril 2003 portant création, attributions et fonctionnement du comité national sur les changements climatiques.

${ }^{855}$ Article 3.5 du décret $n^{\circ} 2003-142$ du 30 avril 2003 portant création, attributions et fonctionnement du Comité National de Lutte contre les Changement Climatiques en République du Bénin.

${ }^{856}$ Article 3.6 du décret $n^{\circ} 2003-142$ du 30 avril 2003 portant création, attributions et fonctionnement du Comité National de Lutte contre les Changement Climatiques en République du Bénin. ${ }^{857}$ Id.

${ }^{858}$ Article 3.8 du décret $n^{\circ} 2003-142$ du 30 avril 2003 portant création, attributions et fonctionnement du Comité National sur les Changement Climatiques en République du Bénin.

${ }^{859}$ Article 3.9 id.

${ }^{860}$ Article 3.10 ibid.

${ }^{861}$ Article 3.11 ibid.
} 
sur les Changements Climatiques, « appuie à la conception et à la formulation de projets ou programmes d'actions à soumettre au Fonds pour 1'Environnement Mondial (FEM) et à d'autres mécanismes de financement, dans le cadre de la mise en œuvre de la Convention Cadre des Nations Unies sur les Changements Climatiques et de ses instruments subséquents ${ }^{862}$.

Le CNCC « représente la plateforme de toutes les Parties prenantes à la lutte contre les changements climatiques au Bénin afin de répondre efficacement aux préoccupations nationales liées aux changements climatiques et d'assurer la prise en compte des changements climatiques dans les différents secteurs de la vie socio-économique du Bénin ${ }^{863}$.

Après la COP $21^{864}$, le Bénin a engagé le processus de renforcement du cadre institutionnel destiné à la lutte contre les changements climatiques. La stratégie béninoise pour internaliser les instruments internationaux de lutte contre les changements climatiques ${ }^{865} \mathrm{a}$ consisté à se conformer aux prescriptions desdits instruments par l'adoption de mesures législatives et règlementaires afin de créer un cadre juridique et institutionnel adapté à la protection du climat. Ce processus a débuté par l'élaboration de la loi n²018-18 portant règlementation des changements climatiques en République du Bénin. Son adoption a permis de renforcer le cadre institutionnel dans le domaine de la lutte contre les changements climatiques. Une disposition de cette $10 i^{866}$ est intervenue pour entériner l'existence du Comité National sur les Changements Climatiques ${ }^{867}$, qui est demeuré un organe de pilotage interministériel de la politique des changements climatiques.

C'est la même loi qui a également créé un autre organe à vocation essentiellement climatique mais, qui s'occupe de la dimension financière de la gestion des changements climatiques, placé sous la tutelle du ministère du Plan.

\section{B- Un organe sous tutelle du ministère du Plan}

L'application des dispositions combinées d'une part, de la Déclaration de Stockholm de 1972 qui recommande le dégagement des ressources financières pour contribuer à la protection

\footnotetext{
${ }^{862}$ Article 2 ibid.

${ }^{863}$ Rapport du Système d'information sur les changements climatiques au Bénin, Première session ordinaire du Comité National sur les changements climatiques au Bénin tenue le 15 juin 2017. Disponible sur le site internet : https://www.changementsclimatiques.bj/premiere-session-ordinaire-du-comite-national.Consulté le 15/01/2022. ${ }^{864} \mathrm{La} 21^{\mathrm{e}}$ Conférence des Parties à la convention cadre sur les changements climatiques tenue à Paris en 2015.

${ }^{865}$ La Convention Cadre des Nations Unies sur les Changements Climatiques et ses accords subséquents.

${ }^{866} \mathrm{~L}$ 'article 77 de la loi n²018-18 du 18 juin 2018 portant changements climatiques au Bénin a entériné la création du Comité National sur les Changements Climatiques.

${ }^{867}$ Le Comité National sur les Changements Climatiques avait été créé en 2003 par décret du Président de la République.
} 
de l'environnement en vue d'amoindrir les charges induites par la planification du développement dans les pays en voie de développement ${ }^{868}$ et d'autre part, la mise en œuvre du principe de la responsabilité commune mais différenciée contenu dans la Convention Cadre des Nations Unies sur les Changements Climatiques, qui oblige les pays développés pollueurs à mettre à la disposition des Etats en voie de développement des ressources technologiques et financières à travers le mécanisme financier ${ }^{869}$, pour permettre à ces derniers de s'acquitter de leurs engagements d'atténuation des émission de gaz à effet de serre ${ }^{870}$ implique des conséquences en terme de gestion administratives de ces ressources. Dès lors que les sources de financement climatique sont établies, il s'est avéré la problématique de la planification, de la mobilisation et de la gestion efficace desdites ressources au niveau de chaque Etat Partie.

Au plan interne, comment le Bénin peut-il parvenir à gérer efficacement la planification des ressources financières dans le cadre de la lutte contre les changements climatiques?

Bien entendu que chaque Etat Partie doit s'inscrire dans une démarche d'élaboration du cadre institutionnel de gestion des ressources financières destinées à la lutte contre les changements climatiques, mais en tenant compte de ses réalités internes. Au Bénin, la rareté d'institutions intéressées par les questions en lien avec le réchauffement climatique dans le domaine de la programmation budgétaire et l'inexistence d'un instrument national de programmation et de suivi des dépenses en faveur des changements climatiques font que, « les dépenses climatiques sont programmées et exécutées dans une relative zizanie se soustrayant ainsi à toute possibilité de suivi ${ }^{871}$.

Pour pallier cette insuffisance, plusieurs recommandations sont intervenues dans le sens de l'amélioration de la gouvernance par la budgétisation des ressources des changements climatiques et par le renforcement des capacités institutionnelles. Par conséquent, il a été élaboré et mis en vigueur le décret n²014-359 qui a donné vie à la Commission de Modélisation Economique des Impacts et de l'Intégration des Changements Climatiques dans le Budget Général de l'Etat (CMEIGB). Cette Commission est placée sous la tutelle du ministère en charge du Plan ${ }^{872}$.

\footnotetext{
${ }^{868}$ Principe 12 de la Déclaration de Stockholm de 1972.

${ }^{869}$ Article 11 de la Convention Cadre des Nations Unies sur les Changements Climatiques.

${ }^{870}$ Article 4-1 id.

${ }^{871}$ Elaboration d'une revue des dépenses publiques et institutionnelles sur les changements climatiques au Bénin Actualités, Changements climatiques, Documentation, Fonds Vert sur le Climat, Publications et rapports https://www.changementsclimatiques.bj/elaboration-dune-revue-des-depenses-publiques-et-institutionnelles-surles-changements-climatiques-au-benin/.Consulté 20/06/2019.

${ }^{872}$ Article 79 de la loi $\mathrm{n}^{\circ} 2018$-18 portant sur la règlementation des changements climatiques en République du Bénin.
} 
Elle a pour «mission de développer des outils et méthodes d'évaluation, de modélisation et de prévision économique des impacts du climat en vue d'une optimisation des stratégies d'adaptation et du développement sobre en carbone et résilient au climat ${ }^{873}$. Ainsi, elle se charge de «l'évaluation de la vulnérabilité de l'économie nationale face aux changements climatiques, afin d'aider à la prise de décisions au niveau de l'Etat ${ }^{874}$. Elle « développe et analyse des arguments économiques en faveur de la résilience climatique» ${ }^{875}$ et développe également un « modèle économique national intégrant des modèles d'évaluation des impacts par secteur de développement ${ }^{876}$. Elle contribue à « l'identification des opportunités nouvelles et innovantes d'investissements prenant en compte les impacts des changements climatiques $\gg 877$.

La Commission de Modélisation Economique des Impacts et de l'Intégration des Changements Climatiques dans le Budget Général de l'Etat est composée des représentants des Ministères, du représentant de la Banque Centrale des Etats de l'Afrique de l'Ouest (BCEAO) et du représentant du Programme des Nations Unies pour le Développement (PNUD) et comprend un Conseil d'orientation, un Comité technique et un Secrétariat permanent ${ }^{878}$. La Commission de modélisation est une institution qui « (...) vient en appui scientifique au Comité National sur les Changements Climatiques, à la Plate-forme Nationale de Réduction des Risques de Catastrophe et d'Adaptation aux Changements Climatiques et aux autres instances nationales intervenant dans le domaine des changements climatiques $\gg{ }^{879}$. Elle a en charge la coordination de «tous les aspects économiques et financiers ${ }^{880}$ concernant la gestion des changements climatiques au Bénin.

\footnotetext{
${ }^{873}$ Article 2 alinéa 1 de la loi n²018-18 portant sur la règlementation des changements climatiques en République du Bénin.

${ }^{874}$ Article 2 troisième tiret du décret $\mathrm{n}^{\circ}$ 2014-359 du 16 juin 2014 portant création de la Commission de Modélisation Economique des Impacts et de 1'Intégration des Changements Climatiques dans le Budget Général de l'Etat.

${ }^{875}$ Article 2 deuxième tiret du décret $\mathrm{n}^{\circ}$ 2014-359 du 16 juin 2014 portant création de la Commission de Modélisation Economique des Impacts et de l'Intégration des Changements Climatiques dans le Budget Général de l'Etat.

${ }^{876} \mathrm{Id}$.

${ }^{877}$ Ibid.

${ }^{878}$ Article 3 de la loi n ${ }^{\circ} 2018$ - 18 portant sur la règlementation des changements climatiques en République du Bénin et le décret $\mathrm{n}^{\circ}$ 2014-359 du 16 juin 2014 portant création de la Commission de Modélisation Economique des Impacts et de l'Intégration des Changements Climatiques dans le Budget Général de l'Etat qui détermine la composition de l'organe.

${ }^{879}$ Article 10 du décret $\mathrm{n}^{\circ}$ 2014-359 du 16 juin 2014 portant création de la Commission de Modélisation Economique des Impacts et de l'Intégration des Changements Climatiques dans le Budget Général de l'Etat.

${ }^{880}$ Article $1^{\text {er }}$ de la loi no $2018-18$ portant sur la règlementation des changements climatiques en République du Bénin.
} 
L'intervention de la loi $\mathrm{n}^{\circ} 2018$-18 portant règlementation des changements climatiques en République du Bénin a permis d'entériner la création de la Commission de Modélisation Economique des Impacts du Climat et d'Intégration des Changements Climatiques au Budget Général de l'Etat en lui concédant les mêmes attributions ${ }^{881}$. Mais elle est conçue comme un organe d'aide à décision, placée sous la tutelle du ministère du Plan ${ }^{882}$ et non plus une institution présidée par le Ministre en charge du Plan.

L'adoption de la loi n²018-18 portant règlementation des changements climatiques en République du Bénin a permis de doter le Bénin de quelques institutions qui sont en adéquation avec l'objectif de lutte contre les changements climatiques.

\section{SECTION II: UN ENCADREMENT INSTITUTIONNEL ADEQUAT}

Le foisonnement d'institutions de protection de l'environnement suscite pour la plupart du temps la question de recherche suivante: «Quelles institutions pour une mise en ouvre effective du droit de l'environnement? ${ }^{883}$. Dans le contexte de notre étude, le questionnement devient : quelles sont les institutions nécessaires à la mise en œuvre effective du droit applicable aux changements climatiques au Bénin?

L'abondance institutionnelle qui a caractérisé le Droit International de l'Environnement a pour conséquence, un effet de contamination des Etats Parties aux diverses conventions de protection de l'environnement. Puisque ces derniers se mirent à créer un nombre important d'institutions, dont le seul but est de contribuer à la protection de l'environnement. Cependant, depuis l'adoption de la Convention Cadre des Nations Unies sur les Changements Climatiques, l'accent est mis sur la problématique de l'encadrement des changements climatiques dans la création des institutions.

En effet, le cadre institutionnel en cours d'élaboration au Bénin est en adéquation avec les objectifs de lutte contre les changements climatiques, car il intègre désormais les aspects les plus pertinents en matière de lutte contre les changements climatiques. Ce cadre institutionnel est plus proche des prescriptions du droit international. Il a pour finalité de contribuer à l'atteinte

\footnotetext{
${ }^{881}$ Article 79 de la loi n²018-18 portant sur la règlementation sur les changements climatiques en République du Bénin.

${ }^{882}$ Id.

${ }^{883}$ Maurice KAMTO, LA MISE EN EUVRE DU DROIT DE L'ENVIRONNEMENT : FORCES ET

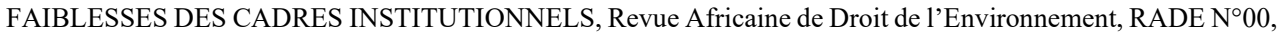
2019. Disponible sur le site internet: https:/www.iucn.org/sites/dev/files/content/documents/rade_ndpdf. Consulté le 05/06/2021.
} 
des objectifs de réduction du gaz à effet de serre et d'adaptation aux effets des changements climatiques.

Ce cadre institutionnel en cours de formation incarne un système de financement orienté vers les objectifs de lutte contre les changements climatiques (Paragraphe 1). Mais également, il intègre les préoccupations des citoyens à la base par, l'environnementalisation de la politique de l'administration territoriale (Paragraphe 2).

\section{Paragraphe 1: Un système de financement orienté}

Ce n'est pas trop prétentieux d'affirmer que la question du financement des changements climatiques relève du «luxe» pour les pays en voie de développement, qui subissent les conséquences du phénomène. La question du financement est un sujet considéré à juste titre dans le cadre de la coopération internationale de la lutte contre les changements climatiques. Compte tenu de la gravité du phénomène qui impose des réductions drastiques des émissions de gaz à effet de serre (GES) sur une dimension planétaire, il est question d'adopter des mesures adéquates pour éradiquer le fléau. Ces mesures consistent entre autres, à la mise en place d'un système de financement international pour assurer le déploiement des technologies d'atténuation du changement climatique.

Le mécanisme financier institué par la Convention Cadre des Nations Unies sur les Changements Climatiques constitue un début de solution inventée par la Communauté internationale, pour appuyer les efforts accomplis par les pays en voie de développement pour ce qui concerne leur engagement pour la réduction des émissions de gaz à effet de serre. Mais, le problème qui se pose, concerne les modalités de fonctionnement dudit mécanisme, qui à terme doit permettre aux Etats en développement de bénéficier de ses prestations. En réponse à cette préoccupation, chaque Etat Partie a développé son arsenal institutionnel pour attirer et gérer les financements climatiques.

Le Bénin aspirant au bénéfice du financement international a organisé à l'interne, un système de financement orienté vers les objectifs de lutte contre les changements climatiques par la création du Fonds National pour l'Environnement et le Climat (FNEC) (A). Ce fonds est alimenté en partie par des ressources provenant des canaux internationaux de financement des changements climatiques (B). 


\section{A- La création du Fonds National pour l'Environnement et le Climat}

En 2003, l'Etat béninois s'est doté d'une institution de financement des activités de protection de l'environnement dénommée « Fonds National pour l'Environnement ${ }^{884}(\mathrm{FNE}) »$. Elle avait pour attribution, le « (...) financement des programmes et projets visant la gestion rationnelle de l'environnement, l'amélioration du cadre de vie et la promotion du développement durable au Bénin» ${ }^{885}$. Cette institution avait été conçue à l'origine pour mobiliser les ressources nationales et internationales destinées au financement des projets dont l'objectif ultime était de contribuer à la protection de l'environnement au Bénin.

Cependant, pour tenir compte des engagements souscrits par le Bénin dans le cadre de la lutte contre les changements climatiques, le document de Stratégie nationale qui avait été élaboré pour contribuer à la mise en œuvre de la Convention Cadre des Nations Unies sur les Changements Climatiques avait indiqué les options prioritaires des « stratégies de réponse du Bénin aux changements climatiques, qui consistent à procéder à l'atténuation aux changements climatiques par la réduction des émissions de gaz à effet de serre et l'augmentation de l'absorption de $\mathrm{CO} 2$, ainsi qu'à l'adaptation aux impacts, par l'adoption des mesures d'ajustement pour élargir la viabilité des activités sociales et économiques dans le seul dessein de réduire leur vulnérabilité aux changements climatiques ${ }^{886}$. Ces mesures couvrent «les secteurs prioritaires suivants: l'énergie, les transports, la planification urbaine, l'industrie, l'agriculture, la foresterie, les ressources en eau et les ressources côtières ${ }^{887}$. Une panoplie d'activité prévue pour être exécutée dans le cadre de la lutte contre les changements climatiques au Bénin.

Seulement, la gestion de ces engagements nécessite la mobilisation d'importantes ressources financières. C'est pourquoi, les attributions du Fonds National pour l'Environnement (FNE) ont subi des modifications au point où l'institution qui déjà, était compétente pour intervenir dans le domaine de la protection de l'environnement est désormais qualifiée pour « financer la lutte contre les effets des changements climatiques et en plus promouvoir le

\footnotetext{
${ }^{884}$ En vertu de la loi n ${ }^{\circ} 94-009$ du 28 juillet 1994 qui régit les offices à caractère social et culturel. Cette institution a été créée par le décret n 2003 - 559 du 24 décembre 2003 portant approbation des statuts du Fonds National pour l'Environnement.

${ }^{885} \mathrm{~L}$ 'article 5 du décret $\mathrm{n}^{\circ} 2008-273$ du 19 mai 2008 portant attributions, l'organisation et le fonctionnement du Fonds, qui modifie celui de 2003 défini les attributions de l'institution financière.

${ }^{886}$ Stratégie nationale de mise en œuvre de la Convention Cadre des Nations Unies sur les Changements Climatiques, 2-4 Les stratégies de réponse aux changements climatiques, p.20.

${ }^{887}$ Stratégie Nationale de mise en œuvre au Bénin de la Convention Cadre des Nations Unies sur les Changements Climatiques, 2-5 Diagnostic et solutions, p.21.
} 
développement durable au Bénin ${ }^{888}$. Le Fonds National pour l'Environnement et le Climat (FNEC) est un instrument financier, «placé sous la tutelle du Ministère du Cadre de Vie et du Développement Durable (MCVDD) ${ }^{889}$. Cette mutation institutionnelle qui augmente les attributions de l'institution financière, l'autorise à diversifier le registre des projets en faveur de ceux concernant les changements climatiques. Avec cette réforme, le Fonds National pour l'Environnement et le Climat (FNEC) dispose désormais des marges de manœuvres nécessaires pour mobilier et gérer des ressources financières de provenance nationale ou internationale, surtout celles provenant du mécanisme financier de la Convention Cadre des Nations Unies sur les Changements Climatiques et destinées au financement des activités de lutte contre les changements climatiques sur toute l'étendue du territoire national ${ }^{890}$. Elle peut aider à faciliter à travers les transactions financières le transfert de technologies et le renforcement des capacités des acteurs intervenant dans les secteurs de protection de l'environnement et du climat.

Depuis $2003^{891}$, persuadé de ce que les changements climatiques constituaient une question de développement dont les effets négatifs n'épargnent aucun secteur vital, aucun écosystème qu'il soit marin, désertique, forestier, aquatique ou terrestre, le FNE avait adopté une méthodologie de mobilisation des ressources internes par l'application du principe « pollueur payeur ${ }^{892}$ qui consistait à faire payer des redevances dénommées «écotaxes » ${ }^{893}$ par les auteurs de pollution environnementale en guise de réparation des préjudices causés à l'environnement. A partir de 2009, cette mesure a été instituée à l'article 9 de la loi n ${ }^{\circ} 2008-09$ du 30 décembre 2008 portant loi de finance pour la gestion 2009 en République du Bénin puis intégrée à l'Article 5 premier tiret du décret n²017- 128 du 27 février 2017 constatant approbation de la création du Fonds National pour l'Environnement et le Climat (FNEC).

\footnotetext{
${ }^{888}$ Article 5 du décret n ${ }^{\circ} 2017-128$ du 17 février 2017 constatant approbation de la création du Fonds National pour l'Environnement et le Climat.

${ }^{889}$ Article 2 du décret $n^{\circ} 2017-128$ du 17 février 2017 constatant approbation de la création du Fonds National pour l'Environnement et le Climat (FNEC).

${ }^{890}$ Selon les prescriptions de l'article 5 alinéa 2 du décret n²017-128 du 17 février 2017 constatant approbation de la création du Fonds National pour l'Environnement et le Climat (FNEC).

${ }^{891}$ C'est l'année au cours de laquelle le FNE a été créé. Puis le FNE est devenu par la suite FNEC.

${ }^{892}$ L'article 16 de la loi cadre sur l'environnement en République du Bénin stipule que « nul ne doit émettre, déposer, rejeter ou permettre, l'émission, le dépôt, le dégagement, l'enfouissement ou le rejet dans l'environnement d'un contaminant au-delà de la quantité ou de la concentration prévue par les lois et les règlements».

${ }^{893}$ Les écotaxes sont des impôts sur les sources de pollution et sur l'exploitation de ressources naturelles non renouvelables.
} 
Les écotaxes sont applicables sur une liste de produits importés ayant d'effet polluant sur l'environnement notamment, le souffre de provenance de l'extérieur chargé au Port Autonome de Cotonou en transit vers la République du Niger ${ }^{894}$.

La pratique qui consiste à financer les projets climatiques à partir des ressources générées par l'écotaxe a valu au Fonds National pour l'Environnement et le Climat (FNEC), son inscription auprès du Fonds d'Adaptation relevant du système du mécanisme financier de la Convention Cadre des Nations Unies sur les Changements Climatiques.

Néanmoins, la taxe sur le souffre en transit vers le Niger qui devrait constituer la cagnotte la plus importante des ressources destinées à financer le fonctionnement du FNEC a été suspendue le $1^{\mathrm{er}}$ janvier $2010^{895}$. Cette situation ajoutée à la faible contribution de 1'Etat à approvisionner en ressources financières le FNEC, font que l'institution est confrontée à l'amenuisement de ces ressources, impactant négativement le déroulement normal de ses activités de financement des projets de lutte contre les changements climatiques.

Par ailleurs, pour tenir compte du «rôle fondamental que les femmes jouent par l'adoption de mode écologiquement rationnel de consommation de production et de gestion des ressources naturelles durables $»^{896}$, le FNEC s'est doté depuis décembre 2016, d'une politique de financement qui tient compte de l'aspect genre ${ }^{897}$. Cette politique est conforme aux objectifs du Fonds d'Adaptation (FA) et du Fonds Vert pour le Climat (FVC) ${ }^{898}$ dont il est partenaire et aussi, elle cadre avec l'esprit de certains accords qui soulignent que les «réponses aux changements climatiques doivent (...) pleinement prendre en compte les conséquences pour les groupes vulnérables, en particulier les femmes et les enfants ${ }^{899}$. La mise en œuvre de la

\footnotetext{
${ }^{894}$ Selon l'article 9 de la loi $n^{\circ} 2008-09$ du 30 décembre 2008 portant loi des finances pour la gestion 2009, les taux applicables aux produits suivants : Piles et accumulateurs $(0,5 \%$ de la valeur c.a.f. ou du coût de production locale); Récipients et emballages jetables, autres que plastiques et contenant divers produits $(0,5 \%$ de la valeur c.a.f.) ; Récipients et emballages jetables vides, autres que plastiques $(0,25 \%$ de la valeur c.a.f.) ; Soufre en régime de transit (25 FCFA par kg) et Tabac et cigarettes ( $5 \%$ de la valeur c.a.f. ou du coût de production locale). ${ }^{895}$ Voir article 8 de l'ordonnance $n^{\circ} 2010-01 \mathrm{du} 1^{\text {er }}$ janvier 2010 portant loi des finances pour la gestion 2010.

${ }^{896}$ Ministère du Cadre de Vie et du Développement Durable (MCVDD), Fonds National pour l'Environnement et le Climat (FNEC), Politique Genre du Fonds National pour l'Environnement et le Climat (FNEC), p.7.

${ }^{897}$ Voir les critères de recevabilité et de présélection des projets (au paragraphe 5.3) dans le document intitulé «GUIDE DE FORMULATION, D'ELIGIBILITE ET DE SELECTION DES PROJETS SOUMIS AU FINANCEMENT DU FONDS NATIONAL POUR L'ENVIRONNEMENT ET LE CLIMAT (FNEC) », janvier 2020, p.15. Disponible sur le site internet: https://www.fnec-benin.org/docs/recueil-guide-eligibility-fnec.pdf. Consulté le 20/12/2021.

${ }^{898}$ Voir les critères de recevabilité et de présélection des projets (au paragraphe 5.3) dans le document intitulé «GUIDE DE FORMULATION, D'ELIGIBILITE ET DE SELECTION DES PROJETS SOUMIS AU FINANCEMENT DU FONDS NATIONAL POUR L'ENVIRONNEMENT ET LE CLIMAT (FNEC) », janvier 2020, p.4. Disponible sur le site internet: https://www.fnec-benin.org/docs/recueil-guide-eligibility-fnec.pdf. Consulté le 20/12/2021.

${ }^{899}$ Notamment, le paragraphe 7 de la Décision 1/CP.16 des Accords de Cancún lors de la COP 16. Disponible sur le site internet: https://unfccc.int/resource/docs/2010/cop16. Consulté le 15/01/2022.
} 
politique basée sur le genre aura pour conséquence de faciliter l'accès de la femme aux ressources financières dans le but d'assurer son autonomisation dans le contexte des changements climatiques.

Malheureusement, l'initiative qui a germé depuis 2003 avec la création du Fonds National pour l'Environnement (FNE) pour donner progressivement corps en 2009 au Fonds National pour l'Environnement et le Climat (FNEC) n'a pas progressé, car la réforme initiée par l'adoption de la loi $\mathrm{n}^{\circ} 2018-18$ portant règlementation sur les changements climatiques en république du Bénin est restée muette sur les conditions de création et de fonctionnement de l'institution financière. Les dispositions contenues dans le TITRE VII intitulé « Du cadre institutionnel » et le TITRE VIII intitulé « Des ressources financières » de ladite loi n'ont pas intégré le Fonds National pour l'Environnement et le Climat (FNEC).

Nonobstant ces faiblesses, le FNEC dispose désormais de la capacité juridique qui lui ouvre la possibilité d'orienter ses actions vers la recherche des financements internationaux ${ }^{900}$.

\section{B- Les financements internationaux}

Les bases juridiques du financement international des changements climatiques ont été élaborées dans la Convention Cadre des Nations Unies sur les Changements Climatiques et matérialisées par l'institution du mécanisme financier ${ }^{901}$. A l'issu de la Conférence de Copenhague sur les changements climatiques tenue en $2009^{902}$, les promesses d'alimentation en ressources financières au profit du mécanisme financier ont été convenues ${ }^{903}$. De toutes les manières, le processus de financement des changements climatiques va connaitre une évolution et donnera lieu a un développement institutionnel pour permettre de mettre à disposition des Etats en développement, des ressources financières qui leurs seront allouées.

\footnotetext{
${ }^{900}$ Voir les dispositions de l'article 5 deuxième tiret du décret no2017- 128 du 27 février 2017 constatant approbation de la création du Fonds National pour l'Environnement et le Climat (FNEC).

${ }^{901}$ Article 11 de la Convention Cadre des Nations Unies sur les Changements Climatiques.

${ }^{902}$ Selon les termes de 1'Accord de Copenhague point 8 du préambule de la Décision 2/CP.15, les Parties sont convenus du présent Accord qui prend effet immédiatement, que "dans l'optique de mesures concrètes d'atténuation et d'une mise en œuvre transparente, les pays développés adhèrent à l'objectif consistant à mobiliser ensemble cent (100) milliards de dollars par an d'ici à 2020 pour répondre aux besoins des pays en développement».

${ }^{903}$ Et les pays développés se sont engagés pour mobiliser cent (100) milliards de dollars par an, jusqu'en 2020 pour soutenir les initiatives d'atténuation des changements climatiques dans les pays vulnérables à l'occasion de la Conférence des Parties agissant comme réunion des Parties à l'Accord de Paris, troisième session, dans les premières communications biennales soumises en application du paragraphe 5 de l'article 9 de l'Accord de Paris, $5^{\mathrm{e}}$ paragraphe de l'introduction. Disponible sur le site internet: https://unfccc.int/sites/default/files.Consulté le $15 / 01 / 2022$.
} 
Il convient alors de s'interroger sur les conditions pouvant faciliter l'accès aux financements climatiques internationaux?

Face aux difficultés de financement des charges induites par la gestion des changements climatiques, la seule alternative qui s'offre pour le Bénin est la possibilité du recours aux financements extérieurs, proposé dans la Convention Cadre des Nations Unies sur les Changements Climatiques. Selon les termes de ladite Convention, il est stipulé que « les pays en développement pourront obtenir des ressources financières par voie bilatérale, régionale ou multilatérale aux fins de l'application de la Convention ${ }^{904}$. Ces ressources sont convergées vers les pays sous « forme de dons ou à des conditions de faveur ${ }^{905}$, notamment par le transfert de technologie $»^{906}$.

Dès lors que plusieurs circuits de financement des climatiques sont proposés par les accords internationaux, il appartient à chaque pays de faire l'option du système financier le plus accessible et capable d'assouvir ses besoins en matière de financement des changements climatiques.

Pour financer les changements climatiques au Bénin, le Législateur qui a imposé des obligations à « l'Etat qui mobilise les ressources financières nécessaires pour l'élaboration, la mise en œuvre et l'évaluation des politiques et stratégies (...) » ${ }^{907}$ a également projeté que $«(\ldots)$ ces ressources proviennent du budget national et entre autres, du Fonds pour l'Environnement Mondial, du Fonds d'Adaptation aux changements climatiques, des Fonds d'investissements climatiques, du Fonds vert pour le climat. Elles peuvent également provenir des partenaires au développement dans le cadre de la mise en œuvre de la coopération internationale et aussi des organisations non gouvernementales et des privés ${ }^{908}$.

Face à cette alternative proposée par le Législateur, le Bénin à défaut de se contenter des maigres ressources internes, n'a de choix que de faire recours au mécanisme financier international établi dans le cadre de la convention, pour rechercher des ressources financières complémentaires aux fins de l'ascension des charges découlant de la gestion des changements climatiques. En application de ces mesures, le Bénin a fait l'option de bénéfice de financement des changements climatiques par les canaux multilatéraux » par la mise en œuvre des relations bilatérales avec d'autres pays. C'est par exemple le cas de la coopération Française avec

\footnotetext{
904 Article 11 paragraphe 5 de la Convention Cadre des Nations Unies sur les Changements Climatiques.

${ }^{905}$ Article 11 de la Convention Cadre des Nations Unies sur les Changements Climatiques.

${ }_{906}$ Article 5 paragraphe 1 de la Convention Cadre des Nations Unies sur les Changements Climatiques.

${ }^{907}$ Article 82 alinéa 1 de la loi ${ }^{\circ} 2018-18$ portant règlementation sur les changements climatiques en République.

908 Article 82 alinéa 2 et 3 de la loi n²018-18 portant règlementation sur les changements climatiques en République du Bénin.
} 
l'Agence Française pour le Développement (AFD). L'AFD est conçue comme une institution de coopération entre la France et environs cent quinze (115) pays du monde pour financer divers projets, qui entre autres concourent à l'éradication des changements climatiques dans le but de la réalisation des Objectifs de Développement Durable ${ }^{909}$. Elle participe au financement des projets d'adaptation par la réalisation d'infrastructures dans les villes dans le cadre de la lutte contre les changements climatiques au Bénin ${ }^{910}$.

Par ailleurs, l'inscription du Fonds National pour l'Environnement et le Climat (FNEC) auprès du Fonds Vert pour le Climat se situe dans le cadre de la mise en œuvre de la coopération bilatérale pour faciliter son accès aux prestations de l'institution financière. Bien entendu, le FVC est un produit né des négociations du Plan d'Action de Bali ${ }^{911}$. Il a été créé en 2010 à Cancun ${ }^{912}$ « comme entité chargée d'assurer le fonctionnement du mécanisme financier de la Convention au titre de l'article $11(\ldots) »{ }^{913}$ pour « soutenir des projets, des programmes, des politiques et d'autres activités dans les pays en développement parties au moyen de guichets de financement thématique» ${ }^{914}$. Il a été rendu fonctionnel en $2011^{915}$.

Profitant des opportunités de financement du changement climatique offertes dans le cadre du Protocole de Kyoto, le Bénin a également entrepris de collaborer avec le Fonds pour l'Adaptation. Le «Fonds pour l'adaptation créé en $2001^{916}$ a pour mission de financer des projets et programmes concrets d'adaptation visant à atténuer les effets néfastes du changement climatique dans les pays en développement, qui sont Parties au Protocole de Kyoto. Il se différencie des autres fonds internationaux intervenant dans les pays en développement par sa spécialité dans le financement de l'adaptation au changement climatique. Sa capacité de mobilisation de ressources financières repose sur la possibilité qui lui est faite de procéder au

\footnotetext{
${ }^{909}$ Information disponible sur le site internet : https://www.afd.fr/fr. Consultée le 05/06/2021.

${ }^{910}$ L'Agence française de développement (AFD) a accordé un montant de 38045506000 FCFA dont 5247656 000 FCFA au titre de subvention au Gouvernement béninois pour le financement du programme d'adaptation des villes au changement climatique. Disponible sur le site internet: https://intellivoire.net/lafd-finance-leprogramme-dadaptation-des-villes-beninoises-au-changement-que. Consulté le 15/01/2022.

${ }^{911}$ Rapport de la treizième $\left(13^{\mathrm{e}}\right)$ session de la Conférence des Parties tenue à Bali du 03 au 15 décembre 2007 Décision 1/CP.13 Plan d'action de Bali. Disponible sur le site internet : https://unfccc.int/resource/doc.Consulté le $15 / 01 / 2022$.

${ }^{912}$ Par la décision 1CP 16 paragraphe 102 Convention Cadre des Nations Unies sur les Changements Climatiques Conférence des Parties sur sa seizième session, tenue à Cancún du 29 novembre au 10 décembre 2010.

${ }^{913}$ Point 102 de la décision 1/CP.16 adoptée par la Conférence des Parties à la Convention Cadre sur les Changements Climatiques, Rapport de la Conférence des Parties sur sa seizième $\left(16^{\mathrm{e}}\right)$ session, tenue à Cancún du 29 novembre au 10 décembre 2010, Mesures prévues par la Conférence des Parties. Disponible sur le site internet : https://unfccc.int/resource/docs/2010/cop16.Consulté le 15/01/2022.

914 Id.

915 Par la Cop de Durban par décision 3/CP.17 paragraphe 3 relative à la mise en place du Fonds vert pour leclimat. Disponible sur le site internet : https://unfccc.int.Consulté le 15/01/2022.

${ }^{916} \mathrm{~A}$ la septième session de la Conférence des Parties à la Convention Cadre des Nations Unies sur les Changements Climatiques (CCNUCC), qui s'est déroulée à Marrakech au Maroc.
} 
prélèvement de redevance dans le cadre du Mécanisme pour un Développement Propre ${ }^{917}$ (MDP) établi par le Protocole de Kyoto. Il est largement représenté dans les pays en développement et facilite l'accès direct au financement. Ces caractéristiques permettent au Fonds pour l'adaptation d'aider efficacement les populations du monde entier à relever les défis du changement climatique en recourant à des systèmes de financement novateurs en mobilisant les organisations de la société civile à tous les niveaux et en faisant participer les pays les plus touchés par le changement climatique. Autrement dit, les pays en développement ont la possibilité à travers leurs propres institutions nationales de mettre en œuvre, d'élaborer et d'exécuter directement les projets qui correspondent le mieux à leur stratégie nationale en matière de lutte contre les changements climatiques et peuvent en revendiquer les résultats. Les effets du changement climatique varient fortement selon les régions et les pays. En conséquence, le Fonds finance des projets et des programmes concernant les ressources en eau, l'agriculture et la sécurité alimentaire, la gestion des zones côtières, la réduction des risques liés aux catastrophes, l'aménagement urbain et rural.

Depuis Juin 2011, le Fonds National pour l'Environnement et le Climat (FNEC) a été admis auprès du Fonds pour l'adaptation comme institution nationale de mise en œuvre pour le Bénin. Dans ce cadre, le Fonds National pour l'Environnement et le Climat (FNEC) accompagne l'encadrement des projets et programmes destinés à bénéficier des ressources de ce Fonds ${ }^{918}$.

\section{Paragraphe 2: L'environnementalisation de l'administration territoriale}

$\mathrm{Au}$ Bénin, les réformes entreprises par les autorités entremêlent parfois la politique de décentralisation du pouvoir de l'Etat et la déconcentration de l'administration et les considérations de protection de l'environnement et de lutte contre les changements climatiques. Il s'ensuit que les politiques territoriales du Bénin sont assujetties au phénomène d'environnementalisation car, les réformes intègrent désormais les considérations environnementales et confèrent de rôle aux acteurs de l'administration territoriale notamment, à l'autorité déconcentrée (A) et aux collectivités locales qui sont désormais impliquées dans la chaine de protection de l'environnement $(\mathbf{B})$.

\footnotetext{
${ }^{917}$ Le Mécanisme de Développement Propre (MDP) est un mécanisme économique de la finance du carbone qui fut élaboré dans le cadre du Protocole de Kyoto. Article 12 dudit Protocole.

918 Fonds pour l'Adaptation, Fond National pour l'Environnement et le Climat, Informations Générales sur le Fonds pour l'Adaptation pour les bénéficiaires disponible sur le site internet : http://fnec-benin.org/wp content/uploads/2017/08/NOTE-dinformations-sur-Fonds-ADPTA.pdf.Consulté le 05/06/2021.
} 


\section{A- Le rôle de l'autorité déconcentrée}

En application de la politique de déconcentration de l'administration publique qui a suivi la réforme constitutionnelle intervenue en $1990^{919}$, certaines mesures législatives ont été élaborées pour permettre de traduire en réalité, l'idée de décongestionnement du pouvoir de l'Etat par la dissémination de l'administration dans toutes les contrées du pays ${ }^{920}$. C'est une réponse qui correspond aux difficultés révélées par la montée de la démographie qui a permis de mettre à nues, les insuffisances de la forme d'organisation étatique qu'est, «l'Etat unitaire $»^{921}$. Une forme d'organisation dans laquelle les structures administratives devant conduire l'action publique sur toute l'étendue du territoire peinent à assurer une couverture intégrale de tous les besoins des citoyens. Ainsi, la mise en œuvre de la politique de déconcentration de l'administration a induit la création de douze (12) départements ${ }^{922}$, administrés chacun par un Préfet ${ }^{923}$ qui représente l'autorité déconcentrée.

\section{Quelles sont les attributions de protection de l'environnement du Préfet?}

Les nombreux textes législatifs et réglementaires élaborés pour encadrer n'importe quel domaine de la vie publique, intègrent accessoirement les données de protection de l'environnement et celles concernant la lutte contre les changements climatiques. C'est dans cette optique que le Préfet identifié comme Chef de Département ${ }^{924}$ dans le cadre de la politique de déconcentration du pouvoir de l'Etat s'est révélé comme un acteur non moins important intervenant dans la chaine de protection de l'environnement et s'est vu confié beaucoup de rôle dans le cadre de la lutte contre les changements climatiques. En effet, en vertu des textes de loi qui organisent l'administration territoriale au Bénin, «le préfet dépositaire de l'autorité de l'Etat dans le département ${ }^{925}$ et chef du Conseil départemental de concertation et de

\footnotetext{
${ }^{919}$ Adoption de la loi constitutionnelle le 11 décembre 1990 après la tenue de la conférence des forces vives de la nation au Bénin.

${ }^{920} \mathrm{Il}$ s'agit de l'ensemble des mesures contenues dans la loi n $97-028$ du 15 janvier 1999 portant organisation territoriale de la République du Bénin.

${ }^{921} \mathrm{C}$ 'est un Etat comportant un centre unique d'impulsion politique auquel la population est conformément soumise sur tout le territoire, les circonscriptions territoriales ne jouissant d'aucune autonomie politique, selon la définition contenue dans Raymond GUILLIEN (Raymond) et VINCENT (Jean), Lexique des termes juridiques, $5^{\mathrm{e}}$ éd., Dalloz, 1981, 567p., p.237.

${ }^{922}$ Article 6 de la loi n ${ }^{\circ} 97-029$ du 15 janvier 1999 portant organisation des communes en République du Bénin.

${ }^{923}$ Un préfet est un haut fonctionnaire nommé par décret du Président de la République, chargé de représenter le pouvoir exécutif dans le département ou la région qu'il administre. Il est placé sous l'autorité du ministre de l'Intérieur. Le terme préfet désigne à la fois la fonction occupée et le grade du titulaire de cette fonction qui est membre du corps préfectoral.

${ }^{924}$ Article 9 de la loi $\mathrm{n}^{\circ}$ 97-028 du 15 janvier 1999 portant organisation de l'administration territoriale de la République du Bénin.

${ }^{925}$ Article 10 de la loi n ${ }^{\circ} 97-028$ id.
} 
coordination est obligatoirement « consulté sur les programmes de développement économique, social et culturel des communes et sur la mise en cohérence de ceux-ci avec les programmes nationaux ${ }^{926}$. Le conseil départemental de concertation et de coordination qu'il dirige, « délibère sur le schéma d'aménagement du territoire et des projets de développement du département et sur les mesures de protection de l'environnement et entre autres, la politique de création et d'utilisation d'équipements collectifs d'intérêt départemental telles que, les forêts classées et les zones cynégétiques ${ }^{927}$.

Le rôle du Préfet dans le domaine de la protection de l'environnement consiste à veiller à l'application de la politique gouvernementale en relation avec les structures décentralisées et déconcentrées de l'Etat dans son ressort territorial. A ce titre, l'autorité déconcentrée « coordonne sous l'autorité du Ministre de l'environnement, les services déconcentrés des administrations civiles de l'Etat dans le Département et représente l'intérêt du ministère de l'Environnement dans son Département de compétence ${ }^{928}$. Il coordonne également «les activités en collaboration avec les structures déconcentrées du ministère telles que, les directions départementales de l'environnement et veille à la réalisation des projets ${ }^{929}$. Il est ainsi le représentant du Ministre de l'environnement et du Gouvernement et chargé de la mise en application de toutes les questions environnementales au niveau déconcentré de l'Etat, c'està-dire dans son Département de compétence et les communes du ressort.

Le Préfet, en sa qualité d'autorité de tutelle des communes du ressort, assure le tutorat par le pouvoir d'approbation que lui confère la $10^{930}$. C'est ce pouvoir qui permet au Préfet de garantir l'application des orientations nationales dans les Communes qui relèvent de sa juridiction. En vertu de cette prérogative, il contribue à la protection de l'environnement et à la lutte contre les changements climatiques en approuvant les politiques, plans, projets, programmes protecteurs de l'environnement à lui soumis par les Communes de ressort pour assurer leur cohérence avec les politiques environnementales élaborées par l'Etat central ${ }^{931}$. Enfin, le Préfet est le principal responsable de la Cellule environnementale instituée au niveau du Département ${ }^{932}$.

\footnotetext{
${ }^{926}$ Article 20 de la loi $n^{\circ} 97-028$ ibid.

927 Ibid.

${ }_{928}$ Article 10 de la loi n97-08 du 15 janvier 1999 portant organisation de l'administration territoriale au Bénin.

${ }^{929}$ Article 11 id.

${ }^{930}$ Article 14 de la loi n97-08 du 15 janvier 1999 portant organisation de l'administration territoriale au Bénin.

${ }^{931}$ L'article 6 de la loi n 98-030 portant loi cadre sur l'environnement en République du Bénin « (...) Il s'assure que les programmes et projets entrepris sur le territoire national sont conformes aux dispositions de la présente loi et en avise les autorités de tutelle le cas échéant (...)».

${ }^{932}$ Cette cellule est créée en vertu de l'article $1^{\text {er }}$ du décret $n^{\circ} 2001-095$ du 20 février 2001 portant création, attributions, organisation et fonctionnement des cellules environnementales en République du Bénin.
} 
Comme les changements climatiques induisent des catastrophes dramatiques qui pèsent sur le développement des nations, la question de la gestion des risques a été une préoccupation à l'occasion du Sommet de Rio de Janeiro de 1992 et a été intégrée sur la liste des engagements que doivent souscrire les Etats Parties à la Convention Cadre des Nations Unies sur les Changements Climatiques ${ }^{933}$. C'est conscient de cette réalité qu'il a été instauré au Bénin des mesures pour prévenir les risques de catastrophe que pourraient provoquer les changements climatiques. Dans ce cadre, c'est d'abord la loi sur les changements climatiques qui a prescrit que «l'Etat assure la prévention et la gestion des risques climatiques et des catastrophes naturelles $»^{934}$, pour ce fait, il « (...) adopte des politiques et stratégies visant la protection des personnes et des biens et assure la préservation du patrimoine culturel et environnemental ${ }^{935}$. Pour la mise en œuvre de cette disposition législative, deux instruments d'application ont été élaborés, il s'agit primo, du décret n²011-834 du 30 décembre 2011 portant création, composition, attributions et fonctionnement de la plate-forme nationale de réduction des risques de catastrophe et d'adaptation au changement climatique en République du Bénin, qui a été élaboré pour instituer un mécanisme de gestion et de coordination au niveau national et local des risques et catastrophes. Ce mécanisme est dénommé, «Plate-forme Nationale de Réduction des Risques de Catastrophe et d'Adaptation au Changement Climatique ${ }^{936}$. Elle a pour mission de «promouvoir l'intégration de la prévention des risques et de la gestion des catastrophes dans les politiques, plans et programmes de développement durable et de réduction de la pauvreté ${ }^{937}$ et puis, de «définir les orientations stratégiques et de valider les programmes établis dans le cadre de la réduction des risques de catastrophe $»^{938}$ et enfin de "faciliter la mobilisation des ressources nécessaires à la mise en œuvre des programmes et projets de prévention des risques, de gestion des catastrophes, de réhabilitation et de développement postcatastrophe ${ }^{939}$. C'est un système de gestion à caractère national mais déconcentré, qui dispose des démembrements dans chaque Département du pays. Les plates-formes « $(\ldots)$ Départementale, Communales et Locales sont chargées de la même mission que la Plate-forme

\footnotetext{
${ }_{933}$ Article 4-1-e de la Convention Cadre des Nations Unies sur les Changements Climatiques.

${ }^{934}$ Article 51 de la loi n ${ }^{\circ} 2018-18$ portant sur la règlementation sur les changements climatiques en République du Bénin.

935 Id.

${ }^{936}$ Article $1^{\mathrm{er}}$ du décret $\mathrm{n}^{\circ} 2011-834$ du 30 décembre 2011 portant création, composition, attributions et fonctionnement de la plate-forme nationale de réduction des risques de catastrophe et d'adaptation au changement climatique en République du Bénin.

${ }^{937}$ Article 2 premier tiret du décret $n^{\circ} 2011-834$ du 30 décembre 2011 portant création, composition, attributions et fonctionnement de la plate-forme nationale de réduction des risques de catastrophe et d'adaptation au changement climatique en République du Bénin.

938 Article 2 deuxième tiret id.

${ }^{939}$ Article 2 troisième tiret ibid.
} 
Nationale, dans le ressort de leurs circonscriptions administratives respectives. Elles exécutent les directives qui leur sont données dans ce sens, par la Plate-forme Nationale ${ }^{940}$ et présidée par le Préfet ${ }^{941}$.

Secundo, le décret n²012-426 du 06 novembre 2012 a été élaboré pour instituer l'Agence Nationale de Protection Civile (ANPC) ${ }^{942}$. Cette institution a pour mission de « contribuer à la mise en œuvre de la politique gouvernementale en matière de réduction des risques de catastrophe ${ }^{943}$. Elle est chargée de «prévenir tout risque de catastrophe sur l'ensemble du territoire national ${ }^{944}$ en veillant à la « préservation de l'environnement » ${ }^{945}$ par l'application méthodique du «Plan National d'Organisation des Secours en cas de Catastrophe ${ }^{946}$. Cette méthode de gestion des risques et catastrophes est dénommée «Plan ORSEC $\gg{ }^{947}$. Ce plan, dans sa mise en œuvre nécessite l'intervention du Préfet qui en est le coordonateur à l'échelle départemental, lorsque le Maire de la commune du ressort ne parvient pas à maitriser la situation ${ }^{948}$. Dans les étapes de gestion de ces catastrophes, le Préfet réunit le Comité départemental pour la protection civile pour recueillir son avis, donne l'alerte générale, met en œuvre le plan ORSEC et rend compte au Ministre en charge de l'intérieur et de la sécurité de l'évolution de la situation. Il met en mouvement les renforts organisés, constitue les postes de commandement et coordonne les opérations de secours. Lorsque la situation est totalement sous contrôle, il lève le plan ORSEC et en rend compte à l'autorité supérieure. Enfin, il procède à l'évaluation générale de la situation en rédigeant le rapport de fin d'opération. Lorsque l'ampleur de la catastrophe dépasse les moyens d'intervention du Département, le préfet en rend compte au Ministre de l'intérieur et de la sécurité publique ${ }^{949}$.

\footnotetext{
${ }^{940}$ Article 12 du n²011-834 du décembre 2011 portant création, composition, attributions et fonctionnement de la plate-forme nationale de réduction des risques de catastrophe et d'adaptation au changement climatique en République du Bénin.

${ }^{941}$ Article 6 id.

${ }_{942}$ Article $1^{\text {er }}$ du décret $n^{\circ} 2012-426$ du 6 novembre 2012 portant création, attributions, organisation et fonctionnement de l'Agence Nationale de Protection Civile (ANPC).

${ }^{943}$ Article 5 id.

${ }^{944}$ Article 5 premier tiret ibid.

945 Article 5 huitième tiret ibid.

${ }^{946}$ Article 5 cinquième tiret ibid.

${ }^{947}$ Selon le dictionnaire Larousse en ligne, le plan ORSEC représente un programme d'organisation des secours à l'échelon départemental, en cas de catastrophe, permettant une mise en œuvre rapide et efficace de tous les moyens nécessaires sous l'autorité du préfet. Disponible sur le site internet : https://www.larousse.fr/dictionnaires/francais. Consulté le 15/01/2022.

${ }^{948}$ Conformément aux dispositions de l'article 76, alinéa 6 de la loi n ${ }^{\circ}$ 97-029 du 15 janvier 1999 portant organisation des Communes en République du Bénin.

${ }^{949}$ Président de la Plate-forme Nationale de réduction de risques et d'adaptation aux changements en vertu de l'article 3 du décret $n^{\circ} 2011-834$ du 30 décembre 2011 portant création, composition, attributions et fonctionnement de la plate-forme nationale de réduction des risques de catastrophe et d'adaptation au changement climatique en République du Bénin.
} 
Enfin, dans la perspective de ce que les Unités Communales pour les Changements Climatiques (UCCC) ${ }^{950}$ seront créées et installées, les textes doivent être élaborés pour conférer le rôle de Coordonnateur desdites cellules dans le département du ressort à chaque préfet.

Le Département correspond à un niveau de l'échelle dans la répartition administrative $\mathrm{du}$ territoire national. A ce niveau, la politique de protection de l'environnement de l'Etat ne connaît pas encore sa phase opérationnelle, car il faut nécessairement que ces actions portées théoriquement à l'échelle départementale soient accompagnées dans la pratique par les collectivités locales qui en assurent sa visibilité à travers des réalisations concrètes sur le terrain.

\section{B- Le rôle de la collectivité locale}

Maurice KAMTO n'a-t-il pas souligné dans son étude que, «les collectivités territoriales décentralisées au Cameroun disposent d'importants pouvoirs d'action en matière de protection de l'environnement, ce qui fait d'elles des instruments de mise en œuvre du droit international de l'environnement $\rangle{ }^{951}$, pour montrer l'importance de l'environnementalisation des politiques territoriales entreprises par les Etats-Parties dans le seul souci de créer des conditions favorables à la mise en œuvre des instruments de protection de l'environnement. C'est pourquoi à Rio de Janeiro en 1992, à l'occasion de la Conférence internationale sur l'environnement, la déclaration issue de la rencontre a rappelé l'importance de la contribution d'un certain nombre d'acteurs notamment, celle de la collectivité locale qui doit jouer un « rôle vital dans la gestion de l'environnement du fait de sa connaissance du milieu et de ses pratiques traditionnelles ${ }^{952}$. Pour cela, les « Etats devraient leur permettre de participer efficacement à la réalisation d'un développement durable $»^{953}$.

L'intérêt à associer la collectivité locale ${ }^{954}$ à la protection de l'environnement réside dans le fait que, le lieu d'application du droit de l'environnement est en réalité l'espace

\footnotetext{
${ }^{950}$ Une recommandation contenue dans la Stratégie Nationale de mise en œuvre au Bénin de la Convention Cadre des Nations Unies sur les Changements Climatiques, pp. 69-70 propose la création de cet organe.

${ }^{951}$ KAMTO (M.), « La mise en œuvre et le suivi du droit international de l'environnement » Rapport introductif général, in Michel PRIEUR (dir.) La mise en œuvre national du droit international de l'environnement dans les pays francophones, Acte des troisièmes journées scientifiques du Réseau «Droit de l'environnement», AUF, Yaoundé, juin 2001.

952 Principe 22 de la Déclaration de Rio sur l'Environnement et le Développement La Conférence des Nations unies sur l'environnement et le développement, Réunie à Rio de Janeiro du 3 au 14 juin 1992.

${ }_{953} \mathrm{Id}$.

${ }^{954}$ Loi n ${ }^{\circ}$ 97-028 du 15 janvier 1999 portant organisation de l'administration territoriale en république du Bénin n'a défini le concept de «Collectivité locale », mais elle précise au titre de son article 21 que les collectivités locales décentralisées sont instituées dans la structure de l'administration territoriale de la République et dotées de la personnalité juridique et de l'autonomie financière ».
} 
géographique occupé et géré par les collectivités locales. La France a marqué un progrès en ce qui concerne l'implication de la collectivité locale dans l'encadrement de l'environnement. Dans ce pays, le droit de « la représentation de la nature par les collectivités territoriales devant le juge judiciaire est reconnu en vertu d'une règlementation existante en la matière ${ }^{955}$. Il s'ensuit que dans un conflit environnemental, « les collectivités locales peuvent se constituer partie civile et obtenir la réparation du préjudice écologique» ${ }^{956}$ devant les juridictions. La preuve de l'implication de la collectivité locale dans la lutte contre les changements climatiques en France en a été donnée à travers une jurisprudence du Conseil d'Etat ${ }^{957}$. Seulement qu'à travers cette jurisprudence, même si l'action tendant à rendre obligatoire la priorité climatique par l'élaboration des mesures législatives, intentée par la commune de Grande-Synthe en France devant le Conseil d'Etat est déclarée irrecevable pour motif d'incompétence, force est de constater que les collectivités locales ont le mérite dans ce pays de s'engager dans la lutte contre les changements climatiques avec la faculté qui leur est offerte d'attraire l'Etat devant une juridiction pour solliciter le respect par lui, de ses engagements souscrits dans la Convention Cadre des Nations Unies sur les Changements Climatiques ${ }^{958}$.

Le concept de «collectivité locale» a été initié dans le contexte béninois par la constitution du 11 décembre 1990 révisée le 07 novembre 2019 en réponse aux difficultés qui entravaient la mise en œuvre de la politique de l'Etat central sur le territoire national ${ }^{959}$. Le Constituant béninois, convaincu de l'importance du rapprochement du service public vers l'administré, a jugé de la nécessité de l'implication des collectivités à la base dans la gestion des affaires de l'Etat. Ainsi, les réformes constitutionnelles ont consacré quelques dispositions à sa création ${ }^{960}$. Dans ce cadre, la politique de l'administration territoriale mise en œuvre au Bénin a retenu la Commune comme le territoire sur lequel la collectivité locale peut exercer sa compétence. Le Bénin compte soixante-dix-sept (77) communes ou collectivités locales réparties en cinq mille deux cent quatre vingt-dix (5290) unités administratives encore appelées

\footnotetext{
${ }^{955} \mathrm{~L}$ 'article L.142-4 du Code de l'environnement. Disponible sur le site internet : https://www.legifrance.gouv.f. Consulté le 15/01/2022.

${ }^{956}$ Id.

${ }^{957}$ Voir décision rendue par le Conseil d'Etat statuant au contentieux (Section du contentieux, $6^{\mathrm{e}}$ et $5^{\mathrm{e}}$ chambres) dans : Affaire $n^{\circ} 427301$, commune de Grande Synthe et autre, le 19 novembre 2020. Publiée au recueil Lebon. Disponible sur le site internet : https://www.conseil-etat.fr/fr/arianeweb/CE/decision/2020-11-19/4271. Consulté le $15 / 01 / 2022$.

${ }^{958}$ Décision rendue par le Conseil d'Etat statuant au contentieux (Section du contentieux, $6^{\mathrm{e}}$ et $5^{\mathrm{e}}$ chambres) dans : Affaire $\mathrm{n}^{\circ}$ 427301, commune de Grande Synthe et autre, le 19 novembre 2020. Publiée au recueil Lebon. Disponible sur le site internet: https://www.conseil-etat.fr/fr/arianeweb/CE/decision/2020-11-19/427301. Consulté le 15/01/2022.

${ }^{959} \mathrm{~L}$ 'article 150 de ladite constitution a créé les collectivités locales. Les 151 à 153 ont définis les modes d'administration des collectivités locales et leurs relations avec l'Etat central.

${ }_{960}$ Articles 150, 151, 152 et 153 de la constitution béninoise du 11 décembre 1990.
} 
«village ou quartier de ville ${ }^{961}$. La commune est le lieu par excellence d'incubation et d'exécution des projets gouvernementaux y compris ceux concernant la protection de l'environnement. Les activités d'atténuation du gaz à effet de serre et la réalisation d'infrastructures résilientes aux effets néfastes des changements climatiques sont visibles sur le territoire communal parce que c'est la commune qui met à disposition le foncier. C'est encore sur le territoire des collectivités locales que se produisent les effets néfastes des changements climatiques, comme les catastrophes naturelles avec les répercutions sur les habitants.

Quelles sont les attributions de la collectivité locale en matière de protection de l'environnement au Bénin?

Les attributions de protection de l'environnement des collectivités locales sont consacrées par des dispositions éparses contenues dans les textes législatifs et réglementaires relatifs à la protection de l'environnement et à la lutte contre les changements climatiques. Les textes de loi sur la décentralisation confèrent aux collectivités locales des compétences propres qui concernent la préservation de l'environnement ${ }^{962}$. L'intervention de la collectivité locale a été confirmée par la Charte sur la gouvernance environnementale qui permet à cette entité de recevoir des compétences en matière de protection de l'environnement provenant de l'Etat central $^{963}$. Ces compétences sont transférées dans le but d'assurer le mieux être à la population pour un encadrement intégral du territoire. Pour accomplir leurs objectifs, les collectivités locales intègrent la politique de l'Etat en matière de protection de l'environnement dans leur Plan de Développement Communal (PDC) ${ }^{964}$ qu'elles élaborent et qui cadre avec la vision de l'Etat en ce qui concerne la protection de l'environnement et ce, conformément à la loi sur les changements climatiques au Bénin, qui stipule que «L'Etat et les collectivités territoriales veillent à l'intégration, dans les politiques, stratégies, budgets et plans de développement

\footnotetext{
${ }^{961}$ Conformément au découpage territorial prévu respectivement par la loi n ${ }^{\circ} 97-028$ du 15 janvier 1999 portant organisation de l'administration territoriale en République du Bénin et la loi n $2013-05$ du 15 février 2013 portant création, organisation, attributions et fonctionnement des unités administratives locales en République du Bénin. ${ }^{962}$ Par exemple, dans la loi n ${ }^{\circ}$ 97-029 du 15 janvier 1999 portant organisation des communes en République du Bénin, les articles 93 à 96 sont consacrés à la protection de l'environnement.

${ }^{963}$ Article 7 de la charte sur la gouvernance du développement adopté les 3 et 4 novembre 2011 à Cotonou. Disponible sur le site internet: http://www.bj.undp.org/content/dam/benin/docs/gouvernance/charte-pour-lagouvernance-du-benin.pdf. Consulté le 15/01/2022.

${ }^{964} \mathrm{Le}$ guide d'élaboration du plan de développement communal publié en novembre 2008 élaboré parle Ministère de la Décentralisation, de la Gouvernance Locale de l'Administration et de l'Aménagement du Territoire (MDGLAAT) défini le PDC comme un document de planification du développement de la commune sur une période de cinq (5) ans. Il est élaboré sur la base d'une méthodologie qui intègre toutes les préoccupations de développement durable, les prévisions et programmations d'ordre social, économique, spatial, environnemental, les préoccupations circonstancielles de la commune et les recommandations issues de l'évaluation du PDC en cours d'achèvement. Celles-ci sont en adéquation avec les orientations stratégiques et programmations sectorielles contenues dans les documents aux niveaux intercommunal, départemental et national. Disponible sur le site internet : https://www.pseau.org/outils/ouvrages/mdglaat_guide. Consulté le 15/01/2022.
} 
communal, des mesures d'adaptation aux changements climatiques et à leurs effets et conséquences négatifs ou visant à atténuer lesdits effets ${ }^{965}$. Cet outil de travail avant d'être mis en œuvre reçoit l'approbation de l'autorité de tutelle ${ }^{966}$ qui s'assure de sa conformité avec les normes en vigueur en matière de politique de protection de l'environnement. Parfois, le refus d'approbation de la politique environnementale de la Commune par l'autorité déconcentrée peut donner lieu à un conflit qui sera déféré devant les formations juridictionnelles statuant en matière administrative ${ }^{967}$. Dans certains cas, ce pouvoir d'approbation manque d'objectivité d'impartialité et se transforme en un instrument de règlement de compte politique. Ce qui entrave les relations de bonne collaboration qui devraient exister entre les démembrements et l'Etat central. Dans ce cas, l'arbitrage des formations juridictionnelles administratives compétentes ne parvient pas souvent à s'imposer à la volonté de l'Etat central qui peut choisir de ne pas exécuter les décisions rendues dans les contentieux qui l'opposent aux collectivités locales.

Cependant, la loi n 98-030 du 12 février 1999 portant loi cadre sur l'environnement en République du Bénin qui définit les bases de la politique en matière d'environnement et organise sa mise œuvre ${ }^{968}$, n'a pas intégré la collectivité locale dans la gestion de la protection de l'environnement. Seulement, les institutions créées par ladite loi ${ }^{969}$ entretiennent des relations avec la collectivité locale dans le cadre de la mise en œuvre de la politique de l'Etat en matière de protection de l'environnement, de gestion des effets des changements climatiques, de reboisement, de protection des ressources naturelles et forestières, de préservation des écosystèmes, de protection des berges et des côtes. A titre l'illustratif, selon la loi n 98-030 du 12 février 1999 portant loi cadre sur l'environnement en République du Bénin l'Agence Béninoise pour l'Environnement (ABE) est investie du devoir d'«assistance et d'appui aux collectivités locales dans le cadre de l'élaboration et de la mise en œuvre de leurs politiques, plans et programmes en matière d'environnement $\gg .{ }^{970}$

\footnotetext{
${ }^{965}$ Article 4 de la loi n ${ }^{\circ} 2018$-18 portant règlementation sur les changements climatiques en République du Bénin.

${ }^{966} \mathrm{~L}$ 'autorité de tutelle des communes est le Préfet du département du ressort qui donne son approbation en vue d'assurer la conformité des documents élaborés avec les politique et stratégie de l'Etat.

${ }^{967}{ }^{\prime}$ 'est ce que dispose l'article 86 de la loi n ${ }^{\circ} 2018$ - 18 portant règlementation sur les changements climatiques en République du Bénin.

${ }^{968}$ Selon les dispositions de l'article premier de la loi $\mathrm{n}^{\circ} 2018-18$ portant règlementation sur les changements climatiques en République du Bénin.

${ }^{969}$ Il s'agit de la Commission Nationale de Développement Durable (CNDD) et de l'Agence Béninoise de l'Environnement (ABE) qui sont respectivement créées par les dispositions des articles 7 et 11 .

${ }^{970}$ L'Article 5 du décret $\mathrm{n}^{\circ}$ 2010-478 du 05 novembre 2010 portant création, attributions, organisation et fonctionnement de l'Agence Béninoise pour l'Environnement défini les missions de l'organe.
} 
Par ailleurs, les valeurs de développement durable recommandent que soient effectuées des études permettant de mesurer l'impact de la réalisation d'un projet sur l'environnement. La loi béninoise fait de la Commune et de ses démembrements, des acteurs incontournables dans le processus d'étude d'impact préalable, avant l'installation des projets d'envergure sur le territoire communal ${ }^{971}$. C'est ce que contient l'article 96 de la loi n98-030 du 12 février 1999 portant loi cadre sur l'environnement en République du Bénin, qui dispose que la Commune donne son avis chaque fois qu'il est envisagé, la création sur son territoire de tout projet susceptible de porter atteinte à l'environnement. L'étude d'impact environnemental est réalisée sur l'initiative de l'Agence Béninoise de l'Environnement (ABE) qui requiert la collaboration inconditionnelle de l'autorité communale ${ }^{972}$, à qui la loi a donné la possibilité d'émettre des avis dans le dossier.

Quelle est la part contributive de la collectivité locale dans la lutte contre les changements climatiques?

Les arbres des forêts constituent des puits de carbone et contribuent à sa fixation à partir de l'atmosphère. Leur contribution dans la réduction des gaz à effet de serre est d'autant plus considérable que le Sommet de Rio de Janeiro de 1992 a consacré à cette matière toute une Déclaration ${ }^{973}$ à défaut de pouvoir élaborer une « convention juridiquement contraignante sur les forêts ${ }^{974}$. C'est la preuve que la protection des forets est placée au rang des préoccupations écologiques internationales et font l'objet de discussion et de négociation à presque tous les Sommets de la Terre. La protection des forêts représente un atout majeur dans le combat contre les changements climatiques. L'application de la Convention Cadre des Nations Unies sur les Changements Climatiques permet de remédier aux problèmes de dérèglement climatique par la multiplication des puits d'absorption des gaz à effet de serre. Pour cela, l'instrument juridique a retenu que chacune des «(...) Parties adopte des politiques nationales ${ }^{975}$ et prend en conséquence les mesures voulues pour atténuer les changements climatiques en limitant ses émissions anthropiques de gaz à effet de serre en protégeant et en renforçant ses puits et réservoirs de gaz à effet de serre» ${ }^{976}$. Le renforcement des puits et réservoirs de gaz à effet de

\footnotetext{
${ }^{971}$ La loi n 98-030 du 12 février 1999 portant loi cadre sur l'environnement en République du Bénin.

${ }^{972} \mathrm{Au}$ Bénin, le Maire représente l'autorité Communale. A ce titre, il représente l'exécutif de la commune (article 24) et il est l'ordonnateur du budget communal (article 8) de la $n^{\circ} 97-029$ du 15 Janvier 1999 Portant organisation des communes en République du Bénin.

${ }^{973}$ Il s'agit de la « Déclaration de Rio sur l’Environnement et le Développement, principes de gestion des forêts ».

${ }^{974}$ Premier paragraphe du préambule de ladite Déclaration.

${ }^{975} \mathrm{Au}$-delà des Etats qui les acteurs classiques du droit international, le terme Partie dans ce contexte s'entend aussi des organisations d'intégration économique régionale.

${ }^{976}$ Article 4 -a de la Convention Cadre des Nations Unies sur les Changements Climatiques.
} 
serre peut être facilité dans le contexte du Bénin par la protection du domaine forestier. Evidemment c'est dans le domaine de la protection des forêts que la règlementation béninoise en matière de protection de l'environnement confère plus de responsabilités aux collectivités locales, qui sont investies de la mission de protection de l'environnement. Il en est de même de l'article 94 de la loi n²013-05 du 27 mai 2013 portant création, organisation, attributions et fonctionnement des unités administratives locales en République du Bénin, qui précise les attributions de protection de l'environnement de la collectivité locale en disposant que «la commune (...) veille à la protection des ressources naturelles, notamment des forêts, des sols de la faune, des ressources hydrauliques, des nappes phréatiques et contribue à leur meilleure utilisation. (...)». Il est stipulé à l'article 89 de ladite loi ${ }^{977}$, que "le chef de village ou de quartier de ville (...) veille à l'application des règlements forestiers et d'aménagement ainsi qu'à la protection des forêts et des espaces verts $\rangle^{978}$. Le chef village ou de quartier de ville «participe et contribue de manière permanente et effective aux activités de préservation de l'environnement et de toutes ses composantes ${ }^{979}$. Le rôle de la collectivité locale dans la protection des forêts a été renforcé par la loi sur les changements climatiques qui stipule que, « l'Etat et les collectivités locales promeuvent les plantations publiques et privées, restaurent les forêts dégradées, ainsi que les espèces en voie de disparition et rationalisent l'exploitation des ressources forestières et fauniques. Les communes sont tenues de créer et d'entretenir des espaces verts, des jardins publics, des parcs et des forêts communales » ${ }^{980}$ mais en plus, « l'Etat et les collectivités territoriales encouragent la mise en place et l'entretien de plantations forestières communautaires ou privées pour renforcer les puits et réservoirs de gaz à effet de serre $»^{981}$.

Enfin, lorsque les Unités Communales pour les Changements Climatiques (UCCC) ${ }^{982}$ seront créées et installées, leur gestion devra relever de la compétence du Maire de la Commune qui en assure le fonctionnement.

\footnotetext{
${ }^{977}$ Il s'agit de la loi n²013-05 du 27 mai 2013 portant création, organisation, attributions et fonctionnement des unités administratives locales en République du Bénin.

978 Article 27 de la loi nº2013-05 du 27 mai 2013 portant création, organisation, attributions et fonctionnement des unités administratives locales en République du Bénin.

${ }^{979}$ Article 49 de la loi n ${ }^{\circ} 2013-05$ du 27 mai 2013 portant création, organisation, attributions et fonctionnement des unités administratives locales en République du Bénin.

${ }_{980}$ Article 54 de la loi portant règlementation des changements climatiques en République du Bénin.

${ }^{981}$ Article 64 de la loi nº2018-18 portant règlementation sur les changements climatiques en République du Bénin.

${ }^{982} \mathrm{La}$ création des Unités Communales pour les Changement Climatiques a été une recommandation contenue dans le document de Stratégie Nationale de mise en œuvre au Bénin de la Convention Cadre des Nations Unies sur les Changements Climatiques. Page 69.
} 
La contribution du Droit international à la lutte contre les changements climatiques a été laborieuse en termes de quantité de normes juridiques élaborées et en nombre d'institutions créées. Ce tableau pourrait laisser planer une lueur d'espoir de parvenir à terme, à bout des innombrables et capricieux défis imposés par les changements climatiques. A ce sujet, le Président $\mathrm{Al}$ Gore ${ }^{983}$ n'a-t-il pas déclaré et affirmé à l'époque ${ }^{984}$ à propos du changement climatique, qu'il était désormais «complètement clair que nous détenons entre nos mains tous les instruments nécessaires pour résoudre la crise climatique (...) ${ }^{985}$ ? Mais, contre toute attente, les changements climatiques progressent, car la contribution du droit à la protection du climat tant espérée et voulue n'a été que modeste.

\footnotetext{
983 Albert Arnold Gore Jr connu sous le nom Al Gore, homme politique américain, vice président de Bill Clinton (1993-2001). Disponible sur le site internet : https://www.larousse.fr/encyclopedie. Consulté le 15/01/2022. Prix Nobel de la paix 2007 partagé avec le GIEC : Groupe d'experts intergouvernemental sur l'évolution du climat. Disponible sur le site internet : https://www.un.org/fr/about-us/nobel-peace-prize/ipcc-al-gore-2007. Consulté le $15 / 01 / 2022$.

${ }_{984} \mathrm{Au}$ moment de sa nomination en 2007.

${ }^{985}$ S. Cypel, « Al Gore appelle au volontarisme sur l'environnement », Le Monde, 11 novembre 2009.
} 



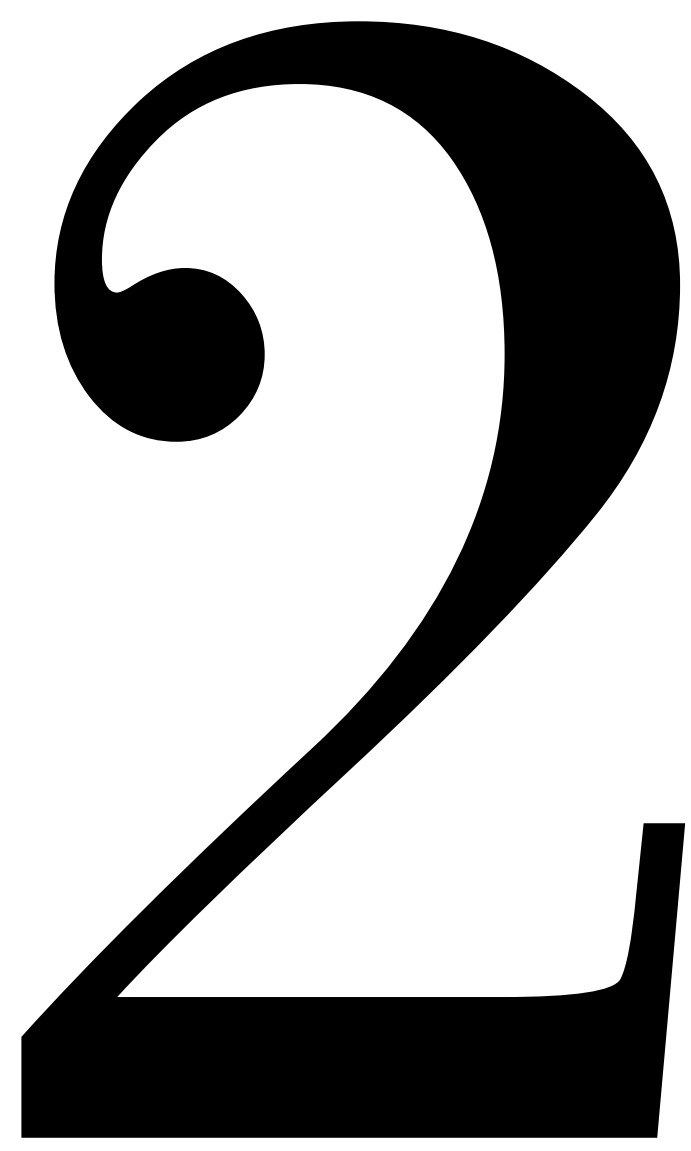




\section{DEUXIEME PARTIE}

\section{UNE CONTRIBUTION MODESTE}


Face à la nécessité d'encadrement d'un domaine donné, le premier réflexe pour le juriste est l'élaboration des normes. L'étape suivante, la plus importante est celle qui consiste à la mise en œuvre des ces normes. C'est d'ailleurs en ce moment que s'aperçoit la qualité et l'efficacité de l'outil juridique élaboré à travers le résultat produit.

Si l'efficacité du droit de protection du climat était conditionnée par le nombre d'instruments internationaux élaborés et souscrits ${ }^{986}$, le défi de la lutte contre les changements climatiques serait déjà conjugué au passé à cause du foisonnement normatif qui a caractérisé le droit climatique, sans compter le nombre impressionnant d'institutions déployés pour son encadrement. Mais en réalité, le foisonnement des normes destinées à la protection du climat ne représente que la face de l'iceberg, c'est « l'arbre qui cache la forêt». Au contraire, la densité normative et la multiplicité institutionnelle n'ont guère émoussé l'ardeur des changements climatiques qui font rage partout et en tout temps.

Néanmoins, ce droit massivement élaboré a contribué dans une proportion limitative à l'encadrement des changements climatiques. Le rapport spécial du Groupe d'Experts Intergouvernemental sur l'Evolution du Climat (GIEC) publié en 2014 en établi la preuve en montrant la faiblesse dudit droit à travers, «(...) les conséquences d’un réchauffement planétaire de $1{ }^{\circ} \mathrm{C}$ qui sont déjà bien réelles comme l'attestent l'augmentation des extrêmes météorologiques, l'élévation du niveau de la mer et la diminution de la banquise arctique» ${ }^{987}$. La baisse de performance qui ressort de la mise à contribution du droit à la lutte contre les changements climatiques est illustrée notamment par la baisse des pluies, la hausse des températures, la multiplication des catastrophes. Le Rapport du Groupe d'Experts Intergouvernemental sur l'Evolution du Climat publié en 2014, pour ce qui concerne les changements observés et leurs causes, constate que «Les changements climatiques récents ont eu de larges répercussions sur les systèmes humains et naturels ${ }^{988}$. Ces signes témoignent de la faiblesse de la contribution du droit à la lutte contre les changements climatiques.

Pour comprendre l'impact du droit sur les changements climatiques, l'étude de l'effectivité dudit droit renseignerait mieux sur sa capacité à relever les défis à lui imposés par le phénomène (TITRE I).

Eventuellement, des perspectives d'amélioration du droit en vue de la protection efficace du climat pourront êtres envisagées (TITRE II).

\footnotetext{
${ }^{986}$ Il s'agit de la Convention Cadre des Nations Unies sur les Changements Climatiques et ses accords subséquents et les décisions issues des COP, des Sommets de la Terre et autres rencontres destinées à la protection de l'environnement et à la lutte contre les changements climatiques.

${ }^{987}$ Septième paragraphe à la page 1 du Résumé à l'intention des décideurs relatif au Rapport spécial sur un réchauffement planétaire de $1,5^{\circ} \mathrm{C}$ (SR15). Disponible sur le site internet : http://www.ipcc.ch/report/sr15/ ou www.ipcc.ch. Consulté le 15/01/2022.

988 Page 2 dudit rapport.
} 


\section{TITRE I:}

\section{L'EFFECTIVITE DE LA PROTECTION}

Face à ce constat désagréable, « après cette phase de frénésie normative durant laquelle il s'agissait de construire un corps de règles et où peu d'attention était portée à sa mise en auvre ${ }^{989}$, il est à cette étape de notre étude judicieux d'évaluer la qualité de l'instrument juridique élaboré, d'examiner les modalités de son application afin de se rendre compte de sa capacité ou non à faire aboutir l'objectif fixé dans la Convention Cadre des Nations Unies sur les Changements Climatiques à savoir, contribuer à « contenir le réchauffement climatique sous la barre des $2^{\circ} \mathrm{C}$ et si possible même à $1,5^{\circ} \mathrm{C}$ par rapport au niveau de l'avant révolution industrielle ${ }^{990}$ en s'assurant pour ce qui concerne les Etats Parties, du respect des engagements souscrits dans l'Accord climat.

Plus concrètement, l'étude de l'effectivité du droit de protection du climat au Bénin permet d'une part, d'appréhender les effets de la mise en œuvre du droit international (CHAPITRE I) puis d'autre part, de se rendre compte de la faible effectivité de la protection du climat (CHAPITRE II).

\footnotetext{
${ }^{989}$ Sandrine Maljean-Dubois, La mise en œuvre du droit international de l'environnement, Centre d'études et de recherches internationales et communautaires, Aix-en-Provence France, 65p., p.8.

${ }_{990}$ Article 2 de l'Accord de Paris conclu en décembre 2015.
} 
TITRE I 


\section{CHAPITRE I: \\ LES EFFETS DU DROIT INTERNATIONAL}

Dans le cadre des réflexions orientées sur le sujet intitulé, « l'influence des conventions internationales sur le droit interne de l'environnement $»,{ }^{991}$ en vue de mesurer ou de faire prendre conscience, l'effectivité et l'efficacité des instruments de protection de l'environnement, le Professeur Michel Prieur souligne que, « Le droit de l'environnement de ce début du siècle est confronté à deux grandes évolutions: son internationalisation et sa constitutionnalisation ${ }^{992}$. Cette mutation induit « une complexité juridique certaine et dans le même temps une incertitude, voir un doute sur les effets juridiques réels» ${ }^{993}$.

Cependant, ce processus d'intégration, de transformation ou d'adaptation de la règlementation nationale en vue de lutter contre les changements climatiques au sein des Etats Parties butte des handicaps. Au-delà de toutes les autres insuffisances évoquées dans le cadre de l'étude, la non intégration des paramètres relatifs aux dimensions notamment, politiques, sociales et économiques, bref, les questions du développement des Etats sont souvent occultées et sont à l'origine des difficultés de la mise en œuvre de la règlementation en matière de protection de l'environnement et de lutte contre les changements climatiques. Le Sommet de Rio de Janeiro de 1992 a considéré à juste titre le développement des Etats comme un aspect essentiel dans la protection de l'environnement et a même innové le concept du développement durable qui «(...) doit faire partie intégrante du processus de développement et ne peut être considérée isolément ${ }^{994}$. La protection de l'environnement suivant le modèle développé par le droit international ne peut être réalisée dans les Etats vulnérables si l'accent n'est pas mis sur le développement. A l'entame de l'édification du droit international, l'instrument élaboré à Stockholm en 1972 privilégie déjà en termes de moyens de protection de l'environnement, des préoccupations en relation avec le développement, car stipule t-il que «Le développement économique et social est indispensable si l'on veut assurer un environnement propice à

\footnotetext{
${ }^{991}$ Michel Prieur. L'influence des conventions internationales sur le droit interne de l'environnement Actes de la réunion constitutive du comité sur l'environnement de l'AHJUCAF, Juin 2008, Porto-Novo, Bénin. pp.291-301.

${ }_{992}$ Id.

${ }^{993}$ Rapport général de Monsieur le Professeur Michel PRIEUR de la réunion constitutive du comité sur l'environnement de l'AHJUCAF Juin 2008, Porto-Novo, Bénin. pp.291-301, p.291.

${ }^{994}$ Principe 4 de la Déclaration de Rio sur l'Environnement et le Développement, Conférence des Nations unies sur l'environnement et le développement, Réunie à Rio de Janeiro du 3 au 14 juin 1992.
} 
l'existence et au travail de l'homme et créer sur la terre des conditions nécessaires à l'amélioration de la qualité de la vie ${ }^{995}$. En effet, le développement s'entend comme le combat pour l'éradication de la pauvreté. Il convient qu'à l'origine de la conception des outils de protection de l'environnement et de lutte contre les changements climatiques que les conditions de développement soient prises en compte. Autrement dit, la réalisation des objectifs de développement contribue à la protection de l'environnement. A titre indicatif, une analyse environnementale en relation avec l'économie illustrée par la Courbe Environnementale de Kuznets ${ }^{996}$ (EKC) a mis en évidence des résultats entre les domaines économique et environnemental. Cette analyse a conduit à la formulation de l'hypothèse selon laquelle, « il existe une corrélation significative entre le revenu et la performance environnementale ${ }^{997} \mathrm{de}$ sorte qu'à un certain niveau de développement économique, peut correspondre une amélioration ou une détérioration de la qualité de l'environnement. Il s'ensuit que la croissance économique doit être source d'amélioration de la qualité de l'environnement dans les pays en voie de développement. Suivant cette vision, MICHAEL FAURE, MORAG GOODWIN FRANZISKA WEBER, partant de ce lien clair qui est établi entre performance environnementale et vitalité économique « suggèrent que l'approche principale pour améliorer la protection de l'environnement, à la fois dans les pays en développement et dans le monde consiste à se concentrer sur l'amélioration de la situation économique des pays pauvres et l'atténuation de la pauvreté» ${ }^{998}$. Malheureusement, les normes internationales élaborées pour faire la promotion aussi bien du développement économique que de la protection environnementale ne sont pas souvent adaptées au contexte africain. Parfois, si ces normes sont convenables, il y a des goulots qui découlent du statut des acteurs en charge de leur mise en œuvre au plan national et le mode de gouvernance qui ne permettent pas d'obtenir les résultats escomptés.

Qu'en est-il du droit positif béninois après l'adhésion du pays aux accords internationaux de protection de l'environnement et de lutte contre les changements climatiques?

\footnotetext{
995 Principe 8 de la Déclaration de Stockholm de 1972.

${ }^{996} \mathrm{C}$ 'est une courbe qui retrace les impacts du développement économique sur l'amélioration de la qualité de l'environnement. La courbe émane des travaux de Grossman et Krueger. Nourry Myriam. La croissance économique est-elle un moyen de lutte contre la pollution ? In: Revue française d'économie, volume 21, n³, 2007. pp. 137-176, p.137-139.

${ }_{997}$ See Dan C. Esty \& Michael E. Porter, National Environmental Performance: An Empirical Analysis of Policy Results and the Determinants, 10 ENV'T \& DEV. ECON. 391 (2005), for an excellent summary of this literature. ${ }^{998}$ Faure, M. G., Goodwin, M. E. A., \& Weber, F. (2010). Bucking the Kuznets curve: Designing effective environmental regulation in developing countries. Virginia Journal of International Law, 51(1), 95-156.
} 
Le droit positif béninois désormais assujetti aux contraintes du droit international a subi par conséquent des transformations provoquées par la ratification des instruments de protection de l'environnement. L'application au plan national de ces nombreux accords a produit des influences sur l'environnement juridique béninois (SECTION I) et a contribué à la modification du cadre institutionnel existant (SECTION II).

\section{SECTION I: L'INFLUENCE SUR L'ENVIRONNEMENT JURIDIQUE BENINOIS}

Selon certains auteurs, l'élaboration du droit national peut se « réaliser par influence directe, par traduction, transposition et incorporation du corpus international ou par influence indirecte, l'intégration des dispositifs internationaux, même lorsque les conventions n'ont pas été ratifiées ou à travers la circulation des idées et des concepts lors des travaux des experts ${ }^{999}$. La Cour de Justice de la Communauté Européenne (CJCE) admet qu'en absence de toute autre règlementation, «Une disposition (...) doit être considérée comme d'application directe lorsque, eu égard à ses termes ainsi qu'à l'objet et à la nature de l'accord, elle comporte une obligation claire et précise, qui n'est subordonnée dans son exécution ou dans ses effets, à l'intervention d'aucun acte ultérieur $\gg{ }^{1000}$. De toutes les manières, dans le cadre de la protection de l'environnement et de la lutte contre les changements climatiques, le droit dispose qu'en absence d'objection, «les Etats doivent promulguer des mesures législatives efficaces en matière d'environnement (...)» ${ }^{1001}$ et s'assurer que «les normes écologiques, les objectifs et priorités pour la gestion de l'environnement sont adaptés à la situation en matière d'environnement et de développement à laquelle elles s'appliquent (...)» ${ }^{1002}$.

Le processus d'internalisation des instruments internationaux à l'environnement juridique en vue de l'édification du droit applicable en matière des changements climatiques enclenché au Bénin a influencé et bouleversé légèrement son ordonnancement juridique ${ }^{1003}$

\footnotetext{
${ }^{999}$ Michel Prieur, «L'influence des conventions internationales sur le droit interne de l'environnement. Actes de la réunion constitutive du comité sur l'environnement de l'AHJUCAF », Jun 2008, Porto-Novo, Bénin. pp.291301., p.291.

${ }^{1000}$ CJCE, 30 septermbre 1987, Delirel c/ ville de Schwabisch Gmund, 12/86, Rec. p. 3752, point 14.

1001 Principe 11 déclaration de Rio de Janeiro de 1992. ${ }^{1002} \mathrm{Id}$.

${ }^{1003}$ L'ordonnancement juridique" ou "ordre juridique", est une expression par laquelle on désigne l'ensemble des règles provenant notamment, de la constitution, de lois, des règlements administratifs qui, à un moment défini et dans un Etat donné, établissent à l'intérieur de cet Etat, le statut des personnes publiques ou privées et qui définissent les rapports juridiques qui existent entre les personnes publiques et les personnes privées, ou entre les personnes publiques entre elles, ou encore entre les personnes privées entre elles. Dictionnaire du droit privé,
} 
(Paragraphe 1). Mais, à terme, ce processus a contribué quelque peu au renforcement du droit positif béninois en matière de protection du climat (Paragraphe 2).

\section{Paragraphe 1: Le bouleversement de l'ordonnancement juridique}

Depuis l'accession du Bénin à la souveraineté internationale le $1^{\mathrm{er}}$ août 1960 jusqu'à la tenue de la Conférence des forces vives de la nation en février $1989^{1004}$, l'ordre juridique établi n'avait pas intégré les préoccupations liées à la protection de l'environnement au point de s'intéresser aux changements climatiques. Cela se justifie par le fait qu'en ce moment, les changements climatiques n'étaient pas une préoccupation pour la Communauté internationale au point d'être considérés par les Etats comme une menace à leur développement. Mais après la consécration constitutionnelle du droit de protection de l'environnement en $1990^{1005}$ et la ratification d'accords internationaux de protection de l'environnement et de lutte contre les changements climatiques intervenue à partir de 1992, le droit international a contribué à forger un arsenal juridique national orienté dans le sens des objectifs de protection de l'environnement et progressivement dirigé vers les ambitions de lutte contre les changements climatiques, induisant par voie de conséquence le bouleversement de l'ordonnancement juridique béninois en matière de protection de l'environnement.

Le Bénin, autrefois dépourvu d'arsenal juridique adapté à la protection de l'environnement a désormais connu une amélioration de son cadre juridique, devenu moderne (A). Par la suite, cet encadrement a été spécifié en faveur de la lutte contre les changements climatiques provoquant ainsi au plan national, l'existence d'un régime juridique essentiellement orienté vers les objectifs de protection du climat (B).

\section{A- L'existence d'un encadrement juridique modernisé}

Le Bénin s'est engagé avec la Communauté internationale dans la protection de l'environnement à partir de 1990, année de la consécration constitutionnelle de la protection de l'environnement. Mais avant cette période, le domaine environnemental était juridiquement

\footnotetext{
élaboré par Serge Braudo, Conseiller honoraire à la Cour d'appel de Versailles, disponible en ligne sur le site internet : https://www.dictionnaire-juridique.com. Consulté le 16/01/2022.

${ }^{1004} \mathrm{La}$ conférence des forces vives de la nation béninoise a été organisée en février 1989 à l'occasion du bouleversement politique survenu dans ce pays. Elle a provoqué l'avènement du renouveau démocratique avec l'adoption d'une nouvelle constitution le 11 décembre 1990. Histoire du Bénin disponible sur le site internet : https://www.gouv.bj/benin/histoire/. Consulté le 16/01/2022.

${ }^{1005} \mathrm{La}$ consécration constitutionnelle du droit de protection de l'environnement est intervenue avec l'adoption d'une nouvelle constitution le 11 décembre 1990.
} 
encadré par un ensemble hétérogène de règles de droit majoritairement traditionnel dit, « droit Coutumier $»^{1006}$, dont le bréviaire était le «Coutumier dahoméen ${ }^{1007}$ et quelques règles éparses élaborées par l'administration pour régir certains domaines de l'environnement. Le Droit coutumier a son fondement dans la constitution béninoise, en ce que la procédure en matière coutumière est citée au même titre que les règles relevant des domaines de compétence du Législateur béninois ${ }^{1008}$. Par conséquent, le droit coutumier était reconnu par les juridictions, il suppléait la loi ou la complétait à condition de ne pas aller à l'encontre d'une autre ${ }^{1009}$. Le coutumier dahoméen n'était pas une codification spéciale concernant l'environnement, mais plusieurs aspects développés à travers ces normes avaient un lien avec la politique de protection de l'environnement de sorte qu'implicitement, l'environnement était protégé par cet outil juridique.

Comment la protection environnementale était organisée sous le régime juridique du «Coutumier dahoméen »?

Dans le coutumier dahoméen, les préoccupations environnementales ont été prises en compte à travers la protection du droit patrimonial ${ }^{1010}$. Le droit patrimonial avait pour nœud gordien la protection de la propriété collective qui obéissait à un certain nombre de principes définis dans le Coutumier dahoméen. Concernant le régime juridique de la propriété collective contenu dans le Coutumier dahoméen, son point 223 dispose que, «La propriété collective du village comprend des terrains indivis censés appartenir personnellement au roi, entretenus par

\footnotetext{
${ }^{1006}$ Le droit coutumier est un ensemble de règles reposant sur la coutume, donc sur l'usage. La coutume peut être définie comme une règle de conduite, une habitude suivie par un groupe social donné. Elle résulte d'un usage plus ou moins prolongé et est transmise de génération en génération. Droit coutumier est disponible sur le site internet : https://justice.ooreka.fr/astuce/voir/525715/droit-coutumier. Consulté le 16/01/2022.

${ }^{1007}$ Le coutumier dahoméen est un document conventionnel édité par circulaire A. P. 128 du 19 mars 1931 par le gouvernement général de l'Afrique Occidentale Française pour le compte de la colonie du Dahomey. Il a été rédigé en tenant compte des principales coutumes du Dahomey, de leurs ressemblances et de leurs différences. Pour chacune des questions, la règle qui a été reconnue comme appartenant à la majorité des coutumes est énoncée en premier lieu, puis les exceptions sont énumérées chacune étant examinée sous le nom de la coutume qui l'admet. La règle générale est donc applicable à toutes les coutumes qui ne sont pas étudiées comme exceptions. Les règles énoncées par le coutumier ne sont pas des articles de code. Elles rappellent la coutume ancienne et marquent les tendances nouvelles. Elles constituent donc l'état de la question à l'époque présente. Mais comme elles admettent une évolution, quant à l'avenir, une des pages laissées en blanc dans la brochure servira à l'inscription, en regard du texte imprimé, des règles qui auront pu être relevées et invoquées au cours de jugements et qui ne figurent pas au coutumier. Droit coutumier est disponible sur le site internet : https://justice.ooreka.fr/astuce/voir/525715/droitcoutumier. Consulté le 16/01/2022.

${ }^{1008}$ Voir article 98 troisième $\left(3^{\mathrm{e}}\right)$ tiret de la constitution béninoise du 11 décembre 1990 révisée le 07 novembre 2019.

${ }^{1009}$ Dans la hiérarchie des normes en absence de loi ou de règlement d'encadrement, recours est fait aux coutumes pour suppléer le vide juridique. Parfois, en cas de règles obscures, la coutume intervient pour en faciliter la compréhension ou aider à l'interprétation.

${ }^{1010}$ Le droit patrimonial est l'ensemble des relations juridiques qui régissent la possession des biens, des droits et des obligations ayant une valeur pécuniaire d'une personne juridique. Disponible sur le site internet: https://www.toupie.org/Dictionnaire/Droit_patrimonial.htm.Consulté le 16/01/2022.
} 
le village et sur lesquels tous ont droit de culture, pacage, affouage, ou de chasse ou de pêche, (...)». Il ressort de ce qui précède que les biens appartenant à une communauté sont gérés par son représentant qui rend compte de l'usufruit ${ }^{1011}$. De toutes les manières, les biens doivent demeurer « ad vitam aeternam » ${ }^{1012}$ dans le patrimoine de la communauté car ils « (...) sont inaliénables (...) ${ }^{1013}$. Le Coutumier dahoméen de part ces principes a posé les bases du « collectivisme » du droit patrimonial ajouté au caractère « inaliénable des biens collectifs ». C'est le régime du collectivisme que soutient Georges Barnabé GBAGO qui déclara dans l'une de ses communications sur le thème: La propriété collective africaine et les défis environnementaux, qu' "Il faut maintenir le régime du collectivisme dans la succession, c'est un gage pour la protection de l'environnement ${ }^{1014}$. Et poursuit en ces termes que, «le régime d'individualisme ne correspond pas aux réalités socioculturelles de l'Afrique, il faut alors préconiser la coexistence du collectivisme et de l'individualisme, pour être en phase avec les réalités actuelles du Bénin $»^{1015}$. Dans tous les cas, la famille de même que la communauté n'a que le fructus ${ }^{1016}$, l'usus ${ }^{1017}$ mais pas l'abusus, ${ }^{1018}$ les biens étant une parfaite propriété de ses auteurs ne pouvaient faire l'objet de vente. Pour renforcer le caractère inaliénable des biens patrimoniaux collectifs, il a été déclaré que certains biens publics sont sacrés selon le point 224 $\mathrm{du}$ Coutumier dahoméen qui dispose, qu' "il existe enfin une espèce de domaine public religieux : bois, rivières, lieux saints, terrains servant à l'inhumation (en cas de mort par maladie contagieuse), inaliénables et sacrés ». Ce qui avait pour conséquence, une limitation dans les transactions foncières.

Même si la protection de l'environnement n'est pas expressément citée dans le Coutumier dahoméen, mais par l'analyse de certaines dispositions, il en ressort que la protection

${ }^{1011}$ L'usufruit signifie en droit réel principal, le démembrement du droit de propriété qui confère à son titulaire le droit d'utiliser la chose et d'en percevoir les fruits, mais non celui d'en disposer, lequel appartient au nupropriétaire. Raymond Guillien, JeanVincent, Serge Guinchard, Gabriel Montagnier et Thierry Debard, Lexique des termes juridiques, Dalloz, 1970, 567p., p.538.

${ }^{1012}$ Mots latins signifiant pour la vie éternelle. Disponible sur le site internet: https://www.larousse.fr/dictionnaires.Consulté le 16/01/2022.

1013 Paragraphe 223 du coutumier Dahoméen.

${ }^{1014}$ AÏVO (Frédéric Joël), FAURE (Michael), GBAGO (Georges Barnabé) et TCHOKA (François), Enjeux économiques et environnementaux des maîtrises foncières en Afrique, $1^{\mathrm{e} e}$ éd., Paris, Anthemis, 2019. 1015 Id.

${ }^{1016}$ En droit civil, mot latin désignant l'un des attributs du droit de propriété sur une chose, le droit d'en percevoir les fruits, au sens large du terme. confère l'ouvrage de : Raymond Guillien, JeanVincent, Serge Guinchard, Gabriel Montagnier et Thierry Debard, Lexique des termes juridiques, Dalloz, 1970, 567p., p.263.

${ }^{1017}$ En droit civil, Les prérogatives attachées à la propriété, droit de détenir et d'utiliser une chose sans percevoir les fruits. confère l'ouvrage de : Raymond Guillien, JeanVincent, Serge Guinchard, Gabriel Montagnier et Thierry Debard, Lexique des termes juridiques, Dalloz, 1970, 567p., p.539.

${ }^{1018}$ L'abusus, mot latin désignant l'un des attributs du droit de propriété, le droit de disposer (disposition juridique par aliénation ou disposition matérielle par la destruction). Confère l'ouvrage de : Raymond Guillien, JeanVincent, Serge Guinchard, Gabriel Montagnier et Thierry Debard, Lexique des termes juridiques, Dalloz, 1970, 567 p., p.4. 
des propriétés, individuelle, familiale et collective consacrée par le Coutumier dahoméen, concourait à la protection de l'environnement. Le maintien de la propriété collective ou du bien indivis dans le giron familial permet de préserver l'environnement, de conserver la biodiversité et de maintenir l'équilibre dans l'écosystème. Il s'ensuit que, les parcelles de terre qui demeurent sous l'emprise familiale sont sensées être protégées par les pratiques endogènes et ancestrales et échappent à la déforestation et aux pratiques culturales peu favorables à la protection de l'environnement. La promotion de la propriété collective au détriment de la propriété individuelle constituait un atout de protection de l'environnement. Ce qui fait que, durant la période qui a précédé l'accession à la souveraineté internationale ${ }^{1019}$, le Dahomey a connu le maintien de vastes étendues de forêts dans l'ensemble du pays avec une stabilité écologique entretenue par le régime juridique du droit patrimonial contenu dans le Coutumier dahoméen.

Y avait-il des possibilités de protection du climat avec le droit traditionnel?

L'analyse exégétique de certaines dispositions du Coutumier dahoméen permet de se rendre compte qu'il y avait des éléments de l'environnement qui sont protégés par le droit traditionnel. A titre illustratif, de vastes étendues de forêts déclarées, "forets sacrées » sont protégées par les pratiques endogènes, les divinités, ces dernières assurent l'intégrité du patrimoine forestier. Dans ces cas, le couvert végétal protégé avec le régime du Coutumier dahoméen contribue à la réalisation des objectifs d'atténuation par l'absorption de gaz à effet de serre. Dans le contexte de la Convention Cadre des Nations Unies sur les Changements Climatiques le patrimoine naturel pouvait contribuer à la mise en œuvre du Mécanisme du Développement Propre (MDP) développé dans le Protocole de Kyoto ${ }^{1020}$ et aider à la réalisation des objectifs du Mécanisme du Développement Durable institué dans 1'Accord de Paris ${ }^{1021}$.

Cependant, le droit traditionnel à tôt fait de présenter ses limites avec l'évolution démographique galopante qui a contribué à l'accentuation de la pression anthropique et l'apparition des changements climatiques. Ce droit était même devenu caduc ${ }^{1022}$ compte tenu des nouvelles prescriptions du droit international de l'environnement et les exigences en matière de lutte contre les changements climatiques. Pendant ce temps, les problèmes environnementaux s'augmentaient, le couvert végétal qui existait depuis le mathusalem a

\footnotetext{
${ }^{1019}$ Le $1^{\text {er }}$ aout 1960 le Dahomey a été indépendant.

${ }^{1020}$ Selon les dispositions de l'article 12 du Protocole de Kyoto.

${ }^{1021}$ Le Mécanisme du Développement Durable institué par l'article 6 dudit Accord.

${ }^{1022}$ Etat de caducité d'un acte juridique valable mais privé d'effet en raison de la souvenance d'un fait postérieurement à sa création. Voir l'ouvrage de Raymond Guillien, JeanVincent, Serge Guinchard, Gabriel Montagnier et Thierry Debard, Lexique des termes juridiques, Dalloz, 1970, 567p., p.77.
} 
commencé par subir les affres de l'Homme. Plusieurs étendues de forêts sont dévastées pour assouvir les besoins de l'Homme sous le regard impuissant des autorités. Celles-ci ne disposent plus d'un arsenal juridique capable de contenir les actes de vandalisme que subissaient les forêts. Tous ces scenarios ont contribué à l'aggravation des changements climatiques et à l'exacerbation de ses effets de sorte que le Dahomey (Bénin) a migré du statut de pays « d'absorption » à celui « d'émission » de gaz à effet de serre.

Face aux difficultés qui découlent de l'état de caducité des outils juridiques d'alors, de nouvelles mesures d'encadrement s'imposent.

A partir de l'année 1980, pour suppléer le caractère limitatif du droit coutumier, le Bénin s'est engagé dans les accords internationaux de protection de l'environnement. Plusieurs conventions ont été signées et ratifiées notamment, la Convention sur l'interdiction d'utiliser des techniques de modification de l'environnement à des fins militaires ou toutes autres fins hostiles, ouverte à la signature le 10 décembre 1976 à New - York et ratifiée le, 30 juin $1986^{1023}$ ; la Convention concernant la protection du patrimoine mondial culturel et naturel adoptée le 16 novembre 1972 à Paris sous l'égide de l'UNESCO et ratifiée le 14 juin $1982^{1024}$; la Convention Cadre des Nations Unies sur les Changements Climatiques ${ }^{1025}$ et la Convention sur

\footnotetext{
${ }^{1023} \mathrm{La}$ Convention sur l'interdiction d'utiliser des techniques de modification de l'environnement à des fins militaires ou toutes autres fins hostiles a été approuvée par l'Assemblée générale de l'Organisation des Nations Unies dans sa résolution 31/72 du 10 décembre 1976. En application de l'alinéa 2 du dispositif de cette résolution, le Secrétaire général a décidé d'ouvrir la Convention à la signature et à la ratification des États du 18 au 31 mai 1977 à Genève (Suisse). Après cette date, la Convention a été transmise au Siège de l'Organisation des Nations Unies à New York, où elle est restée ouverte à la signature des Etats jusqu'au 4 octobre 1978. Elle a été ratifiée par soixante dix-huit (78) Parties dont la République du Bénin. Nations Unies, Recueil des Traités, vol. 1108, p. 151, et notification dépositaire C.N.263.1978.TREATIES-12 du 27 octobre 1978. Disponible sur le site internet : https://treaties.un.org. Consulté le 27/05/2021.

${ }^{1024} \mathrm{Au} 23$ octobre 2020, la Convention concernant la protection du patrimoine mondial culturel a été ratifiée par cent quatre vingt quatorze (194) Parties dont la République du Bénin. Disponible sur site internet: https://whc.unesco.org/fr/etatsparties. Consulté le 27/05/2021.

${ }^{1025} \mathrm{La}$ Convention Cadre des Nations Unies sur les Changements Climatiques a été arrêtée et adoptée par le Comité intergouvernemental de négociation d'une convention-cadre sur les changements climatiques, lors de la deuxième partie de sa cinquième session, tenue à New York du 30 avril au 9 mai 1992. Conformément à son article 20, la Convention a été ouverte à la signature des Etats Membres de l'Organisation des Nations Unies ou membres d'une institution spécialisée des Nations unies ou parties au Statut de la Cour internationale de Justice ainsi que des organisations d'intégration économique régionale à Rio de Janeiro, pendant la Conférence des Nations unies sur l'environnement et le développement, du 4 au 14 juin 1992 et ensuite au Siège de l'Organisation des Nations Unies à New York, du 20 juin 1992 au 19 juin 1993. Elle a été ratifiée par cent quatre vingt dix-sept (197) Parties dont une organisation régionale d'intégration économique et la République du Bénin. Confère : Nations Unies, Recueil des Traités, vol. 1771, p. 107; notifications dépositaires C.N.148.1993.TREATIES-4 du 12 juillet 1993 (procèsverbal de rectification des textes authentiques de la Convention); C.N.436.1993.TREATIES-12 du 15 décembre 1993 (rectificatif à la notification dépositaire C.N.148.1993.TREATIES-4 du 12 juillet 1993); C.N.247.1993.TREATIES-6 du 24 novembre 1993 (procès-verbal de rectification du texte original français); C.N.462.TREATIES-13 du 30 décembre 1993 (rectificatif à la notification dépositaire C.N.247.1993.TREATIES6 du 24 novembre 1993); C.N.544.1997.TREATIES-6 du 13 février 1998 (amendement à la liste de l'annexe I de la Convention); et C.N.1478.2001.TREATIES-2 du 28 décembre 2001 (amendement à la liste de l'annexe II de la Convention)); C.N.237.2010.TREATIES-2 du 26 avril 2010 (adoption d'amendement à la liste figurant à l'Annexe I de l'Accord); C.N.355.2012.TREATIES-XXVII.7 du 9 juillet 2012 (adoption d'amendements à l'annexe I de la
} 
la Diversité Biologique ${ }^{1026}$, conjointement ratifiées le 13 décembre 1993 et la Convention sur la désertification et la sécheresse ratifiée le 28 août $1996^{1027}$. Par cet engagement dans les accords internationaux d'envergure, le Bénin a commencé par opérer la migration de son système juridique vers un droit moderne en matière de protection de l'environnement et de lutte contre les changements climatiques.

Les accords internationaux ont impacté l'ordonnancement juridique béninois et ont contribué à la création d'un corps de règles de droit destiné à la protection de l'environnement, dont le pilier principal est la loi $\mathrm{n}^{\circ}$ 98-030 du 12 février 1999 portant loi cadre sur l'environnement en République du Bénin. La participation du Bénin au Sommet de Rio de Janeiro de 1992 et son adhésion à la Convention Cadre des Nations Unies sur les Changements Climatiques, ont propulsé le Bénin dans le giron de la Communauté internationale, désormais engagée pour le combat contre les changements climatiques. C'est dans ces conditions qu'est intervenue, la loi $\mathrm{n}^{\circ} 2018-18$ portant règlementation des changements climatiques. Cette loi constitue le pilier du régime juridique élaboré par le Législateur pour relever le défi de la lutte contre les changements climatiques au Bénin. Dans le processus d'harmonisation de la coutume avec les principes fondamentaux, tel que prévu dans la constitution béninoise du 11 décembre 1990 révisée le 07 novembre 2019, certaines dispositions du Coutumier dahoméen sont prises en compte par le Législateur béninois. La loi sur les changements climatiques a d'ailleurs valorisé certains aspects du Coutumier dahoméen en donnant la responsabilité à l'Etat de promouvoir les connaissances, le savoir-faire et les capacités endogènes à travers le

Convention) et C.N.81.2013.TREATIES-XXVII.7 du 14 janvier 2013 (entrée en vigueur d'amendements à l'annexe I de la Convention). Disponible sur le site internet : https://treaties.un.org. Consulté le 27/05/2021.

${ }^{1026}$ La Convention sur la Diversité Biologique a été adoptée par le Comité intergouvernemental de négociation d'une Convention sur la diversité biologique, lors de sa cinquième session tenue à Nairobi du 11 au 22 mai 1992. La Convention a été ouverte à la signature à Rio de Janeiro par tous les États et les organisations d'intégration économique régionale du 5 juin 1992 au 14 juin 1992, et au Siège de l'Organisation des Nations Unies, à New York, du 15 juin 1992 au 4 juin 1993. Elle a été ratifiée par 196 Partie dont le Bénin. Confère Nations Unies, Recueil des Traités, vol. 1760, p. 79; et notification dépositaire C.N.29.1996.TREATIES-2 du 18 mars 1996. Disponible sur le site internet : https://treaties.un.org. Consulté le 27/05/2021.

${ }^{1027}$ Convention des Nations unies sur la lutte contre la désertification dans les pays gravement touchés par la sécheresse et/ou la désertification, en particulier en Afrique a été adoptée le 17 juin 1994 par le Comité intergouvernemental de négociation pour l'élaboration d'une Convention internationale sur la lutte contre la désertification dans les pays gravement touchés par la sécheresse ou par la désertification, en particulier en Afrique (créé en vertu de la résolution 47/188³ de l'Assemblée générale des Nations unies en date du 22 décembre 1992), lors de sa cinquième session tenue à Paris. La Convention a été ouverte à la signature à Paris par tous les États et les organisations d'intégration économique régionale, les 14 et 15 octobre 1994. Elle est restée ouverte à la signature au Siège de l'Organisation des Nations unies à New York jusqu'au 13 octobre 1995. Elle a été ratifiée par 197 Partie dont le Bénin. Confère : Nations Unies, Recueil des Traités, vol. 1954, p. 3; notification dépositaire C.N.176.1995.TREATIES-6 du 27 juillet 1995 (procès-verbal de rectification du texte authentique chinois); et C.N.513.2000.TREATIES-9 du 19 juillet 2000 [procès-verbal de rectification du texte authentique russe); et C.N.1490.2000.TREATIES-16 du 6 mars 2001 (adoption de l'annexe V) et C.N.866.2001.TREATIES-5 du 17 septembre 2001. Disponible sur le site internet : https://treaties.un.org. Consulté le 27/05/2021. 
renforcement des capacités et aptitudes des ressources humaines en ordonnant « la mobilisation de ressources financières et matérielles à consacrer à la formation et à la recherche dans le domaine de l'environnement, en particulier, des changements climatiques ${ }^{1028}$. La même loi incite l'Etat à «encourager l'utilisation et la diffusion des connaissances, savoir-faire et pratiques endogènes en adaptant les technologies écologiquement rationnelles et les méthodes d'agriculture et de gestion des terroirs compatibles avec les conditions socio-économiques nationales $\gg .{ }^{1029}$

De tout point de vue, passée l'époque peu glorieuse de l'application du Droit coutumier, l'encadrement juridique de l'environnement avec les outils juridiques modernes a pris son envol et a considérablement évolué au Bénin malgré le faible taux de texte élaboré.

Le droit positif béninois s'approprie de toutes les manières, des principes élaborés au cours des rencontres internationales destinées à la lutte contre les changements climatiques, ce qui a induit l'existence d'un cadre juridique protecteur du climat.

\section{B-L'existence d'un cadre juridique climatique}

Le droit positif béninois a connu une amplification importante depuis que le Bénin s'est engagé pour la mise en œuvre des instruments élaborés à l'occasion de la Conférence des Nations unies sur 1'Environnement et le Développement tenue à Rio de Janeiro en 1992. Cette productivité normative est justifiée par la détermination du Bénin à freiner les désastres climatiques et se traduit notamment, par la multiplication des textes législatifs et réglementaires à objectifs généraux destinés à la protection de l'environnement ${ }^{1030}$. Cet engouement à l'encadrement du domaine de protection de l'environnement est également motivé par la tendance de plus en plus marquée des pouvoirs publics à recourir au droit, comme moyen de lutte contre toute forme de dégradation de l'environnement. En dépit de ce que la constitution béninoise du 11 décembre 1990 révisée par la loi 2019-40 du 07 novembre 2019 a jeté les bases juridiques de la protection de l'environnement à travers les dispositions de ses articles 27, 28, 29, 74 et 98, plusieurs autres textes législatifs de portée générale avec leurs textes réglementaires d'application sont élaborés, pour encadrer l'environnement dans son ensemble,

\footnotetext{
${ }^{1028}$ Article 17 alinéa 2 de la loi $\mathrm{n}^{\circ} 2018-18$ portant sur la règlementation des changements climatiques en République du Bénin. ${ }^{1029}$ Id.

${ }^{1030}$ Il s'agit entre autres de la loi n $98-030$ du 12 février 1999 portant loi cadre sur l'environnement en République du Bénin et de la loi n 93-009 du 02 juillet 1993 portant régime des forêts en République du Bénin.
} 
puis ses composants. Mais spécifiquement, un encadrement orienté dans le sens de la lutte contre les changements climatiques a pris corps.

Face à la nécessité de la préservation de l'environnement contre les effets néfastes des changements climatiques, le Bénin a adopté la loi n²018-18 relative aux changements climatiques.

La loi sur les changements climatiques au Bénin est un ensemble de règles élaborées, qui « (...) s'applique aux écosystèmes continentaux, littoraux et marins ainsi qu'aux eaux connexes et aux établissements humains relevant de la souveraineté de l'Etat ${ }^{1031}$. Cette loi qui comporte quatre vingt dix-huit (98) articles, régit également « (...) toutes les actions, activités, mesures et initiatives entrant dans le cadre de la lutte contre les changements climatiques et leurs effets et conséquences négatifs $\gg{ }^{1032}$. La règlementation béninoise vise «(...) à lutter contre les changements climatiques, ainsi que leurs effets et conséquences négatifs et à accroitre la résilience des communautés vivantes. Elle permet entre autres, de prendre des mesures efficaces de riposte, d'adaptation et d'atténuation en fixant des objectifs précis de développement économique et social durable, de sécurité et d'efficacité énergétique conformément aux dispositions spécifiques des instruments juridiques nationaux et internationaux relatifs aux changements climatiques » ${ }^{1033}$. La loi prescrit que « toute stratégie de développement et leur déclinaison aux niveaux national, départemental, communal et local intègrent la dimension concernant les changements climatiques sans occulter la durabilité environnementale et la réduction des risques de catastrophes naturelles»» ${ }^{1034}$. La loi sur les changements climatiques a entériné l'existence de certaines institutions notamment, le Comité National sur les Changements Climatiques ${ }^{1035}$ et la Commission de Modélisation Economique des Impacts et de l'Intégration des Changements Climatiques dans le Budget Général de l'Etat en leur consacrant quelques dispositions ${ }^{1036}$. Alors que ces institutions avaient été créées par actes règlementaires ${ }^{1037}$.

L'ensemble de ces textes nationaux et internationaux forme un dispositif juridique important qui concourt à donner un corps au Droit de Protection du Climat au Bénin.

\footnotetext{
${ }^{1031}$ Article 2 alinéa 1 de la loi 2018 -18 portant règlementation sur les changements climatiques au Bénin.

${ }^{1032}$ Article 2 alinéa 2 de la loi 2018 -18 portant règlementation sur les changements climatiques au Bénin.

1033 Article 4 de la loi 2018 -18 portant règlementation sur les changements climatiques au Bénin.

1034 Article 7 id.

${ }^{1035}$ Article 77 ibid.

${ }^{1036}$ Article 79 ibid.

${ }^{1037} \mathrm{La}$ Commission de Modélisation Economique des Impacts et de l'intégration des Changements Climatiques dans le Budget Général de 1'Etat avait été créée par le décret n²014-359 du 16 juin 2014. Disponible sur le site internet : https://sgg.gouv.bj/doc/decret-2014-359. Consulté le 16/01/2022.
} 
Seulement que si l'Etat béninois a le mérite d'accomplir un effort important pour doter le pays d'un arsenal juridique spécialement destiné aux changements climatiques, ce régime juridique reste encore imparfait. L'encadrement juridique climatique béninois ne traite pas de manière holistique la problématique de la lutte contre les changements climatiques. Ce texte de loi demeure un peu laconique voire insuffisant pour contribuer à enrayer efficacement les changements climatiques. A titre indicatif, certains aspects pratiques énumérés dans la Convention Cadre des Nations Unies sur les Changements Climatiques, devant induire la réduction substantielle des émissions des gaz à effet de serre n'ont pas été convenablement internalisés. Un regard sur les dispositions de la loi n²018-18 relative aux changements climatiques permet de constater l'absence des mesures d'application des normes relatives à la mise en œuvre de l'article 12 du Protocole de Kyoto relatif au «Mécanisme pour un Développement Propre » et de l'article 6 de l'Accord de Paris instituant le « Mécanisme du Développement Durable ». En effet, puisque la responsabilité principale des Etats en voie de développement dans la mise en œuvre de la Convention Cadre des Nations Unies sur les Changements Climatiques entre autres, consiste à aider les Etats pollueurs à se débarrasser des émissions de gaz à effet de serre ${ }^{1038}$, il serait recommandable que l'encadrement juridique des Etats en voie de développement en l'occurrence celui du Bénin prenne en compte des mesures devant conduire à l'effectivité du « Mécanisme du Développement Propre » ${ }^{1039}$ dans le contexte du Protocole de Kyoto ou du « Mécanisme du Développement Durable » s'agissant de l'Accord de Paris. Surtout que le contexte béninois est propice à l'applicabilité de ces mesures dont l'effectivité dépend des paramètres climatologiques qui sont très favorables au développement du couvert végétal. L'effectivité des mesures de promotion du « Mécanisme du Développement Propre » et celui du « Mécanisme du Développement Durable » peut être profitable aux pays en voie de développement, dans la mesure où, selon les prescriptions de la Convention climat, en s'engageant pour la réduction substantielle des émissions des gaz à effet de serre, cet engagement génère des ressources financières importantes au profit des ces Etats mais en plus le transfert de la technologie devient effectif, lesquels moyens seront déployés dans l'économie pour éradiquer la pauvreté et booster le développement. Autrement dit, l'effectivité de l'action climatique peut entrainer les Etats à économie modeste dans le processus du développement durable.

\footnotetext{
${ }^{1038}$ Article 12 paragraphe 1 du Protocole de Kyoto à la Convention Cadre des Nations Unies sur les Changements Climatiques.

${ }^{1039}$ Id.
} 
Nonobstant ces insuffisances, force est de constater que le processus d'intégration des instruments internationaux au cadre juridique béninois a contribué au renforcement de l'environnement juridique de protection du climat.

\section{Paragraphe 2: Le renforcement de l'environnement juridique}

Selon le Professeur Michel PRIEUR, pour ce qui concerne la mise en œuvre des normes internationales, leur «incorporation dans le droit national peut obliger à des réformes importantes ${ }^{1040}$. Cette assertion ne manque pas de pertinence, car l'intégration de la Convention Cadre des Nations Unies sur les Changements Climatiques au droit positif béninois a induit des modifications de l'encadrement juridique de ce pays, qui a connu une amélioration matérialisée entre autres par l'adoption de la loi $n^{\circ} 2018-18$ portant règlementation sur les changements climatiques en République du Bénin. Cette loi a donné corps à un ensemble de règles qui a contribué au renforcement de l'environnement juridique destiné à la protection du climat.

Cet environnement juridique essentiellement orienté vers les objectifs d'atténuation et d'adaptation aux effets des changements climatiques est constitué entre autres, de mesures de promotion des énergies renouvelables (A) et des mesures dites des «obligations d'intégration des changements climatiques $»(\mathbf{B})$.

\section{A- La promotion des énergies renouvelables}

Les évaluations sur les changements climatiques concernant la période de 1990 à 1992 effectuées par le Groupe d'Experts Intergouvernemental sur l'Evolution du Climat (GIEC) portaient entre autres, sur les questions de «Limitation des émissions nettes imputables au secteur de l'énergie ». Ces études ont été spécifiquement orientées sur la question relative à la production, à l'utilisation et à l'exploitation de l'énergie. Il a été révélé qu'il y a accroissement de la consommation de l'énergie, parce que selon les termes du rapport, « (...) la production et la consommation d'énergie entrent pour environ de moitié dans le forçage radiatif résultant des activités humaines $\gg{ }^{1041}$. A l'origine de l'engouement à la consommation de l'énergie se trouvent, l'explosion démographique, les réformes structurelles opérées dans le domaine de

\footnotetext{
${ }^{1040}$ Michel PRIEUR, «L'influence des conventions internationales sur le droit interne de l'environnement». Actes de la réunion constitutive du comité sur l'environnement de l'AHJUCAF, Juin 2008, Porto-Novo, Bénin, pp.291301.

${ }^{1041}$ Changements climatiques, premier rapport du GIEC élaboré pour la période de 1990 à 1992 sur les évaluations des changements climatiques, Page 138-4.1.
} 
l'économie, le ravitaillement en combustibles fossiles et les possibilités d'exploitation de l'énergie nucléaire de même que le recours aux énergies renouvelables qui constituent des facteurs qui pourront influencer les émissions de gaz à effet de serre et impacter le climat. Les solutions envisageables dans le cadre de ces études sont formulées en termes «d'amélioration du rendement énergétique » et de « l'économie d'énergie » ${ }^{1042}$.

Dans l'hypothèse que l'accroissement de la consommation d'énergie est susceptible de modifier le climat, l'enjeu de l'étude portant sur la question énergétique se trouve dans la méthodologie à adopter pour rechercher les formes d'énergie et les conditions d'utilisation et d'exploitation qui contribuent à l'amélioration du climat. Dans ce cadre, le GIEC a recommandé «l'utilisation efficace des ressources énergétiques notamment, les combustibles fossiles émettant moins de gaz a effet de serre ou de sources d'énergies non fossiles, le développement dans la mesure du possible de sources d'énergies propres et renouvelables comme la biomasse, l'énergie éolienne, l'énergie des vagues, l'énergie hydroélectrique et l'énergie solaire ${ }^{1043}$. Les préoccupations énergétiques ont également transparu dans le Protocole de Kyoto sous forme de solution à la question de la réduction des émissions de gaz à effet de serre de provenance énergétique. En effet, le traité international sur les changements climatiques a prescrit à la charge de chacune des Parties, qu'elle « (...) élabore plus avant des politiques et des mesures en fonction de sa situation nationale, relatives à l'accroissement de l'efficacité énergétique dans les secteurs pertinents de l'économie nationale $\gg{ }^{1044}$. Cette disposition a initié le concept de «l'efficacité énergétique ${ }^{1045}$, comme facteur sur lequel la communauté peut agir pour modifier positivement les données climatiques. Dans le même texte conventionnel, la Communauté internationale a recommandé aux Etats Parties de prendre des mesures en faveur de l'accroissement de «l'efficacité énergétique ». L'institution mondiale qualifiée pour évaluer les changements climatiques et ses effets négatifs projette la possibilité de contenir l'élévation de la température planétaire à $1,5^{\circ} \mathrm{C}$, avec l'organisation des « transitions rapides et profondes

\footnotetext{
${ }^{1042}$ Changements climatiques, premier rapport du GIEC élaboré pour la période de 1990 à 1992 sur les évaluations des changements climatiques, Page 138- 4.1.3.

${ }^{1043}$ Changements climatiques, premier rapport du GIEC élaboré pour la période de 1990 à 1992 sur les évaluations des changements climatiques, p.65-4.0.3.

1044 Selon l'article 2 -a-i du Protocole de Kyoto.

1045 Id.
} 
dans les domaines énergétiques» ${ }^{1046}$. La mise en œuvre de ces mesures permettra de réaliser l'objectif de l'Accord de Paris ${ }^{1047}$ qui conduira à la neutralité climatique.

Au Bénin, la Stratégie Nationale pour la Mise en CEuvre de la Convention Cadre des Nations Unies sur les Changements Climatiques et d'autres études similaires ont permis de faire le même constat que celui contenu dans le rapport du GIEC en ce qui concerne le secteur énergétique, que « la principale source d'émission de gaz à effet de serre est imputable à la consommation de combustible fossile à des fins énergétiques qui aboutissent à la libération de la majeure partie du carbone contenue dans ces produits, sous forme de $\mathrm{CO} 2$ et autres gaz à effet de serre tels que le $\mathrm{CO}$, le $\mathrm{CH} 4$, les hydrocarbures non méthaniques, le $\mathrm{NO} »{ }^{1048}$. Pour faire face aux conséquences néfastes qu'induisent la consommation de certaines formes d'énergies impropres à la qualité du climat, il a été proposé dans le document de Stratégie Nationale pour la Mise en Euvre de la Convention Cadre des Nations Unies sur les Changements Climatiques de «mettre en œuvre des mesures efficaces à moindre coût pour la transition vers des sources d'énergie moins intensives en carbone et d'autres éléments qui ne contiennent pas de carbone telles que les énergies renouvelables» ${ }^{1049}$ comme mesure d'atténuation des émissions de gaz à effet de serre dans le secteur de l'énergie.

Le législateur béninois a eu la lucidité de comprendre que la consécration solennelle du droit à l'environnement ne suffit pas pour que les idéaux prôner à travers la Constitution soient effectifs. En réalité, cette formalité doit être relayée dans un souci d'efficacité par des dispositions législatives et réglementaires adéquates. Il a ainsi entrepris la réforme du cadre juridique de protection du climat en intégrant les exigences internationales concernant le secteur de l'énergie. L'aboutissement de ce processus a été l'adoption de la loi n²018-18 portant règlementation sur les changements climatiques en République du Bénin qui a intégré les préoccupations concernant « l'efficacité énergétique ${ }^{1050}$. En considérant ce concept, cette loi a défini comme objectif de « (...) lutter contre les changements climatiques (...) par la prise

\footnotetext{
${ }^{1046}$ Rapport spécial du GIEC sur les conséquences d'un réchauffement planétaire de $1,5 \quad{ }^{\circ} \mathrm{C}$ par rapport aux niveaux préindustriels et les trajectoires associées d'émissions mondiales de gaz à effet de serre, dans le contexte du renforcement de la parade mondiale au changement climatique, du développement durable et de la lutte contre la pauvreté. Disponible sur le site internet: https://www.ipcc.ch. Consulté le 16/01/2022.

${ }^{1047}$ Article 2 paragraphe 1-a dudit Accord.

${ }^{1048}$ Stratégie Nationale pour la Mise en Euvre de la Convention Cadre des Nations Unies sur les Changements Climatiques, page 25. Disponible sur le site internet : https://unfecc.int. Consulté le 16/01/2022.

${ }^{1049}$ Stratégie Nationale pour la Mise en Euvre de la Convention Cadre des Nations Unies sur les Changements Climatiques, p.20. Disponible sur le site internet : https://unfccc.int. Consulté le 16/01/2022.

${ }^{1050}$ Article premier de la loi ${ }^{\circ} 2018-18$ portant règlementation sur les changements climatiques en République du Bénin défini l'«Efficacité énergétique» comme rapport du rendement énergétique d'un processus de transformation ou d'un système à son intrant énergétique.
} 
des mesures efficaces de riposte, d'adaptation et d'atténuation en fixant des objectifs précis de développement économique et social durable, de sécurité et d'efficacité énergétique (...)»» ${ }^{1051}$. En s'inscrivant dans la ligne tracée par les conventions internationales, le législateur béninois à la recherche de solutions efficaces pour lutter contre les effets pervers des changements climatiques à travers l'élaboration de ladite loi, a aussi indiqué «l'efficacité énergétique » comme moyen d'action de lutte contre les changements climatiques au Bénin. Il convient de préciser que la notion «d'efficacité énergétique » n'est qu'une reprise du Protocole de Kyoto $^{1052}$.

La sécurité et l'efficacité énergétique qui figurent en ligne de mire à l'article 4 de la loi sur les changements climatiques représentent des points névralgiques de l'économie du Bénin en relation avec les changements climatiques. Parce que, les émissions de GES dans le secteur de l'énergie au Bénin sont surtout dues à la « consommation des combustibles fossiles dans les transports et à la consommation de la biomasse-énergie dans les ménages notamment, dans les centres urbains dont l'approvisionnement se fait à partir des coupes de bois sur pied $»^{1053}$, malgré les potentialités relativement importantes dont le pays dispose en matière d'énergies renouvelables. Par rapport à cette problématique, le document de stratégie béninoise de mise en œuvre de la Convention Cadre des Nations Unies sur les Changements Climatiques a défini comme objectif à atteindre, la maîtrise de l'évolution de la demande énergétique par l'exploitation des potentialités nationales et les possibilités de coopération internationale et régionale pour développer les sources d'énergies renouvelables qui correspondent aux prescriptions en matière « d'efficacité énergétique ».

\section{Comment faire pour atteindre les objectifs de l'efficacité énergétique?}

Au-delà de la notion de "l'efficacité énergétique » introduite dans le droit positif béninois, pour régler le problème de l'impact de la consommation de l'énergie sur les changements climatiques, le Législateur béninois a prescrit des mesures de promotion des énergies renouvelables à travers la loi $\mathrm{n}^{\circ} 2018$-18 portant, règlementation sur les changements climatiques en République du Bénin en y consacrant entièrement son chapitre $\mathrm{V}^{1054}$. Selon l'article 37 de la loi béninoise sur les changements climatiques, "l'Etat intègre dans sa politique énergétique des mesures visant la promotion des énergies nouvelles et renouvelables

\footnotetext{
${ }^{1051}$ Article 4 de la loi ${ }^{\circ} 2018-18$ portant règlementation sur les changements climatiques en République du Bénin. 1052 Selon les dispositions de l'article 2-a-i du Protocole de Kyoto.

${ }^{1053}$ Document de Stratégie béninoise de mise en œuvre de la Convention Cadre des Nations Unies sur les Changements Climatiques, p.25. Disponible sur le site internet :https://unfccc.int/sites/default/files/resource. Page consultée le 02/06/2021.

${ }^{1054} \mathrm{Ce}$ chapitre est intitulé « des énergies nouvelles et renouvelables».
} 
de manière à augmenter leur part dans le bilan énergétique, en vue d'atteindre la sécurité énergétique ». En effet, l'accent est mis sur la distribution de l'électricité à partir des sources d'énergies nouvelles et renouvelables ${ }^{1055}$ en ce qui concerne la «production, le transport, le stockage qui se fera en respectant les conditions de sûreté et de sécurité prescrites par les textes en vigueur ${ }^{1056}$. Dans ce cadre, il est prévu à la charge de l'Etat, l'organisation d'un dispositif de «promotion des énergies nouvelles et renouvelables et de l'efficacité énergétique » ${ }^{1057}$ devant se dérouler dans le « respect des normes de protection de la santé publique, de l'environnement, des exigences de compétitivité de l'économie nationale et du développement durable ${ }^{1058}$. La loi a consacré l'ouverture du secteur privé aux domaines des «activités de réalisation, d'exploitation, d'extension de la capacité ou de modification des installations de production d'énergie électrique à partir des sources d'énergies nouvelles et renouvelables» ${ }^{1059}$. $\mathrm{La}$ « production des biocarburants ${ }^{1060}$ a été réglementée ${ }^{1061}$ pour éviter de mettre en péril le respect (...) de la protection et la préservation de l'environnement par l'intensification agricole et l'expansion des cultures ${ }^{1062}$. Cependant, la réussite de la promotion de l'énergie renouvelable comme moyen de lutter contre les changements climatiques dépendra entre autres, de l'efficacité des dites mesures. ${ }^{1063}$

En effet, au-delà de l'aspect juridique de la question concernant les énergies renouvelables, qui constituent une opportunité pour atténuer les émissions des gaz à effet de serre, si le pan juridique a pu trouver quelques solutions dans la législation béninoise à travers certaines dispositions qui sont élaborées pour encadrer la matière, il n'en demeure pas moins que les préoccupations relatives à l'effectivité de ces mesures dépendent plus de l'apport technique et scientifique. Cependant, la combinaison de la technologie et de la science en vue

\footnotetext{
${ }^{1055} \mathrm{Au}$ terme de l'article premier de la loi $\mathrm{n}^{\circ} 2018$-18 portant règlementation sur les changements climatiques en République du Bénin, le groupe de mot "énergies renouvelables » est défini comme "source d'énergie qui se constitue ou se reconstitue plus rapidement qu'elle n'est utilisée. Leur exploitation n'entraîne en aucune façon l'extinction de la ressource initiale et elle est renouvelable à l'échelle humaine ».

${ }^{1056}$ Article 38 de la loi ${ }^{\circ} 2018$-18 portant sur la règlementation sur les changements climatiques en République du Bénin.

${ }^{1057}$ Article 39 alinéa premier de la loi n²018-18 portant sur la règlementation sur les changements climatiques en République du Bénin.

${ }^{1058}$ Article 39 alinéa 2 id.

${ }^{1059}$ Article 40 ibid.

${ }^{1060}$ Les biocarburants couvrent l'ensemble des carburants et combustibles liquides, solides ou gazeux produits à partir de la biomasse et destinés à une valorisation énergétique dans les transports et le chauffage. Disponible sur le site internet :https://www.ecologie.gouv.fr/biocarburants. Consulté, le 16/01/2022.

${ }^{1061}$ Article 41 de la $n^{\circ} 2018-18$ portant sur la règlementation sur les changements climatiques en République du Bénin.

1062 Id.

${ }^{1063}$ Articles 7 à 9 de la loi n²018-18 du 18 juin 12018 relative aux changements climatiques en République du Bénin.
} 
de la réalisation des moyens de production des énergies renouvelables nécessite la mobilisation d'importantes ressources financières dont ne disposent pas les pays en voie développement en général, et puis particulièrement le Bénin. La contribution de la science ou de la technique pour la réalisation des dispositifs pouvant générer les énergies renouvelables n'est pas à occulter dans l'effectivité des mesures de substitution des énergies polluantes par des énergies nouvelles compatibles avec les normes climatiques. Ce qui fait surgir l'éternelle question de l'effectivité du transfert de technologies et de moyens financiers vers les pays en voie de développement, pour atténuer les émissions de gaz à effet de serre. Cette question a été élevée au rang de principe dit « de la responsabilité commune mais différenciée » dans la Convention Cadre des Nations Unies sur les Changements Climatiques ${ }^{1064}$. Il en résulte que la résolution des questions relatives à la qualité d'énergie ne peut être résolue sans la contribution des puissances pollueuses et des Etats qui ont pour responsabilité de contribuer à l'ascension des charges induites par la gestion des changements climatiques.

Nonobstant ces obstacles, le Législateur béninois a élaboré une pile de mesure constituant un régime d'obligation d'intégration pour assurer entre autres, l'effectivité de la transition énergétique.

\section{B- Les obligations d'intégration des changements climatiques}

L'Accord de Paris qui fait suite à la Convention Cadre des Nations Unies sur les Changements Climatiques et au Protocole de Kyoto comporte de nombreuses obligations juridiques de résultats. Bien que les engagements précis de limitation d'émissions de gaz à effet de serre sont laissés à la libre convenance des Parties, parce que ne faisant pas partie de l'accord stricto sensu, chaque Partie a quand même l'obligation d'en établir, de la mettre en œuvre et surtout de la réviser à la hausse tous les cinq (5)ans ${ }^{1065}$. En face de la prescription internationale, l'Etat béninois a affiché sa détermination à accomplir sa part d'engagement convenue dans la Convention Cadre des Nations Unies sur les Changements Climatiques. Cette détermination s'était traduite entre autres par l'élaboration de la Stratégie Nationale de la Mise en CEuvre de la Convention Cadre des Nations Unies sur les Changements Climatiques. L'outil qui devrait aider le Bénin à réaliser les objectifs de ses engagements d'atténuation des gaz à effet de serre et d'adaptation aux effets des changements climatiques a recommandé l'intégration dans les politiques, plans, programmes et projets, des préoccupations liées aux changements

\footnotetext{
${ }^{1064}$ Article 3 principe 1 de la Convention Cadre des Nations Unies sur les Changements Climatiques.

1065 Selon les articles 3 et 4 de l'Accord de Paris.
} 
climatiques. Dans ce cadre, la Commission Nationale du Développement Durable a été chargée de réaliser cette mission ${ }^{1066}$.

Néanmoins, le processus d'intégration des dimensions des changements climatiques tel qu'énoncé dans le document de stratégie de mise en œuvre au Bénin de la Convention Cadre des Nations Unies sur les Changements Climatiques n'est pas effectif. Ce document de stratégie n'a aucune force contraignante. Il est perçu comme un acte administratif courant, qui n'est pas auto-exécutoire. L'autre handicap est que, les prescriptions réglementaires en ce qui concerne l'intégration de la dimension de la protection de l'environnement dans les outils stratégiques ne sont pas étendues au volet concernant les changements climatiques. Mais en plus, le caractère trop généraliste de la loi cadre sur l'environnement ${ }^{1067}$ n'a pas non plus favorisé l'application de la Convention Cadre des Nations Unies sur les Changements Climatiques, alors qu'il était indiqué comme une loi devant concourir à sa mise en œuvre. Cependant, les instruments réglementaires existants ne posent aucune contrainte d'intégration de la dimension concernant les changements climatiques aux acteurs de la protection de l'environnement. Tous ces outils ressemblent à la codification internationale, où certains accords internationaux élaborés qui ne font qu'énoncer des principes, c'est-à-dire des mesures qui sont plutôt des directives que des obligations juridiques. La méthodologie qui a consisté à élaborer des outils de mise en œuvre de la Convention Cadre des Nations Unies sur les Changements Climatiques au Bénin, sans être appuyée de dispositions internes spécifiques en la matière, n'était pas de nature à faciliter l'atteinte des objectifs en ce qui concerne la lutte contre les changements climatiques. Dès lors, se posait la problématique de la qualité de l'outil devant aider à faire intégrer réellement les considérations concernant les changements climatiques dans la gouvernance environnementale au Bénin.

A partir de ce moment, la préoccupation pertinente qui s'affichait était de rechercher par quel moyen de droit, faciliter l'intégration de la dimension concernant les changements climatiques aux instruments de protection de l'environnement au Bénin?

Le Législateur béninois en élaborant la loi $n^{\circ} 2018-18$ sur les changements climatiques ne s'est pas éloigné de la disposition constitutionnelle qui stipule que, « toute personne a droit à un environnement sain, satisfaisant et durable et a le devoir de le défendre, que l'Etat veille à la protection de l'environnement ${ }^{1068}$. En effet, il a défini des responsabilités en matière de la

\footnotetext{
${ }^{1066}$ Article 2 du décret $n^{\circ} 2005-068$ du 14 février 2005 portant attributions, organisation et fonctionnement de la commission nationale du développement durable (CNDD).

${ }^{1067}$ Loi n98-030 portant loi cadre sur l'environnement en République du Bénin. Disponible sur le site internet : https://sgg.gouv.bj/doc/loi-98-030. Consulté le 16/01/2022.

${ }^{1068}$ Article 27 de la constitution béninoise du 11 décembre 1990 révisée le 07 novembre 2019.
} 
gestion de la protection de l'environnement qui sont mises à la charge des acteurs que sont l'Etat, les organisations de la société civile et les citoyens. Mais la particularité de cette loi réside dans la définition de certaines mesures devant à la longue, aboutir à l'intégration des dimensions concernant les changements climatiques à l'encadrement juridique, administratif et institutionnel en République du Bénin. Ce qui fait que, le législateur a concocté diverses mesures pour constituer ce qui peut être qualifié, de « régime juridique d'intégration des changements climatiques » qui est logé au chapitre 3 de la loi n²018-18 portant règlementation des changements climatiques en République du Bénin. Le chapitre 3 de ladite loi a prescrit «des obligations de prise en compte des changements climatiques dans les stratégies et planifications nationales et infranationales mises à la charge de l'Etat, de ses démembrements et des citoyens». Il s'ensuit que, désormais, «toute politique et toute stratégie de développement et leur déclinaison aux niveaux, national, départemental, communal et local devront intégrer la dimension changements climatiques sans occulter la durabilité environnementale et la réduction des risques et catastrophes naturelles ${ }^{1069}$. De même, «Les stratégies et planifications existantes au niveau national et infranational sont révisées pour intégrer également la dimension des changements climatiques» ${ }^{1070}$. Par ailleurs, il est recommandé à travers la loi que des dispositions réglementaires soient élaborées en vue de « l'intégration des conclusions et modalités prévues dans les plans nationaux d'adaptation à la stratégie de développement à faible intensité de carbone et résilient aux changements climatiques $\gg{ }^{1071}$. Les obligations d'intégration des changements climatiques sont également mises à la charge des « (...) collectivités territoriales, qui devront adopter et mettre en œuvre un programme spécial destiné à la réhabilitation des zones dégradées, du fait des effets et conséquences des phénomènes naturels et des actions anthropiques ${ }^{1072}$. Ces obligations incombent aussi au citoyen, car la loi stipule que «toute personne physique vivant définitivement ou séjournant temporairement sur le territoire national ou toute personne morale y ayant établi son siège social a le devoir de développer des activités résilientes aux changements climatiques, qui garantissent la préservation et la sauvegarde de la vie humaine, animale et végétale ${ }^{1073}$.

\footnotetext{
${ }^{1069}$ Article 7 alinéa premier de la loi $\mathrm{n}^{\circ} 2018-18$ portant règlementation sur les changements climatiques en République du Bénin.

${ }^{1070}$ Article 7 alinéa 2 de la loi $n^{\circ} 2018$-18 portant règlementation sur les changements climatiques en République du Bénin.

1071 Article 7 alinéa 3 id.

${ }^{1072}$ Article 13 de la loi n 2018 -18 portant règlementation sur les changements climatiques en République du Bénin ${ }^{1073}$ Article 11 id.
} 
Les changements climatiques deviennent dès lors une matière transversale, qui doit être intégrée obligatoirement à tous les secteurs vitaux de l'Etat sous peine de sanction pénale ${ }^{1074}$.

Cependant, suffit-il de faire obligation aux structures de l'Etat d'intégrer les dimensions concernant les changements climatiques dans les, politique, plans, stratégies, projets et programmes de l'Etat, pour espérer relever les défis qu'impose ce fléau?

Suite à l'érection des mesures d'obligation d'intégration des changements climatiques dans la loi sur les changements climatiques, la modification de l'encadrement institutionnel de protection du climat a été réalisée pour accompagner l'encadrement juridique en gestation.

\section{SECTION II: LA MODIFICATION DE L'ENCADREMENT INSTITUTIONNEL}

L'intégration des instruments du droit international de protection de l'environnement au cadre juridique national a contribué à la modification de l'encadrement institutionnel béninois avec pour conséquence l'émergence de nouveaux acteurs (Paragraphe 1) et le renforcement dudit cadre (Paragraphe 2).

\section{Paragraphe 1: L'émergence de nouveaux acteurs}

La nouvelle règlementation en matière de lutte contre les changements climatiques a fait émerger de nouveaux acteurs qui participent désormais à la mise en œuvre du droit.

Ces nouveaux acteurs peuvent être des citoyens qui sont impliqués soit, personnellement (A) soit, par l'entremise des organisations dénommées, " société civile » qui peuvent apporter leur pierre à l'édifice dans le cadre de la lutte contre les changements climatiques (B).

\section{A- Le citoyen}

Il a été estimé dans le rapport spécial de synthèse sur les changements climatiques du Groupe d'Experts Intergouvernemental sur l'Evolution du Climat (GIEC) publié en 2014 que «(..) les activités humaines ont provoqué un réchauffement planétaire d'environ $1{ }^{\circ} \mathrm{C}$ audessus des niveaux préindustriels, avec une fourchette probable allant de $0,8{ }^{\circ} \mathrm{C}$ à $1,2{ }^{\circ} \mathrm{C}$. Il est probable que le réchauffement planétaire atteindra $1,5^{\circ} \mathrm{C}$ entre 2030 et 2052 s'il continue d'augmenter au rythme actuel» ${ }^{1075}$. Alors que le maintien de cette tendance contrarie les efforts

\footnotetext{
${ }^{1074}$ Voir les dispositions des articles 89 à 96 de la loi nº 2018 -18 portant règlementation sur les changements climatiques en République du Bénin.

${ }^{1075}$ Page 6. dudit rapport.
} 
de la Communauté internationale qui s'est donnée pour objectif de cerner le réchauffement global dans une proportion inférieure « à $+2^{\circ} \mathrm{C}$ comparé aux niveaux préindustriels et si possible à $+1,5^{\circ} \mathrm{C}{ }^{1076}$. Dans ce même rapport, constat a été fait de ce que « L'influence de l'homme sur le système climatique est manifeste et aujourd'hui, les émissions de gaz à effet de serre d'origine humaine sont les plus élevées jamais observées. Les changements climatiques récents ont eu de larges répercussions sur les systèmes humains et naturels ${ }^{1077}$. Curieusement, l'Homme qui se trouve à l'origine de la destruction de l'environnement, c'est encore lui qui subit les affres des changements climatiques. C'est pourquoi, les mesures de protection du climat pour la plupart, sont orientées dans le sens de l'encadrement de l'activité humaine pour empêcher qu'elle ne porte atteinte à l'environnement. Néanmoins, si ces règles ne sont pas assimilées et agréées par les acteurs intéressés, elles peuvent ne pas être appliquées. C'est pourquoi, il convient d'associer tous les acteurs tant, au processus d'élaboration des normes de protection du climat qu'à l'étape de leurs mises en œuvre. C'est une belle manière de répondre au cri d'alarme du Président Chirac qui déclarait ceci, « Notre maison brûle et nous regardons ailleurs $»^{1078}$. Une analyse exégétique de ce passage permet de dire que la dernière personne du pluriel, « Nous » utilisée dans cette phrase, est une invitation adressée à tout le monde sans exclusion, pour s'intéresser à la lutte contre les changements climatiques. C'est une manière de faire appel à tout le monde pour contribuer à la sauvegarde du patrimoine commun de l'humanité, «La planète Terre ». Par ailleurs, si les décisions prises lors des négociations intergouvernementales sur le climat prennent place aux niveaux international et national, c'est au niveau local que la plupart des actions concrètes ont lieu. Au niveau local, se trouvent des citoyens qui de part leurs actions peuvent exercer de pression sur les politiques en indiquant leurs espérances. En d'autres termes, l'effectivité du droit des changements climatiques doit être conditionnée par la participation de tous.

Par quel mécanisme impliquer tous les acteurs à la lutte contre les changements climatiques?

Un principe majeur relevant des règles de bonne gouvernance est l'approche participative qui consiste à impliquer tous les acteurs dans l'œuvre de protection de

\footnotetext{
${ }_{1076}$ Article 2 paragraphe1-a de l'Accord de Paris.

${ }^{1077}$ GIEC : CHANGEMENTS CLIMATIQUES 2014, Conclusions titres du Résumé à l'intention des décideurs, Les changements observés et leurs causes, page 1. Disponible sur le site internet : https://www.ipcc.ch/site. Consulté le 16/01/2022.

${ }^{1078}$ En 2002, Jacques Chirac prononce à Johannesburg un grand discours sur l'urgence climatique dans le monde, avec ses mots «Notre maison brûle, et nous regardons ailleurs». Disponible sur le site internet: https://www.rtl.fr/actu/politique/video-mort-de-jacques-chirac-son-discours. Consulté le 16/01/2022.
} 
l'environnement. Cette forme de gouvernance permet de «tenir compte des intérêts et modes de vie des populations dans l'élaboration des règles de protection de l'environnement et à les associer étroitement à leur mise en œuvre ${ }^{1079}$. Elle permet une implication et une responsabilisation du citoyen dans les actions de promotion et de protection de l'environnement. C'est pourquoi, l'approche participative est préconisée par de nombreux instruments internationaux de protection de l'environnement notamment, la Déclaration de Rio de Janeiro de 1992, qui proclame dans son principe 10 que, «La meilleure façon de traiter les questions d'environnement est d'assurer la participation de tous les citoyens concernés, au niveau qui convient. (...) et leur donner la possibilité de participer aux processus de prise de décision ${ }^{1080}$. En tout état de cause, «Les Etats doivent faciliter et encourager la sensibilisation et la participation du public en mettant les informations à sa disposition (...)» ${ }^{1081}$. Pour renchérir les précédentes dispositions, il est prévu dans la Convention Cadre des Nations Unies sur les Changements Climatiques, « la participation publique à l'examen des changements climatiques et de leurs effets et à la mise au point de mesures appropriées pour y faire face $»^{1082}$. Conformément à ces dispositions, les citoyens sont considérés comme des acteurs inconditionnels dans les négociations sur le climat. ${ }^{1083}$

$\mathrm{Au}$ regard des normes internationales, la personne humaine est sacrée et inviolable et mérite ainsi une protection particulière ${ }^{1084}$. Ce principe est d'ailleurs érigé en norme constitutionnelle en République du Bénin. ${ }^{1085} \mathrm{Il}$ s'ensuit que, toute action pouvant impacter négativement la vie de la personne humaine doit être rigoureusement proscrite, c'est ce que traduit l'article 27 de la constitution béninoise du 11 décembre 1990, révisée par la loi 2019-40 du 07 novembre 2019 qui a érigé le bénéfice d'un environnement sain, satisfaisant et durable, en droit pour le citoyen.

\footnotetext{
${ }^{1079}$ Granier Laurent, Aspects contemporains du droit de l'environnement en Afrique de l'Ouest et centrale, UICN, 2008, 224p., p.32.

${ }^{1080}$ Les principes 4 et 19 de la Déclaration de Stockholm de 1972 sur l'environnement humain reconnaissent également l'importance de l'approche participative en matière de protection de l'environnement. La Stratégie mondiale de la conservation insiste pour sa part, sur l'importance de la participation du public et la nécessité de participer à la planification et à la prise de décision concernant l'utilisation des ressources vivantes afin d'éviter des décisions inconsidérées. Page 42. Disponible sur le site internet : https://portals.iucn.org/library/sites/library. Consulté le 16/01/2022.

$1081 \mathrm{Id}$.

1082 Article 6-a-iii de la Convention Cadre des Nations Unies sur les Changements Climatiques.

${ }^{1083} \mathrm{La}$ Commission nationale du débat public, la Danish Board of Technology et Missions publiques. Vaia Tuuhia (4D) - Déléguée Générale Camille André (GERES) - Chargé de mission Commission Climat et développement. Disponible sur le site internet: http://www.geres.eu/fr/paragraphes-de-vue/779-les-citoyens-moteurs-de-lalutte.Consulté le 16/01/2022.

${ }^{1084}$ L'article 4 de la charte africaine des droits de l'homme et des peuples.

${ }^{1085}$ Article 8 de la constitution béninoise du 11 décembre 1990.
} 
Toutefois, le citoyen doit-il se prévaloir de l'existence d'un droit à son profit sans une contrepartie?

Selon le Professeur M. Prieur, « la protection de l'environnement, si elle est devenue une obligation de l'Etat, est avant tout un devoir des citoyens.» Ainsi, l'utilité que représente l'environnement pour l'homme, tout en jouissant de ses avantages dans les conditions idoines, a l'impérieuse obligation d'observer strictement les règles de protection éditées. Seulement qu'au Bénin, si le principe de la jouissance du droit à la protection de l'environnement est établi au profit du citoyen, les dispositions légales n'ont pas clairement conférés de devoirs et d'obligations directes à l'endroit de ce dernier. Néanmoins, l'implication du citoyen dans l'œuvre de protection de l'environnement a été implicitement évoquée dans la Constitution béninoise, laquelle dispose que « Toute personne a droit à un environnement sain, satisfaisant et durable et a le devoir de le défendre ${ }^{1086}$. Cette disposition a été intégrée dans la loi sur les changements climatiques en ces termes : «l'Etat veille à l'information, à la sensibilisation, à l'éducation du public et au renforcement des capacités des acteurs en vue d'une participation de toute personne résidant sur le territoire national à la résolution des problèmes environnementaux et d'une prise de conscience des menaces et risques liés aux effets négatifs des changements climatiques $»^{1087}$. La participation du citoyen à l'œuvre de protection de l'environnement peut s'effectuer par la garantie de l'accès de ce dernier à l'information sur l'environnement notamment, à travers la vulgarisation de l'information environnementale disponible auprès des autorités et l'accès à certains documents officiels élaborés dans le cadre de la protection de l'environnement. Elle passe également par leur implication à l'élaboration des textes d'encadrement de la protection de l'environnement et enfin, elle se traduit par la mise en œuvre des actions juridictionnelles. Il s'ensuit qu'à la lumière de la législation béninoise en matière de protection de l'environnement et de lutte contre les changements climatiques, le citoyen béninois est établi comme un acteur passif, il n'est pas capable de prendre d'initiative indépendante pour intervenir dans ces domaines.

Néanmoins, en vertu du principe de la liberté d'association, les citoyens peuvent constituer de mouvement associatif classé dans la catégorie de la «société civile» pour contribuer à la protection du climat.

${ }^{1086}$ Article 27 de la constitution béninoise du 11 décembre 1990 .
${ }^{1087}$ Article 24 de la loi n ${ }^{\circ} 2018-18$ de la loi portant règlementation sur les changements climatiques. 


\section{B-La Société civile}

Avec la complexité que revêt la lutte contre les changements climatiques, le cadre étatique ne suffit plus pour répondre aux nombreuses sollicitudes de la matière. Le droit qui régit le domaine « commande par son objet même le dépassement du cadre étatique face à un enjeu liant tous les habitants de la planète ${ }^{1088}$. Ainsi, en dehors de la contribution coutumière des Etats et Organisations gouvernementales internationales, l'apport d'institutions non étatiques en l'occurrence celle de la société civile est souhaitée dans ce domaine précis.

La Société civile a été présente à l'occasion du Sommet de Rio de Janeiro et a participé à l'élaboration de la Convention Cadre des Nations Unies sur les Changements Climatiques (CCNUCC). Seulement, qu'elle n'est pas signataire de ladite Convention et n'est pas non plus assujettie à des engagements formels découlant des négociations. Malgré cela, la Société civile constitue un acteur non négligeable, très sollicité en matière de lutte contre les changements climatiques sur le plan international, parce qu'elle est surtout « porteuse de solutions locales et régionales $\gg{ }^{1089}$. Par ailleurs, la Société civile fait le plaidoyer auprès des Etats pour les motiver à élaborer ou à améliorer des normes tout en leur accordant son appui technique et scientifique. Cependant, leur marge de manœuvre reste limitée parce que si, cette entité a la possibilité d'initier un accord international, elle reste toutefois dépendante d'un sujet de droit international qui devra soutenir cette initiative et qui prendra le relai en organisant une conférence internationale à cet effet. De la même manière, un sujet de droit international peut demander à la société civile d'élaborer un avant-projet de convention ${ }^{1090}$. A titre indicatif, « l'OUA a chargé l'UICN de préparer un projet de convention sur la conservation de la nature. Le résultat ne s'est pas fait longtemps attendre puisque la Convention a été signée à Alger en 1968 (...) et rapidement entrée en vigueur dès $1969 »^{1091}$

Depuis la tenue de l'historique conférence des forces vives de la nation en 1990 que le Bénin a opté pour la démocratie et l'Etat de droit, les libertés sont consacrées en l'occurrence, la liberté d'association comme le dispose l'article 25 de la constitution béninoise du 11 décembre 1990 révisée par la loi 2019-40 du 07 novembre 2019, que «l'Etat reconnait et

\footnotetext{
${ }^{1088}$ Juliette OLIVIER, « Les nouveaux acteurs du droit de l'environnement : Le rôle de l'UICN dans l'élaboration du droit de l'environnement », in Revue Européenne de Droit de l'Environnement n³, 2005, p.274.

${ }^{1089}$ Fiche thématique ${ }^{\circ} 13$ réalisée dans le cadre du projet «Moi, citoyen en PACA, je m'engage pour le climat», page 1. Disponible sur le site internet : http://paca.climatcitoyen.org. Consulté le 16/01/2022..

${ }^{1090}$ Par exemple l'avant projet de la convention de Bonn sur la conservation des espèces migratrices appartenant à la faune sauvage fut rédigé à la demande du gouvernement Ouest-Allemand par l'UICN.

${ }^{1091}$ La Convention africaine sur la conservation de la nature : hâter son entrée en vigueur en vue d'assurer sa mise en œuvre par Mohamed Ali MEKOUAR. Disponible sur le site internet : https://cmsdata.iucn.org. Consulté le $16 / 01 / 2022$.
} 
garantit dans les conditions fixées par la loi, (...), la liberté d'association, (...) ». Ces libertés sont effectives et ont contribué à la naissance de plusieurs mouvements associatifs. Désormais, les organisations de la Société civile se font de plus en plus remarquées dans l'animation de la vie publique. Elles sont présentent dans presque tous les secteurs d'activités et spécifiquement, elles interviennent dans le domaine de la protection de l'environnement. Néanmoins, elles sont en nombre restreint à intervenir dans ce domaine, car la thématique environnementale n'est pas encore mieux appropriée par tous. Cette collaboration prescrite par le droit international et internalisée par le Bénin n'est pas encore perceptible en thème de visibilité et de réactivité que doit revêtir la contribution de cette entité sociale aux actions de la lutte contre les changements climatiques. Cependant, dans d'autres nations, les actions des mouvements associatifs impactent déjà positivement le droit climatique. C'est le cas de la république de France où les actions juridictionnelles des associations ont débouché sur une importante décision rendue par une juridiction nationale, contraignant l'Etat français à respecter ses engagements climatiques souscrits dans le cadre de la Convention des Nations unies sur les Changements Climatiques ${ }^{1092}$. L'une des conséquences qui découle de cette jurisprudence est l'importance de l'implication de la Société civile qui peut s'avérer comme un moyen d'effectivité du droit climatique dans un Etat Partie à la Convention Cadre des Nations Unies sur les Changements Climatiques. En effet, si les juridictions internes se donnent la possibilité, le pouvoir de faire respecter les engagements souscrits par les Etats dans la Convention Cadre des Nations Unies sur les Changements Climatiques, il est important de souligner que ces actions ne peuvent qu'être portées valablement et efficacement par des entités non étatiques devant ces juridictions, compte tenu de leur indépendance vis-à-vis du pouvoir politique.

La loi $\mathrm{n}^{\circ} 98-030$ portant loi cadre sur l'environnement en République du Bénin a prescrit aux organisations non gouvernementales ${ }^{1093}$ la mission de "promouvoir l'information et l'éducation relatives à l'environnement (...)» ${ }^{1094}$, ce qui permet d'une part, de mettre à contribution les compétences dont disposent les organisations de la Société civile dans le cadre

\footnotetext{
${ }^{1092}$ Décision du tribunal administratif de Paris dans l'affaire $N^{\circ} 1904967,1904968,1904972,1904976 / 4-1$, rendue le 03 février 2021: L'action climatique des mouvements associatifs tels que: L'association Oxfam France, l'association Notre Affaire À Tous, la Fondation pour la Nature et l'Homme, l'association Greenpeace France, l'association France Nature Environnement, la Fondation Abbé Pierre, la Fédération nationale de l'agriculture biologique, l'Association Initiatives pour le climat et l'énergie, l'Association nationale pour la protection des eaux et rivières. Disponible sur le site internet : http://paris.tribunal-administratif.fr.Consulté le 16/01/2022.

${ }^{1093}$ Concernant les résolutions consensuelles des états généraux, le concept de la société civile a été décliné ainsi qu'il suit : sont membres de la société civile, les entités suivantes: Associations (associations de jeunes, de développement, de femmes, des chefferies traditionnelles, des confessions religieuses, médias, etc.) ; Organisation Non Gouvernementale ; Organisations socioprofessionnelles et Syndicats. Disponible sur le site internet: https://www.mdscbenin.org/2018/03/08/la-societe-civile-au-benin.Consulté le 16/01/2022.

${ }^{1094}$ Suivant les dispositions de l'article 5 , paragraphe b, c de ladite loi.
} 
de la protection de l'environnement et d'autre part, de la retremper dans son rôle classique où elle peut développer son intervention dans le sens de l'Information, l'Education, la Communication (IEC), la formation, la vulgarisation, les études, l'appui-conseil, l'animation et la participation au diagnostic préalable dans les domaines de protection de l'environnement et de lutte contre les changements climatiques. Elle participe à la veille et à l'éveil des consciences des citoyens et des autorités sur la nécessité de protéger l'environnement et de lutter contre les changements climatiques. Des cadres formels sont créés pour faire participer les ONG à l'exécution des programmes gouvernementaux de protection de l'environnement et de lutte contre les changements climatiques, conformément aux prescriptions de la loi $\mathrm{n}^{\circ} 98-030$ portant loi cadre sur l'environnement en République du Bénin en son article 5 point f qui dispose que «Le gouvernement doit faciliter la création et le fonctionnement d'associations de protection, de défense et de mise en valeur de l'environnement, tant au niveau national que local. Ces organismes peuvent être associés aux actions entreprises par le gouvernement, notamment en matière d'information, d'éducation et de communication des citoyens et être reconnus d'utilité publique ». Pour se conformer aux dispositions de la même loi, l'article 7 a créé la Commission Nationale du Développement Durable (CNDD) qui est un organe intervenant dans le domaine de la protection de l'environnement et composée de membres provenant du Gouvernement et de la Société civile ${ }^{1095}$. Dans cet ordre d'idée, le décret $\mathrm{n}^{\circ} 2003-$ 142 du 30 avril 2003 portant création attributions et fonctionnement du Comité National sur les Changements Climatiques en son article 4, a retenu certaines Organisations Non Gouvernementales ${ }^{1096}$ et les représentants du réseau des ONG intervenant dans le domaine de la protection de l'environnement au niveau départemental comme membre dudit Comité.

La loi ${ }^{\circ} 98-030$ portant loi cadre sur l'environnement en République du Bénin confère beaucoup de prérogatives en matière de poursuite des infractions aux règles de protection de l'environnement à la Société civile qui peu en cas de violation desdites règles, mettre en œuvre l'action publique aux fins de sanctionner l'auteur. A titre illustratif, l'article 109 dispose que, «(...) Les associations compétentes en matière d'environnement, légalement reconnues et représentatives, peuvent mettre en mouvement l'action publique et se constituer partie civile à la condition qu'elles prouvent que les faits incriminés portent préjudice directement ou indirectement à l'intérêt collectif qu'elles représentent $»$. Dans ce cas, le privilège de la mise en

\footnotetext{
1095 Suivant les dispositions de l'article 8 de ladite loi.

1096 BENIN 21, OFEDI ; ABFEM-ONG ; GRAIB.
} 
mouvement de l'action publique est concédé à la société civile concurremment avec le ministère public $^{1097}$.

L'audience publique sur l'environnement est prévue par l'article 96 de la loi n $98-030$ portant loi cadre sur l'environnement en République du Bénin. La procédure de sa mise en œuvre prévue par l'article 100 dispose que « Toute personne physique ou morale peut demander au Ministre de mettre en œuvre la procédure d'audience publique sur l'environnement. (...)». Ces dispositions visent à garantir la consultation de la population sur les questions relatives à l'environnement afin de la faire participer aux décisions qui découlent des projets dont les incidences affectent leur milieu de vie. A travers ces dispositions, la Société civile se trouve encore être la cheville ouvrière dans la procédure de l'évaluation environnementale.

Le principe du financement de la Société civile en guise de son accompagnement par le Gouvernement est retenu à travers la notion d'incitation contenue dans la loi cadre sur la protection de l'environnement, qui dispose en son article 105 que « Des mesures d'incitation fiscale visant à associer le secteur privé (...) pourront être prises par loi des finances(...)»1098. Ces mesures ne sont pas toujours visibles à cause du caractère modeste de l'économie béninoise.

En quoi les actions de la Société civile peuvent-elles contribuer à la résorption des problèmes qui découlent des changements climatiques au Bénin?

Au cours de la phase préparatoire de la COP21, certaines organisations de la Société civile ont organisé des ateliers afin de proposer des solutions dans le cadre de la résorption du phénomène des changements climatiques. A titre illustratif, l'ONG Bénin Environnement vert, un mouvement associatif régi par le droit béninois a proposé un programme intitulé " 10 millions d'âmes, 10 millions d'arbres », qui a consisté à faire planter par chaque Béninois un arbre, lequel projet a reçu le soutient de l'Etat. A cet effet, une journée de l'arbre a été instituée chaque année et se déroule pendant tout le mois de juin où des activités de plantation d'arbre sur toute l'étendue du territoire national se font avec la participation des autorités au plus haut niveau et les citoyens dans leur immense majorité.

\footnotetext{
${ }^{1097}$ Selon les dispositions de l'article premier de la loi n ${ }^{\circ} 2012-15$ du 18 mars 2013 portant code de procédure pénale en République du Bénin, «L'action publique, pour l'application des peines est mise en mouvement et exercée par les magistrats du ministère public (...) ». Mais il y a des lois spéciales qui interviennent et confèrent ces prérogatives à d'autres structures notamment, la société civile. C'est le cas de l'article 109 alinéa 2 de la loi $\mathrm{n}^{\circ}$ 98 -030 portant loi cadre sur l'environnement en République du Bénin qui dispose que « (...) Les associations compétentes en matière d'environnement, légalement reconnues et représentatives peuvent mettre en mouvement l'action publique et se constituer parties civiles (...)».

${ }^{1098}$ Il s'agit de la loi n80-030 du 12 février 1999 portant loi cadre dur l'environnement en République du Bénin.
} 
L'une des actions phares de la participation de la Société civile à la lutte contre les changements climatiques au Bénin est encore celle du «Réseau des Parlementaires béninois sur le Climat et le Développement Durable » ${ }^{1099}$ qui de par son dynamisme a contribué à l'adoption de la loi $\mathrm{n}^{\circ} 2018-18$ portant réglementation des changements climatiques en République du Bénin, qui fut la première en la matière, en hissant le Bénin parmi les tous premiers Etats de l'Afrique a avoir adopté une loi sur les changements climatiques. Malheureusement, la loi ${ }^{\circ} 2018-18$ portant réglementation des changements climatiques en République du Bénin n'a conféré aucun rôle à la Société civile. Elle a seulement engagé « l'Etat à lui donner la formation relative aux méthodes participatives de conservation et d'utilisation durable des ressources naturelles $»^{1100}$. Curieusement le groupe de mot «Société civile ${ }^{1101}$ n'apparait qu'une seule fois dans cet important texte de loi qui comporte quatre-vingt dix-huit (98) articles.

Cependant, la Société civile pouvait entre autres actions se donner les moyens de s'illustrer positivement dans le domaine de l'action juridictionnelle pour amener les juridictions béninoises à contraindre l'Etat à honorer sa part d'engagement souscrit dans la Convention des Nations Unies sur les Changements Climatiques à l'instar des organisations similaires notamment, l'ONG Urgenda aux Pays-Bas. Puisque, grâce à l'intervention de certaines organisations de la Société civile, les Etats comme les Pays-Bas, la France, la Colombie, les Etats-Unis d'Amérique et puis l'Allemagne sont contraints par décisions de justice interne de respecter leur part d'engagement convenu dans la Convention climat. Ce n'est pas encore le cas au Bénin.

Néanmoins sur un autre plan, l'adoption de la loi climatique béninoise a contribué au renforcement du cadre institutionnel béninois de lutte contre les changements climatiques.

\section{Paragraphe 2: Le renforcement du cadre institutionnel}

Le cadre institutionnel béninois est caractérisé par la multiplication d'institutions de protection de l'environnement (A).

Avec l'adoption de la loi $\mathrm{n}^{\circ} 2018-18$ portant réglementation des changements climatiques en République du Bénin, certaines institutions environnementales ont été consolidées (B).

\footnotetext{
${ }^{1099}$ Information disponible sur le site internet : https://assemblee-nationale.bj. Consulté le 08/06/2021.

${ }^{1100}$ Article 17 paragraphe 4 de la loi n²018-18 sur les changements climatiques en République du Bénin.

${ }^{1101}$ Voir article 17 alinéa 4 de la loi n²018-18 sur les changements climatiques en République du Bénin.
} 


\section{A- La multiplication d'institutions}

Depuis son accession à la souveraineté internationale le $1^{\text {er }}$ août 1960 jusqu'à l'avènement de la conférence des forces vives de la nation tenue en février $1990^{1102}$, le Bénin n'a pas véritablement développé un cadre institutionnel approprié à la protection de l'environnement. La gestion du domaine environnemental était une activité partagée par plusieurs institutions de l'Etat. Ce fut à partir de 1990, année d'adoption de la nouvelle constitution $^{1103}$, qui a consacré le principe de la protection de l'environnement ${ }^{1104}$ que des actions ont été entreprises pour asseoir un cadre institutionnel approprié à la protection de l'environnement. Ce processus a abouti à la création du premier département ministériel de protection de l'environnement du Bénin le 29 juillet $1991^{1105}$.

La Constitution béninoise prescrit que, «(..) la loi détermine les principes fondamentaux de la protection de l'environnement et de la conservation des ressources naturelles $(\ldots) »^{1106}$. Cela implique que l'encadrement aussi bien juridique qu'institutionnel en matière de protection de l'environnement devra être fondé sur une base législative. Le cadre législatif n'étant pas encore bien fourmi et face au besoin d'encadrement de l'environnement et la nécessité de lutter contre les changements climatiques, en dehors de l'Agence Béninoise de l'Environnement (ABE) et de la Commission Nationale pour le Développement Durable (CNDD) qui sont créées par la loi cadre sur l'environnement ${ }^{1107}$, les autorités béninoises ont fait usage des mesures réglementaires pour créer quelques institutions devant intervenir dans les domaines de la protection de l'environnement et de la lutte contre les changements climatiques. Beaucoup d'institutions ont été créées notamment, le Comité National sur les Changements Climatiques, créé par décret 2003-142 du 30 avril 2003. Elle a pour mission, « l'appui à la définition des politiques et stratégies nationales et locales de lutte contre les effets néfastes des changements climatiques » et la «promotion des activités relatives à la mise en œuvre des directives politiques des programmes mondiaux, régionaux et nationaux de gestion

\footnotetext{
${ }^{1102}$ C'est à l'occasion de cette conférence que le Bénin a connu un bouleversement politique qui l'a entrainé dans le changement de régime politique et l'adoption d'une nouvelle constitution. Histoire du Bénin disponible sur le site internet : https://www.gouv.bj/benin/histoire/. Consulté le 16/01/2022.

${ }^{1103}$ La Constitution du 11 décembre 1990, laquelle a été révisée le 07 novembre 2019.

${ }^{1104}$ Article 27 Constitution du 11 décembre 1990, laquelle a été révisée le 07 novembre 2019.

${ }^{1105}$ Le « Ministère de l'Environnement, de l'Habitat et de l'Urbanisme (MEHU) » a été créé par décret n 91-176 portant composition du Gouvernement, le «Ministère de l'Environnement, de l'Habitat et de l'Urbanisme (MEHU) ». Disponible sur le site internet: https://sgg.gouv.bj > doc > décret-1991-242. Consulté le 16/01/2022.

${ }^{1106}$ Il s'agit de l'article 98 de la constitution du 11 décembre 1990 révisée par la loi 2019-40 du 07 novembre 2019.

${ }^{1107}$ Les articles 7 à 10 pour ce qui concerne la création de la Commission Nationale pour le Développement Durable (CNDD) et les articles 11 à 14 s'agissant de la création de l'Agence Béninoise pour l'Environnement (ABE) de la loi 98-030 du 12 février 1999, portant loi cadre sur l'environnement en République du Bénin.
} 
des changements climatiques ». Ensuite, la Commission de Modélisation Economique des Impacts et de l'Intégration des Changements Climatiques dans le Budget Général de l'Etat a été créée par le décret n²014-359 en date du 16 juin 2014. Cet organe s’occupe uniquement de l'aspect financier de la gestion des changements climatiques.

Ces deux organes contribuent à la mise en œuvre de la Convention Cadre des Nations Unies sur les Changements Climatiques sur le territoire béninois.

Par ailleurs, il existe une pratique qui a cours au plan international qui consiste à créer un cadre institutionnel lié à une nouvelle convention internationale de protection de l'environnement. Par exemple, chaque instrument international crée sa Conférence des Parties et son secrétariat pour s'occuper de la mise en œuvre de l'instrument juridique. Cette pratique a été également adoptée au Bénin et a contribué à la création de plusieurs institutions. La première institution technique qui a été créée est le Comité National sur les Changements Climatiques institué en 2003 par décret ${ }^{1108}$, pour contribuer à la mise en œuvre de la Convention Cadre des Nations Unies sur les Changements Climatiques. Au cours de la même année, l'Etat béninois a adopté le décret $\mathrm{n}^{\circ}$ 2003-129 du 15 avril 2003 lequel a créé un Comité National chargé de la Coordination et de la mise en œuvre de la Convention de Stockholm sur les Polluants Organiques Persistants ${ }^{1109}$. C'est «est un organe pluridisciplinaire chargé de la gestion coordonnée des polluants organiques persistants au Benin» ${ }^{1110}$. Il est placé sous l'autorité du ministère en charge de l'Environnement ${ }^{1111}$. Lorsque le Bénin a ratifié la Convention de Rio sur la Désertification, il a procédé à la création du Comité National de Lutte contre la Désertification et son secrétariat par décret n²008-727 du 22 décembre 2008 portant création, composition, attributions et fonctionnement du Comité National de Lutte contre Désertification et son Secrétariat Exécutif qui ont pour attributions, le «suivi de la mise en œuvre du Programme d'Action National de Lutte contre la Désertification $»^{1112}$. Particulièrement, appuyé de son secrétariat, le Comité National est l'organe national de mise œuvre de la Convention et à ce titre, « coordonne les actions menées dans le cadre de la mise

\footnotetext{
${ }^{1108}$ Il s'agit du décret $n^{\circ} 2003-I 42$ du 30 avril 2003, portant création, attributions et fonctionnement du comité national sur les changements climatiques. Disponible sur le site internet: https://sgg.gouv.bj > doc > décret-2003142. Consulté le 16/01/2022.

1109 Article $1^{\text {er }}$ dudit décret.

${ }^{1110}$ Article 2 du décret $n^{\circ}$ 2003-129 du 15 avril 2003 a créé le Comité National de Coordination de la mise en œuvre de la Convention de Stockholm sur les Polluants Organiques Persistants. Disponible sur le site internet: https://sgg.gouv.bj > doc > décret-2003-129. Consulté le 16/01/2022.

1111 Article 3 du décret $n^{\circ}$ 2003-129 du 15 avril 2003 a créé le Comité National de Coordination de la mise en œuvre de la Convention de Stockholm sur les Polluants Organiques Persistants.

1112 Article $1^{\text {er }}$ du décret $n^{\circ} 2008-727$ du 22 décembre 2008 portant création, composition, attributions et fonctionnement du Comité National de Lutte contre Désertification et de son Secrétariat Exécutif. Disponible sur le site internet: https://sgg.gouv.bj > doc > décret-2008-727. Consulté le 16/01/2022.
} 
en œuvre du Programme d'Action National de Lutte contre la Désertification ${ }^{1113}{ }^{113}$ L'institution est dirigée par le Ministre en charge de l'environnement ${ }^{1114}$.

De même, l'Agence Nationale de Protection Civile (ANPC), placée sous la tutelle du Ministère de l'Intérieur de la Sécurité Publique et des Cultes est créée par le décret n²012-426 du 06 novembre 2012 qui fixe pour cet organe la mission de, «contribuer à la mise en œuvre de la politique gouvernementale en matière de réduction des risques de catastrophes ${ }^{1115}$. Les activités de cette institution sont en lien avec la gestion des effets négatifs qui découlent des changements climatiques.

Il est important de faire remarquer que ces institutions créées par actes réglementaires sont fragiles et peuvent subir des mutations suivant la volonté du Gouvernement. Ce dernier peut décider de les réformer ou les supprimer selon sa volonté. Ce mode d'existence conditionne aussi leur efficacité, car ces institutions peuvent subir la pression des autorités ou manquer de moyen pour l'accomplissement des missions qui leurs sont attribuées.

Cependant, la volonté d'en découdre avec les changements climatiques et ses effets néfastes n'étant pas émoussée, après la ratification de l'Accord de Paris par le Bénin le 31 octobre $2016^{1116}$, l'adoption de la loi sur les changements climatiques a contribué à la consolidation de certaines institutions qui intervenaient déjà dans la protection du climat.

\section{B- La consolidation d'institutions}

La loi n²018-18 portant règlementation sur les changements climatiques en République du Bénin a prescrit un cadre institutionnel approprié à la gestion des changements climatiques, composé de deux (2) importants organes ${ }^{1117}$. En effet, l'article 77 alinéa 1 de ladite loi dispose qu' "il est créé un Comité National sur les Changements Climatiques, en abrégé (CNCC), placé sous la tutelle du ministère en charge des Changements Climatiques (...)». La même loi définit généralement l'objet de ses relations avec d'autres ministères par la disposition suivante: «Chaque ministère définit les activités relatives aux changements climatiques et présente au

\footnotetext{
1113 Article 2 du décret $\mathrm{n}^{\circ} 2008-727$ du 22 décembre 2008 portant création, composition, attributions et fonctionnement du Comité National de Lutte contre Désertification et de son Secrétariat Exécutif. Disponible sur le site internet: https://sgg.gouv.bj > doc > décret-2008-727. Consulté le 16/01/2022.

1114 Article 3 id.

1115 Article 5 ibid.

${ }^{1116}$ Etat de ratification de la Convention. Disponible sur le site internet: https://treaties.un.org. Consulté le 05/06/2021.

1117 Voir TITRE VII Intitulé « DU CADRE INSTITUTIONNEL » de ladite loi.
} 
Comité National sur les Changements Climatiques son Plan d'Action ${ }^{1118}$. La composition, l'organisation, les attributions et le fonctionnement du Comité National sur les Changements Climatiques sont laissés à la charge du Gouvernement qui prend un décret en Conseil des Ministres pour en fixer les règles ${ }^{1119}$.

Mais en réalité, le Comité National sur les Changements Climatiques institué par la loi $\mathrm{n}^{\circ}$ 2018-18 portant règlementation sur les changements climatiques au Bénin n'est pas différente de l'institution créée par le décret 2003-142 portant création attributions et fonctionnement du Comité National sur les Changements Climatiques. Cette institution menait son existence légale depuis le 30 avril $2003^{1120}$. Sa création avait été recommandée dans le document de stratégie de la mise en œuvre au Bénin de la Convention Cadre des Nations Unies sur les Changements Climatiques $^{1121}$.

La mise en œuvre de la Convention Cadre des Nations Unies sur les Changements Climatiques au Bénin était pilotée par le Comité National sur les Changements Climatiques $(\mathrm{CNCC})^{1122}$. Avec l'entrée en vigueur de la loi $\mathrm{n}^{\circ} 2018-18$ portant règlementation sur les changements climatiques en République du Bénin, le Comité National sur les Changements Climatiques, conserve les mêmes modalités de fonctionnement, ses attributions n'ont pas connu de changement ainsi que la configuration de sa composition. Il en est ainsi parce que le décret $\mathrm{n}^{\circ}$ 2003-142 portant création attributions et fonctionnement du Comité national sur les changements climatiques qui l'a créé n'a pas été abrogé. Seulement que la loi n²018-18 sur les changements climatiques qui est intervenue a entériné l'existence de l'institution climatique.

C'est également par la stipulation suivante : « il est créé un organe d'aide à la décision dénommé, la Commission de Modélisation Economique des Impacts du climat et d'Intégration des Changements Climatiques au Budget Général de l'Etat ${ }^{1123}$ qu'une deuxième institution climatique a été créée par la loi n²018-18 portant règlementation sur les changements climatiques en République du Bénin. Elle est « (...) placée sous la tutelle du ministère en charge

\footnotetext{
${ }^{1118}$ Article 77 alinéa 2 de la loi n²012-18 portant règlementation sur les changements climatiques en République du Bénin. Disponible sur le site internet: https://sgg.gouv.bj > doc > loi-2018-18. Consulté le 16/01/2022.

1119 Article 78 id

${ }^{1120}$ Décret $n^{\circ}$ 2003-142 portant création attributions et fonctionnement du Comité National sur les changements climatiques. Disponible sur le site internet: https://sgg.gouv.bj > doc > décret-2003-142. Consulté le 16/01/2022.

${ }^{1121}$ Stratégie béninoise de mise en œuvre de la Convention Cadre des Nations Unies sur les Changements Climatiques, p.43. Disponible sur le site internet: https://unfccc.int/sites/default/files/resource/Benin. Page consultée le 02/06/2021.

${ }^{1122}$ Article 2 du décret $\mathrm{n}^{\circ}$ 2OO3-I42 du 30 avril $2 \mathrm{OO} 3$ portant création, attributions et fonctionnement du comité national sur les changements climatiques au Bénin. Disponible sur le site internet: https://sgg.gouv.bj > doc > décret-2003-142. Consulté le 16/01/2022.

${ }^{1123}$ Article 79 de la de la loi n²018-18 sur les changements climatiques en République du Bénin. Disponible sur le site internet: https://sgg.gouv.bj > doc > loi-2018-18. Consulté le 16/01/2022.
} 
$d u$ Plan $\gg{ }^{1124}$ et la composition, l'organisation, les attributions et le fonctionnement de ladite Commission « (...) sont fixés par décret pris en Conseil des Ministres $»^{1125}$.

En réalité, c'est aussi par décret qu'avait été créée la Commission de modélisation économique des impacts du climat et d'intégration des changements climatiques au budget général de l'Etat depuis le 16 juin $2014^{1126}$. Elle a pour mission de « développer des outils et méthodes d'évaluation, de modélisation et de prévision économique des impacts du climat en vue d'une optimisation des stratégies d'adaptation et du développement sobre en carbone et résilient au climat à promouvoir ${ }^{1127}{ }^{127}$. Avec l'avènement de la loi sur les changements climatiques, la commission n'a pas subi une profonde mutation en ce qui concerne les modalités de son fonctionnement, ses attributions, son organisation et sa composition sont demeurés sans changement, parce que le décret qui l'a créé à l'origine demeure toujours applicable. Seulement qu'une précision a été faite en ce qui concerne sa tutelle qui, au terme de l'article 79 alinéa 2 de la loi $n^{\circ} 2018-18$ portant règlementation sur les changements climatiques en République du Bénin, relève du ministère en charge du Plan. Par contre, l'article 4 du décret 2014-359 du 16 juin 2014 portant création, attributions, organisation et fonctionnement de la Commission de Modélisation Economique des Impacts et de l'Intégration des Changements Climatiques dans le Budget Général de l'Etat, dispose que le Comité d'Orientation, l'une des structures principales de la Commission est présidée par le Ministre en charge du développement. Il s'ensuit que la loi 2018-18 portant règlementation sur les changements climatiques en République du Bénin a renforcé le degré de normativité des deux (2) institutions en charge de la gestion des changements climatiques au Bénin.

Depuis 1991, année de la création du premier département ministériel en charge de l'environnement, tous les Gouvernements qui se sont relayés à la tête de l'exécutif de l'Etat béninois sont intervenus dans le domaine de la protection de l'environnement par le biais du ministère en charge de l'Environnement. Cette institution était d'habitude créée par décret du Président de la République. Néanmoins, la loi n²018-18 portant règlementation sur les

\footnotetext{
1124 Id.

${ }^{1125}$ Article 80 de la de la loi n²018-18 sur les changements climatiques en République du Bénin. Disponible sur le site internet: https://sgg.gouv.bj > doc > loi-2018-18. Consulté le 16/01/2022.

${ }^{1126}$ Décret $\mathrm{n}^{\circ} 2014-359$ portant création, attributions, organisation et fonctionnement de la Commission de Modélisation Economique des Impacts et de l'Intégration des Changements Climatiques dans le Budget Général de l'Etat (CMEIGB). Disponible sur le site internet: https://sgg.gouv.bj > doc > décret-2014-359. Consulté le $16 / 01 / 2022$.

${ }^{1127}$ Article 2 du décret $\mathrm{n}^{\circ}$ 2014-359 portant création, attributions, organisation et fonctionnement de la Commission de Modélisation Economique des Impacts et de l'Intégration des Changements Climatiques dans le Budget Général de l'Etat (CMEIGB). Disponible sur le site internet: https://sgg.gouv.bj > doc > décret-2014-359. Consulté le $16 / 01 / 2022$.
} 
changements climatiques en République du Bénin a introduit une innovation par la création dudit ministère par l'une de ses dispositions ${ }^{1128}$. Seulement que cette réforme n'est pas encore mise en œuvre compte tenu des contradictions qui relèvent de la méthodologie utilisée par le Législateur qui a rangé la question relative à la création d'un ministère dans le domaine législatif contrairement aux usages qui ont cours en République du Bénin.

L'adoption de la loi n²018-18 portant règlementation sur les changements climatiques en République du Bénin n'a pas contribué a modifié fondamentalement les statuts des institutions intervenant dans la gestion des changements climatiques au Bénin. Elle n'a pas non plus créé de nouvelles institutions. Elle n'a fait qu'entériner l'existence des deux institutions climatiques que sont : le Comité National sur les Changements Climatiques et la Commission de Modélisation Economique des Impacts et de l'Intégration des Changements Climatiques dans le Budget Général de l'Etat.

En effet, l'option faite par le Législateur béninois de consacrer l'existence d'anciennes institutions en charge de la gestion des changements climatiques par une loi permet d'assurer leur durabilité. La consécration législative de la création des ces institutions contribue à leur consolidation. Cette méthodologie du Législateur béninois qui consiste à légiférer dans le domaine de la protection de l'environnement et de la lutte contre les changements climatiques est conforme aux normes prescrites en la matière car, en réalité, la matière environnementale relève du domaine de la loi ${ }^{1129}$. Du point de vue formalisme, le Bénin s'est conformé aux normes établies en matière de légistique. C'est la preuve qu'en matière de gouvernance environnementale, les bases d'un encadrement institutionnel objectif et efficace sont entrain d'être projetées. Ceci emporte plusieurs avantages pour des institutions créées conformément aux dispositions légales. Désormais, ces institutions seront à l'abri des pressions et des actions malveillantes provenant d'autres institutions en l'occurrence, le Gouvernement.

In fine, l'étude révèle que le droit international comme par effet de contamination a contribué aussi bien, quantitativement que qualitativement à l'amélioration de l'encadrement juridique et institutionnel de la protection du climat au Bénin. Mais, la question de l'effectivité de ce droit destiné à relever les défis en matière des changements climatiques demeure entière, car les indicateurs climatiques ne sont pas encourageants et font croire à la faible effectivité dudit droit.

\footnotetext{
${ }^{1128}$ Article 77 de ladite loi.

${ }^{1129}$ Article 98 de la Constitution béninoise du 11 décembre 1990 révisée le 07 novembre 2019.
} 
TITRE I 


\section{CHAPITRE II:}

\section{LA FAIBLE EFFECTIVITE DE LA PROTECTION}

« Les Etats sont à l'origine de la formation du droit et sont également en charge de son exécution ${ }^{1130}$. Dans ces conditions, «(..) le respect du droit par les sujets de droit international est présumé $»{ }^{1131}$. L'idée qui fonde cette présomption découle de l'application de l'article 26 de la Convention de Vienne sur le droit des traités de 1969 qui prescrit, « le Pacta sunt servanda », c'est-à-dire que, « Tout traité en vigueur lie les Parties et doit être exécuté par elles de bonne foi ${ }^{1132}$. Ce qui signifie que les engagements que les Etats ont librement consenti sont, "généralement conformes à leurs intérêts et ils n'ont pas de raison de les méconnaitre $»^{1133}$.

Paradoxalement, ces mêmes Etats qui ont librement consenti des engagements dans le cadre de la coopération internationale s'obstinent de les respecter. Parfois, ils se cachent derrière des motifs à caractère politique, économique ou stratégique pour se dérober de leurs engagements. Ce comportement est préjudiciable à la lutte contre les changements climatiques parce qu'il ne favorise pas l'atteinte de l'objectif qui consiste à « (...) stabiliser conformément aux dispositions pertinentes de la Convention Cadre des Nations Unies sur les Changements Climatiques, les concentrations de gaz à effet de serre dans l'atmosphère à un niveau, qui empêche toute perturbation anthropique dangereuse du système climatique (...). ${ }^{1134}$. Evidemment, les rapports consécutifs du Groupe d'Experts Intergouvernemental sur l'Evolution du Climat (GIEC) n'ont jamais été reluisant par rapport à la réalisation de l'objectif de la Convention.

Cependant, au-delà des motifs personnels brandis par certains Etats Parties pour se soustraire de l'exécution des engagements souscrits, il y aurait certainement des motifs objectifs

\footnotetext{
${ }^{1130}$ Sandrine Maljean-Dubois et Vanessa RICHARD, Mécanisme internationaux de suivi et mise en œuvre des conventions internationales de protection de l'environnement, CERIC, France Vanessa Richard CERIC, France Novembre 2004, p.14.

${ }^{1131}$ COMBACU (J), SUR (S), Droit international public, Paris Montchrestien, $10^{\mathrm{e}}$ éd., 2012, p et s.

1132 Article 26 de la Convention de Vienne sur le droit des Traités de 1969. Disponible sur le site internet: https://legal.un.org/ilc/texts/instruments/french/conve. Consulté le 16/01/2022.

1133 MALJEAN-DUBOIS (S.), «Les enjeux du contrôle dans le droit international de l'environnement et du Protocole de Kyoto en particulier», in MALJEAN-DUBOIS (S.) (dir.), Changements climatiques: les enjeux du contrôle international, op, cit, p. 18.

${ }^{1134}$ Article 2 de la Convention Cadre des Nations Unies sur les Changements Climatiques. Disponible sur le site internet: https://unfccc.int/fr/processus-et-reunions/la-convention. Consulté le 16/01/2022.
} 
qui sont à l'origine des contres performances obtenues dans le cadre de la mise en œuvre de la Convention Cadre des Nations Unies sur les Changements Climatiques.

De toutes les manières, le non respect par les Etats-Parties des dispositions conventionnelles peut donner lieu à des différends susceptibles d'être déférés devant les juridictions compétentes. C'est fort de cela, qu'en dépit de l'activation de la diplomatie en vue du règlement pacifique des différends climatiques internationaux, il est prévu dans le contexte de la Convention Cadre des Nations Unies sur les Changements Climatiques, un contrôle juridictionnel international qui, malheureusement s'avère imparfait (SECTION I). De la même manière, lorsque les difficultés découlent de l'application de la règlementation relative aux changements climatiques en vigueur au sein des Etats Parties, il existe une organisation judiciaire interne pour chaque Etat qui peut s'en occuper. Dans ce cas, les systèmes judiciaires de certains Etats se sont manifestés et ont impacté positivement la mise en œuvre de la Convention Cadre des Nations Unies sur les Changements Climatiques ${ }^{1135}$.

Malheureusement, à l'antipode de cette prouesse, la contribution de la justice de certains Etats, tout comme celui du Bénin à l'action climatique a été mitigée (SECTION II).

\section{SECTION I: LE CONTROLE JURIDICTIONNEL INTERNATIONAL IMPARFAIT}

Selon Dominique CARREAU, « quant le choix a été donné pour un Etat entre la perte du bénéfice de l'appartenance à une Organisation internationale et le maintien de ses options propres, fussent-elles illégales, il a toujours été exercé au profit des priorités nationales ${ }^{1136}$. Il s'ensuit que l'observation présumée des obligations conventionnelles librement consenties par les Etats Parties dans le cadre de l'application des accords internationaux n'est pas toujours respectée. C'est for de cela que la Convention de Vienne sur le droit des traités de 1969 a mis l'accent sur le règlement pacifique des différends internationaux. Elle affirme dans l'une de ses dispositions, "que les différends concernant les traités doivent, comme les autres différends internationaux, être réglés par des moyens pacifiques et conformément aux principes de la

\footnotetext{
${ }^{1135}$ Il s'agit entre autres, des Pays-Bas, de la France, des Etats-Unis d'Amérique, de la Colombie, du Canada et du Brésil.

${ }^{1136}$ Dominique CARREAU, Droit international. Paris, Pedome, 1994, p. 557 cité dans Mécanismes internationaux de suivi et mise en œuvre des conventions internationales de protection de l'environnement Sandrine MaljeanDubois CERIC, France Vanessa Richard CERIC, France Novembre 2004., p.27.
} 
justice et du droit international $»{ }^{1137}$. Cependant, d'autres paramètres provoquent l'intervention de la justice internationale pour assurer le contrôle juridictionnel des différends.

$\mathrm{Au}$ sens du dictionnaire juridique, «le contrôle est une opération consistant à vérifier si un organe public, un particulier ou encore un acte respecte les exigences de leur fonction ou des règles qui s'imposent à eux» ${ }^{1138}$. La finalité du contrôle est d'avoir la certitude que les mesures édictées sont appliquées. Ce contrôle s'opère dans le cadre du droit international avec pour objectif principal est de sanctionner les actions de désobéissance relevées dans le cadre de la mise en œuvre des instruments juridiques internationaux.

Anciennement, « le contrôle de mise en œuvre du droit international était confié à l'Etat lui même ou aux autres Etats ${ }^{1139}$. Cette forme de contrôle paraissait inefficace car les Etats sont «peu portés à se désigner eux-mêmes comme cibles des réactions possibles à sa violation» ${ }^{1140}$.

Cependant, avec l'évolution du droit international, la sanction de l'inobservation constitue un ultime recours. Toutefois, en cas de non application ou de mauvaise application des instruments juridiques internationaux, la justice internationale peut être saisie pour établir les responsabilités. C'est ainsi que les différends qui sont nés de l'application de la Convention Cadre des Nations Unies sur les Changements Climatiques peuvent faire l'objet d'un contrôle juridictionnel international, qui n'est pas tout à fait parfait parce que, accompli devant une juridiction internationale à compétence générale (Paragraphe 1).

Néanmoins, les Etats ont la possibilité ou la faculté de faire recours au mode alternatif de règlement des différents climatiques prévu dans le mécanisme de règlement des différends, établi dans l'accord climat ${ }^{1141}$ (Paragraphe 2).

\section{Paragraphe 1 : Le recours à une juridiction à compétence générale}

En cas de survenance de différends internationaux, pour sauvegarder les relations amicales entre Etats, le droit international privilégie des approches pacifiques avant de donner

\footnotetext{
${ }^{1137}$ Cinquième $\left(5^{\mathrm{e}}\right)$ paragraphe du préambule de la Convention de Vienne sur le droit des traités Faite à Vienne le 23 mai 1969. Disponible sur le site internet:https://legal.un.org/ilc/texts/instruments/french/convent.Consulté le $16 / 01 / 2022$.

${ }^{1138}$ Dictionnaire juridique, Gérard CORNU, p. 263.

${ }^{1139}$ MALJEAN-DUBOIS (S), « Les enjeux du contrôle dans le droit international de l'environnement », p. 18.

${ }^{1140}$ COMBACU (J), SUR (S), Droit international public, Paris Montchrestien, $10^{\mathrm{e}}$ éd., 2012, p et s.

${ }^{1141}$ L'accord climat est essentiellement constitué de la Convention Cadre des Nations Unies sur les Changements Climatiques, le Protocole de Kyoto et de l'Accord de Paris.
} 
la possibilité de passer à tout autre mode de règlement de gestion des conflits. ${ }^{1142}$ Cependant, il n'est pas exclu de recourir à la compétence juridictionnelle pour décanter certains différends comme le stipule l'article 33 de la Charte des Nations unies, qui précise que « les Parties à tout différend, dont la prolongation est susceptible de menacer le maintien de la paix et la sécurité internationale, doivent en rechercher la solution, avant tout, par voie de (...) règlement judiciaire(...)».

Quand bien même il a été convenu de la nécessité d'assurer le contrôle juridictionnel des différends nés de l'application de la Convention Cadre des Nations Unies sur les Changements Climatiques, cet instrument n'a pas créé de juridiction. Mais il a prévu le recours à une juridiction internationale à compétence générale pour intervenir dans le cadre du règlement des différends climatiques. C'est ainsi que la Cour Internationale de Justice (CIJ) a été désignée pour jouer ce rôle ${ }^{1143}(\mathbf{A})$.

Seulement, l'intervention de la Cour Internationale de Justice comme organe juridictionnel de la Convention Cadre des Nations Unies sur les Changements Climatiques n'a pas encore impacté remarquablement l'action de protection du climat dans certains Etats-Parties tout comme la République du Bénin (B).

\section{A- La compétence climatique de la Cour Internationale de Justice}

L'organe judiciaire principal des Nations unies est «(...) La Cour internationale de justice $»^{1144}$. Cette juridiction a été créée en 1945 et siège à La Haye aux Pays-Bas. Son mode de fonctionnement repose sur les bases juridiques élaborées dans le cadre de la règlementation de la Cour permanente de justice internationale ${ }^{1145}$. Elle a compétence pour connaitre les conflits juridiques soumis par les Etats et constitue également un organe consultatif pour les structures et agences internationales agréées par l'Assemblée générale des Nations unies ${ }^{1146}$. La Cour internationale de justice est une juridiction à compétence universelle, car tous les Etats

\footnotetext{
${ }^{1142} \mathrm{~A}$ titre indicatif, l'article $1^{\mathrm{er}}$, paragraphe 2 de la Charte des Nations unies recommande de «Développer entre les nations des relations amicales fondées sur le respect du principe de l'égalité des droits des peuples et de leur droit à disposer d'eux-mêmes et prendre toutes autres mesures propres à consolider la paix du monde». Ainsi suivant le principe 26 de la Déclaration de Rio sur l'environnement et le développement de 1992 «Les Etats doivent résoudre pacifiquement leurs différends en matière d'environnement en employant des moyens appropriés conformément à la Charte des Nations Unies».

${ }_{1143}$ Article 14 paragraphe 2-a de la Convention Cadre des Nations Unies sur les Changements Climatiques.

${ }^{1144}$ L'article 92 de la Charte des Nations unies. Disponible sur le site internet: https://www.un.org/fr/about-us/uncharter.Consulté le 16/01/2022.

1145 Id.

${ }^{1146}$ Selon l'article 96 de la Charte des Nations unies. Disponible sur le site internet: https://www.un.org/fr/aboutus/un-charter.Consulté le 16/01/2022.
} 
membres de l'Organisation des Nations Unies sont assujettis à son statut ${ }^{1147}$. C'est une juridiction permanente indépendante et impartiale, composée de quinze (15) magistrats indépendants élus pour neuf (9) ans, par l'Assemblée générale et le Conseil de sécurité de l'Organisation des Nations Unies ${ }^{1148}$. La Cour internationale de justice n'est compétente que, pour les différends qui opposent les Etats ${ }^{1149}$. En effet, il existe trois (3) moyens de saisine de la Cour. Premièrement, les deux (2) Parties élaborent un accord au fin de soumettre leur différend à la compétence de la Cour ${ }^{1150}$. Deuxièmement, un Etat peut souscrire à une « déclaration facultative de juridiction obligatoire, qui peut se faire purement et simplement sous condition de réciprocité ou pour un délai de réciprocité ${ }^{1151}$. Troisième, la compétence de la Cour est requise « lorsqu'un traité ou une convention en vigueur prévoit le renvoi à une juridiction que devait instituer la Société des Nations (SDN) ou à la Cour Permanente de Justice Internationale (CPJI), la Cour internationale de Justice constituera cette juridiction entre les Parties» ${ }^{1152}$. La compétence de la Cour «s'étend à toutes les affaires que les Parties lui soumettront, ainsi qu'à tous les cas spécialement prévus dans la Charte des Nations unies ou dans les traités et conventions en vigueur(...) ${ }^{1153}$. La mission de la CIJ est « de régler conformément au droit international, les différends qui lui sont soumis $»^{1154}$. Le droit applicable devant la juridiction internationale repose sur « les conventions internationales, soit générales, soit spéciales, établissant des règles expressément reconnues par les Etats en litige, mais également la coutume internationale comme preuve d'une pratique générale, acceptée comme étant le droit.»> ${ }^{1155}$

La Cour internationale de justice a vu sa compétence s'étendre au domaine environnemental par son avis consultatif du 08 juillet $1991,{ }^{1156}$ rendu à la demande de l'Assemblée générale des Nations unies, avis dans lequel la Cour s'est déclarée consciente que «l'environnement est menacée, jour après jour (...) » ${ }^{1157}$, et qu'il «n'est pas une abstraction,

\footnotetext{
${ }^{1147}$ L'article 93 (1) de la Charte des Nations unies adoptée à San Francisco le vingt-six juin mil neuf cent quarantecinq dispose que « Tous les Membres des Nations unies sont ipso facto parties au Statut de la Cour internationale de Justice ». Disponible sur le site internet: https://www.un.org/fr/about-us/un-charter.Consulté le 16/01/2022.

1148 Articles $2 ; 3 ; 4$ et 36 du statut de la Cour Internationale de Justice. Disponible sur le site internet: https://www.icj-cij.org/fr/statut.Consulté le 16/01/2022.

1149 Article 34 alinéa premier du Statut de la CIJ.

1150 Article 36 alinéa 2 id.

1151 Article 36-7 ibid.

1152 Article 37 du Statut de la Cour Internationale de Justice.

${ }_{1153}$ Article 36 id.

${ }^{1154}$ Article. 38 ibid.

${ }^{1155}$ Article 38 alinéa 1 et 2 ibid.

${ }^{1156}$ CIJ, Licéité de la menace ou de l'emploi d'armes nucléaires, Avis consultatif du 08 juillet 1996, Rec. 1996 P. 241.

${ }^{1157}$ Id.
} 
mais l'espace où vivent les êtres humains et dont dépendent la qualité de leur vie et leur santé, $y$ compris pour les générations à venir ${ }^{1158}$. Cette jurisprudence est la preuve de ce que la Cour Internationale de Justice (CIJ) considère désormais la question de la protection de l'environnement comme relevant de sa compétence. Et elle intervient par nécessité de sauvegarder l'environnement au profit des générations présentes et futures. En effet, le 19 juillet 1993, la Cour internationale de justice a redéfini son domaine d'intervention en élargissant sa compétence au secteur environnemental par la constitution d'une chambre spéciale pour les questions d'environnement par application de l'article 26, paragraphe 1 de sont Statut. Cependant, plusieurs années durant ${ }^{1159}$, aucun différend n'a été jugé par cette chambre ${ }^{1160}$ provoquant en 2006 le non renouvèlement de la composition ${ }^{1161}$. Malgré sa spécificité, le recours à la chambre à compétence environnementale est conditionné par la mise en œuvre des dispositions ordinaires qui déterminent la compétence de la Cour ${ }^{1162}$.

Une fois la compétence environnementale de la Cour établie, existe-t-il une relation entre le statut de la Cour Internationale de Justice et la Convention Cadre des Nations Unies sur les Changements Climatiques?

Certains accords internationaux contiennent des « clauses compromissoires ${ }^{1163}$ en vue de régler le problème de compétence juridictionnelle en cas de survenance de différends entre Etats en ce qui concerne les difficultés relevant de l'application desdits accords. C'est dans cette optique que la Convention Cadre des Nations Unies sur les Changements Climatiques dispose, qu'en cas de « différend entre deux (2) ou plusieurs Parties, au sujet de l'interprétation ou de l'application de la Convention, les Parties concernées doivent s'efforcer de régler par voie de négociation ou par tout autre moyen pacifique de leur choix ${ }^{1164}$. Mais elle n'a pas non plus exclu le mode de règlement juridictionnel des litiges environnementaux ${ }^{1165}$.

En réalité, le contrôle juridictionnel parait le seul moyen de garantir l'effectivité des instruments internationaux car, «les sanctions décidées dans un cadre non juridictionnel ne

\footnotetext{
${ }^{1158}$ CIJ, Licéité de la menace ou de l'emploi d'armes nucléaires, Avis consultatif du 08 juillet 1996, Rec. 1996 P. 241.

1159 Treize (13) années d'existence de la chambre spéciale pour les questions d'environnement.

${ }^{1160}$ Chambres spéciale pour les questions d'environnement. Disponible sur le site internet: https://www.icjcij.org/fr/chambres-et-comites. Consulté le 16/01/2022.

${ }^{1161}$ Les membres de la Chambre spéciale pour les questions d'environnement. Disponible sur le site internet : https://www.icj-cij.org/fr/chambres-et-comites. Consulté le 16/01/2022.

1162 Il s'agit des articles 36 et 37 de son statut

${ }^{1163}$ Se définit comme suit, «Clause d'un traité stipulant le recours au règlement arbitral ou judiciaire pour les litiges concernant l'interprétation ou l'application dudit traité ». GUILLIEN (Raymond) et VINCENT (Jean), Lexique des termes juridiques, 5éd., Dalloz, 1981, 567p., p. 99.

${ }^{1164}$ Article 14 paragraphe 1 de la Convention Cadre des Nations Unies sur les Changements Climatiques.

1165 Voir article 14 paragraphe2-a de la Convention Cadre des Nations Unies sur les Changements Climatiques.
} 
suffissent pas toujours à modifier les comportements des Etats»» ${ }^{166}$. C'est pour cela que par anticipation, la Convention Cadre des Nations Unies sur les Changements Climatiques a désigné la Cour international de justice comme instance juridictionnelle susceptible de statuer sur le contrôle de l'application des instruments juridiques internationaux de lutte contre les changements climatiques ${ }^{1167}$.

Il s'ensuit que la compétence de la CIJ est requise dans le cadre du règlement des différends nés de l'application des dispositions de la Convention Cadre des Nations Unies sur les Changements Climatiques, sans qu'elle ne soit une juridiction spécialisée en matière de lutte contre les changements climatiques. La compétence de la Cour peut être également requise pour l'interprétation des dispositions conventionnelles, elle peut toutefois donner des avis consultatifs sur la demande des Parties ${ }^{1168}$.

Quelle est la contribution de la Cour internationale de justice dans la gestion des différends dans le cadre de la mise en cuvre des dispositions de la Convention Cadre des Nations Unies sur les changements climatiques?

En termes de contribution de la Cour internationale de justice à l'évolution du droit international respectivement de protection de l'environnement et des changements climatiques, force est de constater qu'il y a une rareté de la jurisprudence de la Cour Internationale de Justice en matière environnementale. Cette situation est causée par le volontarisme qui caractérise le droit international et la complexité de la procédure applicable devant la juridiction internationale.

Néanmoins, la CIJ saisie de quelques différends a rendu des décisions non moins importantes dans le domaine de la protection de l'environnement. Il s'agit entre autre de l'arrêt rendu par la CIJ le 20 avril 2010, dans l'affaire de construction et du fonctionnement de deux (2) usines de fabrication de pâte à papier sur le fleuve international Uruguay ${ }^{1169}$. Ce fut la première affaire à caractère purement environnemental, jugée par la Cour Internationale de Justice qui dans son arrêt en substance, « reconnait que la pratique de l'étude d'impact sur l'environnement a acquis une valeur coutumière et par conséquent, qu'elle engage tous les Etats. Elle constate cependant que le droit international général ne précise pas l'objet ni le

\footnotetext{
${ }^{1166}$ Sandrine Maljean-Dubois, Mécanismes internationaux de suivi et mise en œuvre des conventions internationales de protection de l'environnement, CERIC, France Vanessa Richard CERIC, France Novembre 2004, p. 27.

${ }^{1167}$ Article 4 paragraphe 2-a de la Convention Cadre des Nations Unies sur les Changements Climatiques. ${ }^{1168} \mathrm{Id}$.

${ }^{1169}$ COUR INTERNATIONALE DE JUSTICE, RECUEIL DES ARRETS, AVIS CONSULTATIFS ET ORDONNANCES, arrêt du 20 avril 2010 : Usines de pâte à papier sur le fleuve Uruguay (Argentine c. Uruguay). Disponible sur le site internet : https://www.icj-cij.org. Consulté le 16/01/2022.
} 
contenu de ces études d'impact. L'arrêt se réfère entre autres, au développement durable et à l'usage équitable et rationnel des ressources en eau transfrontières ${ }^{1170}$. Ce différend a quelque peu, un lien avec les changements climatiques car dans la substance, « la construction d'usine sur un fleuve peut engendrer la pollution fluviale par le déversement des déchets toxiques ou radioactifs dans l'eau et le dégagement dans l'atmosphère des gaz à effet de serre $»^{1171}$. Par cette jurisprudence, la Cour a apporté une contribution non négligeable au droit de protection de l'environnement en reconnaissant comme principe de base à l'élaboration de tout projet, la réalisation des évaluations environnementales et en fait une obligation à la charge de tous les Etats. Cette jurisprudence a contribué à la protection de l'environnement par la prévoyance des situations éventuelles pouvant provoquer sa dégradation par la réalisation de « l'étude d'impact environnementale préalable» à la réalisation des projets d'envergure. Dans ce même cadre, la Cour Internationale de Justice (CIJ) s'est également prononcée dans l'affaire des barrages de Danube Gabcikovo-Nagymaros, «concluant que les Parties devraient examiner à nouveau les effets sur l'environnement de l'exploitation de la centrale Gabcikovo » ${ }^{1172}$.

Malgré le développement rapide du Droit International de l'Environnement, les conflits environnementaux sont rarement déférés devant la Cour internationale de justice. Parmi les cent quatre vingt-quinze (195) affaires dont la Cour internationale de justice est saisie au cours de la période de 2008 à 2012, très peu ont eu pour thème principal, l'interprétation et l'application de règle $\mathrm{du}$ droit international de l'environnement ${ }^{1173}$. D'autres affaires ont soulevé des questions ayant trait entre autres, au Droit international de l'environnement sans que l'objet du litige ait cependant un caractère purement environnemental ${ }^{1174}$. Il s'agit par exemple de " l'affaire où l'avis de la CIJ a été sollicité sur la licéité de la menace ou de l'emploi des armes nucléaires où la CIJ considère que les Etats engagés dans un conflit armé ont le devoir de tenir

\footnotetext{
${ }^{1170}$ La protection de l'environnement dans la jurisprudence de la Cour Internationale de Justice. A propos de l'arrêt des Usines de pâte à papier sur le fleuve Uruguay (20 avril 2010) Emmanuel LA Doussis Professeur adjoint à l'Université d'Athènes.http://www.academia.edu.Consulté le 05/06/2021.

$1171 \mathrm{Id}$.

${ }^{1172}$ COUR INTERNATIONALE DE JUSTICE, RECUEIL DES ARRETS, AVIS CONSULTATIFS ET ORDONNANCES, Projet GabCikovo-Nagymaros (Hongrie/Slovaquie), arrêt, C. I. J. Recueil 1997, p. 7v.

1173 Résumé des arrêts, avis consultatifs et ordonnances de la Cour internationale de Justice 2008-2012 ST/LEG/SER.F/1/Add.5 Nations unies • New York, 2013 ST/LEG/SER.F/1/Add.5 PUBLICATION DES NATIONS UNIES NUMÉRO DE VENTE: F.13.V. ISBN 978-92-http://legal.un.org. Disponible sur le site internet : https://www.icj-cij.org. Consulté le 16/01/2022. $1174 \mathrm{Id}$.
} 
compte des considérations écologiques lorsqu'ils décident de ce qui est nécessaire ou proportionné dans la poursuite d'objectifs militaires légitimes ${ }^{1175}$.

Dans cette optique, la Cour internationale de justice est-elle d'une utilité pour les EtatsParties à la Convention Cadre des Nations Unies sur les Changements Climatiques?

L'étude de l'impact de l'intervention de la justice internationale dans le règlement des différends climatiques où sont impliqués les Etats-Parties à la Convention Cadre des Nations Unies sur les Changements Climatiques a révélé que sa contribution est mitigée.

\section{B- La contribution mitigée de la justice internationale}

En prescrivant dans la Convention Cadre des Nations Unies sur les Changements Climatiques de déférer les différends nés entre deux ou plusieurs Parties en ce qui concerne l'interprétation ou l'application de ladite Convention devant la Cour Internationale de Justice, la Communauté internationale a fait l'option de la reconnaissance de la compétence de la Cour internationale de justice par application de l'article 37 de son Statut ${ }^{1176}$. C'est une décision de haute portée, car le contrôle juridictionnel peut contribuer à assurer l'effectivité de l'application de la Convention Cadre des Nations Unies sur les Changements Climatiques. Dans ce cas, les décisions rendues par la Cour internationale de justice peuvent contribuer à faire évoluer le droit climatique. Selon le Professeur émérite Michel Prieur, «les décisions des juridictions internationales et régionales en matière d'environnement vont comme le droit international conduire par leur effet obligatoire à prendre des mesures nationales d'exécution ${ }^{1177}$. Il s'ensuit que les sentences prononcées par la juridiction internationale peuvent à titre indicatif être orientées dans le sens de contraindre les Etats Parties à la Convention Cadre des Nations Unies sur les Changements Climatiques au respect de leurs engagements. Même en absence de différends, l'existence de cette juridiction peut parfois suffire pour dissuader d'éventuels contrevenant des dispositions de la Convention Cadre des Nations Unies sur les Changements Climatiques.

\footnotetext{
${ }^{1175}$ COUR INTERNATIONALE DE JUSTICE, RECUEIL DES ARRETS, AVIS CONSULTATIFS ET ORDONNANCES, 8 juillet 1996, avis sur la licéité de la menace ou l'emploi des armes nucléaires. Disponible sur le site internet : https://www.icj-cij.org. Consulté le 16/01/2022.

${ }^{1176}$ La disposition stipule que « Lorsqu'un traité ou une convention en vigueur prévoit le renvoi à une juridiction que devait instituer la Société des Nations ou à la Cour permanente de Justice internationale, la Cour internationale de Justice constituera cette juridiction entre les parties au présent Statut ».

${ }^{1177}$ Michel PRIEUR, «L'influence des conventions internationales sur le droit interne de l'environnement». Actes de la réunion constitutive du comité sur l'environnement de l'AHJUCAF, Juin 2008, Porto-Novo, Bénin, pp.291301.
} 
A partir de ce moment, les Etats qui ont ratifié sans réserve particulière contraire à l'esprit de l'article 14 et les paragraphes subséquents de la Convention Cadre des Nations Unies sur les Changements Climatiques ont qualité de justiciable qui leur donne la possibilité d'exercer des voies de recours devant la haute juridiction. La Cour internationale de justice, suivant ses procédures ne peut s'autosaisir, elle ne peut qu'intervenir à condition d'être sollicitée. Il en est de même de la Conférence des Parties qui n'a pas qualité à agir devant la Cour Internationale de Justice (CIJ) pourtant, qu'elle est une institution gardienne de la Convention Cadre des Nations Unies sur les Changements Climatiques.

Les recherches documentaires des jurisprudences de la Cour Internationale de Justice concernant les Etats-Parties, n'ont révélé l'existence de décision rendue dans aucun différend né de l'application ou de l'interprétation de la Convention Cadre des Nations Unies sur les Changements Climatiques. L'analyse de la situation caractérisée par le non déferrement de différends nés de l'application ou de l'interprétation de la Convention Cadre des Nations Unies sur les Changements Climatiques devant la Cour Internationale de Justice (CIJ) trouve sa justification dans la complexité et le volontarisme qui entourent la procédure applicable devant la haute juridiction. Les Etats qui s'engagent dans les accords internationaux sont souvent jaloux des attributs de souveraineté et n'aiment pas privilégier le règlement juridictionnel des différends. Malheureusement, l'accès à ces juridictions demeure fermé à certaines organisations de la société civile et aux individus qui sont pourtant mieux placés pour assurer l'effectivité juridictionnelle de la Convention Cadre des Nations Unies sur les Changements Climatiques. La preuve en a été donnée aux Pays-Bas où l'hyper activité de la Société civile a conduit à la prise des décisions par des juridictions de cet Etat pour le contraindre à respecter son engagement de réduction des émissions des gaz à effet de serre souscrit dans la Convention Cadre des Nations Unies sur les Changements Climatiques. S'agissant de la France, malgré le rejet des nombreuses demandes formulées pour contraindre ce pays à respecter son engagement, le Juge Français a quand même reconnu la pertinence de l'implication des organisations de la société civile dans les actions juridictionnelles et a déclaré leur action recevable ${ }^{1178}$.

\footnotetext{
${ }^{1178}$ Selon la décision du CE, 19 nov. 2020, no 427301, ECLI:FR:CECHR:2020:427301.20201119, l'article 3 dispose que «Les interventions de la Ville de Paris, de la ville de Grenoble, des associations Oxfam France, Greenpeace France et Notre Affaire A Tous et de la Fondation pour la Nature et l'Homme sont admises dans la limite de la recevabilité de la requête de la commune de Grande-Synthe». Disponible sur le site internet: https://www.conseil-etat.fr/fr/arianeweb/CE/decision. Consulté le 16/01/2022.
} 
La justice des Pays-Bas, qui a condamné ce pays à réduire ses émissions de gaz à effet de serre suite à sa saisine par environ neuf cents $(900)$ citoyens ${ }^{1179}$. Cette condamnation passée en force de chose jugée, « pose le principe que le juge national peut enjoindre l'Etat à respecter et à traduire ses engagements climatiques internationaux dans le droit national $»^{180}$ contrairement à la faiblesse qu'affiche la justice internationale en présence de certaines puissances polluantes du monde ${ }^{1181}$.

De même, pour attraire un Etat Partie devant la Cour internationale de justice, le consentement de ceux-ci est requis malgré qu'ils aient ratifiés la Convention. Eu égard à ces goulots, la reconnaissance formelle et l'acceptation de la compétence obligatoire de la dite Cour s'impose. Dans ce cadre, le Royaume des Pays-Bas a fait l'option de la compétence de la Cour internationale de justice en déclarant que, « (...) conformément au paragraphe 2 de l'article 14 de la Convention Cadre des Nations Unies sur les Changements Climatiques, qu'il reconnaît les deux moyens de règlement visés dans ledit paragraphe comme obligatoires à l'égard de toute Partie acceptant la même obligation ${ }^{1182}$. Par contre, pour le Cuba, à propos de l'application de 14 de la Convention cadre, ce pays déclare "qu'en ce qui le concerne, le règlement des différends qui surgiraient entre les Parties au sujet de l'interprétation ou de l'application de la Convention fera l'objet d'une négociation par la voie diplomatique ${ }^{1183}$. Le Cuba a exclu la compétence de la Cour internationale de justice au détriment de procédure non contentieuse.

En matière de justice internationale, le Bénin n'est pas un intrus à la Cour internationale de justice, car ce pays a été bénéficiaire d'une décision issue d'un procès engagé devant cette juridiction contre l'Etat du Niger, dans un différend territorial que la Cour a tranché en faveur du Niger ${ }^{1184}$. Ce conflit a connu son aboutissement avec l'appui de la coopération internationale entre le Bénin et le Niger. Ces deux (2) Etats ce sont entendus pour engager le procès et en accepter le verdict. Mais en ce qui concerne le conflit climatique, il est difficile de mesurer

\footnotetext{
${ }^{1179}$ Commission environnement, .Rapport : renforcer l'efficacité du droit international. Devoirs des Etats, droits des individus, novembre 2015, p. 90 . Disponible sur le site internet: www.leclubdesjuristes.com consulté le 25 juillet 2016.

${ }^{1180}$ Voir les dispositions des articles 280 et 299 du traité sur le fonctionnement de l'UE. Raphaël ROMI, Le juge, la prévention et la résolution des litiges en matière d'environnement, thèse de doctorat, Paris III, multigraphiée, 1994, 432 p. et Sandrine MALJEAN-DUBOIS, L'«observance» du Protocole de Kyoto sur les changements climatiques. Les enjeux du contrôle international du respect des engagements», op. cit., p.2.507.

${ }^{1181}$ Par exemple les Etats-Unis d'Amérique.

${ }^{1182}$ Etat des ratifications de la Convention Cadre des Nations Unies sur les Changements Climatiques. Disponible sur le site internet : https://treaties.un.org. Consulté le 28/05/2021.

${ }^{1183} \mathrm{Id}$.

${ }^{1184}$ Différend frontalier (Bénin/Niger), arrêt, C.I.J. Recueil 2005, p.90. Disponible sur le site internet: https://www.icj-cij.org. Consulté le 16/01/2022.
} 
l'impact des actions de la Cour internationale de justice sur le Bénin, s'agissant de la gestion par la haute juridiction de différend qui opposerait cet Etat avec un autre. Et généralement, il en serait ainsi pour tous les autres Etats Parties à la Convention Cadre des Nations Unies sur les Changements Climatiques, puisque la juridiction internationale n'a jamais été saisie d'un conflit directement lié à l'application de ladite Convention.

Il en est ainsi parce que la faculté concédée aux Etats ${ }^{1185}$ adhérents au statut de la Cour d'ester devant la juridiction internationale et de recourir aux avis consultatifs de ladite Cour au profit des organisations internationales ${ }^{1186}$ constitue un facteur qui ne favorise pas l'éclosion des actions juridictionnelles visant à mettre en cause la responsabilité de l'Etat dans le cadre de l'application de la Convention Cadre des Nations Unies sur les Changements Climatiques. En plus, les organisations non gouvernementales, qui sont plus aptes à porter les intérêts de la population et ceux de leurs Etats ne sont pas autorisés à ester devant cette juridiction. Si cette possibilité existait, le dossier concernant la «découverte d'un contrat entre le Gouvernement d'alors ${ }^{1187}$ et la société SESCO GIBRALTAR LIMITED de déversement au Bénin de déchets dangereux, signé à Cotonou le 12 janvier $1988 »^{1188}$ connaitrait un épilogue judiciaire important. Car le fait que le Gouvernement était au cœur de ce scandale, son inaction pouvait être supplée par l'intervention des Organisations de la Société civile qui vont se battre pour déférer le contentieux devant les juridictions internationales compétentes.

Cependant, il existe en droit international des mesures pour contraindre les Parties à respecter leurs engagements. Par exemple, le droit international admet dans le cadre d'un traité multilatéral, que la réponse à la violation d'une obligation conventionnelle passe par l'application des mesures de suspension comme conséquence de sa violation ${ }^{1189}$. Seulement que cette réaction ne serait efficace que si les Etats ont effectivement un intérêt commun à une mise en œuvre sans faille du traité. Pour ce qui concerne la Convention Cadre des Nations unies sur les Changements Climatiques, les obligations des Parties ne sont pas identiques ni réciproques. Il s'ensuit que l'application de sanction contre un Etat contrevenant les dispositions de ladite Convention serait contre productif, parce que cette mesure peut favoriser l'explosion de la plateforme internationale de protection du climat.

\footnotetext{
${ }^{1185}$ Article $34 \mathrm{du}$ statut de la Cour Internationale de Justice. Disponible sur le site internet : https://www.icjcij.org/fr. Consulté le 16/01/2022.

${ }^{1186}$ Article 96 de la Charte des Nations unies et l'article 65 du Statut de la Cour Internationale de Justice.

${ }^{1187}$ C'était le Gouvernement militaro marxiste dirigé par le Général Mathieu KEREKOU de 1972 à 1990.

${ }^{1188}$ LAWOGNI (Athanase) et de SADELEER (Nicolas), « Droit de l'environnement au Bénin Code officieux de l'environnement », Cotonou - Bruxelles 2002, 406p., p.3.

${ }^{1189}$ Article 60 de la Convention de Vienne sur les Traités de 1969. Disponible sur le site internet: https://legal.un.org/ilc/texts/instruments/french/conven. Consulté le 16/01/2022.
} 
En dehors de la Cour internationale de justice dont la compétence est requise pour accomplir le contrôle juridictionnel de l'application de la Convention Cadre des Nations Unies sur les Changements Climatiques, il est également prévu des modes alternatifs de règlement des différends climatiques.

\section{Paragraphe 2: Le mode alternatif de règlement des différends}

Les conclusions d'une étude ont révélé que, «Presque toutes les conventions internationales de protection de l'environnement incluent une clause classique de règlement des différends prévoyant que, tout différend relatif à l'interprétation et à l'application de la convention sera réglé par des moyens diplomatiques $»{ }^{1190}$. Ce mode de règlement des différends n'a pas suscité d'engouement de la part des Parties à la Convention Cadre des Nations Unies sur les Changements Climatiques qui se sont abstenues d'y souscrire à l'exception des Îles Salomon qui ont déclaré en vertu du paragraphe 2 de l'article 14 de la Convention, « reconnaître comme obligatoire l'arbitrage $(\ldots){ }^{1191}$. Le rejet des Etats est symptomatique de ce que, ce mode de règlement des différends ne conviendrait pas à leur aspiration. Il s'ensuit que la non adhésion massive à ce mode de règlement de différend complique sa mise en œuvre. Cette situation s'ajoute à la non effectivité de la gestion juridictionnelle des différends climatiques devant la Cour Internationale de Justice

Face à la nécessité d'empêcher la dégradation continue de l'environnement, pour cause de conflits ou des difficultés éventuelles qui peuvent entraver la mise en œuvre de la Convention Cadre des Nations Unies sur les Changements Climatiques, le droit international a innové autres modes alternatifs de règlement des différends à titre préventif qui consistent d'une part, au contrôle du respect des engagements $(\mathbf{A})$ et d'autre part, au règlement pacifique $(\mathbf{B})$.

\section{A- Le contrôle du respect des engagements}

Au-delà des recommandations habituelles d' « application » ou de « mise en œuvre » des accords internationaux, l'actualité est focalisée sur la problématique de la «mise en conformité » des actions accomplies pour réaliser les engagements souscrits dans le cadre conventionnel. Puisqu'il ne s'agit plus de se confiner dans la logique du concept de «mise en

\footnotetext{
${ }^{1190}$ Sandrine Maljean-Dubois, Changements climatiques, l'enjeu du contrôle international, La Documentation Française, Paris, 2007, 383p.

${ }^{1191}$ Etat de ratification de la Convention Cadre des Nations Unies sur les Changements Climatiques. Disponible sur le site internet : https://treaties.un.org/Pages. Consulté le 28/05/2021.
} 
œuvre » ou de simple « application des normes conventionnelles » pour espérer atteindre ses objectifs, mais il est question de procéder au contrôle du respect des engagements pour s'assurer de leur conformité par rapport aux objectifs du Traité.

Les questions relatives au respect d'engagements librement souscrits par les Etats se trouvent actuellement au cœur des préoccupations des acteurs du droit de protection de l'environnement. En effet, si le respect est une condition pour atteindre l'efficacité, c'est par ce moyen que l'objectif de la convention peut être atteint. Cependant, Michael G. Faure et Jürgen Lefevere estiment que, "Si un traité est respecté, cela ne signifie pas automatiquement qu'il est efficace pour atteindre l'objectif environnemental pour lequel il a été initialement conçu» ${ }^{1192}$. C'est pourquoi, objectivement, la question de la recherche de l'efficacité des normes doit être évoquée au moment de l'élaboration des instruments internationaux. Elle " dépend de la conception même du traité, des instruments et objectifs contenus dans le traité, ainsi que d'autres facteurs externes, tels que l'évolution des situations politiques ou même des conditions environnementales» ${ }^{1193}$.

Désormais, pour faciliter les questions de mise en œuvre, le droit international a marqué son choix sur l'esprit de coopération dans l'application des normes internationales au détriment de la coercition, parce que le non respect des engagements conventionnels par l'Etat découlerait le plus souvent « d'une difficulté, voire d'une impossibilité de mise en œuvre, pour des raisons techniques ou financières, que de la mauvaise volonté de l'Etat ${ }^{1194}$. En effet, la recherche de l'efficacité a provoqué l'introduction dans les traités internationaux des dispositifs de règlement de différends qui sont conçus pour aider à interpréter ou à solutionner les conflits découlant de leur application. Seulement que, le choix porté sur les méthodes traditionnelles de règlement des différends basées sur l'application des sanctions en cas de violation des normes, la suspension des traités, parfois l'application des mesures coercitives à l'endroit des auteurs des violations présente des limites. D'ailleurs, dans le domaine du Commerce international, l'application des sanctions économiques est devenue non avenue depuis l'intensification des relations commerciales par la mise en œuvre des accords internationaux en la matière. La mise œuvre de ces mesures et le risque pour certains Etats de ne pas affronter leur pair devant les juridictions ne favorisent pas l'application des Traités internationaux.

\footnotetext{
${ }^{1192}$ Compliance with Global Environmental Policy Climate Change and Ozone Layer Cases, Michael G. Faure and Jürgen Lefevere, p.2.

1193 Id.

${ }^{1194} \mathrm{~S}$. Maljean-Dubois et C. Nègre, « La procédure de non-respect du Protocole de Montréal relatif à des substances qui appauvrissent la couche d'ozone », in S. Maljean-Dubois (dir.), Changements climatiques. Les enjeux du contrôle international, préc., p. 348 et 351., p. 334.
} 
C'est pourquoi, il est désormais question de passer à une approche «managériale» de la question de la gestion des différends en tenant compte réellement de la capacité des Parties à appliquer la Convention. De ce point de vue, selon le Professeur Michel PRIEUR, c'est d'abord le protocole de Montréal de 1987 sur la réduction des substances appauvrissant la couche d'ozone qui a innové des «procédures originales et propres au droit de l'environnement (...) il s'agit de l'organisation de compliance commitee ou comité de mise en cuvre ou de non respect $»^{1195}$. Le Protocole de Kyoto qui est un prolongement de la Convention Cadre des Nations Unies sur les Changements Climatiques ne contient pas de clause propre de règlement des différends, mais le traité a prévu que « Les dispositions de l'article 14 de la Convention relatives au règlement des différends s'appliquent mutatis mutandis au (...) Protocole $»^{1196}$. Dans sa substance, l'article 14 paragraphe 1 de la Convention prescrit que les « Parties règlent leurs différends par voie diplomatique $»$. C'est seulement en cas de règlement diplomatique infructueux des différends que les Parties ont la faculté de recourir à un règlement juridictionnel $^{1197}$. En adoptant le Protocole de Kyoto, à travers cet instrument, sont fixés des engagements quantitatifs de réduction des émissions de gaz à effet de serre à la charge des Etats pollueurs ${ }^{1198}$. La Communauté internationale a fourni des moyens d'atteindre l'objectif de réduction de gaz à effet de serre en instituant les mécanismes de flexibilité, notamment, le « Mécanisme de Développement Propre, ${ }^{1199}$ un mécanisme à caractère économique pour animer le marché de transaction sur le gaz carbonique aux fins de la réduction de l'émission du gaz à effet de serre. Les pays en développement à travers la mise en œuvre de ce mécanisme bénéficient des projets dont l'exécution des activités qui en découlent devrait permettre aux puissances pollueuses de s'acquitter de leurs engagements de limitation ou de réduction de gaz à effet de serre ${ }^{1200}$. Mais en plus, le Protocole a exigé «(...) le rapport de la preuve de l'exécution de l'engagement (...)» ${ }^{1201}$ pour s'assurer de l'effectivité de ces objectifs. Pour cela, il a institué « Le contrôle du respect des engagements des Parties». Les moyens de parvenir à réaliser ce contrôle est de s'assurer de la conformité des engagements. Bien entendu, «la

\footnotetext{
${ }^{1195}$ Michel Prieur. L'influence des conventions internationales sur le droit interne de l'environnement. Actes de la réunion constitutive du comité sur l'environnement de l'AHJUCAF, Juin 2008, Porto- Novo, Bénin. pp.291-301., p.297.

1196 Art. 19 du Protocole de Kyoto.

${ }^{1197}$ L'article 14 paragraphe 2 de la Convention Cadre des Nations Unies sur les Changements Climatiques, contient une clause facultative de règlement juridictionnel.

1198 Article 3 paragrahe1 du Protocole de Kyoto.

1199 Article 12 id.

${ }^{1200}$ Article 12 paragraphe 2 du Protocole de Kyoto.

${ }^{1201}$ Article 3 paragraphe 2 et 3 du Protocole de Kyoto.
} 
conformité n'est qu'un indicateur de l'efficacité » ${ }^{1202}$. Cependant, " une plus grande conformité entraînera généralement une amélioration de l'environnement» ${ }^{1203}$. Dans ce cas, il est préférable que les thèmes des engagements ne soient pas superflus. A titre illustratif, le Protocole de Montréal sur les substances qui appauvrissent la couche d'ozone formule des engagements précis à la charge des cibles restreintes et connues. Dans le cas contraire, lorsque les obligations conventionnelles sont formulées sans aucune objectivité, le contrôle du respect devient compliqué ${ }^{1204}$. C'est le cas de l'article 4 (2) (a) de la Convention Cadre des Nations Unies sur les Changements Climatiques qui ne détermine pas d'obligation spécifique à la charge des Parties. Il a fallu l'intervention du Protocole de Kyoto et de l'Accord de Paris pour encadrer à nouveau et de manière claire sans équivoque les engagements à la charge des Parties. Ces instruments ont institué respectivement, le contrôle du respect des engagements pour ce qui concerne le Protocole de Kyoto ${ }^{1205}$ et le cadre de transparence s'agissant de l'Accord de Paris ${ }^{1206}$.

Quelle est la consistance de la procédure applicable en matière du contrôle du respect des engagements?

La procédure du non-respect vise à faciliter le respect par les Etats Parties de l'accomplissement de leurs engagements sans qu'il ne soit besoin de recourir à l'application de la sanction en cas de difficulté d'exécution des obligations conventionnelles. Elle fournit un cadre politique pour des réponses «amiables» à la non-conformité qui ne peut pas être considérée comme «illicite».

Dans le cadre de la mise en œuvre de cette procédure, les sanctions comme l'expulsion, la suspension des droits et privilèges ne sont pas applicables. Il est plutôt souhaitable dans le cadre d'une protection universelle de l'environnement, d'avoir une adhésion massive autour des conventions. Les règles de procédure du respect d'engagement ont été adoptées à Marrakech en 2001 lors de la COP $7^{1207}$ et ont connu leur début d'application après l'entrée en vigueur du Protocole de Kyoto en décembre 2005. Dans le cadre de sa mise en œuvre, le Comité $\mathrm{du}$ respect des engagements apprécie les rapports nationaux et désigne les Etats qui sont

\footnotetext{
1202 Compliance with Global Environmental Policy Climate Change and Ozone Layer Cases, Michael G. Faure and Jürgen Lefevere, p.3.

${ }^{1203}$ id.

${ }^{1204}$ Compliance with Global Environmental Policy Climate Change and Ozone Layer Cases, Michael G. Faure and Jürgen Lefevere, p.7.

1205 Article 18 du Protocole de Kyoto.

1206 Article 15 de l'Accord de Paris.

${ }^{1207}$ Par décision 24/cp.7, 10 novembre 2001 ; fccc/cp/2001/13/Add.3, 21 janvier 2002. La mise en place de ce Comité a été approuvée lors de la Conférence de Montréal en 2005 ; Décision 27/cpm.1.
} 
éligibles pour prendre part aux mécanismes du Protocole de Kyoto. La chambre de facilitation saisie du rapport des Experts n'accuse pas, mais accompagne les Etats dans la mise en œuvre du Protocole. Puisqu'en réalité, la chambre de facilitation est chargée de donner des « conseils et d'apporter une aide aux Parties aux fins de l'application du Protocole et de promouvoir le respect par les Parties, des engagements qu'elles ont pris en vertu du Protocole, compte tenu du principe des responsabilités communes mais différenciées et des capacités respectives des Parties énoncé au paragraphe 1 de l'article 3 de la Convention ${ }^{1208}$. Tandis que, la chambre d'exécution a le pourvoir de suspendre le pays en cause de la participation au mécanisme du Protocole en attendant le règlement de la question de la mise en œuvre.

L'Accord de Paris a aussi institué un mécanisme de respect des obligations pour «faciliter la mise en œuvre et promouvoir le respect des dispositions ( ...)» ${ }^{1209}$ conventionnelles. Dans cet Accord, « L'accent est ainsi mis sur un mécanisme souple de revue par les pairs plutôt qu'un système juridique de sanctions $»{ }^{1210}$. C'est ce que précise l'article 15 paragraphe 2 dudit Accord qui dispose que, ce mécanisme de non-respect est « (...) axé sur la facilitation et fonctionne d'une manière transparente, non accusatoire et non punitive(...) ». Dans ce cadre, une attention singulière est accordée aux Parties quant à leurs capacités respectives à amorcer les charges qui leur incombent. La procédure applicable devant la chambre de l'exécution s'apparente à celle suivie dans le cadre du comité d'observance du Protocole de Kyoto qui est « hybride » ou « quasi juridictionnelle ${ }^{1211}$ et dépasse le cadre d'une procédure diplomatique classique telle que, la conciliation ou la médiation. La chambre de l'exécution peut même trancher des différends et rendre une décision en droit exécutoire et opposable à l'Etat en cause. In fine, « cette procédure non contentieuse vise à mettre à la lumière, les difficultés objectives de caractère technique, scientifique ou économique qui empêchent de bonne foi, de satisfaire totalement aux obligations de la convention ${ }^{1212}$. Dans ce cadre, le non respect des engagements n'induit pas des sanctions à l'encontre de l'Etat contrevenant, il peut simplement être classé sur la liste des «cancres du climat», c'est-à-dire, qu'il peut faire l'objet d'une

\footnotetext{
${ }^{1208}$ Conformément à la Décision 27/CMP.1. Les procédures et mécanismes relatifs au respect des dispositions du Protocole de Kyoto, sont contenues dans la rapport de la première session de la Conférence des Parties agissant comme réunion des Parties au Protocole de Kyoto tenue à Montréal du 28 novembre au 10 décembre 2005, annexe, art. 4.

${ }^{1209}$ Article 15 de l'Accord de Paris.

${ }^{1210}$ L'analyse de Daniel Bodansky, «Reflections on the Paris Conference », postée le 15 décembre 2015. Disponible sur le site internet : http://opiniojuris.org/2015/12/15reflectionson-the-paris-conference/; p.15.

1211 Sandrine MALJEAN-DUBOIS, «L'observance du Protocole de Kyoto sur les changements climatiques. Les enjeux du contrôle international du respect des engagements, in Synthèses IDDRI $n^{\circ} 01,2007$.

${ }^{1212}$ Michel Prieur. L'influence des conventions internationales sur le droit interne de l'environnement. Actes de la réunion constitutive du comité sur l'environnement de l'AHJUCAF, Juin 2008, Porto- Novo, Bénin. pp.291-301., p. 297.
} 
sanction politique ${ }^{1213}$. Les pays en développement peuvent se voir privés de l'assistance financière au titre de l'adaptation en cas de non respect du cadre de transparence des communications de leur $\mathrm{CDN}$ et de leurs mesures d'adaptation bien qu'il ne l'a pas été expressément stipulé dans l'article 7 paragraphe 3 de l'Accord de Paris ${ }^{1214}$.

La mise en ouvre du système de contrôle international de non respect des engagements assure-t-elle l'effectivité de la protection du climat?

Selon MALJEAN-DUBOIS Sandrine, SPENCER Thomas et WEMAERE Matthieu, «Bien que la procédure du contrôle du respect des engagements soit très bien élaborée, le système n'est pas totalement verrouillé » ${ }^{1215}$. Parce que, «Un Etat en grande difficulté pourrait choisir de passer outre ${ }^{1216}$ la réalisation de ses engagement. Certes, il peut encourir l'exclusion des mécanismes du marché, «mais il pourrait en contrepartie cumuler les dettes environnementales jusqu'à ce que celles-ci deviennent irrécouvrables $»^{1217}$. Dans le cas échéant, le risque de voir le pays concerné se retirer de cet Accord est grand ${ }^{1218}$. Malgré ces « prévisions pessimistes, le respect du Protocole de Kyoto a été supérieur à 100\% au cours de la première période d'engagement, la plupart des pays ont même dépassé leurs objectifs d'émission nationaux $»^{1219}$.

Nonobstant les performances obtenues au cours de la première période d'engagement du Protocole de Kyoto, l'enjeu de limitation ou de réduction des gaz à effet de serre demeure encore, car le Protocole de Kyoto n'a pas connu la participation des puissances pollueuses telles que les Etats-Unis d'Amérique ${ }^{1220}$. L'objectif in fine du contrôle du respect des engagements

\footnotetext{
${ }^{1213}$ Sophie Lavallée, «Quelques réflexions sur 1'Accord de Paris et la souveraineté économique des Etats », Vertigo, la revue électronique en sciences de l'environnement, Volume 18 numéro 1, mai 2018, p. 15.

${ }^{1214}$ Sophie Lavallée, "Quelques réflexions sur l'Accord de Paris et la souveraineté économique des Etats ", Vertigo, la revue électronique en sciences de l'environnement, Volume 18 numéro 1, mai 2018, p. 15.

1215 MALJEAN-DUBOIS (Sandrine), SPENCER (Thomas) et WEMAERE (Matthieu), « La forme juridique du futur accord de Paris sur le climat : enjeux et principales options », Pedome, Paris 2015, pp. 177-208. ${ }^{1216}$ Id.

${ }^{1217}$ R. Guesnerie, Kyoto et l'économie de l'effet de serre, Rapport CAE n³9, La Documentation Française, Paris, 2003 , p. 72.

${ }^{1218}$ C'est le cas du Canada qui s'est retiré du Protocole de Kyoto. Voir Note 2 : Conformément au paragraphe 2 de l'article 27 du Protocole de Kyoto à la Convention Cadre des Nations Unies sur les Changements Climatiques, le Gouvernement du Canada a notifié au Secrétaire général de sa décision de se retirer du Protocole de Kyoto, à la date 15 décembre 2011 qui prend effet le15 décembre 2012. Disponible sur le site internet : https://treaties.un.org. Consulté le 17/01/2022.

${ }^{1219}$ See Igor Shishlov, Romain Morel and Valentin Bellassen, "Compliance of the Parties to the Kyoto Protocol in the First Commitment Period," Climate Policy 16, no. 6 (2016): 768-782. This was reportedly also due to the non-participation of the US and Canada and to the fact that the global financial and economic crisis decreased GHG emissions, but also by policies and measures put in place by signatory countries. See also Michael Grubb, "Full Legal Compliance with the Kyoto Protocol's First Commitment Period - Some Lessons," Climate Policy 16, no. 6 (2016): 673-681.

${ }^{1220}$ Etat de ratification du Protocole de Kyoto. Disponible sur le site internet : https://treaties.un.org. Consulté le $17 / 01 / 2022$.
} 
étant d'empêcher l'augmentation des gaz à effet de serre, il s'ensuit que la mise en œuvre du Protocole de Kyoto n'a pu empêcher le réchauffement climatique, qui s'est aggravé avec l'accroissement d'émission supplémentaire de gaz à effet de serre dans l'atmosphère entre la période de 2008 et 2013. Pour certains chercheurs ${ }^{1221}$, cet accroissement supplémentaire représente «la facture climatique d'une faille embarrassante dans le Protocole de Kyoto précisément destiné à lutter contre les émissions de gaz à effet de serre ${ }^{1222}$. Il en est ainsi, parce que malgré le succès du Traité, il ne représente qu'une infirme portion de l'émission mondiale du gaz à effet de serre ${ }^{1223}$. C'est ce problème de légitimité qui a caractérisé le Protocole de Kyoto que l'Accord de Paris est intervenu pour corriger. Et par la même occasion, le mécanisme du respect a été remplacé par un autre mécanisme plus amélioré dénommé « cadre de transparence $»$.

Entre autre mode alternatif de règlement, se trouve le règlement pacifique des différends climatiques prévu par la Convention Cadre des Nations Unies sur les Changements Climatiques.

\section{$B$ - Le règlement pacifique des différends}

La vision de la Communauté internationale en ce qui concerne la promotion des relations amicales entre les Etats, la paix et la sécurité internationale est basée sur la recherche primordiale des solutions pacifiques au règlement des différends ${ }^{1224}$. Cette vision a été matérialisée par l'article 33 de la Charte des Nations unies qui précise que, « les Parties à tout différend dont la prolongation est susceptible de menacer le maintien de la paix et de la sécurité internationale, doivent en rechercher la solution avant tout, par voie de négociation, d'enquête, de médiation, de conciliation, d'arbitrage, de règlement judiciaire, de recours aux organismes ou accords régionaux ou par d'autres moyens pacifiques de leur choix. » La Communauté internationale mobilisée autour de la lutte contre les changements climatiques, a internalisé cette règle en prescrivant qu' « en cas de différend entre deux ou plus de deux Parties au sujet de l'interprétation ou de l'application de la Convention, les Parties concernées s'efforcent de

\footnotetext{
${ }^{1221}$ Ceux qui proviennent du Stockholm Environnent Institute (SEI est une organisation internationale de recherche et de politique à but non lucratif qui s'attaque aux défis de l'environnement et du développement). Site internet de l'organisation est: https://www.sei.org.

${ }^{1222}$ Dans un rapport publié lundi 24 août, le centre de recherche met en lumière les faiblesses de l'un des mécanismes prévus par le traité, entré en vigueur en 2005. https://www.lemonde.fr/climat.

${ }^{1223}$ Comme en témoigne l'état de ratification du Protocole de Kyoto qui n'est pas souscrit par la plupart des puissances pollueuses, notamment, les Etats Unis d'Amérique, le Canada. Disponible sur le site internet: https://treaties.un.org. Consulté le 17/01/2022.

${ }^{1224}$ Article 1 paragraphe 1 de la Charte des Nations Unies.
} 
le régler par voie de négociation ou par tout autre moyen pacifique de leur choix ${ }^{1225}$. Il s'ensuit que la négociation et le règlement pacifique des différends climatiques sont admis comme modes alternatifs de règlement de conflit dans le contexte de la Convention Cadre des Nations Unies sur les Changements Climatiques. Selon le Professeur Laurence Boisson de Chazournes, ce "processus consultatif multilatéral est envisagé en vue d'aider les Parties à surmonter les difficultés rencontrées dans l'application de la Convention et d'empêcher la naissance des différends ${ }^{1226}$. Le même auteur précise que le caractère préventif de la démarche peut «faciliter la compréhension de la Convention et empêcher la naissance de différends » ${ }^{1227}$.

Comment se déroule le règlement pacifique des différends climatiques dans le contexte de l'Accord climat?

Selon les dispositions de la Convention Cadre des Nations Unies sur les Changements Climatiques, la procédure applicable au règlement pacifique ${ }^{1228}$ des différends repose sur la négociation $^{1229}$, une procédure non contraignante, axée sur le volontarisme des Parties. La procédure de règlement pacifique consiste en une communication directe ou indirecte entre deux ou plusieurs Parties en vue d'endiguer, voire de résoudre un litige les opposant, né de l'application de la Convention Cadre des Nations Unies sur les Changements Climatiques. A titre indicatif, en cas de survenance de difficulté d'application entre Etats Parties, il appartient à chacun des membres de faire son option de règlement pacifique par la négociation, qui s'ouvre par la notification de l'existence du différend à la Partie adverse ${ }^{1230}$. « Si, à l'expiration d'un délai de douze (12) mois à compter de la date à laquelle une Partie a notifié à une autre l'existence d'un différend entre elles, les Parties concernées ne sont pas parvenues à régler leur différend en utilisant les moyens décrits au paragraphe 1 de l'article 14 de la Convention Cadre des Nations Unies sur les Changements Climatiques, le différend, à la demande de l'une quelconque des Parties est soumis à la conciliation ${ }^{1231}$. Pour rappel, l'article 14 de la Convention Cadre des Nations Unies sur les Changements Climatiques donne la possibilité de régler les différends par la mise en œuvre des procédures bilatérales classiques de règlement des différends, par la création d'une commission de conciliation à la demande de l'une des Parties au différend ou, si les Etats concernés l'acceptent par la soumission du différend à la

\footnotetext{
${ }^{1225}$ Article 14 paragraphe 1 de la Convention Cadre des Nations Unies sur les Changements Climatiques.

${ }^{1226}$ Laurence Boisson de Chazournes, «L'impact humain sur le changement climatique », United Nations Audiovisual Library of International Law, 2009, p.5.

1227 Id.

${ }^{1228}$ Article 14 paragraphes 5, 6, 7 de la Convention Cadre des Nations Unies sur les Changements Climatiques.

${ }^{1229}$ Article 14 paragraphe 1 de la Convention Cadre des Nations Unies sur les Changements Climatiques.

${ }^{1230}$ Article 14 paragraphe 5 id.

1231 Ibid.
} 
Cour internationale de justice ou à un arbitrage international. Dans le cas échéant, «une commission de conciliation est créée à la demande de l'une des Parties au différend, composée de membres désignés en nombre égal, par chaque Partie concernée et d'un Président choisi conjointement par les membres désignés par les Parties. La Commission présente une recommandation que les Parties examinent de bonne foi ${ }^{1232}$. Nonobstant ce mode de règlement pacifique des différends, la Convention Cadre n'a pas exclu l'option de la procédure judiciaire à laquelle les Etats Parties peuvent recouvrir en cas d'échec des autres moyens prévus à l'article 14 de ladite Convention.

Le règlement pacifique des différends climatiques-a-t-il vraiment contribué à éclore l'action de la justice climatique?

Tout comme l'expérience peut concluante de la Cour internationale de justice, organe juridictionnel de la Convention Cadre des Nations Unies sur les Changements Climatiques, pour le règlement des différends climatiques nés de l'application de la Convention, le cadre du règlement pacifique des différends climatiques n'a pas non plus émergé, car il n'y a pas de différends qui soit tranché suivant ce mode de règlement. La recherche de la jurisprudence a été infructueuse en ce qui concerne des sentences découlant de la mise en œuvre des procédures de négociation ou de conciliation en application de la Convention Cadre des Nations Unies sur les Changements Climatiques. A l'instar de la procédure contentieuse se déroulant devant la Cour internationale de justice, la négociation et la conciliation sont subordonnées à la volonté des Parties qui acceptent se soumettre non seulement à la procédure mais également, elles devront s'engager à subir la sentence qui en découlerait. Le volontarisme qui caractérise les procédures applicables pour ces modes de règlement des différends ne favorise pas l'éclosion de la jurisprudence en la matière.

Malgré les faiblesses notées dans le règlement juridictionnel international des différends climatiques, il y a bien des Etats Parties qui essayent tant bien que mal de relever à l'interne les défis infligés par les changements climatiques en mettant à contribution le système juridictionnel de leur pays. Le comportement de la justice des Etats tels que, les Pays- Bas, les Etats-Unis d'Amérique, la Colombie, le Brésil et la France, qui ce sont illustrées positivement dans la gestion de conflit climatique en intimant des mesures pour contraindre ces Etats respectifs à respecter leur part d'engagement souscrit dans le cadre de la Convention Cadre des Nations Unies sur les Changements Climatiques édifie mieux sur la question. De la même manière, au Bénin, la justice est sollicitée pour assurer la gestion des conflits qui découlent de

\footnotetext{
${ }^{1232}$ Article 14 paragraphe 6 de la Convention Cadre des Nations Unies sur les Changements Climatiques.
} 
la mise en œuvre des dispositions applicables dans le cadre de la lutte contre les changements climatiques, seulement que sa participation à l'action climatique est mitigée.

\section{SECTIONII: LA PARTICIPATION MITIGEE DE LAJUSTICE BENINOISE}

La règlementation sur l'organisation judiciaire béninoise ${ }^{1233}$ n'avait pas intégré la dimension de la protection de l'environnement, ni les règles applicables en matière de gestion des conflits climatiques dans les domaines de compétence des juridictions existantes. Les juridictions étaient créées et réputées compétentes pour les conflits relevant de tous les domaines exceptés, ceux qui concernent les domaines de la protection de l'environnement et des changements climatiques.

La réforme du cadre juridique intervenue après l'intégration de la protection de l'environnement dans la constitution béninoise a provoqué l'intervention de la justice dans le domaine de la protection de l'environnement. En effet, certains textes régissant la protection de l'environnement, la protection de la forêt, la pollution environnementale et la protection du climat ont conférés des attributions à la justice béninoise qui peut désormais intervenir dans l'action de protection de l'environnement et dans la lutte contre les changements climatiques. Seulement que, cette intervention est soldée par des résultats mitigés.

La justice béninoise intervient dans le domaine de la protection de l'environnement, pour contribuer au règlement des conflits environnementaux. Ces conflits sont examinés selon les cas, soit par la Cour constitutionnelle, (Paragraphe 1) ou soit par les juridictions de l'ordre judiciaire (Paragraphe 2).

\section{Paragraphe 1: L'intervention de la Cour constitutionnelle}

Il s'agit dans cette rubrique d'examiner l'effectivité de la compétence environnementale et climatique de la Cour constitutionnelle, qui est impliquée dans le contrôle juridictionnel des actes du domaine de la règlementation environnementale et climatique. L'intervention de la Cour constitutionnelle dans le contrôle juridictionnel du droit applicable aux domaines environnemental et climatique s'effectue de deux manières à savoir :

${ }^{1233}$ La loi n²001-37 du 10 juin 2002 portant organisation judiciaire en République du Bénin et la loi n 2016-15 modifiant et complétant la loi $\mathrm{n}^{\circ}$ 2001-37 du 10 juin 2002 portant organisation judiciaire en République $d u$ Bénin. Disponible sur le site internet : https://legis.cdij.bj > index.php > loi-n-2016-15-modifiant. Consulté le 17/01/2022. 
Premièrement, au Bénin, c'est par le contrôle de constitutionnalité que les accords internationaux sont intégrés au droit positif (A).

Deuxièmement, le dispositif normatif devant contribuer à l'application des accords internationaux, c'est-à-dire les lois et règlements d'application desdits accords et autres actes d'importances sont également exposés au contrôle du juge constitutionnel (B).

\section{A- L'intégration des accords internationaux}

Généralement en droit international, l'Etat en souscrivant aux accords internationaux décide en toute souveraineté de s'accommoder des obligations convenues. Dans ce cas, il est tenu de les mettre en œuvre sur son territoire en s'acquittant de ses obligations ${ }^{1234}$. La particularité des conventions de protection l'environnement et les accords de protection du climat qui établissent des engagements à la charge des Etats Parties n'exempte guère, l'observance de ces formalités requises en matière du droit des relations internationales. La valeur de ces conventions dépend de la prédisposition de l'Etat signataire à les faire appliquer de bonne foi. Mais avant leur mise en œuvre, il faudra les intégrer au droit positif en observant les formalités de ratification qui représentent l'acte matériel d'acceptation des accords internationaux $^{1235}$.

Au Bénin, les engagements internationaux de l'Etat n'ont pas d'effets immédiats, ils obéissent à des mesures de réception interne avant qu'ils soient applicables dans l'ordre juridique national. Le processus d'intégration d'accords internationaux à l'ordre juridique de certains Etats démocratiques tout comme celui du Bénin, obéit au formalisme de la constitutionnalité qui se déroule devant le juge de la juridiction constitutionnelle. Cependant, les juridictions constitutionnelles varient d'un Etat à un autre. En France, le contrôle de constitutionnalité est effectué par le Conseil constitutionnel ${ }^{1236}$, tandis qu'aux Etats-Unis d'Amérique ${ }^{1237}$ et au Bénin, la juridiction de constitutionnalité est représentée par la Cour

\footnotetext{
${ }^{1234}$ Articles 26, 27 et 29 de la Convention de Vienne sur le Droit des traités. Disponible sur le site internet: https://legal.un.org/ilc/texts/instruments/french/conve. Consulté le 16/01/2022.

${ }^{1235}$ Article 11 de la Convention de Vienne sur le Droit des traités. Disponible sur le site internet: https://legal.un.org/ilc/texts/instruments/french/conve. Consulté le 16/01/2022.

${ }^{1236}$ Le Conseil constitutionnel est une institution française créée par la Constitution de la Cinquième République du 4 octobre 1958. Il veille à la régularité des élections nationales et référendums. Il se prononce sur la conformité à la Constitution des lois et de certains règlements dont il est saisi. Il intervient également dans certaines circonstances de la vie parlementaire et publique. Texte intégral de la Constitution du 4 octobre 1958 en vigueur, disponible sur le site internet : https://www.conseil-constitutionnel.fr/le-bloc-de-constitutionnalite/t. Consulté le $17 / 01 / 2022$.

${ }^{1237}$ Article 3, section 1 de la constitution des Etats Unis d'Amérique. Texte intégral de la Constitution américaine en vigueur, disponible sur le site internet : https://mjp.univ-perp.fr/constit/us1787.htm. Consulté le 17/01/2022.
} 
constitutionnelle. En effet, la Cour constitutionnelle du Bénin est la plus haute juridiction de l'Etat en matière constitutionnelle $(\ldots)^{1238}$. Elle a pour mission entre autres, de vérifier la conformité des accords internationaux avec la constitution.

Comment s'effectue le contrôle de constitutionnalité des instruments internationaux de protection de l'environnement et ceux destinés à la lutte contre les changements climatiques au Bénin?

Tout en étant pas opposé à l'intégration d'accords internationaux à son droit positif, des gardes fous sont établies pour organiser méthodiquement le processus de ratification desdits accords par l'Etat béninois. Les accords internationaux de protection de l'environnement et de lutte contre les changements climatiques à l'instar de la Convention Cadre des Nations Unies sur les Changements Climatiques et ses accords subséquents, ont subi le même processus que d'autres conventions internationales dans lesquelles le Bénin s'est engagé.

En effet, le Président de la République négocie les accords internationaux qu'il signe ${ }^{1239}$. L'intégration de ces accords à l'ordonnancement juridique béninois intervient suite à l'adoption d'une « loi d'autorisation de ratification ${ }^{1240}$. Cette loi est entérinée par une décision de conformité à la constitution rendue par la Cour constitutionnelle après sa saisine par le Président de la République ou par un membre de l'Assemblée nationale ${ }^{1241}$. Il s'ensuit que, «Les traités ou accords régulièrement ratifiés ont, dès leur publication, une autorité supérieure à celle des lois, sous réserve pour chaque accord ou traité de son application par l'autre» ${ }^{1242}$. A titre illustratif, la Cour constitutionnelle béninoise, saisie pour contrôle de conformité à la Constitution béninoise de la loi ${ }^{\circ}$ 2018-03 portant autorisation de ratification de l'amendement de Kigali au Protocole de Montréal relatif aux substances appauvrissant la couche d'ozone, signé à Kigali au Rwanda le 15 octobre 2016, a décidé à l'issu de la procédure de contrôle de conformité que, «la loi $n^{\circ} 2018-03$ portant autorisation de ratification de l'amendement de Kigali au protocole de Montréal relatif aux substances appauvrissant la couche d'ozone, signé à Kigali au Rwanda, le 15 octobre 2016, votée par l'Assemblée nationale le 05 janvier 2018 est conforme à la Constitution en toutes ses dispositions.» ${ }^{1243}$ Par cette décision, le Président de la République est autorisée de ratifier l'amendement de Kigali au Protocole de Montréal

\footnotetext{
${ }^{1238}$ Article 114 de la constitution béninoise du 11 décembre 1990, révisée le 07 novembre 2019. ${ }^{1239}$ Id.

${ }^{1240}$ Article 145 de la constitution béninoise du 11 décembre 1990, révisée le 07 novembre 2019.

${ }^{1241}$ Article 146 id.

1242 Article 147 ibid.

1243 Cour constitutionnelle du Bénin, DECISION DCC 18-006 DU 25 JANVIER 2018. Disponible sur le site internet de la Cour constitutionnelle béninoise: https://courconstitutionnelle.bj/decisions-2. Consulté ce $29 / 05 / 2021$.
} 
relatif aux substances appauvrissant la couche d'ozone qui appartient désormais au bloc de constitutionnalité béninoise. Cet instrument de protection du climat devient ainsi un cadre juridique de référence pour le Bénin en matière de protection de la couche d'ozone. De même, les juridictions peuvent s'en servir comme source de droit dans la gestion des conflits environnementaux.

Quelles sont les impacts de la contribution de la Cour constitutionnelle dans la lutte contre les changements climatiques au Bénin?

Depuis sa création ${ }^{1244}$ et sa mise en fonction ${ }^{1245}$, la Cour constitutionnelle béninoise a contribué à l'intégration d'accords internationaux en matière de protection de l'environnement et de lutte contre les changements climatiques au droit positif béninois. Les plus importants sont ; la Convention Cadre des Nations Unies sur les Changements Climatiques, le Protocole de Kyoto et l'Accord de Paris et puis toutes les conventions de la génération de Rio de Janeiro de 1992 que sont: la Convention sur la biodiversité et la Convention sur la lutte contre la désertification. Cette possibilité de diversification du droit offerte par la Constitution béninoise permet de renforcer les sources du droit de protection du climat. Elle contribue à l'enrichissement du droit béninois par l'intégration des sources diverses, dont les normes élaborées au plan international intègrent une vision générale, globale et planétaire pour enrayer les changements climatiques. Cette méthode d'intégration des normes internationales consolide les acquis environnementaux.

L'effectivité de la procédure d'intégration des accords internationaux à la Constitution béninoise participe à la consolidation et à la sécurisation du cadre juridique de lutte contre les changements climatiques au Bénin. Dans ce cas, «si la Cour constitutionnelle saisie par le Président de la République ou par un membre de l'Assemblée nationale a déclaré qu'un engagement international comporte une clause contraire à la constitution, l'autorisation de ratification ne peut intervenir qu'après la révision de ladite constitution $»^{1246}$.

Mais dans la pratique, l'intégration d'accords internationaux à la constitution béninoise n'a jamais donné lieu à une procédure contentieuse, tendant à solliciter ou à ordonner le rejet par l'autorité de la juridiction constitutionnelle de l'autorisation de ratification pour nonconformité à la Constitution béninoise. Cette situation est due au fait que ces procédures sont pour la plupart sollicitées par des personnalités politiques que sont les membres de l'Assemblée

\footnotetext{
${ }^{1244}$ Le 11 décembre 1990, date d'adoption de la constitution béninoise.

${ }^{1245} \mathrm{La}$ Cour a commencé par fonctionner en 1993. Histoire de la Cour constitutionnelle béninoise disponible sur le site internet: https://courconstitutionnelle.bj/decisions-2. Consulté ce 29/05/2021.

1246 Suivant les dispositions de l'article 146 de la constitution béninoise du 11 décembre 1990, révisée le 07 novembre 2019.
} 
nationale et le Président de la république à qui la loi a donné la compétence ${ }^{1247}$, mais paradoxalement, ceux-ci ne pas portent pas toujours l'intérêt de l'immense majorité de la population. De même, la méconnaissance des possibilités offertes par la Constitution béninoise, qui donne l'opportunité de saisine de la Juridiction constitutionnelle aux citoyens béninois et aux organisations de la société civile constitue également un facteur limitatif quant à la progression du droit climatique au Bénin. Il s'ensuit que les accords internationaux qui sont soumis au contrôle de constitutionnalité sont systématiquement intégrés à la constitution par décision du Juge constitutionnel. Mais curieusement, dans les archives de la juridiction constitutionnelle, il n'existe aucune décision de rejet d'autorisation de ratification d'accord international.

Est-ce-que le comportement de la juridiction constitutionnelle tendant à intégrer systématiquement les accords internationaux au droit positif béninois est un gage de sécurité juridique?

Au Bénin, la Juridiction constitutionnelle est une institution politique. Cela se justifie par le mode de désignation de ses représentants qui ont pour la plupart un statut d'homme politique et désignés par des institutions politiques ${ }^{1248}$ Dans l'hypothèse où la juridiction constitutionnelle est contrôlée par une institution de la République ou par le Président de la République, cette institution peut, sans tenir compte des intérêts généraux de la population, rendre des décisions pour satisfaire l'autorité politique. Cette pratique est couramment développée au Bénin sans oublier l'impact de la corruption sur le fonctionnement des institutions de l'Etat. Par contre, aucune pression citoyenne n'a permis d'infléchir la position de la juridiction constitutionnelle ou de lui imprimer une dynamique dans le sens de la protection des intérêts communs et généraux. II s'ensuit que si éventuellement il existe d'accords internationaux qui ne consacrent pas la progression du droit de l'environnement ou du droit climatique, ces accords peuvent passer les mailles du contrôle du juge constitutionnel béninois sans aucun obstacle. Dans ce cas, certains auteurs considèrent que « l'Etat qui adopte un texte national contraire à une convention internationale engage désormais sa responsabilité non seulement au plan international, mais aussi au plan national $»^{1249}$.

En effet, en adoptant les accords internationaux par leur ratification, l'Etat béninois se trouve en présence des normes internationales qui s'équivalent parfois à la règlementation

\footnotetext{
${ }^{1247}$ Article 121146 de la constitution béninoise du 11 décembre 1990, révisée le 07 novembre 2019.

1248 Article 115 id.

${ }^{1249}$ Michel Prieur. L'influence des conventions internationales sur le droit interne de l'environnement. Actes de la réunion constitutive du comité sur l'environnement de l'AHJUCAF, Juin 2008, Porto- Novo, Bénin. pp.291-301., p.297.
} 
interne. En cas de conflit, le juge applique la règle du monisme avec la suprématie de la norme internationale sur la norme interne qui est en vigueur au Bénin ${ }^{1250}$.

Les lois d'application desdits engagements sont élaborées et subissent également le contrôle de constitutionnalité.

\section{B- Le contrôle de constitutionnalité des actes}

La protection de l'environnement est un principe constitutionnel, car il a été inscrit à l'article 27 de la constitution béninoise du 11 décembre 1990 révisée par la loi 2019-40 du 07 novembre 2019 en ces termes, «toute personne a droit à un environnement sain, satisfaisant et durable (...) ». C'est une évolution considérable du droit de protection de l'environnement au Bénin où ce droit a fait l'objet d'une consécration constitutionnelle à l'instar de la France qui en faveur de la révision constitutionnelle du $1^{\text {er }}$ mars 2005 a procédé à la constitutionnalisation du droit de l'environnement par « l'adoption d'une Charte de l'environnement adossée à la Constitution » ${ }^{1251}$. Il en est de même en Espagne où la constitution a prévu que « (...) tous ont le droit de jouir d'un environnement approprié pour développer leur personnalité et le devoir de le conserver $\gg{ }^{1252}$. A la seule différence que le débat sur l'intégration à la Constitution Française suscite un intérêt croissant au point d'engendrer une dynamique de constitutionnalisation progressive des principes environnementaux pouvant prendre en compte les dimensions concernant les changements climatiques.

La reconnaissance par la constitution béninoise du droit à la protection d'un environnement sain ${ }^{1253}$ soulève immédiatement la question de l'effectivité dudit droit et de la problématique de l'accès à la justice. D'abord, le droit à l'environnement sain s'explique en ce que l'Etat a la charge d'assurer « (...) l'encadrement des changements climatiques, le respect de la préservation du système climatique contre les changements climatiques et leurs effets et conséquences négatifs dans tous les secteurs économiques et sociaux fragiles et vulnérables (..) $\gg{ }^{1254}$.

\footnotetext{
${ }^{1250}$ Suivant les dispositions de l'article 147 de la Constitution béninoise du 11 décembre 1990 révisée le 07 novembre 2019.

${ }^{1251}$ Désormais, le Préambule de la Constitution de 1958 prévoit que «le peuple français proclame solennellement son attachement (...) aux droits et devoirs définis dans la Charte de l'environnement de $2004 »$. Disponible sur le site internet du conseil constitutionnel : https://www.conseil-constitutionnel.fr/la-constitution/la-protection-de-lenvironnement. Consulté le 17/01/2022.

${ }^{1252}$ Le premier alinéa de l'article 45 de la Constitution espagnole du 27 décembre 1978 Disponible sur le site internet du conseil constitutionnel: https://www.conseil-constitutionnel.fr/la-constitution/la-protection-de-lenvironnement. Consulté le 17/01/2022.

${ }^{1253}$ Article 27 de la constitution béninoise du 11 décembre 1990, révisée le 07 novembre 2019.

${ }^{1254}$ Article 6 de loi n ${ }^{\circ} 2018$-18 portant règlementation sur les changements climatiques en République du Bénin.
} 
L'effectivité de cette mesure doit être assurée par l'utilisation du pouvoir qui appartient à chaque individu de faire juger ses prétentions par une juridiction et éventuellement, par la Cour constitutionnelle en ce qui concerne la violation du droit du citoyen béninois à un environnement sain. Ce droit est reconnu à travers la Charte africaine des droits de l'Homme et des peuples ${ }^{1255}$. La conception jurisprudentielle des droits de l'homme s'est élargie au concept de la protection de l'environnement, laquelle conception ne s'écarterait pas non plus de la prise en compte de la dimension concernant les changements climatiques qui constituent un domaine de l'environnement global, d'où la notion de droit de l'homme à un environnement sain.

En effet, les autorités administratives, dans le cadre de l'accomplissement de leur mission élaborent des actes réglementaires tels que les décrets, arrêtés et notes ou posent des actes qui parfois, violent les dispositions constitutionnelles. Ceux de ces actes qui sont susceptibles de porter atteinte au droit du béninois à un environnement sain et durable tel que prescrit à l'article 27 de la Constitution béninoise du 11 décembre 1990 révisée par la loi 201940 du 07 novembre 2019, peuvent être déférés devant la Cour constitutionnelle. C'est pourquoi, l'article 117 de la constitution béninoise stipule que, «la Cour constitutionnelle statue obligatoirement sur la constitutionnalité (...) des actes réglementaires censés porter atteinte aux droits fondamentaux de la personne humaine (...)». Les droits fondamentaux de l'homme s'entendent également du droit de l'homme à un environnement sain et satisfaisant. Dans ce cadre, la loi $\mathrm{n}^{\circ} 2018-18$ portant règlementation sur les changements climatiques en République du Bénin a requis « la compétence des juridictions administratives et n'exclut pas la saisine alternative de la Cour constitutionnelle, lorsque dans le cadre de ses attributions, l'autorité chargée de la lutte contre les effets et conséquences négatives des changements climatiques pose un acte ou entreprend toute action qui viole les droits fondamentaux de la personne ${ }^{1256}$.

En réalité, les décisions de justice sont exclues du champ du contrôle de constitutionnalité. Mais parfois, selon certaines études, «Le juge constitutionnel peut $s^{\prime}$ intéresser à elles $»^{1257}$. Selon la jurisprudence de la Cour constitutionnelle, «cette soustraction des décisions de justice du contrôle du juge constitutionnel ne joue que dans la

\footnotetext{
${ }^{1255} \mathrm{~L}$ 'article 24 de ladite charte dispose que « Tous les peuples ont droit à un environnement satisfaisant et global, propice à leur développement». La charte africaine des droits de l'homme et des peuples adoptée par l'Organisation de l'Unité Africaine en 1981 et ratifiée par le Bénin le 20 janvier 1986, fait partie intégrante de la Constitution de la République du Bénin du 11décembre 1990 révisée le 07 novembre 2019 comme le dispose le $7^{\mathrm{e}}$ paragraphe de son préambule.

${ }^{1256}$ Article 88 de la loi n 2018 -18 portant, règlementation sur les changements climatiques en République du Bénin.

${ }^{1257}$ Ahonagnon Noel Gbaguidi, William Kodjoh-Kpakpassou, Gérard AÏVO et Lazard H. HOUNSA, Introduction au Système Juridique et Judiciaire du Bénin, Hauser Global Law School Program, New York University School of Law, New York, Mai 2019.
} 
mesure où ces dernières ne violent pas les droits de la Personne ${ }^{1258}$. Ce développement jurisprudentiel qui a commencé depuis 1996 a connu une clarification le 11 novembre 2003 à travers la décision DCC 03-166 $6^{1259}$ qui a dit et jugé que «les décisions de justice n'étaient pas des actes au sens de l'article 3 al.3 de la constitution, que pour autant qu'elles ne violent pas les droits de l'Homme ». Sachant que l'article 3 al.3 de la constitution béninoise dispose que, « Toute loi, tout texte réglementaire et tout acte administratif contraire à ces dispositions est nul et non avenu. En conséquence, tout citoyen a le droit de se pourvoir devant la Cour constitutionnelle contre les lois, textes et actes présumés anticonstitutionnels $»^{1260}$. Ainsi, les décisions de justice sont déférées à la compétence de l'autorité pour contrôle de constitutionnalité si et seulement si, elles portent atteintes aux droits fondamentaux de l'Homme. Donc, tout acte y compris les décisions de justice qui violerait les droits de la Personne peuvent subir la sanction du juge constitutionnel, qui de part ses prérogatives, déclare l'acte «anticonstitutionnel » ${ }^{1261}$. Bien entendu qu'il s'agit ici «du droit de l'Homme à un environnement sain». Cet élargissement de compétence de la Cour constitutionnelle aux décisions de justice est une opportunité qui peut être saisie pour voir juger à nouveau une décision de justice qui violerait les règles de protection de l'environnement et la règlementation en matière des changements climatiques.

Le droit de saisine de la Cour constitutionnelle appartient à «tout citoyen pour les lois, les textes réglementaires, les actes administratifs et la violation des actes fondamentaux de la Personne humaine (...)» ${ }^{1262}$. Selon ADELOUI Arsène-Joël, «cette particularité de la constitution béninoise participe du dynamisme de l'institution chargée de faire respecter les droits de l'Homme et conditionne la soumission de tous, c'est-à-dire, des pouvoirs publics et des individus au respect de la légalité ${ }^{1263}$. De part ce développement jurisprudentiel, la Cour constitutionnelle à qui appartient la prérogative de protéger les droits fondamentaux de l'Homme s'est imposée comme un acteur important pour le domaine de la protection de l'environnement.

\footnotetext{
1258 Id.

${ }^{1259}$ Recueil des décisions et avis de la cour constitutionnelle, 2003, p. 673 et s.

${ }^{1260}$ Article 3 alinéa 3 de la Constitution béninoise du 11 décembre 1990 modifiée le 07 novembre 2019.

${ }^{1261}$ Contraire ou non conforme à la constitution.

${ }^{1262}$ Article 24 de la loi organique et article 29 du règlement intérieur de la cour constitutionnelle du Bénin. Les textes fondamentaux de la Cour constitutionnelle du Bénin. Disponible sur le site internet: https://courconstitutionnelle.bj/textes-fondamentaux. Consulté le 17/01/2022.

${ }^{1263}$ ADELOUI (Arsène-Joël), Transition démocratique et coopération au développement. Approches comparative Bénin, Mali, Niger et Togo. Thèse de Doctorat, UAC, FADESP, Chaire, UNESCO, droit de l'homme et démocratie, Cotonou, 2006, 169p.
} 
Beaucoup de décisions pertinentes sont rendues par la Cour constitutionnelle suite à la saisine des citoyens, en thème de contribution de la haute juridiction à la concrétisation du droit de protection de l'environnement au Bénin. Ce fut le cas dans l'affaire Eugène Comlan Boya où, la Cour constitutionnelle saisie d'une « plainte contre les Ministres de la Santé Publique, de l'Environnement et Dahouéto Innocent » pour violation de l'article $27^{1264}$ de la constitution, a décidé dans sa sentence que, « l'implantation de volailles par Innocent Dahouéto au milieu des agglomérations viole l'article 27 de la constitution, que les préjudices subies par Eugène Comlan Boya ouvrent droit à réparation, (...) $»^{1265}$. Enfin, saisie par Jonas H. KOUDAHOUA LATOUNDJI, Président directeur général de la société AFRITEC et Claude HOUNDETE, gérant de la Société Africaine de Dragage (SAD), qui forment des recours contre le Ministre de l'énergie, des recherches pétrolières et minières et du développement des énergies renouvelables et le Ministre de l'environnement chargé de la gestion du changement climatique, du Reboisement et de la Protection des ressources naturelles et forestières, la Cour constitutionnelle « déclare qu'il y a violation de l'article 27 de la Constitution » ${ }^{1266}$. L'élément de pertinence des décisions précitées est la sanction infligée par la Cour constitutionnelle, pour violation du droit à un environnement $\operatorname{sain}^{1267}$, tel que contenu dans la Constitution béninoise et l'audace dont a fait preuve la haute juridiction en dénonçant les auteurs, que sont des Ministres du Gouvernement qui sont déclarés coupables de la violation de ce droit.

Néanmoins, derrière ces merveilles que fait la Cour constitutionnelle pour assurer l'effectivité de la protection de l'environnement au Bénin, se cachent des faiblesses qui impactent négativement les actions de cette institution. Il s'agit de la question de la mise en œuvre des décisions rendues par la haute juridiction. La Cour constitutionnelle béninoise rend des décisions exécutoires mais qui pour la plupart, ne sont pas exécutées. Ces décisions qui ont pour autant un caractère définitif, c'est-à-dire qu'elles ne sont susceptibles d'aucun recours suspensif d'exécution s'imposent à toutes les institutions de l'Etat ${ }^{1268}$. Malheureusement, elles ne sont revêtues d'aucune mesure de contrainte, telle que la sanction coercitive pour obliger à

\footnotetext{
${ }^{1264}$ Qui dispose que « Toute personne a droit à un environnement sain, satisfaisant et durable et a le devoir de le défendre. L'Etat veille à la protection de l'environnement».

${ }^{1265}$ Cour constitutionnelle du Bénin : décision DCC 02-065 du 05 juin 2002. Cour constitutionnelle béninoise. Disponible sur le site internet : https:/courconstitutionnelle.bj/decisions-2. Consulté ce 29/05/2021.

${ }^{1266}$ République du Bénin Décision DCC 16-113 DU 28 juillet 2016 : Requérant : Jonas H. KOUDAHOUA, agissant au nom de l'Union pour le développement des riverains d'Akogba de Cotonou (UDRAC), Contrôle de conformité Droit à un environnement sain Autorité de chose jugée Irrecevabilité Violation de la Constitution La Cour constitutionnelle. Disponible sur le site internet : https://courconstitutionnelle.bj/decisions-2. Consulté ce 29/05/2021.

${ }^{1267}$ Article 27 de la constitution béninoise du 11 décembre 1990 révisée le 07 novembre 2019.

${ }^{1268}$ Article 124 aliéna 2 de la constitution béninoise du 11 décembre 1990 révisée le 07 novembre 2019.
} 
cesser de commettre les actes qui violent le droit à un environnement sain. De même, lorsque les décisions de la Cour constitutionnelle donnent la possibilité de bénéfice de réparation de préjudices devant les juridictions de droit commun, il est difficile d'évaluer le dommage environnemental, car il n'y a pas encore de textes consacré à cela. Par ailleurs, le juge Constitutionnel n'a pas non plus la compétence nécessaire pour s'adonner à cet exercice. Ce sont ces faiblesses qui justifient que, Eugène BOYA a saisi à nouveau la Cour d'une plainte tendant à la violation de la décision DCC 02-065 du 05 juin 2002, rendue en sa faveur, par le Ministre de l'Environnement et le Ministre de la Santé Publique, qui « n'ont pas pris des dispositions utiles et efficaces aux termes des articles 33, 34, 35 et 124 de la Constitution pour exécuter ladite décision... ». La Cour a décidé en substance «(...) que le Ministre de l'environnement a violé les articles 35 et 124 de la Constitution.» ${ }^{1269}$

Sur un autre plan, la question de l'accessibilité à la Cour constitutionnelle demeure une préoccupation majeure car, bien qu'il ait reconnu le droit de saisine de la Cour par tout citoyen, ${ }^{1270}$ la mise en œuvre des procédures applicables devant l'institution n'est pas chose aisée. Il en est ainsi parce que, la population béninoise compte un nombre important d'analphabète, environ $40,4 \%{ }^{1271}$. Celle d'entre elle qui est lettrée, est en majorité profane des procédures judiciaires ${ }^{1272}$. Et cela devient encore plus compliqué pour une procédure devant être diligentée dans le domaine de la protection de l'environnement, compte tenu des spécificités de la matière.

Le contrôle de constitutionnalité des actes, contribue t-il à l'effectivité de la lutte contre les changements climatiques au Bénin?

L'élargissement de la compétence environnementale de la Cour constitutionnelle béninoise aux changements climatiques est organisé à travers la loi n²018-18 portant règlementation sur les changements climatiques en République du Bénin. Selon les dispositions de cette loi, « La compétence des juridictions administratives n'exclut pas la saisine alternative de la Cour Constitutionnelle lorsque dans le cadre de ses attributions, l'autorité chargée de la

\footnotetext{
${ }^{1269}$ Cour constitutionnelle du Bénin : Décision n ${ }^{\circ}$ DCC 04-038 en date du 12 mars 2004.Disponible sur le site internet : https://courconstitutionnelle.bj/decisions-2. Consulté ce 29/05/2021.

${ }^{1270}$ Article 122 de la constitution béninoise du 11 décembre 1990, révisée le 07 novembre 2019.

${ }^{1271}$ Voir statistique à la Page 13 du document de synthèse sur les caractéristiques socioculturelles et économiques de la population réalisé au cours de l'année 2017.Institut National de la Statistique et de l'Analyse Economique (INSAE) du ministère en charge du Plan, République du Bénin. Disponible sur le site internet : https://instad.bj/apropos-de-1-insae. Consulté le 17/01/2022.

${ }^{1272}$ Faiblesse notoire du taux de judiciarisassions.
} 
lutte contre les effets et conséquences négatifs des changements climatiques pose un acte ou entreprend toute action qui viole les droits fondamentaux de la personne $»^{1273}$.

Malgré que la juridiction constitutionnelle a une compétence en matière climatique, aucun conflit découlant de l'application de la loi 2018-18 portant règlementation sur les changements climatiques en république du Bénin et de l'application par le Bénin de la Convention Cadre des Nations Unies sur les Changements Climatiques n'a été porté devant cette juridiction $^{1274}$.

Après le contrôle juridictionnel qui s'opère devant la Cour constitutionnelle, les textes réglementant les domaines de protection de l'environnement et des changements climatiques requièrent également la compétence des juridictions classiques pour régler éventuellement certains conflits qui découleraient de leur application.

\section{Paragraphe 2: L'intervention des juridictions}

Au Bénin, le pouvoir judiciaire est indépendant ${ }^{1275}$, il est exercé par les juridictions de droit commun à savoir, les cours et tribunaux créés conformément à la règlementation en vigueur ${ }^{1276}$. La « justice est rendue au nom du peuple béninois » ${ }^{1277}$ à travers les juridictions d'instance et de recours (A) et dans certains cas, par la Haute cour de justice (B) qui disposent de la compétence pour intervenir dans les conflits qui requièrent la mise en œuvre des dispositions applicables à la lutte contre les changements climatiques au Bénin.

\section{A- Les juridictions de droit commun}

La loi n²001-37 du 27 aout 2002 portant organisation judiciaire en république du Bénin classe dans la catégorie des juridictions de droit commun, les tribunaux d'instance largement répartis sur toute l'étendue du territoire national ${ }^{1278}$. Ces juridictions ont compétence en matière pénale, civile, commerciale, sociale et administrative ${ }^{1279}$. Par contre, les Cours d'appel sont des juridictions de recours pour des décisions rendues dans les matières précitées par les juridictions

\footnotetext{
${ }^{1273}$ Article 88 de ladite loi.

${ }^{1274}$ Les recherche de la jurisprudence en cette matière sur le site internet de la Cour constitutionnelle a été infructueuse. Cour constitutionnelle béninoise: https://courconstitutionnelle.bj/decisions-2. Consulté ce $29 / 05 / 2021$

${ }^{1275}$ Article 125 de la constitution béninoise du 11 décembre 1990 et la loi n²001-37 du 27 août 2002 portant organisation judiciaire en République du Bénin modifiée par la loi n²016-15 du 28 juillet 2016.

1276 Id.

1277 Article 126 de la Constitution du 11 décembre 1990 révisée le 07 novembre 2019.

1278 Article 49 de la loi n²001-37 du 27 aout 2002 portant organisation judiciaire en République du Bénin.

1279 Article 49 id.
} 
d'instance statuant en premier ressort ${ }^{1280}$ et puis la Cour suprême est compétente pour des décisions rendues en dernier ressort ${ }^{1281}$ aussi bien par les juridictions d'instance que par les cours d'appel.

Le Juge du droit commun a-t-il compétence en matière de conflit relatif à la lutte contre les changements climatiques au Bénin?

La loi n 98-030 du 12 février 1999 portant, loi cadre sur l'environnement en République du Bénin a consacré le titre V aux volets concernant l'étude d'impact, l'audit environnemental, l'audience publique sur l'environnement, des plans d'urgence et mesures d'incitation qui sont pour la plupart, des activités qui concourent à l'évaluation environnementale. Ces activités ont une connotation essentiellement administrative et les actes posés dans ce cadre, par les promoteurs des projets et l'administration peuvent être sujets à contestation. En prévision des conflits éventuels que peuvent générer ces activités, le Législateur béninois a disposé à travers la loi $\mathrm{n}^{\circ}$ 2018-18 portant, règlementation sur les changements climatiques en République du Bénin que, «les différends relatifs aux actes délivrés ou accomplis par l'administration

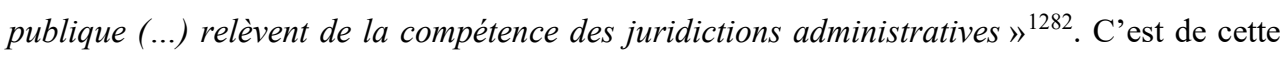
manière que le Législateur a retenu la compétence du juge administratif béninois pour les contentieux qui découlent des actes élaborés par les autorités administratives dans le cadre de la protection de l'environnement et particulièrement pour ceux concernant l'encadrement des changements climatiques. Seulement, la même loi dispose qu' «(...) aucun recours de plein contentieux n'est recevable s'il n'a été précédé d'une tentative de règlement transactionnel à l'initiative du requérant (...) ${ }^{1283}$. Le recours préalable prévu dans ce cas, est une condition sine qua none de recevabilité qui oblige le requérant à solliciter un règlement transactionnel en cas de survenance de conflit ${ }^{1284}$. En règle générale, dans le contentieux administratif ${ }^{1285}$, s'agissant

\footnotetext{
${ }^{1280}$ Jugement contre lequel un appel peut être interjeté, voir GUILLIEN (Raymond) et VINCENT (Jean), Lexique des termes juridiques, $5^{\text {e }}$ éd., Dalloz, 1981, 567p., p. 313.

${ }^{1281}$ Jugement ou arrêt contre lequel aucun appel ne peut être interjeté, seule restant possible l'introduction de voies de recours extraordinaires (recours en révision ou pourvoi en cassation), voir GUILLIEN (Raymond) et VINCENT

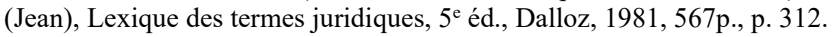

${ }^{1282}$ Article 86 de la loi ${ }^{\circ}$ 2018-18 portant, règlementation sur les changements climatiques en République du Bénin.

${ }^{1283}$ Article 87 de la loi $n^{\circ} 2018-18$ portant, règlementation sur les changements climatiques en République du Bénin.

${ }^{1284} \mathrm{Id}$.

${ }^{1285}$ Le contentieux administratif encore désigné sous le vocable du « recours administratif», par opposition aux recours juridictionnels portés devant des tribunaux, ce recours est porté devant l'administration elle-même en vue de faire annuler l'un de ses actes prétendu illégal ou de demander une réparation pécuniaire. GUILLIEN (Raymond) et VINCENT (Jean), Lexique des termes juridiques, $5^{\mathrm{e}}$ éd., Dalloz, 1981, 567p., p. 446. En cas de non satisfaction, le contentieux est déféré devant le juge administratif par le requérant qui le saisi soit par l'acte d'inaction de l'autorité administrative, soit par la réponse négative de l'autorité administrative. Le contentieux administratif, en tant que branche du droit peut être défini comme l'ensemble des règles juridiques qui régissent
} 
du Recours pour Excès de Pouvoir (REP) ${ }^{1286}$, le recours gracieux qui a valeur de recours préalable ne prend pas forcément la forme d'un règlement transactionnel du contentieux. C'est une innovation du Législateur béninois ${ }^{1287}$. Malgré ces spécificités, le Législateur béninois n’a daigné créer de juridiction spéciale exclusivement compétente en matière de protection de l'environnement et de lutte contre les changements climatiques. Mais il a attribué par la loi, la compétence climatique aux formations juridictionnelles à compétence administrative des Tribunaux de Première Instance (TPI) des Cours d'appel et enfin de la Cour suprême ${ }^{1288}$.

Par ailleurs, la loi n ${ }^{\circ} 2018-18$ portant règlementation sur les changements climatiques en République du Bénin a retenu la compétence des juridictions répressives des tribunaux d'instance qui interviennent en cas d'infraction à ladite loi. Elle dispose que, « la recherche, la constatation et la répression des infractions prévues, (...) ainsi que les règles de procédure obéissent aux dispositions du code de procédure pénale, du code pénal et de la loi-cadre sur l'environnement $»^{1289}$. A titre illustratif, « (...) en cas d'inobservation de la procédure d'étude d'impact, l'intéressé est puni conformément aux dispositions de l'article 114 de la loi » ${ }^{1290}$, également, « est punie d'une amende de Cent vint mille (120.000) à Un million (1.000.000) de francs d'une peine d'emprisonnement de Un (01) à Cinq (05) mois, ou de l'une de ces deux (2) peines seulement, toute personne ayant contrevenue aux dispositions de l'article $21 »^{1291}$. Sachant que l'article 21 dispose que, «L'affectation et l'aménagement du sol à des fins agricoles, industrielles, urbaines ou autres, ainsi que les travaux de recherche ou d'exploitation des ressources du sous-sol, pouvant porter atteinte à l'environnement béninois donne lieu à une

la solution par voie juridictionnelle des litiges administratifs (J-M AUBY et R. DRAGO, Traité de contentieux administratif, tome I). La loi n ${ }^{\circ} 2008-07$ du 28 février 2011 portant code de procédure civile, commerciale, sociale, administrative et des comptes en république du Bénin ne définit pas le contentieux administratif, mais son article 818 énumérer les domaines qui relèvent de ce contentieux par la disposition suivante « La juridiction statuant en matière administrative est compétente pour connaitre du contentieux de tous les actes émanant de toutes les autorités administratives de son ressort. Relèvent de ce contentieux : 1- les recours en annulation pour excès de pouvoir des décisions des autorités administratives (...)».

${ }^{1286}$ Recours juridictionnel dirigé en vue de les faire annuler pour cause d'illégalité, contre des actes unilatéraux soit d'une autorité administrative, soit d'un organisme privé agissant dans le cadre d'une mission de service public. GUILLIEN (Raymond) et VINCENT (Jean), Lexique des termes juridiques, 5ééd., Dalloz, 1981, 567p., p. 446.

${ }^{1287}$ Article 87 de la loi ${ }^{\circ}$ 2018-18 portant, règlementation sur les changements climatiques en République du Bénin.

${ }^{1288}$ La compétence climatique de ces juridictions est définie par l'article 86 de la loi $\mathrm{n}^{\circ} 2018$ portant règlementation des changements climatiques en République du Bénin qui dispose que « Les différends relatifs aux actes délivrés ou accomplis par l'administration publique dans le cadre de la présente loi relèvent de la compétence des juridictions administratives ».

${ }^{1289}$ Article 92 de la loi ${ }^{\circ} 2018$-18 portant, règlementation des changements climatiques en République du Bénin.

${ }^{1290}$ Article 21 de la loi n $98-030$ portant loi cadre sur l’environnement en République du Bénin.

${ }^{1291}$ Article 114 id. 
étude d'impact préalable (...) $»^{1292}$. Ainsi, la compétence climatique des juridictions de droit commun statuant en matière correctionnelle est consacrée par ladite loi.

Tout comme en matière administrative, le règlement transactionnel peut mettre fin à toute forme de poursuite au pénal en cas de violation de la règlementation forestière. Selon l'article 85 de la loi n 93-009 du 02 juillet 1993 portant régime des forêts en République du Bénin, « les poursuites relatives à la règlementation forestière peuvent être arrêtées moyennant l'acceptation et le règlement par le délinquant d'une transaction dument proposée par le directeur des ressources naturelles ou l'un de ses représentants délégué. (...) ». De même, la loi n 98-030 du 12 février 1999 portant, loi cadre sur l'environnement en République du Bénin n'a pas fait exception à la règle, car dispose-t-elle que, « nonobstant les recours à l'action prévue contre les auteurs des infractions environnementales, elle autorise expressément le règlement transactionnel, qui peut mettre fin aux poursuites ${ }^{1293}$. Il en est de même pour la loi $n^{\circ} 2018$ 18 , portant règlementation sur les changements climatiques qui a également admis le règlement transactionnel comme élément pouvant handicaper la mise en œuvre de l'action publique pour l'application de peine à l'auteur de l'infraction climatique devant la juridiction répressive ${ }^{1294}$.

Quel bilan établir de l'impact de l'intervention des juridictions d'instance et de recours sur la lutte contre les changements climatiques au Bénin?

Les données statistiques ne donnent aucun résultat probant quant à la gestion des conflits climatiques par les juridictions de droit commun en république du Bénin. Il n'existe pas non plus de jurisprudence en ce qui concerne l'applicabilité de la Convention Cadre des Nations Unies sur les Changements Climatiques à l'instar de ce qui se passe dans d'autres Etats du monde où certaines juridictions nationales imposent le respect des engagements souscrits dans la Convention Cadre des Nations Unies sur les Changements Climatiques à leur Etat ${ }^{1295}$. Il en est ainsi parce que le « système judiciaire béninois ne fait pas suffisamment la preuve de son indépendance vis-à-vis du pouvoir politique et des groupes de pression ${ }^{1296}$. Il est difficile, voire impossible au Juge béninois de se saisir d'un litige relatif à l'application de la Convention Cadre des Nations Unies sur les Changements Climatiques contre l'Etat et de rendre utilement

\footnotetext{
1292 Article 21 de la loi n $98-030$ portant loi cadre sur l’environnement en République du Bénin.

1293 Article 108 de ladite loi.

1294 Article 93 et 94 de ladite loi.

1295 Allusion faite à la justice des Pays-Bas, de la Colombie, du Brésil, des Etats-Unis d'Amérique et de la France où la justice s'est illustrée comme un instrument d'effectivité du droit climatique international en condamnant ces Etats respectifs à respecter leurs engagements souscrits dans la Convention Cadre des Nations Unies sur les Changements Climatiques.

${ }^{1296}$ Document intitulé «PLAN STRATEGIQUE 2011-2020 DE LA REFORME ADMINISTRATIVE ET INSTITUTIONNELLE DU BENIN », p. 33. Disponible sur le site internet : https://travail.gouv.bj/public/storag. Consulté le 17/01/2022.
} 
une décision pour faire avancer les acquis climatiques sans subir la pression du pouvoir central. Malgré la consécration de la compétence climatique des juridictions béninoises, les diagnostics ont révélé l'existence de trois (3) facteurs essentiels qui concourent à l'inapplicabilité des normes et par ricochet à l'inefficacité de la lutte contre les changements climatiques à savoir : le défaut de compétence en matière du droit des changements climatiques, la corruption des acteurs intervenant tant, dans le processus d'élaboration de la règlementation d'application que, dans le cadre de la mise en œuvre de ladite règlementation et la politisation à outrance de l'administration. Tous ces facteurs combinés ne permettent pas d'une part, la mise en place d'une règlementation climatique durable et efficace et d'autre part, la mise en œuvre efficace des ces règlementations. Cette règlementation se développe au gré des intérêts des acteurs politiques qui ne sont pas forcément en adéquation avec les désidératas des citoyens. Le constat du disfonctionnement des structures administratives et institutionnelles béninoises a été rapporté dans le document intitulé, «Plan stratégique 2011-2020 de la réforme administrative et institutionnelle du Bénin» ${ }^{1297}$, dans lequel il a été consigné que des structures administratives et institutionnelles béninoises «...n'arrivent pas encore à se débarrasser entièrement des dysfonctionnements comme la politisation, la corruption et la concussion, qui ne sont que les résultats de l'absence d'éthique et de l'appropriation personnelle de la chose publique $\gg{ }^{1298}$.

Généralement, les juridictions de droit commun ne sont compétentes que pour connaitre des actions dirigées contre les personnes de toute catégorie sociale. Cependant, la Haute Cour de Justice (HCJ), une autre composante du pouvoir judiciaire au Bénin est investie de la compétence de juger certains auteurs d'infractions environnementales ou climatiques en raison de leur statut politique.

\section{B- La Haute cour de justice}

La justice doit être rendue sans discrimination aucune afin de permettre de poursuivre réellement les auteurs des infractions quelque soit leur rang social ou leur statut professionnel et de rétablir les victimes des effets de la délinquance dans leur droit. Paradoxalement, cette justice est parfois paralysée par l'impunité provoquée par des situations qui découlent du statut

\footnotetext{
${ }^{1297}$ Le Plan stratégique de la réforme administrative et institutionnelle 2011-2020 vise à garantir des services de qualité aux usagers dans tous les domaines de la vie socio-économique et de servir de levier à la croissance économique et au développement. Il est élaboré par le Ministère du Travail, de la Fonctions Publique et des Affaires Sociales. Disponible sur le site internet: https://ravail.gouv.bj/public/storage/documents/PSRAI. Consulté le 17/01/2022.

${ }^{1298}$ Le Plan stratégique de la réforme administrative et institutionnelle 2011-2020, p.33. Disponible sur le site internet : https://travail.gouv.bj/public/storage/documents/PSRAI. Consulté le 17/01/2022.
} 
ou de la personnalité de l'agent pénal. C'est pourquoi, le Conseil de sécurité des Nations unies , dans l'une de ses déclarations, rappelle que, «les Etats sont tenus de se conformer aux obligations qui leur incombent en matière de lutte contre l'impunité, de mener des enquêtes approfondies et de poursuivre les auteurs de crime de guerre, de génocide et de crime contre l'humanité afin de prévenir ces crimes, d'éviter qu'ils ne se reproduisent et de promouvoir une paix durable, la justice, la vérité et la réconciliation» ${ }^{1299}$. Tenant compte de la nécessité du rétablissement de la justice comme étant un gage de maintien de la paix dans le monde entier, les acteurs du droit international de l'environnement ont intégré cette préoccupation dans la Convention Cadre des Nations Unies sur les Changements Climatiques, laquelle fait obligation « (...) aux Parties de préserver le système climatique dans l'intérêt des générations présentes et futures, sur la base de l'équité (...)» ${ }^{1300}$. Le Constituant béninois n'a pas non plus occulté cet aspect en introduisant une disposition dans la Constitution du 11 décembre 1990, révisée par la loi 2019-40 du 07 novembre 2019, qui stipule que « l'Etat assure à tous, l'égalité devant la loi sans distinction d'origine, de race, de sexe, de religion, d'opinion politique ou de position sociale (...) $»^{1301}$. Ces principes d'équité, d'égalité placés dans leur contexte tels que formulés dans les instruments internationaux, visent à bannir la discrimination dans la poursuite des auteurs d'infraction criminelle quelque soit le domaine. En réalité, aucun citoyen du monde ne peut se prévaloir de quelque statut que ce soit pour se soustraire de la justice. C'est pourquoi, il a été institué au Bénin la Haute Cour de Justice pour juger les crimes que peuvent commettre certaines personnalités politiques du pays.

En effet, au titre du pouvoir judiciaire ${ }^{1302}$ la Haute cour de justice a été instituée dans la Constitution béninoise du 11 décembre 1990 révisée par la loi 2019-40 du 07 novembre $2019^{1303}$. Son organisation et son fonctionnement sont régis par les dispositions de la loi organique n93-013 du 10 août 1999 et complétées par le règlement intérieur du 26 novembre 2001 modifié par celui du 04 mai 2007. La Haute cour de justice composée des membres de la Cour constitutionnelle à l'exception de son Président, de six (6) députés élus par l'Assemblée nationale et du Président de la Cour suprême ${ }^{1304}$ est « compétente pour juger le Président de la

\footnotetext{
${ }^{1299}$ La déclaration S/PRST/2014/5 du Conseil de sécurité des Nations unies du 21 février 2014 relative à la «Promotion et renforcement de l'état de droit dans le cadre des activités de maintien de la paix et de la sécurité internationales ». Disponible sur le site internet : https://undocs.org/fr/S/PRST/2014/5. Consulté le 17/01/2022.

${ }^{1300}$ Article 3 de la Convention Cadre des Nations Unies sur les Changements Climatiques.

${ }^{1301}$ Article 26 de la constitution béninoise du 11 décembre 1990, révisée le 07 novembre 2019.

1302 Titre VI id.

1303 Article 135 ibid.

${ }^{1304}$ Article 135 de la Constitution béninoise du 11 décembre 1990, révisée par la loi 2019-40 du 07 novembre 2019 de la Constitution béninoise du 11 décembre 1990, révisée par la loi 2019-40 du 07 novembre 2019.
} 
République et les membres du Gouvernement à raison des faits qualifiés de haute trahison, d'infraction commise dans l'exercice ou à l'occasion de l'exercice de leurs fonctions ${ }^{1305}$.

La Haute cour de justice est-elle compétente pour juger le Président de la République et les membres du Gouvernement, pour des infractions aux règles de protection du climat?

En droit pénal, avant qu'il n'y ait répression, il est indispensable qu'un texte de loi ait prévu les faits ou actions prohibitifs ${ }^{1306}$. C'est ce qu'indique le maxime latin, « Nullum crimen, nulla pæena sine lege », c'est-à-dire qu' « il n'y a aucun crime, aucune peine, sans loi » ${ }^{1307}$. Le principe généré par ce maxime est inscrit à l'article 8 de la Déclaration des droits de l'homme et du citoyen ${ }^{1308}$ et repris à l'article 16 de la constitution béninoise du 11 décembre 1990 révisée par la loi 2019-40 du 07 novembre 2019 de la manière suivante : « Nul ne peut-être arrêté ou inculpé qu'en vertu d'une loi promulguée antérieurement aux faits qui lui sont reprochés. $(\ldots) »^{1309}$. La conséquence qui découle de la mise en œuvre de ce principe dans le contexte béninois, caractérisé par la démocratie et l'Etat de droit suppose que même, s'il y a besoin d'exposer la première personnalité de l'Etat ${ }^{1310}$ comme tout citoyen à la justice pénale, il faut avoir défini au préalable les infractions à travers une loi ou d'autres catégories de textes ${ }^{1311}$.

En effet, au terme de l'article 74 de la Constitution béninoise du 11 décembre 1990 révisée par la loi 2019-40 du 07 novembre 2019, la poursuite du Président de la République ou des membres du Gouvernement ne peut être ordonnée que s'il est reconnu comme auteur, coauteur ou complice de « violations graves et caractérisées des droits de l'homme » et pour " tout acte attentatoire au maintien d'un environnement sain, satisfaisant, durable et favorable au développement $»^{1312}$. Dans le cas échéant, il faudra que les « (...) infractions soient commises dans l'exercice où à l'occasion de l'exercice de sa fonction (...) ». La loi n 2018-18 portant règlementation sur les changements climatiques en République du Bénin munie d'un dispositif pénal, contenu en ses articles 89 à 91 a définie des infractions susceptibles d'être commises dans le cadre de l'application de ladite loi. A titre illustratif, au terme de l'article 90 alinéa 2 de la loi $\mathrm{n}^{\circ}$ 2018-18 portant règlementation sur les changements climatiques en République du

\footnotetext{
${ }^{1305}$ Article 136 de la Constitution béninoise du 11 décembre 1990, révisée par la loi 2019-40 du 07 novembre 2019.

${ }^{1306}$ En vertu de l'application du principe de la légalité des peines et des délits.

1307 Id.

${ }^{1308}$ La Déclaration des droits de l'homme et du citoyen (parfois abrégée en DDHC) de 1789 est un texte fondamental de la Révolution française, qui énonce un ensemble de droits naturels individuels et les conditions de leur mise en œuvre. Ses derniers articles sont adoptés le 26 août 1789. Disponible sur le site internet: https://www.legifrance.gouv.fr. Consulté le 17/01/2022.

${ }^{1309}$ Le principe de la légalité des délits et peines.

${ }^{1310}$ Le Président de la République est ainsi désigné en République du Bénin.

${ }^{1311}$ Les actes réglementaires (Décret et arrêté).

${ }^{1312}$ Article 3 de la loi n $93-013$ du 10 Août 1999 portant loi organique pour la Haute Cour de Justice.
} 
Bénin, « toute autorité publique, nommée ou élue, qui entreprend de s'opposer ou s'oppose, directement ou indirectement aux politiques, stratégies, programmes ou projets (...) d'adaptation de la communauté nationale au climat, à ses variabilités, à ses changements et l'atténuation de ses effets et conséquences négatifs, est punie d'une peine d'emprisonnement de cinq (05) ans à vingt (20) ans de travaux forcés et d'une amende de cinq millions (5.000.000) à cent millions (100.000.000) de francs CFA». Cet article fait allusion à « toute autorité élue » sans distinction pouvant être poursuivie en cas de sa violation. L'autorité élue au Bénin peut être assimilée dans ce contexte au « Président de la République » qui détient son mandat de la souveraineté du peuple des suites des élections qui sont organisées périodiquement ${ }^{1313}$. Quant à l'autorité nommée, elle peut être assimilable aux «Membres du Gouvernement ${ }^{1314}$ ou certains membres de la Cour constitutionnelle ${ }^{1315}$ ou d'autres cadres, qui le sont sur l'initiative du Président de la République.

Mais, la procédure applicable pour la mise en œuvre de l'action publique ${ }^{1316}$ pour l'application des sanctions contre le Président de la République sont d'une part, des règles de procédure prévues par la loi n²018-14 du 02 juillet 2018 modifiant et complétant la loi $\mathrm{n}^{\circ}$ 2012-15 du 18 mars 2013 portant code de procédure pénale en République du Bénin et d'autre part, l'ensemble des dispositions contenues dans le TITRE IX intitulé « DU CONTENTIEUX, DES INFRACTIONS ET DES SANCTIONS » de la loi $\mathrm{n}^{\circ} 2018-18$ portant règlementation sur les changements climatiques en République du Bénin y compris certaines dispositions constitutionnelles telles que les articles $78 ; 136$ à 138 de la constitution béninoise du 11 décembre 1990 révisée le 07 novembre 2018.

L'avènement de la Haute Cour de Justice a-t-elle été d'une utilité pour la lutte contre les changements climatiques au Bénin?

Depuis la mise en vigueur de la Constitution béninoise le 11 décembre 1990, la Haute Cour de Justice n'a été installée que le 15 février $2001^{1317}$. Cette institution juridictionnelle n'a

\footnotetext{
${ }^{1313} \mathrm{Au}$ terme de l'article 42 de la constitution béninoise du 11 décembre 1990 révisée le 07 novembre 2019, « Le Président de le République est élu au sulfurage universel direct pour un mandat de cinq (5) ans, renouvelable une seule fois.

${ }^{1314} \mathrm{Au}$ terme de l'article alinéa 3 de la constitution béninoise du 11 décembre 1990 révisée le 07 novembre 2019 «Il nomme (...) les membres du Gouvernement (...)».

${ }^{1315}$ L'article 56 de la de la Constitution béninoise du 11 décembre 1990 révisée le 07 novembre 2019 donne les prérogatives au Président de la République de nommer trois (3) membres de la Cour constitutionnelle.

${ }^{1316} \mathrm{Au}$ terme de l'Article $1^{\text {er }}$ de la loi n ${ }^{\circ} 2012-15$ du 18 mars 2013 portant code de procédure pénale en République du Bénin, «l'action publique » est une prérogative appartenant à la société, déléguée au ministère public afin de faire déclarer la culpabilité et sanctionner une personne physique ou morale, auteur d'une infraction à la loi pénale. Elle est mise en mouvement et exercée par les représentants du ministère public.

${ }^{1317}$ Histoire de la Haute cour de justice disponible sur le site internet de la Haute Cour de Justice du Bénin hcjbenin.org. Consulté le 17/01/2022.
} 
connu aucun dossier relatif au crime climatique ${ }^{1318}$. Il en est ainsi à cause des insuffisances contenues dans les textes applicables devant cette institution juridictionnelle qui pourtant, s'était révélée comme un joyau pour les Béninois. Elle était réputée pour sonner le glas de l'impunité au Sommet de l'Etat, malheureusement cet objectif n'a pas été atteint car il existe des goulots qui empêchent la mise en œuvre de l'action publique au niveau de cette institution. Par exemple, dans le cadre de la poursuite des infractions, « les plaintes et les dénonciations contre le Président de la République et les membres du Gouvernement sont adressées au Président de l'Assemblée nationale ». Dans ce cas, l'aboutissement de la procédure dépend du bon vouloir de ce dernier, car dans le système institutionnel béninois, il y a parfois emprise de la politique sur le fonctionnement normal des institutions ${ }^{1319}$. Mais le paradoxe est que, ceux qui ont plus de propension à défendre les intérêts généraux tout comme les citoyens et les Organisations Non Gouvernementales (ONG) ne sont pas habilités à saisir directement la Haute cour de justice. Si, l'article 137 de la Constitution béninoise dispose que, « La Haute cour de justice est liée par la définition des infractions et par la détermination des sanctions résultant des lois pénales en vigueur à l'époque des faits ${ }^{1320}$, mais il existe un goulot à la mise en œuvre de cette disposition qui découle du fait que. «la décision de poursuite puis la mise en accusation du Président de la République et des membres du Gouvernement doit être votée à la majorité des deux tiers $(2 / 3)$ des députés composant l'Assemblée nationale» ${ }^{1321}$. En somme, il faut constater dans la pratique que la sanction criminelle ainsi que la procédure de mise en accusation du Président de la République sont difficilement réalisable à cause des obstacles juridico-politiques.

Compte tenu de l'impossibilité qu'affiche cette institution à offrir les missions régaliennes pour lesquelles elle a été créée, l'opinion souhaite sa suppression estimant que la Haute cour de justice est une institution budgétivore qui garantie l'impunité au Président de la République et aux membres du Gouvernement. Malheureusement, l'occasion de la révision de la constitution béninoise du 11 décembre 1990 intervenue le 07 novembre 2019 n'a pas été saisie pour opérer les modifications nécessaires sur les dispositions consacrées à la Haute cour

\footnotetext{
${ }^{1318}$ Les recherches de jurisprudence au niveau de la Haute cour de justice ont été infructueuses.

${ }^{1319}$ Dans la rubrique : 6.2.1 Poursuite de la séparation des fonctions politiques de celles administratives et techniques du Plan stratégique de la réforme administrative et institutionnelle 2011-2020, il est indiqué que «La politisation des fonctions techniques et administratives constitue un frein pour la promotion d'une administration moderne et professionnelle soucieuse des résultats et corrélativement de la qualité des services et de l'intérêt général », p.68. Disponible sur le site internet: https://ravail.gouv.bj/public/storage/documents. Consulté le $17 / 01 / 2022$.

${ }^{1320}$ Article 137 de la Constitution du 11 décembre 1990 révisée le 07 novembre 2019.

${ }^{1321}$ Les dispositions de l'article 137 alinéa 2 de la Constitution béninoise du 11 décembre 1990, révisée le 07 novembre 2019 .
} 
de justice dans le sens de faciliter l'application des procédures de poursuite des délinquants écologiques $^{1322}$. Ce qui fait qu'en l'état, même si la juridiction est saisie de motif de crime écologique susceptible d'être commis par les hautes personnalités de l'Etat, elle ne pourra statuer utilement.

Cependant, l'existence de la Haute cour de justice dans le contexte béninois ne manque pas de pertinence dans la mesure où, elle constitue un instrument de dissuasion qui permet de semer la crainte de poursuite au pénal dans la conscience des autorités politiques qui violeraient les règles de protection de l'environnement au Bénin.

La mobilisation de la Communauté internationale à travers l'élaboration des normes, la création d'institutions d'encadrement et puis la détermination des autorités béninoises n'ont pas suffit pour faire progresser la lutte contre les changements climatiques au Bénin. La question d'effectivité de la lutte contre les changements climatiques dans ce pays demeure encore un casse-tête dans la mesure où, le fléau au lieu de reculer s'amplifie davantage. Mais, il y a de très bonnes perspectives devant aider à l'amélioration de la lutte contre les changements climatiques au Bénin.

${ }^{1322}$ Il s'agit des articles 135 à 138 de la constitution béninoise du 11 décembre 1990, révisée le 07 novembre 2019. 



\section{TITRE II:}

\section{LES PERSPECTIVES DU DROIT CLIMATIQUE}

\section{AU BENIN}

La montagne n'a-t-elle pas accouché d'une souris, lorsqu'en face d'un fléau aussi gigantesque que titanesque comme celui des changements climatiques contre lequel les acteurs du droit international ont opposé un impressionnant arsenal juridico-institutionnel ingénieusement et massivement élaboré, néanmoins le phénomène perdure et s'aggrave de plus en plus. La preuve de cette contreperformance est contenue dans le rapport spécial publié en 2014 par le Groupe d'Experts Intergouvernemental sur l'Evolution du Climat (GIEC), lequel rapport concluait que la planète subit déjà les conséquences d'une augmentation de $1^{\circ} \mathrm{C} \mathrm{du}$ réchauffement climatique ${ }^{1323}$. Le même rapport informe la Communauté internationale sur la gravité de la moindre hausse du réchauffement qui impliquerait de plus grands risques pour la planète. Les prévisions de ce rapport sont telles que, "Les changements climatiques vont amplifier les risques existants et en engendrer de nouveaux pour les systèmes naturels et humains. Ces risques qui ne sont pas répartis uniformément, sont généralement plus grands pour les populations et les communautés défavorisées de tous les pays, quel que soit leur niveau de développement. Selon les prévisions, l'intensité croissante du réchauffement climatique augmenterait la probabilité d'incidences graves généralisées et irréversibles pour les personnes, les espèces et les écosystèmes. Des émissions élevées et ininterrompues auraient des incidences essentiellement néfastes sur la biodiversité, les éco-services et le développement économique. Ce qui aggraveraient les risques liés aux moyens de subsistance à l'alimentation et à la sécurité des personnes ${ }^{1324}$. C'est pourquoi, il est suggéré à travers ce rapport, qu'il y a des avantages certains à limiter l'augmentation du réchauffement climatique à $1,5^{\circ} \mathrm{C}$ plutôt qu'à $2^{\circ} \mathrm{C}$

Si le pari de la lutte contre les changements climatiques n'est pas gagné au plan international, le sujet demeure une préoccupation pour les Etats Parties à la Convention Cadre

\footnotetext{
${ }^{1323}$ Le rapport spécial publié en 2014 par le Groupe d'Experts Intergouvernemental sur l'Evolution du Climat. Les estimations actuelles indiquent que le seuil de ce réchauffement, par rapport aux températures préindustrielles, est supérieur à environ $1{ }^{\circ} \mathrm{C}, \mathrm{p} .80$. Disponible sur le site internet: https://www.ipcc.ch/reports. Consulté ce 30/05/2021.

${ }^{1324}$ Le Rapport spécial publié en 2014 par le Groupe d'Experts Intergouvernemental sur l'Evolution du Climat, point 2-3, p. 69. Disponible sur le site internet : https://www.ipcc.ch/reports. Consulté ce 30/05/2021.
} 
des Nations Unies sur les Changements Climatiques qui jusque là, peinent à trouver la porte de sortie, car les différents outils élaborés ne leur permet pas de relever les innombrables défis à eux imposés par le fléau. A titre illustratif, le Bénin Etat signataire de ladite Convention à l'instar d'autres nations du monde, n'a pu réussir à atteindre son objectif celui d'enrayer les effets néfastes des changements climatiques qui continuent de perturber la tranquillité des citoyens. Il s'ensuit que l'effectivité du droit de protection du climat voulue n'est pas encore une réalité, nonobstant les efforts accomplis par les autorités qui ont doté ce pays d'un arsenal juridico-institutionnel plus ou moins adapté à relever les défis qu'impose le fléau.

En effet, la persistance des défis imposés par les changements climatiques au plan interne suscite l'interrogation suivante: La réussite de la lutte contre les changements climatiques au Bénin n'est-elle pas d'abord conditionnée par l'effectivité du droit international des changements climatiques?

Puisque le caractère global et planétaire des changements climatiques influence les paramètres climatiques de chaque Etat du monde, de la même manière, ces Etats peuvent être impactés par des initiatives supranationales tendant à lutter contre le phénomène. Dans ce cas, si la lutte contre les changements climatiques connait une réussite au plan international, ce résultat devrait être répercuté sur les Etats Parties, car le droit climatique élaboré au plan international est susceptible d'influencer l'arsenal juridique et institutionnel des Etats Parties. Si le droit international climatique est bien raffiné, il doit réconforter les Etats Parties qui disposeront d'outil adéquat pour mener à bien la lutte contre les changements climatiques. Par contre, les difficultés de mise en œuvre de ce droit au plan supranational ne peuvent faciliter l'atteinte des objectifs de lutte contre les changements climatiques au plan interne.

Nonobstant les difficultés qui entravent la mise en œuvre efficace du droit des changements climatiques, l'espoir est toujours permis de voir un jour relever les défis imposés par ce fléau, mais à la condition que des perspectives soient projetées en vue d'un meilleur encadrement.

Eu égard à ce qui précède, les perspectives d'amélioration de la lutte contre les changements climatiques au Bénin dépendent d'abord de l'amélioration de l'encadrement international (CHAPITRE I) et puis évidemment du renforcement de la gouvernance climatique interne (CHAPITRE II). 


\section{CHAPITRE I:}

\section{L'AMELIORATION DE L'ENCADREMENT INTERNATIONAL}

Dans une perspective d'amélioration de la lutte contre les changements climatiques au Bénin, en tenant compte de l'objet de la matière qui dépasse le cadre étatique, il faudra de tout point de vue projeter des solutions qui intègrent la dimension planétaire du phénomène.

En effet, la détermination de la Communauté internationale à lutter contre les changements climatiques est parfois concluante parce que sanctionnée par certaines avancées notables, notamment, l'existence d'un cadre juridique climatique assez cohérent matérialisé par l'adoption de la Convention Cadre des Nations Unies sur les Changements Climatiques et ses instruments subséquents et la mise en place d'institutions appropriées perfectibles. La Communauté internationale se mobilise également de manière permanente à l'occasion des Conférences des Parties, des Sommets ou d'autres rencontres pour raffiner davantage le cadre juridique international de lutte contre les changements climatiques.

A l'occasion des futures rencontres internationales, la Communauté internationale devra poursuivre l'œuvre de raffinement du droit international climatique dans le seul dessein d'atteindre l'objectif de la Convention Cadre des Nations Unies sur les Changements Climatiques. Cet objectif ne peut être atteint que si la Communauté internationale se concentre à enrayer les difficultés et les insuffisances relevées dans l'encadrement institutionnel international de mise en œuvre du droit climatique devra être renforcé (SECTION I).

Mais en plus, compte tenu du caractère général, global, puis planétaire des changements climatiques, il convient de structurer la coopération internationale en faveur de l'action climatique (SECTION II).

\section{SECTION I: LE RENFORCEMENT DU CADRE INSTITUTIONNEL}

La Convention Cadre des Nations Unies sur les Changements Climatiques et le Protocole de Kyoto ont donné naissance à des institutions d'encadrement qui, à l'épreuve des faits ont permis de réaliser des exploits à l'instar du mécanisme du contrôle du respect qui s'est avéré comme un excellent organe de mise en œuvre de la Convention Cadre des Nations Unies sur les Changements Climatiques à travers son mode opératoire caractérisé par la souplesse. Mais, les attentes ne sont pas comblées parce que le Protocole de Kyoto qui s'est avéré comme 
un excellent cadre normatif qui a permis de faire progresser la lutte contre les changements climatiques ne couvre qu'une infime partie de l'émission mondiale des gaz à effet de serre.

C'est pourquoi l'Accord de Paris est intervenu pour résorber certaines faiblesses d'encadrement relevées au cours de la mise en œuvre de la Convention Cadre des Nations Unies sur les Changements Climatiques et le Protocole de Kyoto.

Pour mieux appréhender le sujet afin de faire concrètement des projections d'amélioration, il convient dans le cadre de notre étude, d'exposer d'abord les améliorations apportées par l'Accord de Paris à l'encadrement institutionnel (Paragraphe 1) avant d'envisager des propositions de son perfectionnement (Paragraphe 2).

\section{Paragraphe 1: Les améliorations dans l'Accord de Paris}

Lors de la COP21 tenue à Paris en décembre 2015, les Parties ont adopté un accord historique pour lutter contre les changements climatiques. Cet accord devrait contribuer à hâter et augmenter les actions et les investissements nécessaires à un avenir durable à faible intensité de carbone.

«L'Accord de Paris est en effet d'une grande complexité architecturale et normative $»^{1325}$ et repose sur les principes fondamentaux de la Convention, Cadre des Nations Unies sur les Changements Climatiques notamment, le principe de la responsabilité commune mais différenciée initié par l'article 3 de ladite Convention, lequel est qualifié d'« article de l'équité ${ }^{1326}$. L'Accord indique une nouvelle voie pour atteindre les objectifs de lutte contre les changements climatiques.

Ainsi, l'Accord de Paris «vise à renforcer la riposte mondiale à la menace des changements climatiques ${ }^{1327}$ à travers le « renforcement des capacités d'adaptation aux effets néfastes des changements climatiques ${ }^{1328}$ en "rendant les flux financiers compatibles avec un profil d'évolution vers un développement à faible émission de gaz à effet de serre et résilient aux changements climatiques $»^{1329}$.

\footnotetext{
${ }^{1325}$ Petit, Y., Rapport introductif « La lutte contre le réchauffement climatique après l'Accord de Paris : quelles perspectives? », », La revue électronique en sciences de l'environnement, VertigO, 2018.

${ }^{1326}$ Y. Petit, « Les mots de la COP21 : justice climatique », op. cit. ; sur ce principe, v. S. Lavallée, « Le principe des responsabilités communes, mais différenciées à Rio, Kyoto et Copenhague. Essai sur la responsabilité de protéger le climat », Études internationales, ${ }^{\circ} 1$, mars 2010, pp. 51-78.

${ }^{1327}$ Article 2 paragraphe 1 de l'Accord de Paris.

${ }^{1328}$ Article 2 paragraphe 1-b id.

${ }^{1329}$ Article 2 paragraphe 1-c de l'Accord de Paris.
} 
Pour atteindre ces objectifs ambitieux, l'Accord de Paris a contribué à l'amélioration du cadre institutionnel par la création d'un cadre technologique ${ }^{1330}$ (A) et par l'innovation d'un cadre de transparence ${ }^{1331}(\mathbf{B})$.

\section{A- La création d'un cadre technologique}

L'importance de la technologie en relation avec les changements climatiques n'est plus à démontrer, car elle contribue « (...) à accroître la résilience aux changements climatiques et

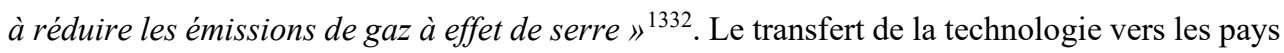
en voie de développement et la mise au point de technologie qui leur sont propre, ont été reconnues au Sommet de Rio de Janeiro de 1992. Consciente de ce que la technologie peut contribuer à un meilleur rendement énergétique pour maitriser et minimiser les émissions de gaz à effet de serre, la Communauté internationale l'a non seulement recommandé, mais a ordonné son transfert vers les Etats qui en ont besoin ${ }^{1333}$. La Convention Cade des Nations Unies sur les Changements Climatiques a prescrit qu'il est nécessaire que, « les pratiques et procédés qui permettent de maîtriser, de réduire ou de prévenir les émissions anthropiques des gaz à effet de serre soient mis au point, appliqués et diffusés par voie de transfert de technologies $»^{1334}$.

Selon Martin Khor, le transfert de technologie « peut concerner l'achat et l'acquisition de matériel et du savoir-faire nécessaire à son utilisation, à son entretien et à sa maintenance, la capacité de fabriquer la technologie grâce à l'ingénierie inverse, l'adaptation de la technologie aux conditions locales et enfin, la conception et la fabrication de produits originaux ${ }^{1335}$. L'Action 21 a même renchéri en ce qui concerne la mise au point du transfert de technologie en indiquant qu' « il est essentiel de pouvoir disposer d'une masse critique de capacité en matière de recherche et de développement pour diffuser et utiliser efficacement les technologies écologiquement rationnelles, ainsi que pour en créer sur le plan local» ${ }^{1336}$. Pour Martin Khor, « Le transfert de technologie ne se résume pas à l'importation ni à l'acquisition

\footnotetext{
${ }^{1330}$ Article 10 paragraphe 4 de l'Accord de Paris.

${ }^{1331}$ Article 13 paragraphe 1 id.

${ }^{1332}$ Article 10 paragraphe 1 ibid.

1333 Paragraphe 25 du préambule et article 4 de la Convention Cadre des Nations Unies sur les Changements Climatiques.

${ }^{1334}$ Article 4 paragraphe1-c de la Convention Cadre des Nations Unies sur les Changements Climatiques.

${ }^{1335}$ Martin Khor, Quel lien y a-t-il entre la lutte contre les changements climatiques, le transfert de technologie et les droits de propriété intellectuelle et où en sont les négociations à leur sujet, Document de recherche, Centre Sud, 2012, 62p. , p.10.

${ }^{1336}$ Paragraphe 13 du chapitre 34 de Action 21.
} 


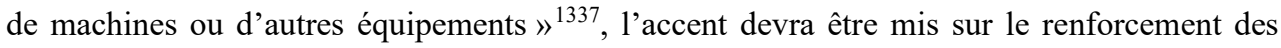
capacités. C'est pourquoi, en plus du transfert de technologie fortement soutenu dans l'Accord de Paris, ce traité recommande le renforcement des capacités ${ }^{1338}$.

L'application du principe des responsabilités communes mais différenciées a permis de désigner les pays développés, grands pollueurs comme les créanciers du droit de transfert de technologie au profit des pays en développement par la stipulation conventionnelle suivante, «les pays développés Parties (...) prennent toutes les mesures possibles en vue d'encourager, de faciliter et de financer selon les besoins, le transfert ou l'accès de technologies et de savoirfaire écologiquement rationnels aux autres Parties et plus particulièrement à celles d'entre elles qui sont des pays en développement afin de leur permettre d'appliquer les dispositions de la Convention. Dans ce processus, les pays développés Parties soutiennent le développement et le renforcement des capacités et technologies propres aux pays en développement Parties. Les autres Parties et organisations en mesure de le faire, peuvent également aider à faciliter le transfert de ces technologies $\gg{ }^{1339}$. La mise en œuvre efficace de ce principe devra permettre de rétablir à terme, la « justice climatique ${ }^{1340}$ suivant la vision tracée dans l'Accord de Paris.

La mise au point et le transfert de technologie sont également évoqués dans plusieurs dispositions de la Convention Cadre des Nations Unies sur les Changements Climatiques en particulier, dans le paragraphe 3 de l'article 4 qui stipule que « les pays développés fournissent les ressources financières nécessaires aux pays en développement notamment, aux fins de transferts de technologie, pour couvrir la totalité des coûts supplémentaires convenus entraînés par l'application des mesures ». Quant au paragraphe 7 de l'article 4 de la même convention indique que, «le respect des engagements pris par les pays en développement sera conditionné par l'efficacité avec laquelle les pays développés exécuteront leurs propres obligations relativement aux ressources financières et au transfert de technologie. Selon Martin Khor ${ }^{1341}$, pour que la mise au point et le transfert de technologies puissent avoir lieu, « il faudra faire dissiper les inquiétudes relatives au manque d'infrastructures, les lois et règlementations

\footnotetext{
${ }^{1337}$ Martin Khor, Quel lien y a-t-il entre la lutte contre les changements climatiques, le transfert de technologie et les droits de propriété intellectuelle et où en sont les négociations à leur sujet, Document de recherche, Centre Sud, 2012, 62p. , p.10.

${ }^{1337}$ Paragraphe 13 du chapitre 34.

${ }^{1338}$ Article 6 paragraphe 8 de l'Accord de Paris.

${ }^{1339}$ Article 4 paragraphe 5 de la Convention Cadre des Nations Unies sur les Changements Climatiques.

${ }^{1340}$ Quatorzième $\left(14^{\mathrm{e}}\right)$ paragraphe du préambule de l'Accord de Paris.

${ }^{1341}$ Né en 1951 à Penang, Malaisie, Martin Khor est un économiste, journaliste et directeur du Third World Network, basé en Malaisie. Il est également directeur exécutif de Centre Sud depuis le $1^{\text {er }}$ mars 2009. C'est un acteur actif de l'alter mondialisme, de la société civile malaise et des réseaux citoyens du Tiers Monde. Il a été invité en 1999 et 2000 au Forum économique mondial. Il participe régulièrement aux Forums sociaux mondiaux (FSM) notamment en 2002 et 2003.
} 
inadaptées, le manque de personnel qualifié, l'insuffisance de moyens financiers, la méconnaissance des questions technologiques, le coût élevé des contrats de licence, les problèmes provenant des fournisseurs de matériel et les droits de propriété intellectuelle $»^{1342}$.

En somme, beaucoup de conditions seront à remplir pour que le transfert de technologie devienne réalité. Mais l'observance de ces conditions est difficilement réalisable.

Conscientes de l'importance de la technologie dans la mise en œuvre de la Convention Cadre des Nations Unies sur les Changements Climatiques, les Parties ont pris l'engagement d' " encourager et de soutenir par leur coopération la mise au point, l'application et la diffusion, notamment par voie de transfert de technologies pratiques et procédés qui permettent de maîtriser, de réduire ou de prévenir les émissions anthropiques des gaz à effet de serre non réglementés par le Protocole de Montréal dans tous les secteurs pertinents, tout comme ceux de l'énergie, des transports, de l'industrie, de l'agriculture, des forêts et de la gestion des déchets» ${ }^{1343}$.

Pour faciliter le transfert de technologie, l'«organe subsidiaire de conseil scientifique et technologique ${ }^{1344}$ a été créé et a reçu pour mission, de « recenser les technologies et savoirfaire de pointe, novateurs et performants et d'indiquer les moyens d'en encourager le développement et d'en assurer le transfert» ${ }^{1345}$.

Malgré les prescriptions de la Convention cadre, les recommandations des Conférences des Parties et l'existence d'un mécanisme chargé de faciliter le transfert de la technologie vers les pays en voie de développement ${ }^{1346}$, « très peu de technologies sans incidence sur le climat ont été transférées au titre de la Convention Cadre des Nations Unies sur les Changements Climatiques $»^{1347}$.

Alors que, la non effectivité du transfert de technologie compromet la mise en œuvre de la Convention Cadre des Nations Unies sur les Changements Climatiques. Dans ces conditions, les pays en développement ne pourront réaliser les objectifs de leurs engagements au titre de la Convention, car la réalisation de ceux-ci est conditionnée par la disponibilité de la technologie. L'Accord de Paris « prenant acte des efforts entrepris pour déployer et diffuser la technologie,

\footnotetext{
${ }^{1342}$ Martin Khor, Quel lien y a-t-il entre la lutte contre les changements climatiques, le transfert de technologie et les droits de propriété intellectuelle et où en sont les négociations à leur sujet, Document de recherche, Centre Sud, 2012, 62p., p.10.

${ }^{1343}$ Article 4 paragraphe 1-c de la Convention Cadre des Nations Unies sur les Changements Climatiques.

${ }^{1344}$ Article 9 paragraphe 1 de la Convention Cadre des Nations Unies sur les Changements Climatiques.

1345 Article 9 paragraphe 2 de la Convention Cadre des Nations Unies sur les Changements Climatiques.

${ }^{1346}$ En application de l'article 20 de la Convention Cadre des Nations Unies sur les Changements Climatiques.

${ }^{1347}$ Martin Khor, Quel lien y a-t-il entre la lutte contre les changements climatiques, le transfert de technologie et les droits de propriété intellectuelle et où en sont les négociations à leur sujet, Document de recherche, Centre Sud, 2012, 62p. , p.9.
} 
renforcer l'action de coopération concernant la mise au point et le transfert de technologies $»^{1348}$, la Communauté internationale a «partagé une vision à long terme de l'importance qu'il y a, à donner pleinement effet à la mise au point et au transfert de technologie de façon à accroître la résilience aux changements climatiques et à réduire les émissions de gaz

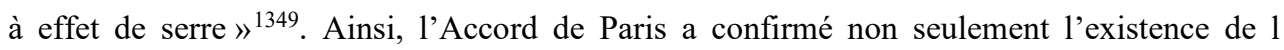
«'Organe subsidiaire de conseil scientifique et technologique» ${ }^{1350}$, mais a réitéré ses attributions qui «s'appliquent mutatis mutandis à l'Accord de Paris» ${ }^{1351}$. Ce mécanisme technologique devra «concourir à l'application de l'Accord de Paris» ${ }^{1352}$. Mais en plus, l'Accord de Paris est intervenu pour renforcer le mécanisme technologique par la « (...) création d'un cadre technologique chargé de donner des directives générales aux travaux du mécanisme technologique visant à promouvoir et faciliter une action renforcée en matière de mise au point et de transfert de technologies de façon à appuyer la mise en œuvre de l'Accord(...)» ${ }^{1353}$. L'article 10 de l'Accord de Paris défini les attributions des organes de technologie qui fonctionnent en complémentarité.

Le processus d'amélioration du mécanisme de mise en œuvre de la Convention Cadre des Nations Unies sur les Changements Climatiques est également orienté dans le sens du renforcement des conditions de gestion des engagements de réduction des émissions des gaz à effet de serre qui s'est matérialisé par l'innovation du cadre de transparence.

\section{B-L'innovation d'un cadre de transparence}

Dans le recueil des traités des Nations unies, le Protocole de Kyoto a été identifié comme, « un cadre novateur dans la lutte contre les changements climatiques $»{ }^{1354}$. Nonobstant ces contre performances, le Protocole de Kyoto est réputé comme, «l'un des mécanismes les plus aboutis de mise en œuvre et de contrôle du droit international» ${ }^{1355}$. Cependant, dans la perspective d'un accord global élargi à plusieurs Etats, principalement les puissances pollueuses, un nouveau modèle d'accord fédérateur a été adopté.

\footnotetext{
1348 Article 10 paragraphe 2 de l'Accord de Paris.

1349 Article 10 paragraphe1 id.

${ }^{1350}$ Article 18 paragraphe 1 ibid.

${ }^{1351}$ Id.

${ }^{1352}$ Article 10 paragraphe 3 de l'Accord de Paris.

${ }^{1353}$ Article 10 paragraphe 4 id.

${ }^{1354}$ Nations Unies, Convention-cadre des Nations unies sur les changements climatiques, Recueil des traités, vol. 1771, $\mathrm{n}^{\circ}$ 30822. Disponible sur le site internet : https://treaties.un.org > pages > Vie. Consulté le 17/01/2022. ${ }^{1355}$ Id.
} 
Il s'agit de l'Accord de Paris a apporté une solution novatrice qui correspond aux nombreuses préoccupations concernant la mise en œuvre efficace de la Convention Cadre des Nations Unies sur les Changements Climatiques. Le Traité de Paris n'est pas muni d'un dispositif qui sanctionne les cas avérés de non-conformité d'Etats qui ne respectent pas leurs engagements en ce qui concerne la réalisation de leurs contributions nationales de limitation d'émission de gaz à effet de serre. Il en est ainsi parce que, le traité international repose «sur un mécanisme souple de revue par les pairs plutôt qu'un système juridique de sanctions »"1356. C'est plutôt un mécanisme de promotion et de facilitation du contrôle du respect des dispositions de l'accord, qui est institué suivant l'article 15. Le but poursuivi, est de « renforcer la confiance mutuelle et de promouvoir une mise en œuvre efficace dudit Accord à travers un nouveau mécanisme de contrôle » ${ }^{1357}$ dénommé « cadre de transparence» ${ }^{1358}$. Le cadre de transparence est un mécanisme institutionnel qui « accorde aux pays en développement Parties qui en ont besoin, compte tenu de leurs capacités, une certaine flexibilité dans la mise en œuvre » ${ }^{1359}$ de l'accord climat. Le mécanisme s'appuie sur les « dispositifs relatifs à la transparence prévus en vertu de la Convention et les renforce en tenant compte de la situation particulière des pays les moins avancés et des petits Etats insulaires en développement et doit être mis en œuvre d'une façon qui soit axée sur la facilitation, qui ne soit ni intrusive ni punitive, qui respecte la souveraineté nationale et qui évite d'imposer une charge excessive aux Parties.» ${ }^{1360}$ Il permet de faire une gestion transparente de la politique de réduction des émissions de gaz à effet de serre et s'intéresse également aux actions devant faciliter la mise en œuvre de l'accord ${ }^{1361}$. Il s'appuie sur l'expérience acquise et est conçu comme un instrument essentiel pour la mise en œuvre effective de l'Accord de Paris. Dans le cas d'espèce, il s'agit en effet de vérifier que les engagements pris par les pays en matière de réduction de gaz à effet de serre pour atteindre l'objectif d'une hausse des températures inférieure à $2{ }^{\circ} \mathrm{C}$, voire proche de $1,5^{\circ} \mathrm{C}$ seront bien tenus. Dans cette optique, l'article 13 de l'Accord de Paris oblige chaque Partie signataire à fournir régulièrement «les informations nécessaires au suivi des progrès accomplis dans la mise en œuvre et la réalisation de sa contribution déterminée au niveau national». L'Accord de Paris se distingue des accords précédents sur le climat sur le point de ce que l'obligation de transparence s'applique sans discrimination à tous les Etats Parties

\footnotetext{
${ }^{1356}$ Analyse de Daniel Bodansky, «Reflections on the Paris Conférence», Mayer, 2016, p. 15. Disponible sur le site internet : http://opiniojuris.org/2015/12/15/reflections-on-the-paris-conference/. Consulté le 17/01/2022.

${ }_{1357}$ Accord de Paris, articles 13-1.

1358 Id.

${ }^{1359}$ Article 13 paragraphe 2 de l'Accord de Paris.

${ }^{1360}$ Article 13 paragraphe 3 de l'Accord de Paris.

${ }^{1361}$ Article 13 de l'Accord de Paris.
} 
contrairement au Protocole de Kyoto qui ne vise que les Etats développés et émergeants. Cet Accord, contrairement au protocole de Kyoto qui a institué un «système d'observance », a élaboré un système de « Monitoring, reporting and verification or measurable, reportable and verifiable $»^{1362}$ applicable à toutes les Parties. Le cadre de transparence s'efforce de donner « une image claire de l'appui fourni et de l'appui reçu par chaque Partie concernée dans le contexte des mesures prises à l'égard des changements climatiques au titre des articles 4, 7, 9, 10 et 11 et dans la mesure du possible, une vue d'ensemble de l'appui financier global fourni pour éclairer le bilan mondial prévu à l'article $14 .{ }^{1363}$ Son objectif in fine est de projeter et de « fournir une image claire des mesures relatives aux changements climatiques à la lumière de l'objectif énoncé à l'article 2 de la Convention notamment, en éclairant et en suivant les progrès accomplis par chaque Partie en vue de s'acquitter de sa contribution déterminée au niveau national et de mettre en œuvre ses mesures d'adaptation notamment, les bonnes pratiques, les priorités, les besoins et les lacunes, afin d'éclairer le bilan mondial» ${ }^{1364}$. Le cadre de transparence de l'Accord de Paris s'apparente au mécanisme de non respect du Protocole de Kyoto suivant les caractéristiques et modalités de son fonctionnement. Ce mécanisme s'inspire en partie des procédures applicables devant la chambre de facilitation mise en place pour faciliter le respect des dispositions du Protocole de Kyoto. L'institutionnalisation du cadre de transparence dans l'Accord de Paris vient renforcer les acquis institutionnels en matière du contrôle des engagements de réduction des émissions des gaz à effet de serre.

Cependant, le « cadre de transparence » tout comme le mécanisme du «contrôle de respect» des engagements ne serait pas non plus une panacée dans le cadre de la lutte contre les changements climatiques. C'est à l'épreuve de l'effectivité de son fonctionnement qu'il pourra être jugé efficace ou non.

Dans le cas où certains Etats Parties s'obstineront à satisfaire les besoins de contrôle du cadre de transparence, la mise en œuvre de la responsabilité internationale de l'Etat pour dommage écologique parait le dernier rempart, le moyen le mieux indiqué pour contraindre les Etats développés et les pays en voie de développement à s'acquitter de leurs obligations conventionnelles. Seulement, la mise en œuvre de cette procédure dépend encore de la volonté des Etats à se soumettre à la compétence de la Cour internationale de justice. Néanmoins, dans la perspective de ce que sera amélioré le cadre juridictionnel avec l'avènement d'une justice

\footnotetext{
${ }^{1362}$ YVES Petit, «Les risques environnementaux globaux et les transformations de la souveraineté », SFDI, Colloque de Nancy, l'Etat dans la mondialisation, 2013, Pedome, p.205 et s.

${ }^{1363}$ Article 13 paragraphe 6 de l'Accord de Paris.

${ }^{1364}$ Article 13 paragraphe 5 id.
} 
internationale climatique plus contraignante, la possibilité de recourir à la justice pour faire respecter les dispositions de la Convention Cadre des Nations Unies sur les Changements Climatiques sera une réalité. Ainsi, finira la complaisance dans la mise en œuvre des obligations conventionnelles en ce qui concerne les Etats Parties de la Convention Cadre des Nations Unies sur les Changements Climatiques. C'est à ce prix que les Etats Parties pourront s'acquitter volontairement ou sous l'effet de la contrainte, leurs parts d'obligations.

Mais, le renforcement de l'architecture institutionnelle de la Convention Cadre des Nations Unies sur les Changements Climatiques prévu dans l'Accord de Paris n'est pas suffisant car, certaines faiblesses relevées lors de la mise en œuvre de la Convention ne sont pas prises en compte par l'Accord de Paris. C'est pourquoi notre étude envisage le perfectionnement du cadre institutionnel international.

\section{Paragraphe 2: Le perfectionnement du cadre institutionnel}

Les Experts du GIEC ont abordé la question du renforcement du cadre institutionnel de lutte contre les changements climatiques. Ces derniers ont fait état de ce que « la mise en œuvre d'options efficaces d'adaptation et d'atténuation pourrait nécessiter la mise en place de nouvelles institutions et l'adoption de nouvelles dispositions institutionnelles applicables à diverses échelles ${ }^{1365}$. Ainsi, la réforme du cadre normatif entreprise à l'occasion de la COP21 tenue à Paris en 2015 qui a débouché sur la création de nouvelles institutions d'encadrement des changements climatiques a contribué à remédier en partie à certaines faiblesses constatées dans l'encadrement institutionnel de la lutte contre les changements climatiques. Il s'ensuit que le travail reste encore à parfaire car, le cadre de notre étude a révélé certaines insuffisances qu'il faille corriger dans l'encadrement institutionnel. A titre illustratif, la question de la capacité réelle de gestion efficace des institutions des Nations unies que sont entre autres, le Programme des Nations Unies pour l'Environnement et la Cour Internationale de Justice, qui ont vu leur compétence s'étendre au domaine des changements climatiques par l'effet de l'application de la Convention Cadre des Nations Unies sur les Changements Climatiques demeure.

Certaines préoccupations relatives à l'encadrement tout comme celles relevant du domaine de la gestion des conflits climatiques ne sont pas parfaitement solutionnées à l'occasion des réformes opérées en 2015 au cours de la tenue de la COP21. Pour cela, il convient donc de palier ces insuffisances par la création d'une nouvelle organisation internationale

\footnotetext{
${ }^{1365}$ Page 14 du Rapport spécial du GIEC 2014. Disponible sur le site internet: https://www.ipcc.ch/reports. Consulté ce 30/05/2021.
} 
environnementale (A) et d'une juridiction internationale environnementale spécialisée en matière des changements climatiques $(\mathbf{B})$.

\section{A- La création d'une organisation internationale environnementale}

Le Programme des Nations Unies pour l'Environnement (PNUE), seule institution relevant du Système des Nations unies ayant pour compétence de coordonner les actions de protection de l'environnement sur toute la planète, s'est acquitté de sa mission dans les limites des possibilités que lui ont permis son statut. Cette institution n'est qu'un «Programme », contrairement à d'autres institutions spécialisées de l'ONU notamment, l'Organisation Mondiale du Commerce (OMC) et l'Organisation Mondiale de la Santé (OMS), qui sont des institutions privilégiées pourvues de moyens pour l'accomplissement de leurs missions respectives. Le Programme des Nations Unies pour l'Environnement (PNUE) manque de financement pour les immenses programmes mis à sa charge. La situation de son siège sis à Nairobi au Kenya ne facilite pas son rayonnement compte tenu de son écart des centres de décision. Ce qui rend le PNUE dépendant vis-à-vis d'autres institutions. Cette faiblesse a été reconnue par les autorités en charge de l'institution qui ont fait transparaitre cette opinion à travers la Déclaration de Nairobi sur le rôle et le mandat du PNUE en ces termes « Nous reconnaissons que nous dépendons des systèmes et des ressources naturelles pour notre existence ${ }^{1366}$.

Le PNUE a obtenu des succès importants de par son investissement dans l'élaboration des accords internationaux sur la protection de l'environnement notamment, le Protocole de Montréal sur la protection de la couche d'ozone ${ }^{1367}$, la Convention de Bâle sur les mouvements transfrontières de déchets dangereux ${ }^{1368}$, la Convention sur la diversité biologique ${ }^{1369}$ ainsi que

\footnotetext{
${ }^{1366} \mathrm{SS} . \mathrm{XII} / 8$ : Déclaration ministérielle à l'occasion du 40 e anniversaire du Programme des Nations unies pour l'environnement. Paragraphe 4. Disponible sur le site internet : https://wedocs.unep.org.Consulté le 17/01/2022.

${ }^{1367}$ Le Protocole de Montréal est un accord multilatéral international sur l'environnement qui fait suite à la convention de Vienne sur la protection de la couche d'ozone adoptée le 22 mars 1985. Il a pour objectif de réduire et à terme d'éliminer complètement les substances qui réduisent la couche d'ozone.

${ }^{1368}$ La Convention de Bâle sur le contrôle des mouvements transfrontières de déchets dangereux et de leur élimination est un traité international qui a été conçu afin de réduire la circulation des déchets dangereux entre les pays. Il s'agissait plus particulièrement d'éviter le transfert de déchets dangereux des pays développés vers les Pays en développement (PED). La convention a aussi pour but de minimiser la quantité et la toxicité des déchets produits, et d'aider les PED à gérer de façon raisonnable les déchets, nocifs ou pas, qu'ils produisent. La Convention a été ouverte à la signature le 22 mars 1989, et est entrée en vigueur le 5 mai 1992. Une liste des Parties et de leur disposition par rapport au traité se trouve sur le site du Secrétariat de la Convention (archive). Sur 166 États Parties à la convention, seuls, Haïti et les Etats-Unis ont signé la Convention mais ne l'ont pas ratifiée. Disponible sur le site internet : https://legal.un.org.Consulté le 17/01/2022.

${ }^{1369}$ La Convention sur la diversité biologique (CDB) est un traité international adopté lors du Sommet de la Terre à Rio de Janeiro en 1992, avec trois buts principaux : 1- la conservation de la biodiversité ;2- l'utilisation durable
} 
la Convention Cadre des Nations Unies sur les Changements Climatiques ${ }^{1370}$ et ses instruments subséquents. Mais cet exploit réalisé par l'Organisation est contrasté par des contreperformances. La preuve en est que, les responsables de l'organisation, tout en reconnaissant les exploits accomplis par le Programme des Nations Unies pour l'Environnement se déclarent, « préoccupés par la dégradation continue de l'environnement, qui met en péril les systèmes et ressources naturels» ${ }^{1371}$. La faiblesse de l'institution est accentuée par les mutations intervenues dans son mode de fonctionnement, puisqu'elle s'était fixée à l'origine comme priorité, la mise en œuvre des stratégies. Ce changement de paradigme correspond aux aspirations de certains Etats qui veulent avoir des réalisations palpables en lieu et place des traités. Le revirement dans la méthode d'intervention du PNUE permet à certains Etats d'obtenir des financements auprès des institutions partenaires.

A partir du moment où l'institution faitière s'est distancée de sa mission originelle qui consistait au «développement de normes et le renforcement des capacités institutionnelles » ${ }^{1372}$, elle n'est plus apte à «coordonner les efforts des organismes internationaux de protection de l'environnement ${ }^{1373}$. Ainsi, contre toute attente, le Programme des Nations Unies pour l'Environnement (PNUE) n'est pas parvenu à se transformer en un centre de résolution en matière de protection internationale de l'environnement. Alors qu'en réalité, l'institution a été conçue dans l'esprit d'être établie comme, «la principale autorité mondiale de l'environnement, qui fixe l'ordre du jour de l'environnement mondial, favorise la mise en œuvre cohérente de la dimension environnementale du développement durable au sein du système des Nations unies et sert comme un des défenseurs de l'environnement mondial $»^{1374}$.

Face aux faiblesses qu'affiche le PNUE en matière de gestion de la protection internationale de l'environnement, quelles sont les mesures alternatives envisageables pour

de ses éléments ; 3-le partage juste et équitable des avantages découlant de l'exploitation des ressources génétiques. Son objectif est de développer des stratégies nationales pour la conservation et l'utilisation durable de la diversité biologique. Il est considéré comme le document clé concernant le développement durable. Disponible sur le site internet : https://www.un.org/fr/observances/biological-diversity-day/convention.Consulté le 17/01/2022.

${ }^{1370}$ La Convention Cadre des Nations Unies sur les Changements Climatiques (CCNUCC) a été adoptée au cours du Sommet de la Terre de Rio de Janeiro en 1992 par 154 Etats auxquels il faut ajouter la totalité des membres de la Communauté européenne. Elle est entrée en vigueur le 21 mars 1994. Etat de ratification disponible sur le site internet : https://treaties.un.org. Consulté le 17/01/2022.

${ }^{1371}$ SS.XII/8 : Déclaration ministérielle à l'occasion du quarantième anniversaire du Programme des Nations unies pour l'environnement. Paragraphe 4. Disponible sur le site internet: https://wedocs.unep.org. Consulté le $17 / 01 / 2022$.

${ }^{1372}$ Id.

${ }^{1373}$ Ibid.

${ }^{1374}$ SS.XII/8 : Déclaration ministérielle à l'occasion du quarantième anniversaire du Programme des Nations unies pour l'environnement. Paragraphe 4. Disponible sur le site internet: https://wedocs.unep.org. Consulté le 17/01/2022. 
améliorer l'encadrement institutionnel international dans un contexte de dégradation continue de l'environnement ajouté aux problèmes engendrés par les changements climatiques?

L'échec du PNUE n'a pas laissé indifférent les acteurs du droit international qui se sont mobilisés autour de la problématique de la qualité de la gouvernance institutionnelle environnementale. Cette question est d'autant plus préoccupante parce que l'institution était créée pour gérer la protection de l'environnement, mais elle a été rattrapée par les changements climatiques qui ont rallongé ses attributions. Même si cette institution a contribué à assurer les bases d'un régime juridique climatique avec l'adoption de la Convention Cadre des Nations Unies sur les Changements Climatiques et ses accords subséquents, des réformes du cadre institutionnel s'imposent à cause des nouvelles donnes provoquées par la survenance des changements climatiques. A l'évidence, les réflexions allant dans le sens de l'amélioration de la qualité institutionnelle devront intégrer désormais les données concernant les changements climatiques. C'était à juste titre qu'à l'occasion de la douzième $\left(12^{\mathrm{e}}\right)$ session extraordinaire du Forum ministériel mondial sur l'environnement tenu le 22 février 2012, Mme Graciela Muslera ${ }^{1375}$ engageait les participants « (...) à réclamer d'une seule voix un cadre institutionnel plus robuste pour le développement durable et la gouvernance de l'environnement ${ }^{1376}$

En effet, les Etats membres de l'Union européenne ont suggéré de modifier le PNUE en une nouvelle institution qui pourra être dénommée «Organisation des Nations Unies pour l'Environnement (ONUE) ». En s'alignant sur la proposition des Etats de l'Union européenne, la France supporte l'idée de « la création d'une organisation mondiale de l'environnement (OME), un projet qu'elle porte depuis plusieurs années avec plus ou moins de force selon les périodes et soutenu par plusieurs pays, notamment les Etats africains $\gg{ }^{1377}$. L'initiative française avait pris corps en 2012 et orientée dans le sens de l'activation de la diplomatie pour appuyer l'idée de création de l'Organisation des Nations Unies pour l'Environnement (ONUE) ${ }^{1378}$. La proposition française avait pour finalité, de contribuer à l'amélioration de la gouvernance mondiale en matière de gestion et de protection de l'environnement. Certaines opinions

\footnotetext{
${ }^{1375}$ Ministre uruguayenne du logement, de l'aménagement du territoire et de l'environnement et Présidente par intérim du Conseil d'administration/Forum ministériel mondial sur l'environnement.

${ }^{1376}$ Nations unies, Conseil d'administration du Programme des Nations Unies pour l'Environnement Douzième session extraordinaire du Conseil d'administration/ Forum ministériel mondial sur l'environnement Nairobi, 20 22 février 2012, Compte rendu du Conseil d'administration. Paragraphe 6., p.2. Disponible sur le site internet : https://wedocs.unep.org. Consulté le 17/01/2022.

${ }^{1377}$ MALAJEAN-DUBOIS(Sandrine), WEMAERE (Matthieu), La Conférence des Nations unies sur le développement durable Rio+20, Annuaire français de relations internationales, Paris, La Documentation française, 2014, pp.721-735, p.732.

${ }^{1378}$ Carine Seghier, ACTU ENVIRONNEMENT Transformer le PNUE en agence spécialisée des Nations unies pour l'environnement Gouvernance. Disponible sur le site internet: https:/www.actu-environnement.com Consulté le 17/01/2022.
} 
favorables à la création de l'Organisation des Nations Unies pour l'Environnement (ONUE) considèrent que cette nouvelle organisation contribuerait à porter les préoccupations environnementales dans le giron du système des Nations unies pour faire profiter à la cause environnementale des privilèges et moyens adéquats à l'ascension des charges ${ }^{1379}$. La nouvelle organisation à créer devra bénéficier du statut «d'institution spécialisée » des Nations unies, qui lui permettrait de «jouir d'une autonomie budgétaire » et de « déterminer la plupart des détails de son programme de travail ${ }^{1380}$. Il est envisagé en ce qui concerne les attributions de l'ONUE, qu'elle se chargerait de converger les connaissances scientifiques en matière environnementale et donner des orientations stratégiques sur la gestion de la protection de l'environnement mondiale. A l'opposé d'autres institutions spécialisées du système des Nations unies, tout comme l'OMC, elle ne serait pas investie du pouvoir juridictionnel pour trancher les différends environnementaux. L'organisation faitière naissante, devra administrer « plusieurs accords environnementaux et de nombreuses organisations internationales à compétence environnementale afin d'assurer leur efficacité managériale » ${ }^{1381}$.

Cependant, il y a des opposants à ce projet qui résistent contre sa réalisation au point où le Professeur Maurice KAMTO cherchait à « comprendre la résistance opposée par certains Etats à l'idée de transformer le PNUE en Organisation Mondiale de l'Environnement $(\mathrm{OME}){ }^{1382}{ }^{132}$. Visiblement, en face de l'option qui consiste à créer une nouvelle organisation internationale environnementale, il y a des inquiétudes qui naissent aux niveaux de certains Etats qui pensent que cette nouvelle initiative contribuera à la promotion de la politique environnementale au détriment d'autres secteurs d'activités. Pour ces motifs, ils ne sont pas favorables pour l'éclosion d'un nouveau cadre institutionnel devant s'occuper de la question de la gestion de l'environnement. Ils suggèrent au contraire, en lieu et place de la création d'une nouvelle organisation, le renforcement des capacités du Programme des Nations Unies pour l'Environnement. Malgré cette opposition, le soutien pour la création de l'Organisation des Nations Unies pour l'Environnement (ONUE) se manifeste de plus en plus à travers des initiatives collectives et individuelles. A titre illustratif, le Groupe des Amis de

\footnotetext{
${ }^{1379}$ EU Presidency Speaking Points on Item 4 (e) - United Nations Reform: 24th Session of the Governing Council/Global Ministerial Forum of UNEP, 5 - 9 February 2007, Nairobi.

${ }^{1380}$ NATIONS UNIES EP UNEP/GCSS.XII/14 Conseil d'administration du Programme des Nations Unies pour l'Environnement Douzième session extraordinaire du Conseil d'administration/ Forum ministériel mondial sur l'environnement Nairobi, 20-22 février 2012 Compte rendu du Conseil d'administration. Disponible sur le site internet : https://wedocs.unep.org. Consulté le 17/01/2022.

${ }^{1381}$ Global Compact- International environmental governance and the United Nations Envrionment Organization (UNEO) projet - Speech by M.

1382 Maurice KAMTO, La mise en œuvre du droit de l'environnement : forces et faiblesses des cadres institutionnels» Revue Africaine de Droit de l'Environnement $\mathrm{N}^{\circ} 01,2014$, p. 30.
} 
l'ONUE composé de cinquante- deux (52) Etats, y compris ceux de l'Union européenne et des pays de chaque zone géographique de la planète a été constitué pour promouvoir la création de l'ONUE $^{1383}$. Face à cette proposition d'amélioration du cadre institutionnel, les avis sont divergents quant à la nature de la réforme à opérer avec le PNUE.

Pour permettre de relever les défis liés à la fois, à la protection de l'environnement et à la lutte contre les changements climatiques, quelle serait l'option idéale entre, créer une nouvelle Organisation pour l'Environnement ou réhabiliter le Programme des Nations Unies pour l'Environnement (PNUE)?

Une analyse objective de la situation du déclin du PNUE permet de comprendre que la faiblesse de cette organisation a d'abord son origine dans les textes régissant sa création et puis les pratiques qui ont été développées dans le cadre du fonctionnement de l'institution. Les attributions du PNUE étaient orientées vers les objectifs généraux de protection de l'environnement, ce fut plus tard que l'organisation a été investie des missions concernant les changements climatiques. De ce point de vue, des réformes s'imposent. Mais quel type de réforme? Réformer le PNUE conduirait à retoucher fondamentalement l'acte de création de l'institution. Dans le cas échéant, il n'est pas envisagé non plus de « conférer au Programme des Nations Unies pour l'Environnement (PNUE) les pouvoirs de surveillance et de sanction qui lui font défaut $\gg{ }^{1384}$. Cet exercice de réforme engendrerait beaucoup de complications sans que les objectifs qui en sont à l'origine ne soient atteints. Dans ces conditions, l'option la plus simple serait de remplacer le PNUE par une nouvelle organisation internationale environnementale qui sera créée. Cette proposition parait objective car, elle permettra de régler de manière holistique et durable les problèmes d'encadrement de la protection de l'environnement tout en intégrant la dimension concernant les changements climatiques.

A propos des réformes institutionnelles, les débats sont avancés dans le cadre de la gouvernance climatique internationale. Il était consigné dans les actes du Sommet de Rio+20 « la création prochaine d'une instance politique de haut niveau qui pourrait, entre autre, exercer une action mobilisatrice, donner des orientations et formuler des recommandations aux fins du développement durable et promouvoir la cohérence et la coordination des politiques de

\footnotetext{
${ }^{1383}$ Le groupe des Amis de l'ONUE a été créé pour accompagner le changement du Programme des Nations Unies pour l'Environnement (PNUE) en Organisation des Nations Unies pour l'Environnement. Information disponible sur le site internet https://www.actu-environnement.com/ae/news/onue_agadir_pnue_2499.php4. Consulté le $18 / 01 / 2022$

${ }^{1384}$ MALAJEAN-DUBOIS(Sandrine), WEMAERE (Matthieu), La Conférence des Nations unies sur le développement durable Rio+20, Annuaire français de relations internationales, Paris, La Documentation française, 2014, pp.721-735, p.732.
} 


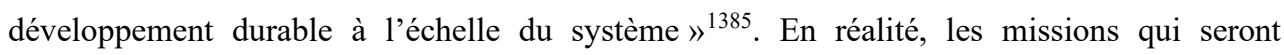
assignées à cette institution s'apparentent à celles qu'accompli déjà, le Programme des Nations Unies pour l'Environnement (PNUE) ${ }^{1386}$. Cette réforme aura pour conséquence la substitution $\mathrm{du}$ «foisonnement institutionnel» par ce que certains auteurs ont qualifié de «cohérence institutionnelle » qui se manifestera par le regroupement des divers organismes dédiés à la protection de l'environnement et à la lutte contre les changements climatiques au niveau international, dans le cadre d'une organisation unique ${ }^{1387}$.

A priori, la création d'une nouvelle institution internationale environnementale, qui toutefois peut être désignée sous le vocable, «Organisation des Nations Unies pour l'Environnement (ONUE) », placée sous le contrôle du système des Nations unies, jouissant des mêmes privilèges que les institutions spécialisées serait un excellent projet. Ce qui permettra de palier les déficits de fonctionnement qui a caractérisé le PNUE. Et désormais, les volets concernant les domaines, environnementaux et climatiques seront considérés comme des attributions principales du système des Nations unies. De même, tous les accords internationaux relatifs à la protection de l'environnement et à la lutte contre les changements climatiques seront coordonnés dans le même cadre institutionnel. En considérant le mode de fonctionnement du système des Nations unies, qui dispose d'une audience dans l'opinion internationale, sa fermeté dans la gestion de ses affaires, sa capacité de mobilisation, son management, l'immensité de ses ressources, humaine, matérielle et financière et la force exécutoire des résolutions de son assemblée générale, l'innovation d'une organisation environnementale inscrite dans son organigramme peut constituer un atout pour l'effectivité du droit international des changements climatiques.

Cependant, la création de cette institution internationale peut heurter l'opposition des pays tels que, les Etats-Unis d'Amérique, le Brésil, la Fédération de Russie, l'Inde et la Chine, c'est-à-dire, les cinq (5) principaux pays émetteurs de gaz à effet de serre ${ }^{1388}$. Ces pays sont hostiles à l'idée de réduction de l'émission de gaz à effet de serre qui est perçu selon eux comme un facteur de régression de l'économie.

Mais en attendant de voir un jour concrétiser le projet de création d'une nouvelle organisation environnementale en remplacement du Programme des Nations Unies pour

\footnotetext{
${ }^{1385}$ Baba HAMADY DEME, « RIO+20 ET L'AVENIR DE LA GOUVERNANCE DE L'ENVIRONNEMENT EN AFRIQUE », Revue Africaine de Droit de l'Environnement n 00, 2012, Université Jean Moulin Lyon 3. p.41. $1386 \mathrm{Id}$.

1387 KAMTO (Maurice), La mise en œuvre du droit de l'environnement: forces et faiblesses des cadres institutionnels, RADE N01, 2014, p.30, 36. Disponible sur le site internet : https://www.iucn.org/sites/dev/files. Consulté le 18/01/2022.

${ }^{1388}$ Alister Doyle, « 46 nations call for tougher U.N. environment rôle », Reuters, 3 février 2007.
} 
l'Environnement, il convient de mener également des réflexions en ce qui concerne la question de la gestion des conflits environnementaux qui intègre les dimensions relatives aux changements climatiques et le statut personnel des acteurs en charge de la gestion de la protection de l'environnement ou intervenant dans le cadre de la lutte contre les changements climatiques, autres que les Etats ou organisations gouvernementales internationales. Cette réflexion peut éventuellement permettre d'aborder la problématique de la gestion efficace des différends et déboucher sur la proposition de création d'une juridiction internationale spécialisée respectivement dans les domaines, de la protection de l'environnement et de la gestion des changements climatiques.

\section{B-La création d'une juridiction internationale spécialisée}

Lorsque le besoin de faire encadrer la Convention Cadre des Nations Unies sur les Changements Climatiques par un organe juridictionnel international pour assurer son effectivité avait été manifesté à l'occasion du Sommet de Rio de Janeiro de 1992, les préoccupations relatives à l'efficacité de cette juridiction pour contribuer à l'avènement d'une justice climatique avec ses spécificités n'avaient guère transparu dans le choix effectué par la Communauté internationale au moment de l'élaboration de la Convention Cadre des Nations Unies sur les Changements Climatiques. Malheureusement, la Cour Internationale de Justice (CIJ) établie dans ces conditions pour administrer les différends générés par l'application de ladite convention a fait preuve de ses limites ${ }^{1389}$. Le bilan de son intervention comme organe juridictionnel de la Convention Cadre des Nations Unies sur les Changements Climatiques est mitigé ${ }^{1390}$. Il en est ainsi parce qu'elle est confrontée à des difficultés liées à la procédure moins contraignante applicable devant elle. Mais en plus, cette juridiction à compétence générale a vu ses attributions s'élargir aux changements climatiques, un domaine autre que celui dans lequel elle intervient habituellement, par application de l'article 14 paragraphe 2 de la Convention Cadre des Nations Unies sur les Changements Climatiques. Ce qui contrarie l'espoir de la Communauté internationale quant à l'atteinte de l'objectif de la Convention en ce qui concerne la gestion efficace des différends climatiques.

Paradoxalement, au moment où la justice internationale parait inefficace face aux règlements des différends climatiques, la preuve de l'effectivité de la justice climatique est

\footnotetext{
${ }^{1389}$ L'article 14-2.a de la Convention Cadre des Nations Unies sur les Changements Climatiques a institué la soumission des différends nés de l'application de la Convention à la Cour internationale de justice.

${ }^{1390}$ Voir supra la rubrique (B- Le contrôle mitigée de la justice internationale, pp. 240-243).
} 
donnée dans certains pays. Les juridictions de certains Etats Parties à la Convention Cadre des Nations Unies sur les Changements Climatiques se sont illustrées de la plus belle manière à régler des litiges climatiques. Aux Pays-Bas, il existe une jurisprudence importante dans un conflit climatique qui a opposé l'ONG Urgenda au Gouvernement Néerlandais ${ }^{1391}$. L'affaire a connu son épilogue le 20 décembre 2019 lorsque la Cour suprême de ce pays a confirmé la décision de la Cour d'appel de La Haye dans laquelle il a été affirmé que l'Etat agissait " illégalement et en violation du devoir de diligence,» ${ }^{1392}$ en ce qui concerne l'acquittement des obligations des Pays-Bas, souscrits dans la Convention Cadre des Nations Unies sur les Changements Climatiques, sans prononcer de sanctions contre lui ${ }^{1393}$. Cette décision n'a fait que rappeler le devoir de diligence dont les Etats Parties à la Convention Cadre des Nations Unies sur les Changements Climatiques sont tributaires. Ce devoir de diligence selon la Conférence de l'Association de Droit International se traduit comme, «l'obligation qui incombe aux Etats de faire preuve de diligence raisonnable pour éviter, minimiser et réduire les dommages environnementaux et autres causés par le changement climatique ${ }^{1394}$. L'enjeu de ce conflit climatique est bien perçu par la Juridiction suprême qui a délibéré en ces termes: «Cette affaire concerne la question de savoir si l'Etat néerlandais est obligé de réduire les émissions de gaz à effet de serre du sol néerlandais d'au moins 25\% d'ici la fin de l'an 2020 par rapport à l'an 1990 et si le tribunal peut ordonner à l'Etat de le faire "1395. Cette jurisprudence, oblige désormais les Pays-Bas à prendre des mesures pour assurer la protection des citoyens contre les conséquences néfastes de la modification climatique. Elle révèle pour l'opinion le besoin et la possibilité de recourir à la justice interne pour faire respecter les règles

\footnotetext{
${ }^{1391}$ Dans une procédure historique, quelque neuf cents (900) Néerlandais avaient entamé une action en justice contre leur gouvernement avec l'aide de l'ONG Urgenda, active dans la défense de l'environnement. Ces citoyens demandaient que La Haye prenne des mesures pour réduire les émissions de $\mathrm{CO} 2$ aux Pays-Bas de $40 \%$ d'ici à 2020, par rapport aux niveaux de 1990.

${ }^{1392}$ DECISION COUR SUPRÊME DES PAYS-BAS, CHAMBRE CIVILE Numéro 19/00135 date 20 décembre 2019 dans l'affaire de L'ÉTAT DES PAYS-BAS (MINISTĖRE DES AFFAIRES ÉCONOMIQUES ET DU CLIMAT), ayant son siège social à La Haye, demandeur en cassation (Pays-Bas), ci-après dénommé : l'État, avocats : K. Teuben, M.W. Scheltema et J.W.H. van Wijk. Disponible sur le site internet: https://equalacademy.eu/uploads/resources_files/URGENDA_ARRET_COUR_SUPREME_PB. Consulté le 18/01/2022.

${ }^{1393}$ Le jugement avait été confirmé par la Cour d'appel de La Haye pour le motif que l'Etat agissait « illégalement » et faisait « trop peu pour prévenir un changement climatique dangereux ».

${ }^{1394}$ International Law association, résolution 2/2014 : Déclaration de principes juridiques relatifs au changement climatique, voir projet d'article 3 intitulé : développement durable en son paragraphe 5.

${ }^{1395}$ DECISION COUR SUPRÊME DES PAYS-BAS, CHAMBRE CIVILE Numéro 19/00135 date 20 décembre 2019 dans l'affaire de L'ÉTAT DES PAYS-BAS (MINISTĖRE DES AFFAIRES ÉCONOMIQUES ET DU CLIMAT), ayant son siège social à La Haye, demandeur en cassation (Pays-Bas), ci-après dénommé : l'État, avocats : K. Teuben, M.W. Scheltema et J.W.H. van Wijk. Disponible sur le site internet: https://equalacademy.eu/uploads/resources_files/URGENDA_ARRET_COUR_SUPREME_PB_2. Consulté le 18/01/2022.
} 
de protection du climat et dénote du vide créé par l'inefficacité de la justice climatique internationale.

Sous un autre angle, cette jurisprudence révèle la possibilité dont disposent les juridictions nationales pour contribuer à la mise en œuvre du principe de prévention initié par le droit international de l'environnement, qui impose aux Etats Parties à la Convention Cadre des Nations Unies sur les Changements Climatiques de prendre des mesures pour atténuer l'émission des gaz à effet de serre. Il s'ensuit qu'après les prouesses réalisées au plan sous régional par la Cour Européenne des Droits de l'Homme (CEDH), certaines justices nationales deviennent désormais des éléments d'effectivité de la Convention Cadre des Nations Unies sur les Changements Climatiques.

Si les actions des justices nationales sont capable d'obliger les Etats Parties à respecter leurs engagements souscrits dans la Convention Cadre des Nations Unies sur les Changements Climatiques, que faut-il faire pour rendre effective la justice climatique au plan international?

Pour régler le problème d'effectivité et d'efficacité de la justice internationale, la création d'une juridiction internationale environnementale spécialisée s'impose et se justifie, par le fait que la lutte contre les changements climatiques comporte des spécificités qui requièrent des mesures particulières de protection. La Cour internationale de justice a déjà fait ses preuves et a montré ses limites en ce qui concerne la gestion des différends climatiques. A partir des expériences des juridictions internationales qui se sont faites remarquer dans les domaines de la protection de l'environnement ${ }^{1396}$, des propositions de création de juridiction climatique peuvent être conçues sur la base de l'expérience tirée du fonctionnement de l'Organe de Règlement des Différends (ORD) de l'Organisation Mondiale du Commerce (OMC) qui impose l'observance des règles de l'Organisation commerciale sous peine d'application de sanctions à l'égard des Etats contrevenant. Une telle institution ne sera pas privée de légitimité dans le cadre de l'Organisation des Nations Unies dont l'ultime finalité, selon l'article 1 de sa Charte est, « de maintenir la paix et la sécurité dans le monde ». Cette juridiction internationale aura compétence à régler les différends et problèmes relatifs à l'application de la Convention Cadre des Nations Unies sur les Changements Climatiques avec la différence que la possibilité sera donnée à toute personne, physique, morale, publique et privée de saisir ladite juridiction en cas de violation des règles édictées par la Convention. De la même manière, les personnes physique ou morale de statut public ou privé qui ne respectent pas les normes de pollution et d'émissions établies au niveau mondial par des accords internationaux du type Convention

\footnotetext{
${ }^{1396}$ Notamment, le Tribunal international du droit de la mer (TIDM).
} 
Cadre des Nations Unies sur les Changements Climatiques, le Protocole de Kyoto et l'Accord de Paris pourront être poursuivies et sanctionnées lorsque leur responsabilité sera judiciairement établie. Il s'agira dans le cas d'espèce de rompre avec le moule traditionnel du droit international public dont l'exclusivité des actions est concédée aux Etats. Il faut donner désormais l'opportunité des poursuites aux citoyens et à toute autre entité distincte de l'Etat ayant intérêt à agir à contribuer à la protection de l'environnement et à la lutte contre les changements climatiques. Dans ce cas, la nouvelle juridiction qui sera créée tiendra compte de ce que les Etats et organisations internationales bénéficient déjà des opportunités juridictionnelles en matière de poursuite des infractions écologiques devant la Cour Internationale de Justice, pour orienter sa compétence à l'endroit des personnes physiques ou morales qu'elles soient du droit public ou privé autre que l'Etat lorsqu'elles violent les dispositions de la Convention climat.

Certaines organisations non gouvernementales et Etats se mobilisent pour la réalisation de ce projet qui permet d'assouvir leur désir de faire planer à l'échelle planétaire, la crainte de réprimande pour inciter à l'adoption des comportements écologiques qui favorisent la réduction de la pollution. La nouvelle juridiction aura une compétence essentiellement environnementale voire climatique et se chargera de juger, les individus, les autorités Etatiques et les multinationales auteurs de crime contre l'environnement par la violation de la Convention Cadre des Nations Unies sur les Changements Climatiques et l'ensemble des Conventions qui concourent à la réduction des émissions du gaz à effet de serre. Elle va coexister avec la Cour Internationale de Justice (CIJ) qui déjà, est compétente pour juger les Etats Parties.

Y a-t-il d'obstacle à la création d'une juridiction spécialisée à compétence climatoenvironnementale?

La seule inquiétude face à la réalisation du projet de création d'une nouvelle juridiction internationale à compétence climato-environnementale consisterait à l'opposition de certains Etats notamment, les Etats-Unis d'Amérique qui sont hostiles à tout projet de justice internationale sans oublier leur réticence quant à l'application de la Convention Cadre des Nations Unies sur les Changements Climatiques.

La décision de création d'une juridiction internationale climato-environnementale ne peut être prise que par la Conférence des Parties à la Convention Cadre des Nations Unies sur les Changements Climatiques et entérinée par résolution de l'Assemblée générale des Nations unies conformément aux dispositions de la Charte des Nations. 
Si le projet de création d'une juridiction internationale climato-environnementale venait à être concrétisé, son existence permettra de semer la crainte de la sanction dans la conscience des autorités étatiques qui désormais, ne peuvent plus s'aventurer à engager virtuellement les Etats dans des conventions internationales sans se préoccuper de leur mise en œuvre au risque de subir des sanctions personnelles. Par ailleurs, les sanctions qui seront prononcées contre les violateurs des conventions internationales, serviront d'exemple pour d'autres. Par conséquent, la plupart d'Etats engagés dans la Convention Cadre des Nations Unies sur les Changements Climatiques seront désormais respectueux des engagements convenus compte de la lucidité dont fera preuve les dirigeants qui auront pour responsabilité d'engager leurs Etats dans les accords internationaux.

Passé cette étape, il convient également de structurer la coopération internationale en faveur de l'action climatique.

\section{SECTION II: LA STRUCTURATION DE LA COOPERATION INTERNATIONALE}

Oeuvrer pour la réduction consistante des émissions de gaz à effet de serre, requiert une coopération internationale pour faciliter les transferts de technologies bas-carbone, de compétences, de financements et tous autres moyens qui peuvent encourager l'action climatique et la réduction de son coût. C'est grâce à cette coopération agissante que les négociations sur le climat ont abouti à l'élaboration du précieux instrument universel qu'est la Convention Cadre des Nations Unies sur les Changements Climatiques et les accords subséquents.

Néanmoins, cet effort de coopération qui a prévalu au niveau planétaire doit être renforcé à toutes les échelles afin de servir de moyen de promotion de solutions transversales et innovantes pour la réduction des émissions des gaz à effet de serre.

Dans le cas d'espèce, il s'agira de se servir de la coopération internationale pour décentraliser l'instrument juridique international au profit des Etats Parties à la Convention Cadre des Nations Unies sur les Changements Climatiques par incorporation de l'outil juridique aux divers instruments de coopération. Ce qui pourra faciliter le rapprochement du droit climatique au plan régional (Paragraphe 1) et puis, la communautarisation du droit climatique à ce niveau (Paragraphe 2). 


\section{Paragraphe 1: Le rapprochement du droit climatique au plan régional}

Après l'adoption de la Convention Cadre des Nations Unies sur les Changements Climatiques(CCNUCC) au Sommet de la Terre de Rio de Janeiro en 1992 et son entrée en vigueur le 21 mars 1994, le traité climatique compte cent quatre-vingt dix-sept (197) Parties ${ }^{1397}$. Quant au Protocole de Kyoto adopté le 11 décembre 1997, entré en vigueur le 16 février 2005 a connu cent quatre vingt-douze (192) Etats signataires ${ }^{1398}$. De même, l'Accord de Paris qui a été approuvé par l'ensemble des cent quatre-vingt-quinze (195) délégations le 12 décembre 2015 et entré en vigueur le 04 novembre 2016, connait cent quatre vingt-douze (192) Parties ${ }^{1399}$. Ces données statistiques témoignent du caractère universel de la Convention climat qui a été adopté par la plupart des Etats de la planète. Ce qui présageait déjà d'un succès sans équivoque de la lutte contre les changements climatiques par la réalisation de l'objectif de la Convention Cadre des Nations Unies sur les Changements Climatiques ${ }^{1400}$. Malheureusement, ce ne fut pas le cas.

Malgré la légitimité dont bénéficie l'Accord climat, chaque Etat jouissant des prérogatives que lui confère la souveraineté internationale, après s'être engagé individuellement dans sa ratification, est appelé à mettre en œuvre les dispositions dudit Accord sur son territoire. La gestion isolée et individuelle de la lutte contre les changements climatiques ne permet donc pas d'obtenir les résultats attendus. Par contre, la gestion efficace des changements climatiques requiert des mesures communes applicables au-delà des frontières géographiques de chaque Etat Partie. La Communauté internationale à travers l'instrument climatique a d'ailleurs créé les conditions normatives favorisant l'éclosion des communautés régionales dans la mise en œuvre du droit climatique en réaffirmant que, « le principe de la souveraineté des Etats doit présider à la coopération internationale destinée à faire face aux changements climatiques $»^{1401}$.

Le cadre d'organisation régional répond aux exigences de solidarité restreinte entre un groupe limité d'Etats qui conviennent de leurs intérêts collectifs. D'ailleurs, l'attachement des Etats à la souveraineté internationale n'a pas été un handicap pour les initiatives communautaires qui ce sont multipliées à foison partout dans le monde entier, le phénomène s'est développé particulièrement sur le Continent africain. Ces organisations pour la plupart s'impliquent de plus en plus dans la vie publique et surtout dans le secteur de l'environnement

\footnotetext{
${ }^{1397}$ Etat de ratification des traités. Disponible sur le site internet: https://treaties.un.org/pages. Consulté le $31 / 05 / 2021$.

1398 Id.

1399 Ibid.

${ }^{1400}$ Il s'agit de l'objectif fixé par la Communauté internationale à l'article 2 de ladite Convention.

${ }^{1401}$ Paragraphe 10 du préambule de la Convention Cadre des Nations Unies sur les Changements Climatiques.
} 
et se consacrent à la lutte contre les changements climatiques. Mais en plus, le cadre communautaire apparaît comme un niveau convenable à la production normative et de facilitation de la mise en œuvre efficace du droit.

Dès lors apparait la préoccupation suivante : Comment arriver à formuler une réponse régionale orientée vers les objectifs de lutte contre les changements climatiques?

Un colloque organisé sur le « renforcement de l'effectivité du droit de l'environnement en Afrique a recommandé d'«Adapter et d'endogénéiser le droit de l'environnement en Afrique $»^{1402}$. Pour atteindre cet objectif, il faut mettre à contribution les canaux régionaux ou communautaires pour rapprocher le droit international de protection du climat vers les Etats signataires de la Convention Cadre des Nations Unies sur les Changements Climatiques. Dans cette perspective, le Bénin relevant de l'Union Africaine (UA) et de la Communauté Economique des Etats de l'Afrique de l'Ouest (CEDEAO), doit pouvoir en principe bénéficier des solutions communes et transfrontalières à ce défi global à la seule condition que ces organisations internationales soient investies d'un rôle plus accru dans la mise en œuvre des instruments internationaux de lutte contre les changements climatiques. Pour y parvenir, il faudra œuvrer pour l'ancrage de l'Union Africaine (UA) dans le droit climatique (A). Cette approche de gestion et d'encadrement décentralisée des changements climatiques est aussi valable pour la Communauté Economique des Etats de l'Afrique de l'Ouest (CEDEAO) qui devra réceptionner dans son droit positif, le droit climatique (B).

\section{A- L'ancrage de l'Union africaine dans le droit climatique}

Les changements climatiques sont aperçus sur le continent africain comme l'un des défis les plus importants à relever sans quoi, le développement auquel aspirent les Etats de cette région ne peut être réalité. Paradoxalement, ce continent a une infirme responsabilité dans la dégradation de l'environnement, mais en subi les effets néfastes. La pertinence de l'infirme contribution et des préjudices subies sont relayés dans le document de stratégie africaine de lutte contre les changements climatiques qui a rapporté la preuve de ce que, le contient africain, «porte le plus grand fardeau et subisse les pires effets dévastateurs provoqués par des externalités du monde moderne ${ }^{1403}$. Cependant, certains Etats africains et pour la plupart, endurent exagérément et différemment les effets négatifs du changement climatique.

\footnotetext{
${ }^{1402}$ Septième $\left(7^{\mathrm{e}}\right)$ recommandation, Revue Africaine de Droit de l'Environnement $\mathrm{n}^{\circ}$ 01-2014, p.17.

${ }^{1403}$ Septième $\left(7^{\mathrm{e}}\right)$ recommandation, Revue Africaine de Droit de 1'Environnement $\mathrm{n}^{\circ}$ 01-2014, p.17.
} 


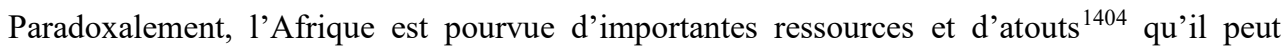
mettre à contribution dans le cadre de la recherche de solutions pour conjurer les changements climatiques $^{1405}$.

L'Union Africaine (UA), une organisation continentale chargée de "promouvoir l'unité, la solidarité, la cohésion et la coopération entre les peuples d'Afrique et entre les Etats africains ${ }^{1406}$, n'a pas occulté les questions des changements climatiques dans ses préoccupations. L'organisation régionale a entrepris des actions tendant à assurer la représentation du contient dans les instances internationales de lutte contre les changements climatiques. Dans ce cadre, plusieurs institutions ont été créées notamment, le Groupe Africain de Négociateurs (AGN) qui a vu le jour en 1992 et investi de la mission « de promouvoir les intérêts de l'Afrique dans les négociations sur les changements climatiques ». L'Afrique à travers l'Union africaine a marqué sa présence au Sommet de Rio de Janeiro sur l'environnement et le développement en 1992 et a pris part à l'organisation de certaines activités des Conférences des Parties à la Convention Cadre des Nations Unies sur les Changements Climatiques. Elle était également présente à la COP21 tenue à Paris en 2015.

Par ailleurs, l'Union africaine a développé une vision commune et partagée de la gestion des défis qu'imposent les changements climatiques auxquels le continent fait face. Cette vision consiste à «mettre en œuvre des programmes et projets liés aux changements climatiques et d'atteindre des objectifs de développement, y compris les Objectifs du millénaire pour le développement, en particulier pour lutter contre la pauvreté en mettant l'accent sur la réalisation de la sécurité alimentaire, en particulier pour les groupes les plus vulnérables ${ }^{1407}$. Pour harmoniser les points de vue de l'Union africaine autour de cette vision, l'organisation régionale s'appuie sur le Comité des Chefs d'Etat et de Gouvernement sur les Changements Climatiques (CAHOSCC $)^{1408}$ qu'elle a créé en 2009 et investi de la mission d" " Accorder la plus grande attention politique aux enjeux du changement climatique sur le continent pour lui permettre d'avoir une position commune et parler d'une seule voix sur les questions du changement

\footnotetext{
${ }^{1404}$ Notamment, la forêt, l'eau, la jeunesse de la population, le taux de croissance en constante progression.

${ }^{1405}$ Répondre au changement climatique en Afrique - Gouvernance en Afrique base.afriquegouvernance.net/docs/cp_climat_final_verselectronique.pdf.

${ }^{1406}$ Premier paragraphe du préambule de l'Acte Constitutif de l'Union Africaine (UA).

${ }^{1407}$ Stratégie Africaine sur les Changements Climatique, p.28. Disponible sur le site internet: https://www.uneca.org. Consulté le 19/01/2022.

${ }^{1408}$ Réponse au changement climatique en Afrique, une approche par la gouvernance des territoires. Disponible sur le site internet: http://base.afrique-gouvernance.net/docs/cp_climat_final_verselectronique.pdf.Consulté le $18 / 01 / 2022$.
} 
climatique» ${ }^{1409}$. A l'appuie du développement institutionnel auquel a donné lieu l'engagement de l'organisation régionale dans la lutte contre les changements climatiques, les Chefs d'Etats et de Gouvernements de l'Union africaine ont fait adopter, en plus du document de stratégie africaine de lutte contre les changements climatiques, la décision sur la «position africaine commune sur le changement climatique ${ }^{1410}$.

A l'origine, l'Union africaine s'est donnée la compétence environnementale à travers son acte de création par les stipulations suivantes : «le Conseil exécutif assure la coordination et décide des politiques dans les domaines (...) de protection de l'environnement (...) "1411. Mais aussi, l'organisation régionale s'est invitée dans la gestion des catastrophes qui constitue un volet non moins important relatif aux effets néfastes des changements climatiques par sa contribution à l' " action humanitaire et réaction et secours en cas de catastrophe ${ }^{1412}$. Puis l'acte constitutif de l'Union africaine intervenu après avoir créé un Comité en charge de l'environnement ${ }^{1413}$. Malgré les efforts déployés par l'Union africaine en ce qui concerne l'encadrement institutionnel des changements climatiques, la tendance du rythme d'aggravation du fléau sur le contient n'est pas inversée.

Comment faire pour outiller davantage l'Union africaine pour lui permettre de mieux assouvir son engagement à combattre les changements climatiques?

La quasi-totalité des Etats du continent ont ratifié la Convention Cadre des Nations Unies sur les Changements Climatiques et les accords subséquents. Cependant, l'Union africaine qui est sensée rapprocher le droit climatique auprès des Etats membres de l'organisation n'est pas suffisamment outillée pour implémenter le droit climatique sur le continent africain. L'Union africaine qui a fait du « droit » un élément régulateur des politiques et stratégies, ne s'est pas juridiquement engagée dans le domaine des changements climatiques contrairement à «l'Union européenne qui s'est dotée progressivement d'un droit communautaire étoffé constitué de directives contraignantes d'application directe dans ses Etats

\footnotetext{
${ }^{1409}$ Stratégie Africaine sur les Changements Climatique, p.27. Disponible sur le site internet: https://www.uneca.org. Consulté le 19/01/2022.

${ }^{1410}$ La décision a été adoptée par la Quinzième $\left(15^{\mathrm{e}}\right)$ session ordinaire du Conseil exécutif de l'Union Africaine à Syrte, Grande Jamahiriya Arabe Libyenne Populaire et Socialiste le $1^{\text {er }}$ juillet 2009. Disponible sur le site internet : https://au.int/sites/default/files/decisions. Consulté le 18/01/2022.

${ }^{1411}$ Article 13 paragraphe 1-e de l'acte constitutif de l'union africaine. Disponible sur le site internet: https://au.int/sites/default/files/page. Consulté le 18/01/2022. 1412 Id.

${ }^{1413}$ Article 14 paragraphe $1-\mathrm{d}$ de l'acte constitutif de l'union africaine. Disponible sur le site internet: https://au.int/sites/default/files/page. Consulté le 18/01/2022.
} 
membres» ${ }^{1414}$. Alors que toute volonté de lutter contre les changements climatiques, malgré le caractère holistique du phénomène, devra nécessairement s'appuyer sur des normes juridiques. Cependant l'Union africaine s'est engagée dans le domaine de la lutte contre les changements climatiques sans que son acte constitutif n'ait évoqué expressément les dispositions relatives à ce fléau.

Nonobstant les initiatives prises au plan continental et régional et évoquant la nécessité de définir une position africaine commune, les Etats membres de l'Union africaine sont individuellement représentés au niveau de la Communauté internationale engagée dans la lutte contre les changements climatiques en vertu de leur engagement personnel. L'organisation continentale qui est sensée portée leurs intérêts n'est pas signataire de la Convention Cadre des Nations Unies sur les Changements Climatiques. Or, pris individuellement, les Etats africains ne sont pas en mesure de défendre convenablement leurs intérêts. Il s'ensuit que l'état actuel de la règlementation de l'Union africaine ne lui permet pas de faire émerger sa position, alors que les Etats africains représentés par l'Union africaine ont une position peu confortable dans les négociations internationales sur les changements climatiques. En réalité, la nécessité d'encadrement de la lutte contre les changements recommande qu'au-delà d'élaboration ou d'adoption d'outil du genre «document de stratégie » ${ }^{1415}$, des perspectives d'encadrement juridique pertinentes de la lutte contre les changements climatiques au plan régional soient envisagées.

Eu égard à ce qui précède, il convient que l'organisation faitière s'implique davantage dans la lutte contre les changements climatiques pour se donner la capacité juridique à assurer la représentation des Etats membres au niveau de la Conférence des Parties à la Convention Cadre des Nations Unies sur les Changements Climatiques, en adhérant à ladite Convention.

Quelles sont les possibilités qu'offre la Convention Cadre des Nations Unies sur les Changements Climatiques pour admettre en son sein une organisation régionale comme l'Union africaine?

Par la prescription selon laquelle «La Convention est soumise à la ratification, à l'acceptation, à l'approbation ou à l'adhésion des Etats et des organisations d'intégration économique régionale» ${ }^{1416}$, sont autorisées à ratifier la convention climatique, les organisations

\footnotetext{
${ }^{1414}$ KAMTO (Maurice), LA MISE EN OEUVRE DU DROIT DE L'ENVIRONNEMENT : FORCES ET FAIBLESSES DES CADRES INSTITUTIONNELS, Revue Africaine de Droit de 1'Environnement $n^{\circ} 01,2014$, p.34, p.39.

${ }^{1415} \mathrm{~A}$ l'instar du document de stratégie africaine de lutte contre les changements climatiques. Disponible sur le site internet : https://www.uneca.org. Consulté le 19/01/2022.

${ }^{1416}$ L'article 22 paragraphe 1 de la Convention Cadre des Nations Unies sur les Changements Climatiques.
} 
d'intégration. Sous d'autres cieux, c'est la mise en œuvre de cette disposition qui a permis à l'Union européenne d'adopter la Convention Cadre des Nations Unies sur les Changements Climatiques le 21 décembre $2013^{1417}$. Cet acte de haute portée accompli par l'Union européenne a eu des répercussions positives sur le continent Européen, car elle a permis de créer le droit climatique européen qui se trouve être un modèle dans le monde entier. Mais en plus, au-delà du cadre continental, le Droit climatique européen a connu une expansion au point de devenir selon un auteur, « par son caractère supranational et ses mécanismes de contrôle et de sanction, notamment juridictionnels, (...) l'instrument d'effectivité et d'efficacité du droit conventionnel international du climat ${ }^{1418}$. Cette possibilité d'accession à la Convention climat est aussi valable pour l'Union africaine, mais il y a quelques difficultés textuelles que l'organisation internationale devra régler en amont. Elle doit pourvoir prendre des mesures pour adapter son dispositif normatif de manière à recevoir des habilitations qui permettent aux dirigeants d'engager au nom et pour le compte des Etats membres, l'organisation régionale dans les accords internationaux et plus précisément ceux concernant la protection de l'environnement et la lutte contre les changements climatiques.

Lorsque ces préalables seront réglés, en tant que Partie au régime international climatique, l'Union africaine restera liée par ses engagements climatiques internationaux conformément à ses propres normes juridiques. Ce qui permettra de déboucher sur des perspectives d'africanisation du droit international climatique avec de répercussions évidentes telles que la redéfinition des attributions des institutions de l'Union africaine à savoir : la Cour de Justice de l'Union Africaine (CJUA), et la Commission Africaine des Droits de l'Homme et des Peuples $(\mathrm{CADHP})^{1419}$ pour intégrer la dimension concernant les changements climatiques à leurs statuts respectifs.

In fine, lorsque 1'Union africaine va intégrer le concert des Nations engagées dans la lutte contre les changements climatiques, sur la base de l'application du principe de « l'effet obligatoire des dispositions $\mathrm{du}$ droit international $»^{1420}$, le régime international climatique

\footnotetext{
${ }^{1417}$ Etat de ratification de la Convention disponible sur le site internet https://treaties.un.org. Le 31/05/2021.

${ }^{1418}$ Coffi Dieudonné ASSOUVI «L'INFLUENCE DU DROIT INTERNATIONAL DES CHANGEMENTS CLIMATIQUES SUR LE DROIT DE L'ENVIRONNEMENT DE L'UNION EUROPEENNE », THESE DE DOCTORAT EN DROIT PUBLIC, Université de Limoges, Faculté de Droit et des Sciences Economiques, 2018, 667 p., p.9.

1419 Article 30 de la Charte africaine des droits de l'homme et des peuples, 1981.

${ }^{1420}$ L'article 26 de la Convention de Vienne sur le droit des traités de 1969, qui stipule «PACTA SUNT SERVANDA » qui signifie que Tout traité en vigueur lie les Parties et doit être exécuté par elles de bonne foi. En vertu de ce principe, le traité climatique une fois ratifié par l'Union africaine va s'imposer non seulement à l'organisation mais produira des effets sur le droit interne de chaque Etat membre de l'Organisation. Il s'ensuit que les engagements souscrits par l'Union africaine dans la Convention Cadre des Nations Unies sur les Changements Climatiques obligent les Etats membres.
} 
produira des effets juridiques envers les Etats membres. Le droit africain va primer sur les normes internationales concernant la lutte contre les changements climatiques transposées et intégrées, aux ordres juridiques internes de chaque Etat membre. Ainsi, le droit de l'Union africaine deviendra l'instrument pouvant assurer l'effectivité du droit climatique en Afrique.

La logique selon laquelle le droit international climatique devrait être rapproché des Etats Parties pour leur assurer un meilleur encadrement dans un cadre global et élargi est aussi valable pour les organisations régionales communautaires qui sont nombreuses sur le continent africain. Le cas de la CEDEAO intéresse également notre étude, car le Bénin en est membre. Cette communauté régionale devra nécessairement adhérer à la Convention Cadre des Nations Unies sur les Changements Climatiques.

\section{B- L'adhésion de la CEDEAO à la Convention climat $^{1421}$}

La ratification massive de la Convention Cadre des Nations Unies sur les Changements Climatiques témoigne de la motivation des Etats concernés à relever collectivement les défis qu'imposent les changements climatiques. Seulement que dans la réalité, pour chaque Etat Partie, après avoir transposé le droit international climatique en des réalités juridiques internes, se trouve seul à appliquer sur son territoire le droit climatique. La codification unilatérale et la mise en œuvre sur l'espace territorial de chaque Etat ne permettent pas toujours d'atteindre les résultats escomptés. Cette méthodologie est contraire au concept de gestion collective de la protection de l'environnement prônée à la Conférence de Stockholm de 1972 à travers sa Déclaration qui recommande que «les Etats (...) veillent à ce que les organisations internationales jouent un rôle coordonné, efficace et dynamique dans la préservation et l'amélioration de l'environnement $»{ }^{1422}$. Mais en plus, ces organisations internationales « $(\ldots)$ devraient prendre des mesures sur les moyens de parer aux conséquences économiques que peut avoir, au niveau national et international, l'application des mesures de protection de l'environnement ${ }^{1423}$. Pour appuyer le concept de gestion collective du domaine de protection de l'environnement, la Déclaration de Rio de Janeiro de 1992 a prohibé toute action unilatérale provenant d'un Etat pour régler un problème écologique au-delà de ses frontières et a indiqué

\footnotetext{
${ }^{1421}$ La convention climat est une manière de désigner la Convention Cadre des Nations Unies sur les Changements Climatiques et ses accords subséquents.

1422 Principe 25 de la déclaration de Stockholm de 1972.

${ }^{1423}$ Principe 11 id.
} 
que «Les mesures de lutte contre les problèmes écologiques transfrontières ou mondiaux devraient, autant que possible, être fondées sur un consensus international $»{ }^{1424}$.

C'est la vision de gestion collective du domaine de l'environnement prônée à Stockholm en 1972 qui a émergé à la Conférence de Rio de Janeiro en 1992 et a permis de mobiliser et de faire consensus sur la nécessité de lutter contre le réchauffement de la planète. Cette position a été renforcée par les différents rapports élaborés par le Groupe d'Experts Intergouvernemental sur l'Evolution du Climat (GIEC), lesquels ont indiqué qu'au delà des Etats, il y a une impérieuse nécessité de comprendre et d'agir aux échelles régionales et sous régionales. Déjà dans leur premier rapport, les Experts du GIEC en recommandant le modèle de traité climatique convenable dans le cadre de la lutte contre les changements climatiques, indiquaient que cette Convention devra contenir « (...) non seulement des obligations générales consenties par les parties, mais également (...) des dispositions pour des ententes bilatérales, multilatérales et régionales ou des accords compatibles avec la Convention et les annexes ou les protocoles, notamment la possibilité pour des groupes de pays de satisfaire aux exigences sur une base régionale ou sous-régionale ${ }^{1425}$. Ainsi, la Convention Cadre des Nations Unies sur les Changements Climatiques et les accords subséquents ont fourni les moyens pour faciliter le regroupement des Etats autour de la cause environnementale en développant un cadre juridique axé sur la coopération internationale.

Dans le contexte de la Convention Cadre des Nations Unies sur les Changements Climatiques, l'implication des organisations régionales dans la gestion de l'environnement facilitera non seulement la mise en œuvre des dispositions applicables aux changements climatiques, mais va aider à mobiliser des ressources et moyens nécessaires pour assouvir les charges qu'induira la gestion des changements climatiques.

Qu'en est-il de l'implication juridique de la CEDEAO dans la gestion des changements climatiques en Afrique de l'ouest?

En matière d'encadrement juridique du secteur de l'environnement dans un espace plus large que le domaine territorial d'un Etat, la Communauté Economique des Etats de l'Afrique de l'Ouest (CEDEAO) a progressé comparativement à d'autres organisations sous-régionales africaines, notamment, la Communauté Economique et Monétaire des Etats de l'Afrique Centrale (CEMAC) et la Communauté Economique des Etats de l'Afrique Centrale

\footnotetext{
${ }^{1424}$ Principe 12 de la Déclaration de Rio de Janeiro de 1992.

${ }^{1425}$ Changements climatiques: Premier rapport des évaluations du GIEC de la période 1990-1992, p.160. Disponible sur le site internet : https://www.ipcc.ch. Consulté le 18/01/2022.
} 
$(\mathrm{CEEAC})^{1426}$. Ainsi, la CEDEAO a déjà au moment de sa création pris des mesures normatives visant la protection de l'environnement ${ }^{1427}$ par la création d'institutions d'encadrement ${ }^{1428}$. Selon le traité révisé de la CEDEAO, «Les Etats membres s'engagent à protéger, préserver et améliorer l'environnement naturel de la Région et coopérer en cas de désastre naturel ${ }^{1429}$. Seulement que chemin faisant, l'organisation régionale qui ambitionnait réaliser la prospérité de la communauté dans son espace géographique est assujettie aux effets des changements climatiques qui risquent d'annihiler ses efforts. Car l'enjeu des changements climatiques n'est pas seulement écologique, mais également économique et social et peuvent produire des effets néfastes importants sur le développement durable de tous les pays. Sur ce point, le quatrième $\left(4^{e}\right)$ rapport d'évaluation du Groupe d'Experts Intergouvernemental sur l'Evolution du Climat (GIEC) publié en 2007 a précisé qu' «Il est très probable que les changements climatiques risquent de ralentir les progrès accomplis sur la voie du développement durable, soit directement par une exposition accrue à leurs effets néfastes, soit indirectement par une altération de la capacité d'adaptation ${ }^{1430}$.

C'est pourquoi, en dehors du cadre formel international de lutte contre les changements climatiques, les pays de la sous région se sont engagés dans des actions concrètes de lutte contre les effets adverses des changements climatiques. La preuve est que, la plupart des Etats membres de la CEDEAO ont déjà élaboré les Programmes d'Action Nationaux d'Adaptation (PANA $)^{1431}$. De même, la CEDEAO a encouragé une concertation intergouvernementale en vue de la gestion des risques de catastrophes qui peuvent être liées aux changements climatiques.

Malgré les efforts accomplis par la CEDEAO dans le domaine de la protection de l'environnement, certaines difficultés qui découlent de la non intégration des changements climatiques dans les statuts de l'organisation régionale se profilent. Bien entendu que

\footnotetext{
${ }^{1426}$ KAMTO (Maurice), LA MISE EN OEUVRE DU DROIT DE L'ENVIRONNEMENT : FORCES ET

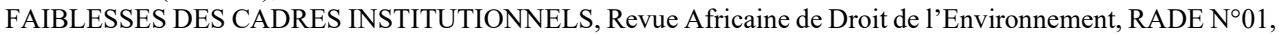
2014, p.34, p.39.

${ }^{1427}$ Article 3 paragraphe 1 du traité révisé de la CEDEAO. Disponible sur le site internet: https://www.ecowas.int/droit-de-la-cedeao/traites. Consulté le 18/01/2022.

${ }^{1428}$ Depuis 2008, il a été créé au sein de la CEDEAO une Direction de l'Environnement et des règlements communautaires ont été adoptés en matière de pesticides, de semences, de sécurité des aliments etc. Voir KAMTO (Maurice), LA MISE EN OEUVRE DU DROIT DE L'ENVIRONNEMENT : FORCES ET FAIBLESSES DES CADRES INSTITUTIONNELS, Revue Africaine de Droit de 1'Environnement n ${ }^{\circ} 01,2014$, p.34, p.39.

${ }^{1429}$ Article 29 du traité révisé de la CEDEAO. Disponible sur le site internet : https://www.ecowas.int/droit-de-lacedeao/traites. Consulté le 18/01/2022.

${ }^{1430}$ Page 28 dudit rapport. Disponible sur le site internet : https://www.ipcc.ch/site/asset. Consulté le 18/01/2022.

${ }^{1431}$ Comité permanent Inter-Etats de Lutte Contre la Sécheresse dans le Sahel Centre Régional AGRHYMET Bulletin Mensuel Le Sahel face aux changements climatiques Enjeux pour un développement durable. Disponible sur le site internet : http://www.agrhymet.ne/PDF/BM2010/specialChC.pdf. Consulté le 18/01/2022.
} 
l'organisation régionale s'est donnée la compétence environnementale par extension de ses attributions classiques, jusqu'à présent, son intervention dans le domaine de la lutte contre les changements climatiques n'est pas encore précisée dans les statuts de l'organisation régionale. Alors que tous les Etats membres de la Communauté ont ratifié la Convention Cadre des Nations Unies sur les Changements Climatiques ${ }^{1432}$.

Comment rendre efficace les actions de lutte contre les changements climatiques dans le cadre de la CEDEAO?

La lutte contre les changements climatiques ne pouvant se mener dans un espace restreint, le GIEC a indiqué dans son rapport spécial que, « Le renforcement des institutions et l'amélioration de la coordination et de la coopération en matière de gouvernance peuvent aider à surmonter les obstacles qui s'opposent, à l'échelle régionale, aux efforts d'atténuation, d'adaptation et de réduction des risques de catastrophe $»{ }^{1433}$. La Communauté internationale, tout en étant consciente « que le caractère planétaire des changements climatiques requiert de tous les Pays qu'ils coopèrent le plus possible (...) », ${ }^{1434}$ a laissé des ouvertures pour faciliter l'adhésion à la Convention Cadre des Nations Unies sur les Changements Climatiques des organisations régionales à vocation économique comme le stipule la disposition suivante : «la Convention est soumise à la ratification, l'acceptation, l'approbation ou l'adhésion des Etats et des organisations d'intégration économique régionale $\gg{ }^{1435}$. La réception du droit climatique dans le cadre régional parait une réponse idoine à cette préoccupation. Dans ce cas, la CEDEAO doit intégrer la Convention Cadre des Nations Unies sur les Changements Climatiques. Cette ouverture peut permettre de rapprocher le droit climatique vers les Etats membres. Pour y procéder, il suffira que les Chefs d'Etats et les Gouvernements des Etats membres de ladite institution décident à l'occasion d'un Sommet, de l'intégration de la dimension concernant les changements climatiques aux normes communautaires, puis d'autoriser l'organisation à adopter au nom de la Communauté des engagements internationaux. Ce qui permettra à l'organisation régionale de marquer son adhésion à la Convention Cadre des Nations Unies sur les Changements Climatiques par sa signature.

En procédant ainsi, le traité réformé de la CEDEAO servira désormais de cadre juridique régional de lutte contre les changements climatiques. Par conséquent, l'institution

\footnotetext{
${ }^{1432}$ Voir état des ratifications de la Convention disponible sur le site internet : https://unfccc.int/fr/process-andmeetings/the-convention/status-of-ratification/etat-des-ratifications-de-la-convention. Consulté le 18/01/2022.

${ }^{1433}$ Page 105 du rapport spécial GIEC 2014. Disponible sur le site internet : https://www.ipcc.ch/reports. Consulté ce $30 / 05 / 2021$.

${ }^{1434}$ Septième $\left(7^{\mathrm{e}}\right)$ paragraphe du préambule de la Convention Cadre des Nations Unies sur les Changements Climatiques.

${ }^{1435}$ Article 22 paragraphe 1 de la Convention Cadre des Nations Unies sur les Changements Climatiques.
} 
juridictionnelle notamment, la Cour de justice de la CEDEAO qui est un excellent cadre de règlement des différends environnementaux, par l'effet de l'extension de ses attributions des droits de l'homme à l'environnement verra sa compétence complétée par la dimension concernant les changements climatiques. La CEDEAO sera intégrée comme organisation internationale, Partie à la Convention Cadre sur les Changements Climatiques et participera aux Conférences des Parties en tant que membre à part entière. Elle pourra porter la voix des Etats membres et peser de son poids dans les prises de décision lors des négociations sur l'accord climat. Le cadre régional servira d'instrument d'effectivité du droit climatique dans cet espace géographique.

L'une des conséquences qui découleront de l'adhésion de l'organisation régionale à la convention climat est que cette organisation et les Etats Membres peuvent « (...) se mettre d'accord pour agir conjointement(...), au moment de communiquer leurs contributions déterminées au niveau national $(\ldots) »^{1436}$.

Les réformes proposées par notre étude en vue de l'intégration des dispositions applicables aux changements climatiques au cadre normatif et institutionnel de la Communauté Economique des Etats de l'Afrique de l'Ouest (CEDEAO), pourront faciliter la communautarisation du droit climatique dans cet espace géographique.

\section{Paragraphe 2: La communautarisation du droit climatique}

Il est recommandé pour un fléau réputé planétaire, complexe et nécessitant l'apport d'importantes ressources de communautariser les moyens de protection pour espérer obtenir un résultat satisfaisant. Dans cette optique, lorsque la Communauté Economique des Etats de l'Afrique de l'Ouest (CEDEAO) va intégrer le concert des Nations en lutte contre les changements climatiques par l'adoption de la Convention climat, il conviendrait qu'elle ne s'arrête en si bon chemin, mais de continuer l'œuvre de construction du droit climatique en travaillant pour la mise en œuvre de la politique d'harmonisation des législations nationales en matière de lutte contre les changements climatiques au sein de ladite communauté (A). Mais en plus, l'organisation communautaire devra veiller à la bonne gouvernance climatique dans cet espace régional (B).

\section{A- L'harmonisation des législations nationales}

${ }^{1436}$ Paragraphe 16 de l'article 4 de l'Accord de Paris. 
Après leur adhésion à la Convention Cadre des Nations Unies sur les Changements Climatiques, chaque Etat Membre de la Communauté Economique des Etats de l'Afrique de l'Ouest (CEDEAO) élabore une législation interne d'application qu'il met en œuvre sur son territoire. Ces Etats en élaborant leur règlementation poursuivent comme objectif, le respect de l'engagement souscrit dans la Convention Cadre des Nations Unies sur les Changements Climatiques. Somme toute, les différentes législations qui sont intervenues pour internaliser le droit climatique sont toutes orientées vers les objectifs de réduction d'émission de gaz à effet de serre. Il résulte de cette méthodologie, que l'élaboration et l'application des mesures législatives et réglementaires peuvent correspondre aux réalités endogènes de ces Etats. Cependant, ces mesures vues d'ensemble à l'échelle régionale sont parfois disparates, divergentes et contradictoires ou controversées. Alors que les actions entreprises par chacun de ces Etats se situant sous un même espace régional devraient concourir à la réalisation de l'objectif de la Convention Cadre des Nations Unies sur les Changements Climatiques. Malheureusement, la mise en œuvre sur le territoire de chaque Etat de ces diverses mesures engendrera des difficultés de coordination de l'action climatique dans la sous-région ouest africaine. L'application du droit climatique dans ces conditions ne permet pas d'atteindre les objectifs de la Convention Cadre des Nations Unies sur les Changements Climatiques. Contrairement aux attentes de réduction considérable des émissions de gaz à effet de serre et d'atténuation des changements climatiques, le phénomène progresse avec pour conséquence la dégradation continue de l'environnement malgré les multiples efforts fournis par les Etats de la Communauté.

L'analyse de la situation révèle que la gestion solitaire et unilatérale des changements climatiques ne cadre pas avec la vision de ce que les changements climatiques sont un phénomène planétaire et global. Ainsi, la solution pour les éradiquer devrait passer par l'élaboration des mesures communes, collectives et globales et leur mise en œuvre devant se dérouler dans un cadre global. A titre illustratif, « en ce qui concerne la responsabilité et l'indemnisation des victimes de la pollution et d'autres dommages écologiques que les activités menées dans les limites de la juridiction de ces Etats ou sous leur contrôle causent à des régions situées au-delà des limites de leur juridiction (...)» ${ }^{1437}$ la Déclaration de Stockholm de 1972 a prescrit que « les Etats doivent coopérer pour développer le droit international (...) » ${ }^{1438}$. Cette préoccupation a été prise en compte dans le cadre de la Déclaration de Rio de Janeiro de 1992

${ }^{1437}$ Principe 22 de la déclaration de Stockholm de 1972.
${ }^{1438}$ Id. 
qui oblige les Parties à « (...) coopérer diligemment et plus résolument pour développer davantage le droit international concernant la responsabilité et l'indemnisation en cas d'effets néfastes de dommages causés à l'environnement dans des zones situées au-delà des limites de leur juridiction par des activités menées dans les limites de leur juridiction ou sous leur contrôle $\gg{ }^{1439}$. En procédant ainsi, le droit international a posé le jalon des règles devant régir les relations entre les Etats voisins en matière de protection de l'environnement. Partant de ces règles, un Etat de part les activités qu'il exerce sur le territoire relevant de sa juridiction ne doit pas constituer une entrave à la mise en œuvre des règles de protection de l'environnement de l'Etat voisin. Autrement dit, le droit international de l'environnement reconnait pour chaque Etat souverain, le droit d'utiliser ses propres ressources ${ }^{1440}$, mais détermine des limites pour que des cas de dommage environnemental ne soient pas causés au territoire d'un Etat voisin ou limitrophe. Il s'ensuit que, l'application des mesures disparates dans le même espace géographique provoquerait parfois des conflits internationaux du fait de la violation des règles de protection de l'environnement de l'Etat voisin.

Eu égard à ce qui précède, comment coordonner l'application des règles de protection du climat dans l'espace géographique de la CEDEAO sans perturber les limitrophes?

La dimension internationale des solutions pour régler les problèmes environnementaux n'est plus à démontrer comme l'affirme Michel PRIEUR, ce dernier affirme qu' «Il y a peu de place en droit de l'environnement pour des solutions purement nationales ${ }^{1441}$. Pour ce qui concerne la Communauté Economique des Etats de l'Afrique de l'Ouest (CEDEAO), un ambitieux cadre de référence d'actions pour une lutte concertée dans la sous région contre la destruction de l'environnement, à l'opposé de certaines organisations internationales qui ne relèvent pas du domaine de la protection de l'environnement, a d'ores et déjà au moment de sa création pris des mesures normatives visant à réaliser les objectifs pertinentes de l'organisation, par l'orientation de ses actions dans le sens de l'harmonisation des politiques en vue de la protection de l'environnement ${ }^{1442}$. A titre illustratif, l'article 3 point 2-b du traité révisé de la CEDEAO inscrit dans ses objectifs «l'harmonisation et la coordination des politiques en vue de la protection de l'environnement». Et puis, l'acte additionnel A/SA.4/12/08 portant adoption de la politique environnementale de la CEDEAO adopté le, 19 décembre 2008 a été consacré à la question de l'harmonisation des politiques environnementales. Certaines dispositions de

\footnotetext{
${ }^{1439}$ Principe 22 de la déclaration de Stockholm de 1972.

${ }^{1440}$ Principe 2 de la Déclaration de Rio de Janeiro de 1992 sur l'Environnement et le Développement.

1441 PRIEUR (M) « Pourquoi revue juridique de l'environnement ?», RJE n¹. 1976, p.3.

${ }^{1442}$ Article 3 paragraphe 1 du traité révisé de la CEDEAO.
} 
l'acte additionnel obligent les «Etats membres qui s'engagent à mettre en cohérence leurs politiques nationales de l'environnement avec la politique environnementale de la CEDEAO afin d'assurer la réalisation de ses objectifs ${ }^{1443}$. Mais il a été également prescrit à la charge de la CEDEAO qu'elle veille à l'harmonisation des textes réglementaires relatifs à la gestion de l'environnement ${ }^{1444}$. Selon Sandrine Maljean Dubois et Mathieu Wemaëre, « Les politiques de lutte contre les changements climatiques doivent en effet être régulées voire harmonisées à l'échelle internationale». ${ }^{145}$ Le colloque international sur la mise en œuvre du droit de l'environnement en Afrique organisé à Abidjan, du 29 au 31 octobre 2013 autour du thème, « Mécanismes institutionnels et financiers de mise en œuvre du Droit de l'Environnement dans la perspective du développement durable » a aussi abordé la question de la protection de l'environnement dans les espaces géographiques régionaux en Afrique et a recommandé $\mathrm{d}$ '"Harmoniser les politiques et les législations nationales en matière de protection de l'environnement en Afrique ${ }^{1446}$.

Nonobstant la convergence de point de vue autour de la question de l'harmonisation et de la coordination des politiques de protection de l'environnement dans l'espace régional, aucun bilan d'effectivité de ces mesures n'existe. Mais en plus, contrairement à la pratique qui a cours au sein de la CEDEAO, aucun organe n'a été créé pour veiller à la mise en œuvre de la politique d'harmonisation voulue.

Il est a remarqué que la CEDEAO a l'avantage que tous les Etats Membres ont souscrit à la Convention Cadre des Nations Unies sur les Changements Climatiques seulement que, le Législateur communautaire n'a pas encore mis l'accent sur le domaine des changements climatiques. A titre illustratif, les dispositions concernant les changements climatiques ne figurent pas dans l'acte fondateur de la communauté ${ }^{1447}$. Il n'existe non plus un document de politique de planification consacré à la lutte contre les changements climatiques à l'instar de l'Acte additionnel A/SA.4/12/08 portant adoption de la politique environnementale de la

\footnotetext{
${ }^{1443}$ Article 10 de l'acte additionnel A/SA.4/12/08 portant adoption de la politique environnementale de la CEDEAO adopté le 19 décembre 2008 et publié dans le Journal Officiel de la CEDEA en décembre 2008. Disponible sur le site internet : http://ecowasmedia.akomantoso.com/akn. Consulté le 18/01/2022.

${ }^{1444}$ Article 12 de l'acte additionnel A/SA.4/12/08 portant adoption de la politique environnementale de la CEDEAO adopté le 19 décembre 2008 et publié dans le Journal Officiel de la CEDEA en décembre 2008. Disponible sur le site internet : http://ecowasmedia.akomantoso.com. Consulté le 18/01/2022.

${ }^{1445}$ Sandrine Maljean Dubois et Matthieu Wemaëre, COP21 : La diplomatie climatique de Rio 1992 à Paris 2015 A. Pedone, France, 2015, 332p., , p.19.

${ }^{1446}$ Voir Revue Africaine de Droit de l'Environnement $n^{\circ} 01-2014$, neuvième recommandation sur l'effectivité du droit de l'environnement en Afrique à la page 18 .

${ }^{1447}$ Traité révisé de la CEDEAO. Disponible sur le site internet : https://www.ecowas.int/droit-de-la-cedeao/traite.. Consulté le 18/01/2022.
} 
$\mathrm{CEDEAO}^{1448}$. Il s'ensuit que cette situation ne permet pas à la Communauté régionale d'avoir un droit de regard sur les législations des Etats Membres dans le domaine de la lutte contre les changements climatiques.

Eu égard à ce qui précède, quelle est la méthodologie à adopter pour asseoir et développer la politique d'harmonisation des règles de protection du climat au sein de la Communauté Economique des Etats de l'Afrique de l'Ouest (CEDEAO)?

Pour parvenir à mettre en cohérence les politiques des Etats Membres de la CEDEAO en matière de lutte contre les changements climatiques, l'organisation faitière devrait disposer d'un cadre normatif de référence sur lequel les Etats Membres vont arrimer leur politique d'encadrement du réchauffement climatique. Mais force est de constater que, la Communauté régionale ne dispose pas de base juridique pour la mise en œuvre de la politique d'harmonisation des législations nationales en matière de lutte contre les changements climatiques. Pour atteindre ces objectifs, il convient donc que le Législateur communautaire procède à l'adoption de la politique de lutte contre les changements climatiques par l'élaboration d'un protocole additionnel pour être en phase avec sa méthodologie de mise en œuvre des traités et Conventions internationaux.

Ainsi, en se dotant d'un cadre normatif général de la politique d'harmonisation des législations nationales en matière de lutte contre les changements climatiques, la CEDEAO se dote d'un instrument d'élaboration et de contrôle de mise en œuvre des législations nationales en la matière. Ce cadre normatif servira de base référentielle pour les Etats qui se verront obliger de suivre les lignes directives tracées par le Législateur communautaire en matière de lutte contre les changements climatiques.

Cependant, faire peser toute la question de règlementation climatique sur l'harmonisation du droit climatique dans le cadre régional sans un minimum de précaution serait illusoire. Il faudra désormais penser à la promotion de la bonne gouvernance climatique dans le cadre de la coopération régionale.

\section{B- La promotion de la bonne gouvernance climatique}

Lorsque la Communauté Economique des Etats de l'Afrique de l'Ouest (CEDEAO) va signer la Convention Cadre des Nations Unies sur les Changements Climatiques, cette adhésion vaudra pour le compte de la Communauté, son engagement pour l'atténuation des émissions de

\footnotetext{
${ }^{1448}$ Cet instrument avait été adopté le 19 décembre 2008 et publié dans le Journal Officiel de la CEDEA en décembre 2008. Disponible sur le site internet : http://ecowasmedia.akomantoso.com/akn. Consulté le 18/01/2022.
} 
gaz à effet de serre et l'adaptation aux effets du réchauffement climatique. C'est l'idée que véhicule l'Accord de Paris à travers l'une de ses dispositions que, «Toute organisation régionale d'intégration économique qui devient Partie (...) est liée par toutes les obligations découlant de l'Accord (...)» ${ }^{1449}$, par conséquent, l'organisation régionale «(...) établit, communique et actualise les contributions déterminées au niveau national successives qu'elle prévoit de réaliser(...) ${ }^{1450}$. Par ailleurs, la Convention Cadre des Nations Unies sur les Changements Climatiques stipule que, «(..) les organisations régionales d'intégration économique et leurs Etats membres qui se sont mis d'accord pour agir conjointement (...), notifient au secrétariat (...) le niveau d'émission attribué à chaque Partie pendant la période considérée au moment de communiquer leurs contributions déterminées au niveau national $(\ldots) »{ }^{1451}$. En tout état de cause, « (...) chaque Etat membre de cette organisation régionale d'intégration économique à titre individuel et conjointement avec l'organisation régionale d'intégration économique, est responsable de son niveau d'émission indiqué dans l'accord communiqué $(\ldots) »^{1452}$.

A partir de ce moment, l'application des dispositions de la Convention Cadre des Nations Unies sur les Changements Climatiques devient une préoccupation majeure pour la Communauté régionale. Parce que les Etats Membres seront toujours tentés de prétexter de leur « souveraineté » pour ne pas s'acquitter de leur part d'obligation communautaire dans le cadre régional. Parfois, les difficultés économiques auxquelles ils sont confrontés ne leur permettra pas d'accomplir de bonne foi leur part d'engagement conventionnel. Les raisons de convenance politique qui ont poussé certains Etats à s'engager au plan international peuvent également constituer un handicap à la mise en œuvre des accords au plan régional. Ainsi donc, la récurrente question d'effectivité des normes climatiques élaborées ou souscrites pour conjurer le réchauffement climatique va refaire surface au plan régional. Dans ce cas, le risque de ce que le droit climatique transposé dans le cadre régional s'affiche comme un gadget pour orner le cadre normatif de la communauté régionale d'intégration économique sans pour autant produire les effets escomptés est à craindre.

Dans ces conditions, que faut-il faire pour que le droit climatique soit effectif au sein de la Communauté Economique des Etats de l'Afrique de l'Ouest (CEDEAO)?

\footnotetext{
1449 Article 20 paragraphe 2 de l'Accord de Paris.

1450 Article 4 id.

${ }^{1451}$ Article 4 paragraphe 16 de l'Accord de Paris.

${ }^{1452}$ Article 4 paragraphe 18 de l'Accord de Paris.
} 
Cette question se résume à la problématique de la mise en œuvre efficace du droit applicable aux changements climatiques dans le cadre de l'Organisation régionale d'intégration économique qu'est la CEDEAO. La réponse à cette question dépend de la capacité de l'institution à faire respecter les règles qu'elle s'est donnée. A l'origine, la CEDEAO avait été créée pour des objectifs essentiellement économiques. A la tête de l'institution régionale se trouve la Conférence des Chefs d'Etat et de Gouvernement des Etats Membres qui a pour mission, d'assurer la direction et le contrôle général de la Communauté ${ }^{1453}$ et peut «prendre toutes mesures nécessaires en vue du développement progressif de celle-ci et de la réalisation de ses objectifs ${ }^{1454}$. A ce titre, elle est chargée de « déterminer la politique générale et les principales orientations de la Communauté, donner des directives, harmoniser et coordonner les politiques économiques, scientifiques, techniques, culturelles et sociales des Etats Membres » ${ }^{1455}$ et puis « saisir en cas de besoin la Cour de justice de la communauté lorsqu'elle constate qu'un Etat Membre n'a pas honoré l'une de ses obligations ou qu'une institution de la Communauté a agi en dehors des limites de sa compétence ou a excédé les pouvoirs qui lui sont conférés, par une décision de la Conférence ou par un règlement du Conseil » ${ }^{1456}$. Les « décisions de la Conférence ont force obligatoire à l'égard des Etats Membres et des institutions de la Communauté, elles sont exécutoires de plein droit soixante (60) jours après la date de leur publication dans le Journal Officiel de la Communauté ${ }^{1457}$. En somme, l'institution régionale s'est dotée d'une panoplie de mesures fortes pour assurer l'effectivité du droit dans son espace.

Par ailleurs, l'institution régionale a créé par des actes conventionnels, des mécanismes qui fonctionnent sous le contrôle de la Conférence des Chefs d'Etat et de Gouvernement des Etats Membres pour encadrer des secteurs d'activités spécifiques notamment, le « Mécanisme de Prévention, de Gestion, de Règlement des Conflits, de Maintien de la Paix et de la Sécurité, » lequel a été renforcé par le Protocole A/SP1/12/01 sur la démocratie et la bonne gouvernance. ${ }^{1458}$.

L'architecture institutionnelle telle que conçue, apparait à priori comme un excellent instrument d'effectivité du droit dans le cadre régional, parce qu'elle est conçue avec des

\footnotetext{
${ }^{1453}$ Article 7 paragraphe 1 du traité révisé de le CEDEAO.

${ }^{1454}$ Article 7 paragraphe 2 id.

1455 Article 7 paragraphe 3-a du traité révisé de le CEDEAO.

${ }^{1456}$ Article 7 paragraphe 3-g id.

${ }^{1457}$ Article 9 paragraphe 4 et 6 du traité révisé de le CEDEAO..

${ }^{1458}$ Ce dernier est un Protocole additionnel au Protocole relatif au mécanisme de prévention, de gestion, de règlement des conflits, de maintien de la paix et de la sécurité. Disponible sur le site internet: https://www.eisa.org/pdf/ecowas2001protocol1.pdf. Consulté le 18/01/2022.
} 
mécanismes pour contraindre les Etats à respecter leurs engagements et à défaut, les sanctions peuvent intervenir.

Mais, des inquiétudes demeurent par rapport à la profusion des variables, des acteurs et des défis induits par la gestion des changements climatiques. La situation de la fluctuation des paramètres de mise en œuvre du droit climatique qui s'est révélée comme une tendance généralisée à l'échelle planétaire n'a pas non plus épargné le cadre de la coopération régionale. En réalité, la fluctuation des paramètres entrant en ligne de compte pour la lutte contre les changements climatiques et l'application différenciée du droit climatique qui en est la conséquence, découlent de ce que parfois, «Un grand nombre d'Etats africains se trouvent déchirés entre les impératifs internes de leur survie et les normes internationales de plus en plus contraignantes qui visent à normaliser les comportements des Etats pour atteindre des objectifs communs ${ }^{1459}$. Faudrait-il rappeler que certaines situations qui découlent du niveau de développement des Etats en voie de développement qui pour la plupart situés sur le contient africain et plus particulièrement en Afrique subsaharienne, ne sont pas occultées par le droit international qui reconnait que pour «Les pays en voie de développement, la plupart des problèmes d'environnement sont causés par le sous-développement ${ }^{1460}$.

Dans la perspective d'une réponse aux préoccupations soulevées, Kamal BENZAIDA qui analyse la problématique de la mise œuvre des normes internationales spécifiquement pour tenir compte du contexte du sous-développement qui caractérise le contient africain, propose «la bonne gouvernance» comme «Un des modèles qui peut sans doute être utilisé comme cadre pour la conception de stratégies efficaces pour faire face aux obstacles de taille du genre des changements climatiques» ${ }^{1461}$. La gouvernance climatique internationale a été conçue sur la base de ce modèle parce qu'elle requiert, «l'application du principe de la responsabilité commune mais différenciée et des capacités respectives des Parties ${ }^{1462}$. L'application de ce principe permet d'exempter les Etats sous-développés des charges financières qui sont imposées aux Etats pollueurs. Par contre, dans l'accord global, les Etats en développement ont la responsabilité de développer les puits d'absorption de gaz à effet de serre.

La bonne gouvernance « incarne généralement un ensemble de principes qui peuvent être adaptés à la réalité et aux contraintes de chaque pays» ${ }^{1463}$. En considérant ce concept, les

${ }^{1459}$ Kamal BENZAIDA, Prospects and actions in Africa to cope with climate change effects, Genava Global Policy $\mathrm{N}^{\circ} 3,2020$.

${ }^{1460}$ Paragraphe 4 du préambule de la Déclaration de Stockholm de 1972.

${ }^{1461}$ Kamal BENZAIDA, Prospects and actions in Africa to cope with climate change effects, Genava Global Policy $\mathrm{N}^{\circ} 3,2020$.

${ }^{1462}$ Article 3 paragraphe 1 de la Convention Cadre des Nations Unies sur les Changements Climatiques.

${ }^{1463}$ Article 3 paragraphe 1 de la Convention Cadre des Nations Unies sur les Changements Climatiques. 
mesures applicables pour le règlement des défis imposés par les changements climatiques peuvent ne pas être les mêmes dans les Etats Membres de l'organisation régionale. Chaque Etat dispose de son diagnostic qui admet des solutions endogènes voire spécifiques. Bien entendu que le cadre global, général permet de converger les moyens, de définir des stratégies globales qui intègrent la vision planétaire des changements climatiques, cependant, il n'est pas exclu qu'une solution qui est bonne pour un Etat ne puisse pas être transposée dans un autre pays sans précaution. Parfois, les problèmes ou difficultés endogènes peuvent dans certaines mesures admettre des solutions spécifiques voire endogènes. Dans ce cas encore, faudrait-il mettre en œuvre des « solutions flexibles» ${ }^{1464}$ en application des règles de bonne gouvernance. Il s'ensuit que, bien que la politique sous-régionale admette l'uniformisation des politiques et stratégies de protection de l'environnement et éventuellement l'uniformisation des normes d'encadrement des changements climatiques, les mesures applicables dans chaque Etat peuvent varier selon les cas et la «particularité de chaque contexte» ${ }^{1465}$. Ainsi, la mise en œuvre des règles de bonne gouvernance permettront d'éviter les difficultés de mise en œuvre du droit climatique dans l'espace régional. La pratique des règles de bonne gouvernance est recommandée dans le cadre de la CEDEAO. La particularité que requiert la gestion des changements climatiques recommandent à ce que les Etats de ladite Communauté soient assistés dans la gestion des difficultés qu'induit la mise en œuvre des dispositions applicables aux changements climatiques. Mais en amont, les mesures à intervenir devront intégrer les préoccupations concernant leur effectivité. Ce qui permet de tenir compte des situations de chaque Etat dans l'élaboration des normes.

\section{Comment promouvoir la bonne gouvernance climatique dans le cadre régional?}

Pour que la CEDEAO prenne efficacement en charge la gestion des changements climatiques, il faudra procéder à la création d'un mécanisme de promotion de la bonne gouvernance climatique au sein de la Communauté. Ce mécanisme aura pour mission de veiller au respect des engagements souscrits par la communauté régionale en privilégiant les solutions non punitives. Ce contrôle sera orienté vers les engagements souscrits par chaque Etat Membre dans le cadre de la Convention Cadre des Nations Unies sur les Changements Climatiques. Le mécanisme sera conçu à l'image de la «procédure de respect » instituée dans le Protocole de Kyoto ou prendra la forme du «cadre de transparence » comme celui qui est institué dans l'Accord de Paris. Dans cette perspective, l'institution régionale devra réviser les textes

\footnotetext{
${ }^{1464}$ Article 3 paragraphe 1 de la Convention Cadre des Nations Unies sur les Changements Climatiques. ${ }^{1465}$ Article 3 paragraphe 1 de la Convention Cadre des Nations Unies sur les Changements Climatiques.
} 
fondamentaux de sa création ${ }^{1466}$ et y incorporer les dimensions concernant les changements climatiques.

Lorsque l'encadrement international sera amélioré, il faudra que chaque Etat Partie à la Convention Cadre des Nations Unies sur les Changements Climatiques se consacre à l'amélioration de son encadrement interne pour espérer relever les défis que lui impose son engagement dans ladite Convention. Dans cette optique, le Bénin devra engager des réformes pour renforcer la gouvernance climatique en cours.

${ }^{1466}$ Le traité révisé de la CEDEAO. Disponible sur le site internet: https://www.ecowas.int/droit-de-lacedeao/traite.. Consulté le 18/01/2022. 


\section{CHAPITRE II:}

\section{LE RENFORCEMENT DE LA GOUVERNANCE CLIMATIQUE INTERNE}

Par l'élaboration du régime international de protection du climat, la Communauté internationale a commencé par coordonner la gestion des solutions à la lutte contre les changements climatiques par l'application de la Convention Cadre des Nations Unies sur les Changements Climatiques.

Malgré ces efforts d'encadrement au plan international, il est difficile d'occulter l'épineuse question de la «mise en œuvre du droit international de l'environnement dans les pays pauvres, une problématique cruciale qui est loin d'être achevée ${ }^{1467}$, parce que le droit international et particulièrement celui des changements climatiques est «(..) mû par des intérêts individuels des Etats et en fonction du rapport de leur puissance respective» ${ }^{1468}$ et aussi ébranlé par des situations qui découlent des conditions du sous développement auxquelles certains Etats sont assujettis. Ce qui fait qu'il y a une application différenciée des normes internationales de lutte contre les changements climatiques avec pour conséquence la non atteinte des objectifs convenus.

Dans ce contexte où le droit international peine à relever les défis qu'imposent les changements climatiques, le Bénin impacté par les effets néfastes des changements climatiques et résolument engagé dans la lutte contre le fléau, a tout comme d'autres Etats Parties à la Convention Cadre des Nations Unies sur les Changements Climatiques développé un arsenal juridico-institutionnel qui a présenté ses limites. Il ne s'agit pas en réalité d'une défaillance de la gouvernance climatique béninoise. En l'état, il serait même précoce d'en arriver à une conclusion pareille, car le droit climatique béninois est en plein processus de construction tout comme le droit international des changements climatiques qui subi à l'occasion de chaque COP des améliorations.

Néanmoins, la gouvernance climatique béninoise a besoin d'être renforcée. Et ce renforcement se fera d'une part, par la promotion des mesures devant contribuer à la

\footnotetext{
${ }^{1467}$ KAMTO (Maurice), LA MISE EN OEUVRE DU DROIT DE L'ENVIRONNEMENT : FORCES ET FAIBLESSES DES CADRES INSTITUTIONNELS, Revue Africaine de Droit de l'Environnement $N^{\circ} 01,2014$, p.35.

${ }^{1468}$ Ruiz Fabri Hélène. Le droit dans les relations internationales. In: Politique étrangère, $\mathrm{n}^{\circ} 3-4,2000$, pp. 659672., p. 660 .
} 
participation inclusive à l'action climatique (SECTION I) et d'autre part, par l'amélioration de l'encadrement juridique (SECTION II).

\section{SECTION I : LA PROMOTION DES MESURES DE PARTICIPATION INCLUSIVE}

Les problèmes provoqués par les modifications climatiques et la gestion de ses effets néfastes sont à la fois pluridisciplinaires, multisectoriels et trans-sectoriels. Ces problèmes impactent la société sans exclusion et tous les aspects relatifs à la vie ${ }^{1469}$. A l'instar des défis globaux et les problèmes qui appellent une intervention collective, la lutte contre les changements climatiques nécessite une approche de gestion partenariale car, plusieurs acteurs avec des compétences diverses sont appelés à mettre ensemble leurs efforts pour combattre le fléau. N'est ce pas le motif pour lequel la Déclaration sur l'environnement et le développement issue de la Conférence des Nations Unies sur l'Environnement et le Développement, réunie à Rio de Janeiro du 03 au 14 juin 1992 a prescrit que, « la meilleure façon de traiter les questions d'environnement est d'assurer la participation de tous les citoyens concernés, au niveau qui convient (...) $»^{1470}$. Il s'agira de faire participer à l'action climatique non seulement, les institutions de l'Etat et ses démembrements mais en plus, les personnes morales relevant du secteur public ou privé notamment, les multinationales, les entreprises, les médias, les organisations de la société civile et même l'opinion publique et les citoyens. C'est pourquoi à l'occasion de la seizième $\left(16^{\mathrm{e}}\right)$ session de la Conférence des Parties de la Convention Cadre des Nations Unies sur les Changements Climatiques tenue à Cancún du 29 novembre au 10 décembre 2010, la Communauté internationale a reconnu que de «multiples Parties prenantes doivent être mobilisées aux niveaux mondial, régional, national et local, qu'il s'agisse de gouvernements, y compris les administrations infranationales et locales, d'entreprises privées ou de la société civile, dont les jeunes et les personnes handicapées et que l'égalité des sexes et la participation effective des femmes et des peuples autochtones sont d'une grande importance pour agir efficacement sur tous les aspects des changements climatiques ${ }^{1471}$. Michel Prieur a laissé entendre que, «la démocratie était une condition indispensable de lutte

\footnotetext{
${ }^{1469}$ Evaluation du Comité National sur les Changements Climatiques Volume 1: Diagnostic et Plan de Renforcement des Capacités Octobre 2016 CNCC - Changements Climatiques. Disponible sur le site internet : https://www.changementsclimatiques.bj. Consulté le 02/06/2021.

${ }^{1470}$ Principe 10 de ladite convention.

${ }^{1471}$ Paragraphe 7 du préambule du Rapport de la Conférence des Parties sur sa seizième session, tenue à Cancún du 29 novembre au 10 décembre 2010. Additif. Deuxième partie: Mesures prévues par la Conférence des Parties à sa seizième session. Disponible sur le site internet : https://unfccc.int/fr/node/6527. Consulté le 18/01/2022 .
} 
contre la pauvreté et un instrument de promotion du développement durable ${ }^{1472}$. Le rapport Brundtland a également conclu à l'association des communautés à diverses échelles au processus du « développement durable» ${ }^{1473}$, ce qui permet de renforcer les initiatives de l'Etat, son intervention dans le domaine de la protection de l'environnement et de la lutte contre les changements climatiques. In fine, la mise en œuvre des règles de démocratie dans le domaine environnemental permettra aux différentes couches de la société de garantir, « le devoir solennel de protéger et d'améliorer l'environnement pour les générations présentes et futures» ${ }^{1474}$.

Ce management voulu par la Communauté internationale a commencé par s'imposer à l'ordre juridique des Etats Parties à la Convention Cadre des Nations Unies sur les Changements Climatiques. C'est pourquoi, la charte béninoise sur la gouvernance environnementale a été élaborée dans le sens de « (...) favoriser la recherche de processus et de solutions pour une gestion partagée de l'environnement (...)» ${ }^{1475}$. De même, la loi $n^{\circ} 2018$-18 portant règlementation sur les changements climatiques en République du Bénin a prescrit que, « l'Etat organise la synergie des actions de tous les acteurs concernés par la lutte contre les effets négatifs des changements climatiques. Dans ce cadre, il élabore des outils et méthodes d'approche participative qui permettent d'impliquer davantage les différents acteurs dans le processus de recherche et d'actions, prenant en compte les spécificités du milieu concerné ${ }^{1476}$. De tout point de vue, le principe de participation inclusive des acteurs au domaine de protection de l'environnement est un acquis au Bénin, seulement que les conditions de la mise à contribution de ces acteurs à l'action climatique ne sont pas encore élucidées.

Comment faire pour rendre effectifl'intervention des acteurs non étatiques dans la lutte contre les changements climatiques au Bénin?

La réponse à cette question passe par l'élaboration d'un cadre juridique dont le principal objectif sera, d'impulser la participation inclusive à l'action climatique. En effet, il convient

\footnotetext{
${ }^{1472}$ Michel PRIEUR, Démocratie et droit de l'Environnement et du développement, Revue juridique de l'Environnement/Année/1993/1/pp.23-30.

${ }^{1473}$ Le développement durable est une conception de la croissance économique qui s'inscrit dans une perspective de long terme et qui intègre les contraintes liées à l'environnement et au fonctionnement de la société. Selon la définition donnée dans le rapport de la Commission mondiale sur l'environnement et le développement de l'Organisation des Nations unies, dit rapport Brundtland, où cette expression est apparue pour la première fois en 1987, « le développement durable est un développement qui répond aux besoins du présent sans compromettre la capacité des générations futures de répondre aux leurs ».

${ }^{1474}$ KAMTO (Maurice), LA MISE EN OEUVRE DU DROIT DE L'ENVIRONNEMENT : FORCES ET FAIBLESSES DES CADRES INSTITUTIONNELS, Revue Africaine de Droit de l'Environnement RADE $\mathrm{N}^{\circ} 01$, 2014, p.37.

${ }^{1475}$ Article $1^{\text {er }}$ de la charte nationale sur la gouvernance environnementale en République du Bénin adoptée par décret n²004-273 du 02 mai 2004. Disponible sur le site internet: https://sgg.gouv.bj/doc/decret-2004273/download. Consulté le 18/01/2022.

${ }^{1476}$ Article 57 de la loi n²018-18 portant règlementation sur les changements climatiques en République du Bénin.
} 
dans le cas d'espèce d'encadrer juridiquement le rôle des acteurs non étatiques, (Paragraphe 1) qui seront désormais appelés à collaborer avec des structures administratives comme les démembrements de l'Etat, qu'il faudra aussi impliquer dans la mouvance d'acteurs intervenant dans le domaine de la lutte contre les changements climatiques (Paragraphe 2).

\section{Paragraphe 1: L'encadrement des acteurs non étatiques}

Si Michel PRIEUR a insisté sur la nécessité de mobiliser les acteurs sociaux autour de la cause de la protection de l'environnement en indiquant que, «Les législations nationales doivent faciliter la création de groupes sociaux ayant pour but la protection de la nature et de l'environnement $\gg{ }^{1477}$, des arrangements normatifs devront être également opérés pour impliquer ces groupes sociaux dans l'encadrement de l'environnement. Cette position a été renforcée dans l'Accord de Paris qui a reconnu non seulement, "l'importance de la participation des pouvoirs publics à tous les niveaux, mais il reconnait également la participation de divers acteurs, conformément aux législations nationales respectives des Parties à la lutte contre les changements climatiques ${ }^{1478}$. C'est par ce moyen que le citoyen peut contribuer à la transparence dans un processus décisionnel engagé par l'autorité publique en ce qui concerne la gestion de la protection de l'environnement ou de la lutte contre les changements climatiques.

$\mathrm{Au}$ Bénin, les changements climatiques posent un problème majeur aux décideurs nationaux en ce qui concerne l'encadrement des rôles des acteurs non étatiques devant intervenir dans ce domaine. En effet, en dépit de la reconnaissance légale de la participation d'acteurs non étatiques à la lutte contre les changements climatiques, il est à noter que leurs contributions sont mitigées. Il en est ainsi parce que, aucun texte n'établit avec précision le contenu des rôles que ces acteurs sont appelés à jouer dans le cadre de la lutte contre les changements climatiques. Alors que, nulle n'ignore leur capacité à booster le domaine climatique parce qu'ils en ont fait leur preuve dans d'autres secteurs d'activités.

Il convient donc de corriger cette insuffisance en procédant à un encadrement plus accru et plus explicite du rôle d'acteurs non étatiques dans le cadre de la lutte contre les changements climatiques. Par exemple, l'amélioration de l'encadrement juridique concernant l'intervention de la société civile dans le domaine de la protection du climat peut aider à ce que cette entité

\footnotetext{
${ }^{1477}$ PRIEUR (Michel), Démocratie et droit de l'environnement et du développement. In: Revue Juridique de l'Environnement, $\mathrm{n}^{\circ} 1,1993$, pp. 23-30., p.12.

${ }^{1478}$ Quinzième $\left(15^{\mathrm{e}}\right)$ paragraphe du Préambule de l'Accord de Paris 2015.
} 
soit transformée en acteur clé de la lutte contre les changements climatiques (A). Il en sera ainsi pour les acteurs économiques dont le cadre juridique de collaboration avec l'Etat devra être renforcé $(\mathbf{B})$.

\section{A- La transformation de la Société civile en acteur clé}

Le droit international a recommandé que les Parties doivent «encourager la participation (...) des organisations non gouvernementales $»{ }^{1479}$ au processus d'atténuation des changements climatiques. Puisque les autorités publiques ne sortent de leur inaction et considèrent à juste titre les réels problèmes de l'environnement qu'en cas de survenance de catastrophes et lorsqu'elles sont en contact avec les « impulsions des organisations de la société civile et de l'opinion publique» ${ }^{1480}$. Ces catégories d'acteurs peuvent contribuer à l'action de protection de l'environnement en adoptant leur méthode habituelle de travail qui consiste à la régulation et au suivi-évaluation des politiques gouvernementales. Car, c'est notoire que les déclarations publiques et plaidoyers de la société civile influent généralement sur les décisions qui doivent être prises au sommet de l'Etat. C'est pourquoi, le rapport Brundtland a recommandé que, «les Organisations Non Gouvernementales (ONG) doivent avoir le droit et s'engager à participer au développement, à la surveillance et à la vérification du droit international au niveau local, national, régional et international ». Par exemple, certaines organisations de la société se sont constituées en bloc de soutien à une action judiciaire entreprise contre l'Etat français, visant à lui imposer le respect de ses engagements climatiques ${ }^{1481}$. Une recommandation similaire a été faite au plan régional dans le sens de « Promouvoir le renforcement des partenariats entre la société civile, les Etats et les ONG internationales cuvrant dans le domaine de l'environnement pour une meilleure mobilisation des ressources financières en vue d'une mise en æuvre effective du droit de l'environnement en Afrique » ${ }^{1482}$.

Pour mieux cerner le sujet, ne convient-il pas de définir le concept de la Société- civile dans le contexte du droit béninois pour appréhender ses composantes?

\footnotetext{
${ }^{1479}$ Article 4 paragraphe 1-i de la Convention Cadre des Nations Unies sur les Changements Climatiques. ${ }^{1480}$ BOISGALLAIS (AS) et KABORE (GL), Livre blanc des ONG françaises. Pour l'environnement et le développement, construire la démocratie internationale, Paris, Bruxelles, CEDI, 1992 - 95 p.

${ }^{1481}$ Il s'agit de quatre (4) Organisations Non Gouvernementales (Greenpeace, Oxfam, la Fondation pour la nature et l'homme, l'association Notre affaire à tous). Disponible sur le site internet : https://www.greenpeace.fr/laffae/. Consulté le 18/01/2022.

${ }^{1482}$ Cinquième $\left(5^{\mathrm{e}}\right)$ recommandation sur « l'effectivité du droit de l'environnement en Afrique », Revue Africaine de Droit de 1'Environnement n01-2014, p.19.
} 
Selon les dispositions réglementaires en vigueur en République du Bénin, la Société civile « représente les formes de structuration, d'interdépendance et de solidarité relativement autonome par rapport à l'Etat à travers laquelle, les individus satisfont leurs besoins. Elle comprend les ménages, les associations de développement, les divers groupements, les Organisations Non Gouvernementales, les sociétés privées, les confessions religieuses et autres composantes du corps social ${ }^{1483}$. Cette entité est reconnue au Bénin d'une part, sur le fondement de l'article 25 de la constitution béninoise du 11 décembre 1990 révisée le 07 novembre 2019 qui consacre la liberté d'association et d'autre part, sur la base de la loi française du $1^{\text {er }}$ juillet 1901 relative au contrat d'association, rendue applicable en 1948 par décret aux Etats de l'Afrique Occidentale Française (AOF) dont le Dahomey devenu Bénin ${ }^{1484}$.

Quel est le degré d'implication de la Société civile dans la lutte contre les changements climatiques au Bénin?

En considérant l'ampleur des effets néfastes des changements climatiques au Bénin, la loi prescrit à la charge de l' Etat d'organiser « la synergie des actions de tous les acteurs concernés par la lutte contre les effets négatifs des changements climatiques ». L'Etat a l'obligation d'élaborer des «outils et méthodes d'approche participative qui permettent d'impliquer davantage les différents acteurs dans le processus de recherche et d'actions, prenant en compte les spécificités du milieu concerné ${ }^{1485}$. Certains textes de loi confèrent à la société civile quelques attributions environnementales. C'est le cas de la charte nationale sur la gouvernance environnementale qui, « (...) en reconnaissant l'évolution des rôles en matière d'environnement au niveau de l'Etat(...) » a également reconnu le rôle de la « (...) société civile (...) $»^{1486}$. Cependant, le rôle de la Société civile dans la lutte contre les changements climatiques n'y est pas clairement défini. La Société civile est souvent confinée en arrière plan et réduite dans son renforcement structurel. La loi a inclus la Société civile dans certaines institutions de protection du climat ${ }^{1487}$ tout comme, le Comité National sur les Changements

\footnotetext{
${ }^{1483}$ Article 3 de la charte nationale sur la gouvernance environnementale en République du Bénin adoptée par décret n²004-273 du 02 mai 2004. Disponible sur le site internet: https://sgg.gouv.bj/doc/decret-2004273/download. Consulté le 18/01/2022.

${ }^{1484}$ L'Afrique-Occidentale française (A.-O.F.) était une Fédération qui regroupa de 1895 à 1958 le Sénégal, la Mauritanie, le Soudan (ancien Haut-Sénégal-Niger), la Haute-Volta, le Niger, la Guinée française, la Côte d'Ivoire et de Dahomey; $4425000 \mathrm{~km}^{2}$. La capitale en était Dakar. Disponible sur le site internet: https://www.larousse.fr/encyclopedie. Consulté le 18/01/2022.

${ }^{1485}$ Article 57 de la loi n²018-18 portant règlementation sur les changements climatiques en République du Bénin. ${ }^{1486}$ Article 2 de la charte nationale sur la gouvernance environnementale en République du Bénin adoptée par décret n²004-273 du 02 mai 2004. Disponible sur le site internet: https://sgg.gouv.bj/doc/decret-2004273/download. Consulté le 18/01/2022.

${ }^{1487}$ Article 8 de la Loi n ${ }^{\circ}$ 98-030 du 12 février 1999 portant loi cadre sur l'environnement en République du Bénin. Disponible sur le site internet : https://sgg.gouv.bj/doc/loi-98-030. Consulté le 16/01/2022.
} 
Climatiques $(\mathrm{CNCC})^{1488}$, qui regorge plusieurs associations. De même, la loi cadre sur l'environnement en République du Bénin a conféré à la Société civile, les prérogatives de mise en œuvre de l'action publique en cas d'infraction à la règlementation sur la protection de l'environnement et la possibilité de se constituer partie civile pour la réclamation éventuelle de réparation de préjudice. Dans ce cas encore, elle doit prouver que les faits incriminés préjudicient directement ou indirectement à l'intérêt collectif qu'elle représente ${ }^{1489}$. Il s'ensuit que les conditions d'organisation de la participation de la Société civile à l'action judiciaire sont difficiles. Par ailleurs, la loi n²018-18 portant règlementation sur les changements climatiques en République du Bénin, n’a consacré qu'un seul article à la Société civile par la stipulation suivante : « l'Etat promeut les connaissances, savoir-faire et capacités endogènes en (...) appuyant la formation des vulgarisateurs et des membres des organisations de la société civile, (..), aux méthodes participatives de conservation et d'utilisation durable des ressources naturelles $\gg{ }^{1490}$.

Somme toute, les infirmes attributions conférées à la société civile par la règlementation environnementale et climatique ne lui permettent pas d'accomplir efficacement son rôle de protecteur des citoyens exposés aux effets néfastes des changements climatiques. Mais en plus, la société civile est exposée aux difficultés de fonctionnement dues à la précarité de ses moyens. Ces facteurs amenuisent la capacité d'intervention de cette entité dans le domaine de la lutte contre le réchauffement climatique, alors que l'expertise de la Société civile est recherchée dans ce domaine.

Par contre à l'échelle internationale, la preuve de l'importance de l'intervention de la Société civile dans la lutte contre les changements climatiques peut être perçue à travers le rôle de la prestigieuse organisation internationale de la Société civile, l’Union Mondiale pour la Nature $(\mathrm{UICN})^{1491}$ qui contribue aux côtés des Etats et des organisations internationales à

\footnotetext{
1488 Telles que BENIN21, OFEDI, ABFEM-ONG, GRAIR.

${ }^{1489}$ Article 109 de la loi n $98-030$ portant loi cadre sur l'environnement en République du Bénin. Disponible sur le site internet : https://sgg.gouv.bj/doc/loi-98-030. Consulté le 16/01/2022.

${ }^{1490}$ Article 17 paragraphe 4 de la loi n²018-18 portant sur les changements climatiques en République du bénin.

${ }^{1491}$ L'Union internationale pour la conservation de la nature (UICN, en anglais IUCN) est l'une des principales organisations non gouvernementale mondiale consacrées à la conservation de la nature. Sa mission est d'influencer, d'encourager et d'assister les sociétés du monde entier, dans la conservation de l'intégrité et de la biodiversité de la nature, ainsi que de s'assurer que l'utilisation des ressources naturelles est faite de façon équitable et durable. Fondée le 5 octobre 1948 à la suite d'une conférence internationale tenue à Fontainebleau, en France, elle s'intitulait, à l'origine, Union internationale pour la protection de la nature (UIPN), mais elle a été rebaptisée sous son nom actuel en 1956. Son siège est situé à Gland, en Suisse. Elle réunit plusieurs États et agences gouvernementales, plus de 1100 ONG et plus de 10000 experts et scientifiques. Elle emploie plus d'un millier de personnes dans le monde travaillant sur plusieurs centaines de projets. Elle a aidé plus de 75 pays à préparer et appliquer des stratégies en matière de conservation et de diversité biologique. Statuts et Règlement de l'UICN. Disponible sur le site internet : https://www.iucn.org/fr. Consulté le 18/01/2022.
} 
l'élaboration du droit international de l'environnement. Cette organisation internationale peut influencer le processus d'élaboration des normes en imprimant une orientation. Elle a fait ses preuves dans le cadre de « l'élaboration de la grande majorité des traités à portée internationale ou régionale auprès des organisations internationales ${ }^{1492}$. Les occasions d'adoption de la Convention d'Aarhus du 25 juin $1998^{1493}$ et de la Convention Cadre des Nations Unies sur les Changements Climatiques de Rio de Janeiro en 1992, ont permis de reconnaitre l'efficacité de l'intervention des organisations de la Société civile dans les domaines de la protection de l'environnement et de la lutte contre le réchauffement climatique.

Comment faire pour remettre la Société civile béninoise dans son rôle de protecteur des intérêts des citoyens dans le cadre de la lutte contre les changements climatiques, à l'image de ce que font certaines composantes de la Société civile à l'échelle internationale?

L'Agenda 21 National a proposé dans sa section III ${ }^{1494}$ une large participation du public à la prise de décisions et à l'évaluation des effets de tout programme de développement lié à l'environnement et a suggéré des solutions au désintérêt qu'affichent les organisations de la Société civile dans le cadre de la protection de l'environnement et aussi dans la lutte contre les changements climatiques. En réalité, il ne s'agit pas d'un désintérêt manifeste, mais au contraire, la passivité des organisations de la Société civile dans le domaine de la lutte contre les changements climatiques découle de l'insuffisance qui caractérise l'arsenal juridique qui les encadre. La Société civile n'est pas inscrite au cœur des préoccupations de lutte contre les changements climatiques comme institution dont l'apport peut être significatif. Au contraire, le cadre juridique destiné à la lutte contre les changements climatiques a été presque muet sur son rôle.

Pour remettre en scène la Société civile dans le concert d'acteurs devant intervenir activement et efficacement dans la lutte contre les changements climatiques au Bénin, il convient de redéfinir ses attributions par un encadrement législatif essentiellement consacré à son rôle dans ledit domaine. En plus, les attributions environnementales conférées à la Société civile par certaines dispositions législatives devront être étendues aux dimensions concernant

\footnotetext{
${ }^{1492}$ Olivier Juliette. Les nouveaux acteurs du droit de l'environnement. Le rôle de l'UICN dans l'élaboration du droit de l'environnement. In: Revue Européenne de Droit de l'Environnement, n³, 2005. pp. 274-296., p.275.

La Convention sur l'accès à l'information, la participation du public au processus décisionnel et l'accès à la justice en matière d'environnement a été adoptée à Aarhus (Danemark), 25 juin 1998 et entrée en vigueur le 30 octobre 2001 , conformément au paragraphe 1 de l'article 20. Elle compte trente neuf (39) signataires et quarante-sept (47) Parties. Nations Unies, Recueil des Traités, vol. 2161, p. 447. Disponible sur le site internet : https://treaties.un.org. Consulté le 18/01/2022.

${ }^{1494}$ Un agenda 21 local est un projet territorial de développement durable, porté par une collectivité locale et qui prend la forme d'un programme d'actions (programme d'actions pour le $21^{\mathrm{e}}$ siècle). Disponible sur le site internet : https://www.un.org/esa/sustdev/documents/agenda21/fre. Consulté le 18/01/2022.
} 
les changements climatiques. A l'occasion de la révision des textes de loi qui encadrent la protection de l'environnement et la lutte contre les changements climatiques, les attributions obscures, imprécises, parfois difficiles conférées à la société civile devront être améliorées. Ces réformes devront prendre en compte les conditions de financement et les moyens de fonctionnement de cette entité pour lui garantir les conditions d'indépendance, sans quoi, ses actions risquent de ne pas être efficaces. Le Législateur au bout du processus devra inscrire dans l'encadrement juridique béninois, plusieurs mesures qui contribuent à remettre en scène la Société civile à travers son rôle d'éveilleur de conscience, de protecteur de l'action publique au profit de la population et la transformer à terme, en acteur clé de la lutte contre les changements climatiques. Pour conférer plus d'autonomie aux groupes sociaux, Michel PRIEUR a même suggéré d'« introduire des avantages fiscaux au profit des adhérents pour éviter que les budgets de ces groupements ne soient totalement dépendants des subventions des pouvoirs publics $»^{1495}$.

L'une des conclusions à laquelle est parvenue l'Institut International pour l'Environnement et le Développement (IIED) au Bénin pour ce qui concerne l'encadrement de la Société civile est que, « Lorsque l'encadrement juridique relatif à l'intervention de la Société civile dans le domaine de la lutte contre les changements climatiques sera adopté, la Société civile accomplira un rôle déterminant dans l'adoption de nouvelles lois, politiques ou stratégies sur les changements climatiques en poussant les gouvernements à tenir leurs engagements, en identifiant l'absence de réactions gouvernementales coordonnées aux changements climatiques et en s'assurant que les pauvres et les plus vulnérables ne sont pas oubliés dans l'élaboration des politiques ${ }^{1496}$. Lorsque le cadre d'intervention de la Société civile dans le domaine de la lutte contre les changements climatiques sera renforcé, cette entité prendra une part active dans les processus décisionnels de l'Etat qui engagent les citoyens pour faire prévaloir non seulement, les intérêts de ces derniers, mais aussi ceux de l'Etat. A titre illustratif, la décision d'interdiction controversée de commercialisation de bois provenant des forêts entre temps rapportée par le Gouvernement béninois sous l'effet de la pression de la population peut être remise en vigueur avec la contribution de la Société civile qui devra participer en collaboration avec le Gouvernement à son amélioration et puis à sa vulgarisation avant son application. Cette entité va se constituer en interface entre l'Etat et les citoyens et se fera porte voix de la population au niveau des autorités qui intègrent leurs préoccupations aux décisions qui les

\footnotetext{
${ }^{1495}$ Michel PRIEUR, « Démocratie et droit de l'environnement et du développement », R.J.E, 1-1993, p. 12.

${ }^{1496}$ Fruits des réflexions menées par l'Institut International pour l'Environnement et le Développement (IIED) et CARE avec le concours de Climate Capacity Consortium.
} 
engagent. Et puis, elle jouera son rôle d'éveilleur de conscience et de sensibilisateur pour expliquer le bien fondé de la décision gouvernementale d'interdiction de commercialisation de bois provenant des forêts, qui ne vise que la protection de l'environnement pour le bien être de la population. Ces actions combinées de la Société civile en collaboration avec les institutions de l'Etat peuvent contribuer à faciliter l'application des mesures de protection de l'environnement en général et celles relatives au reboisement en particulier.

Eu égard à ce qui précède, la Société civile devra faire l'objet d'un encadrement spécifique sur l'initiative du Législateur béninois qui renforcera sa participation à l'action de lutte contre les changements climatiques. La même initiative devra être étendue aux acteurs économiques par le renforcement du cadre juridique de leur collaboration avec l'Etat, dans le domaine climatique.

\section{B- Le renforcement du cadre de collaboration du secteur public et privé}

Pour atténuer les impacts négatifs des changements climatiques, le droit international a prescrit des mesures d'adaptation ${ }^{1497}$. Certains Experts ont estimé que compte tenu de son ampleur, l'adaptation aux effets néfastes des changements climatiques ne peut pas être du seul ressort des Etats ${ }^{1498}$. Par conséquent, il faut l'intervention coordonnée à la fois des autorités étatiques et celle des partenaires relevant du secteur privé. Ainsi, les entreprises et les investisseurs privés doivent prendre part au processus d'atténuation des changements climatiques. La Déclaration de Rio de Janeiro de 1992 a abordé la question dans la même logique en envisageant que « la mise en œuvre des politiques et programmes nationaux en matière de gestion de conservation et d'exploitation écologiquement viable des forêts, notamment, dans les pays en développement devrait être appuyée par une coopération financière et technique internationale y compris par l'intermédiaire du secteur privé $(\ldots){ }^{1499}$.

Dès lors que la règlementation internationale est favorable à l'implication des acteurs privés dans le domaine de la gestion des changements climatiques les Etats Parties se trouvent dans l'obligation d'élaborer un cadre juridique interne pour organiser l'intervention de cette entité dans l'élaboration et la mise en œuvre du droit applicable dans ces matières respectives.

\footnotetext{
${ }^{1497}$ Article 4 paragraphe 1 (b et e) de la Convention Cadre des Nations Unies sur les Changements Climatiques. ${ }^{1498}$ Crawford, A. et Church, C. (2019). Impliquer le secteur privé dans les processus nationaux de planification de l'adaptation. Winnipeg, Canada : Institut international du développement durable. Extrait de www.napglobalnetwork.org. Consulté le 18/01/2022.

${ }^{1499}$ Paragraphe 8 point $\mathrm{c}$ de la Déclaration de principes, non juridiquement contraignantes mais faisant autorité, pour un consensus mondial sur la gestion, la conservation et l'exploitation écologiquement viable de tous les types de forêts. Disponible sur le site internet: https:/www.un.org/french/events/rio92/aconf15126vol3f.htm. Consulté le 18/01/2022.
} 
La République du Bénin dispose d'un cadre juridique à caractère général, ${ }^{1500}$ qui met en contact les acteurs économiques et l'Etat, dans le but de l'accomplissement de « (...) tout contrat ou toute convention (..) ${ }^{1501}$ à l'occasion de l'exécution des marchés conclus dans le cadre partenarial, partenariat public-privé. Ce contrat de partenariat public-privé peut avoir pour objet, la « conception des ouvrages, équipements ou biens immatériels ainsi que, des prestations de service concourant à l'exercice par l'autorité contractante, de la mission de service public dont elle est chargée $\gg{ }^{1502}$. Seulement, la réalisation de ces ouvrages peut avoir des répercutions sur l'environnement du fait, du non respect des règles de protection de l'environnement. De même, les technologies qui sont déployées dans le cadre des réalisations des ouvrages ou infrastructures peuvent ne pas être compatibles avec les normes de protection de l'environnement, contribuant ainsi à la pollution de l'atmosphère. C'est pourquoi, dans le cadre de l'élaboration de la loi sur le partenariat public-privé ${ }^{1503}$, le Législateur béninois soucieux de l'impact négatif que peut induire la réalisation de certains ouvrages ou projets d'envergure sur l'environnement, a fait figurer des dispositions de protection de l'environnement dans ladite loi. Au nombre de ces mesures, figure l'étude d'impact environnementale ${ }^{1504}$. Il s'agit de l'évaluation environnementale qui est une conditionnalité de mise en œuvre des projets d'envergure en vue de prévenir les impacts environnementaux au Bénin. Dans le processus de sélection d'acteurs économiques devant participer à la réalisation de marchés publics, un cahier de charges contenant les clauses de protection de l'environnement est inclus dans le Dossier d'Appel d'Offre (DAO) ${ }^{1505}$. Ainsi, «Le porteur de l'offre spontanée soumet à l'autorité contractante un dossier comportant entre autres conditions, une étude d'impact environnemental $(\ldots) »^{1506}$. Il s'ensuit que les questions de protection de l'environnement sont mises au cœur des relations qui lient le secteur privé au secteur public au Bénin.

\footnotetext{
${ }^{1500}$ Loi n²016-24 du 11 octobre 2016 portant cadre juridique du partenariat public-privé en République du Bénin. Disponible sur le site internet : https://sgg.gouv.bj > doc > loi-2016-24. Consulté le 18/01/2022.

${ }^{1501}$ Article 3 de la loi n ${ }^{\circ} 2016-24$ du 11 octobre 2016 portant cadre juridique du partenariat public-privé en République du Bénin. Disponible sur le site internet : https://sgg.gouv.bj > doc > loi-2016-24. Consulté le $18 / 01 / 2022$.

${ }^{1502}$ Article premier alinéa 8 de la loi n²016-24 du 11 octobre 2016 portant cadre juridique du partenariat publicprivé en République du Bénin.

${ }^{1503} \mathrm{Id}$.

${ }^{1504}$ Articles 21 et 43 de la loi nº8-030 portant loi cadre sur l'environnement en République du Bénin.

${ }^{1505}$ Article 12 de la loi no $2016-24$ du 11 octobre 2016 portant cadre juridique du partenariat public-privé en République du Bénin. Disponible sur le site internet : https://sgg.gouv.bj > doc > loi-2016-24. Consulté le $18 / 01 / 2022$.

${ }^{1506}$ Article 22 la loi nº2016-24 du 11 octobre 2016 portant cadre juridique du partenariat public-privé en République du Bénin. Disponible sur le site internet : https://sgg.gouv.bj > doc > loi-2016-24. Consulté le $18 / 01 / 2022$.
} 
La loi n²016-24 du 11 octobre 2016 portant cadre juridique du partenariat public-privé en République du Bénin a fait une brève incursion dans le domaine des changements climatiques en mettant l'accent sur la technologie comme étant un critère substantiel d'éligibilité d'acteurs économiques ${ }^{1507}$. Selon les prescriptions légales, les technologies doivent pouvoir être en conformité avec les normes de protection de l'environnement et ne doivent contribuer en aucun cas à l'émission de gaz à effet de serre. Il s'agit dans le cas d'espèce, des technologies écologiquement rationnelles c'est-à-dire, des «technologies qui protègent l'environnement, qui sont moins polluantes, qui utilisent toutes les ressources plus durablement, qui recyclent une partie plus importante de leurs déchets et produits et traitent les déchets résiduels d'une manière plus acceptable que les technologies qu'elles ont remplacées et qui sont compatibles avec des priorités socio-économiques, culturelles et environnementales définies au plan national $»^{1508}$. Il s'agit, d'une « technologie d'atténuation et d'adaptation ${ }^{1509}$.

Par ailleurs, puisque « l'économie des Etats en développement Parties à la Convention Cadre des Nations Unies sur les Changements Climatiques est tributaire de la production de l'utilisation et de l'exportation de combustibles fossiles, du fait des mesures prises pour limiter les émissions de gaz à effet de serre ${ }^{1510}{ }^{15}$, les règlementations élaborées au niveau des Etats contribuent à la promotion des énergies renouvelables pour substituer à l'avenir les énergies fossiles ${ }^{1511}$. A l'image de l'encadrement international, la loi sur les changements climatiques impose le « développement des sources d'énergies nouvelles et renouvelables, qui doit se faire dans le respect des normes de protection de la santé publique, de l'environnement, des exigences de compétitivité de l'économie nationale et du développement durable ${ }^{1512}{ }^{12}$. Pour assurer l'effectivité de cette mesure, la loi sur le partenariat public privé dispose que, «la Cellule d'Appui au Partenariat Public Privé (CAPPP) émet un avis portant sur (...) les modalités de transfert de technologie» ${ }^{1513}$. Ainsi, « (...) le contrat de partenariat public-privé est conclu avec le candidat ayant présenté l'offre économiquement plus avantageuse sur la base de la

\footnotetext{
${ }^{1507}$ Des acteurs économiques devant participer à la réalisation des ouvrages pour le compte de l'Etat.

${ }^{1508}$ Article $1^{\text {er }}$ de la loi n ${ }^{\circ} 2018-18$ portant règlementation sur les changements climatiques en République du Bénin. ${ }^{1509}$ Id.

${ }^{1510}$ Dix-neuvième $\left(19^{\mathrm{e}}\right)$ paragraphe du préambule de la Convention Cadre des Nations Unies sur les Changements Climatiques.

${ }^{1511}$ Les énergies renouvelables englobent un ensemble hétérogène de technologies. Les divers types d'énergies renouvelables peuvent fournir de l'électricité, de l'énergie thermique ou de l'énergie mécanique et produire des combustibles susceptibles de répondre à de multiples besoins en matière de services énergétiques. Selon le rapport spécial sur les sources d'énergie renouvelable et l'atténuation du changement climatique, page7. Disponible sur le site internet : https://www.ipcc.ch. Consulté le 18/01/2022.

${ }^{1512}$ Article 39 de la loi n ${ }^{\circ} 2018-18$ portant sur la règlementation sur les changements climatiques en République du Bénin.

${ }^{1513}$ Article 23 de la loi no $2016-24$ du 11 octobre 2016 portant cadre juridique du partenariat public-privé en République du Bénin.
} 
combinaison optimale de différents critères d'évaluation prévus dans le dossier d'appel d'offres et pouvant être liés, notamment (...) au respect des normes environnementales $»^{1514}$.

Si l'adoption de la loi $n^{\circ} 2016-24$ du 11 octobre 2016 portant cadre juridique du partenariat public-privé en République du Bénin et la loi n²018-18 portant règlementation sur les changements climatiques en République du Bénin, constituent une avancée en matière d'encadrement des changements climatiques au Bénin, il se trouve que ces lois ne permettent pas de définir avec précision le cadre de collaboration du secteur public-privé. Car, en énumérant certains domaines de l'économie qui peuvent contribuer à l'atténuation des changements climatiques, ces lois n'ont pas définies clairement, le rôle des acteurs privés dans le cadre de leur collaboration avec le secteur public en relation avec les objectifs de lutte contre les changements climatiques. Ce qui fait qu'il est difficile d'appréhender à juste titre, l'impact positif de l'intervention des acteurs économiques dans le domaine de la lutte contre les changements climatiques.

Que faut-il faire pour améliorer les performances des acteurs économiques dans le domaine de la lutte contre les changements climatiques au Bénin?

Pour régler fondamentalement le problème de l'implication des acteurs économiques dans la lutte contre les changements climatiques au Bénin, la loi n²016-24 du 11 octobre 2016 portant cadre juridique du partenariat public-privé en République du Bénin doit être modifiée dans le sens de l'élargissement de son champ d'application au domaine des changements climatiques. De même, il est nécessaire de procéder à la relecture de la loi nº2018-18 portant règlementation sur les changements climatiques en République du Bénin pour créer un cadre législatif qui définit le rôle du secteur privé en relation avec l'Etat dans le domaine de la lutte contre les changements climatiques. Il convient également de rendre le cadre technique, financier et juridique attractif pour le secteur privé afin de faciliter la promotion des énergies renouvelables. La réforme du cadre juridique permettra aux acteurs du secteur privé de jouer pleinement leur partition dans la réalisation des objectifs que l'Etat béninois s'est fixés dans le cadre de la lutte contre les changements climatiques.

Pour parfaire l'encadrement juridique, il faut aussi impliquer les démembrements de l'Etat dans le domaine de la lutte contre les changements climatiques.

${ }^{1514}$ Article 37 id. 


\section{Paragraphe 2: L'implication des démembrements de l'Etat}

Dans le but d'assurer la couverture du territoire national, le Bénin a élaboré une politique de décentralisation du pouvoir de l'Etat et de déconcentration de l'administration, qui a été mise en vigueur en $1999^{1515}$. Cette politique répond quelque peu, aux exigences internationales en ce qui concerne la dissémination des normes de protection de l'environnement sur toute l'étendue du territoire national. Ces réformes initiées ont conféré des attributions de protection de l'environnement aux structures décentralisées et déconcentrées de l'Etat.

Par contre, bien qu'il ait été prescrit « la déclinaison de l'action climatique aux niveaux national, départemental, communal et local ${ }^{1516}$ par la règlementation nationale en matière de lutte contre les changements climatiques, aucune disposition légale n'a attribué de rôle aux démembrements de l'Etat en la matière. Ce qui fait que ces derniers ne parviennent pas à intervenir efficacement dans le domaine de la lutte contre les changements climatiques, alors qu'ils disposent des atouts qui peuvent être utiles dans le cadre de la riposte contre les changements climatiques.

Comment organiser l'intervention des démembrements de l'Etat dans le domaine de la lutte contre les changements climatiques au Bénin?

L'implication des démembrements de l'Etat dans le domaine de la lutte contre les changements climatiques au Bénin sera rendue possible par l'élaboration d'un encadrement juridique qui attribue des compétences climatiques à la collectivité locale (A) et à l'autorité déconcentrée de l'Etat (B).

\section{A- L'attribution de compétence climatique à la Collectivité locale}

Au moment où les manifestations négatives des changements climatiques se retournent de plus en plus contre les communautés de base, les solutions orientées et portées par ces communautés sont plus indiquées. Il y a nécessité de ramener le droit climatique à la base dans le cadre des collectivités territoriales. En effet, les acteurs de la collectivité territoriale, peuvent en plus de leurs attributions classiques être transformés en acteurs de lutte contre les changements climatiques, étant entendu que les problèmes climatiques se posent et se vivent à la base et que les actions concernant la mise en œuvre du droit applicable à la lutte contre les changements climatiques ne peuvent être réalisées que, sur le territoire communal, propriété de

\footnotetext{
${ }^{1515}$ Année de la mise en vigueur de la loi n $97-028$ du 15 janvier 1999 portant organisation de l'administration territoriale de la République du Bénin.

${ }^{1516} \mathrm{~L}$ 'article 7 de la loi n ${ }^{\circ} 2018-18$ portant règlementation sur les changements climatiques en République du bénin.
} 
la collectivité locale. Selon l'article 2 de la loi n $97-029$ du 15 janvier 1999 portant organisation des communes en République du Bénin, «la commune (...) est l'expression de la décentralisation et le lieu privilégié de la participation des citoyens à la gestion des affaires publiques locales». Les citoyens sont fixés sur le territoire communal que la collectivité locale administre librement par des conseils élus ${ }^{1517}$. En réalité, les collectivités locales sont des facteurs « $d u$ civisme local $»^{1518}$ dans la mesure où elles «favorisent la consultation et la participation des habitants à l'action publique de développement » ${ }^{1519}$. Selon «Action $21 »{ }^{1520}$, les collectivités locales doivent désormais être «missionnées pour suivre la planification des besoins et fixer les orientations et la réglementation locale qui concourent à l'application des politiques adoptées en matière de lutte contre les changements climatiques aux échelons national et international ${ }^{1521}$. Ainsi, le territoire communal pourra servir de base pour la promotion des actions de protection du climat. A titre indicatif, dans le cadre de la réalisation des contributions déterminées au niveau national, aux fins de l'atteinte de l'objectif de la Convention Cadre des Nations Unies sur les Changements Climatiques, l'Accord de Paris préconise que les Parties doivent prendre des mesures pour conserver et le cas échéant, renforcer les puits et réservoirs de gaz à effet de serre comme (..) notamment, les forêts ${ }^{1522}$. Ainsi donc, le Protocole de Kyoto a accordé une place importante à la gestion durable des forêts en recommandant à «chacune des Parties visées à l'annexe I pour s'acquitter de ses engagements chiffrés en matière de limitation et de réduction prévus à l'article 3 , de façon à promouvoir le développement durable (...) applique et/ou élabore (...) des politiques et des mesures (...) de protection et de renforcement des puits et des réservoirs de gaz à effet de serre (...) de promotion des méthodes durable de gestion forestière, de boisement et de reboisement (...) ». Il s'agit dans le cas d'espèce de renforcer les formations forestières existantes. Ce

\footnotetext{
1517 Article 153 de la constitution béninoise du 11 décembre 1990, révisée le 07 novembre 2019.

${ }^{1518}$ Michel PRIEUR, « Démocratie et droit de l'environnement et du développement », R.J.E, 1-1993, p. 25. ${ }^{1519}$ Id.

${ }^{1520}$ Action 21 est un guide qui concourt à la mise en œuvre du développement durable pour le $21^{\mathrm{e}}$ siècle. Il a été élaboré à l'occasion du Sommet de Rio de Janeiro de 1992. Plusieurs Nations ont décidé de l'appliquer au niveau national, régional et local. Disponible sur le site internet: https:/www.un.org/french/ga/special. Consulté le $18 / 01 / 2022$.

${ }^{1521}$ Le point 28-1 d'Action 21.

1522 Article 20 paragraphe 1 de l'Accord de Paris.
} 
renforcement peut être effectué à travers certaines techniques, notamment, l'agroforesterie ${ }^{1523}$ et la sylviculture ${ }^{1524}$, qui ne peuvent être réalisées que sur le territoire communal.

Malheureusement, c'est sur ce territoire de la collectivité locale que les politiques de mise en œuvre de la protection de l'environnement et de lutte contre les changements climatiques sont dangereusement démantelées par la réalisation de projets dont le seul objectif est la modernisation du cadre de vie. Dans ces conditions, la dégradation des ressources environnementales s'accélère.

En effet, la loi n²018-18 portant règlementation sur les changements climatiques en République du Bénin, en disposant que "l'Etat et les collectivités territoriales veillent à l'intégration, dans les politiques, stratégies, budgets et plans de développement communal, des mesures d'adaptation aux changements climatiques et à leurs effets et conséquences négatifs

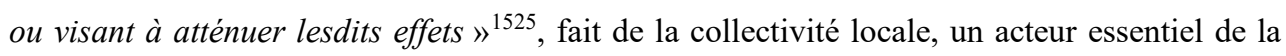
lutte contre les changements climatiques. Parce qu'en réalité, « ce sont les collectivités locales qui construisent, surveillent les processus de planification, fixent les orientations et la réglementation locale en matière d'environnement et apportent leur concours à l'application des politiques environnementales adoptées à l'échelon national ou infranational. Elles jouent au niveau administratif le plus proche de la population, un rôle essentiel dans l'éducation, la mobilisation et la prise en compte des préoccupations du public en faveur du développement durable $»^{1526}$. En effet, des actions tendant à contribuer à l'atténuation des changements climatiques par le développement des puits et réservoirs d'absorption de gaz à effet de serre telles que l'afforestation $^{1527}$, la promotion d'énergie propre encore appelée énergie verte ${ }^{1528}$,

\footnotetext{
${ }^{1523} \mathrm{~L}$ 'agroforesterie est une pratique qui consiste en l'utilisation des terres sur lesquelles des plantes ligneuses sont cultivées sur des parcelles exploitées pour des productions agricoles et/ou animales, selon la définition retenue à l'article $1^{\text {er }}$ de la loi ${ }^{\circ} 2018-18$ portant règlementation sur les changements climatiques en République du Bénin. ${ }^{1524}$ La sylviculture signifie culture des arbres. Elle peut être pratiquée soit par reboisement systématiquement soit par afforestation selon la définition retenue à l'article $1^{\text {er }}$ de la loi $n^{\circ} 2018-18$ portant règlementation sur les changements climatiques en République du Bénin.

${ }^{1525}$ Article 9 de la loi $n^{\circ} 2018-18$ portant règlementation sur les changements climatiques en République du Bénin. ${ }^{1526}$ Action 21, Chapitre 28 Principes d'action 28.1. Disponible sur le site internet : https://www.un.org/french/ga. Consulté le 18/01/2022.

${ }^{1527}$ Selon l'article $1^{\text {er }}$ de la loi n ${ }^{\circ} 2018-18$ portant règlementation sur les changements climatiques « Afforestation ou boisement : Plantation d'arbres ayant pour but d'établir un état boisé sur une surface longtemps restée dépourvue d'arbre ou n'ayant éventuellement jamais (aux échelles humaines de temps) appartenu à l'aire forestière. Elle se distingue du reboisement en ce que celui-ci est réalisé sur une surface boisée peu de temps auparavant ».

${ }^{1528}$ Selon l'article $1^{\text {er }}$ de la loi $\mathrm{n}^{\circ} 2018-18$ portant règlementation sur les changements climatiques « Une source d'énergie dont l'exploitation ne produit que des quantités négligeables de polluants par rapport à d'autres sources plus répandues et considérées comme plus polluantes. Le concept d'énergie propre est distinct de celui d'énergie renouvelable : le fait qu'une énergie se reconstitue n'implique pas que les déchets d'exploitation de cette énergie disparaissent, ni le contraire. Les sources d'énergie suivantes sont généralement citées comme énergie propre : énergie géothermique, haute ou basse énergie ; énergie éolienne ; énergie hydroélectrique ; énergie solaire ; biomasse ; énergie marémotrice, énergie des vagues, énergies hydroliennes, ...etc.»
} 
peuvent être entreprise sur le territoire dirigé par la collectivité locale. Ce qui peut faciliter la réalisation des engagements souscrits par le Bénin dans le cadre de la Convention Cadre des Nations Unies sur les Changements Climatiques.

Comment faire pour transformer le territoire communal en un milieu propice pour le développement des puits et réservoirs d'absorption de gaz à effet de serre afin de contribuer à l'atténuation des changements climatiques?

La loi n97-029 du 15 janvier 1999 portant organisation des communes en République du Bénin prescrit en son article 84 que «la commune élabore et adopte son Plan de Développement Communal (PDC)». Le Plan de Développement Communal est un « document de planification du développement de la commune sur une période de cinq (5) ans qui exprime les priorités retenues par l'ensemble des composantes sociales de la commune ${ }^{1529}$. Il constitue un « instrument de cadrage de la politique communale et de mise en œuvre des actions de développement à court et moyen terme par le Conseil communal et portées par tous ${ }^{1530}$. Ce document est élaboré sur la base d'une « méthodologie qui intègre toutes les préoccupations de développement durable, les prévisions et programmations d'ordre social, économique, spatial, environnemental, les préoccupations circonstancielles de la commune $\gg{ }^{1531}$ qui sont conformes aux orientations de l'Etat. Seulement que le Plan de Développement Communal (PDC) ne contenait que des informations concernant la protection de l'environnement, mais pas celles relatives aux changements climatiques. Compte tenu de son importance, il est possible d'agir sur cet instrument de mise en œuvre de la politique de développement de la commune pour atteindre l'objectif de lutte contre les changements climatiques, par l'intégration de la dimension concernant les changements climatiques, conformément aux stipulations de la loi $\mathrm{n}^{\circ}$ 2018-18 portant règlementation sur les changements climatiques en République du Bénin ${ }^{1532}$.

Par conséquent, la loi n 97-029 du 15 janvier 1999 portant organisation des communes en République du Bénin devra être révisée pour recevoir l'intégration de la dimension concernant les changements climatiques. Elle devra préciser davantage les domaines de compétence des collectivités locales qui seront étendues désormais aux changements climatiques.

\footnotetext{
${ }^{1529}$ République du Bénin, Ministère de la Décentralisation, de la Gouvernance Locale, de l'Administration et de l'Aménagement du Territoire (MDGLAAT), Guide d'élaboration du plan de développement communal actualisé version https://www.ancb-benin.org/Document_pdf/Guide_PDC. Consulté le 08/06/2021.

1530 Id.

1531 Ibid.

${ }^{1532}$ Il de l'ensemble des dispositions contenues dans le Chapitre III : Des obligations de prise en compte des changements climatiques dans les stratégies et planifications nationales et infranationales.
} 
Par ailleurs, pour ce qui concerne la mise en œuvre des plans d'efficacité énergétique, « l'Etat doit également veiller au développement et à l'utilisation de sources d'énergie propre et alternative aux combustibles fossiles en initiant au profit des élus locaux le droit à l'utilisation de l'énergie renouvelable» ${ }^{1533}$. Inversement, l'autorité communale doit édicter des mesures tendant à l'encadrement de cette activité sur son territoire dans l'intérêt de la population.

Les collectivités territoriales qui recevront désormais des attributions en matière de lutte contre les changements climatiques exerceront leurs compétences climatiques sous la tutelle du Préfet, qui représente l'autorité déconcentrée, laquelle doit aussi être investie des compétences climatiques.

\section{B-L'attribution de compétence climatique à l'autorité déconcentrée}

L'Etat en tant qu'acteur principal du droit international, participe aux négociations et ratifie les accords internationaux en matière de lutte contre les changements climatiques. Passée cette étape, il s'investit au plan national dans l'élaboration des mesures législatives et réglementaires et dans la création d'institutions de mise en œuvre de la règlementation sur les changements climatiques. Mais en plus, «l'Etat et les collectivités locales (...) veillent à l'intégration dans les politiques, stratégies, budgets et plans de développement communal des mesures d'adaptation aux changements climatiques et à leurs effets et conséquences négatifs ou visant à atténuer lesdits effets» ${ }^{1534}$. Ils « (...) adoptent et mettent en œuvre un plan annuel d'alerte précoce sur les phénomènes résultant des changements climatiques ou y afférents. Ils créent, renforcent et assurent de façon durable le fonctionnement des équipements et systèmes d'observation et de surveillance météorologiques, climatologiques, hydrologiques et océanographiques. L'Etat élabore, adopte et met en œuvre un programme national pour améliorer les connaissances et capacités en la matière ${ }^{1535}$. Il « (...) appuie les collectivités locales dans la prise de toutes les mesures visant à accroître la résilience des populations locales ${ }^{1536}$. Mais également, certaines interdictions ont été édictées, tout comme, « (...) des mesures pour prévenir, réduire, combattre et maîtriser les effets négatifs des changements climatiques $(\ldots) »^{1537}$. A titre indicatif, « (...) l'Etat s'abstient de transférer directement ou indirectement le préjudice ou les risques de ses activités anthropiques d'un département à un

\footnotetext{
${ }^{1533}$ Article 8 de la loi n²018-18 portant règlementation sur les changements climatiques en République du Bénin.

1534 Article 9 id.

1535 Article 22 ibid.

${ }^{1536}$ Article 10 de la loi n²018-18 portant règlementation sur les changements climatiques e République du Bénin.

${ }^{1537}$ L'article 18 id.
} 
autre, d'une commune à une autre et à ne pas substituer un type de pollution d'ampleur supérieure ou égale à un autre ${ }^{1538}$. Il s'avère que le rôle de l'Etat dans la mise en œuvre de la règlementation élaborée dans le cadre de la lutte contres les changements climatiques est d'une importance croissante. La loi a programmé à la charge de l'Etat beaucoup de mesures qui sont constituées des obligations de faire ou de ne pas faire des actions dans le cadre de la lutte contre les changements climatiques sur toute l'étendue du territoire national. Toutes ces mesures sont la déclinaison des dispositions contenues dans l'article 27 de la Constitution béninoise ${ }^{1539}$ dont l'objectif finaliste est de faire garantir par l'Etat un environnement sain, satisfaisant et durable à la population.

Cependant, il y a des difficultés de fonctionnement de l'administration caractérisées par la lenteur et la centralisation des activités gouvernementales qui font craindre que, les activités prévues dans le cadre de la lutte contre les changements climatiques pour être exécutées sur toute l'étendue du territoire national, ne connaissent pas leur aboutissement.

Comment faire pour disséminer les actions climatiques de l'Etat central sur toute l'étendue du territoire national?

Pour que les mesures édictées par le Législateur béninois dans le cadre de la lutte contre les changements climatiques impactent toute la population, il faut mettre à contribution les structures déconcentrées de l'Etat. Ces institutions vont servir d'interface entre l'Etat central et les collectivités de base pour répercuter sur ces dernières, les actions climatiques entreprises par l'Etat central. C'est en ce sens que la politique de déconcentration de l'administration publique qui a suivi la réforme constitutionnelle intervenue en $1990^{1540}$ s'avère nécessaire. Les actes juridiques qui sont consacrés à l'application de cette politique ont conféré des compétences de mise en œuvre de la politique gouvernementale sur le territoire national, aux Préfets des départements. Seulement que la règlementation ne leur a conféré que d'infirme attribution en matière de protection de l'environnement. Et même, ces compétences environnementales qui leurs sont attribuées par les textes épars, n’ont pas défini de manière étanche les limites de leur intervention. L'article 20 de la loi n $97-028$ du 15 janvier 1999 portant organisation de l'administration territoriale de la République du Bénin, en énumérant les attributions environnementales du Préfet en relation avec les communes qui relèvent de son ressort, par la stipulation suivante, que «le conseil départemental de concertation et de

\footnotetext{
1538 Ibid.

${ }^{1539}$ Constitution du 11 décembre 1990 révisée le 07 novembre 2019.

${ }^{1540}$ Adoption de la loi constitutionnelle le 11 décembre 1990 après la tenue de la conférence des forces vives de la nation béninoise en février 1990.
} 
coordination est obligatoirement consulté sur les programmes de développement économique, social et culturel des communes et sur la mise en cohérence de ceux-ci avec les programmes nationaux, (...) sur le schéma d'aménagement du territoire et les projets de développement du département; les mesures de protection de l'environnement; (...) les forêts classées et les zones cynégétiques (...)», confine le Préfet dans un rôle d'organe consultatif. Mais en plus, aucune disposition de la loi sus-indiquée ne mentionne les mesures relatives à la lutte contre les changements climatiques qui relèvent de la compétence du Préfet. Ainsi, l'autorité départementale ne parvient pas à s'affirmer comme étant un acteur au premier plan de la protection de l'environnement dans son département. Son incursion dans le domaine de la lutte contre les changements climatiques n'est que de fait, parce que le cadre juridique devant régir son intervention dans ce domaine n'existe pas.

Pour que la politique du Gouvernement béninois en matière de lutte contre les changements climatiques soit implémentée dans toutes les contrées du pays, il faut mettre à contribution le Préfet en sa qualité de représentant d'organe déconcentré de l'Etat en lui donnant, en plus de ses attributions, le statut d'acteur climatique.

Actuellement, le Bénin compte douze (12) Départements administrés chacun par un Préfet ${ }^{1541}$. Ceux-ci constituent des interfaces entre l'administration centrale et les collectivités de base. Le rayonnement des actions de lutte contre les changements climatiques à travers le territoire national, peut être facilité par la mise à contribution efficace de l'autorité déconcentrée. Compte tenu de ce qui précède, il convient alors de renforcer l'arsenal juridique qui encadre le rôle du Préfet de département, par son implication dans le domaine de la lutte contre les changements climatiques, en procédant à la relecture de la loi n $97-028$ du 15 janvier 1999 portant organisation de l'administration territoriale de la République du Bénin et la loi ${ }^{\circ}$ 97-029 du 15 Janvier 1999 portant organisation des communes en République du Bénin, pour élargir le champ de compétence du Préfet aux domaines des changements climatiques. De même, la loi n²018-18 portant règlementation sur les changements climatiques en République du Bénin doit être révisée pour intégrer le préfet comme acteur devant participer à la dissémination de la politique du pourvoir central. En procédant ainsi, le Préfet deviendra un acteur actif de la lutte contre les changements climatiques et pourra mieux accomplir sa fonction de tutelle des communes de son ressort surtout en ce qui concerne la mise en œuvre de la règlementation sur les changements climatiques par les collectivités locales. L'intervention du

\footnotetext{
${ }^{1541}$ Les articles 6 et 9 de la loi n ${ }^{\circ}$ 97-029 du 15 Janvier 1999 portant organisation des communes en République du Bénin.
} 
Préfet permettra de faire émerger la politique gouvernementale en matière de lutte conte les changements climatiques partout sur toute l'étendue du territoire national.

Dans le cas où les compétences climatiques seront affectées aux démembrements de l'Etat pour accentuer leur intervention dans le domaine de la lutte contre les changements climatiques, il faudra procéder à l'amélioration de l'encadrement climatique.

\section{SECTION II : L'AMELIORATION DE L'ENCADREMENT CLIMATIQUE}

Tout en fixant un objectif uniforme et commun à toute la Communauté internationale, celui de contenir et de réduire sensiblement les risques et les effets des changements climatiques par « (...) l'élévation de la température moyenne de la planète nettement en dessous de $2{ }^{\circ} \mathrm{C}$ et en poursuivant l'action menée pour limiter l'élévation de la température à $1,5^{\circ} \mathrm{C}$ par rapport aux niveaux préindustriels, l'Accord de Paris, « (...) vise à renforcer la riposte mondiale à la menace des changements climatiques, $(\ldots){ }^{1542}$. Pour contenir le phénomène dans les limites fixées, le cadre normatif et institutionnel de la Convention Cadre des Nations Unies sur les Changements Climatiques a été amélioré. Cet effort de raffinement de l'instrument élaboré par la Communauté internationale pour lutter contre les changements climatiques doit être entrepris au niveau de chaque Partie.

Le Bénin devra s'engager dans les réformes tendant à spécifier son environnement juridique (Paragraphe 1) et le cadre institutionnel, destinés à la lutte contre les changements climatiques (Paragraphe2).

\section{Paragraphe 1: La spécification de l'environnement juridique}

Pour tenir compte de ce que l'environnement juridique destiné à la lutte contre les changements climatiques contient plusieurs textes de loi qui ne sont élaborés exclusivement pour la lutte contre les changements climatiques, il convient d'aménager le cadre juridique dans l'objectif de sa spécification. Il s'agira en effet dans cette rubrique, d'envisager la réforme du cadre législatif (A) et de projeter la révision de la Constitution béninoise (B) en vue de l'intégration de la dimension concernant les changements climatiques.

${ }^{1542}$ Article 2 paragraphe 1-a de l'Accord de Paris. 


\section{A- La réforme du cadre législatif}

Le traité international sur le climat ${ }^{1543}$ actualisé à l'occasion de la COP21 tenue à Paris en $2015^{1544}$ a reconnu «(...) la nécessité d'une riposte efficace et progressive à la menace pressante des changements climatiques (...) ${ }^{1545}$. C'est pourquoi l'Accord de Paris a prescrit que, «les Etats doivent promulguer des mesures législatives efficaces en matière d'environnement. Et que les normes écologiques et les objectifs et priorités pour la gestion de l'environnement devraient être adaptés à la situation en matière d'environnement et de développement à laquelle ils s'appliquent (...) ${ }^{1546}$. Cette disposition laisse transparaitre la nécessité pour chaque Etat Partie d'opérer des réformes du cadre législatif destiné à la lutte contre les changements climatiques.

Au-delà de ce que le Bénin est signataire dudit Accord et devra s'engager pour son application, il y a des motifs qui découlent de la faiblesse de l'encadrement juridique et du fonctionnement défaillant de l'administration béninoise qui justifient la nécessité de rendre perfectible le droit de protection du climat dans ce pays. D'où, la nécessité d'une réforme du cadre législatif destiné à la lutte contre les changements climatiques.

Quelles sont les orientations des réformes à envisager?

Nombreuses sont des lois qui sont élaborées pour la protection de l'environnement et mises à contribution dans le cadre de la lutte contre les changements climatiques au Bénin. Ces lois, pour autant qu'elles soient pertinentes, ne sont pas pour la plupart efficaces dans le contexte des changements climatiques. A titre indicatif, selon le décret n²004-273 du 12 mai 2004 portant approbation de la charte nationale sur la gouvernance environnementale au Bénin, «(...) la loi cadre sur l'environnement est la base de l'action de la politique en matière d'environnement (...) $»^{1547}$. De même, l'article $1^{\text {er }}$ de la loi cadre sur l'environnement précise davantage son caractère fondamental en matière d'encadrement de la protection de l'environnement, et complète que la même loi «(...) organise la mise en æuuvre de la politique en matière d'environnement (...)» ${ }^{1548}$. Il ressort de ces dispositions, que l'élément basique du cadre législatif de protection de l'environnement au Bénin est sans nul doute la loi cadre sur l'environnement ${ }^{1549}$. Certaines dispositions de cette loi sont utilisées dans le cadre de la lutte

\footnotetext{
${ }^{1543}$ La Convention Cadre des Nations Unies sur les Changements Climatiques adoptée à Rio de Janeiro en 1992.

${ }^{1544}$ L'actualisation a été faite par l'adoption de l'Accord de Paris en 2015.

1545 Paragraphe 5 du préambule de l'Accord de Paris de 2015.

${ }^{1546}$ Principe 11 de la Déclaration de Rio sur l'Environnement et le Développement de Rio de Janeiro de 1992.

${ }^{1547}$ Article 4 dudit décret.

${ }^{1548}$ Loi n $98-030$ du 12 février 1999 portant loi cadre sur l'environnement en République du Bénin.

${ }^{1549} \mathrm{C}^{\prime}$ 'est-à-dire la loi n ${ }^{\circ} 98-030$ du 12 février 1999 portant loi cadre sur l'environnement en République du Bénin.
} 
contre les changements climatiques, alors qu'elles n'ont aucun rapport avec les changements climatiques. Par exemple, l'évaluation environnementale ou l'étude d'impact environnemental préalable est prévue par la loi cadre sur l'environnement ${ }^{1550}$. Mais les dispositions qui organisent la réalisation de l'étude d'impact environnementale n'ont jamais fait allusion aux dimensions concernant les changements climatiques. Il convient donc, de corriger cette insuffisance par l'intégration de la dimension concernant les changements climatiques dans la loi cadre sur l'environnement au Bénin.

Toutefois, à titre d'innovation, il serait judicieux d'élaborer une loi cadre sur les changements climatiques qui va servir comme pilier dans l'arsenal juridique d'encadrement des changements climatiques au Bénin et coexister avec la loi $\mathrm{n}^{\circ} 2018-18$ portant règlementation sur les changements climatiques en République du Bénin. L'adoption d'une loi cadre sur les changements climatiques viendra revaloriser la qualité de l'encadrement juridique et permettra d'organiser les actions de protection du climat dans un cadre coordonné et suivant des objectifs définis dans le cadre de la mise en œuvre de la politique de l'Etat en matière de lutte contre les changements climatiques.

Certaines mesures prises au Bénin en vue de l'atténuation des changements climatiques sont concentrées autour du domaine de la protection des forêts. La gestion des forêts étant régie par la loi n 93-009 du 02 juillet 1993 portant régime des forêts en République du Bénin. Malheureusement, cette loi ne contient aucune disposition concernant les changements climatiques. Il convient alors de procéder à la relecture de ladite loi, pour d'une part y intégrer les mesures devant régir, la relation entre la protection de la forêt et la lutte contre les changements climatiques, et d'autre part, comme l'a suggéré un auteur, d'envisager des dispositions nouvelles en vue de « l'intégration des entreprises à la lutte contre les changements climatiques $»^{1551}$.

En outre, l'adoption de la loi $\mathrm{n}^{\circ} 2018-18$ portant règlementation sur les changements climatiques en République du Bénin n'a pas suffit pour régler tous les aspects concernant la lutte contre les changements climatiques. A titre illustratif, le Législateur béninois n'a pas encore élaboré des mesures pour garantir l'effectivité de la participation de la société civile à la lutte contre les changements climatiques. Il s'ensuit que le Législateur devra intervenir pour initier d'autres mesures climatiques plus enrichissantes, qui prennent en compte certains aspects de la protection du climat qui ne sont pas régis par les lois en vigueur notamment, les mesures

\footnotetext{
${ }^{1550}$ Article 21 de la loi n ${ }^{\circ} 98-030$ portant loi cadre sur l'environnement en République du Bénin.

${ }^{1551}$ Sophie Lavallée, «Quelques réflexions sur l'Accord de Paris et la souveraineté économique des Etats », Vertigo, la revue électronique en science de l'environnement, Volume 18 numéro 1, mai 2018, p. 13.
} 
concernant le domaine de « l'utilisation des énergies renouvelables aux fins d'atténuation des changements climatiques $»$.

Par ailleurs, la loi ${ }^{\circ} 2018-18$ portant règlementation sur les changements climatiques en République du Bénin a introduit une confusion dans le système de gouvernance au Bénin par la création du «ministère en charge des Changements Climatiques ${ }^{1552}$, contrairement aux prescriptions et à la coutume. La création de « ministère » est une prérogative du Président de la République concédée par l'article 54 de la constitution béninoise du 11 décembre 1990 révisée le 07 novembre 2019 qui dispose que, «le Président de la République (...) nomme, après avis consultatif du bureau de l'Assemblée nationale, les membres du gouvernement ; il fixe leurs attributions et met fin à leurs fonctions (...) ». En l'état, le ministère en charge des Changements Climatiques créé dans ces conditions risque de ne pas fonctionner compte tenu du précédent à l'origine de sa création, parce que les pouvoirs politiques sont jaloux de leurs prérogatives et ne tolèrent point l'empiètement de leur domaine. C'est un aspect dont il faut tenir compte lors de la révision de la loi ${ }^{\circ} 2018-18$ portant règlementation sur les changements climatiques en République du Bénin soit, en supprimant la mesure ou soit, en élaborant d'autres dispositions complémentaires pour contraindre l'exécutif à rendre effectif la disposition de la loi qui a créé le ministère en charge des Changements Climatiques. Par rapport à la même préoccupation, il est possible de faire recours à l'arbitrage de la Cour constitutionnelle qui intervient également dans la « régulation du fonctionnement des institutions et de l'activité des pouvoir publics $»^{1553}$. Après sa saisine, cette juridiction pourra rendre une décision pour situer les autorités compétentes sur la conduite à tenir en ce qui concerne l'option de création du ministère en charge de la gestion des changements climatiques.

Si la réforme est bien raffinée, elle va déboucher sur des mesures qui rendent autonome le droit climatique béninois. Ainsi, l'encadrement juridique qui en découlera devra à lui seul suffire pour régler les problèmes relevant du domaine, sans qu'il soit besoin de faire recours à des règles autres que celles qui sont destinées au domaine de la lutte contre les changements climatiques.

Cependant, il serait illusoire d'envisager objectivement la réforme du cadre législatif dans le contexte béninois caractérisé par un niveau de développement moins élevé sans étudier les conditions de sa mise en œuvre. Au titre de la constitution béninoise, les prérogatives législatives sont exercées par les députés à l'assemblée nationale qui ont « l'initiative des lois

\footnotetext{
${ }^{1552}$ Article 77 de la loi n²018-18 portant règlementation sur les changements climatiques en République du Bénin. ${ }^{1553}$ Article 114 de la constitution béninoise du 11 décembre 1990 révisée le 07 novembre 2019.
} 
concurremment au Président de la République ${ }^{1554}$. Seulement que «Les propositions et amendements déposés par les députés ne sont pas recevables lorsque leur adoption peut avoir pour conséquence soit une diminution des ressources publiques soit, la création ou l'aggravation d'une charge publique à moins qu'elles ne soient accompagnées d'une proposition d'augmentation de recettes ou d'économies équivalentes $\gg{ }^{1555}$. La lecture de cette disposition permet de comprendre que le pouvoir dont dispose les députés pour légiférer est limité dans la mesure où il est conditionné par la disponibilité des moyens financiers. Alors que la réforme du cadre législatif envisagée devra nécessiter des ressources financières pour sa réalisation.

Comment lever les difficultés relatives aux aspects financiers qui conditionnent la réalisation des réformes qui pourtant sont nécessaires pour améliorer la gouvernance climatique au Bénin?

Dans ce cas, l'alternative qui s'offre pour le Bénin par exemple, est la mise à contribution de l'économie circulaire, « un modèle de croissance résiliente ${ }^{1556}$, un concept qui « consiste à recycler les produits et les matériaux et à les réutiliser dans un processus industriel qui permet une utilisation optimale des déchets» ${ }^{1557}$. L'économie circulaire est utilisée comme, «un élément central à la fois stratégique à long terme pour parvenir à une neutralité climatique en Europe» ${ }^{1558}$. Elle peut être expérimentée en Afrique et surtout au Bénin, car elle relève des réalités des pays en voie de développement et est compatible avec les réalités en matière de lutte contre les changements climatiques. Ce secteur d'activité est en plein essor au Bénin, mais ne bénéficie d'aucun encadrement juridique, alors qu'il brasse d'importantes ressources financières. Il constitue un manque à gagner pour l'économie béninoise, parce que ce secteur peut générer d'importantes ressources qui serviront à amorcer les réformes du cadre législatif de protection du climat. Il convient donc de mettre en avant les réformes du genre pour faciliter la disponibilité des ressources pouvant servir à accompagner des réformes dans le cadre de la lutte contre les changements climatiques.

La réforme du cadre législatif relatif à la lutte contre les changements climatiques pourra également être facilitée, si la constitution béninoise est réformée et adaptée aux dimensions concernant les changements climatiques.

\footnotetext{
${ }^{1554}$ Article 105 de la constitution béninoise du 11 décembre 1990 révisée le 07 novembre 2019.

${ }^{1555}$ Article 107 id.

${ }^{1556}$ BENZAIDA (Kamal), Prospects and Actions in Africa to cope with Climate Change Effects, Géneva Global Policy Briefs n³, 2020.

${ }^{1557}$ Id.

${ }^{1558}$ Ibid.
} 


\section{B- La réforme de la constitution béninoise}

La constitution est «l'ensemble des règles écrites ou coutumières qui déterminent la forme de l'Etat, la dévolution et l'exercice du pouvoir $\gg{ }^{1559}$. Elle représente la norme supérieure qui précise les matières qui relèvent du domaine législatif. A titre illustratif, l'article 98 de la constitution béninoise du 11 décembre 1990 révisée le 07 novembre 2019 dispose que, « (...) La loi détermine les principes fondamentaux (...) de la protection de l'environnement et de la conservation des ressources naturelles (...) ». Plusieurs dispositions dont, les articles 27, 28, 29 et 74 sont consacrées au volet de protection de l'environnement. Il en ressort que les règles devant régir le domaine de protection de l'environnement ont leur fondement juridique dans la constitution. Cela étant, toute réforme du cadre législatif envisagée en ce qui concerne le domaine des changements climatiques se fera en application des dispositions constitutionnelles en la matière.

Si la Constitution béninoise du 11 décembre 1990 est « réputée comme l'une des constitutions africaines les plus vertes, pour avoir élevé la protection de l'environnement au rang de droit constitutionnel» ${ }^{1560}$ et permis de lancer le Bénin sur le chantier de la protection de l'environnement, malheureusement en l'état, aucune disposition de cette constitution n'a tenu compte de la dimension concernant les changements climatiques. Son contenu n'est pas paramétré pour servir à encadrer le domaine du réchauffement climatique. Il en est ainsi parce que cette constitution a été élaborée en 1990, soit environ deux (2) décennies après la tenue de la Conférence de Stockholm de 1972. L'avènement du Sommet de Rio de Janeiro en 1992 a contribué au bouleversement de l'ordonnancement juridique international par la prise en compte des changements climatiques dans l'élaboration des normes de protection de l'environnement. Cependant, la constitution béninoise du 11 décembre 1990 qui devrait subir les effets de l'influence du droit international climatique est demeurée sans modification, alors que le Bénin a adhéré à la convention climat. Paradoxalement, la révision de ladite constitution intervenue le 07 novembre 2019 n'a pas non plus intégré les préoccupations concernant les changements climatiques.

La loi fondamentale béninoise qualifiée au temps jadis de " constitution la plus verte de la sous-région ouest africaine " permet-elle en l'état de relever les défis qu'imposent les changements climatiques?

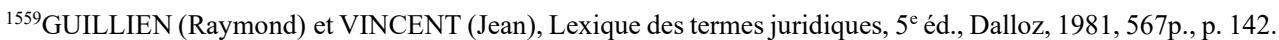
${ }^{1560}$ Michel PRIEUR, La mise en œuvre nationale du droit international de l'environnement dans les pays francophones, Actes des troisièmes journées scientifiques du Réseau- Droit de l'environnement de la francophonie Yaoundé (Cameroun), 14-15 juin 2001.
} 
En raison de l'incorporation par la ratification des accords internationaux de protection du climat ${ }^{1561}$ à son corpus juridique, le droit positif béninois en la matière présage de sa capacité à fournir les outils juridiques nécessaires pour faire face aux enjeux majeurs des changements climatiques. Mais en réalité, ce n'est qu'une vue d'esprit, parce que à l'évidence, il n'a pas été toujours facile de développer les bases d'un droit climatique autonome en absence de sources ou principes constitutionnellement établies. S'il est évident que les règles de protection de l'environnement peuvent être étendues aux domaines des changements climatiques, ces dernières admettent des spécificités qui méritent de prendre des dispositions appropriées. Dans ces conditions, sans le réaménagement de la Constitution, il ne saurait y avoir une réforme fondamentale du cadre législatif relatif à la lutte contre les changements climatiques.

Que faut-il pour réaliser une réforme fondamentale des dispositions applicables à la lutte contre les changements climatiques?

Pour répondre aux questions d'encadrement efficace de la lutte contre les changements climatiques au Bénin, la réforme constitutionnelle s'impose. Mais ce n'est pas une nécessité impérative sans quoi la lutte contre les changements climatiques ne peut continuer. Car, ce qui est à craindre est lié aux controverses que soulève la question de la révision de la constitution dans un Etat démocratique à économie modeste. Et surtout qu'au Bénin, il y a une particularité relative à la recherche du consensus imposée par la jurisprudence sans quoi le principe de révision de la Constitution ne peut être admis ${ }^{1562}$. Alors que la recherche du consensus est difficilement réalisable à cause des controverses et des contradictions que suscitent les questions relatives aux réformes à caractère politique. Cette solution relève d'une vision futuriste en matière de planification des mesures à prendre pour consolider la gestion des changements climatiques au Bénin. Lorsque les conditions seront favorables pour la révision constitutionnelle, l'Etat béninois pourra s'engager sur cette piste pour asseoir les bases juridiques et institutionnelles du domaine des changements climatiques par l'élaboration des principes généraux qui devront gouverner désormais le domaine. L'objectif in fine, sera de faire figurer l'action en faveur de la lutte contre le réchauffement climatique parmi les principes fondateurs de la République béninoise dans la constitution. C'est aussi une exigence la loi n²018-18 portant règlementation sur les changements climatiques en République du Bénin, qui recommande la «prise en compte des changements climatiques dans les stratégies et

\footnotetext{
${ }^{1561}$ Le Bénin a ratifié la Convention Cadre des Nations Unies sur les Changements Climatiques et ses accords subséquents. Etat de ratification de la Convention Cadre des Nations Unies sur les Changements Climatiques. Disponible sur le site internet : https://treaties.un.org.Consulté le 18/01/2022.

${ }^{1562}$ Selon la décision $n^{\circ}$ DCC 06-074 du 8 juillet 2006 de la Cour constitutionnelle du Bénin. Cour constitutionnelle béninoise : https://courconstitutionnelle.bj/decisions-2. Consulté ce 29/05/2021.
} 
planifications nationales et infranationales ${ }^{1563}$. Dans la pratique, il convient d'élever dans la loi fondamentale, la lutte contre les changements climatiques au rang de principe constitutionnel par l'élargissement du contenu de l'article 98 de la constitution du 11 décembre 1990 révisée le 07 novembre 2019 aux dispositions concernant les changements climatiques. Car, c'est cet article qui détermine les matières qui relèvent du domaine de la loi.

La création de l'Autorité Nationale des Changements Climatiques (ANCC), telle que proposée dans le cadre de notre étude, ainsi que les modalités de désignation des membres de ladite juridiction, le mandat et aussi les règles applicables devant elle, sont des aspects qui devront figurer dans la constitution amendée. Il en sera de même en ce qui concerne le Comité National sur les Changements Climatiques (CNCC), qui devra être pris en compte à l'occasion de la révision de la constitution.

L'antécédent provoqué par la loi n²018-18 portant règlementation sur les changements climatiques en République du Bénin qui a créé le Ministère des Changements Climatiques ${ }^{1564}$, alors que cette attribution relève de la compétence du Président de la République pourra être corrigé par l'insertion de la mesure dans la constitution. De la même manière, l'intégration de la dimension de lutte contre les changements climatiques dans la constitution permettra à la Cour constitutionnelle, à l'Assemblée nationale à l'Autorité nationale des changements climatiques ${ }^{1565}$ ou aux juridictions compétentes de prendre en compte au mieux, les impacts sur le climat dans la mise en œuvre des dispositions applicables à la lutte contre les changements climatiques. L'ancrage de ces institutions climatiques dans la constitution va leur conférer la pérennité. Elle contribuera à conforter leur autorité et le caractère exécutoire des décisions qu'elles sont appelées à prendre dans le cadre de l'exercice des activités relevant de leur compétence.

La réussite de la révision de la Constitution en faveur de l'intégration des dispositions applicables aux changements climatiques va permettre de disposer d'une base juridique constitutionnelle appropriée à la thématique pour générer un encadrement institutionnel amélioré.

\footnotetext{
${ }^{1563}$ Chapitre 3 de la loi n ${ }^{\circ} 2018$-18 portant règlementation sur les changements climatiques en République du Bénin. 1564 Son article 77.

${ }^{1565}$ Voir infra la rubrique (A- La création d'une autorité des changements climatiques, p.344 à 347).
} 


\section{Paragraphe 2 : L'amélioration du cadre institutionnel}

Le cadre institutionnel élaboré pour la mise en œuvre de la Convention Cadre des Nations Unies sur les Changements Climatiques à l'épreuve des réalités objectives induites par le fléau planétaire a révélé quelques faiblesses. N'est ce pas le constat fait par M. Youba Sokona, coprésident du Groupe de travail III du GIEC, qui déclare, qu' «Il est possible, sur le plan technique, de passer à une économie à faible teneur en carbone. Mais ce qui manque, ce sont des politiques et des institutions appropriées(...) ${ }^{1566}$. L'Organisation Internationale de la Francophonie (OIF), s'est également préoccupée de la problématique du renforcement du cadre institutionnel de protection du climat et a recommandé de «prendre d'urgence des mesures pour lutter contre les changements climatiques et leurs répercussions par l'amélioration, des capacités institutionnelles en ce qui concerne l'adaptation aux changements climatiques, l'atténuation de leurs effets et la réduction de leur impact et les systèmes d'alerte rapide ${ }^{1567}$.

Face à ces préoccupations institutionnelles diversement exprimées, la réponse de la Communauté internationale ne s'est pas fait attendre. L'Accord de Paris de 2015, tout en contribuant à l'amélioration du cadre institutionnel international ${ }^{1568}$ a également recommandé aux Etats Parties, « de renforcer les dispositifs institutionnels, notamment, ceux relevant de la Convention, qui concourent à l'application dudit Accord (...) ${ }^{1569}$. Inéluctablement, le raffinement du droit climatique à l'occasion de la COP21 à Paris en 2015, doit avoir des retombés sur les institutions des Etats Parties.

En visitant le cadre institutionnel béninois de protection du climat, lequel n'est pas à l'abri des austérités opposées par les changements climatiques parce que, parfois inadéquat ou désuet, notre étude propose que le Bénin s'inscrive dans le sens de la recommandation de l'Accord de Paris en ce qui concerne le renforcement du cadre institutionnel. En effet, il doit prendre des mesures pour engager des réformes du cadre institutionnel aux fins de le renforcer. Cette réforme doit avoir pour objectif de construire une architecture institutionnelle essentiellement orientée vers les objectifs de lutte contre les changements climatiques. Elle

\footnotetext{
${ }^{1566}$ GROUPE D'EXPERTS INTERGOUVERNEMENTAL SUR L'EVOLUTION DU LCIMAT COMMUNIQUÉ DE PRESSE DU GIEC, Conclusion du cinquième rapport d'évaluation: Les changements climatiques pourraient avoir des incidences irréversibles et dangereuses, mais il existe des options pour en limiter les effets, publié le 2 novembre 2014 13e paragraphe, p.2. Disponible sur le site internet : https://archive.ipcc.ch. Consulté le 01/06/2021.

${ }^{1567}$ Le point 13 de l'objectif 2030. Disponible sur le site internet: https://www.objectif2030.org/objectif/13. Consulté le 01/06/2021.

${ }^{1568}$ Création du cadre de transparence et du cadre technologique.

${ }^{1569}$ Article 7 paragraphe7-b de l'Accord de Paris de 2015.
} 
donnera lieu à la création d'une l'Autorité Nationale des Changements Climatiques (ANCC) (A) et à la restructuration du Comité National des Changements Climatiques (CNCC) (B).

\section{A- La création de l'Autorité Nationale des Changements Climatiques}

La loi n²018-18 portant règlementation sur les changements climatiques en République du Bénin dispose en son article 86 que, « les différends relatifs aux actes délivrés ou accomplis par l'administration publique (...) relèvent de la compétence des juridictions administratives ». La même loi en son article 88 stipule que, « la compétence des juridictions administratives n'exclut pas la saisine alternative de la Cour constitutionnelle lorsque, dans le cadre de ses attributions, l'autorité chargée de la lutte contre les effets et conséquences négatifs des changements climatiques pose un acte ou entreprend toute action qui viole les droits fondamentaux de la personne ». Par ces dispositions, la loi précitée a posé les bases juridiques $\mathrm{du}$ contrôle juridictionnel en matière de lutte contre les changements climatiques par la désignation des juridictions compétentes pour statuer sur les cas de violation des dispositions de ladite loi. Cependant, à défaut de créer de nouvelles juridictions pour gérer les litiges climatiques, la loi $\mathrm{n}^{\circ} 2018-18$ portant règlementation sur les changements climatiques en République du Bénin a simplement requis la compétence des juridictions de droit commun ${ }^{1570}$ notamment, les tribunaux d'instance en élargissant leur champ de compétence au domaine de la lutte contre les changements climatiques ${ }^{1571}$. Alors que l'organisation judiciaire de la République du Bénin telle que conçue, n'est pas adaptée à la gestion des conflits environnementaux, encore moins, les litiges climatiques ${ }^{1572}$. Lorsque les juridictions de droit commun sont saisies des conflits environnementaux ou climatiques, ses acteurs n'ont pas l'expertise nécessaire pour juger ces différends. L'institution judiciaire n'est pas aussi adaptée au règlement des conflits climatiques avec les procédures qui sont applicables devant elle.

Dans ces conditions, la réforme des institutions, judiciaire et juridictionnelle est une condition sine qua none pour l'amélioration de la lutte contre les changements climatiques au Bénin. Cette réforme doit être orientée dans le sens de la professionnalisation de l'architecture

\footnotetext{
${ }^{1570}$ Articles 86,88 et 92 de la loi n²018-18 portant règlementation sur les changements climatiques en République du Bénin

${ }^{1571}$ Les compétences climatiques des tribunaux de droit commun sont déclinées dans les dispositions des articles 86,88 et 95 de la loi $n^{\circ} 2018-18$ portant règlementation sur les changements climatiques en République du Bénin. ${ }^{1572}$ La loi n ${ }^{\circ} 2001-37$ du 27 août 2002 portant organisation judiciaire en République du Bénin modifiée par la loi n²016-15 du 28 juillet 2016 ne prend pas en compte les spécificités que revêtent la protection de l'environnement et la lutte contre les changements climatiques.
} 
institutionnelle ${ }^{1573}$ et sera soldée par l'existence d'une organisation judiciaire qui intègre les dimensions concernant l'action climatique. Mais, en considérant la faible capacité financière du Bénin, il sera difficile pour ce pays de faire l'option de la séparation étanche des institutions en créant des juridictions climatiques distinctes des juridictions de droit commun. Pour éviter d'engendrer des réformes couteuses à l'Etat, toute chose qui peut empêcher d'atteindre les objectifs de la réforme, il serait mieux de mettre à contribution les juridictions de droit commun en élargissant leur champ de compétence au domaine climatique par l'élaboration des textes spécifiques. Dans ce cas, il sera créé auprès des juridictions classiques des formations juridictionnelles à compétence climatique au sommet duquel se trouvera, l'Autorité Nationale des Changements Climatiques (ANCC). Ces formations juridictionnelles spécialisées, doivent être animées par des spécialistes de la question climatique.

L'Autorité Nationale des Changements Climatiques sera la plus haute juridiction de l'Etat en matière de protection du climat. Elle va suppléer la Cour constitutionnelle ${ }^{1574}$, une juridiction dont la compétence est élargie au domaine climatique ${ }^{1575}$. Les décisions de l'Autorité Nationale des Changements Climatiques ne seront susceptibles d'aucun recours. Elles s'imposeront au pouvoir, exécutif, législatif, ainsi qu'à toutes les juridictions. Elle pourra intervenir comme juridiction de cassation des décisions rendues en dernier ressort par les formations juridictionnelles des Tribunaux d'instance et des Cours d'appel, compétentes en matière de conflit climatique et elle assurera le contrôle de légalité des actes administratifs élaborés par le Comité National sur les Changements Climatiques. L'Autorité Nationale des Changements Climatiques sera consultée par le Gouvernement, plus généralement sur toutes les matières administratives et juridictionnelles relevant des domaines des changements climatiques. Elle peut, à la demande du Chef de l'Etat être chargée de la rédaction et de la modification de tout texte législatif et réglementaire relatif à la protection du climat préalablement à leur examen par le parlement. Le Président de l'Autorité Nationale des Changements Climatiques, sera élu par ses pairs pour une durée déterminée, parmi les

\footnotetext{
${ }^{1573}$ Propositions contenues dans le Document d'évaluation du comité national sur les changements climatiques effectuées par le ministère du cadre de vie et du développement durable de la République du Bénin en collaboration avec le Programme de Nations Unies pour le Développement (PNUD) Octobre 2016. Disponible sur le site internet : https://www.changementsclimatiques.bj/.../Evaluation-CNCC-V1-Diagnostic. Consulté le 19/01/2022. ${ }^{1574} \mathrm{Au}$ terme des dispositions de l'article 114 de la constitution béninoise du 11 décembre 1990 révisée le 07 novembre 2019, « La Cour constitutionnelle est la plus haute juridiction de l'Etat en matière constitutionnelle. Elle est juge de la constitutionnalité de la loi et elle garantit les droits fondamentaux de la personne humaine et les libertés publiques. Elle est l'organe régulateur du fonctionnement des institutions et de l'activité des pouvoirs publics $»$.

${ }^{1575}$ Les dispositions de l'article 88 de la loi n²018-18 portant règlementation sur les changements climatiques en République du Bénin attribuent des compétences climatiques à la Cour constitutionnelle.
} 
magistrats et les juristes ayant acquis d'expérience professionnelle dans les domaines de protection de l'environnement et du droit applicable aux changements climatiques. Il sera inamovible pendant la durée de son mandat, qui ne sera renouvelé qu'une seule fois. Les fonctions de Président de l'Autorité Nationale des Changements Climatiques ne seront pas compatibles avec la qualité de membre du Gouvernement, l'exercice de tout mandat électif de tout emploi public, civil ou militaire, de toute autre activité professionnelle, ainsi que de toute fonction de représentation nationale. Il sera élaboré une loi qui déterminera le statut des magistrats et Juristes de l'Autorité Nationale des Changements Climatiques.

Comment procéder à la création de l'Autorité Nationale des Changements Climatiques?

Pour réaliser ce projet, il faudra procéder à la révision de la loi fondamentale pour y intégrer la création de l'institution, puis élaborer une loi spéciale qui régit les procédures applicables devant ladite juridiction et une autre loi qui régit le statut du personnel de cette juridiction. Enfin, il faudra envisager la modification de la loi n²001 - 37 du 27 août 2002 portant organisation judiciaire en République du Bénin, modifiée par la loi n²016 - 15 du 28 juillet 2016 pour intégrer la nouvelle juridiction. Les modalités de mise en œuvre de cette réforme institutionnelle dépendront de l'engagement des différents acteurs intervenant dans la lutte contre les changements climatiques. Il faudra compter avec l'aide des organisations de la société civile, l'Etat et les partenaires au développement pour organiser des activités de sensibilisation d'éducation aux fins d'appeler l'attention des citoyens béninois et celle des autorités sur le caractère pernicieux des changements climatiques et la nécessité d'opérer de profondes réformes. Car, si les autorités se disent conscientes de l'extrême gravité du phénomène, tel n'est pas le cas en ce qui concerne l'immense majorité de la population qui connait à peine le fléau et pourtant, en subit les conséquences néfastes. Si les actions de sensibilisation portent leurs fruits, le projet de création de l'Autorité Nationale en charge des Changements Climatiques deviendra réalité avec l'approbation du peuple seul détenteur de la souveraineté.

Sur le volet, du renforcement du cadre institutionnel, il faudra également restructurer le Comité National des Changements Climatiques (CNCC).

\section{B- La restructuration du Comité National des Changements Climatiques}

Attributaire des missions techniques relativement à ses compétences, le Comité National sur les Changements Climatiques à l'épreuve des faits, s'est avéré comme une institution qui 
n'est pas capable de combler les attentes. L'étude évaluative de la performance du Comité National sur les Changements Climatiques ${ }^{1576}$ a indiqué les facteurs qui affectent son fonctionnement et impactent négativement ses performances. Selon cette étude, l'institution est affaiblie par l'insuffisance des capacités humaines matérielles et financières.

Les difficultés de fonctionnement que connait le Comité National sur les Changements Climatiques découlent des conditions de sa création. Selon l'article 77 de la loi n²018-18 portant règlementation des changements climatiques en République du Bénin, le «(...) Comité National sur les Changements Climatiques est placé sous la tutelle du ministère en charge des Changements Climatiques (...) ». Il ressort de l'analyse de cette disposition que, la position de subordination qu'occupe le Comité National sur les Changements Climatiques, dans l'organigramme du ministère en charge de l'Environnement, est une source de difficulté. Dans son fonctionnement, cette institution de par sa composition est sujette à des collaborations difficiles avec ses membres qui ne sont que des émanations de divers ministères ${ }^{1577}$, chacun étant jaloux de ses prérogatives. Mais en plus, aucun texte n'a clarifié la portée des décisions qui sont prises par cette institution qui est assimilée à un simple organe technique consultatif, dont les avis n'obligent pas le Ministre de tutelle. C'est ce que précise d'ailleurs l'article $3 \mathrm{du}$ décret n²003-142 du 30 avril 2003 portant création, attributions et fonctionnement du Comité National sur les Changements Climatiques en République du Bénin qui dispose que, «placé sous l'autorité du Ministre chargé de l'environnement, le Comité national a pour attributions (...) l'émission d'avis sur les questions juridiques, scientifiques technologiques et autres relatives à l'évolution du climat(...)».

Par ailleurs, le Comité National sur les Changements Climatiques a un caractère ad hoc. Ce statut juridique ne permet pas à cet organe d'être performant, parce que, quelque soit la célérité et l'importance que requiert la gestion des changements climatiques, l'article $6 \mathrm{du}$ décret n`2003-142 du 30 avril 2003 portant création, attributions et fonctionnement du Comité National sur les Changements Climatiques prescrit que cette institution ne peut que «(...) se réunir en session ordinaire deux fois dans l'année au cours des mois de juin et décembre (...) ». Les dispositions textuelles qui régissent le fonctionnement de l'institution n'offrent pas de

\footnotetext{
${ }^{1576}$ Cette étude a été effectuée en octobre 2016 par le Ministère du Cadre de Vie et du Développement Durable (MCVDD), en collaboration avec le Programme des Nations Unies pour le Développement (PNUD). Disponible sur le site internet: https://www.changementsclimatiques.bj/.../Evaluation-CNCC-V1-Diagnostic-et-PR-1.doc. Consulté le 01/05/2021.

${ }^{1577}$ Article 4 du décret $n^{\circ} 2003-142$ du 30 avril 2003 portant création, attributions et fonctionnement du comité national sur les changements dispose que « Le Comité National sur les Changements Climatiques est composé des représentants des départements ministériels et structures non gouvernementales (...)»
} 
possibilité au Comité National sur les Changements Climatiques pour statuer sur des cas urgents. Si, la Communauté internationale a senti le besoin d'organiser le secteur des changements climatiques en une véritable filière accompagnée d'institutions adéquates, l'organe ad' hoc que représente le Comité National sur les Changements Climatiques ne cadre pas avec les objectifs internationaux en la matière.

Que faut-il faire pour permettre au Comité National sur les Changements Climatiques d'accomplir avec efficacité sa mission?

Les études évaluatives réalisées par l'Etat béninois pour jauger la capacité du Comité National sur les Changements Climatiques à gérer efficacement les changements climatiques, ont suggéré de transformer le cadre institutionnel destiné à la lutte contre les changements climatiques en un assemblage d'institutions professionnalisées. Cependant les détails ne sont pas fournis sur l'opportunité de la création d'une institution du genre, « Autorité Nationale en charge des Changements Climatiques ». Le document d'évaluation de la performance du Comité National sur les Changements Climatiques a révélé in fine la vision de l'Etat béninois en ce qui concerne le développement du cadre institutionnel en matière de lutte contre les changements climatiques. Selon les éléments de contribution de cette évaluation, la vision du développement du cadre institutionnel de lutte contre les changements climatiques se décline en deux (2) étapes, dont la première consiste à doter l'Etat «d'organes et d'organismes suffisamment proactifs, efficaces, efficients ${ }^{1578}$. Mais en plus, ces institutions devront être « animées par des personnes d'une compétence avérée, spécialistes des divers domaines d'intervention ${ }^{1579}$. L'ensemble des ces institutions doivent disposer de la «capacité de prévoir, d'impulser, d'élaborer et de gérer les politiques et stratégies, de réguler et de coordonner toutes les activités initiées et mises en œuvre, d'évaluer et d'assurer la veille stratégique ${ }^{1580}$. Pour cela, il convient que, «le cadre institutionnel de lutte contre les changements climatiques soit doté d'une administration distincte, indépendante, performante et

\footnotetext{
${ }^{1578}$ Propositions contenues dans le document d'évaluation du comité national sur les changements climatiques effectuées par le ministère du cadre de vie et du développement durable de la République du Bénin en collaboration avec le Programme de Nations unies pour le Développement (PNUD) Octobre 2016. CNCC - Changements Climatiques sur le site internet: https://www.changementsclimatiques.bj/../Evaluation-CNCC-V1-Diagnostic.Consulté le 01/05/2021.

1579 Propositions contenues dans le document d'évaluation du comité national sur les changements climatiques effectuées par le ministère du cadre de vie et du développement durable de la République du Bénin en collaboration avec le Programme de Nations unies pour le Développement (PNUD) Octobre 2016 sur le site internet : https://www.changementclimatique.bj.Consulté le 01/05/2021.

${ }^{1580}$ Document d'évaluation du comité national sur les changements climatiques effectué par le ministère du cadre de vie et du développement durable de la République du Bénin en collaboration avec le Programme de Nations unies pour le Développement (PNUD) Octobre 2016.
} 
autonome ${ }^{1581}$. Le secteur des changements climatiques « devra disposer de son administration spécialisée, son réseau de compétence en personnel, avoir les moyens pour assurer son fonctionnement et le financement des projets relatifs aux changements climatiques $»^{1582}$. L'institution doit être investie de large pourvoir de décision, dont les actes ne sont attaquables que devant l'Autorité Nationale en charge des Changements Climatiques (ANCC), organe de contrôle juridictionnel dans le domaine des litiges climatiques ${ }^{1583}$.

Pour apporter une réponse innovante et pertinente à l'ensemble de ces défis, l'évaluation de la performance du Comité national sur les changements climatiques suggère, "une refonte totale de l'institution de prise en charge de la lutte contre les effets néfastes des changements climatiques $»^{1584}$. Dans ce cas, deux (2) options sont envisageables, dont la première qui ne devra pas inciter à des réformes en profondeur, parce qu'elle ne requiert pas la modification en profondeur du décret 2016-501 portant attributions, organisation et fonctionnement du Ministère du Cadre de Vie et du Développement Durable. Il s'agira dans ce cas de procéder à la réformation totale du Comité National sur les Changements Climatiques qui peut changer de dénomination pour devenir "Commission Nationale sur les Changements Climatiques $(C N C C) »^{1585}$. Cette réforme se conforme à celle opérée au niveau du ministère en charge de l'Environnement et fondée sur le décret n² 2016-501 du 11 Août 2016 portant attributions, organisation et fonctionnement de ce ministère ${ }^{1586}$. La seconde option plus délicate compte tenu des modifications qui en découleront consiste, «à créer le Conseil Béninois sur les Changements Climatiques(CBCC), qui sera un organe pluridisciplinaire, directement rattaché au ministère en charge de l'Environnement qui en assure la Présidence» ${ }^{1587}$. Le Conseil aura pour mission, « le suivi de la mise en œuvre de la Convention Cadre des Nations Unies sur les Changements Climatiques et ses documents juridiques connexes ${ }^{1588}$. Il sera un «organe

\footnotetext{
${ }^{1581} \mathrm{Id}$.

1582 Document d'évaluation du comité national sur les changements climatiques effectué par le ministère du cadre de vie et du développement durable de la République du Bénin en collaboration avec le Programme de Nations unies pour le Développement (PNUD) Octobre 2016.

${ }^{1583}$ L'autorité Nationale en charge des changements climatiques est une institution à caractère juridictionnel qui est prévue pour être créée dans le cadre de notre étude. Voir supra, p.344 et s.

${ }^{1584}$ Proposition contenue dans l'étude évaluative du comité national sur les changements climatiques effectué par le ministère du cadre de vie et du développement durable de la République du Bénin en collaboration avec le Programme de Nations unies pour le Développement (PNUD) Octobre 2016.

${ }^{1585}$ Id.

${ }^{1586}$ Proposition contenue dans l'étude évaluative du comité national sur les changements climatiques effectué par le ministère du cadre de vie et du développement durable de la République du Bénin en collaboration avec le Programme de Nations unies pour le Développement (PNUD) Octobre 2016.

${ }^{1587}$ Proposition contenue dans l'étude évaluative du comité national sur les changements climatiques effectué par le ministère du cadre de vie et du développement durable de la République du Bénin en collaboration avec le Programme de Nations unies pour le Développement (PNUD) Octobre 2016.

${ }^{1588}$ Id.
} 
d'étude, d'analyse, de coordination, de concertation, d'éducation, de sensibilisation, de gestion et de suivi des différentes activités identifiées dans le cadre de la lutte contre les effets néfastes des changements climatiques ${ }^{1589}$. En d'autres termes, le Conseil Béninois sur les Changements Climatiques (CBCC) sera un « organe hiérarchico-fonctionnel composé d'une équipe de direction sous l'autorité du Ministre en charge de l'environnement qui conseille, supervise et coordonne les activités, vérifie leur conformité avec la vision, les politiques et stratégies du Gouvernement ${ }^{1590}$. Cette institution va exécuter certaines «activités prévues dans les différentes options climatiques, telles que le reboisement des berges et des espaces déboisés et coordonner les Unités Communales pour les Changements Climatiques $(\mathrm{UCCC}) »^{1591}$.

Enfin, pour palier les contradictions et les difficultés de fonctionnement qui découlent de la pluralité de représentants d'institutions de l'Etat au sein du Comité National sur les Changements Climatiques, il convient de reformer l'article 4 du décret n ${ }^{\circ} 2003-142$ du 30 avril 2003 portant création, attributions et fonctionnement du Comité National sur les Changements Climatiques en désignant comme membre de cette institution, des personnalités qualifiées pour leur expertise dans les domaines de la science, de l'économie et de la transition ou de l'efficacité énergétique et des changements climatiques.

En tout cas, la meilleure proposition de restructuration sera celle qui contribue à sortir la lutte contre les changements climatiques au plan organisationnel, "des arcanes d'une administration aux règles rigides pour conquérir une large autonomie organisationnelle et décisionnelle qui lui permette de s'investir totalement dans les questions liées aux changements climatiques ${ }^{1592}$. Seulement, malgré leur pertinence, aucune des deux (2) options proposées par l'étude évaluative ${ }^{1593}$ pour améliorer les performances du Comité National sur les Changements Climatiques (CNCC) ne permet de sortir l'institution des carcans administratifs tel que suggéré. Malgré les mesures de renforcement proposées pour améliorer la performance de l'institution afin de la rénover, elle demeure toujours sous tutelle du ministère en charge de

\footnotetext{
${ }^{1589}$ Ibid.

${ }^{1590}$ Ibid.

${ }^{1591}$ Une recommandation contenue dans la Stratégie Nationale de mise en œuvre au Bénin de la Convention Cadre des Nations Unies sur les Changements Climatiques.

${ }^{1592}$ Proposition contenue dans l'étude évaluative du comité national sur les changements climatiques effectué par le ministère du cadre de vie et du développement durable de la République du Bénin en collaboration avec le Programme de Nations unies pour le Développement (PNUD) Octobre 2016.Disponible sur le site internet: https://www.changementsclimatiques.bj/.../Evaluation-CNCC-V1-Diagnostic. Consulté le 08/10/2019.

${ }^{1593}$ Du Comité National sur les Changements Climatiques.
} 
l'Environnement. Une tutelle qui n'est pas favorable à une gestion efficace des changements climatiques.

Pour opérer «une rénovation institutionnelle nécessaire» ${ }^{1594}$, selon les termes de Stéphane DOUMBE-BILLE, il faudra procéder à la révision de la loi n²018-18 portant règlementation sur les changements climatiques en République du Bénin, en ses articles 77 et 78 de manière à sortir le Comité National sur les Changements Climatiques de l'organigramme du ministère en charge de l'Environnement et placer l'institution sous le contrôle du Président de la République. En France, une institution administrative similaire en l'occurrence le « Haut Conseil pour le Climat » en charge de la gestion des changements climatiques est créé par décret n²019-439 du 14 mai 2019 puis placée sous l'autorité du Président de la République ${ }^{1595}$. Il serait souhaitable, dans le cadre de cette réforme institutionnelle de muer le Comité National sur les Changements Climatiques en une « Agence Nationale sur les Changements Climatiques (ANCC)». Car, la consonance « Comité » ne donne que l'impression d'une structure ad hoc ou précaire, alors que l'extrême gravité que revêtent les changements climatiques mérite que des réformes intègrent également les moindres détails concernant la dénomination de la structure de gestion des changements climatiques. N'est-ce pas les mêmes motivations qui font qu'en France, il a été préféré dénommer l'institution en charge de la gestion des changements climatiques, «Haut Conseil pour le Climat ${ }^{1596}$. L'article 88 de la loi n ${ }^{\circ} 2018-18$ portant règlementation sur les changements climatiques en République du Bénin a déjà posé des jalons dans le sens de l'institutionnalisation de «l'autorité chargée de la lutte contre les effets et conséquences négatifs des changements climatiques » qui est semblable à la proposition de création de l'«Agence Nationale sur les Changements Climatiques (ANCC)», organe essentiellement administratif dont les actes ne sont attaquables que devant la juridiction constitutionnelle ${ }^{1597}$. Cette proposition s'apparente au modèle d'institution que les Experts du GIEC ont recommandé à « Chaque pays en développement d'envisager de créer (...) pour assurer la coordination nationale de toutes ses activités relatives au climat ${ }^{1598}$, car estimentils que « cela serait utile, notamment pour la diffusion de l'information, l'élaboration et la mise

\footnotetext{
${ }^{1594}$ DOUMBE-BILLE (S.), «Evolution des institutions et des moyens de mise en œuvre du droit de l'environnement et du développement », pp. 33 et s.

${ }^{1595}$ Art. D. 132-1. du décret $n^{\circ} 2019-439$ du 14 mai 2019 relatif au Haut Conseil pour le climat en France. Disponible sur le site internet : https://www.legifrance.gouv.fr/jorf/id/JORFTEXT. Consulté le 19/01/2022.

1596 Id.

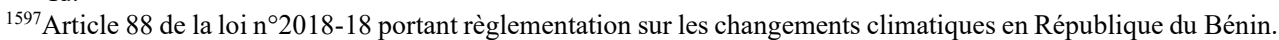
${ }^{1598}$ Premier rapport du GIEC, Paragraphe 15 du résumé destiné aux décideurs établi par le Comité spécial du GIEC pour la participation des pays en développement, Résumé directif, p.165. Disponible sur le site internet: https://www.ipcc.ch.Consulté le 19/01/2022.
} 
en œuvre de programme de recherche et de surveillance ou encore, pour le choix des orientations et la formulation des stratégies» ${ }^{1599}$. Il ne reste qu'à entériner cette mesure par une législation complémentaire.

En somme, l'amélioration de la gouvernance climatique au Bénin va aider ce pays, qui s'est déjà investi volontiers dans la lutte contre les changements climatiques, malgré la précarité de ses moyens à accomplir sa part d'obligation souscrit dans la Convention Cadre des Nations Unies sur les Changements Climatiques. Mais, la réalisation des propositions d'améliorations émises à travers notre étude, dépendra plus de la volonté des autorités politiques, car c'est encore la politique qui impulse les réformes dans ce pays.

${ }^{1599} \mathrm{Id}$. 


\section{CONCLUSION}

Les changements climatiques confirmés par des études scientifiques climatologistes ${ }^{1600}$ se manifestent par des phénomènes météorologiques extrêmes ${ }^{1601}$. Provoqués par les gaz à effet de serre (GES) d'origine anthropique ${ }^{1602}$, les changements climatiques constituent un problème mondial, global et planétaire ${ }^{1603}$ dont les conséquences transfrontalières n'épargnent aucun pays, même si certaines régions paraissent plus fragiles et vulnérables que d'autres. Selon les évaluations du GIEC, les risques des changements climatiques «ne sont pas uniformément répartis » ${ }^{1604}$, ils sont " généralement plus grands pour les populations et les communautés défavorisées de tous les pays, quel que soit leur niveau de développement ${ }^{1605}$. C'est pourquoi, lutter contre les changements climatiques est devenu un défi majeur pour les organisations internationales face à un fléau aux conséquences néfastes qui «représente une menace immédiate et potentiellement irréversible pour les sociétés humaines et la planète ${ }^{1606}$.

Pour l'encadrement de la lutte contre les changements climatiques, recours a été fait à l'outil juridique. Cet encadrement s'est matérialisé par l'élaboration des règles et principes à caractères déclaratoires ou parfois contraignants qui ont abouti à la construction d'un régime juridique international de protection du climat dont les piliers principaux sont, la Convention Cadre des Nations Unies sur les Changements Climatiques adoptée à l'occasion du Sommet de Rio de Janeiro tenu en 1992, le Protocole de Kyoto intervenu en 1997, 1'Accord de Copenhague adopté à l'occasion de la COP15 en 2009 et puis, l'Accord de Paris adopté à la COP21 en 2015. Le régime international de lutte contre les changements climatiques est édifié sur la base de «l'équité et en fonction des responsabilités communes mais différenciées et des capacités respectives des Etats Parties ${ }^{1607}$ à la Convention Cadre des Nations Unies sur les Changements Climatiques, dans le but de protéger le climat dans l'intérêt de l'humanité, surtout

\footnotetext{
${ }^{1600}$ Les évaluations du GIEC de 1990 et 1992 contenues dans son premier rapport d'évaluation. Disponible sur le site internet : https://www.ipcc.ch/reports. Consulté ce 30/05/2021.

${ }^{1601}$ Deuxième rapport d'évaluation du GIEC changements climatiques 1995 Page 34, point 3.4. Disponible sur le site internet : https://www.ipcc.ch/reports. Consulté le 30/05/2021.

1602 Paragraphe 3 du préambule de la Convention Cadre des Nations Unies sur les Changements Climatiques.

${ }^{1603}$ Paragraphe 7 du préambule de la Convention Cadre des Nations Unies sur les Changements Climatiques.

1604 Rapport spécial d'évaluation du GIEC Changements climatiques publié en 2014, page 13. \{2.3\}. Disponible sur le site internet : https://www.ipcc.ch/reports. Consulté le 30/05/2021.

${ }^{1605} \mathrm{Id}$.

${ }^{1606}$ Rapport spécial d'évaluation du GIEC Changements climatiques publié en 2014, page 51. Disponible sur le site internet : https://www.ipcc.ch/reports. Consulté le 30/05/2021.

${ }^{1607}$ Un principe contenu dans l'article 4 paragraphe 1 de la Convention Cadre des Nations Unies sur les Changements Climatiques.
} 
celui concernant les générations actuelles et futures. Ce régime juridique a reçu une adhésion massive des Etats de la planète ${ }^{1608}$.

Formellement, la lutte contre les changements climatiques fut désormais engagée à partir de l'année 1992, sur la base d'un objectif précis, celui « (...) de stabiliser, conformément aux dispositions pertinentes de la Convention Cadre des Nations Unies sur les Changements Climatiques, les concentrations de gaz à effet de serre dans l'atmosphère à un niveau qui empêche toute perturbation anthropique dangereuse du système climatique $(\ldots) »^{1609}$. En effet, « (...) il conviendrait d'atteindre ce niveau dans un délai suffisant pour que les écosystèmes puissent s'adapter naturellement aux changements climatiques, que la production alimentaire ne soit pas menacée et que le développement économique puisse se poursuivre d'une manière durable.» ${ }^{1610}$ Ensuite, cet objectif a été d'abord quantifié et chiffré dans le cadre du Protocole de Kyoto « (...), en vue de réduire le total des émissions de gaz à effet de serre d'au moins 5\% par rapport au niveau de 1990 au cours de la période d'engagement allant de 2008 à $2012 »{ }^{1611}$. L'objectif de réduction du gaz à effet de serre défini dans le cadre du Protocole de Kyoto a été reconduit pour la deuxième $\left(2^{\mathrm{e}}\right)$ période d'engagement allant de 2013 à 2020 suite à l'intervention de l'Accord de Doha ${ }^{1612}$. Enfin, l'objectif de réduction des émissions de gaz à effet de serre a été renchéri dans le cadre de l'Accord de Paris par « (...) l'élévation de la température moyenne de la planète nettement en dessous de $2^{\circ} \mathrm{C}$ et en poursuivant l'action menée pour limiter l'élévation de la température à $1,5{ }^{\circ} \mathrm{C}$ par rapport aux niveaux préindustriels, étant entendu que cela réduirait sensiblement les risques et les effets des changements climatiques $»^{1613}$.

Nombreuses sont les institutions diversement composées qui ont été mises à contribution pour aider à l'élaboration et à la mise en œuvre du droit climatique. Certaines institutions internationales en raison de la nature des missions qu'elles accomplissent, lesquelles établissent un lien avec l'existence de l'humanité, s'autorisent à s'ingérer dans le domaine de la protection de l'environnement et contribuent à la lutte contre les changements

\footnotetext{
${ }^{1608}$ Excepté le Protocole de Kyoto qui a été marginalisé par les puissances pollueuses et ratifié par cent quatre vingt douze (192) Parties, la Convention Cadre des Nations Unies sur les Changements Climatiques a été ratifié par cent quatre vingt dix-sept (197) Parties et puis l'Accord de Paris a été ratifié par cent quatre vingt onze (191) Parties.

${ }^{1609}$ Article 2 de la Convention Cadre des Nations Unies sur les Changements Climatiques. ${ }^{1610}$ Id.

1611 Article 3 paragraphe 1 du Protocole de Kyoto.

1612 Décision 1/CP.18 adoptée par la Conférence des Parties à la Convention Cadre des Nations Unies sur les Changements Climatiques lors de sa $18^{\mathrm{e}}$ session tenue à Doha en 2012. Disponible sur le site internet : https://unfccc.int/resource/docs/2012/cop18/fre/08a01f.pdf. Consulté le 19/01/2022.

${ }^{1613}$ Article 2 paragraphe 1-a de 1'Accord de Paris.
} 
climatiques. En ligne de mire se trouve l'Organisation des Nations Unies (ONU) qui s'est intéressée au fléau que constitue le dérèglement climatique, en raison de la relation intrinsèque qui existe entre l'environnement, la paix et les droits de l'Homme. L'ONU a directement contribué à la mise en place du régime juridique international de protection du climat par l'adoption par son assemblée générale de certaines résolutions qui ont servi de base juridique pour l'élaboration des normes internationales de lutte contre les changements climatiques. L'ONU intervient également par l'entremise d'institution qu'elle a contribué à créer, en l'occurrence, le Programme des Nations Unies pour l'Environnement (PNUE). Cette dernière, en collaboration avec l'Organisation Mondiale de la Météorologie (OMM) a contribué à la création du GIEC avec l'appui de l'Assemblée générale des Nations unies. Cette institution s'illustre par la qualité de son expertise exclusivement destinée à la résolution des problèmes liés aux changements climatiques. C'est suite au rapport alarmant et aux recommandations du GIEC, après des évaluations effectuées sur les changements climatiques concernant la période de 1990 et 1992 que le Sommet de la Terre de Rio de Janeiro de 1992 avait été organisé ${ }^{1614}$. Les deux (2) institutions à savoir, le PNUE et le GIEC ont collaboré et contribuer à asseoir les bases d'un cadre intergouvernemental de lutte contre les changements climatiques par l'adoption de la Convention Cadre des Nations Unies sur les Changements Climatiques. Cette Convention a été complétée par la suite par le Protocole de Kyoto et par l'Accord de Paris. Elle subira au cours des années, des modifications dans le sens de son amélioration.

Par ailleurs, quelques institutions non étatiques sont également intervenues pour contribuer à l'élaboration du droit de protection du climat, c'est le cas notamment, de l'Union Mondiale pour la Nature (UICN). Une impressionnante organisation associative internationale à vocation environnementale, dont l'expertise a été d'une grande importance dans le processus d'élaboration des instruments de protection de l'environnement.

La Convention Cadre des Nations Unies sur les Changements Climatiques a donné ellemême naissance à la Conférence des Parties (COP), organe suprême de ladite convention ${ }^{1615}$ qui encadre sa mise en œuvre. Le traité climatique a également entériné l'existence du GIEC, qui a reçu mandat de contribuer à sa mise en œuvre par sa contribution scientifique et technique.

\footnotetext{
1614 A l'occasion de la Conférence des Nations unies sur l'Environnement et le Développement tenue à Rio de Janeiro en 1992, les Parties présentes à ladite Conférence se sont déclarées "Conscientes des utiles travaux d'analyse menés par nombre d'États sur les changements climatiques et des contributions importantes apportées par l'Organisation météorologique mondiale, le Programme des Nations Unies pour l'environnement et d'autres organes, organisations et organismes des Nations Unies, ainsi que par d'autres organismes internationaux et intergouvernementaux, à l'échange des résultats de la recherche scientifique et à la coordination de la recherche ${ }^{1614}$. Sur le site internet : https://www.ipcc.ch/reports. Consulté ce 30/05/2021.

1615 Article 7 paragraphe 2 de la Convention Cadre des Nations Unies sur les Changements Climatiques.
} 
Néanmoins, pour palier toute difficulté qui découlerait de l'application et de l'interprétation de la Convention Cadre des Nations Unies sur les Changements Climatiques, en dehors de ce que le droit a prescrit que, les Parties «s'efforcent de régler par voie de négociation ou par tout autre moyen pacifique de leur choix les conflits, le contrôle juridictionnel international a été recommandé ${ }^{1616}$. C'est ainsi que les différends nés de l'application de la Convention Cadre des Nations Unies sur les Changements Climatiques peuvent être déférés devant la Cour Internationale de Justice $(\mathrm{CIJ})^{1617}$, une juridiction internationale à compétence générale relevant du système des Nations unies. La Convention Cadre des Nations Unies sur les Changements Climatiques a prescrit la compétence climatique à cette institution en la désignant comme son organe juridictionnel ${ }^{1618}$. Toutefois, le contrôle juridictionnel peut être également effectué par, l'Organisation Mondiale pour le Commerce (OMC) qui peut participer de toutes les manières au règlement des différends climatiques dont le fond est commercial sans qu'elle ne soit investie à l'origine des missions en relation avec la protection de l'environnement. Son domaine de prédilection étant la régulation du commerce international.

D'importants moyens financiers de même que la technologie sont indispensables et devront être apportés par les pays développés pollueurs au profit des pays en développement moins pollueurs, mais vulnérables aux effets néfastes des changements climatiques, pour les accompagner dans leurs efforts de lutter contre le fléau ${ }^{1619}$. C'est pourquoi la Communauté internationale a initié dans l'outil juridique, le mécanisme financier pour faciliter la mobilisation des ressources financières. En effet, pour déployer ces ressources dans les Etats Parties, plusieurs institutions ont été créées dans le contexte de la Convention Cadre des Nations Unies sur les Changements Climatiques à savoir : le Fond Vert pour le Climat et le Fond pour l'Adaptation. Auparavant, dans le cadre de la Déclaration de Stockholm de 1972, le Fond Mondial pour l'Environnement (FEM) avait été créé pour financer les activités de protection de l'environnement. Il a reçu mission pour intervenir dans le cadre de la lutte contre les changements climatiques ${ }^{1620}$.

Dans un contexte caractérisé par un phénomène climatique qui n'épargne aucun pays quel qu'en soit son niveau de développement, le besoin d'une riposte commune et générale pour éradiquer le fléau planétaire se manifeste de plus en plus. Le droit international climatique étant

\footnotetext{
${ }^{1616}$ Article 14 paragraphes 1 et 2 de la Convention Cadre des Nations Unies sur les Changements Climatiques.

${ }^{1617}$ Article 14 point 2-a de la Convention Cadre des Nations Unies sur les Changements Climatiques.

1618 Article 14 paragraphe 2-a de la Convention Cadre des Nations Unies sur les Changements Climatiques.

${ }^{1619}$ Article 9 paragraphe1 de l'Accord de Paris.

${ }^{1620} \mathrm{Au}$ terme de l'article 21 paragraphe 3 de la Convention Cadre des Nations Unies sur les Changements Climatiques « Le Fonds pour l'environnement mondial (...) sera l'entité internationale chargée d'assurer à titre provisoire le fonctionnement du mécanisme financier $(\ldots) »$.
} 
lui-même le produit de la coopération internationale, a recommandé dans la plupart des instruments internationaux la mise en œuvre de la coopération à diverses échelles pour lutter contre ce phénomène. C'est pourquoi, plusieurs organisations communautaires ou régionales telles que, l'Union Africaine (UA) et la Communauté Economique des Etats de l'Afrique de l'Ouest (CEDEAO) se sont intéressées aux changements climatiques. Dans le cas contraire, ces organisations peuvent voir leurs objectifs contrarier par les effets néfastes du fléau qui n'épargnent aucune institution. Cependant, pour la plupart de ces organisations, elles n'étaient pas créées pour les objectifs de protection de l'environnement, ni pour lutter contre les changements climatiques. L'Union africaine sans être Partie de la Convention Cadre des Nations Unies sur les Changements Climatiques, s'est engagée dans la lutte contre les changements climatiques par la mise en place d'institutions telles que le Groupe Africain de Négociateurs (AGN) investi de la mission «de promouvoir les intérêts de l'Afrique dans les négociations sur les changements climatiques», et le Comité des Chefs d'Etat et de Gouvernement sur les Changements Climatiques (CAHOSCC) $)^{1621}$ qui a pour mission d'" Accorder la plus grande attention politique aux enjeux du changement climatique sur le continent pour lui permettre d'avoir une position commune et parler d'une seule voix sur les questions du changement climatique» ${ }^{1622}$. Par ailleurs, l'organisation continentale s'est dotée d'outil de planification en l'occurrence, le «document de stratégie africaine sur les changements climatiques $»{ }^{1623}$ qui sert de base pour des actions à entreprendre sur le continent africain. Quant à la CEDEAO, cette organisation régionale s'invite dans le débat climatique par l'accomplissement de certaines activités qui intègrent le cadre de la lutte contre les changements climatiques. A titre indicatif, conjointement avec l'Union Mondiale pour la Nature (UICN), la Communauté Economique des Etats de l'Afrique de l'Ouest (CEDEAO) a participé à l'élaboration et à la mise en œuvre d'un programme régional sur les changements climatiques $^{1624}$.

Cependant, l'objectif défini dans la Convention Cadre des Nations Unies sur les Changements Climatiques ne peut être atteint que si, le droit international savamment et

\footnotetext{
${ }^{1621}$ Réponse au changement climatique en Afrique, une approche par la gouvernance des territoires. Disponible sur le site internet: http://base.afrique-gouvernance.net/docs/cp_climat_final_verselectronique.pdf. Consulté le $05 / 06 / 2021$

1622 Stratégie Africaine sur les Changements Climatique, p.27. Disponible sur le site internet: https://www.uneca.org. Consulté le 19/01/2022.

${ }^{1623} \mathrm{Ce}$ document est intervenu suite à l'adoption au Sommet de juillet 2009 à Syrte (Libye) de la Recommandation du Conseil exécutif à se prononcer sur le changement climatique en Afrique (EX.CL/Déc.500 (XV) Rev.1).

${ }^{1624} \mathrm{La}$ réalisation de ce programme est centrée sur les résultats produits dans le cadre d'un autre programme dénommé « Initiative pour la Réduction de la Pauvreté et la Gestion de l'Environnement (PREMI) ». Disponible sur le site internet : https://www.iucn.org/km/node. Consulté le 19/01/2022.
} 
massivement élaboré s'est déployé et mis en œuvre sur les territoires des Etats Parties. C'est pourquoi, tous les Etats signataires de la convention climat ${ }^{1625}$ ont élaboré unilatéralement et distinctement leur droit climatique interne. C'est le cas de la République du Bénin, qui compte une population d'environ 10741458 habitants, ${ }^{1626}$ un Etat côtier d'Afrique occidentale, étendue sur une superficie de $114763 \mathrm{~km}^{2}$, qui débouche sur l'océan atlantique au sud et a comme limitrophes, le Nigeria à l'est, le Togo à l'ouest, le Niger et le Burkina-Faso au nord ${ }^{1627}$, qui est confrontée aux manifestations des changements climatiques. Ce pays est menacé par la montée de l'eau des courants marins, l'érosion côtière, l'inondation, la perturbation de la pluviométrie, la déforestation et la désertification. Le Bénin dispose d'une politique qui se soucie de plus en plus des changements climatiques, car les autorités sont conscientes des risques qu'ils font courir aux générations présentes et futures. Pour s'engager dans la lutte contre les changements climatiques, le Bénin a adopté la méthodologie qui consiste à engager l'Etat dans les accords internationaux de protection de l'environnement notamment, la Convention Cadre des Nations Unies sur les Climatiques. Mais avant, il a d'abord souscrit aux instruments déclaratoires tels que, la Déclaration de Stockholm de 1972, la Charte mondiale pour la nature adoptée par les Nations unies en 1982, et les Déclarations de Rio de Janeiro de $1992^{1628}$. Tenant compte de la multidisciplinarité et de la complexité des changements climatiques, le Bénin a ratifié la Convention des Nations Unies sur la Biodiversité et la Convention des Nations Unies sur la Désertification adoptées à Rio de Janeiro en 1992. Lorsque le 30 juin 1994 le Bénin ratifiait la Convention Cadre des Nations Unies sur les Changements Climatiques, ce pays faisait la preuve de sa détermination à se rallier à la Communauté internationale pour contribuer à la lutte contre les changements climatiques par l'atténuation des gaz à effet de serre. Mais au-delà de cette détermination, l'Etat béninois a entrepris le processus d'internalisation desdits instruments par la définition des stratégies de lutte contre les changements climatiques qui s'est matérialisée par l'élaboration de plusieurs documents de stratégie dont celui intitulé, «Stratégie béninoise de mise en œuvre de la Convention Cadre des Nations Unies sur les Changements Climatiques ». Puis avait suivi l'adoption de la loi n²018-18 portant règlementation sur les changements

\footnotetext{
${ }^{1625}$ Il s'agit cent quatre vingt seize (196) Etats qui ont ratifié la Convention Cadre des Nations Unies sur les Changements Climatiques. Etat de ratification de la Convention Cadre des Nations Unies sur les Changements Climatiques, disponible sur le site internet : https://treaties.un.org/pages. Consulté le 09/01/2022.

${ }^{1626}$ Enquêtes de INSAE - Institut National de la Statistique et de l'Analyse Économique [archive]. Disponible sur le site internet : www.insae-bj.org. Consulté le 12 novembre 2019.

${ }^{1627}$ Information générale disponible sur le site officiel du gouvernement béninois : http://www.gouv.bj.Consulté le $02 / 06 / 2021$.

${ }^{1628}$ Il s'agit de la Déclaration de Rio sur l'environnement et le développement, principes de gestion des forêts et la Déclaration de Rio sur l'environnement et le développement.
} 
climatiques en République du Bénin. Cet outil juridique ajouté à ceux élaborés dans le cadre de la protection de l'environnement constituent l'univers juridique qui est mis à contribution pour lutter contre les changements climatiques au Bénin.

Dans le processus d'internalisation des instruments internationaux destinés à lutter contre les changements climatiques, le droit international a fortement influencé l'encadrement juridique Béninois. L'adhésion du Bénin à la Convention climat à favoriser l'éclosion du droit climatique dans ce pays. Le droit jadis traditionnel a été modernisé sous l'effet des instruments internationaux. Et ce droit a évolué jusqu' à l'élaboration d'un encadrement juridique spécialement destiné à la lutte contre les changements climatiques. Cet encadrement juridique est matérialisé par l'adoption de la loi n²018-18 portant règlementation sur les changements climatiques en République du Bénin. Cependant, cette loi ne constitue pas une panacée, les mesures élaborées ne sont pas tout à fait ce qu'il faut pour mener à bien une lutte efficace contre le fléau.

En appui à l'encadrement juridique, le Bénin a développé une architecture institutionnelle de lutte contre les changements climatiques, dont le pilier principal est le ministère chargé de la protection de l'Environnement, qui représente la plaque tournante de l'exécution de la politique climatique de l'Etat. Mais surtout, la réussite de la mission assignée à ce Ministère dépend de la qualité de l'expertise que lui apportent les institutions techniques telles que, l'Agence Béninoise pour l'Environnement (ABE), le Comité National pour le Développement Durable (CNDD), la Commission de Modélisation Economique des Impacts du Climat et d'Intégration des Changements Climatiques au Budget Général de l'Etat (CMEIGB), l'Agence Nationale de Protection Civile et le Comité National sur les Changements Climatiques (CNCC). Le besoin d'encadrement du financement des changements climatiques a poussé le Bénin à organiser à l'interne, un système de financement thématique orienté vers les objectifs de lutte contre les changements climatiques, par la création du Fonds National pour l'Environnement et le Climat (FNEC). Les réformes entreprises par les autorités béninoises entremêlent parfois, la politique de décentralisation du pouvoir de l'Etat et la déconcentration de l'administration et les considérations de protection de l'environnement et de lutte contre les changements climatiques. Ces réformes ont révélé de nouveaux potentiels acteurs tels que, le Préfet du département, les « autorités locales » ${ }^{1629}$ et les citoyens qui sont impliqués dans la chaine de protection du climat, pour servir de relais aux activités gouvernementales de protection du climat sur toute l'étendue du territoire national. Par ailleurs, l'organisation

${ }^{1629}$ Le Maire et les chefs des Unités villageoises. 
judiciaire béninoise ${ }^{1630}$ n'avait pas intégré la dimension de la protection de l'environnement et de la lutte contre les changements climatiques dans les domaines de compétence des juridictions béninoises. Néanmoins, les juridictions qui étaient créées pour exercer leur compétence générale, ont subies l'influence du régime juridique international de protection de l'environnement et de lutte contre les changements climatiques. Ainsi, certains textes nationaux régissant la protection de l'environnement, la protection de la forêt, la pollution environnementale et la protection du climat et même la constitution béninoise ont conférés des attributions environnementales et climatiques aux organes juridictionnels tels que, les Tribunaux d'instance, les Cours d'appel, la Cour suprême, la Cour Constitutionnelle et la Haute cour de justice.

Malgré l'effort accompli d'une part, par la Communauté internationale à travers l'élaboration et la mise à contribution d'un arsenal juridico-institutionnel savamment et massivement élaboré et d'autre part, par certains Etats Parties à la Conventions Cadre des Nations Unies sur les Changements Climatiques qui ont œuvré pour le redéploiement, du droit climatique et sa mise en œuvre sur leurs territoires respectifs, les résultats sont loin de combler les attentes. Les changements climatiques continuent d'éprouver tous les dispositifs d'encadrement élaborés. Pour se rendre compte de la réalité de la lutte contre les changements climatiques, l'étude de l'effectivité du droit applicable en la matière, dans le contexte béninois a révélé les innombrables défis auxquels sont confrontés les acteurs en ce qui concerne la qualité de la gouvernance. Cette étude a aussi permis de rendre visible les maigres résultats qui ont sanctionné les efforts permanents et colossaux accomplis dans le cadre de la lutte contre les changements climatiques.

En effet, les diagnostics ont révélé des éléments de faible effectivité aux niveaux national et international.

Au plan international, le régime climatique international bien élaboré contient quelques faiblesses qui ne facilitent pas sa mise en œuvre. A titre illustratif, l'engouement que certains Etats manifestent en souscrivant aux accords internationaux n'empêche ces derniers de renier leurs engagements lorsque la mise en œuvre ne les arrange pas. Le cas des Etats-Unis d'Amérique édifie mieux sur la question de l'application des accords internationaux, qui donne l'impression de ce que les conventions internationales constituent un assemblage de normes apparemment contraignantes, qui confèrent l'impunité aux acteurs indélicats. Il ne peut en être

${ }^{1630}$ La loi n ${ }^{\circ} 2001-37$ du 10 juin 2002 portant organisation judiciaire en République du Bénin et la loi n ${ }^{\circ} 2016-15$ modifiant et complétant la loi $\mathrm{n}^{\circ}$ 2001-37 du 10 juin 2002 portant organisation judiciaire en République du Bénin. Disponible sur le site internet : https://legis.cdij.bj > index.php > loi-n-2016-15-modifiant. Consulté le 17/01/2022. 
autrement parce que, ces engrangements librement souscrits en apparence, cachent des motivations politiques, des intérêts économiques et participent parfois à la mise en œuvre des stratégies par certains Etats. Mais ils ratifient ces accords sans avoir la volonté de s'acquitter de leurs obligations. Par contre, si certains Etats ont la volonté d'appliquer les accords internationaux, ils manquent parfois de ressources nécessaires à affecter pour leur mise en œuvre ${ }^{1631}$. Ce déficit d'engagement dont fait preuve certains Etats, relève des « déficiences qui sont parfois imputables aux conditions de sous-développement qui posent des problèmes graves ${ }^{1632}$ dont la résolution nécessite la mise à disposition « d'importants moyens humains, matériels et financiers $»^{1633}$. C'est à cause de ces difficultés de mise en œuvre de la Convention Cadre des Nations Unies sur les Changements Climatiques, surtout ce qui concerne l'acquittement des engagements souscrits par les Etats que le Protocole de Kyoto élaboré en 1997, entré en vigueur en 2005 est intervenu pour instaurer « la procédure de non respect ». Ce mécanisme est une approche managériale de gestion non punitive des difficultés qui découlent de la mise en œuvre de l'Accord climat. Malheureusement, cet accord a été boycotté par certaines puissances pollueuses ${ }^{1634}$. A ces faiblesses normatives, s'ajoute également le caractère imparfait de l'encadrement institutionnel international. Par exemple, le contrôle juridictionnel international est un élément qui peut contribuer à assurer l'effectivité du Droit International Climatique dans les Etats concernés par la Convention Cadre des Nations Unies sur les Changements Climatiques. La Cour Européenne des Droits de l'Homme (CEDH) en a donné la preuve au sein de la Communauté européenne par les nombreuses jurisprudences élaborées qui confortent l'effectivité du droit climatique en Europe. Malheureusement, la gestion des différends nés de l'application de la Convention Cadre des Nations Unies sur les Changements Climatiques devant la Cour Internationale de Justice n'est pas effective parce que, cette juridiction internationale a une compétence générale et les procédures applicables devant elle, sont moins contraignantes. Cette juridiction s'est enfermée dans le rigorisme du droit international public qui ne facilite pas la poursuite des Etats et n'est pas compétent pour juger les personnes, qu'elles soient de statut publique ou privé. Parfois, certains Etats prétextant de leur souveraineté s'obstinent à se soumettre à la compétence de cette juridiction, tout comme le cas de la France ou les Etats -Unis d'Amérique qui sont hostiles au concept de juridiction

\footnotetext{
1631 Edith Brown Weiss et Harold K. Jacobson, «Engager les pays: renforcer le respect des accords environnementaux », Cambridge, Londres, The MIT Press, 1998.

1632 Principe 9 de la Déclaration de Stockholm du 5 au 16 juin 1972.

${ }^{1633}$ Id.

${ }^{1634}$ Les Etats-Unis d'Amérique et le Canada.
} 
internationale ${ }^{1635}$. Ces difficultés subsistent et empêchent la mise en œuvre efficace de la Convention Cadre des Nations Unies sur les Changements Climatiques avec pour conséquence, la non effectivité du transfert de technologie et le faible résultat qui découle de la mise en œuvre du mécanisme financier. Il s'ensuit que les pays en développement ne parviennent pas à réaliser les objectifs de leurs engagements pour n'avoir pas bénéficié de moyens prévus au titre de la Convention.

Au Bénin, l'encadrement élaboré pour lutter contre les changements climatiques n'est pas à la hauteur de l'enjeu des défis imposés par le phénomène. Cet encadrement est parfois désuet ou muet sur certains aspects importants du phénomène. Pour ce qui concerne les conflits climatiques, les juridictions béninoises sont dépourvues de compétence scientifiques voire techniques et de texte de loi adaptable à l'enjeu de lutte contre les changements climatiques dans un contexte de sous développement. Il existe des motifs qui découlent de la qualité des textes de loi applicables en matière climatique qui pour la plupart sont imparfaits, mais en plus, le personnel judiciaire n'est pas outillé. Ce qui a pour conséquence, une participation mitigée de la justice béninoise à l'action de protection du climat. Ce qui fait que la justice béninoise ne s'est pas faite remarquée dans l'effectivité du droit climatique, contrairement au regain d'initiatives observé dans certains Etats où la justice nationale s'est illustrée de la plus belle manière en rendant des décisions tendant à contraindre l'Etat à respecter sa part d'engagement souscrit dans la Convention Cadre des Nations Unies sur les Changements Climatiques ${ }^{1636}$. Ces justices nationales se sont illustrées comme des instruments d'effectivité du droit international climatique.

A cela, s'ajoutent les faiblesses qui découlent de l'intervention de l'organe principal de gestion des changements climatiques au Bénin, c'est-à-dire, le Comité National sur les Changements Climatiques (CNCC), dont le fonctionnement n'est pas à la hauteur des enjeux et les défis que représentent les changements climatiques pour la société béninoise. Ceci est du au fait que cette institution est hébergée et fonctionne sous la tutelle du ministère en charge de la protection de l'Environnement. La situation de subordination, de précarité financière et la diversité de provenance de ses membres, sont autant de goulot qui entravent son fonctionnement normal.

\footnotetext{
${ }^{1635}$ A l'instar des initiatives de démobilisation entreprise par les Etats pour empêcher la mise en œuvre du Statut de Rome qui créé la Cour Pénale Internationale en négociant non seulement une résolution au sein du Conseil de Sécurité pour limiter la compétence de la CPI à leur égard, mais aussi pour solliciter des négociations d'accords bilatéraux d'impunité pour éviter toute remise à la Cour de leurs ressortissants. KOUEMBEU TAGNE (Jean Jacques), La Cour pénale internationale et les USA, Facultés Universitaires Saint Louis de Bruxelles, Bruxelles, Belgium, 2008, 19pp., p.4.

${ }^{1636}$ Il s'agit des Pays-Bas, des Etats-Unis d'Amérique, la Colombie, le Brésil et la France.
} 
Malgré les goulots qui contrastent les efforts accomplis par la Communauté internationale et par certains Etats Parties, l'espoir de relever les défis imposés par les changements climatiques est permis. Il est possible d'envisager des solutions pour une amélioration des conditions de protection du climat. En effet, les solutions envisageables doivent d'une part, aider la Communauté internationale à atteindre l'objectif de réduction des émissions de gaz à effet de serre dans la limite fixée par la Convention ${ }^{1637}$ et d'autre part, faciliter le respect des engagements librement souscrits par chaque Etat Partie, dans la Convention Cadre des Nations Unies sur les Changements Climatiques.

En effet, l'édifice normatif international de protection du climat sans être exhaustif, est relativement au complet car, le droit international est bien élaboré et continue d'être amélioré. Désormais, la Communauté internationale doit davantage consacrer son énergie autour de la question de la mise en œuvre qu'à la définition de nouvelles règles ${ }^{1638}$. Mais la question de la mise en œuvre concerne le cadre d'application, c'est-à-dire l'architecture institutionnel destinée à rendre effectif le droit applicable aux changements climatiques. Sur ce plan, l'Accord de Paris qui vise à accroître la capacité des pays à faire face aux impacts du changement climatique et à rendre les flux financiers compatibles avec un faible niveau d'émissions de gaz à effet de serre, comporte déjà beaucoup d'amélioration en termes de solutions aux difficultés d'application de la Convention Cadre des Nations Unies sur les Changements Climatiques. A titre illustratif, pour ce qui concerne l'encadrement institutionnel, le cadre technologique et le cadre de transparence sont innovés et puis le renforcement des capacités est prévu pour être mis en place afin d'appuyer l'action pour la mise en œuvre dudit accord.

Cependant, la Communauté internationale doit œuvrer davantage pour l'amélioration du cadre institutionnel destiné à la mise en œuvre des dispositions applicables aux changements climatiques pour l'atteinte des objectifs globaux d'éradication de la planète Terre, des changements climatiques et leurs effets néfastes. Dans ce cadre, l'étude a projeté la création d'une organisation internationale qui aura la charge de la gestion de la protection de l'environnement et de la lutte contre les changements climatiques, une institution faitière devant remplacer désormais le Programme des Nations Unies pour l'Environnement (PNUE) ${ }^{1639}$. Ce dernier ayant fait preuve de ses limites quant à la gestion holistique du domaine de protection de l'environnement et celui des changements climatiques. Nombreux sont les Etats qui se

\footnotetext{
${ }^{1637}$ Article 2 de la Convention Cadre des Nations Unies sur les Changements Climatiques.

1638 Sandrine Maljean-Dubois et Vanessa Richard, Mécanismes internationaux de suivi et mise en œuvre des conventions internationales de protection de l'environnement, CERIC, France N 09/2004, 55pp., p37.

${ }^{1639}$ Voir rubrique supra, pp. 284-290 (A- La création d'une organisation internationale environnementale).
} 
mobilisent au tour du projet de création de cette organisation qui semble faire l'unanimité, malgré l'inquiétude que cela suscite auprès de certains Etats qui craignent que le domaine de la protection de l'environnement et celui de la lutte contre les changements climatiques deviennent les activités de prédilection du Système des Nations unies au détriment des préoccupations de développement. Il est également envisagé la création d'une juridiction internationale pour se charger du contrôle juridictionnel des matières environnementales et des changements climatiques $^{1640}$. Cette juridiction va coexister avec la Cour Internationale de Justice, organe juridictionnel de la Convention Cadre des Nations Unies sur les Changements Climatiques. Contrairement à la Cour Internationale de Justice, qui n'est compétent que pour juger uniquement les Etats, la nouvelle juridiction à vocation environnementale et climatique se chargera de juger les autorités publiques, les multinationales et autres sujets susceptibles de commettre l'écocide. Cependant, le projet de création de cette juridiction peut heurter l'opposition de certaines puissances pollueuses et surtout celle des Etats-Unis d'Amérique qui sont hostiles au concept de juridiction internationale ${ }^{1641}$.

Par ailleurs, œuvrer pour la réduction substantielle des émissions de gaz à effet de serre nécessite la dynamisation de la coopération internationale pour faciliter les transferts de technologies bas-carbone, de compétences, de financements et tous autres moyens, qui peuvent encourager l'action climatique et la réduction de son coût. Cet effort de coopération qui a prévalu au plan international soutenu par le traité climatique, doit se renforcer à toutes les échelles de la société. Il s'agira d'utiliser ce moyen pour rapprocher le droit climatique vers les Etats signataires de la Convention par les canaux régionaux et communautaires. Dans cette perspective, le Bénin relevant de l'Union Africaine (UA) et de la Communauté Economique des Etats de l'Afrique de l'Ouest (CEDEAO) doit pouvoir en principe bénéficier des solutions communes et transfrontalières à ce défi global à la seule condition que ces organisations internationales soient investies d'un rôle plus accru dans la mise en œuvre des instruments internationaux de lutte contre les changements climatiques. D'où, la nécessité d'œuvrer pour l'ancrage de l'Union africaine dans le droit climatique. Cette approche de gestion et d'encadrement décentralisée des changements climatiques est aussi valable pour la CEDEAO qui doit adhérer en tant qu'organisation régionale d'intégration économique la Convention Cadre des Nations Unies sur les Changements Climatiques.

\footnotetext{
${ }^{1640}$ Voir supra, pp. 290-294 (B- La création d'une juridiction internationale spécialisée).

1641 Voir supra, pp. 361-362 note 1635.
} 
L'un des aspects le plus important de la mise en œuvre du droit de protection de l'environnement est le concept de la «bonne gouvernance », qui a été un succès dans le cadre de la mise en œuvre du Protocole de Kyoto à travers la procédure de non respect ${ }^{1642}$. Une approche managériale de l'application des conventions environnementales qui permet d'aider les Etats à surmonter les difficultés auxquelles ils sont confrontés sans pour autant apercevoir le défaut du respect d'engagement comme un fait illicite pour eux. Cette méthode d'application des instruments internationaux de lutte contre les changements climatiques doit faire école dans les cadres de coopération régionale et sous-régionale et s'étendre également aux Etats Parties pour susciter une mobilisation générale autour de la lutte contre les changements climatiques.

Dans la règlementation béninoise des changements climatiques, la loi n ${ }^{\circ} 2018-18$ portant règlementation sur les changements climatiques en République du Bénin a initié plusieurs mesures, dont celles dites «d'intégrations de la dimension concernant les changements climatiques » dans les projets, programmes, plans et aux cadres, législatif et réglementaire. Cependant, cette loi qui est la seule réglementation en la matière n'est pas à la hauteur des enjeux provoqués par les défis des changements climatiques. Le cadre juridique béninois destiné à la lutte contre les changements climatiques pour répondre aux exigences actuelles doit subir de profondes réformes en vue de son amélioration.

Dans les perspectives d'amélioration de la lutte contre les changements climatiques, le Bénin doit envisager mettre en place une architecture institutionnelle climatique qui sera constituée de deux (2) ordres d'institutions, l'un à caractère juridictionnel au sommet duquel se trouvera, l'Autorité Nationale en charge des Changements Climatiques (ANCC) qui sera créée ${ }^{1643}$. Elle sera la plus haute juridiction de l'Etat en matière de protection de l'environnement et des changements climatiques. Quant au Comité National sur les Changements Climatiques (CNCC), sera restructuré avec les mêmes attributions ${ }^{1644}$. Cette réforme doit aboutir à terme, à l'amélioration de la gouvernance climatique. Les réformes envisagées doivent contribuer à la participation inclusive à l'action climatique notamment, par la transformation de la Société civile en acteur clé dans la mise en œuvre du droit climatique et l'implication des citoyens à travers la définition du cadre juridique de collaboration avec l'Etat et ses démembrements.

\footnotetext{
${ }^{1642}$ La procédure de non- respect est instituée dans le cadre du Protocole de Kyoto par l'article 18. 1643 Voir supra, pp. 344-347(A-L'Autorité Nationale en charge des Changements Climatiques).

1644 Voir supra, pp. 347-352(B- La restructuration du Comité National des Changements Climatiques).
} 
Enfin, en dépit des difficultés et faiblesses relevées dans la mise en œuvre du droit climatique, le Bénin est un petit pays en termes de superficie, mais représente un atout considérable dans le giron des Etats engagés dans la lutte contre les changements climatiques. Parce que, lorsque les mesures mélioratives seront adoptées, le territoire béninois pourra constituer un espace considérable d'hébergement des gaz à effet de serre à cause des conditions climatiques favorables au développement du couvert végétal. Mais en plus, la détermination des autorités qui ne cessent de rechercher des solutions pour conjurer le mal planétaire constitue un facteur qui présage d'un avenir climatique radieux pour le Bénin. 


\section{IMPACT DE LA THESE}

L'étude portant sur les changements climatiques et les conditions d'élaboration et d'application des règles en la matière présente un double intérêt scientifique et pratique.

Scientifiquement, la thèse a révélé la richesse du droit international des changements climatiques qui a été savamment et massivement élaboré par la Communauté internationale, laquelle a établi un régime juridique climatique, contraignant, évolutif et perfectible, construit autour d'un pilier principal qu'est la Convention Cadre des Nations Unies sur les Changements Climatiques avec l'objectif ultime, de stabiliser conformément aux dispositions pertinentes de ladite Convention les concentrations de gaz à effet de serre dans l'atmosphère à un niveau qui empêche toute perturbation anthropique dangereuse du système climatique.

La richesse du droit international climatique s'est également révélée à travers l'encadrement institutionnel des changements climatiques, qui a connu la participation des plus importantes organisations internationales du monde telle que, l'Organisation des Nations Unies (ONU) qui s'est invitée dans la lutte contre les changements à cause des menaces que le fléau représente pour la paix et l'humanité. Cette organisation mondiale a institué le Programme des Nations Unies pour l'Environnement qui représente l'autorité en matière de protection internationale de l'environnement. Avec l'avènement des changements climatiques, cette institution a présenté des limites compte tenu de son statut et aussi de la précarité des ressources dont elle dispose pour gérer ses activités. Pour remédier cette insuffisance institutionnelle, notre étude a proposé la création d'une nouvelle organisation mondiale ancrée dans le système des Nations unies qui a compétence à intervenir dans la protection internationale de l'environnement et la gestion des changements climatiques.

Les changements climatiques ont également bénéficié de l'encadrement technique du Groupe d'Expert Intergouvernemental sur l'Evolution du Climat (GIEC) qui est spécialisé dans l'évaluation des changements climatiques. Cette institution apporte son expertise dans le cadre de la Convention Cadre des Nations Unies sur les Changements Climatiques et a réalisé plusieurs rapports qui ont contribué à l'élaboration du régime juridique climatique.

Cette contribution institutionnelle a été matérialisée par l'intervention de la plus prestigieuse organisation de la société civile, l'Organisation Mondiale de la Nature (UICN) qui a contribué à l'élaboration du droit international climatique par l'influence qu'elle exerce sur le processus d'élaboration des normes et par l'expertise qu'elle y apporte. 
En outre, l'étude a révélé la contribution des Organisations régionales des continents africain et européen qui se sont invitées dans la lutte contre les changements climatiques par l'appropriation des procédures en la matière. L'Union Africaine (UA) et la Communauté Economiques des Etats de l'Afrique de l'Ouest (CEDEAO), sans être à l'origine des organisations ayant inscrit dans leur statut, la protection de l'environnement et la lutte contre les changements climatiques, se sont invitées dans le débat en posant plusieurs actes dans ce cadre notamment, la définition des stratégies de lutte et la participation aux activités de plusieurs organismes internationaux de protection de l'environnement et de lutte contre les changements climatiques. Quant à l'Union Européenne (UE), elle a marqué la différence en intégrant la Convention Cadre des Nations Unies sur les Changements Climatiques en 2013 et s'est illustrée comme un instrument d'effectivité du droit climatique dans ses Etats Membres.

Cependant, pour ce qui concerne le Bénin, pays ayant ratifié la Convention Cadre des Nations Unies sur les Changements Climatiques depuis 1993, la volonté affichée par les autorités pour lutter contre les changements climatiques n'a pas suffit pour obtenir un droit interne laborieux. Néanmoins, les efforts fournis ont contribué à doter ce pays d'une importante loi sur les changements climatiques, qui le propulse dans le giron des premiers Etats africains à se doter d'une loi exclusive sur les changements climatiques.

Pratiquement, la thèse, tout en révélant les forces du droit international climatique et la qualité de l'encadrement institutionnel, a ressorti quelques faiblesses inhérentes à l'encadrement qui ne favorisent l'atteinte de l'objectif de la Convention Cadre des Nations Unies sur les Changements Climatiques. En effet, les efforts de la Communauté internationale sont contrastés par l'application défectueuse de certaines normes, notamment, le principe de la responsabilité commune mais différenciée des Parties. L'application de ce principe est sujette à controverse, car contrairement à ses stipulations, les Etats Parties à la Convention ne parviennent pas toujours à s'acquitter de leur engagement, celui qui consiste pour les Pays développés pollueurs à mettre des ressources financières et de la technologie à la disposition des Pays en développement, qui devront à leur tour contribuer, à la réduction des émissions de gaz à effet de serre. Si les normes élaborées pour lutter contre les changements climatiques sont d'une certaine qualité, il n'en demeure pas moins que leur mise œuvre rencontre des obstacles parfois, politique, économique ou stratégique. De ce point vu, la Communauté internationale devra continuer à rechercher des solutions négociées et acceptées par les Parties.

Par ailleurs, en réalité, la justice internationale devrait constituer un élément d'effectivité du droit international climatique, cependant, c'est le phénomène contraire qui 
s'observe avec la Cour Internationale de Justice (CIJ) organe juridictionnel de la Convention Cadre des Nations Unies sur les Changements Climatiques. La contribution de la Cour Internationale de Justice (CIJ) au règlement des différends climatiques a été mitigée. Aucun résultant probant n'est ressorti des entrailles de cette juridiction qui n'est pas adaptée aux spécificités des changements climatiques. Une proposition de création de juridiction internationale spécialisée pour palier cette insuffisance est faite. La nouvelle juridiction aura une compétence essentiellement environnementale voire climatique et se chargera de juger, les individus, les autorités Etatiques et les multinationales auteurs de crime contre l'environnement par la violation de la Convention Cadre des Nations Unies sur les Changements Climatiques et l'ensemble des Conventions qui concourent à la réduction des émissions du gaz à effet de serre.

Par contre, il y a résurgence de la justice de certains pays qui prennent de l'initiative et font preuve d'audace pour suppléer d'une manière ou d'une autre, les failles de la justice internationale en prenant des décisions pour obliger les Etats à respecter leurs engagements souscrits dans le cadre de la Convention Cadre des Nations Unies sur les Changements Climatiques. C'est le cas des Pays-Bas dans l'affaire : Urgenda contre Pays-Bas, par laquelle il a été imposé à l'Etat Néerlandais le respect de ses engagements de réduction de l'émission des gaz à effet de serre souscrit dans la Convention Cadre des Nations Unies sur les Changements Climatiques. Par l'élaboration de cette jurisprudence, la justice interne s'illustre désormais comme un instrument d'effectivité du droit international climatique. Par contre au Bénin, la justice n'a pas encore marqué son intervention dans le domaine des changements alors qu'elle en a la compétence. Il y a plusieurs motifs qui en sont à la base, mais la plus plausible découle de l'indépendance de la justice.

Compte tenu de l'importance de l'environnement et sa protection pour l'humanité toute entière, la mise en œuvre des solutions proposées dans notre étude devrait contribuer à l'atteinte de l'objectif d'atténuation des changements climatiques et de ses effets pour le grand bonheur de l'humanité. Ils s'en suivront, la régression des catastrophes naturelles, la diminution de la pauvreté, de la maladie et des guerres, en un mot l'amélioration des conditions d'existence de l'homme sur terre. Le Bénin pourra atteindre les objectifs de son engagement souscrit dans la Convention Cadre des Nations Unies sur les Changements Climatiques. Les béninois pourront vivre mieux à cause de l'amélioration de l'économie, la régression du chômage, le recul de l'érosion côtière et de la désertification. En un mot les conditions du développement durable vont commencer par s'installer. 


$$
\text { A }
$$




\section{ANNEXES}





\begin{abstract}
ANNEXE I
REGLEMENTATION BENINOISE SUR LES CHANGEMENTS CLIMATIQUES
\end{abstract}


ANNEX I 
ACTE LEGISLATIF 


\section{REPUBLIQUE DU BENIN}

FRATERNITE - JUSTICE - TRAVAIL

ASSEMBLEE NATIONALE

\section{Loi $n^{\circ}$ 2018-18 relative aux changements climatiques en République du Bénin.}

L'Assemblée nationale a délibéré et adopté en sa séance du 18 juin 2018, la loi dont la teneur suit :

TITRE PREMIER

DES DISPOSITIONS GENERALES

CHAPITRE PREMIER

\section{DES DEFINITIONS ET DU CHAMP D'APPLICATION}

Article $\mathbf{1}^{\mathrm{er}}$ : Au sens de la présente loi, on entend par :

- Adaptation aux changements climatiques : Ajustement des systèmes naturels ou des systèmes humains face à un nouvel environnement ou un environnement changeant.

L'adaptation aux changements climatiques indique l'ajustement des systèmes naturels ou humains en réponse à des stimuli climatiques présents ou futurs ou à leurs effets, afin d'atténuer les effets négatifs ou d'exploiter des opportunités bénéfiques.

On distingue divers types d'adaptation, notamment l'adaptation anticipée et réactive, l'adaptation publique et privée et l'adaptation autonome et planifiée ;

- Afforestation ou boisement : Plantation d'arbres ayant pour but d'établir un état boisé sur une surface longtemps restée dépourvue d'arbre ou n'ayant éventuellement jamais (aux échelles humaines de temps) appartenu à l'aire forestière. Elle se distingue du reboisement en ce que celui-ci est réalisé sur une surface boisée peu de temps auparavant ;

- Agriculture Intelligente face au Climat : Elle a pour objet de renforcer la capacité des systèmes agricoles, de contribuer à la sécurité alimentaire, en intégrant le besoin d'adaptation et le potentiel d'atténuation dans les stratégies de développement de l'agriculture durable ;

- Aléa: Phénomène dangereux, substance, activité humaine ou condition pouvant causer la mort, des blessures ou d'autres effets sur la santé, des dommages aux biens, des pertes de moyens de subsistance et de services, des perturbations socio-économiques ou des dommages écologiques ;

- Atténuation : Intervention anthropique pour réduire les sources ou augmenter les puits de gaz à effet de serre ;

- Atténuation des effets de la sécheresse : Activités liées à la prévision de la sécheresse et visant à réduire la vulnérabilité de la société et des systèmes naturels face à la sécheresse dans le cadre de la lutte contre la désertification ;

- Audit énergétique : Démarche initiale essentielle pour garantir une bonne définition des actions de maîtrise de l'énergie dans les entreprises. L'étude approfondie des différents postes consommateurs d'énergie permet de mettre en évidence des gisements d'économie d'énergie et de déterminer les actions et les investissements envisageables pour les exploiter 
aux meilleurs coûts. Ils peuvent être autonomes ou être intégrés à un audit environnemental plus large ;

- Capacité d'adaptation : Capacité d'ajustement d'un système face aux changements climatiques (y compris à la variabilité et aux extrêmes climatiques) afin d'atténuer les effets potentiels, d'exploiter les opportunités ou de faire face aux conséquences ;

- Capacité d'atténuation : Structures et conditions sociales, politiques et économiques nécessaires pour une atténuation efficace ;

- Catastrophes naturelles : Catastrophes qui résultent d'un événement naturel : séisme, éruption volcanique, mouvements de terrain, inondation, tempête, cyclone, orages, etc. Elles désignent également les effets dommageables d'un phénomène brutal, durable ou intense ; d'origine naturelle ou humaine ;

- Changements climatiques : Variations attribuées directement ou indirectement à une activité humaine altérant la composition de l'atmosphère mondiale ;

- Déboisement : Conversion d'une forêt en zone non forestière ;

- Déforestation : Changement d'utilisation de la terre induit par le déplacement de la forêt par des humains pour différents buts ;

- Dégradation des terres : Diminution ou la disparition, dans les zones arides, semiarides et subhumides sèches, de la productivité biologique ou économique et de la complexité des terres cultivées non irriguées, des terres cultivées irriguées, des parcours, des pâturages, des forêts ou des surfaces boisées du fait de l'utilisation des terres ou d'un ou de plusieurs phénomènes, notamment de phénomènes dus à l'activité de l'homme et à ses modes de peuplement, tels que :

a- l'érosion des sols causée par le vent et/ou l'eau,

b- la détérioration des propriétés physiques, chimiques et biologiques ou économiques des sols, c- la disparition à long terme de la végétation naturelle.

- Désertification: Dégradation des terres dans les zones arides, semi-arides et subhumides sèches par suite de divers facteurs, parmi lesquels les variations climatiques et les activités humaines ;

- Ecosystème: Système d'organismes vivants en interaction, ainsi que leur environnement physique ;

- Ecosystèmes fragiles : Ce sont des écosystèmes sensibles, avec des caractéristiques et des ressources uniques en leur genre. Ils comprennent les déserts, les terres semi-arides, les montagnes, les terrains marécageux, les petites îles et certaines régions côtières. La plupart de ces écosystèmes sont d'intérêt régional, car ils débordent les frontières nationales ;

- Endémique : Limité ou propre à un endroit ou à une région. En ce qui concerne la santé humaine, endémique peut se rapporter à une maladie ou un agent présent ou généralement prévalent en permanence au sein d'une population ou d'une zone géographique ;

- Effets néfastes/négatifs des Changements climatiques : Modifications de l'environnement physique ou des biotopes dues à des changements climatiques et qui exercent des effets nocifs significatifs sur la composition, la résistance ou la productivité des écosystèmes naturels et aménagés, sur le fonctionnement des systèmes socio-économiques ou sur la santé et le bienêtre de l'homme ; 
- Efficacité énergétique : Rapport du rendement énergétique d'un processus de transformation ou d'un système à son intrant énergétique ;

- Emissions : Libération de gaz à effet de serre ou de précurseurs de tels gaz dans l'atmosphère au-dessus d'une zone et au cours d'une période donnée ;

- Emissions anthropiques : Emissions de gaz à effet de serre, d'aérosols et de précurseurs de gaz à effet de serre ou d'aérosols dues aux activités humaines. Au nombre de ces activités figurent l'utilisation de combustibles fossiles, le déboisement, les changements d'affectation des terres, la production animale, la fertilisation, la gestion des déchets et les processus industriels ;

- Energie nouvelle : Energie renouvelable mais de nouvelle génération, on peut citer l'énergie solaire, la biomasse pour l'électricité ;

- Energie primaire : Energie présente dans les ressources naturelles (charbon, pétrole brut, lumière solaire, uranium, par exemple) qui n'a pas encore fait l'objet d'aucune conversion ou transformation anthropique ;

- Energie propre encore appelée énergie verte : Une source d'énergie dont l'exploitation ne produit que des quantités négligeables de polluants par rapport à d'autres sources plus répandues et considérées comme plus polluantes.

Le concept d'énergie propre est distinct de celui d'énergie renouvelable : le fait qu'une énergie se reconstitue n'implique pas que les déchets d'exploitation de cette énergie disparaissent, ni le contraire. Les sources d'énergie suivantes sont généralement citées comme énergie propre : énergie géothermique, haute ou basse énergie ; énergie éolienne ; énergie hydroélectrique ; énergie solaire ; biomasse ; énergie marémotrice, énergie des vagues, énergies hydroliennes,...etc ;

- Emballage non biodégradable : Emballage qui ne peut être converti en dioxyde de carbone ou méthane, en eau et en biomasse sous l'effet des micro-organismes qui l'utilisent comme nutriment ;

- Energies renouvelables: Source d'énergie qui se constitue ou se reconstitue plus rapidement qu'elle n'est utilisée. Leur exploitation n'entraîne en aucune façon l'extinction de la ressource initiale et elle est renouvelable à l'échelle humaine.

On peut ainsi retenir comme énergie renouvelable : l'énergie éolienne, l'énergie hydraulique, l'énergie solaire, la biomasse produite par photosynthèse, et une partie des énergies marines. Il en est de même pour l'énergie due à la gravité ou à la géodynamique interne ;

- Erosion côtière : Processus de dégradation et de transformation du relief, et donc des roches, qui est aussi causé par tout agent externe. Elle résulte de l'action combinée des vagues, du vent, des courants et des flores fixatrices des sables et vases ;

- Etablissements humains : Endroit ou zone de peuplement ;

- Exposition : Rythme et ampleur de la variabilité climatique et du changement climatique : changement des températures/précipitations, apparition de fortes pluies, vents violents, etc. Evaluer l'exposition consistera donc à évaluer l'ampleur des variations climatiques auxquelles le territoire devra faire face, ainsi que la probabilité d'occurrence de ces variations climatiques ;

- Financement structuré : Ensemble des activités et produits mis en place pour apporter des financements aux acteurs économiques, tout en réduisant le risque grâce à l'utilisation de 
structures complexes. On y inclut la subordination des créances pour créer une dette senior, mezzanine et equity, et mieux relier le risque effectif de la créance à sa rémunération. La titrisation participe également aux financements structurés, en permettant de transformer un actif non liquide en un titre liquide, et donc en apportant des financements nouveaux à l'entreprise ;

- Gaz à effet de serre: Constituants gazeux de l'atmosphère, tant naturels qu'anthropiques, qui absorbent et émettent des radiations à des longueurs d'ondes spécifiques dans le spectre du rayonnement infrarouge émis par la surface de la terre, l'atmosphère et les nuages. La vapeur d'eau $\left(\mathrm{H}_{2} \mathrm{O}\right)$, le dioxyde de carbone $\left(\mathrm{CO}_{2}\right)$, l'oxyde d'azote $\left(\mathrm{N}_{2} \mathrm{O}\right)$, le méthane $\left(\mathrm{CH}_{4}\right)$, l'ozone $\left(\mathrm{O}_{3}\right)$ sont les principaux gaz à effet de serre dans l'atmosphère. Il existe également des gaz à effet de serre résultant des activités humaines, tels que les halocarbures et autres substances contenant du chlore et du bromure, qui sont réglementés par le Protocole de Montréal. Outre le $\mathrm{CO}_{2}$, le $\mathrm{N}_{2} \mathrm{O}$ et le $\mathrm{CH}_{4}$, le Protocole de Kyoto règlemente l'hexafluorure de soufre $\left(\mathrm{SF}_{6}\right)$, les hydrofluorocarbures (HFC) et les perfluorocarbures (PFC), qui sont eux aussi des gaz à effet de serre ;

- Gestion durable des terres : Adoption de systèmes d'utilisation des terres qui, par des pratiques d'aménagement appropriées, permet aux usagers des terres d'optimiser les avantages économiques et sociaux tirés de la terre tout en conservant ou en renforçant les fonctions de soutien écologique des ressources ;

- Gestion intégrée des zones côtières : Approche intégrée en faveur d'une gestion durable des zones côtières, prenant en compte tous les habitats et toutes les utilisations ;

- Gestion du risque : Plans, mesures ou politiques visant à réduire la probabilité et/ou les conséquences des risques ;

- Impacts des changements climatiques : Effets défavorables ou bénéfiques des changements climatiques sur les systèmes naturels et les systèmes humains. Selon que l'on tient compte ou non de l'adaptation, on peut établir une distinction entre impacts potentiels et impacts résiduels ;

- Inondation : Submersion par l'eau débordant du lit normal d'un cours d'eau ou d'autres masses d'eau, ou accumulation d'eau sur des zones qui ne sont pas normalement submergées. On englobe sous ce terme les crues fluviales, les crues éclair, les crues en milieu urbain, les inondations pluviales, les débordements d'égouts, les inondations côtières et les crues de rupture de lacs glaciaires ;

- Insécurité alimentaire : Situation qui existe lorsque les personnes n'ont pas un accès sûr à des denrées alimentaires et nutritives en quantité suffisante pour garantir une croissance et un développement normaux et une vie active et saine. Elle peut résulter de l'absence de denrées, d'un pouvoir d'achat insuffisant, d'une mauvaise distribution, ou d'une mauvaise utilisation des aliments au niveau domestique. L'insécurité alimentaire peut être chronique, saisonnière ou transitoire ;

- Installation: Toute source fixe, susceptible d'être génératrice d'atteinte à l'environnement, quel que soit son propriétaire ;

- Lutte contre la désertification : Activités qui relèvent de la mise en valeur intégrée des terres dans les zones arides, semi-arides et subhumides sèches, en vue d'un développement durable et qui visent à :

a- prévenir et/ou réduire la dégradation des terres,

b- remettre en état les terres partiellement dégradées,

c- restaurer les terres désertifiées ; 
- Matériaux locaux : Matériaux de fabrication locale réalisés par la communauté, respectant les normes sanitaires, environnementales et sociales;

- Moyens d'existence : Moyens d'existence englobent les capacités, les avoirs, y compris les ressources matérielles et sociales incluses, et les activités requises pour subsister.

Les moyens d'existence sont durables lorsqu'ils peuvent faire face à des pressions et à des chocs et s'en remettre tout en maintenant ou en améliorant, aujourd'hui et demain, leurs capacités et leurs avoirs, sans toutefois amoindrir la réserve de ressources naturelles ;

- Pêche responsable encore appelée pêche durable ou soutenable (ancien terme) : La pêche responsable consiste en l'application à la pêche des principes du développement durable tels que reconnus par la communauté internationale à Rio de Janeiro en juin 1992. Elle fait appel à la dynamique des populations de la pêche et prescrit des méthodes de capture qui atténuent la surpêche, les prises accessoires et la destruction des fonds ;

- Performance énergétique : Quantité d'énergie effectivement consommée ou estimée dans le cadre d'une utilisation standardisée à partir de valeurs de référence ;

- Pluviométrie : Etude des précipitations, de leur nature et distribution, et des techniques utilisées pour leur mesure. Elle désigne à la fois la pluviosité elle-même, c'est-àdire la quantité d'eau de pluie tombée sur une région donnée, pendant une période déterminée, et la fréquence de ces précipitations, ainsi que la mesure de la pluviosité ;

- Précaution: Mesure et stratégie de prudence, de prévoyance minutieuse que l'on observe pour permettre que les activités de lutte contre les effets néfastes des changements climatiques puissent produire les impacts escomptés ;

- Puits : Tout processus, activité ou mécanisme, naturel ou artificiel, qui élimine de l'atmosphère un gaz à effet de serre, un aérosol ou un précurseur de gaz à effet de serre ;

- Pyranomètre : Capteur de flux thermique utilisé pour la mesure de la quantité d'énergie solaire en lumière naturelle. Utilisé surtout en météorologie, il permet la mesure de la puissance du rayonnement solaire total en watts par mètre carré. Il est sensible dans un domaine spectral de 300 à 2500 nanomètres selon le filtre utilisé ;

- Reboisement : Plantation de forêts sur des terres anciennement forestières, mais converties à d'autres usages ;

- Recherche et développement : Activités visant à élaborer de nouveaux procédés de production ou produits, associés à des analyses et des mesures informant les utilisateurs potentiels de leurs usages possibles ;

- Régénération : Renouvellement de peuplements forestiers par des moyens naturels tels que les semences sur place ou à côté de peuplements forestiers, ou déposées par le vent, les oiseaux ou les animaux, ou artificiels tels que les plantations de semis d'arbres ou l'ensemencement direct ;

- Renforcement de capacités : En matière d'adaptation aux changements climatiques, le renforcement des capacités consiste à améliorer les compétences techniques et les moyens institutionnels des intervenants, afin de leur permettre de participer à toutes les initiatives destinées, notamment, à favoriser l'adaptation aux changements climatiques et la recherche sur le sujet ;

- Réservoir : Un ou plusieurs constituants du système climatique qui retiennent un gaz à effet de serre ou un précurseur de gaz à effet de serre ; 
- Résilience : Capacité des systèmes sociaux, économiques ou environnementaux à faire face à une perturbation, une tendance ou un événement dangereux, leur permettant d'y réagir ou de se réorganiser de façon à conserver leur fonction essentielle, leur identité et leur structure, tout en gardant leurs facultés d'adaptation, d'apprentissage et de transformation ;

- Résilience écologique : Degré selon lequel des perturbations peuvent être absorbées par un système avant qu'il passe d'un état à un autre. La stabilité est l'autre concept associé, définie comme la tendance d'un système à retourner à une position d'équilibre après une perturbation ;

- Résilience sociale : Capacité des groupes ou communautés à s'adapter et à apprendre à faire face à des stress et à des perturbations externes d'ordre politique, social, économique ou environnemental ;

- Ressources énergétiques renouvelables : Sources d'énergie qui, dans un cadre temporel court par rapport aux cycles naturels de la terre, sont durables et qui incluent des technologies sans carbone, de type énergie solaire, hydroélectrique et éolienne, ainsi que des technologies à bilan de carbone neutre, telle la biomasse ;

- Risque : Probabilité et magnitude d'occurrence d'une perturbation ou d'un stress dans une région en un temps donné ;

- Risque climatique : Interaction de trois composantes : l'aléa climatique, l'exposition des populations, milieux et activités à cet aléa et la vulnérabilité. L'aléa climatique est un événement susceptible de se produire et pouvant entraîner des dommages sur les populations, les activités et les milieux. Il s'agit soit d'extrêmes climatiques, soit d'évolutions à plus ou moins long terme. Quant à la vulnérabilité aux aléas climatiques, elle caractérise le degré auquel un système peut être affecté négativement par les effets de ces aléas. L'impact d'un risque climatique est la mesure des conséquences de la manifestation d'un risque climatique sur un territoire et/ou un secteur donné ;

- Sensibilité : Proportion dans laquelle un système exposé aux changements climatiques est susceptible d'être affecté, favorablement ou défavorablement, par la manifestation du changement. Elle décrit l'environnement naturel ou physique du territoire et dépend de multiples paramètres, tels que la densité de population, le profil démographique, l'occupation du sol, l'aménagement de la terre, etc. L'exposition et la sensibilité forment l'impact potentiel du changement climatique qui se produit sans tenir compte de la capacité de la population locale à s'adapter aux effets ;

- Scénario d'atténuation : Description plausible de la réponse future du système étudié, comme suite à la mise en œuvre de politiques et de mesures d'atténuation. La représentation vraisemblable et souvent simplifiée du futur climat, fondée sur un ensemble intrinsèquement cohérent de relations climatologiques, établie pour l'étude explicite des conséquences possibles des changements climatiques anthropiques, et composante fréquente des modèles sur les incidences.

Les projections climatiques constituent fréquemment la matière première des scénarii climatiques, mais, en général, ces derniers nécessitent des données complémentaires de type données climatiques réelles. Un "scénario de changements climatiques" est la différence entre un scénario climatique et le climat réel ;

- Sécheresse : Période de temps anormalement sec suffisamment long pour causer un grave déséquilibre hydrologique ;

- Source : Tout processus ou activité qui libère dans l'atmosphère un gaz à effet de serre, un aérosol ou un précurseur de gaz à effet de serre ; 
- Système climatique : Ensemble englobant l'atmosphère, l'hydrosphère, la biosphère et la géosphère, ainsi que leurs interactions ;

- Taxe sur les émissions : Redevance imposée par un gouvernement sur chaque unité d'émission d'équivalent $\mathrm{CO}_{2}$ par une source soumise à taxe ;

- Technologies écologiquement rationnelles : Technologies qui protègent l'environnement, sont moins polluantes, utilisent toutes les ressources plus durablement, recyclent une partie plus importante de leurs déchets et de leurs produits, et traitent les déchets résiduels d'une manière plus acceptable que les technologies qu'elles ont remplacées, et qui sont compatibles avec des priorités socio-économiques, culturelles et environnementales définies au plan national. Elles s'entendent comme des technologies d'atténuation et d'adaptation, des technologies dures et douces ;

- Technique de goutte à goutte encore appelée "micro-irrigation": Méthode d'irrigation utilisée en zone aride. Elle réduit au minimum l'utilisation de l'eau et de l'engrais. L'eau s'égoutte lentement vers les racines des plantes soit en coulant à la surface du sol soit en irriguant directement la rhizosphère par un système de tuyaux ; c'est le goutte-à-goutte enterré.

Le goutte à goutte peut également utiliser des dispositifs appelés tête de microvaporisation qui pulvérisent de l'eau sur une petite zone ; c'est la micro aspersion. La microirrigation est employée presque exclusivement en utilisant de l'eau potable car les règlementations interdisent généralement de pulvériser de l'eau non potable ;

- Transition énergétique : Phase de transformation qui doit permettre de passer progressivement d'un système énergétique basé essentiellement sur les énergies fossiles, telles que le charbon, le pétrole, le gaz naturel, les matières radioactives, qui sont par nature limitées, à des sources énergétiques moins centralisées, diversifiées et renouvelables : éolienne, solaire, hydraulique, géothermique, marémotrice, biomasse, ... etc ;

- Variabilité climatique : Variations de l'état moyen et d'autres statistiques du climat à des échelles temporelles et spatiales au-delà des phénomènes climatiques individuels. La variabilité peut être due à des processus internes naturels au sein du système climatique, variabilité interne, ou à des variations des forçages externes anthropiques ou naturels, variabilité externe ;

- Vulnérabilité aux changements climatiques : Degré auquel un système risque de subir ou d'être affecté négativement par les effets négatifs des changements climatiques, y compris la variabilité climatique et les phénomènes extrêmes. La vulnérabilité dépend du caractère, de l'ampleur et du rythme des changements climatiques auxquels un système est exposé, ainsi que de sa sensibilité et de sa capacité d'adaptation ;

- Vulnérabilité actuelle : Evaluation des risques connus, avec l'objectif de réduire les dangers et d'identifier des actions d'atténuation des risques et pour la gestion des risques ;

- Vulnérabilité future : Evaluation des risques connus et potentiels avec l'objectif d'estimer des dangers et d'identifier des capacités et des actions d'adaptation ;

- Zone humide encore appelée "milieu humide," : Région où le principal facteur d'influence du biotope et de sa biocénose est l'eau. Elle peut être côtière ou marine. Selon l'article $1^{\text {er }}$ de la Convention de Ramsar en 1971, « les zones humides sont des étendues de marais, de fagnes, de tourbières ou d'eaux naturelles ou artificielles, permanentes ou temporaires, où l'eau est stagnante ou courante, douce ou salée, y compris des étendues d'eau marine dont la profondeur à marée basse n'excède pas six mètres. »; 
- Zone résidentielle : Zone urbaine appartenant à un quartier où l'habitat est la fonction prépondérante et où l'espace public est conçu pour être partagé dans la perspective d'une véritable coexistence des différentes catégories d'usagers. Les piétons y sont prioritaires et les jeux d'enfants autorisés ;

Article 2 : La présente loi s'applique aux écosystèmes continentaux, littoraux et marins ainsi qu'aux eaux connexes et aux établissements humains relevant de la souveraineté de l'Etat.

Les dispositions de la présente loi sont applicables à toutes les actions, activités, mesures et initiatives entrant dans le cadre de la lutte contre les changements climatiques et leurs effets et conséquences négatifs.

Article 3 : La présente loi est également applicable aux domaines d'activités ci-après :

1- 1'agriculture et la gestion des terroirs ;

2- la production de l'électricité et l'efficacité énergétique ;

3- la gestion intégrée des ressources en eau;

4- la gestion des forêts ;

5- la gestion des écosystèmes naturels et fragiles ;

6- les transports terrestres, maritimes, fluviaux et aériens ;

7- les industries ;

8- la prévention et la lutte contre la pollution environnementale et la santé publique ;

9- la prévention et la lutte contre l'érosion côtière ;

10- la gestion durable des terres ;

11- la gestion des zones humides, des écosystèmes côtiers et des ressources marines ;

13- les risques climatiques et les catastrophes naturelles ;

14- les établissements humains et les infrastructures ;

15- les échanges commerciaux.

\section{CHAPITRE II \\ DES OBJECTIFS ET DES PRINCIPES GENERAUX}

Article 4 : La présente loi vise à lutter contre les changements climatiques ainsi que leurs effets et conséquences négatifs et d'accroitre la résilience des communautés vivantes. Elle permet entre autres de prendre des mesures efficaces de riposte, d'adaptation et d'atténuation en fixant des objectifs précis de développement économique et social durable, de sécurité et d'efficacité énergétiques, conformément aux dispositions spécifiques des instruments juridiques nationaux et internationaux relatifs aux changements climatiques.

Article 5 : Les objectifs environnementaux fixés par la présente prescription sont entre autres :

1- la protection des êtres et établissements humains, des animaux et des végétaux contre les menaces globales que sont : les gaz à effet de serre, l'altération de la couche d'ozone, la perte de la diversité biologique, la gestion des espaces pastoraux et des conflits y associés, la déforestation, le déboisement, la désertification et la sécheresse ;

2- la lutte contre la pollution de l'air, des sols, des eaux marines et continentales superficielles et souterraines ; 
3- la gestion écologiquement rationnelle des ressources non renouvelables et de tous les types de déchets ;

4- la réduction des risques de catastrophes.

Article 6 : L'État, en tant que garant du droit des populations à un environnement sain, assure dans toutes les initiatives portant sur les changements climatiques, le respect des principes ciaprès :

1- préserver le système climatique contre les changements climatiques et leurs effets et conséquences négatifs dans tous les secteurs économiques et sociaux fragiles et vulnérables;

2- prendre des mesures de précaution pour prévoir, prévenir ou atténuer les causes des changements climatiques et en limiter les effets et conséquences négatifs ;

3- prendre toutes les dispositions pour s'adapter au nouveau contexte climatique ;

4- œuvrer pour un développement durable en intégrant dans les programmes et projets nationaux de développement des mesures destinées à faire face aux changements climatiques ;

5- rendre responsables, directement ou indirectement, l'auteur ou les auteurs de tout acte ou activité susceptible d'entrainer des perturbations du climat avec des effets et conséquences négatifs.

\section{CHAPITRE III}

\section{DES OBLIGATIONS DE PRISE EN COMPTE DES CHANGEMENTS CLIMATIQUES DANS LES STRATEGIES ET PLANIFICATIONS NATIONALES ET INFRA-NATIONALES}

Article 7 : Toute politique et toute stratégie de développement et leur déclinaison aux niveaux national, départemental, communal et local intègrent la dimension changements climatiques sans occulter la durabilité environnementale et la réduction des risques de catastrophes naturelles.

Les stratégies et planifications existantes au niveau national et infranational sont révisées pour intégrer la dimension changements climatiques.

Des dispositions réglementaires sont prises en vue de l'intégration des conclusions et modalités prévues dans les Plans nationaux d'adaptation à la stratégie de développement à faible intensité de carbone et résilient aux changements climatiques.

Article 8: L'Etat adopte des politiques et stratégies intégrées axées simultanément sur la promotion, en partenariat avec divers centres de recherche, d'études pour le développement, de scénarios climatiques pour les différentes zones agro-écologiques, la réhabilitation des écosystèmes dégradés, la régénération du couvert végétal, l'amélioration de la productivité des terres dégradées et le changement progressif de mode de production et de consommation aboutissant à la réduction des déchets et à l'amélioration du cadre de vie et de travail à tous les niveaux, en particulier au niveau des communautés de base ou collectivités locales.

L'Etat assure l'adoption et la mise en œuvre des mesures fiscales incitatives et une politique de crédit destinée aux producteurs et aux consommateurs en vue de promouvoir et de soutenir les investissements dans le domaine des technologies, des procédés et des produits à faible émission de gaz à effet de serre.

L'Etat, veille à la mise en œuvre des plans d'efficacité énergétique ainsi que le développement et l'utilisation de sources d'énergie propre et alternatives aux combustibles fossiles. 
Article 9 : L'Etat et les collectivités territoriales veillent à l'intégration, dans les politiques, stratégies, budgets et plans de développement communal, des mesures d'adaptation aux changements climatiques et à leurs effets et conséquences négatifs ou visant à atténuer lesdits effets.

\section{TITRE II}

\section{DES DISPOSITIONS ET OBLIGATIONS GENERALES CHAPITRE PREMIER DES DISPOSITIONS GENERALES}

Article 10 : L'Etat prend toutes les mesures appropriées pour sauvegarder les processus écologiques et les systèmes biologiques, préserver la diversité biologique et génétique, et assurer l'utilisation durable des ressources naturelles.

Il protège et préserve les écosystèmes rares ou fragiles, les espèces rares et menacées ou en voie d'extinction de la faune ou de la flore et leurs habitats.

Il prend toutes les mesures appropriées visant à promouvoir et à renforcer la collaboration et la coopération aux niveaux sous régional, régional ou international afin d'assurer la préservation et l'amélioration de la gestion des ressources naturelles des systèmes biologiques et géologiques.

Il appuie les collectivités locales dans la prise de toutes les mesures visant à accroître la résilience des populations locales.

Article 11 : Toute personne physique vivant définitivement ou séjournant temporairement sur le territoire national, ou toute personne morale y ayant établi son siège social, a le devoir de développer des activités résilientes aux changements climatiques qui garantissent la préservation et la sauvegarde de la vie humaine, animale et végétale.

\section{CHAPITRE II}

\section{DES OBLIGATIONS GENERALES}

Article 12: L'Etat adopte les politiques et stratégies appropriées pour la prévention, la réduction, et la maîtrise des effets liés aux changements climatiques et aux catastrophes naturelles. Dans ce cadre, il s'associe à toutes les compétences nationales et internationales nécessaires.

Article 13 : L'Etat et les collectivités territoriales adoptent et mettent en œuvre un programme spécial destiné à la réhabilitation des zones dégradées du fait des effets et conséquences des phénomènes naturels et des actions anthropiques.

\section{TITRE III}

\section{DES POLITIQUES ET MESURES \\ CHAPITRE PREMIER \\ DES POLITIQUES}

Article 14 : L'Etat, en vue d'une prévention et d'une protection prend des dispositions pour :

1- l'acquisition d'équipements de mesures spécifiques météorologiques, climatologiques, agro-climatologiques, hydrologiques, océanographiques, biologiques ; 
2- la mise à disposition du personnel spécialisé pour la maintenance desdits équipements, l'acquisition et la gestion des données à des fins de planification de développement ;

3- la protection des données sensibles liées aux changements climatiques ;

4- la production et l'actualisation périodiques de rapports sur les priorités et les besoins en matière de mise en œuvre et d'appui, aux projets et aux mesures relatifs au climat à intégrer dans la planification nationale de développement ;

5- la prise en compte systématique de la dimension des changements climatiques dans les études d'impact environnemental et social.

\section{CHAPITRE II \\ DES MESURES}

Article 15 : L'Etat prend des mesures de protection du système climatique en adoptant des stratégies et mesures pour la réduction des gaz à effet de serre d'origine anthropique inhérents au modèle d'industrialisation, de développement urbain, agricole et pastoral.

Il met en œuvre des mesures spécifiques d'adaptation pour protéger l'environnement.

Article 16 : L'Etat prend les mesures pour :

1- l'évaluation préliminaire ainsi que la publication des faits, incidents, phénomènes ou fléaux constatés, notamment des effets probables ou existants des changements climatiques ;

2- l'information, dans les meilleurs délais possibles, des organismes sous régionaux, régionaux et internationaux compétents des faits, incidents, phénomènes ou fléaux liés aux changements climatiques observés sur le territoire national ;

3- la détermination au niveau national, des mesures efficaces pour faire face aux faits, incidents, phénomènes ou fléaux observés, ainsi que de l'assistance qui pourrait être nécessaire ;

4- la consultation, si nécessaire, d'autres Etats ou organismes compétents pour la détermination des mesures à prendre et des moyens à déployer pour faire face à la situation observée.

Article 17 : L'Etat promeut les connaissances, savoir-faire et capacités endogènes en :

1- renforçant les capacités et aptitudes des ressources humaines ;

2- mobilisant davantage les ressources financières et matérielles consacrées à la formation et à la recherche dans le domaine de l'environnement, en particulier des changements climatiques ;

3- créant et/ou renforçant les services de production de données hydro-climatiques, météorologiques, océanographiques, environnementales et biologiques pour une meilleure diffusion des technologies ;

4- appuyant la formation des vulgarisateurs et des membres des organisations de la société civile, y compris ceux des organisations paysannes, aux méthodes participatives de conservation et d'utilisation durable des ressources naturelles ;

5- encourageant l'utilisation et la diffusion des connaissances, savoir-faire et pratiques endogènes, et en adaptant les technologies écologiquement rationnelles et les méthodes d'agriculture et de gestion des terroirs compatibles avec les conditions socio-économiques nationales ; 
6- adoptant et faisant exécuter des programmes d'écocitoyenneté ainsi que des programmes de formation adaptés à l'utilisation des sources d'énergie de substitution, en particulier des sources d'énergies renouvelables, et en fournissant les technologies adéquates afin de réduire, voire éliminer, la dépendance à l'égard du bois de feu et du charbon de bois ;

7- adoptant également des formules propres à promouvoir de nouveaux moyens d'existence ou de subsistance, y compris la formation en vue de l'acquisition de nouvelles qualifications en matière, entre autres, de gestion, de collecte et d'analyse des données, de diffusion et d'utilisation des informations sur les changements climatiques fournies par les systèmes d'alerte précoce et de la production des cultures alimentaires.

Article 18: En prenant des mesures pour prévenir, réduire, combattre et maîtriser les effets négatifs des changements climatiques, l'Etat s'abstient de transférer, directement ou indirectement, le préjudice ou les risques de ses activités anthropiques d'un département à un autre, d'une commune à une autre et à ne pas substituer un type de pollution, d'ampleur supérieure ou égale, à un autre.

\section{TITRE IV}

\section{DES ENGAGEMENTS}

\section{CHAPITRE PREMIER}

\section{DE LA COOPERATION SCIENTIFIQUE, TECHNIQUE ET DE LA RECHERCHE- DEVELOPPEMENT}

Article 19: L'Etat entretient les liens de coopération dans les domaines de la recherche scientifique, technique et technologique, de la surveillance et de l'échange de données et autres informations endogènes, scientifiques dans le cadre de l'application de la présente loi.

Il adopte et exécute, des programmes et projets de recherche et de surveillance de manière à renforcer et consolider, entre autres, sa capacité et son aptitude dans les domaines de l'anticipation et de la riposte compatibles.

Il soutient la mise en place de réseaux nationaux de centres et d'instituts de recherche et de laboratoires spécialisés d'application pour accompagner les échanges avec le monde extérieur dans ces domaines.

Article 20 : L'Etat encourage, appuie et renforce les activités qui :

1- aident à mieux comprendre les processus qui aboutissent aux changements climatiques de même que l'impact et le rôle respectif des facteurs naturels et humains qui en sont la cause ;

2- facilitent l'élaboration des politiques et stratégies nationales d'adaptation, d'atténuation et de précaution ;

3- visent à satisfaire les besoins spécifiques des populations victimes des changements climatiques et à découvrir et appliquer des solutions susceptibles d'améliorer les conditions de vie et de travail dans les zones fragiles, dégradées et vulnérables ;

4- peuvent promouvoir les connaissances, savoir-faire et pratiques endogènes ;

5- accordent une attention particulière à la recherche socio-économique participative, et tiennent compte, des rapports entre la pauvreté et les migrations dues à des facteurs écologiques et aux changements climatiques.

Article 21: L'Etat octroie des allocations budgétaires annuelles pour la recherchedéveloppement aux fins du renforcement des capacités nationales de recherche, 
d'expérimentation et de vulgarisation des laboratoires, centres et instituts existants ou à créer, en vue de l'acquisition de technologies et outils appropriés pour la lutte contre les changements climatiques et leurs effets et conséquences négatifs.

Il finance la mise en place d'une base de données sur le système climatique, le système numérique d'informations sur les changements climatiques, la protection de l'environnement et la réduction des risques.

\section{CHAPITRE II \\ DE LA RECHERCHE, DE L'OBSERVATION SYSTEMATIQUE ET DE L'EVALUATION D'IMPACT ENVIRONNEMENTAL}

Article 22 : L'Etat et les collectivités locales adoptent et mettent en œuvre un plan annuel d'alerte précoce sur les phénomènes résultant des changements climatiques ou y afférents.

Ils créent, renforcent et assurent de façon durable le fonctionnement des équipements et systèmes d'observation et de surveillance météorologiques, climatologiques, hydrologiques et océanographiques.

L'Etat élabore, adopte et met en œuvre un programme national pour améliorer les connaissances et capacités en la matière.

Article 23 : La mise en œuvre de toute politique, de toute stratégie, de tout plan, de tout programme et de tout projet de développement susceptibles de nuire à l'environnement est subordonnée à une étude d'impact environnemental et social qui intègre les changements climatiques.

\section{CHAPITRE III}

\section{DE L'EDUCATION, DE LA FORMATION ET DE LA COMMUNICATION}

Article 24 : L'Etat veille à l'information, à la sensibilisation, à l'éducation du public et au renforcement des capacités des acteurs en vue d'une participation de toute personne résidant sur le territoire national à la résolution des problèmes environnementaux et d'une prise de conscience des menaces et risques liés aux effets négatifs des changements climatiques.

L'Etat et les collectivités locales adoptent et mettent en œuvre une stratégie de formation conséquente de ressources humaines et d'éducation généralisée en matière de protection et de gestion intégrée des ressources en eau en vue du succès des politiques nationale et locale de gestion intégrée des ressources en eau.

Article 25 :L'éducation relative aux changements climatiques, à l'environnement et à la réduction des risques climatiques et des catastrophes naturelles est intégrée dans les programmes et curricula de formation de toutes les institutions d'enseignement et de formation, de la maternelle à l'université.

L'Etat intègre la gestion rationnelle des ressources en eau dans les programmes d'enseignement, de formation et de recherche de tous les ordres d'enseignement formels et non formels.

TITRE V

DE L'ADAPTATION

CHAPITRE PREMIER

DE LA GESTION INTEGREE DES RESSOURCES EN EAU 
Article 26 : L'Etat et les collectivités locales veillent à un accès et à une répartition équitables, un approvisionnement suffisant, une utilisation équilibrée et efficiente et une exploitation durable de toutes les ressources en eau.

A cette fin, ils prennent les mesures nécessaires à la planification et à la gestion des ressources en eau, y compris l'élaboration et la mise en application de schémas directeurs d'aménagement et de gestion des eaux.

Ils mobilisent les eaux de surface aux fins d'adaptation aux changements climatiques en construisant des ouvrages appropriés.

Ils veillent aussi au maintien et à l'entretien des équipements et ouvrages hydrauliques pour assurer la sécurité sanitaire des consommateurs.

Article 27 : L'Etat et les collectivités locales veillent à la mise en œuvre des politiques, stratégies, programmes et projets de protection et de gestion intégrée des ressources en eau.

Ils procèdent à l'actualisation périodique de la réglementation en matière de protection et de gestion intégrée des ressources en eau aux fins de l'adaptation à la dynamique démographique et à l'évolution des besoins en eau.

Article 28 : L'Etat et les collectivités locales prennent les mesures nécessaires pour favoriser le recyclage et l'utilisation des eaux usées et pour lutter contre l'exploitation et l'usage abusifs des ressources en eau en particulier dans les zones vulnérables et sujettes à la sécheresse.

Article 29: L'Etat et les collectivités locales procèdent, périodiquement, à l'inventaire de l'impact des changements climatiques sur tous les programmes et projets de mise en valeur des ressources hydriques.

\section{CHAPITRE II}

\section{DE LA PREVENTION ET DE LA LUTTE CONTRE L'EROSION CÔTIERE}

Article 30 : L'Etat prend les mesures pour prévenir, réduire, combattre et maîtriser l'érosion côtière due aux activités anthropiques nuisibles et dangereuses, telles que le comblement des zones humides en vue de la récupération des terres, le prélèvement du sable marin et l'érection ou la construction des infrastructures sur la côte.

Il encourage et promeut la collecte, l'analyse, la gestion, l'utilisation et la diffusion à tous les niveaux de données relatives à l'érosion côtière.

Article 31 : L'Etat assure l'évaluation du degré de vulnérabilité des personnes et des biens face aux aléas et risques propres aux zones côtières.

Il assure la restauration de l'écosystème marin et promeut une technologie appropriée d'extraction du sel combinant les énergies renouvelables.

Il organise des campagnes de sensibilisation, d'information, de communication et de formation au profit des populations sur la menace et les dangers de l'élévation du niveau de la mer.

Article 32 : L'Etat adopte et met en œuvre une politique de formation et de renforcement continu des capacités des ressources humaines en matière de protection, de gestion et de réhabilitation des zones côtières dégradées. 


\section{CHAPITRE III \\ DE LA GESTION DES ZONES HUMIDES, DES ECOSYSTEMES CÔTIERS ET DES RESSOURCES MARINES}

Article 33 : L'Etat et les collectivités locales veillent à la mise en œuvre des politiques, stratégies, programmes et projets de protection et de gestion intégrée des zones humides.

Ils adoptent des mesures en vue de définir et de mettre en œuvre un programme intégré de gestion des zones humides et des zones côtières visant à préserver les écosystèmes et à protéger les communautés locales vivant dans les milieux les plus vulnérables.

Ils procèdent à l'actualisation périodique de la réglementation en matière de protection et de gestion intégrée des zones.

Ils veillent également à la conservation, à l'exploitation et à la transformation des ressources des zones humides et des écosystèmes marins côtiers.

\section{CHAPITRE IV}

\section{DE L'AGRICULTURE, DE LA FORESTERIE ET DE LA SECURITE ALIMENTAIRE}

Article 34 : L'Etat crée les conditions pour l'accès aux terres cultivables, aux ressources pastorales et halieutiques pour assurer une agriculture de conservation et une agriculture intelligente face au climat.

Article 35 : L'Etat adopte et met en œuvre des politiques et programmes de gestion, de conservation et d'exploitation, écologiquement durables, de tous les types de forêt.

Article 36 : L'Etat met en place des systèmes agro-météorologiques et renforce les systèmes d'alerte précoce et de gestion des catastrophes naturelles dans les zones de production et dans les bassins fluviaux en République du Bénin.

\section{CHAPITRE V}

\section{DES ENERGIES NOUVELLES ET RENOUVELABLES}

Article 37 : L'Etat intègre dans sa politique énergétique des mesures visant la promotion des énergies nouvelles et renouvelables de manière à augmenter leur part dans le bilan énergétique, en vue d'atteindre la sécurité énergétique.

Article 38 : La production, le transport, le stockage et la distribution de l'électricité à partir des sources d'énergies nouvelles et renouvelables se font dans le respect des conditions de sûreté et de sécurité prescrites par les textes en vigueur.

Article 39 : L'Etat met en place des instruments et mécanismes de promotion des énergies nouvelles et renouvelables et de l'efficacité énergétique.

Le développement des sources d'énergies nouvelles et renouvelables se fait dans le respect des normes de protection de la santé publique, de l'environnement, des exigences de compétitivité de l'économie nationale et du développement durable.

Article 40: Les activités de réalisation, d'exploitation, d'extension de la capacité ou de modification des installations de production d'énergie électrique à partir des sources d'énergies nouvelles et renouvelables sont ouvertes au secteur privé conformément aux dispositions des textes en vigueur. 
Article 41 : Le développement et la promotion des filières de biocarburant ne doivent pas mettre en péril le respect des principes de :

1- 1'accès équitable des populations aux terres agricoles ;

2- la sécurité alimentaire ;

3- la protection et la préservation de l'environnement, notamment par la réduction des émissions de gaz à effet de serre.

Un décret pris en Conseil des ministres fixe les conditions et modalités de culture, d'achat et de collecte de biomasse en vue de la production de biocarburant, la transformation de la biomasse en biocarburant, le mélange des biocarburants à des hydrocarbures fossiles raffinés, l'importation et l'exportation des biocarburants non mélangés.

\section{CHAPITRE VI \\ DU RENFORCEMENT DU RESEAU D'OBSERVATION SYSTEMATIQUE DU SYSTEME CLIMATIQUE}

Article 42 : L'Etat prend des mesures pour étendre les réseaux et moderniser les stations de mesures climatologiques, hydrologiques, océanographiques et météorologiques.

Il œuvre pour le renforcement du réseau d'observation systématique, pour la capacitation à la gestion et pour une meilleure administration des bases de données issues des stations.

Un décret pris en Conseil des ministres fixe les catégories d'équipements nécessaires et leur répartition sur le territoire national.

Article 43 : L'Etat prend des dispositions pour recruter un personnel qualifié pour :

1- l'observation ;

2- 1'analyse des données et les projections climatologiques ;

3- l'entretien des équipements et des réseaux.

\section{CHAPITRE VII}

\section{DE LA PREVENTION ET DE LA LUTTE CONTRE LA POLLUTION ENVIRONNEMENTALE ET SANTE PUBLIQUE}

Article 44 : L'Etat prend des mesures en vue d'éliminer les substances nuisibles à la santé humaine, animale et végétale dans l'environnement, notamment dans l'eau, l'air et le sol.

Article 45: L'Etat veille à l'installation et au fonctionnement des centres, laboratoires et équipements de surveillance et de contrôle de la qualité de l'air, des eaux et du sol en zones urbaines et rurales.

Il assure le respect des normes sanitaires et sécuritaires y afférentes.

Article 46 : Toute personne est tenue de respecter, dans son processus de production, de commercialisation et de distribution, toutes les normes de sécurité sanitaire des produits destinés à l'alimentation humaine.

Les produits alimentaires ne répondant pas aux normes sanitaires et sécuritaires sont systématiquement retirés du marché et détruits par les structures compétentes conformément aux procédés technologiques écologiquement rationnels. 


\section{CHAPITRE VIII \\ DES TRANSPORTS PROPRES ET DES INDUSTRIES}

Article 47 : L'Etat prend des dispositions pour encourager l'importation et l'utilisation des moyens de transport les moins polluants.

Il prend des mesures pour faciliter l'acquisition de véhicules motorisés neufs.

Il encourage les promoteurs privés et les collectivités territoriales à développer le transport en commun.

Un décret pris en Conseil des ministres fixe les caractéristiques techniques de véhicules motorisés pouvant être admis à circuler sur le territoire national.

Article 48 : L'Etat et les collectivités territoriales veillent à l'implantation des industries dans les zones industrielles réglementairement prévues.

L'Etat adopte et met en œuvre une politique de délocalisation des industries se retrouvant en pleine agglomération.

Article 49: L'implantation d'une entreprise industrielle est subordonnée à la réalisation préalable de l'étude d'impact environnemental, sans préjudice de toutes autres procédures en vigueur en la matière.

Les équipements et matériels industriels sont soumis au contrôle de l'efficacité énergétique effectué par les structures compétentes du Ministère en charge de l'Energie.

\section{CHAPITRE IX}

\section{DES RISQUES CLIMATIQUES ET DES CATASTROPHES NATURELLES}

Article 50 : L'Etat réalise et actualise périodiquement une cartographie des risques climatiques au niveau national.

Article 51 : L'Etat assure la prévention et la gestion des risques climatiques et des catastrophes naturelles.

Il adopte des politiques et stratégies visant la protection des personnes et des biens et assure la préservation du patrimoine culturel et environnemental.

Article 52 : L'Etat et les collectivités locales adoptent et mettent en œuvre une politique de collecte, de conservation et d'utilisation des eaux de pluie à des fins agricoles, pastorales, sylvicoles, piscicoles et de construction.

Ils promeuvent la création des lacs artificiels, la construction des retenues d'eau et la vulgarisation des techniques d'irrigation.

Ils renforcent la politique d'adduction et d'approvisionnement en eau potable dans les zones rurales et périurbaines.

Article 53 : L'Etat met en place un système national d'alerte précoce des inondations, appuie les actions des collectivités locales destinées à prévenir et à gérer les risques et les effets des inondations à l'échelle locale notamment par la réalisation des ouvrages adéquats en amont des basses vallées.

Il définit les normes de construction d'habitations, d'infrastructures et d'ouvrages d'art spécifiques à chaque localité, facilite et vulgarise, entre autres, la technique de relèvement et 
accompagne, le cas échéant, l'évacuation temporaire des populations en cas de survenance d'inondation.

\section{CHAPITRE $X$ \\ DE LA DEFORESTATION ET DU DEBOISEMENT}

Article 54 : L'Etat et les collectivités locales promeuvent les plantations publiques et privées, restaurent les forêts dégradées, ainsi que les espèces en voie de disparition et rationalisent l'exploitation des ressources forestières et fauniques.

Les communes sont tenues de créer et d'entretenir des espaces verts, des jardins publics, des parcs et des forêts communales.

Article 55 : La pratique de l'agriculture en dehors des zones affectées à cet effet est interdite dans les forêts protégées et classées.

Tous les modes et moyens d'exploitation susceptibles d'affecter négativement la nature ou la qualité des sols, de la faune et de la flore sont interdits.

Article 56 : L'Etat promeut les foyers améliorés et les appareils de cuisson à base d'énergies nouvelles et renouvelables.

Article 57 : L'Etat organise la synergie des actions de tous les acteurs concernés par la lutte contre les effets négatifs des changements climatiques.

Il élabore des outils et méthodes d'approche participative qui permettent d'impliquer davantage les différents acteurs dans le processus de recherche et d'actions, prenant en compte les spécificités du milieu concerné.

\section{TITRE VI}

DE L'ATTENUATION

CHAPITRE PREMIER

\section{DE L'AGRICULTURE, DE LA FORESTERIE ET DE LA GESTION DES TERROIRS}

Article 58 : L'Etat prend des mesures incitatives à la promotion d'une agriculture intelligente face au climat.

Article 59 : L'Etat favorise la conservation et le renforcement des puits et réservoirs de gaz à effet de serre par la reconstitution des galeries forestières, l'enrichissement des forêts, la sauvegarde des forêts sacrées et l'afforestation.

Article 60: Tout producteur agricole est tenu d'éviter l'usage d'intrants agricoles non homologués, les pratiques culturales nuisibles à l'environnement, les feux de végétation tardifs ainsi que la déforestation, qui accélèrent l'appauvrissement et la dégradation des sols.

Article 61 : L'Etat soutient la recherche agronomique pour l'amélioration des itinéraires techniques et des pratiques optimisant l'atténuation dans le secteur agricole.

Il favorise, par diverses mesures incitatives, l'utilisation d'intrants homologués pour une gestion durable de la fertilité des sols. 


\section{CHAPITRE II \\ DE LA GESTION DES DECHETS}

Article 62 : L'Etat adopte et met en œuvre une politique visant à promouvoir des modes de production et de consommation viables. Il met en place un plan directeur national afin de réduire la production de déchets.

Article 63 : L'Etat accompagne les collectivités locales dans la mise en œuvre des plans, programmes et projets de valorisation et de gestion intégrée et durable des déchets par filière.

\section{CHAPITRE III}

\section{DES ENERGIES NOUVELLES ET RENOUVELABLES}

Article 64 : L'État et les collectivités territoriales encouragent la mise en place et l'entretien de plantations forestières communautaires ou privées pour renforcer les puits et réservoirs de gaz à effet de serre.

Article 65 : L'Etat crée les conditions visant à promouvoir les investissements publics et privés en technologies d'énergies renouvelables, en particulier les technologies photovoltaïques, éoliennes et thermiques.

\section{CHAPITRE IV}

\section{DE LA PRODUCTION DE L'ENERGIE ELECTRIQUE ET DE L'EFFICACITE ENERGETIQUE}

Article 66 : L'Etat adopte et met en œuvre une politique de production de l'énergie électrique en vue de la réalisation, de l'autosuffisance nationale en matière énergétique.

Article 67 : Un décret pris en Conseil des ministres fixe, les règles de performance énergétique portant sur l'orientation, l'éclairage, l'isolation, les flux thermiques, l'utilisation des énergies nouvelles et renouvelables et des matériaux locaux en matière de construction.

Article 68: L'Etat assure progressivement l'accessibilité de tous les ménages à l'énergie électrique dans toutes les localités du territoire national.

Article 69 : L'État et les collectivités territoriales veillent à la prise en compte de l'efficacité énergétique avant de délivrer le permis de construire ou le permis d'aménager.

Article 70 : L'Etat veille :

1- à la sécurité en approvisionnement en énergie électrique ;

2- à la définition des critères de sûreté du système énergétique pour l'électricité ;

3- à la prise des dispositions spécifiques pour se prémunir des risques systémiques ;

4- à l'amélioration continue de l'efficacité énergétique ;

5- au développement de l'exploitation des énergies nouvelles et renouvelables ;

6- au renforcement de capacités professionnelles dans le domaine de l'énergie et à la spécialisation en fonction des besoins du secteur. 
Article 71 : L'Etat veille à la réduction progressive de la dépendance de tous les bâtiments, en particulier résidentiels, administratifs et commerciaux, en matière de consommation de l'énergie fossile et à la promotion des énergies nouvelles et renouvelables.

Il assure, à cet effet, l'harmonisation des objectifs de la politique énergétique et ceux de la politique nationale de gestion des changements climatiques.

Article 72 : L'Etat définit les secteurs où les performances énergétiques et environnementales sont respectées en matière de construction, d'installation et d'aménagement de standing.

Article 73 : L'Etat prend des dispositions pour un audit obligatoire et périodique pour le contrôle de l'émission de gaz à effet de serre.

L'audit d'émission des gaz à effet de serre s'applique à tous les secteurs consommateurs d'énergie.

Les conditions d'assujettissement à l'audit d'émission des gaz à effet de serre ainsi que son contenu et sa périodicité sont fixés par décret pris en Conseil des ministres.

Article 74 : La construction de tout bâtiment de standing élevé est soumise à une étude préalable d'impact environnemental qui intègre l'efficacité énergétique.

Article 75 : L'importation, le stockage, la commercialisation, la distribution et l'utilisation de l'amiante sont interdits.

Article 76 : Toutes les activités émettrices de gaz à effet de serre sont sujettes à une taxe dénommée "taxe carbone".

Les modalités de fixation du montant et de prélèvement de la taxe carbone sont définies par la loi des finances.

\section{TITRE VII}

\section{DU CADRE INSTITUTIONNEL}

\section{CHAPITRE PREMIER}

\section{DU COMITE NATIONAL SUR LES CHANGEMENTS \\ CLIMATIQUES (CNCC)}

Article 77 : Il est créé un Comité national sur les changements climatiques, en abrégé (CNCC), placé sous la tutelle du Ministère en charge des changements climatiques.

Chaque ministère définit les activités relatives aux changements climatiques et présente au Comité national sur les changements climatiques son Plan d'Action.

Article 78 : La composition, l'organisation, les attributions et le fonctionnement du Comité national sur les changements climatiques sont fixés par décret pris en Conseil des ministres.

\section{CHAPITRE II}

\section{DE LA COMMISSION DE MODELISATION ECONOMIQUE, DES IMPACTS}

\section{DU CLIMAT ET D'INTEGRATION DES CHANGEMENTS CLIMATIQUES}

\section{AU BUDGET GENERAL DE L'ETAT}

Article 79 : Il est créé un organe d'aide à la décision dénommé la Commission de modélisation économique des impacts du climat et d'intégration des changements climatiques au budget général de l'Etat.

Cette Commission est placée sous la tutelle du ministère en charge du plan. 
Article 80 : La composition, l'organisation, les attributions et le fonctionnement de la Commission de modélisation économique des impacts du climat et d'intégration des changements climatiques au budget général de l'Etat sont fixés par décret pris en Conseil des ministres.

\section{CHAPITRE III}

\section{DE L'INFORMATION NATIONALE SUR LES EMISSIONS DE GAZ A EFFET DE SERRE ET DE LA NOTIFICATION INTERNATIONALE}

Article 81 : L'Etat prend des dispositions pour informer l'opinion publique sur l'état de l'inventaire national des émissions et des réductions de gaz à effet de serre par un rapport biennal. Ce rapport est transmis à l'Assemblée nationale.

Il communique au Secrétariat Exécutif les progrès réalisés au plan national dans le cadre de la mise en œuvre de la Convention-Cadre des Nations unies sur les changements climatiques.

\section{TITRE VIII}

\section{DES RESSOURCES FINANCIERES}

CHAPITRE PREMIER

\section{DE LA MOBILISATION DES RESSOURCES FINANCIERES}

Article 82 : L'Etat mobilise les ressources financières nécessaires pour l'élaboration, la mise en œuvre et l'évaluation des politiques et stratégies rentrant dans le cadre de l'application de la présente loi.

Ces ressources proviennent du budget national et entre autres du fonds pour l'environnement mondial, du fonds d'adaptation aux changements climatiques, des fonds d'investissements climatiques, du fonds vert pour le climat.

Elles peuvent également provenir des partenaires au développement bilatéraux et multilatéraux, non gouvernementaux et privés.

Article 83 : L'Etat renforce ses liens de coopération avec les partenaires au développement et les organisations et institutions compétentes en vue d'obtenir l'assistance technique, financière et autres dans les domaines de la lutte contre les changements climatiques leurs effets et conséquences négatifs pour la gestion écologiquement rationnelle de ses écosystèmes.

\section{CHAPITRE II}

\section{DES REGLES DE GESTION FINANCIERE}

Article 84 : L'Etat veille, dans le cadre du financement des politiques, stratégies, programmes et projets liés à la lutte contre les changements climatiques et leurs effets et conséquences négatifs, au respect des principes de transparence, de traçabilité et d'effectivité.

Article 85 : L'Etat, conformément aux dispositions de la Convention-Cadre des Nations Unies sur les Changements Climatiques et de l'Accord de Paris, communique périodiquement au Secrétariat Exécutif de la Convention, les informations financières sur les ressources budgétaires allouées et les appuis extérieurs reçus.

La communication visée à l'alinéa précédent fournit des informations sur l'utilisation des ressources financières mises à disposition et des appuis extérieurs reçus, y compris les appuis entrant dans le cadre des mesures de transparence, ainsi que de leur impact et des résultats obtenus. 
Le Gouvernement communique à l'Assemblée Nationale et à l'opinion publique, dans les trois (03) mois qui suivent la fin de l'année budgétaire, les ressources consacrées au financement des actions de lutte contre les effets et conséquences négatifs des changements climatiques.

\section{TITRE IX}

\section{DU CONTENTIEUX, DES INFRACTIONS ET DES SANCTIONS CHAPITRE PREMIER DU CONTENTIEUX}

Article 86: Les différends relatifs aux actes délivrés ou accomplis par l'administration publique dans le cadre de la présente loi relèvent de la compétence des juridictions administratives.

Article 87 : La procédure suivie devant les juridictions visées à l'article 92 de la présente loi est celle prévue par les lois en vigueur.

Toutefois, aucun recours de plein contentieux ne sera recevable, s'il n'a été précédé d'une tentative de règlement transactionnel à l'initiative du requérant. Celui-ci adresse, le cas échéant, à la partie adverse une demande accompagnée au besoin d'une offre.

Article 88 : La compétence des juridictions administratives n'exclut pas la saisine alternative de la Cour Constitutionnelle lorsque dans le cadre de ses attributions, l'autorité chargée de la lutte contre les effets et conséquences négatifs des changements climatiques pose un acte ou entreprend toute action qui viole les droits fondamentaux de la personne.

\section{CHAPITRE II}

\section{DES INFRACTIONS ET SANCTIONS}

Article 89 : Est punie d'une peine d'emprisonnement d'un (01) an à deux (02) ans et d'une amende de cinq cent mille (500.000) francs CFA à un million (1.000.000) de francs CFA ou de l'une de ces deux peines seulement, toute personne qui commet un délit écologique en provoquant un feu de végétation tardif sur un terrain lui appartenant ou non.

En cas de récidive, ces peines sont portées au double.

Article 90 : Est punie d'une peine d'emprisonnement de cinq (05) ans à vingt (20) ans de travaux forcés et d'une amende de cinq millions (5.000.000) à cent millions (100.000.000) de francs CFA :

1- Toute personne qui entreprend de s'opposer ou s'oppose, directement ou indirectement, aux politiques, stratégies, programmes ou projets définis dans le cadre de la présente loi en vue de favoriser l'adaptation de la communauté nationale au climat, à ses variabilités, à ses changements et l'atténuation de ses effets et conséquences négatifs ;

2- toute autorité publique, nommée ou élue, qui entreprend de s'opposer ou s'oppose, directement ou indirectement, aux politiques, stratégies, programmes ou projets définis dans le cadre de la présente loi en vue de favoriser l'adaptation de la communauté nationale au climat, à ses variabilités, à ses changements et l'atténuation de ses effets et conséquences négatifs ;

3- toute personne qui omet ou néglige de protéger la collectivité contre les risques sévères et persistants résultant des changements climatiques ou liés à leurs effets et conséquences négatifs ;

4- toute personne qui acquiert ou prend à bail, cède ou met en bail par elle-même ou par un intermédiaire, un bien du domaine public situé dans une zone à écosystème fragile ou dégradé ; 
5- toute personne qui émet, dépose, dégage, enfouit, rejette ou favorise l'émission, le dépôt, le dégagement, l'enfouissement ou le rejet dans l'environnement d'un contaminant au-delà de la quantité ou de la concentration prévue par les lois et règlements en vigueur en République du Bénin.

Article 91: Les infractions de faux et usage de faux et complicité commises dans le cadre de la présente loi sont punies conformément aux dispositions du code pénal.

Article 92 : La recherche, la constatation et la répression des infractions prévues par la présente loi et ses textes d'application ainsi que les règles de procédure obéissent aux dispositions du code de procédure pénale, du code pénal et de la loi-cadre sur l'environnement.

Article 93 : Les infractions prévues par la présente loi sont constatées par les officiers et agents de la police de l'environnement et les agents assermentés ayant compétence à assurer la protection de l'environnement.

Elles ne peuvent l'être par les officiers et agents de police judiciaire qu'à défaut des fonctionnaires spécialisés visés à l'alinéa précédent.

Sauf en matière criminelle, ces fonctionnaires spécialisés ou leur hiérarchie sont tenus d'engager une transaction avec les personnes mises en cause. Le cas échéant, le montant de la transaction ne peut être inférieur aux quatre-cinquièmes $\left(4 / 5^{\mathrm{me} e}\right)$ du maximum de l'amende fixée par la présente loi et doit être payé sous huitaine.

Article 94 : En matière criminelle, ou en cas de non règlement de l'amende transactionnelle, les personnes mises en cause, ensemble avec la procédure et les pièces à conviction, sont présentées sans délai au procureur de la République territorialement compétent. Celui-ci est tenu, selon le cas, d'ouvrir une information ou de saisir le tribunal correctionnel après avoir interrogé les personnes mises en cause sur leur identité et sur les faits.

Un avertissement leur est donné en caractères apparents sur le mandat de dépôt ou la convocation par le procureur de la République, de ce qu'elles sont tenues de faire connaitre au tribunal au plus tard cinq (5) jours avant l'audience, les éléments à leur décharge.

Dans tous les cas, ces personnes sont dispensées de la détention provisoire si elles consignent au greffe de la juridiction une somme d'argent équivalent à la totalité du maximum de l'amende fixée par la loi.

Article 95 : Le tribunal correctionnel statue à l'audience d'évocation et au plus tard dans un délai d'ajournement qui ne saurait excéder un (1) mois.

Article 96: Le recouvrement des amendes peut être poursuivi sur le patrimoine des personnes civilement responsables.

\section{TITRE X}

\section{DES DISPOSITIONS TRANSITOIRES ET FINALES}

Article 97 : Toute personne physique ou morale dispose d'un délai de six (06) mois pour se conformer aux dispositions de la présente loi après sa promulgation.

Article 98 : La présente loi est exécutée comme loi de l'Etat.-

Porto-Novo, le 18 juin 2018

Le Président de l'Assemblée nationale

Adrien HOUNGBEDJI 
A C T E

R E G L E M E N T A I R E 
ANNEX I 


\section{REPUBLIQUE DU BENIN}

\section{PRESIDENCE DE LA REPUBLIQUE}

\section{DECRET No 2OO3-I42 DU 30 AVRII, 2003}

Portant création, attributions et fonctionnement du comité national sur les changements climatiques.

Vu la loi n' 90-032 du 11 décembre 1990 portant constitution de la République du Bénin ; Vu la loi n'2001-033 du22 novembre 2001 portant autorisation d'adhésion de la République du Bénin au protocole de Kyoto relative à la Convention Cadre des Nations Unies sur les changements climatiques ;

Vu la loi n' 98-030 du 12 févier 1999 portant loi-cadre sur l'environnement en République du Bénin ;

Vu la proclamation le 03 avril 2001 par la Cour Constitutionnelle des résultats définitifs de l'élection présidentielle du 22 mars 2001 ;

Vu le décret n' 2001- I 70 du 07 mai 2001 portant composition du gouvernement et le décret n ${ }^{\circ}$ 2002-082 du 20 février 2002 qui l'a modifié ;

Vu le décret n" 2003-072 du 05 mars 2003 portant attributions, organisation et fonctionnement du Ministère de l'Environnement de l'Habitat et de l'Urbanisme ;

Vu le décret 93-304 du 13 décembre 1993 portant ratification de la

Convention-cadre des Nations-Unies sur les changements climatiques en République du Bénin

Vu le décret 2001-543 du 17 décembre 2001 portant ratification de l'adhésion du Bénin au protocole de Kyoto relative à la Convention-cadre des Nations-Unies sur les changements climatiques ;

Vu le décret no 87-083 du 17 avril 1987 portant création et fonctionnement du Conseil Supérieur de la Météorologie en République du Bénin ;

Sur proposition du Ministre de l'Environnement, de 1'Habitat et de l'Urbanisme ;

Le Conseil des Ministres entendu en sa séance du 30 janvier 2003 ;

\section{DECRETE}

Article $1^{\text {er }}$ : Il est institué en République du Bénin un Comité National sur les Changements Climatiques (CNCC).

Article 2: Le Comité National sur les Changements Climatiques (CNCC) est un organe pluridisciplinaire, chargé du suivi et de l'appui à la mise en œuvre de la Convention-Cadre sur les Changements Climatiques et de tous les instruments juridiques connexes à cette Convention 
notamment le Protocole de Kyoto ainsi que de l'étude de toutes questions scientifiques, technologiques et autres relatives à l'évolution du climat.

Article 3 : Placé sous l'autorité du Ministère chargé de l'environnement, le Comité National a pour attributions :

- l'information et la sensibilisation sur les changements climatiques et leurs impacts ;

- le suivi et l'évaluation de la mise en œuvre de la Convention Cadre sur les changements climatiques et de ses instruments juridiques subséquents ;

- 1 'appui à la préparation de la participation du Bénin aux sessions des organes de la Convention (Conférence des Parties, Organes subsidiaires);

- la mise en exécution effective des décisions de la Conférence des Parties à la Convention-Cadre sur les Changements Climatiques (organe suprême de la Convention)

- le suivi et le contrôle du processus d'élaboration des Communications Nationales du Bénin sur les Changements Climatiques, requises en application des dispositions de l'article 12.5 de la Convention;

- le suivi et le contrôle du processus d'élaboration et de mise en œuvre de la Stratégie Nationale sur les Changements Climatiques ;

- l'appui à la coordination des études et recherches sur les inventaires des gaz à effet de serre, la vulnérabilité et l'adaptation aux changements climatiques, ainsi que sur les options d'atténuation ;

- la participation à l'évaluation des besoins technologiques nécessaire à la mise en œuvre de la Convention sur les Changements Climatiques et en matière de renforcement des capacités ;

- l'appui à la préparation et l'organisation de conférences, séminaires, ateliers, colloques, et tables rondes sur des sujets relatifs aux changements climatiques ;

- l'émission d'avis sur les questions juridiques, scientifiques, technologiques, méthodologiques et autres relatives à l'évolution du climat; l'étude des indicateurs nécessaires à la prévision des changements climatiques ;

- l'appui à la conception et à la formulation de projets ou programmes d'actions à soumettre au Fonds pour l'environnement Mondial et à d'autres mécanismes de financement dans le cadre de la mise en œuvre de la Convention et de ses instruments juridiques subséquents. 
Article 4 : Le Comité National sur les Changements Climatiques est composé des représentants des Départements ministériels et structures non gouvernementales ci-après :

- Ministère chargé de l'Environnement (Direction de l'Environnement, Agence Béninoise pour l'Environnement) ;

- Ministère chargé des Transports (Direction de la Météorologie Nationale ;

- Directions des Transports Terrestres, Port Autonome de Cotonou);

- Ministère chargé de l'Agriculture, de l'Elevage et de la Pêche (Direction de l'Agriculture, Direction des Forêts et Ressources Naturelles, Direction des Pêches, Institut National de la Recherche Agronomique du Bénin, Centre National d'Agro Pédologie) ;

- Ministère chargé de l'industrie (Direction de l'industrie);

- Ministère chargé du Commerce ;

- Ministère chargé de l'Enseignement Supérieur et de la Recherche Scientifique (Université d'Abomey-Calavi : Département de Géographie et de l'Aménagement du Territoire, Département de Chimie et de Physique ; Centre Béninois pour la Recherche Scientifique et Technique, Faculté des Sciences Agronomiques, Collège Polytechnique Universitaire) ;

- Ministère chargé de l'Hydraulique et de l'Energie (Direction de l'Hydraulique, Direction de l'Energie) ;

- Ministère chargé de la Santé ;

- Ministère chargé des Affaires Etrangères et de l'intégration Africaine (Direction des Organisations internationales);

- Ministère chargé du Plan (DCRE, Cellule NEX, CBDD);

- Ministère chargé de l'intérieur (Direction de la Prévention et de la Protection Civile);

- Ministère chargé de la Communication ;

- Ministère chargé de la Justice et de la Législation ;

- Ministère chargé des Finances (Direction Générale du Budget) ;

- Organisations Non Gouvernementales (BENIN 21, OFEDI, ABFEM-ONG, GRAIB, représentant du réseau des ONG au niveau départemental)

- Secteur privé (Chambre de Commerce et d'industrie du Bénin).

Article 5 : Les organes du Comité National sont :

1 - l'Assemblée Générale ;

2 - le Secrétariat Permanent; 
3 - les Equipes Techniques.

Article 6 : L'ensemble des membres sus cités à l'article 4 forment l'Assemblée Générale du CNCC qui est son organe de décision. Elle exerce la plénitude des attributions définies à l'article 3 ci-dessus. Elle adopte le Budget du CNCC et contrôle son exécution par le Secrétariat Permanent défini à l'article 7. Elle se réunit en session ordinaire deux fois dans l'année en juin et décembre, la session de juin étant une session budgétaire de l'année à venir et celle de décembre, une session bilan et de contrôle de la gestion du Secrétariat Permanent. Elle prend ses décisions à la majorité absolue de ses membres.

Cependant, en cas de nécessité, des sessions extraordinaires peuvent être convoquées sur l'initiative du Président.

Dans son fonctionnement, l'Assemblée Générale peut faire appel à toutes personnes ressources en raison de la nature des dossiers à étudier.

Article 7 : L'organe d'exécution des décisions du CNCC est le Secrétariat Permanent. Il est composé ainsi qu'il suit :

1- Un Secrétaire Exécutif, chargé de coordonner les activités du Comité, Point Focal National de la Convention-Cadre des Nations Unies sur les Changements Climatiques ;

2- Un Secrétaire chargé des questions scientifiques, techniques et technologiques, représentant le Ministère chargé de l'Enseignement Supérieur et de la Recherche Scientifique ;

3- Un Secrétaire chargé des activités de collecte, de gestion et d'échange de données d'observation et d'informations sur le système climatique, représentant le Ministère chargé des Transports et Travaux Publics ;

4- Un Secrétaire chargé du suivi des projets et programmes relatifs à la mise en œuvre de la Convention, représentant le Ministère chargé du Plan;

5- Un Rapporteur chargé de la préparation des réunions et de la rédaction des comptes rendus et rapports d'activités du Comité National, représentant le Ministère chargé de l'Environnement.

Article 8: Le Secrétariat Permanent a pour mission :

- l'organisation des activités du CNCC;

- la coordination des activités des équipes techniques ;

- la gestion administrative et financière ; 
- la préparation et la consolidation des plans périodiques (triennal, annuel, trimestriel...) du CNCC ;

- la préparation de la participation du Bénin aux sessions des organes de la Convention (Conférence des Parties, Organes subsidiaires);

- la coordination des études et recherches sur les inventaires des gaz à effet de serre, la vulnérabilité et l'adaptation aux changements climatiques, ainsi que sur les options d'atténuation ;

- l'évaluation des besoins technologiques requis pour la mise en œuvre de la Convention et en matière de renforcement des capacités ;

- la préparation et l'organisation de conférences, séminaires, ateliers, colloques, et tables rondes sur les changements climatiques;

Article 9: Le CNCC comprend en son sein trois équipes techniques qui sont les organes auxiliaires du Secrétariat Permanent :

- Une équipe technique chargée de l'élaboration, du suivi et de la mise en ouvre des Communications Nationales ;

- Une équipe technique chargée des études, de l'élaboration, du suivi et de la mise en œuvre des Programmes d'Actions Nationaux aux fins d'Adaptation (PANA) et autres stratégies dans le domaine des Changements Climatiques;

- Une équipe technique chargée du Mécanisme pour un Développement Propre et autres mécanismes à mettre en place.

Chaque équipe est dirigée par un Président et un rapporteur élus en son sein.

Article 10 : Les charges afférentes au fonctionnement du CNCC sont imputables au budget national et aux ressources extérieures allouées à la mise en œuvre de la Convention-Cadre sur les Changements Climatiques.

Article 11: Les membres du Comité National sur les changements climatiques sont nommés par arrêté du Ministre chargé de l'environnement, sur proposition de leurs structures de tutelle.

Article 12 : Le Ministre de l'Environnement, de l'Habitat et de l'Urbanisme et le Ministre des

Finances et de l'Economie sont chargés, chacun en ce qui le concerne, de la mise en æuvre et de l'application du présent décret.

Article 13 : Le présent décret qui abroge toutes dispositions antérieures contraires, sera publié au Journal Officiel. 
Fait à Cotonou, le 10 avril 2OOJ

Par le Président de la République,

Chef de l'Etat. Chef du Gouvernement,

Mathieu KEREKOU.-

Le Ministre des Finances et de l'Economie,

Grégoire LAOUROU.-

Le Ministre d'Etat Chargé de la Coordination de l'Action Gouvernementale, de la Prospective et du Développement,

Bruno AMOUSSOU.-

Le Ministre de l'Environnement, de l'Habitat et de 1'Urbanisme,

Luc- Marie Constant GNACADJA.- 


\section{ANNEXE II \\ Tableau récapitulatif des Accords de protection de l'environnement souscrits par le Bénin}


ANNEX II 


\section{D'ORDRE}

\begin{tabular}{|c|c|c|c|c|c|c|}
\hline \multirow[b]{2}{*}{ ㄹ. } & \multirow{2}{*}{$\begin{array}{c}\text { TITRE DE } \\
\text { L'INSTRUMENT }\end{array}$} & \multicolumn{2}{|c|}{ ADOPTION } & \multicolumn{2}{|c|}{ DATE } & \multirow[t]{2}{*}{ OBJET } \\
\hline & & 常 & 疍 & 可产 & 党 & \\
\hline 01 & $\begin{array}{l}\text { Convention sur le criquet } \\
\text { migrateur africain }\end{array}$ & 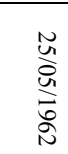 & $\begin{array}{l}\pi \\
\stackrel{\pi}{0} \\
0\end{array}$ & & 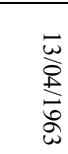 & Prévention de criquet migrateur en Afrique \\
\hline 02 & $\begin{array}{l}\text { Convention de Ramsar sur } \\
\text { les zones humides }\end{array}$ & 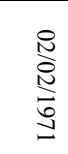 & 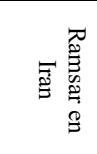 & & 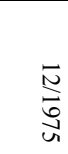 & $\begin{array}{r}\text { Conservation et l'utilisation rationnelle des } \\
\text { zones humides. }\end{array}$ \\
\hline 02 & $\begin{array}{l}\text { Convention sur la prévention } \\
\text { des pollutions par les navires }\end{array}$ & 疍 & 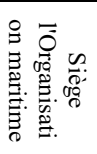 & & 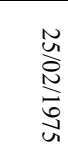 & $\begin{array}{r}\text { Prévention de la pollution en milieu marin par } \\
\text { l'élaboration des procédures et règles } \\
\text { techniques de conception d'équipement et } \\
\text { d'inspection des navires }\end{array}$ \\
\hline & $\begin{array}{c}\text { Convention sur le commerce } \\
\text { Iiternational des espèces de } \\
\text { Faune et de Flore Sauvages } \\
\text { menacées d'extinction } \\
\text { (CITES) }\end{array}$ & 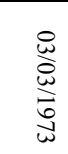 & 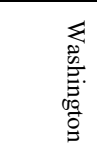 & 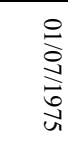 & 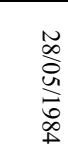 & $\begin{array}{r}\text { Protection des espèces de flore et de faune en } \\
\text { voie de disparition }\end{array}$ \\
\hline 06 & $\begin{array}{l}\text { Convention sur la } \\
\text { conservation des espèces } \\
\text { migratrices appartenant à la } \\
\text { faune sauvage (CMS) }\end{array}$ & 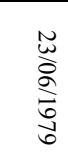 & $\begin{array}{l}\text { to } \\
0 \\
\vdots\end{array}$ & 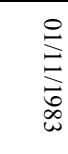 & 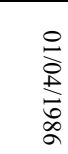 & $\begin{array}{r}\text { Conservation des espèces migratrices } \\
\text { appartenant à la faune sauvage }\end{array}$ \\
\hline 10 & $\begin{array}{l}\text { Convention d'Abidjan } \\
\text { relative à la coopération en } \\
\text { matière de protection et de } \\
\text { mise en valeur du milieu } \\
\text { marin des zones côtières et } \\
\text { de la région de l'Afrique de } \\
\text { l'ouest et du centre }\end{array}$ & 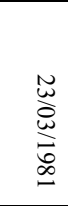 & : & 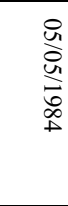 & פ্ & $\begin{array}{l}\text { Protection du milieu marin et des zones } \\
\text { côtières de certains Etats de l'Afrique }\end{array}$ \\
\hline 12 & $\begin{array}{l}\text { Convention de Vienne sur la } \\
\text { protection de la couche } \\
\text { d'ozone }\end{array}$ & 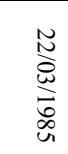 & 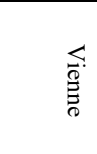 & & 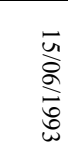 & Protection de la couche d'ozone \\
\hline 13 & $\begin{array}{c}\text { Protocole de Montréal relatif } \\
\text { aux substances appauvrissant } \\
\text { la couche d'ozone }\end{array}$ & 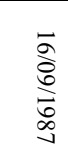 & 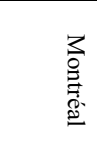 & & 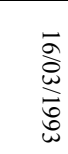 & $\begin{array}{r}\text { Règlementation des substances nuisibles à } \\
\text { l'ozone }\end{array}$ \\
\hline 15 & $\begin{array}{c}\text { Convention de Bâle sur le } \\
\text { contrôle des mouvements } \\
\text { transfrontières de déchets } \\
\text { dangereux et de leur } \\
\text { élimination }\end{array}$ & 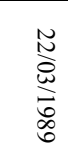 & 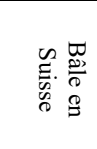 & 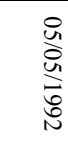 & 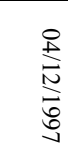 & $\begin{array}{l}\text { Règlementation des mouvements } \\
\text { transfrontières de déchets dangereux }\end{array}$ \\
\hline
\end{tabular}




\begin{tabular}{|c|c|c|c|c|c|c|}
\hline 14 & $\begin{array}{l}\text { Convention de Bamako sur } \\
\text { l'interdiction d'importer en } \\
\text { Afrique des déchets } \\
\text { dangereux et sur le contrôle } \\
\text { des mouvements } \\
\text { transfrontières et la gestion } \\
\text { des déchets dangereux } \\
\text { produits en Afrique }\end{array}$ & $\stackrel{\substack{0 \\
ٍ}}{\stackrel{2}{ٍ}}$ & 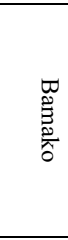 & $\begin{array}{l}\frac{N}{O} \\
\frac{1}{2} \\
D \\
\infty\end{array}$ & 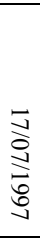 & $\begin{array}{r}\text { Interdiction d'importer en Afrique des } \\
\text { déchets dangereux et le contrôle des } \\
\text { mouvements transfrontières et la gestion des } \\
\text { déchets dangereux produits en Afrique }\end{array}$ \\
\hline 07 & $\begin{array}{c}\text { Convention Cadre des } \\
\text { Nations Unies sur les } \\
\text { Changements Climatiques }\end{array}$ & 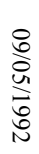 & 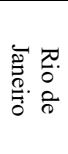 & $\underset{\substack{\infty \\
ٍ}}{\stackrel{N}{O}}$ & 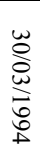 & $\begin{array}{l}\text { Contribution à l'atténuation des changements } \\
\text { climatiques par la réduction des gaz à effet de } \\
\text { serre }\end{array}$ \\
\hline 09 & $\begin{array}{c}\text { Convention Cadre des } \\
\text { Nations Unies sur la diversité } \\
\text { biologique }\end{array}$ & 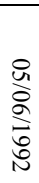 & 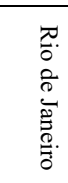 & 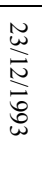 & 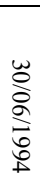 & $\begin{array}{r}\text { Conservation de la diversité biologique, } \\
\text { l'utilisation durable de ses éléments et le } \\
\text { partage juste et équitable des avantages } \\
\text { découlant de l'exploitation des ressources } \\
\text { génétiques. }\end{array}$ \\
\hline 08 & $\begin{array}{l}\text { Convention Cadre des } \\
\text { Nations Unies sur la lutte } \\
\text { contre la désertification }\end{array}$ & 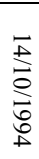 & 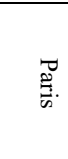 & 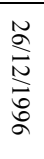 & $\begin{array}{l}\text { No } \\
\stackrel{0}{\circ} \\
\stackrel{\infty}{\sigma} \\
\stackrel{0}{\circ}\end{array}$ & Lutte contre la désertification \\
\hline 17 & Protocole de Kyoto & 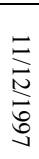 & $\underset{\substack{\pi \\
\vdots \\
0}}{\pi}$ & 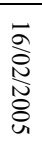 & 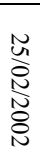 & $\begin{array}{r}\text { Protocole additionnel à la Convention Cadre } \\
\text { des Nations Unies sur les Changements } \\
\text { Climatiques }\end{array}$ \\
\hline 04 & $\begin{array}{l}\text { Convention de Stockholm sur } \\
\text { les polluants organiques } \\
\text { persistants (POPs) }\end{array}$ & $\begin{array}{l}\frac{N}{N} \\
\frac{N}{N} \\
\frac{N}{0} \\
0\end{array}$ & 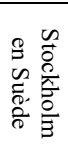 & 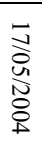 & 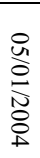 & 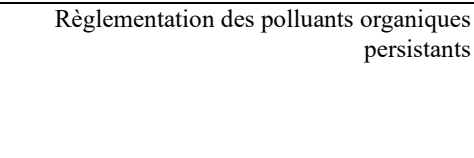 \\
\hline 18 & Accord de Paris & $\frac{\substack{n \\
\frac{N}{N}}}{\frac{N}{N}}$ & 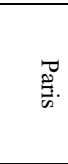 & 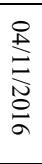 & 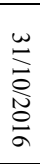 & $\begin{array}{r}\text { Protocole additionnel à la Convention Cadre } \\
\text { des Nations Unies sur les Changements } \\
\text { Climatiques }\end{array}$ \\
\hline
\end{tabular}




\section{BIBLIOGRAPHIE}


Bibliographie 


\section{REFERENCES BIBLIOGRAPHIQUES}

\section{Ouvrages généraux}

BOISGALLAIS (AS) et KABORE (GL), Livre blanc des ONG françaises. Pour l'environnement et le développement. Construire la démocratie internationale, Paris, Bruxelles, CEDI, 1992 - 95 p.

CARREAU (Dominique), DROIT INTERNATIONAL, 5ème édition, Paris, A. Pedone, 2009, 633p.

COMBACU (Jean), SUR (Serge), Droit international public, 13 éd., Paris, L.G.D.J, 2019, $896 \mathrm{p}$.

CORNU (Gérard), Dictionnaire juridique, 12éd., Paris, PUF, 2018, 1103p.

DAILlIER (Patrick), FORTEAU (Mathias) et PELLET (Alain), Droit international Public, Paris, LGDJ, 8ème éd., 2009.

DUPUY (Pierre-Marie) et KERBRAT (Yann), Droit international public, 15e édition, Paris, Dalloz, 2020, 962p.

FROMAGEAU (J.) et CORNU (M), La genèse du Droit de l'environnement : Fondements et enjeux internationaux, L'Harmattan, 2001, vol 1.

FALLOUX (François) et TABOT (Lee), Crise et opportunité. Environnement et développement en Afrique, G. P. Maisonneuve \& La Rose, Paris, 1992, 444p., pp 290-291

GAUDUSSON (Jean du Bois), CONAC (Gérard) et DESSOUCHES (Christine), Les constitutions africaines, Tome1, Bruylant, Bruxelles, 2003.

GUILLIEN (Raymond) et VINCENT (Jean), Lexique des termes juridiques, $5^{\mathrm{e}}$ éd., Dalloz, 1981, 567p.

GRANIER (Laurent), Aspects contemporains du droit de l'environnement en Afrique de l'Ouest et centrale, UICN, Gland, Suisse, 2010, 242p.

GUILLAUMET (Jean-Louis), Désertification et déforestation : Note synthétique sur l'état des connaissances, Nanterre, Horizon, 1996, 494p.

GUTWIRTH, (S.), Pour une approche plus générale des rapports entre la science et le droit (avec entre autres des illustrations dans le domaine du droit de l'informatique, de la protection de la jeunesse, de l'internement, de la "collocation" et du droit de l'environnement), Bruxelles/Anvers, 1993, 846 p.

KAMTO (M.), Droit de l'environnement en Afrique, Paris, Edicef, 1996, 416p.

KISS (A.), Droit International de l'Environnement, Bruxelles, $1^{\text {ère }}$ éd. Pedome, 1969, 229p.

LAWOGNI (Athanase) et de SADELEER (Nicolas), Droit de l'environnement au Bénin Code officieux de l'environnement, Cotonou- Bruxelles, 2002, 406p.

MALJEAN DUBOIS (Sandrine), Quel droit pour l'environnement? Hachette Supérieur, Espagne, 2008, 158p. 
MARTIN (Holdgate M.), the Green Web A Union for World Conservation, Earthscan, London, 1999, 308p.

OLIVIER (Juliette), L'Union mondiale pour la nature (UICN) une organisation singulière au service du droit de l'environnement, Bruxelles, Bruylant, 2005, 372p.

PRIEUR (Michel), Droit de l'environnement, $8^{\mathrm{e}}$ éd, Paris, Précis Dalloz, 2019, 1394p.

ROBERT (Paul), Dictionnaire Alphabétique et Analogique de la Langue Française, LE PETIT ROBERT 1, LE ROBERT, Paris, 1997.

ROUSSEAU (Charles), Príncipes Généraux du Droit International Public. Tome I: Introduction; Sources. Publié en ligne par Cambridge University Press, Paris: A. Pedone, 2017 $1944 \mathrm{p}$.

SALMON (Jean), Dictionnaire de droit international public, Bruylant, Bruxelles, 2011, 1200p.

SANDS (Philippe), Jacqueline Peel; Adriana Fabra; Ruth Mackenzie, Principles of international environmental law, 4th edition, Cambridge University Press, August 2018, 1032p.

\section{Ouvrages spécialisés}

MALJEAN-DUBOIS (Sandrine), Les enjeux du contrôle international, La Documentation Française, Paris, 2007, 383p.

MALJEAN-DUBOIS (Sandrine) et WEMAERE (Matthieu), La diplomatie climatique de Rio 1992 à Paris 2015, Pedone, 332p.

De Perthuis, C., Et pour quelques degrés de plus ... Nos choix économiques face au risque climatique, Pearson, Paris 2009, 306p.

\section{Thèses}

ADELOUI (Arsène-Joël), Transition démocratiques et coopération au développement. Approches comparative Bénin, Mali, Niger et Togo. Thèse de Doctorat, UAC, FADESP, Chaire, UNESCO, droit de l'homme et démocratie, Cotonou, 2006.

ROMI (Raphaël), Le juge, la prévention et la résolution des litiges en matière d'environnement, Thèse de doctorat, Paris III, multigraphiée, 1994, 432 p.

TCHOCA FANIKOUA (François), La contribution du droit pénal de l'environnement à la répression des atteintes à l'environnement au bénin, Thèse de doctorat en Droit, Université de Maastricht, 2012, 445p.

\section{Articles, communications et actes de colloque}

ABBAS, M., Economie politique globale des changements climatiques. Grenoble: Presse Universitaires de Grenoble, 2010.

ALLARD (Patrick), « Malaise dans la climatisation, le changement climatique et la sécurité des États », Annuaire français des relations internationales nº 6 , 2005, pp. 942-951. 
ALEXANDRE (Kiss), « Les traités-cadres. Une technique juridique caractéristique du droit international de l'environnement », Annuaire français de droit international, 1993.

ALEXANDRE (Kiss.), « Cinq années de droit international de l'environnement », RJE, $\mathrm{n}^{\circ}$ $4 / 2001$.

ALEXANDRE (Charles Kiss) et JEAN (Didier Sicault), Conférence des Nations unies sur l'environnement (Stockholm, 5/16 juin 1972) Annuaire de Droit International, 1972, p.610.

AÏVO (Frédéric Joël), FAURE (Michael), GBAGO (Georges Barnabé) et TCHOKA (François), Enjeux économiques et environnementaux des maîtrises foncières en Afrique, $1^{\mathrm{e}}$ éd., Paris, Anthemis, 2019, p.296.

BARNOSKY (A. D.), "Approaching a state shift in Earth's biosphere », Nature, vol. CDLXXXVI, 7 juin 2012, pp. 52-58.

Benoit (Mayer), «Un examen des lignes directrices de la Commission du droit international sur la protection des atmosphères », Melbourne Journal of International Law, 2019.

BERTHAUD (Pierre), CAVARD (Denise), et CRIQUI (Patrick), Le régime international pour le climat, vers la consolidation ou l'effondrement? In Revue française d'économie. Vol. $19 \mathrm{n}^{\circ} 2,2004$.

BODANSKY (Daniel) et O'CONNOR (Sandra Day), Reflections on the Paris Conference, Mayer, 2016.

BENZAIDA (G Kamal), Perspectives et actions en Afrique pour faire face aux effets du changement climatique, GPB N³/2020, Maroc, 2020.

BELOW (R.) et Wallemacq (P.), Annual Disaster Statistical Review 201, CRED, 2018.

DOYLE (Alister), « 46 nations call for tougher U.N. environment rôle », Reuters, 3 février 2007.

DESPAX (Michel), Droit de l'environnement, Litec, 1980, p.879 .

DOMINIQUE (Bourg), «L'impératif écologique », L'indépendance d'Esprit, décembre 2009.

DOUMBE-BILLE (S.) et KISS (A. C.), « La Conférence des Nations unies sur l'environnement et le développement ", AFDI, 1992, pp. 823-843.

DOUMBE-BILLE (Stéphane), Evolution des institutions et des moyens de mise en œuvre du droit de l'environnement et du développement. In: Revue Juridique de l'Environnement $\mathrm{n}^{\circ} 1$, 1993. pp. 31-44.

DOUMBE-BILLE (Stéphane), Droit international de la faune et des aires protégées : importance et implications pour l'Afrique, Etudes juridiques de la FAO en ligne, n²0, 2001.

Dow, K., et Downing, T, The Atlas of Climate Change: Mapping the World's greatest Challenge (éd. 3e). Los Angeles: University of California Press, 2011.

Ellul Jacques, Sur l'artificialité du droit et le droit d'exception, Archives de philosophie du Droit, 1963. 
ENCINAS DE MUNAGORI (R.), «Expert et expertise », in Dictionnaire de la culture juridique, Paris, PUF, 2003.

FAURE (Michael) ; GOODWIN (Morag) et WEBER (Franziska), Bucking the Kuznets Curve: Designing Effective Environmental Regulation in Developing Countries, USA, Virginia Journal of International Law, 2010, 51p, pp. 95-156.

Faure, M. G., \& Lefevere, J. G. J. (2014). Compliance with global environmental policy: climate change and ozone layer cases. In R. S. Axelrod, \& S. D. VanDeveer (Eds.), The global environment. Institutions, law, and policy (4th ed. ed., pp. 110-132).

FOUCARD (Stéphane), « La biosphère mondiale à la veille d'une crise "irréversible” », Le Monde, 2012

FOURRIER (J), « Remarques générales sur les températures du globe terrestre et des espaces planétaires », Annales de Chimie et de physique, 1824, vol.27, p. 136-167.

GODARD (O.), Stratégies industrielles et conventions d'environnement : de l'univers stabilisé aux univers controversés, Environnement, Economie, Actes de colloques, Paris 15-16 février 1993, Ed. de 1'INSEE méthodes, Paris, (Coll. 'INSEE Méthodes n³9-40'), décembre, pp.145-174.

Grubb (Michael), Full Legal Compliance with the Kyoto Protocol's First Commitment Period - Some Lessons, Climate Policy 16, no. 6 (2016): pp. 673-681.

HAMADY DEME (Baba), RIO+20 ET L'AVENIR DE LA GOUVERNANCE DE L'ENVIRONNEMENT EN AFRIQUE, Revue Africaine de Droit de l'Environnement $n^{\circ} 00$ 2012, 2012, Centre de droit international, Université Jean Moulin Lyon 3.

JAWORSKI (V.), La Charte constitutionnelle de l'environnement face au droit pénal, in RJE $\mathrm{n}^{\circ}$ spécial 2005.

Joanna Lewis, "China», dans Climate Change and National Security: A Country-Leve/ Analysis, sous la dir. de Daniel Moran. (Washington, D.C.: Georgetown University Press, 2011).

JOHN (T. Ackerman), «Climate Change, National Security, and the Quadrennial Defense Review », Strategic Studies Quarterly, printemps, 2008, pp. 56-96.

KAMTO (Maurice), Les conventions régionales sur la conservation de la nature et des ressources naturelles en Afrique et leur mise en œuvre. In: Revue Juridique de l'Environnement, n4, 1991. pp. 417-442.

KAMTO (Maurice), « Singularité du droit international de l'environnement », in Mélanges en hommage à Alexandre Kiss, les hommes et l'environnement, Editions Frison-Roche, 1998.

KAMTO (Maurice), « La mise en œuvre et le suivi du droit international de l'environnement » Rapport introductif général, Acte des troisièmes journées scientifiques du Réseau «Droit de l'environnement », AUF, Yaoundé, juin 2001.

KAMTO (Maurice), La mise en œuvre du droit de l'environnement : Forces et faiblesse des cadres institutionnelles, RADE n01-2014, 2014.

Ahonagnon Noel Gbaguidi, William Kodjoh-Kpakpassou, Gérard AÏVO et Lazard H. HOUNSA, Introduction au Système Juridique et Judiciaire du Bénin, Hauser Global Law School Program, New York University School of Law, New York, Mai 2019. 
KOUEMBEU TAGNE (Jean Jacques), La Cour pénale internationale et les USA, Facultés Universitaires Saint Louis de Bruxelles, Bruxelles, Belgium, 2008, 19p.

KHOR (Martin), Quel lien y a-t-il entre la lutte contre les changements climatiques, le transfert de technologie et les droits de propriété intellectuelle et où en sont les négociations à leur sujet ?», Centre Sud, 2012.

LAM (V. Trien), « Les enjeux juridiques de l'intégration des pays en développement dans le régime climatique post-Kyoto, 23 RQDI 31, 2010, pp. 88-89.

Laurence Boisson de Chazournes, «L'impact humain sur le changement climatique », United Nations Audiovisual Library of International Law, 8p., 2009.

LAVIEILLE (Jean-Marc) et OLIVIER (Mazaudoux), " Droit international public et droit international de l'environnement », Les Cahiers du CRIDEAU, $n^{\circ} 16$, 2008. In: Revue Juridique de l'Environnement, $n^{\circ} 3,2008$.

LAVALLEE (Sophie), « Le principe des responsabilités communes, mais différenciées à Rio, Kyoto et Copenhague. Essai sur la responsabilité de protéger le climat », Études internationales, $\mathrm{n}^{\circ} 1$, mars 2010, pp. 51-78.

LAVALLEE (Sophie), "Quelques réflexions sur 1'Accord de Paris et la souveraineté économique des Etats », Vertigo, la revue électronique en sciences de l'environnement, Volume 18 numéro 1 , mai 2018.

LITIM (Nadia), Le PNUE : Quel rôle joue-t- il pour l'environnement ? Dans la REVUE ELMOFAKER N 13 de l'Université- Skikda, 1955.

M. Torre-Schaub, Le principe de précaution dans la lutte contre le réchauffement climatique: entre croissance économique et protection durable, Revue européenne de droit de l'environnement, $\mathrm{n}^{\circ} 2,2002$.

MALJEAN-DUBOIS (Sandrine) et RICHARD (Vanessa), Mécanisme internationaux de suivi et mise en œuvre des conventions internationales de protection de l'environnement, Aixen-Provence, 2003.

MALJEAN-DUBOIS (Sandrine) et RICHARD (Vanessa), Mécanismes internationaux de suivi et mise en œuvre des conventions internationales de protection de l'environnement, France, CERIC, 2004.

MALJEAN-DUBOIS (Sandrine), La mise en route du Protocole de Kyoto à la Convention Cadre des Nations Unies sur les Changements Climatiques, Annuaire Français de Droit International, 2005.

MALJEAN-DUBOIS (Sandrine), L'«observance» du Protocole de Kyoto sur les changements climatiques. Les enjeux du contrôle international du respect des engagements ", Synthèse $n^{\circ} 01 / 2007$, Institut du développement durable et des relations internationales, Paris, $4 \mathrm{p}$.

Maljean-Dubois Sandrine et Lavanya Rajamani, La mise en œuvre du droit international de l'environnement, Académie de droit international de La Haye, La Haye, Martinus Nijhoff, 2011.

MALAJEAN-DUBOIS (Sandrine), WEMAERE (Matthieu), La Conférence des Nations unies sur le développement durable Rio+20, Annuaire français de relations internationales, Paris, La Documentation française, 2014, pp.721-735. 
MALJEAN-DUBOIS (Sandrine) et WEMAERE (Matthieu), Cop21 ? La diplomatie climatique de Rio 1992, Pedome, Paris 2015.

MALJEAN-DUBOIS (Sandrine), SPENCER (Thomas) et WEMAERE (Matthieu), "La forme juridique du futur accord de Paris sur le climat : enjeux et principales options », Pedome, Paris 2015.

MALJEAN-DUBOIS (Sandrine), Quel droit international face au changement climatique? SFDE, 2017.

MALJEAN-DUBOIS (Sandrine) ; RAJAMANI (Lavanya), L'Accord de Paris sur les changements climatiques du12 décembre 2015. Annuaire Français de Droit International, CNRS, 2017.

Michael Mastrandera et Stephen Schneider, "Climate Change Science Overview », dans Climale Change: Science and Policy sous la dir. de Stephen Schneider. (Washington, D.C.: Island Press, 2010).

MOUTONDO (Emmanuel G.), Les lois-cadres environnementales dans les pays francophones d'Afrique, in Aspects contemporains du droit de l'environnement en Afrique de 1'ouest et centrale, UICN, droit et politique de l'environnement nº69, 2008.

OUATTARA (B.), Le Rolle des organisations sous-régionales dans le développement du droit de l'environnement: l'exemple de l'UEMOA», in L. Granier (coord.), Aspects contemporains du droit de l'environnement en Afrique de l'ouest et centrale, UICN, Gland, 2008, pp. 177-196.

OLIVIER (Juliette), « Les nouveaux acteurs du droit de l'environnement : Le rôle de l'UICN dans l'élaboration du droit de l'environnement» in Revue Européenne de Droit de l’Environnement n³, 2005, pp.274-296.

PATRICK (Allard), « Malaise dans la climatisation, le changement climatique et la sécurité des États », Annuaire français des relations internationales, ${ }^{\circ}$ 6, 2005, pp. 942-951.

PHILIPPE (Ambrosi) et STEPHANE (Hallegate), " Changement climatique et enjeux de sécurité », Les cahiers de la sécurité, $\mathrm{n}^{0}$ 58, 2005, pp. 297-318.

PRIEUR (Michel), Pourquoi une revue juridique de l'environnement ?, In: Revue Juridique de l'Environnement, ${ }^{\circ} 1,1976$, pp. 3-4.

PRIEUR (Michel), Démocratie et droit de l'environnement et du développement. In: Revue Juridique de l'Environnement, $\mathrm{n}^{\circ} 1,1993$, pp. 23-30.

PRIEUR (Michel) (dir.), La mise en œuvre national du droit international de l'environnement dans les pays francophones, Acte des troisièmes journées scientifiques du Réseau «Droit de l'environnement », AUF, Yaoundé, juin 2001.

PRIEUR (Michel), « Mondialisation et droit de l'environnement », Rio de Janeiro, 2002, pp. $13-22$.

PRIEUR (Michel), Vers un droit de l'environnement renouvelé, Les Cahiers du Conseil constitutionnel, 2004, $\mathrm{n}^{\circ} 15$. 
PRIEUR (Michel), L'influence des conventions internationales sur le droit interne de l'environnement, Actes de la réunion constitutive du comité sur l'environnement de l'AHJUCAF, Juin 2008, Porto-Novo, Bénin, pp.291-301.

PRIEUR (Michel), Le juge au secours des indiens et de la forêt, France Forum, n77, 2020.

ROCHE (Céline), Droit de L'Environnement, 2eme Edition, Paris, Gualino éditeur, 2006.

ROLIN (Henri), "Les principes de droit international public (Volume 77)", in: Collected Courses of the Hague Academy of International Law. Consulted online on 03 June 2021 $<$ http://dx.doi.org/10.1163/1875-8096_pplrdc_A9789028611429_04> First published online: 1950.

RUIZ FABRI (Hélène), Le droit dans les relations internationales. In: Politique étrangère, $\mathrm{n}^{\circ} 3$ 4, 2000, pp. 659-672.

SYLVAIN Cypel, « $\mathrm{Al}$ Gore appelle au volontarisme sur l'environnement », Le Monde, 11 novembre 2009.

Shishlov (See Igor), Morel (Romain) and Bellassen (Valentin), Compliance of the Parties to the Kyoto Protocol in the First Commitment Period," Climate Policy 16, no. 6 (2016): 768-782.

Staehelin ,J., F. Tummon, A. Stenke, C. Arndt et P. Bratfisch, « Modifications de la couche d'ozone suite au changement climatique », SPARC Faktenblatt, 2014, 4p.

STEFAN C. Aykut et AMI Dahan, 2014, Gouverner le climat? 20 ans de négociations internationales, Paris, Sciences Po Les Presses.

Siobhan Mc Inerney-Lankford, Mac Darrow, and Lavanya Rajamani,Human rights and climate change: are view of the international legal dimensions, Banque Mondiale, 2011.

Stephen Humphreys (dir.), Humanrights and climate change, Cambridge University Press, 2010 .

VAN LANG (Agathe), Droit de l'environnement, Paris, PUF, 2016.

Weiss (Edith Brown) et Jacobson (Harold K.), « Engager les pays: renforcer le respect des accords environnementaux », Cambridge, Londres, The MIT Press, 1998.

YVES Petit, «Les risques environnementaux globaux et les transformations de la souveraineté », SFDI, Colloque de Nancy, l'Etat dans la mondialisation, 2013, Pedome.

YVES Petit, Rapport introductif « La lutte contre le réchauffement climatique après l'Accord de Paris: quelles perspectives?», VertigO - la revue électronique en sciences de l'environnement, Volume 18 numéro 1, mai 2018.

\section{Sites internet}

FAO : www.fao.org.

ONU : https://treaties.un.org.

UICN : https://www.iucn.org.

\section{Traités et déclarations}


Convention sur le droit des traités entre Etats, Vienne, 23 mai 1969, Doc.NU. A/Conf.39/27, 1969. Sur le site internet: https://treaties.un.org/doc/source/docs/A_CONF.39_11_Add.2F.pdf. Consulté le 04/06/2021.

Protocole à la Convention de Vienne sur la protection de la couche d'ozone, Montréal, 1987, UN. Treaty Series, $\mathrm{n}^{\circ} 19$, 1990. Sur le site internet: https://ise.unige.ch/isdd/IMG/pdf/convention_de_vienne.pdf. Consulté le 04/06/2021.

Convention sur le Diversité Biologique, 5 juin 1992, Doc. UNEP/Bo.Div/N7-INC.5/4.

Convention Cadre des Nations Unies sur les Changements Climatiques a été adoptée au siège des Nations unies, à New York, le 9 mai 1992 (en ligne sur le site internet https://unfccc.int/resource/docs/convkp/convfr.pdf) consulté le 05 aout 2020.

Accord de l'Organisation Mondiale du Commerce (OMC), Marrakech, 15 avril 1994 (en ligne sur le site internet https://www.wto.org/french/res_f/booksp_f/wtoagreement_f.pdf) consulté le 13 mars 2021.

Protocole de Kyoto à la Convention Cadre des Nations Unies sur les Changements Climatiques (en ligne sur le site https://unfccc.int/resource/docs/convkp/kpfrench.pdf) consulté le 05 aout 2020 .

Accord de Paris (en ligne sur le site internet https://unfccc.int/fr/process-and-meetings/theparis-agreement/l-accord-de-paris) consulté le 05 aout 2020.

Nations unies , Déclaration de Stockholm de la Conférence des Nations unies sur l'environnement, 1972, (en ligne), disponible sur le site : http ://www.unep.org/Documents (consulté le 30 juin 2015), Doc.NU A/Conf.48/14/Rev.1.

Charte Mondiale de la Nature, Résolution 37/7 des Nations unies , 28 octobre 1982, UN Doc. $\mathrm{A} / 398$.

Nations unies, Déclaration de Rio de Janeiro sur l'environnement et le développement, Rio, 13 juin 1992, (en ligne), disponible sur le site internet : http ://www.un.org/french/events/rio92/rio-fphtm (consulté le 05 aout 2020).

Nations unies, Déclaration de principe non juridiquement contraignantes mais faisant autorité, pour un consensus mondial sur la gestion, la conservation et l'exploitation écologiquement viable de tous les types de forêts, Rio, juin 1992, (en ligne), disponible sur le site internet : http ://www.un.org/french/events/rio92/rio-fp.htm (consulté le 28 juillet 2020); NU Doc.A/Conf.151/26,vol.III.

Nations unies, Développement durable, Action 21, Rio, juin 1992, (en ligne), disponible sur le site internet : http ://www.un.org/french/events92/agenda21/index.html (consulté le 28 juillet 2020); NU Doc. a/Conf.151/26.

\section{Résolutions d'assemblée générale des Nations unies (Disponible sur le site : $\quad$ https://www.un.org/fr/sections/documents/general- assemblyresolutions.)}

Résolution A/RES/2850 du 20 décembre 1971 sur la Conférence des Nations unies sur l'environnement.

Résolution A/RES/2849 du 20 décembre 1971 sur le développement et l'environnement. 
Résolution 299715 décembre 1972 relatives aux dispositions institutionnelles et financières concernant la coopération internationale dans le domaine de l'environnement.

Résolution A/RES/3129 en date du 13 décembre 1973 relative à la Coopération dans le domaine de l'environnement en matière de ressources naturelles partagées par deux ou plusieurs Etats.

Résolution A/RES/3264 du 09 décembre 1974 relative à l'interdiction d'agir sur l'environnement et le climat à des fins militaires et autres incompatibles avec le maintien de la sécurité internationale, le bien-être et la santé de l'être humain.

Résolution A/RES/31/72 du 10 décembre 1976 relative à la Convention sur l'interdiction d'utiliser des techniques de modification de l'environnement à des fins militaires ou toutes autres fins hostiles.

Résolution 37/7 de l'Assemblée générale des Nations unies , "Charte mondiale de la nature » 1982.

Résolution 42/187 en date 11 décembre 1987 relative à l'adoption du rapport de la Commission mondiale pour l'environnement et le développement.

Résolution $\mathrm{A} / \mathrm{RES} / 42 / 186$ du 11 décembre 1987 relative à l'étude des perspectives en matière d'environnement jusqu'à l'an 2000 et au-delà.

Résolution 43/53 du 6 décembre 1988 relative à « Protection du climat mondial pour les générations présentes et futures.

Résolution A/RES/43/53 du 6 décembre 1988 relative à la Protection du Climat mondial pour les générations présentes et futures.

Résolution A/RES/44/207 du 22 décembre 1989 relative à la protection du climat mondial pour les générations présentes et futures.

Résolution 45/212 du 21 décembre 1990 relative à protection du climat mondial pour les générations présentes et futures.

Décisions de la Conférence des Parties à la Convention Cadre des Nations Unies sur les Changements Climatiques. (Disponible sur le https://unfecc.int/fr.)

Décision 6/CP.1, Organes subsidiaires créés par la Convention, FCCC/CP/1995/7/Add.1.

Décision 14 / CP.1 sur les liens institutionnels entre le Secrétariat de la Convention et l'Organisation des Nations Unies et les questions administratives connexes: note / du Secrétaire exécutif. FCCC / CP / 1996/6 / Add.2.

Décision 1/CP.3, Adoption du Protocole de Kyoto à la Convention Cadre des Nations Unies sur les Changements Climatiques, FCCC/CP/1997/7/Add.1. 
Décision 16/CP/7., Lignes directrices pour l'application de l'article 6 du Protocole de Kyoto, FCCC/CP/2001/13/Add.2.

Décision 17/CP.7, Modalités et procédures d'application d'un mécanisme du développement propre tel que défini à l'article 12 du Protocole de Kyoto, FCCC/CP/2001/13/Add.2.

Décision 18/CP.7, Modalités, règles et lignes applicables à l'échange de droits d'émission (article 17 du Protocole de Kyoto), FCCC/CP2001/13/Add.2.

Décision 1/CP.17, Création d'un groupe de travail spécial de la plate-forme de Durban pour une action renforcée, FCCC/CP/2011/9/Add.1.

Décision 3 / CP.17, Lancement du Fonds vert pour le climat, FCCC / CP / 2011/9 / Add.1.

Décision 1/CP.18, Résultat convenu conformément au Plan d'action de Bali, FCCC/CP/2012/8/Add.1.

Décision 1/CP.21, Adoption de l'Accord de Paris, FCCC/CP/2015/10/Add.1.

\section{Décisions de la Conférence des Parties au Protocole de Kyoto (Disponible sur le https://unfecc.int/fr.)}

Décision 24/CP.7, CCC/CP/2001/13/Add.3 21 janvier 2002, Procédures et mécanismes relatifs au respect des dispositions du Protocole de Kyoto.

Décision 27 / CMP.1, Procédures et mécanismes relatifs à la conformité dans le cadre du protocole de Kyoto, FCCC / KP / CMP / 2005/8 / Add.3.

\section{Rapport méthodologique du GIEC (disponible sur www.ipcc.ch)}

GIEC : Premier rapport sur les Changements climatiques, les évaluations du GIEC de 1990 à 1992.

GIEC : Deuxième rapport sur les Changements climatiques, les évaluations du GIEC de 1995.

GIEC : Troisième rapport sur les Changements climatiques, les évaluations du GIEC de 2001.

GIEC : Quatrième rapport sur les Changements climatiques, les évaluations du GIEC de 2007.

GIEC : Cinquième rapport spécial publié en 2014 par le Groupe d'Experts Intergouvernemental sur l'Evolution du Climat.

\section{Autre rapport}

Cellule Etat de l'Environnement wallon (2007) : Rapport analytique sur l'état de l'environnement wallon 2006-2007, MRW, DGRNE, Namur, 736 pp. 


\section{Actes d'institution communautaire}

UA : Statut de la Cour Africaine de Justice. Sur le site internet https://au.int/fr/treaties/protocole-sur-le-statut-de-la-cour-africaine-de-justice-et-desdroits-de-lhomme. Consulté le 02/06/2021.

UA : Acte constitutif de l'Union africaine. Sur le site internet : https://au.int/fr/treaties/protocole-sur-les-amendements-lacte-constitutif-de-lunion-africaine. Consulté le 02/06/2021.

UA: Charte Africaine des Droits de l'Homme et des Peuples. Sur le site internet: https://au.int/fr/treaties/charte-africaine-des-droits-de-lhomme-et-des-peuples. Consulté le 02/06/2021.

CEDEAO : Traité révisé. Sur le site internet: https://www.ecowas.int/wpcontent/uploads/2015/02/Traite-Revise.pdf. Consulté le 02/06/2021.

CEDEAO : Acte additionnel A/SA.4/12/08 portant adoption de la politique environnementale de la CEDEAO adopté le 19 décembre 2008.

UE : Convention européenne des droits de l'homme. Sur le site internet : https://www.coe.int/fr/web/human-rights-convention/the-convention-in-1950. Consulté le $02 / 06 / 2021$.

\section{Traité et déclaration}

Déclaration des droits de l'homme et du citoyen de 1789. Sur le site internet: https://www.legifrance.gouv.fr/contenu/menu/droit-national-en-

vigueur/constitution/declaration-des-droits-de-1-homme-et-du-citoyen-de-1789. Consulté le 02/06/2021.

Déclaration Universelle des Droits de l'Homme de 1948. Sur le site internet: https://www.un.org/fr/universal-declaration-human-rights. Consulté le 02/06/2021.

Charte mondiale de la nature adoptée en 1982 par l'Assemblée générale des Nations unies .

Déclaration de Rio de Janeiro sur l'environnement et le développement, adopté en 1992. Sur le site internet: https://www.un.org/french/events/rio92/aconf15126vollf.htm. Consulté le 02/06/2021.

Déclaration S/PRST/2014/5 du Conseil de sécurité des Nations unies relative à la Promotion et renforcement de l'état de droit dans le cadre des activités de maintien de la paix et de la sécurité internationales. Sur le site internet : https://www.un.org/securitycouncil/fr/content/statementsmade-president-security-council-2014. Consulté le 02/06/2021.

\section{Décision d'institution spécialisée de l'ONU}

Conseil des droits de l'homme : NATIONS UNIES, Assemblée générale, Conseil des droits de l'homme, Droits de l'homme et changement climatique, A/HRC/7/L.21/Rev.1, 20 mars 2008 . 


\section{Jurisprudences de juridiction internationale}

Liste des arrêts, décisions et avis consultatifs disponible sur le site internet: https://echr.coe.int/Pages/home.aspx? $=$ caselaw\& c=fre. Consulté le 04/06/2021.

CEDH, arrêt 16798/90 du 09.12.1994, Affaire Respect de la vie familiale, Lopez Ostra C/ Espagne.

CEDH 9, arrêt décembre 1994, Lopez Ostra c. Espagne (Requête n 16798/90), « la Cour a jugé que les bruits et les odeurs d'une station d'épuration pouvaient affecter le bien-être d'une personne et le droit au respect de son domicile. A un certain degré d'intensité, cela représente une violation du droit au respect de la vie privée et familiale, prévu à l'article 8 CEDH ».

Les arrêts, avis consultatifs et ordonnances disponible sur le site internet : https://www.icjcij.org/fr/decisions/judgment/2003/2021/desc. Consulté le 04/06/2021.

CIJ : Licéité de la menace ou de l'emploi d'armes nucléaires, Avis consultatif du 08 juillet 1996, Rec. 1996 P. 241.

CIJ : Projet Gabcikovo-Nagymaros (Hongrie/Slovaquie), arrêt, C. I. J. Recueil 1997.

CIJ : Différend frontalier (Bénin/Niger), arrêt, C.I.J. Recueil 2005,p.90.

CIJ, Arrêt de 2010 dans Pulp Mills, (n16) para 204.

Répertoire de jurisprudence disponible sur le site internet: https://curia.europa.eu/jcms/jcms/Jo2_7046/fr/. Consulté le 04/06/2021.

CJCE, 30 septermbre 1987, Delirel c/ ville de Schwabisch Gmund, 12/86, Rec. p. 3752, point 14.

OMC/Organe d'appel, États-Unis - Normes concernant l'essence nouvelle et ancienne formules (Plainte de la République Bolivarienne du Venezuela) (1996), OMC Doc WT/DS2/8 aux pp 1719, en ligne: OMC <www.wto.org/french/tratop_f/dispu_f/cases_f/ds2_f.htm> [ÉtatsUnis - Essence]. Page consulté ce 02/06/2021.

OMC : Gabrielle Marceau, « The New WTO TBT jurisprudence in US -Clove Cigarettes, WTO US-Tuna II, and US-COOL » (2013) 8:1 Asian J WTO \& Intl Health L \& Policy 1.

OMC : Organe d'appel, États-Unis - Mesures affectant la production et la vente des cigarettes aux clous de girofle (Plainte de l'Indonésie) (2012), OMC Doc WT/DS406/AB/R, en ligne : OMC < $<w w$ w.wto.org/french. Page consulté ce 02/06/2021.

OMC : Organe d'appel, États-Unis - Prohibition à l'importation de certaines crevettes et de certains produits à base de crevette (Plainte de l'Inde et al.) (1998), OMC Doc WT/DS58/AB/R, en ligne : OMC <www.wto.org/french/tratop_f $/$ dispu_f/cases_f $/$ ds58_f.htm $>$ [États-Unis - Crevettes]. Page consulté ce 02/06/2021.

OMC : Gabrielle Marceau, «L'affaire CE - Amiante et la nouvelle jurisprudence de l'Organe d'appel concernant les risques à la Santé » (2000) 38 ACDI 213.

OMC: Organe d'appel, Brésil-Mesures visant l'importation de pneumatiques rechapés (Plainte des communautés européennes) (2007), OMC Doc WT/DS332/AB/R, en ligne : OMC $<$ www.wto.org/french/tratop_f/dispu_f/cases_f/ds332_f.htm $>\quad$ [Brésil-Pneumatiques rechapés]. Page consulté ce $02 / 06 / 2021$.

OMC: Organe d'appel, États-Unis-Normes concernant l'essence nouvelle et ancienne formules (Plainte de la République Bolivarienne du Venezuela) (1996), OMC Doc WT/DS2/8 
aux pp 17-19, en ligne: OMC <www.wto.org/french/tratop_f/dispu_f/cases_f/ds2_f.htm> [États-Unis-Essence]. Page consulté ce 02/06/2021.

\section{Rapport d'organe spécialisé des Nations unies}

PNUD : Rapport mondial sur le développement humain 2007/2008, La lutte contre le changement climatique : un impératif de solidarité humaine dans un monde divisé, New York, 2007, p. v. Sur le site internet: http://hdr.undp.org/sites/default/files/hdr_20072008_fr.pdf. Consulté le 04/06/2021.

ONU : Rapport de la Commission environnement : Renforcer l'efficacité du droit international. Devoirs des Etats, droits des individus, novembre 2015. Sur le site internet: https://globalpactenvironment.org/uploads/2.-Synth\%C3\%A8se-du-rapport_10-pages1.pdf.

Consulté le 04/06/2021.

UICN-CMAP, TNC, PNUD, WCS, la Banque mondiale et le WWF: « Solutions Naturelles Les aires protégées nous aident à faire face aux changements climatiques ", (en ligne) https://www.iucn.org. Page consulté ce 02/06/2021.

UICN : Rapport général sur l'influence des conventions internationales sur le droit interne de l'environnement de Monsieur Michel PRIEUR Professeur émérite à l'Université de Limoges, président du Centre International de Droit comparé de l'environnement, vice-président de la Commission de droit de l'environnement à l'Union mondiale de la Nature. Sur le site internet : https://www.iucn.org/sites/dev/files/content/documents/rade_n00.pdf. Consulté le 04/06/2021.

Conférence de Stockholm de 1972: Rapport de la Conférence des Nations unies sur l'Environnement Stockholm, 5 au 16 juin 1972, Première recommandation relative aux «Résolutions sur les dispositions institutionnelles et financières ». Sur le site internet: https://www.un.org/fr/conferences/environment/stockholm1972. Consulté le 04/06/2021.

COP/CCCC : Rapport de la première session de la Conférence des Parties agissant comme réunion des Parties au Protocole de Kyoto tenue à Montréal du 28 novembre au 10 décembre 2005, Doc. N.U. FCCC/KP/CMP/2005/8/Add.3 (30 mars 2006). Sur le site internet: https://unfccc.int/resource/docs/2005/cmp1/eng/08a02.pdf. Consulté le 04/06/2021.

$\mathrm{COP} / \mathrm{CCCC}$ : Rapport de la quinzième session de la Conférence des Parties tenue à Copenhague du 7 au 19 décembre 2009 Additif Deuxième partie: Mesures prises par la Conférence des Parties à sa quinzième session Décision 2/CP.15. Sur le site internet : https://unfccc.int/resource/docs/2009/cop15/fre/11a01f.pdf. Consulté le 04/06/2021.

Protocole de Montréal : Rapport de la première session de la Conférence des Parties agissant comme réunion des Parties au Protocole de Kyoto tenue à Montréal du 28 novembre au 10 décembre 2005. Sur le site internet : https://unfccc.int/fr/node/4253. Consulté le 04/06/2021.

COP/CCCC : Guesnerie (R.), Kyoto et l'économie de l'effet de serre, Rapport CAE n³9, La Documentation Française, Paris, 2003. Sur le site internet: https://www.caeeco.fr/staticfiles/pdf/039.pdf. Consulté le 04/06/2021. 


\section{Documents fondamentaux d'institutions spécialisées de l'ONU}

UNESCO : Convention pour la protection du patrimoine mondial, culturel et naturel adopté par les Etats membres de l'UNESCO en 1972. Disponible sur le site internet : https://whc.unesco.org/fr/conventiontexte. Consulté le 04/06/2021.

OMC : Accord sur les Obstacles Techniques au Commerce, 15 avril 1994, 1868 RTNU 120 (entrée en vigueur: $1^{\text {er }}$ janvier 1995) [Accord OTC]. Disponible sur le site internet : https://www.wto.org/french/tratop_f/tbt_f/tbt_f.htm. Consulté le 04/06/2021.

OMC : Accord général sur les tarifs douaniers et le commerce, 30 octobre 1947, 58 RTNU 187 (entrée en vigueur: $1^{\mathrm{er}}$ janvier 1948) [GATT]. Disponible sur le site internet : https://www.wto.org/french/tratop_f/tbt_f/tbt_f.htm. Consulté le 04/06/2021.

\section{Documents fondamentaux d'organisation internationale non gouvernementale}

Statuts et Règlement de l'UICN disponible sur le site https://www.iucn.org. Consulté le 02$06 / 2021$.

\section{Textes du Bénin ○ Lois}

Loi n ${ }^{\circ}$ 93-009 du 02 juillet 1993 portant protection des forêts en République du Bénin. Sur le site internet : https://legis.cdij.bj/index.php/loi-n-93-009-du-2-juillet-1993-portant-r-gime-desfor-ts-en-r-publique-du-b-nin. Consulté le 02/06/2021.

Loi n94-009 du 28 juillet 1994 qui régit les offices à caractère social et culturel et scientifique en République du Bénin. Sur le site internet : https://legis.cdij.bj/index.php/loi-n-94-009-du28-juillet-1994-portant-cr-ation-organisation-et-fonctionnement-des-offices-caract-res-socialculturel-et-scientifique. Consulté le 02/06/2021.

Loi n98-030 du 12 février 1999 portant loi cadre sur l'environnement en République du Bénin. Sur le site internet: https://legis.cdij.bj/index.php/loi-n-98-030-portant-loi-cadre-surlenvironnement-en-r-publique-du-b-nin. Consulté le 02-06/2021.

Loi n ${ }^{\circ}$ 97-029 du 15 janvier 1999 portant organisation des communes en République du Bénin. Sur le site internet: https://legis.cdij.bj/index.php/loi-n-97-029-du-15-janvier-1999-portantorganisation-des-communes-en-r-publique-du-b-nin. Consulté le 02-06/2021.

Loi $\mathrm{n}^{\circ}$ 97-028 du 15 janvier 1999 portant organisation de l'administration territoriale de la République du Bénin. Sur le site internet: https://legis.cdij.bj/index.php/loi-n-97-028-15janvier-1999-portant-organisation-de-ladministration-territoriale-de-la-r-publique-du-b-nin.

Consulté le 02-06/2021.

Loi n93-013 du 10 août 1999 portant loi organique pour la Haute Cour de Justice. Sur le site internet: https://legis.cdij.bj/index.php/loi-n-93-013-du-10-ao-t-1999-portant-loi-organiquede-la-haute-cour-de-justice. Consulté le 02-06/2021. 
Loi N ${ }^{\circ} 2016-15$ modifiant et complétant la loi $N^{\circ} 2001-37$ du 27 août 2002 portant organisation judiciaire en République du Bénin. Sur le site internet : https://legis.cdij.bj/index.php/loi-n2016-15-modifiant-et-compl-tant-la-loi-n-2001-37-du-27-ao-t-2002-portant-organisationjudiciaire-en-r-publique-du-b-nin. Consulté le 02-06/2021.

Loi ${ }^{\circ} 2008-09$ du 30 décembre 2008 portant loi de finances pour la gestion 2009 en République du Bénin. Sur le site internet: https://legis.cdij.bj/index.php/loi-n-2008-09-portant-loi-definances-pour-la-gestion-2009. Consulté le 02-06/2021.

Ordonnance $\mathrm{n}^{\circ} 2010-01 \mathrm{du}$ 1er janvier 2010 portant loi des finances pour la gestion de $2010 \mathrm{en}$ République du Bénin. Sur le site internet: https://sgg.gouv.bj/doc/ordonnance-2010-01. Consulté le 02-06/2021.

Loi n ${ }^{\circ}$ 2013-05 du 15 février 2013 portant création, organisation, attributions et fonctionnement des unités administratives locales en République du Bénin. Sur le site internet: https://legis.cdij.bj/index.php/loi-n-2013-05-portant-cr-ation-organisation-attributions-etfonctionnement-des-unit-s-administratives-locales-en-r-publique-du-b-nin. Consulté le 02$06 / 2021$.

Loi $n^{\circ} 2016-24$ du 11 octobre 2016 portant cadre juridique du partenariat public-privé en République du Bénin. Sur le site internet : https://legis.cdij.bj/index.php/loi-n-2016-24-portantcadre-juridique-du-partenariat-public-priv-en-r-publique-du-b-nin. Consulté le 02-06/2021.

Loi ${ }^{\circ} 2018-18$ en date du 18 juin 2018 portant règlementation sur les changements climatiques au Bénin. Sur le site internet : https://sgg.gouv.bj/doc/loi-2018-18. Consulté le 02-06/2021.

\section{○ Décrets}

Décret N 91-176 du 29 juillet 1991portant composition Gouvernement, remplacé par le Décret $\mathrm{N}^{\circ}$ 91-204 du 05 Septembre 199. Sur le site internet : https://sgg.gouv.bj/doc/decret-1991-176. Consulté le 02-06/2021.

Décret $\mathrm{n}^{\circ} 95-47$ du 20 février 1995 portant organisation, attributions, et fonctionnement de l'Agence Béninoise pour l'Environnement. Sur le site internet : https://sgg.gouv.bj/doc/decret1995-47. Consulté le 02/06/2021.

Décret $n^{\circ}$ 99-641 du 30 décembre 1999 portant attributions organisation et fonctionnement de la Commission Nationale du Développement Durable. Sur le site internet: https://sgg.gouv.bj/doc/decret-1999-641. Consulté le 02/06/2021.

Décret n²001-095 du 20 février 2001 portant création, attributions, organisation et fonctionnement des cellules environnementales en République du Bénin. Sur le site internet : https://sgg.gouv.bj/doc/decret-2001-095. Consulté le 02/06/2021.

Décret n ${ }^{\circ}$ 2003-129 du 15 avril 2003 a créé le Comité National de Coordination de la mise en œuvre de la Convention de Stockholm sur les Polluants Organiques Persistants. Sur le site internet : https://sgg.gouv.bj/doc/decret-2003-129. Consulté le 02/06/2021.

Décret 2003-142 du 30 avril 2003 portant création, attributions et fonctionnement du Comité National sur les Changements Climatiques (CNCC). Sur le site internet : https://sgg.gouv.bj/doc/decret-2003-142. Consulté le 02/06/2021.

Décret $\mathrm{n}^{\circ}$ 2007-493 du 02 novembre 2007, portant attributions, organisation et fonctionnement du Ministère de l'Environnement et de la Protection de la Nature. Sur le site internet: https://sgg.gouv.bj/doc/decret-2007-493. Consulté le 02/06/2021. 
Décret $n^{\circ} 2008-727$ du 22 décembre 2008 portant création, composition, attributions et fonctionnement du Comité National de Lutte contre Désertification et de son Secrétariat Exécutif. Sur le site internet : https://sgg.gouv.bj/doc/decret-2008-727. Consulté le 02/06/2021.

Décret $n^{\circ} 2010-478$ du 05 novembre 2010, portant création, attributions, organisation et fonctionnement de l'Agence Béninoise pour l'Environnement. Sur le site internet: https://sgg.gouv.bj/doc/decret-2010-478. Consulté le 02/06/2021.

Décret n²011-834 du 30 décembre 2011 portant création, composition, attributions et fonctionnement de la plate-forme nationale de réduction des risques de catastrophe et d'adaptation au changement climatique en République du Bénin. Sur le site internet: https://sgg.gouv.bj/doc/decret-2011-834. Consulté le 02/06/2021.

Décret n²012-426 du 6 novembre 2012 portant création, attributions, organisation et fonctionnement de l'Agence Nationale de Protection Civile. Sur le site internet: https://sgg.gouv.bj/doc/decret-2012-426. Consulté le 02/06/2021.

Décret $n^{\circ}$ 2014-359 du 16 juin 2014 portant création de la Commission de Modélisation Economique des Impacts et de l'Intégration des Changements Climatiques dans le Budget Général de 1'Etat. Sur le site internet: https://sgg.gouv.bj/doc/decret-2014-359. Consulté le 02/06/2021.

Décret ${ }^{\circ} 2016-264$ du 06 avril 2016 portant composition du Gouvernement. Sur le site internet : https://sgg.gouv.bj/doc/decret-2016-264. Consulté le 02/06/2021.

Décret n²016-292 du 17 mai 2016 fixant la structure -type des ministères en République du Bénin. Sur le site internet : https://sgg.gouv.bj/doc/decret-2016-292. Consulté le 02/06/2021.

Décret $n^{\circ} 2016-501$ du 11 aout 2016 portant attributions, organisation et fonctionnement du Ministère du Cadre de Vie et du Développement Durable en République du Bénin. Sur le site internet : https://sgg.gouv.bj/doc/decret-2016-501. Consulté le 02/06/2021.

Décret $n^{\circ}$ 2017-128 du 27 février 2017 constatant approbation de la création du Fonds National pour l'Environnement et le Climat (FNEC). Sur le site internet : https://sgg.gouv.bj/doc/decret2017-128. Consulté le 02/06/2021.

Coutumier Dahoméen, institué par Circulaire 128 A.P. du 19 mars 1931 relative à la codification des coutumes indigènes par le Gouvernement général de l'Afrique occidentale française. Colonie du Dahomey.

\section{○ Document de stratégie}

Stratégie béninoise de mise en œuvre de la Convention Cadre des Nations Unies sur les Changements Climatiques. Site internet :https://unfccc.int/sites/default/files/resource/Benin\%. Page consultée le 02/06/2021.

Programme d'Action National d'Adaptation aux Changements Climatiques du Bénin (PANABENIN). Site le internet: https://www.changementsclimatiques.bj/actualites/programmedaction-national-dadaptation-aux-changements-climatiques-du-benin-pana-benin-2008.htm. Consulté de 02/06/2021.

Etudes Nationales de Perspectives à Long Terme « Bénin 2025». Disponible sur le site internet : http://dsa-flash.viabloga.com/files//BEN_2025_ALAFIA.pdf.

Programme d'Action du Gouvernement 2016-2021. Sur le site internet : https://www.gouv.bj. Consulté ce $02 / 06 / 2021$. 


\section{○ Décision de la Cour constitutionnelle}

Décision DCC 02-065 du 05 juin 2002. Cour constitutionnelle béninoise. Sur le site internet : https://courconstitutionnelle.bj/decisions-2. Consulté ce 29/05/2021.

Décision DCC 04-038 en date du 12 mars 2004. Sur le site internet: https://courconstitutionnelle.bj/decisions-2. Consulté ce 29/05/2021.

Décision DCC 16-113 du 28 juillet 2016. Sur le site internet : https://courconstitutionnelle.bj/decisions-2. Consulté ce 29/05/2021.

Décision DCC 18-006 du 25 Janvier 2018. Sur le site internet : https://courconstitutionnelle.bj/decisions-2. Consulté ce 29/05/2021. 



\section{CURRICULUM VITAE}

ETAT CIVIL

Nom : YEKINI

Prénom : Amidou

Date et lieu de Naissance: : 01/01/1969 à Avrankou (BENIN)

Situation Matrimoniale : Célibataire

Nationalité : Béninoise

Téléphone : (+229) 97479401

E-mail : yekiniamidou@yahoo.fr

SITUATION PROFESSIONNELLE

Profession : Greffier

Poste occupé : Chef Service de la Codification au ministère de la Justice

STATUT ACTUEL

Doctorant en droit à l'Université de Maastricht aux Pays-Bas et à l'Université d'Abomey Calavi au Bénin

FORMATION UNIVERSITAIRE

\begin{tabular}{|c|c|c|c|}
\hline $\begin{array}{c}\text { ANNEE } \\
\text { D'ETUDE }\end{array}$ & FILIERE & ETABLISSEMENT & DIPLOME OBTENU \\
\hline $2014-2015$ & $\begin{array}{l}\text { Droit International et } \\
\text { Comparé de } \\
\text { l'Environnement (DICE) }\end{array}$ & $\begin{array}{c}\text { Faculté de Droit et des } \\
\text { Sciences Economiques } \\
\text { de l'Université de } \\
\text { Limoges en France en } \\
\text { partenariat avec } \\
\text { l'Agence Universitaire } \\
\text { de la Francophonie }\end{array}$ & $\begin{array}{r}\text { Master } 2 \text { Droit International et } \\
\text { Comparé de l'Environnement } \\
\text { (DICE) }\end{array}$ \\
\hline $15 / 05 / 2006$ & Droit & $\begin{array}{l}\text { Faculté de Droit et des } \\
\text { Sciences Politiques à } \\
\text { 1'Université d'Abomey- } \\
\text { Calavi au Bénin }\end{array}$ & Maitrise es-sciences Juridiques \\
\hline $2003-2004$ & Droit & $\begin{array}{l}\text { Faculté de Droit et des } \\
\text { Sciences Politiques à } \\
\text { 1'Université d'Abomey- } \\
\text { Calavi au Bénin }\end{array}$ & $\begin{array}{r}\text { Licence en droit des affaires } \\
\text { carrières judiciaires }\end{array}$ \\
\hline $1999-2000$ & Droit & $\begin{array}{l}\text { Faculté de Droit et des } \\
\text { Sciences Politiques à } \\
\text { l'Université d'Abomey- } \\
\text { Calavi au Bénin }\end{array}$ & $\begin{array}{r}\text { Capacité en Droit et Sciences } \\
\text { Economiques }\end{array}$ \\
\hline \multicolumn{4}{|c|}{ FORMATION ENSEIGNEMENT SECONDAIRE ET PRIMAIRE } \\
\hline 1988 & Classe de $3^{\mathrm{e}}$ & $\begin{array}{l}\text { CEG DAVIE à Porto- } \\
\text { Novo au Bénin }\end{array}$ & $\begin{array}{r}\text { Brevet d'Etude du Premier } \\
\text { Cycle (BEPC) }\end{array}$ \\
\hline 1982 & Cours Moyen 2 (CM2) & $\begin{array}{c}\text { Ecole Primaire Publique } \\
\text { Avrankou au Bénin }\end{array}$ & $\begin{array}{r}\text { Contrôle des Etudes de Fin } \\
\text { d'Enseignement de Base } \\
\text { (CEFEB) }\end{array}$ \\
\hline
\end{tabular}




\begin{tabular}{|c|c|c|c|}
\hline \multicolumn{4}{|c|}{ FORMATION PROFESSIONNELLE } \\
\hline 2008-2009 & $\begin{array}{c}\text { La filière Greffe de l'option } \\
\text { Magistrature et Carrières } \\
\text { Judiciaires }\end{array}$ & $\begin{array}{c}\text { Ecole Nationale } \\
\text { d'Administration et de } \\
\text { la Magistrature de } \\
\text { 1'Université d'Abomey- } \\
\text { Calavi au Bénin }\end{array}$ & $\begin{array}{r}\text { Certificat d'Aptitude à la } \\
\text { Profession de Greffier(CAPG) }\end{array}$ \\
\hline \multicolumn{4}{|c|}{ STAGE PROFESSIONNEL } \\
\hline $\begin{array}{ll}12 / 03 & \text { au } \\
19 / 03 / 2012 & \\
\text { et } & \\
20 & \text { au } \\
26 / 02 / 2018 & \end{array}$ & $\begin{array}{c}\text { Formation sur "' } \\
\text { International Standard } \\
\text { Minimum Rules for } \\
\text { Treatment of Prisoners } \\
\text { (Règles Nelson Mandela), }\end{array}$ & $\begin{array}{l}\text { Centre de détention des } \\
\text { Nations Unies du } \\
\text { Tribunal Pénal } \\
\text { International pour le } \\
\text { Rwanda (TPIR), sis à } \\
\text { Arusha en Tanzanie }\end{array}$ & Attestation de fin de stage \\
\hline \multicolumn{4}{|c|}{ COMPETENCE LINGUISTIQUE } \\
\hline Langue écrite et $p$ & lée & & Français \\
\hline
\end{tabular}

Je certifie exacts tous les renseignements ci-dessus mentionnés

Fait à Porto-Novo, le 06 février 2022

YEKINI Amidou 


\section{TABLE DES MATIERES}




\section{TABLE DES MATIERES}

\section{AVERTISSEMENT}

DEDICACES

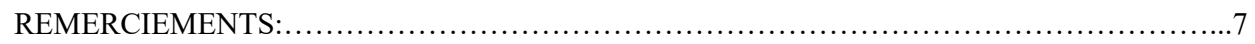

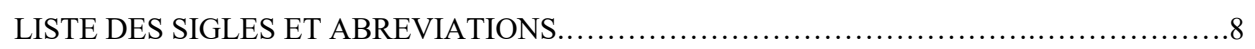

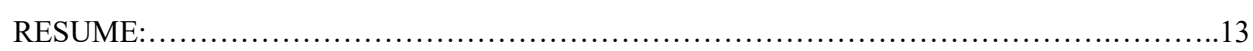

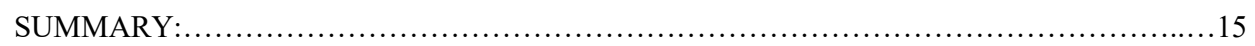

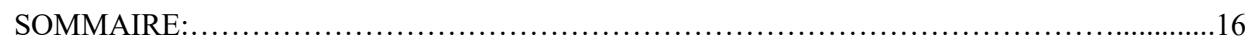

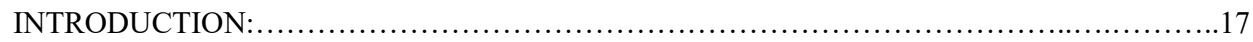

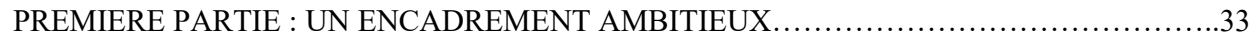

TITRE I: LES REGLES DE PROTECTION DU CLIMAT .....................................

CHAPITRE I: L'APPORT DU DROIT INTERNATIONAL .................................41

SECTION I: L'EUVRE D'ACTEURS DU DROIT ...............................................41

Paragraphe 1 : Une construction progressive..............................................42

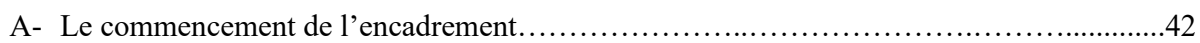

B- L'amélioration des normes de protection..................................................... 47

Paragraphe 2: L'élaboration du régime international du climat...............................53

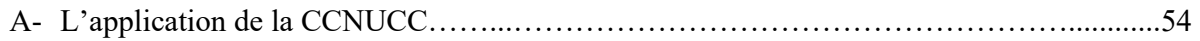

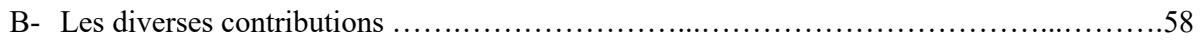

SECTION II: LA CONTRIBUTION D'ACTEURS EXTERIEURS.............................64

Paragraphe 1: L'influence sur l'élaboration du droit............................................65

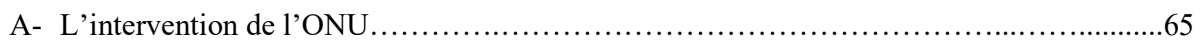

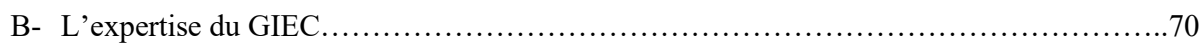

Paragraphe $2:$ La participation à la mise en œuvre du droit......................................... 74

A- Les acteurs non étatiques......................................................... 74

B- L'expérience de l'UICN....................................................... 78

CHAPITRE II: LES MESURES NORMATIVES INTERNES ............................... 83

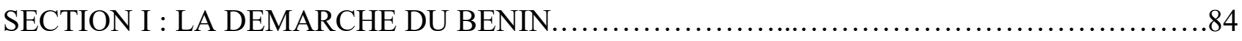

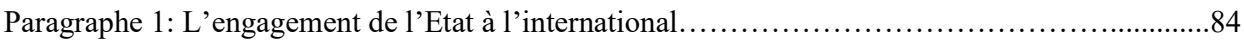

A- L'internalisation d'instruments déclaratoires....................................... 85

B- La ratification d'accords internationaux......................................... 89

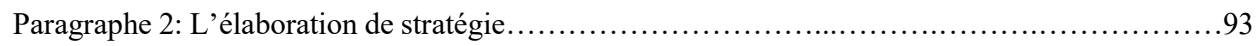




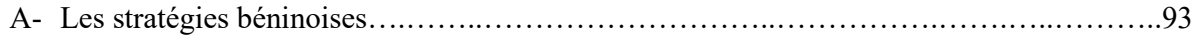

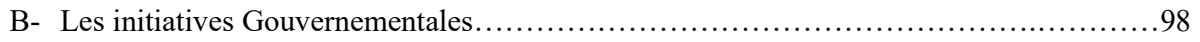

SECTION II : UN ENCADREMENT JURIDIQUE PERTINENT, MAIS INADEQUAT...........103

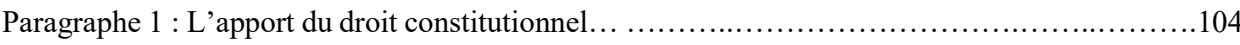

A- La protection constitutionnelle de l'environnement...................................104

B- L'environnement, objet de droit de l'homme......................................... 108

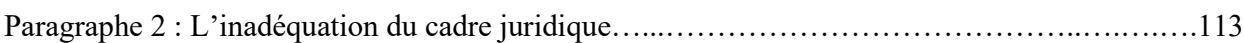

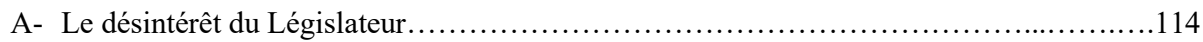

B- L'accroissement d'une législation aux objectifs généraux.............................. 117

TITRE II: LES INSTITUTIONS DE PROTECTION DU CLIMAT................................ 123

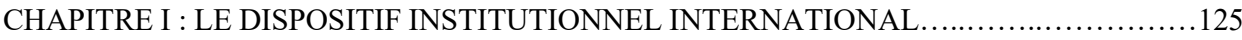

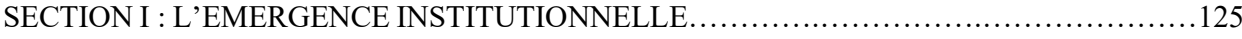

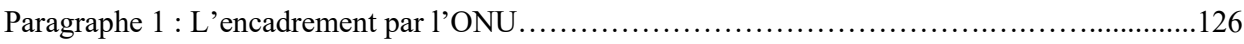

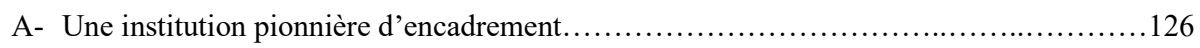

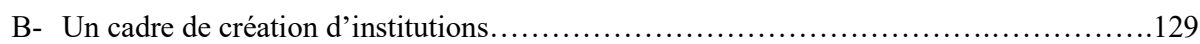

Paragraphe 2 : L'existence d'un cadre institutionnel climatique.................................133

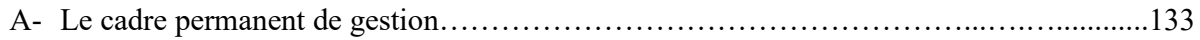

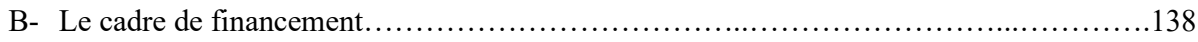

SECTION II : L'ENVIRONNEMENTALISATION D'INSTITUTIONS ........................ 141

Paragraphe 1 : L'intervention d'organisations internationales.................................. 142

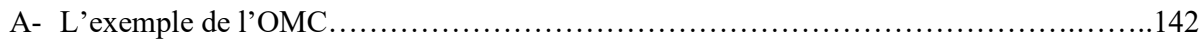

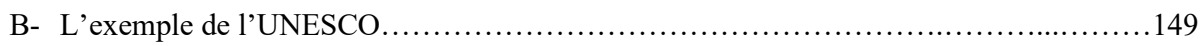

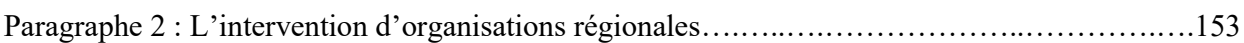

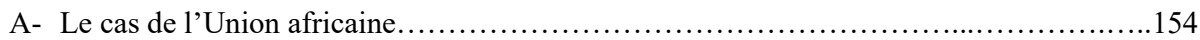

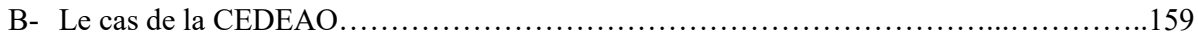

CHAPITRE II : LE DISPOSITIF INSTITUTIONNEL INTERNE.............................

SECTION I: UN CADRE INSTITUTIONNEL PERTINENT .............................. 166

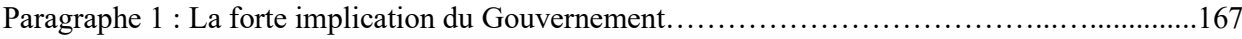

A- L'intervention du ministère de l'Environnement ...................................... 168

B- L'accompagnement d'organes techniques.......................................... 172

1-L'Agence Béninoise pour l'Environnement................................... 173

2-La Commission Nationale du Développement Durable................................175

Paragraphe 2 : La création d'un réseau institutionnel de climat...................................176

A- Un organe relevant du ministère de l'Environnement.................................177

B- Un organe sous tutelle du ministère du Plan............................................ 180

SECTION II : UN ENCADREMENT INSTITUTIONNEL ADEQUAT $\ldots \ldots \ldots \ldots \ldots \ldots \ldots \ldots \ldots \ldots$ 
Paragraphe 1 : Un système de financement orienté........................................... 184

A- La création du Fonds National pour l'Environnement et du Climat........................185

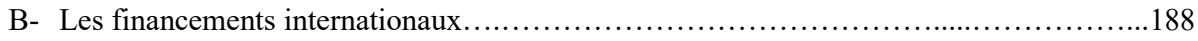

Paragraphe 2 : L'environnementalisation de l'administration territoriale........................191

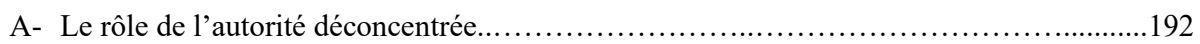

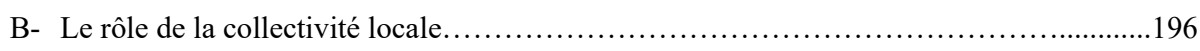

DEUXIEME PARTIE : UNE CONTRIBUTION MODESTE......................................205

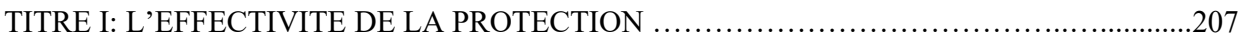

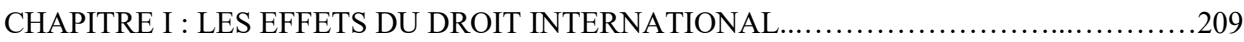

SECTION I: L'INFLUENCE SUR L'ENVIRONNEMENT JURIDIQUE BENINOIS...........211

Paragraphe 1: Le bouleversement de l'ordonnancement juridique............................212

A- L'existence d'un encadrement juridique modernisé....................................212

B- L'existence d'un cadre juridique climatique..........................................218

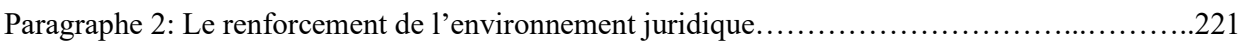

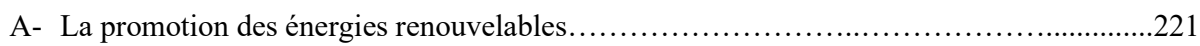

B- Les obligations d'intégration des changements climatiques.............................226

SECTION II: LA MODIFICATION DE L'ENCADREMENT INSTITUTIONNEL ...............229

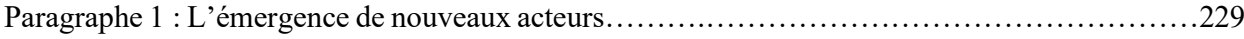

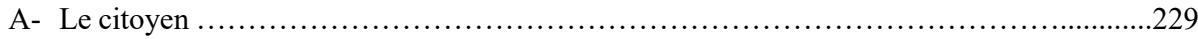

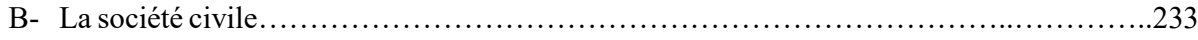

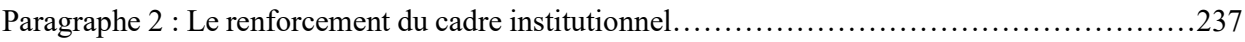

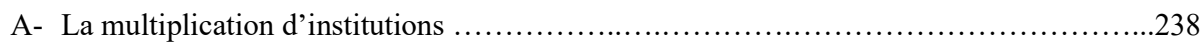

B- La consolidation d'institutions................................................... 240

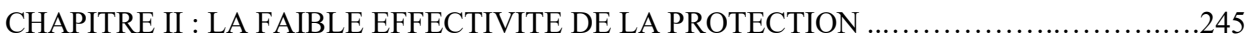

SECTION I: LE CONTROLE JURIDICTIONNEL INTERNATIONAL IMPARFAIT.............246

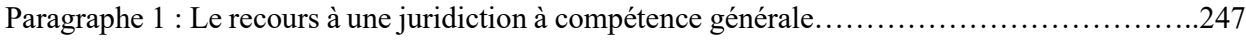

A- La compétence climatique de la Cour Internationale de Justice..........................2248

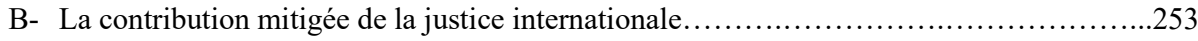

Paragraphe $2:$ Le mode alternatif de règlement des différends..............................257

A- Le contrôle du respect des engagements..........................................257

B- Le règlement pacifique des différends.......................................263

SECTION II: LA PARTICIPATION MITIGEE DE LA JUSTICE BENINOISE...................266

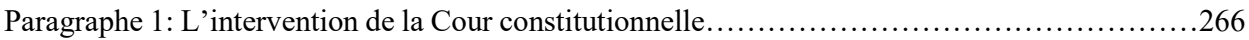

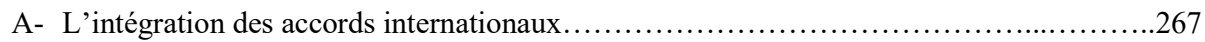

B- Le contrôle de constitutionnalité des actes. 
Paragraphe 2 : L'intervention des juridictions .276

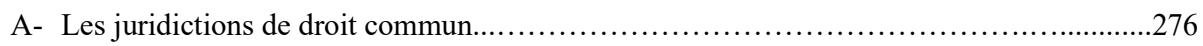

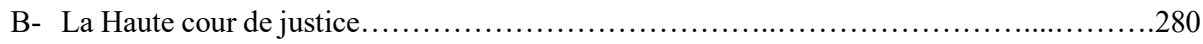

TITRE II : LES PERSPECTIVES DU DROIT CLIMATIQUE AU BENIN ......................287

CHAPITRE I: L'AMELIORATION DE L'ENCADREMENT INTERNATIONAL ...............289

SECTION I: LE RENFORCEMENT DU CADRE INSTITUTIONNEL ............................289

Paragraphe 1: Les innovations dans l'Accord de Paris......................................................................290

A- La création d'un cadre technologique..............................................291

B- L'innovation d'un cadre de transparence....................................................294

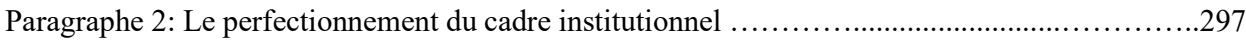

A- La création d'une organisation internationale environnementale .........................298

B- La création d'une juridiction internationale spécialisée................................304

SECTION II : LA STRUCTURATION DE LA COOPERATION INTERNATIONALE...............308

Paragraphe $1:$ Le rapprochement du droit climatique au plan régional.............................309

A- L'ancrage de l'Union africaine dans le droit climatique...................................... 310

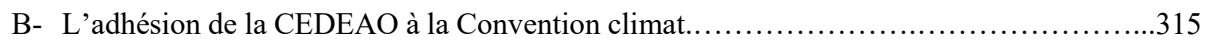

Paragraphe 2 : La communautarisation du droit climatique.......................................

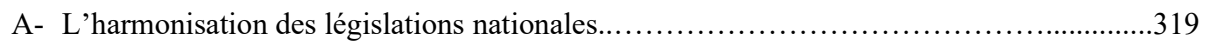

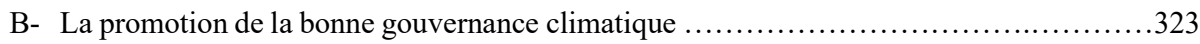

CHAPITREII:LERENFORCEMENTDELAGOUVERNANCECLIMATIQUEINTERNE. .......................

SECTION I : LA PROMOTION DES MESURES DE PARTICIPATION INCLUSIVE............330

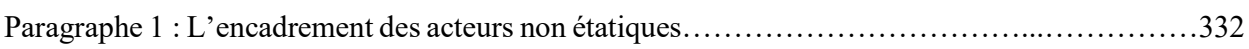

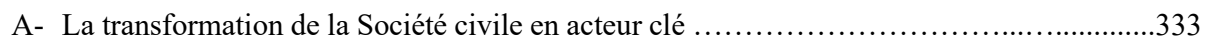

B- Le renforcement du cadre de collaboration du secteur public et privé.......................338

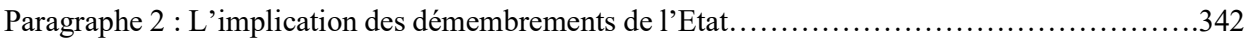

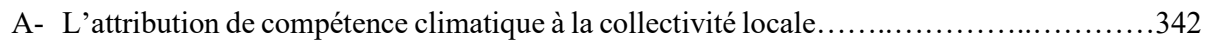

B- L'attribution de compétence climatique à l'autorité déconcentrée .........................346

SECTION II : L'AMELIORATION DE L'ENCADREMENT CLIMATIQUE...................349

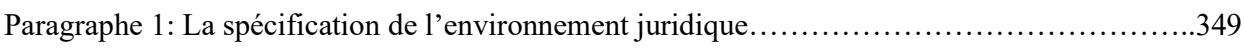

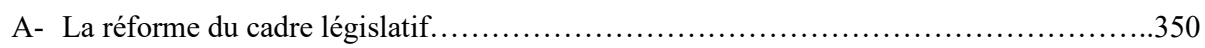

B- La réforme de la constitution béninoise........................................ 354

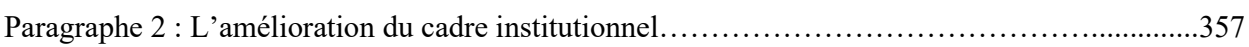

A- La création de l'Autorité Nationale des Changements Climatiques.....................................358

B- La restructuration du Comité National des Changements Climatiques .....................360

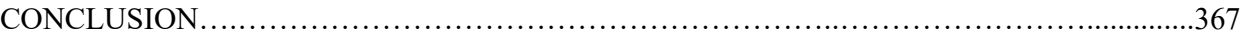

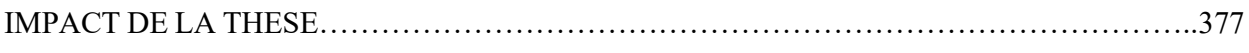




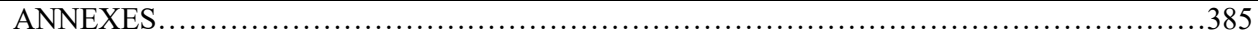

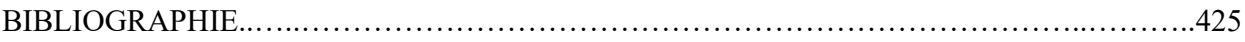

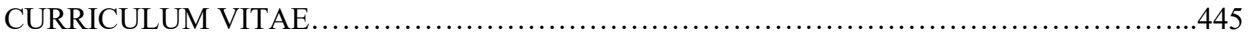

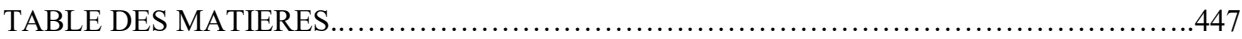





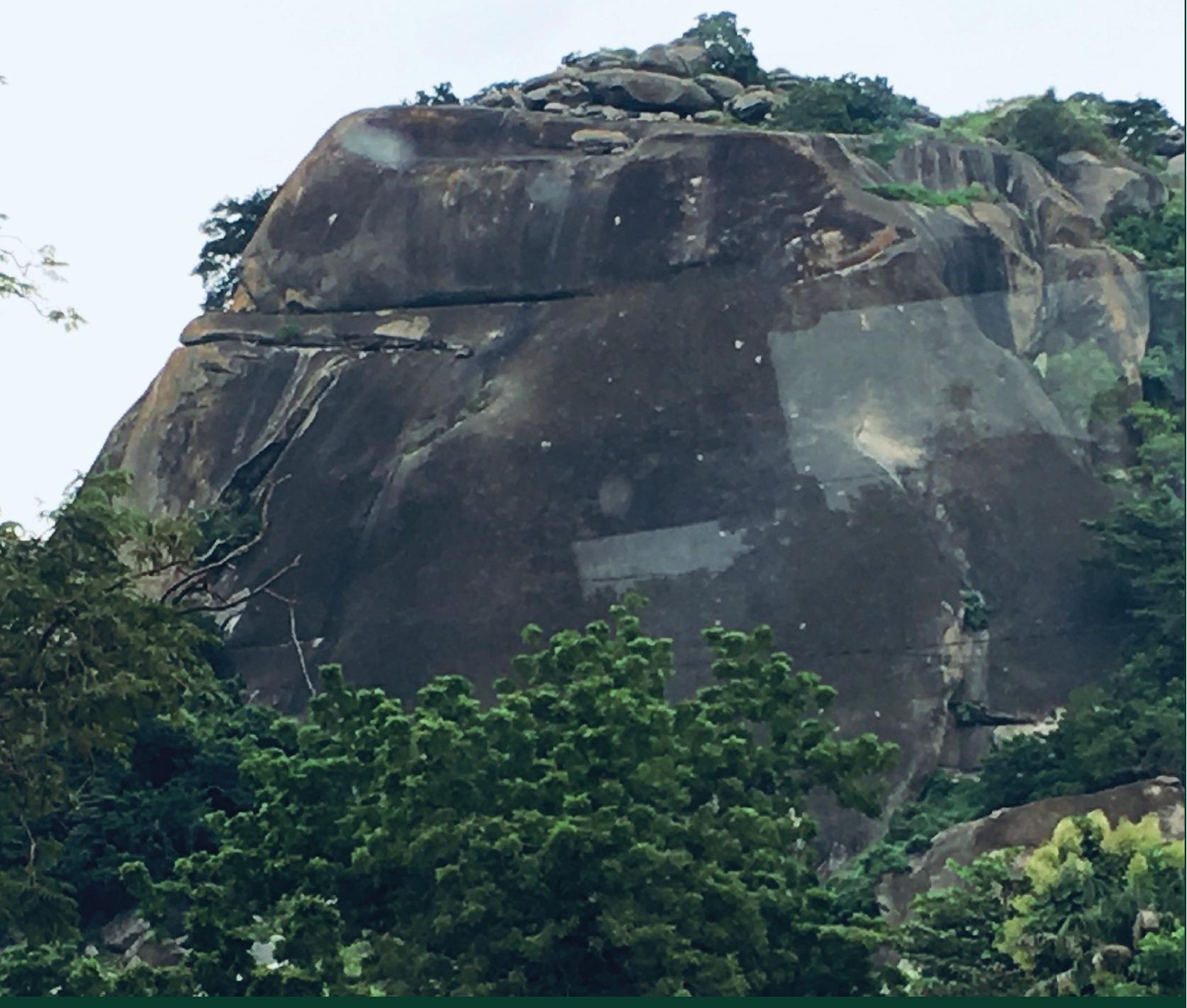

